\title{
Dirac's Observables for the Rest-Frame Instant Form of Tetrad Gravity in a Completely Fixed 3-Orthogonal Gauge.
}

\author{
Roberto De Pietri \\ Dipartimento di Fisica, Campus Universitario \\ Universita' di Parma \\ Viale delle Scienze \\ 43100 Parma, Italy \\ E-mail: DEPIETRI@PR.INFN.IT \\ and \\ Luca Lusanna \\ Sezione INFN di Firenze \\ L.go E.Fermi 2 (Arcetri) \\ 50125 Firenze, Italy \\ E-mail LUSANNA@FI.INFN.IT \\ and \\ Luca Martucci \\ Dipartimento di Fisica \\ Universita' di Milano I \\ via G.Celoria 16 \\ 20133 Milano \\ E-mail MARTUCCI@MI.INFN.IT \\ and \\ Stefano Russo \\ Condominio dei Pioppi 16 \\ 6916 Grancia (Lugano) \\ Svizzera
}

Abstract 
We define the rest-frame instant form of tetrad gravity restricted to Christodoulou-Klainermann spacetimes. After a study of the Hamiltonian group of gauge transformations generated by the 14 first class constraints of the theory, we define and solve the multitemporal equations associated with the rotation and space diffeomorphism constraints, finding how the cotriads and their momenta depend on the corresponding gauge variables. This allows to find a quasi-Shanmugadhasan canonical transformation to the class of 3-orthogonal gauges and to find the Dirac observables for superspace in these gauges. The construction of the explicit form of the transformation and of the solution of the rotation and supermomentum constraints is reduced to solve a system of elliptic linear and quasi-linear partial differential equations. We then show that the superhamiltonian constraint becomes the Lichnerowicz equation for the conformal factor of the 3-metric and that the last gauge variable is the momentum conjugated to the conformal factor. The gauge transformations generated by the superhamiltonian constraint perform the transitions among the allowed foliations of spacetime, so that the theory is independent from its $3+1$ splittings. In the special 3-orthogonal gauge defined by the vanishing of the conformal factor momentum we determine the final Dirac observables for the gravitational field even if we are not able to solve the Lichnerowicz equation. The final Hamiltonian is the weak ADM energy restricted to this completely fixed gauge.

October 25, 2018

Typeset using REVTEX 


\section{INTRODUCTION}

In a previous paper [1] a new parametrization of arbitrary cotetrads has been introduced to achieve a simplification of the 14 first class constraints of tetrad gravity starting from the ADM action for metric gravity [5] with the 4-metric expressed in terms of cotetrads.

As said in Ref. [1], this has been done in the special class of non-compact spacetimes $M^{4}$, which:

i) are globally hyperbolic 4-manifolds f $M^{4} \approx R \times \Sigma$, so that a Hamiltonian formulation is possible;

ii) are asymptotically flat at spatial infinity, so that Poincaré charges may be defined [6];

iii) admit a spin structure, i.e. are parallelizable and have a trivial orthonormal frame bundle $F\left(M^{4}\right)=M^{4} \times S O(3,1)$ and coframe bundle $L\left(M^{4}\right)=M^{4} \times S O(3,1)$, so that tetrads and cotetrads are globally defined;

iv) have the Cauchy surfaces $\Sigma_{\tau}$ that are topologically trivial, geodesically complete Riemannian 3-manifolds $\left(\Sigma_{\tau},{ }^{3} g\right)$ and diffeomorphid to $R^{3}, \Sigma_{\tau} \approx R^{3}$;

$\mathrm{v})$ have the non-compact Riemannian 3-manifolds $\left(\Sigma_{\tau},{ }^{3} \mathrm{~g}\right)$ not admitting isometries .

This new formulation of tetrad gravity has been introduced with the aim to study the Hamiltonian group of gauge transformations and to perform the canonical reduction of the theory to a completely fixed gauge with the identification of the physical degrees of freedom (Dirac observables [7] 9]) of the gravitational field. This would conclude the research program aiming to express the four interactions only in terms of canonical cases of Dirac's observables". The program is based on the Shanmugadhasan canonical transformations [11]:

\footnotetext{
${ }^{1}$ See the papers 造团, quoted as I, II, III in what follows, for a preliminary presentation of many results.

${ }^{2} \Sigma$ is an abstract model of spacelike Cauchy surface. These spacetimes admit regular foliations with orientable, complete, non-intersecting spacelike 3-manifolds: the leaves of the foliation are the embeddings $i_{\tau}: \Sigma \rightarrow \Sigma_{\tau} \subset M^{4}, \vec{\sigma} \mapsto z^{\mu}(\tau, \vec{\sigma})$, where $\vec{\sigma}=\left\{\sigma^{r}\right\}, \mathrm{r}=1,2,3$, are local coordinates in a chart of the $C^{\infty}$-atlas of the abstract 3-manifold $\Sigma$ and $\tau: M^{4} \rightarrow R, z^{\mu} \mapsto \tau\left(z^{\mu}\right)$, is a global timelike future-oriented function labelling the leaves (surfaces of simultaneity). In this way, one obtains $3+1$ splittings of $M^{4}$ and the possibility of a Hamiltonian formulation.

${ }^{3}$ The parallelizable spacelike hypersurfaces $\Sigma_{\tau}$ of simultaneity have trivial orthonormal frame bundle $F \Sigma_{\tau}=\Sigma_{\tau} \times S O(3)$ and coframe bundle $L \Sigma_{\tau}=\Sigma_{\tau} \times S O(3)$, so that triads and cotriads are globally defined.

${ }^{4}$ Therefore the 3-manifolds $\Sigma_{\tau}$ admit global coordinate systems.

${ }^{5}$ This requires that triads, cotriads and 3-spin connections belong to suited weighted Sobolev spaces to avoid Gribov ambiguities.

${ }^{6}$ See Ref. [10] for such a canonical reduction of the electromagnetic, weak and strong interactions in Minkowski spacetime.
} 
if a system has first class constraints at the Hamiltonian level凹, then, at least locally, one can find a canonical basis with as many new momenta as first class constraints (Abelianization of first class constraints), with their conjugate canonical variables as Abelianized gauge variables and with the remaining pairs of canonical variables as pairs of canonically conjugate Dirac's observables of. Putting equal to zero the Abelianized gauge variables defines a local gauge of the model. If a system with constraints admits one (or more) global Shanmugadhasan canonical transformations, one obtains one (or more) privileged global gauges in which the physical Dirac observables are globally defined and globally separated from the gauge degrees of freedom 9 . These privileged gauges (when they exist) can be called generalized Coulomb or radiation gauges. Second class constraints [12], when present, are also taken into account by the Shanmugadhasan canonical transformation [11].

In flat spacetime the problem of how to covariantize this kind of canonical reduction is solved by using Dirac reformulation (see the book in Ref. [7]) of classical field theory on spacelike hypersurfaces foliating 10 Minkowski spacetime $M^{4}$. In this way one gets parametrized Minkowski field theory with a covariant 3+1 splitting of flat spacetime and already in a form suited to the transition to general relativity in its ADM canonical formulation $\square$. The price is that one has to add as new configuration variables the embeddings $z^{(\mu)}(\tau, \vec{\sigma})$ of the spacelike hypersurface $\Sigma_{\tau}\left[2\right.$ and then define the fields on $\Sigma_{\tau}$ so that they know the hypersurface $\Sigma_{\tau}$ of $\tau$-simultaneity [13. Then one rewrites the Lagrangian of the given isolated system in the form required by the coupling to an external gravitational field, makes the previous $3+1$ splitting of Minkowski spacetime and interpretes all the fields of the system as the new fields on $\Sigma_{\tau}$ (they are Lorentz scalars, having only surface indices). Instead of considering the 4-metric as describing a gravitational field [4], here one replaces the 4-metric with the the induced

\footnotetext{
${ }^{7}$ So that its dynamics is restricted to a presymplectic submanifold of phase space.

${ }^{8}$ Canonical basis of physical variables adapted to the chosen Abelianization; they give a trivialization of the BRST construction of observables.

${ }^{9}$ For systems with a compact configuration space this is in general impossible.

${ }^{10}$ The foliation is defined by an embedding $R \times \Sigma \rightarrow M^{4},(\tau, \vec{\sigma}) \mapsto z^{(\mu)}(\tau, \vec{\sigma})[(\mu)$ are flat Minkowski indices], with $\Sigma$ an abstract 3-surface diffeomorphic to $R^{3}$ : this is the classical basis of TomonagaSchwinger quantum field theory.

${ }^{11}$ See also Ref. [13], where a theoretical study of this problem is done in curved spacetimes.

${ }^{12}$ The only ones carrying Lorentz indices; the scalar parameter $\tau$ labels the leaves of the foliation and $\vec{\sigma}$ are curvilinear coordinates on $\Sigma_{\tau}$.

${ }^{13}$ For a Klein-Gordon field $\phi(x)$, this new field is $\tilde{\phi}(\tau, \vec{\sigma})=\phi(z(\tau, \vec{\sigma}))$ : it contains the non-local information about the embedding.

${ }^{14}$ Therefore as an independent field as it is done in metric gravity, where one adds the Hilbert action to the action for the matter fields.
} 
metric $g_{A B}[z]=z_{A}^{(\mu)} \eta_{(\mu)(\nu)} z_{B}^{(\nu)}$ on $\Sigma_{\tau}$ 四 and considers the embedding coordinates $z^{(\mu)}(\tau, \vec{\sigma})$ as independent fields $\square$. From this Lagrangian, besides a Lorentz-scalar form of the constraints of the given system, we get four extra primary first class constraints $\mathcal{H}_{\mu}(\tau, \vec{\sigma}) \approx 0$ implying the independence of the description from the choice of the foliation with spacelike hypersufaces. Therefore the embedding variables $z^{(\mu)}(\tau, \vec{\sigma})$ are the gauge variables associated with this kind of general covariance. If we interpret the unit normal $l^{\mu}(\tau, \vec{\sigma})$ at $\Sigma_{\tau}$ as the unit 4 -velocity of a timelike observer at $(\tau, \vec{\sigma})$, the foliation with leaves $\Sigma_{\tau}$ identifies a congruence of timelike surface-forming accelerated observers. In special relativity, it is convenient to restrict ourselves to arbitrary spacelike hyperplanes $z^{(\mu)}(\tau, \vec{\sigma})=x_{s}^{(\mu)}(\tau)+b_{r}^{(\mu)}(\tau) \sigma^{r}$, which are associated with a congruence of timelike inertial observers. Since the hyperplanes are described by only 10 variables ॠ, we remain only with ten first class constraints determining the 10 variables conjugate to the hyperplane 18 in terms of the variables of the system.

If we now consider only the set of configurations of the isolated system with timelike [9] 4-momenta, we can restrict the description to the so-called Wigner hyperplanes orthogonal to $p_{s}^{(\mu)}$ itself. To get this result, we must boost at rest all the variables with Lorentz indices by using the standard Wigner boost $L_{(\nu)}^{(\mu)}\left(p_{s}, \stackrel{\circ}{p}_{s}\right)$ for timelike Poincaré orbits, and then add the gauge-fixings $b_{r}^{(\mu)}(\tau)-L_{r}^{(\mu)}{ }_{r}\left(p_{s}, \stackrel{\circ}{p}_{s}\right) \approx 0$. Since these gauge-fixings depend on $p_{s}^{(\mu)}$, the final canonical variables, apart $p_{s}^{(\mu)}$ itself, are of 3 types: i) there is a non-covariant canonical external center-of-mass variable $\tilde{x}_{s}^{(\mu)}(\tau)$; ii) all the 3 -vector variables become Wigner spin

${ }^{15} \mathrm{~A}$ functional of $z^{(\mu)}$; here we use the notation $\sigma^{A}=\left(\tau, \sigma^{r}\right) ;(\mu)$ is a flat Minkowski index; $z_{A}^{(\mu)}=\partial z^{(\mu)} / \partial \sigma^{A}$ are flat cotetrad fields on Minkowski spacetime with the $z_{r}^{(\mu)}$, s tangent to $\Sigma_{\tau}$.

${ }^{16}$ This is not possible in metric gravity, because in curved spacetimes, given the embeddings $z^{\mu}(\tau, \vec{\sigma}), \Sigma \rightarrow \Sigma_{\tau}$, the $z_{A}^{\mu} \neq \partial z^{\mu} / \partial \sigma^{A}$ 's are not cotetrad fields. In tetrad gravity, given a resolution of the 4-metric in non-holonomic cotetrads (the configurational degrees of freedom of tetrad gravity), ${ }^{4} g_{\mu \nu}={ }^{4} E_{\mu}^{(\alpha)}{ }^{4} \eta_{(\alpha)(\beta)}{ }^{4} E_{\nu}^{(\beta)}$, and the $\Sigma_{\tau^{-}}$adapted 4-metric ${ }^{4} g_{A B}(\tau, \vec{\sigma})=$ $z_{A}^{\mu}(\tau, \vec{\sigma})^{4} g_{\mu \nu}(z(\tau, \vec{\sigma})) z_{B}^{\nu}(\tau, \vec{\sigma})$, the $\Sigma_{\tau}$-adapted cotetrads replacing the $z_{A}^{(\mu)}$ 's of the flat case are ${ }^{4} F_{A}^{(\alpha)}(\tau, \vec{\sigma})=z_{A}^{\mu}(\tau, \vec{\sigma}){ }^{4} E_{\mu}^{(\alpha)}(z(\tau, \vec{\sigma}))$ : they depend simultaneously on the embedding and on the non-holonomic cotetrads and can be found only a posteriori.

${ }^{17}$ An origin $x_{s}^{(\mu)}(\tau)$ and, on it, three orthogonal spacelike unit vectors $b_{r}^{(\mu)}(\tau)$ generating the fixed constant timelike unit normal $l^{(\mu)}=b_{\tau}^{(\mu)}=\epsilon^{(\mu)}(\nu)(\rho)(\sigma) b_{\check{1}}^{(\nu)}(\tau) b_{\check{2}}^{(\rho)}(\tau) b_{\check{3}}^{(\sigma)}(\tau)$ to the hyperplane.

${ }^{18}$ They are a 4-momentum $p_{s}^{(\mu)}$ and the six independent degrees of freedom hidden in a spin tensor $S_{s}^{(\mu)(\nu)}$.

${ }^{19} \epsilon p_{s}^{2}>0 ; \epsilon= \pm 1$ according to the chosen convention for the Lorentz signature of the metric $\eta^{(\mu)(\nu)}=\epsilon(+---)$.

${ }^{20}$ It is only covariant under the little group of timelike Poincaré orbits like the Newton-Wigner position operator. 
13 -vectors 2 ; iii) all the other variables are Lorentz scalars. Only four 1st class constraints are left: one of them identifies the invariant mass of the isolated system, to be used as Hamiltonian, while the other three are the rest-frame conditions implying the vanishing of the internal (i.e. inside the Wigner hyperplane) total 3-momentum.

We obtain in this way a new kind of instant form of the dynamics (see Ref. [14]), the Wigner-covariant 1-time rest-frame instant form [15,10,16] with a universal breaking of Lorentz covariance independent from the isolated system under investigation. It is the special relativistic generalization of the non-relativistic separation of the center of mass from the relative motions $\left[H=\frac{\vec{P}^{2}}{2 M}+H_{\text {rel }}\right]$.

As shown in Refs. [15, 17], the rest-frame instant form of dynamics automatically gives a physical ultraviolet cutoff in the spirit of Dirac and Yukawa for all the rotating configurations of an isolated system: it is the Møller radius [18] $\rho=\sqrt{-\epsilon W^{2}} / \epsilon P^{2}=|\vec{S}| / \sqrt{\epsilon P^{2}}$ [2], namely the classical intrinsic radius of the worldtube, around the covariant non-canonical FokkerPryce 4-center of inertia $Y_{s}^{(\mu)}$, inside which the non-covariance of the canonical 4-center of mass $\tilde{x}_{s}^{(\mu)}$ is concentrated. At the quantum level $\rho$ becomes the Compton wavelength of the isolated system multiplied its spin eigenvalue $\sqrt{s(s+1)}, \rho \mapsto \hat{\rho}=\sqrt{s(s+1)} \hbar / M=$ $\sqrt{s(s+1)} \lambda_{M}$ with $M=\sqrt{\epsilon P^{2}}$ the invariant mass and $\lambda_{M}=\hbar / M$ its Compton wavelength. Therefore, the criticism to classical relativistic physics, based on quantum pair production, concerns the testing of distances where, due to the Lorentz signature of spacetime, one has intrinsic classical covariance problems: it is impossible to localize the canonical 4-center of mass $\tilde{x}_{s}^{(\mu)}$ of the system in a frame independent way. Let us remember 15 that $\rho$ is also a remnant in flat Minkowski spacetime of the energy conditions of general relativity: since the Møller non-canonical, non-covariant 4-center of energy $R_{s}^{(\mu)}$ has its non-covariance localized inside the same worldtube with radius $\rho$ (it was discovered in this way) [18], it turns out that for an extended relativistic system with the material radius smaller than its intrinsic radius $\rho$ one has: i) its peripheral rotation velocity can exceed the velocity of light; ii) its classical energy density cannot be positive definite everywhere in every frame.

Now, the real relevant point is that this ultraviolet cutoff determined by $\rho$ exists also in Einstein's general relativity (which is not power counting renormalizable) in the case of asymptotically flat spacetimes, taking into account the Poincaré Casimirs of its asymptotic ADM Poincaré charges 20 at spatial infinity.

Therefore in Ref. [6], after a review of ADM metric gravity, of spacetimes asymptotically flat at spatial infinity, of supertranslations and of ADM strong and weak asymptotic Poincaré charges, the definition of the rest-frame instant form of metric gravity was given. This is possible only when the requirement of absence of supertranslations, needed for the

${ }^{21}$ Boosts in $M^{4}$ induce Wigner rotations on them.

${ }^{22} W^{2}=-\epsilon P^{2} \vec{S}^{2}$ is the Pauli-Lubanski Casimir when $\epsilon P^{2}>0$.

${ }^{23}$ When supertranslations are eliminated with suitable boundary conditions; let us remark that Einstein and Wheeler use closed universes because they don't want to introduce (non Machian) boundary conditions, but in this way they loose Poincaré charges and the possibility to make contact with particle physics and to define spin. 
existence of a well defined and unique asymptotic Poincaré group $\mathcal{P}_{(\infty)}$, is imposed. In this way the allowed foliations of the spacetime $M^{4}$ have the leaves $\Sigma_{\tau}$ approaching Minkowski hyperplanes at spatial infinity in an angle-independent way, the allowed atlas of coordinates of $M^{4}$ must be compatible with asymptotic Minkowski Cartesian coordinates and the allowed diffeomorphisms of $M^{4}$ in Diff $M^{4}$ are restricted to tend to $\operatorname{Diff}_{I} M^{4} \times \mathcal{P}_{(\infty)}$ at spatial infinity in an angle-independent way ${ }^{2}$. The class of Christodoulou-Klainermann spacetimes [19] is selected: in them the strong ADM 3-momentum vanishes identically so that the vanishing of the weak ADM 3-momentum is equivalent to three first class constraints defining the rest frame of the universe. Therefore, the rest-frame instant form of metric gravity may be consistently defined. The Wigner hyperplanes of Minkowski spacetime are replaced by the so-called Wigner-Sen-Witten hypersurfaces, which asymptotically tend in a directionindependent way to Minkowski hyperplanes orthogonal to the weak ADM 4-momentum. In presence of matter the Wigner-Sen-Witten hypersurfaces tend to the Wigner hyperplanes for the same matter in Minkowski spacetime when the Newton constant vanishes: in this way we get the deparametrization of general relativity.

It will be shown in this paper that in a similar way we can define the rest-frame instant form of tetrad gravity restricted to Christodoulou-Klainermann spacetimes.

Then we can study the component connected with the identity of the Hamiltonian group of gauge transformations, whose generators are the 14 first class constraints of tetrad gravity given in Ref. [1], following the scheme developed for Yang-Mills theory [17]. Since seven constraints are already Abelianized, this study is concentrated on rotations, space diffeomorphisms and gauge transformations generated by the superhamiltonian constraint. The main problem in general relativity is the lack of control on the group manifold of diffeomorphisms groups. Also the interpretation of the gauge transformations generated by the superhamiltonian constraint is given following Ref. [6]: they change the foliation, so that the theory is independent from the $3+1$ splittings of spacetime like it happens in parametrized Minkowski theories.

The next step is the study of the gauge transformations generated by the rotation and space diffeomorphism constraints. By solving the associated multitemporal equations 10,17 we can find how the cotriads and their momenta depend upon the gauge angles and the gauge parameters of pseudo-diffeomorphisms (passive diffeomorphisms), which are the Abelianized gauge variables associated with these six constraints. This allows to Abelianize these six constraints and to find the quasi-Shanmugadhasan canonical transformation (it is a point canonical transformation) corresponding to the class of 3-orthogonal gauges: only the superhamiltonian constraint has not been Abelianized at this stage. We are able to find a canonical basis of Dirac observables with respect to these six constraints and to write a system of elliptic linear and quasi-linear partial differential equations, whose solution would give the expression of the cotriad momenta in terms of the gauge variables and of the Dirac observables in these gauges. To solve these equations is equivalent to the solution of the supermomentum constraints of metric gravity, namely to find York's gravitomagnetic potential and how the extrinsic curvature of the 3-surfaces depends on it in the 3-orthogonal gauges. We write the equations for the determination of the shift functions in the 3-orthogonal

${ }^{24}{ }^{2}$ if $f_{I} M^{4}$ are the diffeomorphisms which reduce to the identity at spatial infinity. 
gauges.

Then with a canonical transformation the previous canonical basis of Dirac observables (it is a canonical basis for superspace) is put in a form convenient for starting the search of the final gauge variable conjugate to the superhamiltonian constraint. As already said in Ref. [6], from the study of the Gauss law associated with the superhamiltonian constraint (the ADM strong and weak energies are the charges) it turns out that this gauge variable is the momentum conjugate to the conformal factor of the 3-metric. Therefore, the superhamiltonian constraint is an equation for the conformal factor of the 3-metric, the Lichnerowicz equation.

A special 3-orthogonal gauge, replacing the maximal slicing condition in our approach, is defined by putting equal to zero the momentum conjugate to the conformal factor of the 3metric as a gauge fixing constraint. Even if we do not know the solution of the Lichnerowicz equation, in this gauge we can identify a canonical basis of the final Dirac observables, namely two pairs of conjugate variables describing the gravitational field in this special completely fixed 3-orthogonal gauge. This is the first time that the canonical reduction of gravity can be pushed till the end: the weak ADM energy restricted to this gauge is the Hamiltonian for the Dirac observables. Finally we write the equation for the lapse and shift functions associated with this special gauge.

Then we study the Wigner-Sen-Witten hypersurfaces of the rest-frame instant form of tetrad gravity and we write the equations, whose solution would allow to find the embedding of these hypersurfaces into the spacetime. We also show the existence of preferred dynamical asymptotic inertial observers to be identified with the fixed stars.

We refer to Ref [6] for the discussion of the interpretational problems with the observables in general relativity, for the problem of time and for the quantization in a completely fixed gauge, because the treatment of these topics is the same in metric and tetrad gravity.

Finally some comments on inertial effets in Minkowski spacetime, like the ones in noninertial frames in Newtonian gravity, as distinct from the gravitational field can be made by imposing the vanishing of the Dirac observables of the gravitational field: this defines the void spacetimes including Minkowski spacetime in Cartesian coordinates.

In Section II, after a review of Hamitonian tetrad gravity and of the rest-frame instant form of metric gravity, we define the rest-frame instant form of tetrad gravity.

In Section III we study the Hamiltonian group of gauge transformations of tetrad gravity, whose component connected to the identity is generated by its 14 first class constraints.

In Section IV we define and solve the multitemporal equations associated with rotations and space diffeomorphism constraints, finding how the cotriads and their momenta depend on the corresponding gauge variables.

In Section $\mathrm{V}$ we find the quasi-Shanmugadhasan canonical transformation adapted to 3-orthogonal gauges.

In Section VI, after a further canonical transformation, we rewrite the superhamiltonian constraint, restricted to 3-orthogonal gauges, as the Lichnerowicz equation for the conformal factor of the 3-metric and we define a special completely fixed 3-orthogonal gauge, whose final Dirac observables for the gravitational field are then identified.

In Section VII we study the embedding of the Wigner-Sen-Witten hypersurfaces in the given spacetime.

In Section VIII we define and study void spacetimes as those spacetimes in which there 
is no gravitational field (meant as source of tidal effects), but only inertial effects like in non-inertial frames in Newton gravity.

In the Conclusions we make some comments about completely fixed gauges and on the open problems and perspectives.

In Appendix A there is the Hamiltonian tetrad gravity expression of relevant 3-tensor in the special 3-orthogonal gauge.

In Appendix B there is the Hamiltonian tetrad gravity expression of the ADM Poincaré charges in the special 3-orthogonal gauge. 


\section{THE REST-FRAME INSTANT FORM OF TETRAD GRAVITY.}

\section{A. Review of the New Parametrization of Tetrad Gravity and of its Constraints.}

Refs. 20 22 are used for the background in differential geometry. A spacetime is a timeoriented pseudo-Riemannian (or Lorentzian) 4-manifold $\left(M^{4},{ }^{4} g\right)$ with signature $\epsilon(+---)$ $(\epsilon= \pm 1)$ and with a choice of time orientation 20 . In Appendix A of Ref. [1] we give a review of notions on 4-dimensional pseudo-Riemannian manifolds, tetrads on them and triads on 3manifolds, which unifies many results, scattered in the literature, needed not only for a well posed formulation of tetrad gravity but also for the further study of its canonical reduction.

As shown in Section II and in Appendix A of Ref. [1], in the family of $\Sigma_{\tau}$-adapted frames and coframes on $M^{4}$, we can select special tetrads and cotetrads ${ }_{(\Sigma)}^{4} \check{E}_{(\alpha)}$ and ${ }_{(\Sigma)}^{4} \check{\theta}^{(\alpha)}$ also adapted to a given set of triads ${ }^{3} e_{(a)}^{r}$ and cotriads ${ }^{3} e_{r}^{(a)}={ }^{3} e_{(a) r}$ on $\Sigma_{\tau}$

$$
\begin{aligned}
& { }_{(\Sigma)}^{4} \check{E}_{(\alpha)}^{\mu}=\left\{{ }_{(\Sigma)}^{4} \check{E}_{(o)}^{\mu}=l^{\mu}=\hat{b}_{l}^{\mu}=\frac{1}{N}\left(b_{\tau}^{\mu}-N^{r} b_{r}^{\mu}\right) ;{ }_{(\Sigma)}^{4} \check{E}_{(a)}^{\mu}={ }^{3} e_{(a)}^{s} b_{s}^{\mu}\right\}, \\
& { }_{(\Sigma)}^{4} \check{E}_{\mu}^{(\alpha)}=\left\{{ }_{(\Sigma)}^{4} \check{E}_{\mu}^{(o)}=\epsilon l_{\mu}=\hat{b}_{\mu}^{l}=N b_{\mu}^{\tau} ;{ }_{(\Sigma)}^{4} \check{E}_{\mu}^{(a)}={ }^{3} e_{s}^{(a)} \hat{b}_{\mu}^{s}\right\}, \\
& { }_{(\Sigma)}^{4} \check{E}_{(\alpha)}^{\mu} \quad{ }^{4} g_{\mu \nu} \stackrel{4}{(\Sigma)} \check{E}_{(\beta)}^{\nu}={ }^{4} \eta_{(\alpha)(\beta)}, \\
& { }_{(\Sigma)}^{4} \underset{\tilde{\tilde{E}}_{(\alpha)}^{A}}{A}{ }_{(\Sigma)}^{4} \check{E}_{(\alpha)}^{\mu} b_{\mu}^{A}, \quad \Rightarrow \quad{ }_{(\Sigma)}^{4} \check{\tilde{E}}_{(o)}^{A}=\epsilon l^{A}, \\
& { }_{(\Sigma)}^{4} \check{\tilde{E}}_{(o)}^{\tau}=\frac{1}{N}, \quad{ }_{(\Sigma)}^{4} \check{\tilde{E}}_{(a)}^{\tau}=0 \\
& { }_{(\Sigma)}^{4} \check{\tilde{E}}_{(o)}^{r}=-\frac{N^{r}}{N}, \quad{ }_{(\Sigma)} \check{\tilde{E}}_{(a)}^{r}={ }^{3} e_{(a)}^{r} \text {; } \\
& { }_{(\Sigma)}^{4} \check{\tilde{E}}_{A}^{(\alpha)}={ }_{(\Sigma)}^{4} \check{E}_{\mu}^{(\alpha)} b_{A}^{\mu}, \quad \Rightarrow \underset{(\Sigma)}{4} \check{\tilde{E}}_{A}^{(o)}=l_{A}, \\
& { }_{(\Sigma)}^{4} \check{\tilde{E}}_{\tau}^{(o)}=N, \quad \stackrel{4}{(\Sigma)} \underset{\tilde{E}}{\check{\tilde{E}}_{\tau}^{(a)}}=N^{r 3} e_{r}^{(a)}=N^{(a)} \\
& { }_{(\Sigma)}^{4} \check{\tilde{E}}_{r}^{(o)}=0, \quad{ }_{(\Sigma)}^{4} \check{\tilde{E}}_{r}^{(a)}={ }^{3} e_{r}^{(a)}, \\
& { }_{(\Sigma)}^{4} \check{E}_{(\alpha)}^{A}{ }^{4} g_{A B} \stackrel{4}{4} \check{E}_{(\beta)}^{B}={ }^{4} \eta_{(\alpha)(\beta)} .
\end{aligned}
$$

Here $b_{r}^{\mu}$ and $b_{\mu}^{r}$ are defined in Eqs.(A3) of Ref [1] [26] and $l^{\mu}(\tau, \vec{\sigma})$ is the unit normal to $\Sigma_{\tau}$ at $\vec{\sigma}$. $N$ and $N^{r}$ are the standard lapse and shift functions.

\footnotetext{
${ }^{25}$ I.e. there exists a continuous, nowhere vanishing timelike vector field which is used to separate the non-spacelike vectors at each point of $M^{4}$ in either future- or past-directed vectors.

${ }^{26}$ Instead of local coordinates $x^{\mu}$ for $M^{4}$, we use local coordinates $\sigma^{A}$ on $R \times \Sigma \approx M^{4}$ $\left[x^{\mu}=z^{\mu}(\sigma)\right.$ with inverse $\left.\sigma^{A}=\sigma^{A}(x)\right]$, i.e. a $\Sigma_{\tau^{-}}$adapted holonomic coordinate basis for vector fields $\partial_{A}=\frac{\partial}{\partial \sigma^{A}} \in T(R \times \Sigma) \mapsto b_{A}^{\mu}(\sigma) \partial_{\mu}=\frac{\partial z^{\mu}(\sigma)}{\partial \sigma^{A}} \partial_{\mu} \in T M^{4}$, and for differential one-forms
} 
We have also shown the components of these tetrads and cotetrads in the holonomic basis ${ }_{(\Sigma)}^{4} \check{\tilde{E}}_{r}^{(o)}=0$ is the Schwinger time gauge condition [23]).

We define our class of arbitrary cotretads ${ }^{4} E_{\mu}^{(\alpha)}(z(\sigma))$ on $M^{4}$ starting from the special $\Sigma_{\tau^{-}}$and cotriad-adapted cotetrads ${ }_{(\Sigma)}^{4} \check{E}_{\mu}^{(\alpha)}(z(\sigma))$ by means of the formula

$$
\begin{aligned}
{ }^{4} E_{\mu}^{(\alpha)}(z(\sigma)) & =L_{(\beta)}^{(\alpha)}(V(z(\sigma)) ; \stackrel{\circ}{V})_{(\Sigma)}^{4} \check{E}_{\mu}^{(\beta)}(z(\sigma)) \\
\left(\begin{array}{c}
{ }^{4} E_{\mu}^{(o)} \\
{ }^{4} E_{\mu}^{(a)}
\end{array}\right)(z(\sigma)) & =\left(\begin{array}{cc}
\sqrt{1+\sum_{(c)} \varphi^{(c) 2}} & -\epsilon \varphi_{(b)} \\
\varphi^{(a)} & \delta_{(b)}^{(a)}-\epsilon \frac{\varphi^{(a)} \varphi_{(b)}}{1+\sqrt{1+\sum_{(c)} \varphi^{(c) 2}}}
\end{array}\right)(z(\sigma))\left(\begin{array}{l}
l_{\mu} \\
{ }^{3} e_{s}^{(b)} b_{\mu}^{s}
\end{array}\right)(\sigma) \\
{ }^{4} E_{\tau}^{(o)}(z(\sigma)) & =\sqrt{1+\sum_{(c)} \varphi^{(c) 2}(\sigma)} N(\sigma)+\sum_{(a)} \varphi^{(a)}(\sigma) N^{(a)}(\sigma) .
\end{aligned}
$$

Here $L^{(\alpha)}{ }_{\beta)}(V(z(\sigma)) ; \stackrel{\circ}{V})$ are the components of the standard Wigner boost for timelike Poincaré orbits (see Ref. [24]), which in the tangent space at each point of $M^{4}$ connects the timelike 4-vectors $\stackrel{\circ}{V}^{(\alpha)}=l^{\mu}(z(\sigma))_{(\Sigma)}^{4} \check{E}_{\mu}^{(\alpha)}(z(\sigma))=(1 ; \overrightarrow{0})$ and $V^{(\alpha)}(z(\sigma))=$ $L_{(\beta)}^{(\alpha)}(V(z(\sigma)) ; \stackrel{\circ}{V}) \stackrel{\circ}{V} \stackrel{(\beta)}{\stackrel{\text { def }}{=}} l^{\mu}(z(\sigma))^{4} E_{\mu}^{(\alpha)}(z(\sigma))$.

Let ${ }^{4} E_{(\alpha)}^{\mu}(z)$ and ${ }^{4} E_{\mu}^{(\alpha)}(z)$ be arbitrary tetrads and cotetrads on $M^{4}$. Let us define the point-dependent Minkowski 4-vector ${ }^{27} V^{(\alpha)}(z(\sigma))=l^{\mu}(z(\sigma))^{4} E_{\mu}^{(\alpha)}(z(\sigma))$ (assumed to be future-pointing), which satisfies $V^{(\alpha)}(z(\sigma))^{4} \eta_{(\alpha)(\beta)} V^{(\beta)}(z(\sigma))=\epsilon$.

$d x^{\mu} \in T^{*} M^{4} \mapsto d \sigma^{A}=b_{\mu}^{A}(\sigma) d x^{\mu}=\frac{\partial \sigma^{A}(z)}{\partial z^{\mu}} d x^{\mu} \in T^{*}(R \times \Sigma)$. The induced 4-metric and inverse 4metric become in the new basis ${ }^{4} g_{\mu \nu}=b_{\mu}^{A}{ }^{4} g_{A B} b_{\nu}^{B}=\epsilon\left(N^{2}-{ }^{3} g_{r s} N^{r} N^{s}\right) \partial_{\mu} \tau \partial_{\nu} \tau-\epsilon^{3} g_{r s} N^{s}\left(\partial_{\mu} \tau \partial_{\nu} \sigma^{r}+\right.$ $\left.\partial_{\nu} \tau \partial_{\mu} \sigma^{r}\right)-\epsilon^{3} g_{r s} \partial_{\mu} \sigma^{r} \partial_{\nu} \sigma^{s}=\epsilon l_{\mu} l_{\nu}-\epsilon^{3} g_{r s}\left(\partial_{\mu} \sigma^{r}+N^{r} \partial_{\mu} \tau\right)\left(\partial_{\nu} \sigma^{s}+N^{s} \partial_{\nu} \tau\right),{ }^{4} g_{A B}=\left\{{ }^{4} g_{\tau \tau}=\right.$ $\left.\epsilon\left(N^{2}-{ }^{3} g_{r s} N^{r} N^{s}\right) ;{ }^{4} g_{\tau r}=-\epsilon{ }^{3} g_{r s} N^{s} ;{ }^{4} g_{r s}=-\epsilon^{3} g_{r s}\right\}=\epsilon\left[l_{A} l_{B}-{ }^{3} g_{r s}\left(\delta_{A}^{r}+N^{r} \delta_{A}^{\tau}\right)\left(\delta_{B}^{s}+N^{s} \delta_{B}^{\tau}\right)\right]$, ${ }^{4} g^{\mu \nu}=b_{A}^{\mu 4} g^{A B} b_{B}^{\nu}=\frac{\epsilon}{N^{2}} \partial_{\tau} z^{\mu} \partial_{\tau} z^{\nu}-\frac{\epsilon N^{r}}{N^{2}}\left(\partial_{\tau} z^{\mu} \partial_{r} z^{\nu}+\partial_{\tau} z^{\nu} \partial_{r} z^{\mu}\right)-\epsilon\left({ }^{3} g^{r s}-\frac{N^{r} N^{s}}{N^{2}}\right) \partial_{r} z^{\mu} \partial_{s} z^{\nu}=\epsilon\left[l^{\mu} l^{\nu}-\right.$ $\left.{ }^{3} g^{r s} \partial_{r} z^{\mu} \partial_{s} z^{\nu}\right],{ }^{4} g^{A B} \stackrel{N^{4}}{=}\left\{{ }^{4 \tau}=\frac{\epsilon}{N^{2}} ;{ }^{4} g^{\tau r}=-\frac{\epsilon N^{r}}{N^{2}} ;{ }^{4} g^{r s}=-\epsilon\left({ }^{3} g^{r s}-\frac{N^{r} N^{s}}{N^{2}}\right)\right\}=\epsilon\left[l^{A} l^{B}-{ }^{3} g^{r s} \delta_{r}^{A} \delta_{s}^{B}\right]$. For the unit normals we have $l^{A}=l^{\mu} b_{\mu}^{A}=N^{4} g^{A \tau}=\frac{\epsilon}{N}\left(1 ;-N^{r}\right)$ and $l_{A}=l_{\mu} b_{A}^{\mu}=N \partial_{A} \tau=$ $N \delta_{A}^{\tau}=(N ; \overrightarrow{0})$. We introduced the 3 -metric of $\Sigma_{\tau}$ : ${ }^{3} g_{r s}=-\epsilon^{4} g_{r s}$ with signature $(+++)$. If ${ }^{4} \gamma^{r s}$ is the inverse of the spatial part of the 4-metric $\left({ }^{4} \gamma^{r u}{ }^{4} g_{u s}=\delta_{s}^{r}\right)$, the inverse of the 3metric is ${ }^{3} g^{r s}=-\epsilon^{4} \gamma^{r s}\left({ }^{3} g^{r u}{ }^{r} g_{u s}=\delta_{s}^{r}\right) .{ }^{3} g_{r s}(\tau, \vec{\sigma})$ are the components of the first fundamental form of the Riemann 3-manifold $\left(\Sigma_{\tau},{ }^{3} g\right)$ and we have the following form for the line element in $M^{4}: d s^{2}={ }^{4} g_{\mu \nu} d x^{\mu} d x^{\nu}=\epsilon\left(N^{2}-{ }^{3} g_{r s} N^{r} N^{s}\right)(d \tau)^{2}-2 \epsilon^{3} g_{r s} N^{s} d \tau d \sigma^{r}-\epsilon^{3} g_{r s} d \sigma^{r} d \sigma^{s}=$ $\epsilon\left[N^{2}(d \tau)^{2}-{ }^{3} g_{r s}\left(d \sigma^{r}+N^{r} d \tau\right)\left(d \sigma^{s}+N^{s} d \tau\right)\right]$.

${ }^{27}$ The point-dependent

flat 4-vector $V^{(\alpha)}(z(\sigma))=\left(V^{(o)}(z(\sigma))=+\sqrt{1+\sum_{r} V^{(r) 2}(z(\sigma))} ; V^{(r)}(z(\sigma)) \stackrel{\text { def }}{=} \varphi^{(r)}(\sigma)\right)$ depends only on the three functions $\varphi^{(r)}(\sigma)$. One has $\varphi^{(r)}(\sigma)=-\epsilon \varphi_{(r)}(\sigma)$ since ${ }^{4} \eta_{r s}=-\epsilon \delta_{r s}$; having the Euclidean signature $(+++)$ for both $\epsilon= \pm 1$, we shall define the Kronecker delta as $\delta^{(i)(j)}=\delta_{(j)}^{(i)}=\delta_{(i)(j)}$. 
The $\varphi^{(a)}(\sigma)=V^{(a)}(z(\sigma))=l^{\mu}(z(\sigma))^{4} E_{\mu}^{(a)}(z(\sigma))$ are the three parameters of the Wigner boost.

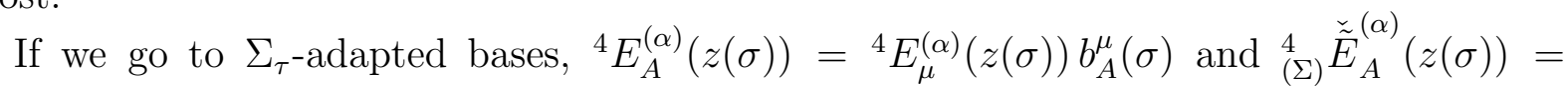
${ }_{(\Sigma)}^{4} \check{E}_{\mu}^{(\alpha)}(z(\sigma)) b_{A}^{\mu}(\sigma)$, one has

$$
\begin{aligned}
& \left(\begin{array}{c}
{ }^{4} E_{A}^{(o)} \\
{ }^{4} E_{A}^{(a)}
\end{array}\right)=\left(\begin{array}{cc}
\sqrt{1+\sum_{(c)} \varphi^{(c) 2}} & -\epsilon \varphi_{(b)} \\
\varphi^{(a)} & \delta_{(b)}^{(a)}-\epsilon \frac{\varphi^{(a)} \varphi_{(b)}}{1+\sqrt{1+\sum_{(c)} \varphi^{(c) 2}}}
\end{array}\right) \times \\
& \left(\begin{array}{l}
{ }_{(\Sigma)}^{4} \underset{\tilde{E}_{A}^{(o)}}{(o)}=(N ; \overrightarrow{0}) \\
{ }_{(\Sigma)}^{4} \check{\tilde{E}}_{A}^{(b)}=\left(N^{(b)}={ }^{3} e_{r}^{(b)} N^{r} ;{ }^{3} e_{r}^{(b)}\right)
\end{array}\right), \\
& { }^{4} E_{r}^{(o)}(z(\sigma))=\sum_{(a)} \varphi^{(a)}(\sigma){ }^{3} e_{r}^{(a)}(\sigma), \\
& { }^{4} E_{\tau}^{(a)}(z(\sigma))=\varphi^{(a)}(\sigma) N(\sigma)+\sum_{(b)}\left[\delta_{(b)}^{(a)}-\epsilon \frac{\varphi^{(a)}(\sigma) \varphi_{(b)}(\sigma)}{1+\sqrt{1+\sum_{(c)} \varphi^{(c) 2}(\sigma)}}\right] N^{(b)}(\sigma), \\
& { }^{4} E_{r}^{(a)}(z(\sigma))=\sum_{(b)}\left[\delta_{(b)}^{(a)}-\epsilon \frac{\varphi^{(a)}(\sigma) \varphi_{(b)}(\sigma)}{1+\sqrt{1+\sum_{(c)} \varphi^{(c) 2}(\sigma)}}\right]^{3} e_{r}^{(b)}(\sigma), \\
& \Rightarrow{ }^{4} g_{A B}={ }^{4} E_{A}^{(\alpha)}{ }^{4} \eta_{(\alpha)(\beta)}{ }^{4} E_{B}^{(\beta)}={ }_{(\Sigma)}^{4} \check{E}_{A}^{(\alpha)}{ }^{4} \eta_{(\alpha)(\beta)}{ }_{(\Sigma)}^{4} \check{E}_{B}^{(\beta)}= \\
& =\epsilon\left(\begin{array}{cc}
\left(N^{2}-{ }^{3} g_{r s} N^{r} N^{s}\right) & -{ }^{3} g_{s t} N^{t} \\
-{ }^{3} g_{r t} N^{t} & -{ }^{3} g_{r s}
\end{array}\right), \\
& \left(\begin{array}{l}
{ }^{4} E_{(o)}^{\mu} \\
{ }^{4} E_{(a)}^{\mu}
\end{array}\right)=\left(\begin{array}{cc}
\sqrt{1+\sum_{(c)} \varphi^{(c) 2}} & -\varphi^{(b)} \\
\epsilon \varphi_{(a)} & \delta_{(a)}^{(b)}-\epsilon \frac{\varphi_{(a)} \varphi^{(b)}}{1+\sqrt{1+\sum_{(c)} \varphi^{(c) 2}}}
\end{array}\right)\left(\begin{array}{l}
l^{\mu} \\
b_{s}^{\mu 3} e_{(b)}^{s}
\end{array}\right), \\
& \left(\begin{array}{l}
{ }^{4} E_{(o)}^{A} \\
{ }^{4} E_{(a)}^{A}
\end{array}\right)=\left(\begin{array}{cc}
\sqrt{1+\sum_{(c)} \varphi^{(c) 2}} & -\varphi^{(b)} \\
\epsilon \varphi^{(a)} & \delta_{(a)}^{(b)}-\epsilon \frac{\varphi_{(a)} \varphi^{(b)}}{1+\sqrt{1+\sum_{(c)} \varphi^{(c) 2}}}
\end{array}\right)\left(\begin{array}{l}
{ }_{(\Sigma)}^{4} \check{\tilde{E}}_{(o)}^{A}=\left(1 / N ;-N^{r} / N\right) \\
\check{\tilde{E}}_{(\Sigma)}^{A}{ }_{(b)}^{A}=\left(0 ;{ }^{3} e_{(b)}^{r}\right)
\end{array}\right), \\
& { }^{4} E_{(o)}^{\tau}(z(\sigma))=\sqrt{1+\sum_{(c)} \varphi^{(c) 2}(\sigma)} \frac{1}{N(\sigma)}, \\
& { }^{4} E_{(o)}^{r}\left(z(\sigma)=-\sqrt{1+\sum_{(c)} \varphi^{(c) 2}(\sigma)} \frac{N^{r}(\sigma)}{N(\sigma)}-\varphi^{(b)}(\sigma)^{3} e_{(b)}^{r}(\sigma),\right. \\
& { }^{4} E_{(a)}^{\tau}(z(\sigma))=\epsilon \frac{\varphi_{(a)}(\sigma)}{N(\sigma)},
\end{aligned}
$$




$$
\begin{aligned}
& { }^{4} E_{(a)}^{r}(z(\sigma))=-\epsilon \varphi_{(a)}(\sigma) \frac{N^{r}(\sigma)}{N(\sigma)}+\sum_{(b)}\left[\delta_{(a)}^{(b)}-\epsilon \frac{\varphi_{(a)}(\sigma) \varphi^{(b)}(\sigma)}{1+\sqrt{1+\sum_{(c)} \varphi^{(c) 2}(\sigma)}}\right]{ }^{3} e_{(b)}^{r}(\sigma), \\
\Rightarrow{ }^{4} g^{A B}= & { }^{4} E_{(\alpha)}^{A}{ }^{4} \eta^{(\alpha)(\beta)}{ }^{4} E_{(\beta)}^{B}={ }_{(\Sigma)}^{4} \check{E}_{(\alpha)}^{A}{ }^{4} \eta_{(\alpha)(\beta)} \stackrel{4}{4} \check{E}_{(\beta)}^{B}= \\
= & \epsilon\left(\begin{array}{cc}
\frac{1}{N^{2}} & -\frac{N^{s}}{N^{2}} \\
-\frac{N^{r}}{N^{2}} & -\left({ }^{3} g^{r s}-\frac{N^{r} N^{s}}{N^{2}}\right)
\end{array}\right) .
\end{aligned}
$$

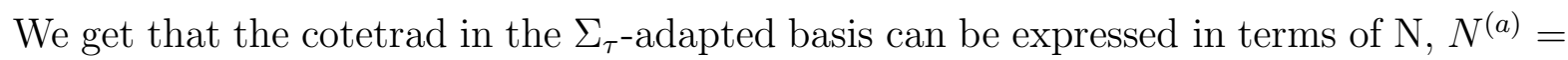
${ }^{3} e_{s}^{(a)} N^{s}=N_{(a)}, \varphi^{(a)}$ and ${ }^{3} e_{r}^{(a)}\left[{ }^{3} g_{r s}=\sum_{(a)}{ }^{3} e_{(a) r}{ }^{3} e_{(a) s}\right]$

From ${ }_{(\Sigma)}^{4} \check{\tilde{E}}_{A}^{(\alpha)}(z(\sigma))=\left(L^{-1}\right)^{(\alpha)}{ }_{(\beta)}(V(z(\sigma)) ; \stackrel{\circ}{V})^{4} E_{A}^{(\beta)}(z(\sigma))$ and $\underset{(\Sigma)}{4} \check{\tilde{E}}_{(\alpha)}^{A}(z(\sigma))=$ ${ }^{4} E_{(\beta)}^{A}\left(L^{-1}\right)^{(\beta)}{ }_{(\alpha)}(V(z(\sigma)) ; \stackrel{\circ}{V})$ it turns out [24] that the flat indices $(a)$ of the adapted tetrads ${ }_{(\Sigma)}^{4} \check{E}_{(a)}^{\mu}$ and of the triads ${ }^{3} e_{(a)}^{r}$ and cotriads ${ }^{3} e_{r}^{(a)}$ on $\Sigma_{\tau}$ transform as Wigner spin 1 indices under point-dependent $\mathrm{SO}(3)$ Wigner rotations $R^{(a)}{ }_{(b)}(V(z(\sigma)) ; \Lambda(z(\sigma)))$ associated with Lorentz transformations $\Lambda^{(\alpha)}{ }_{(\beta)}(z)$ in the tangent plane to $M^{4}$ in the same point ${ }^{28}$. Instead the index $(o)$ of the adapted tetrads ${ }_{(\Sigma)}^{4} \check{E}_{(o)}^{\mu}$ is a local Lorentz scalar in each point. Therefore, the adapted tetrads in the $\Sigma_{\tau}$-adapted basis should be denoted as ${ }_{(\Sigma)}^{4} \check{\tilde{E}}_{(\bar{\alpha})}^{A}$, with $(\bar{o})$ and $A=(\tau, r)$ Lorentz scalar indices and with $(\bar{a})$ Wigner spin 1 indices; we shall go on with the indices $(o),(a)$ without the overbar for the sake of simplicity. In this way the tangent planes to $\Sigma_{\tau}$ in $M^{4}$ are described in a Wigner covariant way, reminiscent of the flat rest-frame covariant instant form of dynamics introduced in Minkowski spacetime in Ref. [15].

Therefore, an arbitrary tetrad field, namely a (in general non-geodesic) congruence of observers' timelike worldlines with 4 -velocity field $u^{A}(\tau, \vec{\sigma})={ }^{4} E_{(o)}^{A}(\tau, \vec{\sigma})$, can be obtained with a pointwise Wigner boost from the special surface-forming timelike congruence whose 4-velocity field is the normal to $\Sigma_{\tau}, l^{A}(\tau, \vec{\sigma})=\epsilon_{(\Sigma)}^{4} \check{\tilde{E}}_{(o)}^{A}(\tau, \vec{\sigma})$ 区ु. ${ }^{4} E_{(\alpha)}^{A}$

We can invert Eqs.(2.3) to get $\mathrm{N}, N^{r}={ }^{3} e_{(a)}^{r} N^{(a)}, \varphi^{(a)}$ and ${ }^{3} e_{(a)}^{r}$ in terms of the tetrads

$$
\begin{aligned}
N & =\frac{1}{\sqrt{\left[{ }^{4} E_{(o)}^{\tau}\right]^{2}-\sum_{(c)}\left[{ }^{4} E_{(c)}^{\tau}\right]^{2}}} . \\
N^{r} & =-\frac{{ }^{4} E_{(o)}^{\tau}{ }^{4} E_{(0)}^{r}-\sum_{(c)}{ }^{4} E_{(c)}^{\tau}{ }^{4} E_{(c)}^{r}}{\left[{ }^{4} E_{(0)}^{\tau}\right]^{2}-\sum_{(c)}\left[{ }^{4} E_{(c)}^{\tau}\right]^{2}} \\
\varphi_{(a)} & =\frac{\epsilon^{4} E_{(a)}^{\tau}}{\sqrt{\left[{ }^{4} E_{(o)}^{\tau}\right]^{2}-\sum_{(c)}\left[{ }^{4} E_{(c)}^{\tau}\right]^{2}}}
\end{aligned}
$$

$$
\begin{aligned}
& { }^{28} R_{(\beta)}^{(\alpha)}(\Lambda(z(\sigma)) ; V(z(\sigma)))=\left[L(\stackrel{\circ}{V} ; V(z(\sigma))) \Lambda^{-1}(z(\sigma)) L(\Lambda(z(\sigma)) V(z(\sigma)) ; \stackrel{\circ}{V})\right]^{(\alpha)}{ }_{(\beta)}= \\
& \left(\begin{array}{cc}
1 & 0 \\
0 & R^{(a)}{ }_{(b)}(V(z(\sigma)) ; \Lambda(z(\sigma)))
\end{array}\right) \text {. }
\end{aligned}
$$

${ }^{29}$ It is associated with the $3+1$ splitting of $M^{4}$ with leaves $\Sigma_{\tau}$. 


$$
\begin{aligned}
{ }^{3} e_{(a)}^{r}= & \sum_{(b)} B_{(a)(b)}\left({ }^{4} E_{(b)}^{r}+N^{r}{ }^{4} E_{(b)}^{\tau}\right) \\
& B_{(a)(b)}=\delta_{(a)(b)}-\frac{{ }^{4} E_{(a)}^{\tau}{ }^{4} E_{(b)}^{\tau}}{{ }^{4} E_{(0)}^{\tau}\left[{ }^{4} E_{(0)}^{\tau}+\sqrt{\left.\left[{ }^{4} E_{(0)}^{\tau}\right]^{2}-\sum_{(c)}\left[{ }^{4} E_{(c)}^{\tau}\right]^{2}\right]}\right.} .
\end{aligned}
$$

If ${ }^{3} e^{-1}=\operatorname{det}\left({ }^{3} e_{(a)}^{r}\right)$, then from the orthonormality condition we get ${ }^{3} e_{(a) r}={ }^{3} e\left({ }^{3} e_{(b)}^{s}{ }^{3} e_{(c)}^{t}-\right.$ $\left.{ }^{3} e_{(b)}^{t}{ }^{3} e_{(c)}^{s}\right)$ 邽 and it allows to express the cotriads in terms of the tetrads ${ }^{4} E_{(\alpha)}^{A}$. Therefore, given the tetrads ${ }^{4} E_{(\alpha)}^{A}$ (or equivalently the cotetrads ${ }^{4} E_{A}^{(\alpha)}$ ) on $M^{4}$, an equivalent set of variables with the local Lorentz covariance replaced with local Wigner covariance are the lapse N, the shifts $N^{(a)}=N_{(a)}={ }^{3} e_{(a) r} N^{r}$, the Wigner-boost parameters $\varphi^{(a)}=-\epsilon \varphi_{(a)}$ and either the triads ${ }^{3} e_{(a)}^{r}$ or the cotriads ${ }^{3} e_{(a) r}$.

The independent variables in metric gravity have now the following expression in terms of $\mathrm{N}, N^{(a)}=N_{(a)}={ }^{3} e_{(a)}^{r} N_{r}, \varphi^{(a)}=-\epsilon \varphi_{(a)},{ }^{3} e_{r}^{(a)}={ }^{3} e_{(a) r}$ 范

$$
\begin{gathered}
N, \quad N_{r}={ }^{3} e_{r}^{(a)} N_{(a)}={ }^{3} e_{(a) r} N_{(a)}, \\
{ }^{3} g_{r s}={ }^{3} e_{r}^{(a)} \delta_{(a)(b)}{ }^{3} e_{s}^{(b)}={ }^{3} e_{(a) r}{ }^{3} e_{(a) s},
\end{gathered}
$$

so that the line element of $M^{4}$ becomes

$$
\begin{aligned}
d s^{2} & =\epsilon\left(N^{2}-N_{(a)} N_{(a)}\right)(d \tau)^{2}-2 \epsilon N_{(a)}{ }^{3} e_{(a) r} d \tau d \sigma^{r}-\epsilon^{3} e_{(a) r}{ }^{3} e_{(a) s} d \sigma^{r} d \sigma^{s}= \\
& =\epsilon\left[N^{2}(d \tau)^{2}-\left({ }^{3} e_{(a) r} d \sigma^{r}+N_{(a)} d \tau\right)\left({ }^{3} e_{(a) s} d \sigma^{s}+N_{(a)} d \tau\right)\right] .
\end{aligned}
$$

The extrinsic curvature takes the form 52

$$
\begin{aligned}
{ }^{3} K_{r s} & =\hat{b}_{r}^{\mu} \hat{b}_{s}^{\nu 3} K_{\mu \nu}=\frac{1}{2 N}\left(N_{r \mid s}+N_{s \mid r}-\partial_{\tau}{ }^{3} g_{r s}\right)= \\
& =\frac{1}{2 N}\left({ }^{3} e_{(a) r} \delta_{s}^{w}+{ }^{3} e_{(a) s} \delta_{r}^{w}\right)\left(N_{(a) \mid w}-\partial_{\tau}{ }^{3} e_{(a) w}\right) \\
{ }^{3} K_{r(a)} & ={ }^{3} K_{r s}{ }^{3} e_{(a)}^{s}=\frac{1}{2 N}\left(\delta_{(a)(b)} \delta_{r}^{w}+{ }^{3} e_{(a)}^{w}{ }^{3} e_{(b) r}\right)\left(N_{(b) \mid w}-\partial_{\tau}{ }^{3} e_{(c) w}\right), \\
{ }^{3} K & =\frac{1}{N}{ }^{3} e_{(a)}^{r}\left(N_{(a) \mid r}-\partial_{\tau}{ }^{3} e_{(a) r}\right)
\end{aligned}
$$

The ADM action in the new variables is obtained from the metric gravity ADM action and has the form

${ }^{30}$ With $(a),(b),(c)$ and $r, s, t$ cyclic.

${ }^{31} \gamma=\operatorname{det}\left({ }^{3} g_{r s}\right)=\left({ }^{3} e\right)^{2}=\left(\operatorname{det}\left(e_{(a) r}\right)\right)^{2}$.

${ }^{32} N_{(a) \mid r}={ }^{3} e_{(a)}^{s} N_{s \mid r}=\partial_{r} N_{(a)}-\epsilon_{(a)(b)(c)}{ }^{3} \omega_{r(b)} N_{(c)}$ with ${ }^{3} \omega_{r(b)}$ being the 3 -spin connection, see Eq.(A22) of Ref. [1]. 


$$
\begin{aligned}
\hat{S}_{A D M T} & =\int d \tau \hat{L}_{A D M T}= \\
& =-\frac{\epsilon c^{3}}{16 \pi G} \int d \tau d^{3} \sigma\left\{N^{3} e \epsilon_{(a)(b)(c)}{ }^{3} e_{(a)}^{r}{ }^{3} e_{(b)}^{s}{ }^{3} \Omega_{r s(c)}+\right. \\
& \left.+\frac{{ }^{3} e}{2 N}\left({ }^{3} G_{o}^{-1}\right)_{(a)(b)(c)(d)}{ }^{3} e_{(b)}^{r}\left(N_{(a) \mid r}-\partial_{\tau}{ }^{3} e_{(a) r}\right)^{3} e_{(d)}^{s}\left(N_{(c) \mid s}-\partial_{\tau}{ }^{3} e_{(c) s}\right)\right\}
\end{aligned}
$$

where we introduced the flat inverse Wheeler-DeWitt supermetric

$$
\begin{aligned}
&\left({ }^{3} G_{o}^{-1}\right)_{(a)(b)(c)(d)}= \delta_{(a)(c)} \delta_{(b)(d)}+\delta_{(a)(d)} \delta_{(b)(c)}-2 \delta_{(a)(b)} \delta_{(c)(d)}, \\
& \Downarrow \\
&{ }^{3} G_{o(a)(b)(c)(d)=}{ }^{3} G_{o(b)(a)(c)(d)}={ }^{3} G_{O(a)(b)(d)(c)}={ }^{3} G_{o(c)(d)(a)(b)}= \\
&= \delta_{(a)(c)} \delta_{(b)(d)}+\delta_{(a)(d)} \delta_{(b)(c)}-\delta_{(a)(b)} \delta_{(c)(d)}, \\
& \frac{1}{2}{ }^{3} G_{o(a)(b)(e)(f)} \frac{1}{2}{ }^{3} G_{o(e)(f)(c)(d)}^{-1}=\frac{1}{2}\left[\delta_{(a)(c)} \delta_{(b)(d)}+\delta_{(a)(d)} \delta_{(b)(c)}\right] .
\end{aligned}
$$

The new action does not depend on the 3 boost variables $\varphi^{(a)}$, contains lapse $\mathrm{N}$ and modified shifts $N_{(a)}$ as Lagrange multipliers, and is a functional independent from the second time derivatives of the fields.

After the definition of the canonical momenta, whose Poisson brackets are

$$
\begin{aligned}
& \left\{N(\tau, \vec{\sigma}), \tilde{\pi}^{N}\left(\tau, \vec{\sigma}^{\prime}\right)\right\}=\delta^{3}\left(\vec{\sigma}, \vec{\sigma}^{\prime}\right), \\
& \left\{N_{(a)}(\tau, \vec{\sigma}), \tilde{\pi}_{(b)}^{\vec{N}}\left(\tau, \vec{\sigma}^{\prime}\right)\right\}=\delta_{(a)(b)} \delta^{3}\left(\vec{\sigma}, \vec{\sigma}^{\prime}\right), \\
& \left\{\varphi_{(a)}(\tau, \vec{\sigma}), \tilde{\pi}_{(b)}^{\vec{\varphi}}\left(\tau, \vec{\sigma}^{\prime}\right)\right\}=\delta_{(a)(b)} \delta^{3}\left(\vec{\sigma}, \vec{\sigma}^{\prime}\right), \\
& \left\{{ }^{3} e_{(a) r}(\tau, \vec{\sigma}),{ }^{3} \tilde{\pi}_{(b)}^{s}\left(\tau, \vec{\sigma}^{\prime}\right)\right\}=\delta_{(a)(b)} \delta_{r}^{s} \delta^{3}\left(\vec{\sigma}, \vec{\sigma}^{\prime}\right), \\
& \left\{{ }^{3} e_{(a)}^{r}(\tau, \vec{\sigma}),{ }^{3} \tilde{\pi}_{(b)}^{s}\left(\tau, \vec{\sigma}^{\prime}\right)\right\}=-{ }^{3} e_{(b)}^{r}(\tau, \vec{\sigma})^{3} e_{(a)}^{s}(\tau, \vec{\sigma}) \delta^{3}\left(\vec{\sigma}, \vec{\sigma}^{\prime}\right), \\
& \left\{{ }^{3} e(\tau, \vec{\sigma}),{ }^{3} \tilde{\pi}_{(a)}^{r}\left(\tau, \vec{\sigma}^{\prime}\right)\right\}={ }^{3} e(\tau, \vec{\sigma})^{3} e_{(a)}^{r}(\tau, \vec{\sigma}) \delta^{3}\left(\vec{\sigma}, \vec{\sigma}^{\prime}\right),
\end{aligned}
$$

we find ten primary constraints and four secondary ones

$$
\begin{aligned}
& \tilde{\pi}_{(a)}^{\vec{\varphi}}(\tau, \vec{\sigma}) \approx 0, \\
& \tilde{\pi}^{N}(\tau, \vec{\sigma}) \approx 0, \\
& \tilde{\pi}_{(a)}^{\vec{N}}(\tau, \vec{\sigma}) \approx 0, \\
{ }^{3} \tilde{M}_{(a)}(\tau, \vec{\sigma})= & \epsilon_{(a)(b)(c)}{ }^{3} e_{(b) r}(\tau, \vec{\sigma})^{3} \tilde{\pi}_{(c)}^{r}(\tau, \vec{\sigma})=\frac{1}{2} \epsilon_{(a)(b)(c)}{ }^{3} \tilde{M}_{(b)(c)}(\tau, \vec{\sigma}) \approx 0, \\
\Rightarrow & { }^{3} \tilde{M}_{(a)(b)}(\tau, \vec{\sigma})=\epsilon_{(a)(b)(c)}{ }^{3} \tilde{M}_{(c)}(\tau, \vec{\sigma})= \\
= & { }^{3} e_{(a) r}(\tau, \vec{\sigma})^{3} \tilde{\pi}_{(b)}^{r}(\tau, \vec{\sigma})-{ }^{3} e_{(b) r}(\tau, \vec{\sigma})^{3} \tilde{\pi}_{(a)}^{r}(\tau, \vec{\sigma}) \approx 0, \\
\hat{\mathcal{H}}(\tau, \vec{\sigma})= & \epsilon\left[\frac{c^{3}}{16 \pi G}{ }^{3} e \epsilon_{(a)(b)(c)}{ }^{3} e_{(a)}^{r}{ }^{3} e_{(b)}^{s}{ }^{3} \Omega_{r s(c)}-\right. \\
- & \left.\frac{2 \pi G}{c^{3}{ }^{3} e}{ }^{3} G_{o(a)(b)(c)(d)}{ }^{3} e_{(a) r}{ }^{3} \tilde{\pi}_{(b)}^{r}{ }^{3} e_{(c) s}{ }^{3} \tilde{\pi}_{(d)}^{s}\right](\tau, \vec{\sigma})=
\end{aligned}
$$




$$
\begin{aligned}
& =\epsilon\left[\frac{c^{3}}{16 \pi G}{ }^{3} e^{3} R-\frac{2 \pi G^{3}}{c^{3}{ }^{3} e} G_{o(a)(b)(c)(d)}{ }^{3} e_{(a) r}{ }^{3} \tilde{\pi}_{(b)}^{r}{ }^{3} e_{(c) s}{ }^{3} \tilde{\pi}_{(d)}^{s}\right](\tau, \vec{\sigma}) \approx 0, \\
\hat{\mathcal{H}}_{(a)}(\tau, \vec{\sigma}) & =\left[\partial_{r}{ }^{3} \tilde{\pi}_{(a)}^{r}-\epsilon_{(a)(b)(c)}{ }^{3} \omega_{r(b)}{ }^{3} \tilde{\pi}_{(c)}^{r}\right](\tau, \vec{\sigma})={ }^{3} \tilde{\pi}_{(a) \mid r}^{r}(\tau, \vec{\sigma}) \approx 0, \\
& \Rightarrow \hat{H}_{(c)}=\int d^{3} \sigma\left[N \hat{\mathcal{H}}-N_{(a)} \hat{\mathcal{H}}_{(a)}\right](\tau, \vec{\sigma})= \\
& =-{ }^{3} e_{(a)}^{r}(\tau, \vec{\sigma})\left[{ }^{3} \tilde{\Theta}_{r}+{ }^{3} \omega_{r(b)}{ }^{3} \tilde{M}_{(b)}\right](\tau, \vec{\sigma}) \approx 0,
\end{aligned}
$$

or

$$
\begin{aligned}
{ }^{3} \tilde{\Theta}_{r}(\tau, \vec{\sigma}) & =-\left[{ }^{3} e_{(a) r} \hat{\mathcal{H}}_{(a)}+{ }^{3} \omega_{r(a)}{ }^{3} \tilde{M}_{(a)}\right](\tau, \vec{\sigma})= \\
& =\left[{ }^{3} \tilde{\pi}_{(a)}^{s} \partial_{r}{ }^{3} e_{(a) s}-\partial_{s}\left({ }^{3} e_{(a) r}{ }^{3} \tilde{\pi}_{(a)}^{s}\right)\right](\tau, \vec{\sigma}) \approx 0 .
\end{aligned}
$$

We see that the first seven constraints are already Abelianized: our parametrization of the cotetrads is equivalent to a Shanmugadhasan canonical transformation Abelianizing the Lorentz boosts.

It can be checked (see Ref. [1]) that the superhamiltonian constraint $\hat{\mathcal{H}}(\tau, \vec{\sigma}) \approx 0$ coincides with the ADM metric superhamiltonian one $\tilde{\mathcal{H}}(\tau, \vec{\sigma}) \approx 0$, where also the ADM metric supermomentum constraints is expressed in terms of the tetrad gravity constraints.

It is convenient to replace the constraints $\hat{\mathcal{H}}_{(a)}(\tau, \vec{\sigma}) \approx 0$ 阿 with the 3 constraints ${ }^{3} \tilde{\Theta}_{r}(\tau, \vec{\sigma}) \approx 0$ generating space pseudo-diffeomorphisms on the cotriads and their conjugate momenta.

We can get the following phase space expression of the extrinsic curvature, of the ADM canonical momentum ${ }^{3} \tilde{\Pi}^{r s}$ and the following decomposition of the cotriad momentum

$$
\begin{aligned}
{ }^{3} K_{r s} & =\frac{\epsilon 4 \pi G}{c^{3}{ }^{3} e}{ }^{3} G_{o(a)(b)(c)(d)}{ }^{3} e_{(a) r}{ }^{3} e_{(b) s}{ }^{3} e_{(c) u}{ }^{3} \tilde{\pi}_{(d)}^{u}, \\
{ }^{3} K & =-\frac{\epsilon 8 \pi G}{c^{3} \sqrt{\gamma}}{ }^{3} \tilde{\Pi}=-\frac{\epsilon 4 \pi G^{3}}{c^{3}{ }^{3} e} e_{(a) r}{ }^{3} \tilde{\pi}_{(a)}^{r}, \\
{ }^{3} \tilde{\Pi}^{r s} & =\frac{1}{4}\left({ }^{3} e_{(a)}^{r}{ }^{3} \tilde{\pi}_{(a)}^{s}+{ }^{3} e_{(a)}^{s}{ }^{3} \tilde{\pi}_{(a)}^{r}\right), \\
{ }^{3} \tilde{\pi}_{(a)}^{r} & ={ }^{3} e_{(b)}^{r}{ }^{3} e_{(b) s}{ }^{3} \tilde{\pi}_{(a)}^{s}=\frac{1}{2}{ }^{3} e_{(b)}^{r}\left[{ }^{3} e_{(a) s}{ }^{3} \tilde{\pi}_{(b)}^{s}+{ }^{3} e_{(b) s}{ }^{3} \tilde{\pi}_{(a)}^{s}\right]-\frac{1}{2}{ }^{3} \tilde{M}_{(a)(b)}{ }^{3} e_{(b)}^{r} \equiv \\
& \left.\equiv \frac{1}{2} e_{(b)}^{r}{ }^{3} e_{(a) s}{ }^{3} \tilde{\pi}_{(b)}^{s}+{ }^{3} e_{(b) s}{ }^{3} \tilde{\pi}_{(a)}^{s}\right], \\
{ }^{3} \tilde{\pi}_{(a)}^{r} & \partial_{\tau}{ }^{3} e_{(a) r} \equiv \frac{1}{2}\left[{ }^{3} e_{(a) s}{ }^{3} \tilde{\pi}_{(b)}^{s}+{ }^{3} e_{(b) s}{ }^{3} \tilde{\pi}_{(a)}^{s}\right]^{3} e_{(b)}^{r} \partial_{\tau}{ }^{3} e_{(a) r} \equiv \\
& \equiv{ }^{3} \tilde{\pi}_{(a)}^{r} N_{(a) \mid r}-\frac{4 \pi G N^{3}}{c^{3}{ }^{3} e} G_{o(a)(b)(c)(d)}{ }^{3} e_{(a) s}{ }^{3} \tilde{\pi}_{(b)}^{s}{ }^{3} e_{(c) r}{ }^{3} \tilde{\pi}_{(d)}^{r} .
\end{aligned}
$$

\footnotetext{
${ }^{33}$ They are of the type of SO(3) Yang-Mills Gauss laws, because they are the covariant divergence of a vector density.
} 
After ignoring a surface term (see next Subsection) we get the Dirac Hamiltonian (the $\lambda(\tau, \vec{\sigma})$ 's are arbitrary Dirac multipliers)

$$
\begin{aligned}
\hat{H}_{(D)}= & \hat{H}_{(c)}+\int d^{3} \sigma\left[\lambda_{N} \tilde{\pi}^{N}+\lambda_{(a)}^{\vec{N}} \tilde{\pi}_{(a)}^{\vec{N}}+\lambda_{(a)}^{\vec{\varphi}} \tilde{\pi}_{(a)}^{\vec{\varphi}}+\mu_{(a)}{ }^{3} \tilde{M}_{(a)}\right](\tau, \vec{\sigma})= \\
= & \hat{H}_{(c)}^{\prime}+\int d^{3} \sigma\left[\lambda_{N} \tilde{\pi}^{N}+\lambda_{(a)}^{\vec{N}} \tilde{\pi}_{(a)}^{\vec{N}}+\lambda_{(a)}^{\vec{\varphi}} \tilde{\pi}_{(a)}^{\vec{\varphi}}+\hat{\mu}_{(a)}{ }^{3} \tilde{M}_{(a)}\right](\tau, \vec{\sigma}), \\
& \hat{H}_{c}=\int d^{3} \sigma\left[N \hat{\mathcal{H}}-N_{(a)} \hat{\mathcal{H}}_{(a)}\right](\tau, \vec{\sigma}), \\
& \hat{H}_{(c)}^{\prime}=\int d^{3} \sigma\left[N \hat{\mathcal{H}}+N_{(a)}{ }^{3} e_{(a)}^{r}{ }^{3} \tilde{\Theta}_{r}\right](\tau, \vec{\sigma}),
\end{aligned}
$$

where we replaced $\left[\mu_{(a)}-N_{(b)}{ }^{3} e_{(b)}^{r}{ }^{3} \omega_{r(a)}\right](\tau, \vec{\sigma})$ with the new Dirac multipliers $\hat{\mu}_{(a)}(\tau, \vec{\sigma})$.

All the constraints are first class because the only non-identically vanishing Poisson brackets are

$$
\begin{aligned}
\left\{{ }^{3} \tilde{M}_{(a)}(\tau, \vec{\sigma}),{ }^{3} \tilde{M}_{(b)}\left(\tau, \vec{\sigma}^{\prime}\right)\right\} & =\epsilon_{(a)(b)(c)}{ }^{3} \tilde{M}_{(c)}(\tau, \vec{\sigma}) \delta^{3}\left(\vec{\sigma}, \vec{\sigma}^{\prime}\right), \\
\left\{{ }^{3} \tilde{M}_{(a)}(\tau, \vec{\sigma}),{ }^{3} \tilde{\Theta}_{r}\left(\tau, \vec{\sigma}^{\prime}\right)\right\} & ={ }^{3} \tilde{M}_{(a)}\left(\tau, \vec{\sigma}^{\prime}\right) \frac{\partial \delta^{3}\left(\vec{\sigma}, \vec{\sigma}^{\prime}\right)}{\partial \sigma^{r}}, \\
\left\{{ }^{3} \tilde{\Theta}_{r}(\tau, \vec{\sigma}),{ }^{3} \tilde{\Theta}_{s}\left(\tau, \vec{\sigma}^{\prime}\right)\right\} & =\left[{ }^{3} \tilde{\Theta}_{r}\left(\tau, \vec{\sigma}^{\prime}\right) \frac{\partial}{\partial \sigma^{s}}+{ }^{3} \tilde{\Theta}_{s}(\tau, \vec{\sigma}) \frac{\partial}{\partial \sigma^{r}}\right] \delta^{3}\left(\vec{\sigma}, \vec{\sigma}^{\prime}\right), \\
\left\{\hat{\mathcal{H}}(\tau, \vec{\sigma}),{ }^{3} \tilde{\Theta}_{r}\left(\tau, \vec{\sigma}^{\prime}\right)\right\} & =\hat{\mathcal{H}}\left(\tau, \vec{\sigma}^{\prime}\right) \frac{\partial \delta^{3}\left(\vec{\sigma}, \vec{\sigma}^{\prime}\right)}{\partial \sigma^{r}}, \\
\left\{\hat{\mathcal{H}}(\tau, \vec{\sigma}), \hat{\mathcal{H}}\left(\tau, \vec{\sigma}^{\prime}\right)\right\} & =\left[{ }^{3} e_{(a)}^{r}(\tau, \vec{\sigma}) \hat{\mathcal{H}}(a)(\tau, \vec{\sigma})+\right. \\
& \left.+{ }^{3} e_{(a)}^{r}\left(\tau, \vec{\sigma}^{\prime}\right) \hat{\mathcal{H}}_{(a)}\left(\tau, \vec{\sigma}^{\prime}\right)\right] \frac{\partial \delta^{3}\left(\vec{\sigma}, \vec{\sigma}^{\prime}\right)}{\partial \sigma^{r}}= \\
& =\left\{\left[{ }^{3} e_{(a)}^{r}{ }^{3} e_{(a)}^{s}\left[{ }^{3} \tilde{\Theta}_{s}+{ }^{3} \omega_{s(b)}{ }^{3} \tilde{M}_{(b)}\right]\right](\tau, \vec{\sigma})+\right. \\
& \left.+\left[{ }^{3} e_{(a)}^{r}{ }^{3} e_{(a)}^{s}\left[{ }^{3} \tilde{\Theta}_{s}+{ }^{3} \omega_{s(b)}{ }^{3} \tilde{M}_{(b)}\right]\right]\left(\tau, \vec{\sigma}^{\prime}\right)\right\} \frac{\partial \delta^{3}\left(\vec{\sigma}, \vec{\sigma}^{\prime}\right)}{\partial \sigma^{r}} .
\end{aligned}
$$

The Hamiltonian gauge group has the 14 first class constraints as generators of infinitesimal Hamiltonian gauge transformations connected with the identity. In particular $\tilde{\pi}_{(a)}^{\vec{\varphi}}(\tau, \vec{\sigma}) \approx 0$ and ${ }^{3} \tilde{M}_{(a)}(\tau, \vec{\sigma}) \approx 0$ are the generators of the $R^{3} \times S O(3)$ subgroup replacing the Lorentz subgroup with our parametrization, while ${ }^{3} \tilde{\Theta}_{r}(\tau, \vec{\sigma}) \approx 0$ are the generators of the infinitesimal pseudo-diffeomorphisms in Diff $\Sigma_{\tau}$.

\section{B. Review of the Rest-Frame Instant Form of Metric Gravity.}

In Ref. [25] and in the book in Ref. [7] (see also Ref. [26]), Dirac introduced asymptotic Minkowski Cartesian coordinates

$$
z_{(\infty)}^{(\mu)}(\tau, \vec{\sigma})=x_{(\infty)}^{(\mu)}(\tau)+b_{(\infty) \check{r}}^{(\mu)}(\tau) \sigma^{\check{r}}
$$


in $M^{4}$ at spatial infinity $S_{\infty}=\cup_{\tau} S_{\tau, \infty}^{2}$ 蛋. For each value of $\tau$, the coordinates $x_{(\infty)}^{(\mu)}(\tau)$ labels an arbitrary point, near spatial infinity chosen as origin. On it there is a flat tetrad $b_{(\infty) A}^{(\mu)}(\tau)=\left(_{(\infty)}^{(\mu)}=b_{(\infty) \tau}^{(\mu)}=\epsilon^{(\mu)}{ }_{(\alpha)(\beta)(\gamma)} b_{(\infty) \grave{1}}^{(\alpha)}(\tau) b_{(\infty) \check{2}}^{(\beta)}(\tau) b_{(\infty) \check{3}}^{(\gamma)}(\tau) ; b_{(\infty) \check{r}}^{(\mu)}(\tau)\right)$, with $l_{(\infty)}^{(\mu)} \tau$-independent, satisfying $b_{(\infty) A}^{(\mu)}{ }^{4} \eta_{(\mu)(\nu)} b_{(\infty) B}^{(\nu)}={ }^{4} \eta_{A B}$ for every $\tau$. There will be transformation coefficients $b_{A}^{\mu}(\tau, \vec{\sigma})$ from the adapted coordinates $\sigma^{A}=\left(\tau, \sigma^{\check{r}}\right)$ to the coordinates $x^{\mu}=z^{\mu}\left(\sigma^{A}\right)$ in an atlas of $M^{4}$, such that in a chart at spatial infinity one has $z^{\mu}(\tau, \vec{\sigma}) \rightarrow \delta_{(\mu)}^{\mu} z_{(\infty)}^{(\mu)}(\tau, \vec{\sigma})$ and $b_{A}^{\mu}(\tau, \vec{\sigma}) \rightarrow \delta_{(\mu)}^{\mu} b_{(\infty) A}^{(\mu)}(\tau)$ 阿. The atlas $\mathcal{C}$ of the allowed coordinate systems of $M^{4}$ is assumed to have this property.

Dirac [25] and, then, Regge and Teitelboim [26] proposed that the asymptotic Minkowski Cartesian coordinates $z_{(\infty)}^{(\mu)}(\tau, \vec{\sigma})=x_{(\infty)}^{(\mu)}(\tau)+b_{(\infty) \check{r}}^{(\mu)}(\tau) \sigma^{\check{r}}$ should define 10 new independent degrees of freedom at the spatial boundary $S_{\infty}$ (with ten associated conjugate momenta), as it happens for Minkowski parametrized theories [15, 10, 16, 27,28] (see Appendix A of Ref. [6] for a review), when the extra configurational variables $z^{(\mu)}(\tau, \vec{\sigma})$ are reduced to 10 degrees of freedom by the restriction to spacelike hyperplanes, defined by $z^{(\mu)}(\tau, \vec{\sigma}) \approx x_{s}^{(\mu)}(\tau)+b_{\tilde{r}}^{(\mu)}(\tau) \sigma^{\check{r}}$.

In Dirac's approach to metric gravity the 20 extra variables of the Dirac proposal can be chosen as the set: $x_{(\infty)}^{(\mu)}(\tau), p_{(\infty)}^{(\mu)}, b_{(\infty) A}^{(\mu)}(\tau)$ 四, $S_{(\infty)}^{(\mu)(\nu)}$, with the non-vanishing Dirac brackets $\left\{b_{A}^{(\rho)}, S_{(\infty)}^{(\mu)(\nu)}\right\}={ }^{4} \eta^{(\rho)(\mu)} b_{A}^{(\nu)}-{ }^{4} \eta^{(\rho)(\nu)} b_{A}^{(\mu)},\left\{S_{(\infty)}^{(\mu)(\nu)}, S_{(\infty)}^{(\alpha)(\beta)}\right\}=C_{(\gamma)(\delta)}^{(\mu)(\nu)(\beta)(\beta)} S_{(\infty)}^{(\gamma)(\delta)}$, of Ref. [29, 15], implying the orthonormality constraints $b_{(\infty) A}^{(\mu)}{ }^{4} \eta_{(\mu)(\nu)} b_{(\infty) B}^{(\nu)}={ }^{4} \eta_{A B}$. Moreover, $p_{(\infty)}^{(\mu)}$ and $J_{(\infty)}^{(\mu)(\nu)}=x_{(\infty)}^{(\mu)} p_{(\infty)}^{(\nu)}-x_{(\infty)}^{(\nu)} p_{(\infty)}^{(\mu)}+S_{(\infty)}^{(\mu)(\nu)}$ satisfy a Poincaré algebra. In analogy with Minkowski parametrized theories restricted to spacelike hyperplanes, one introduces 10 extra first class constraints of the type

$$
p_{(\infty)}^{(\mu)}-P_{A D M}^{(\mu)} \approx 0, \quad S_{(\infty)}^{(\mu)(\nu)}-S_{A D M}^{(\mu)(\nu)} \approx 0,
$$

with $P_{A D M}^{(\mu)}, S_{A D M}^{(\mu)(\nu)}$ related to the ADM Poincaré charges [5] $P_{A D M}^{A}, J_{A D M}^{A B}$ and 10 extra Dirac multipliers $\tilde{\lambda}_{(\mu)}(\tau), \tilde{\lambda}_{(\mu)(\nu)}(\tau)$, in front of them in the Dirac Hamiltonian. The origin $x_{(\infty)}^{(\mu)}(\tau)$ is going to play the role of an external decoupled observer with his parametrized clock.

If we replace $p_{(\infty)}^{(\mu)}$ and $S_{(\infty)}^{(\mu)(\nu)}$, whose Poisson algebra is the direct sum of an Abelian algebra of translations and of a Lorentz algebra, with the new variables (with indices adapted to $\Sigma_{\tau}$ )

$$
p_{(\infty)}^{A}=b_{(\infty)(\mu)}^{A} p_{(\infty)}^{(\mu)}, \quad J_{(\infty)}^{A B} \stackrel{\text { def }}{=} b_{(\infty)(\mu)}^{A} b_{(\infty)(\nu)}^{B} S_{(\infty)}^{(\mu)(\nu)}\left[\neq b_{(\infty)(\mu)}^{A} b_{(\infty)(\nu)}^{B} J_{(\infty)}^{(\mu)(\nu)}\right],
$$

the Poisson brackets for $p_{(\infty)}^{(\mu)}, b_{(\infty) A}^{(\mu)}, S_{(\infty)}^{(\mu)(\nu)}$, imply

\footnotetext{
${ }^{34}$ Here $\left\{\sigma^{\check{r}}\right\}$ are global coordinate charts of the atlas $\mathcal{C}_{\tau}$ of $\Sigma_{\tau}$, not matching the spatial coordinates $z_{(\infty)}^{(i)}(\tau, \vec{\sigma})$.

${ }^{35}$ For $r \rightarrow \infty$ one has ${ }^{4} g_{\mu \nu} \rightarrow \delta_{\mu}^{(\mu)} \delta_{\nu}^{(\nu)}{ }^{4} \eta_{(\mu)(\nu)}$ and ${ }^{4} g_{A B}=b_{A}^{\mu}{ }^{4} g_{\mu \nu} b_{B}^{\nu} \rightarrow b_{(\infty) A}^{(\mu)}{ }^{4} \eta_{(\mu)(\nu)} b_{(\infty) B}^{(\nu)}=$ ${ }^{4} \eta_{A B}$

${ }^{36}$ With $b_{(\infty) \tau}^{(\mu)}=l_{(\infty)}^{(\mu)} \tau$-independent and coinciding with the asymptotic normal to $\Sigma_{\tau}$, tangent to $S_{\infty}$
} 


$$
\begin{aligned}
& \left\{p_{(\infty)}^{A}, p_{(\infty)}^{B}\right\}=0, \\
& \left\{p_{(\infty)}^{A}, J_{(\infty)}^{B C}\right\}={ }^{4} g_{(\infty)}^{A C} p_{(\infty)}^{B}-{ }^{4} g_{(\infty)}^{A B} p_{(\infty)}^{C}, \\
& \left\{J_{(\infty)}^{A B}, J_{(\infty)}^{C D}\right\}=-\left(\delta_{E}^{B} \delta_{F}^{C}{ }^{4} g_{(\infty)}^{A D}+\delta_{E}^{A} \delta_{F}^{D}{ }^{4} g_{(\infty)}^{B C}-\delta_{E}^{B} \delta_{F}^{D}{ }^{4} g_{(\infty)}^{A C}-\delta_{E}^{A} \delta_{F}^{C}{ }^{4} g_{(\infty)}^{B D}\right) J_{(\infty)}^{E F}= \\
& =-C_{E F}^{A B C D} J_{(\infty)}^{E F},
\end{aligned}
$$

Therefore, we get the algebra of a realization of the Poincaré group (this explains the notation $\left.J_{(\infty)}^{A B}\right)$ with all the structure constants inverted in the sign (transition from a left to a right action).

This implies that, after the transition to the asymptotic Dirac Cartesian coordinates the Poincaré generators $P_{A D M}^{A}, J_{A D M}^{A B}$ in $\Sigma_{\tau}$-adapted coordinates should become a momentum $P_{A D M}^{(\mu)}=b_{(\infty) A}^{(\mu)} P_{A D M}^{A}$ and only an $\mathrm{ADM}$ spin tensor $S_{A D M}^{(\mu)(\nu)}$ ఛ7.

As shown in Ref. [6], the first problem with the ADM metric gravity Hamiltonian, whose canonical part contains the superhamiltonian and supermomentum secondary first class constraints, is that it must be finite and differentiable [31]. Since in front of the secondary constraints there are the lapse and shift functions, they are the parameters of the gauge transformations generated by these constraints. To separate the improper gauge transformations from the proper ones (like in Yang-Mills theory [17]), we shall assume the existence of a global coordinate system $\left\{\sigma^{\check{r}}\right\}$ on $\Sigma_{\tau}$, in which we introduce the following decomposition of the lapse and shift functions isolating their asymptotic part from the bulk one

$$
\begin{aligned}
N(\tau, \vec{\sigma}) & =N_{(a s)}(\tau, \vec{\sigma})+m(\tau, \vec{\sigma}), \\
N_{\check{r}}(\tau, \vec{\sigma}) & =N_{(a s) \check{r}}(\tau, \vec{\sigma})+m_{\check{r}}(\tau, \vec{\sigma}), \\
N_{(a s)}(\tau, \vec{\sigma}) & =-\tilde{\lambda}_{(\mu)}(\tau) l_{(\infty)}^{(\mu)}-l_{(\infty)}^{(\mu)} \tilde{\lambda}_{(\mu)(\nu)}(\tau) b_{(\infty) \check{s}}^{(\nu)}(\tau) \sigma^{\check{s}}= \\
& =-\tilde{\lambda}_{\tau}(\tau)-\frac{1}{2} \tilde{\lambda}_{\tau \check{s}}(\tau) \sigma^{\check{s}}, \\
N_{(a s) \check{r}}(\tau, \vec{\sigma}) & =-b_{(\infty) \check{r}}^{(\mu)}(\tau) \tilde{\lambda}_{(\mu)}(\tau)-b_{(\infty) \check{r}}^{(\mu)}(\tau) \tilde{\lambda}_{(\mu)(\nu)}(\tau) b_{(\infty) \check{s}}^{(\nu)}(\tau) \sigma^{\check{s}}= \\
& =-\tilde{\lambda}_{\check{r}}(\tau)-\frac{1}{2} \tilde{\lambda}_{\check{r} \check{s}}(\tau) \sigma^{\check{s}},
\end{aligned}
$$

with $m(\tau, \vec{\sigma}), m_{\check{r}}(\tau, \vec{\sigma})$, given by Eqs.(3.8) of Ref. [6] $]^{38}$ and with the asymptotic parts $N_{(a s)}$,

\footnotetext{
${ }^{37}$ To define an angular momentum tensor $J_{A D M}^{(\mu)(\nu)}$ one should find an external center of mass of the gravitational field $\left.X_{A D M}^{(\mu)}{ }^{3} g,{ }^{3} \tilde{\Pi}\right]$ (see Ref. 30,28 for the Klein-Gordon case) conjugate to $P_{A D M}^{(\mu)}$, so that $J_{A D M}^{(\mu)(\nu)}=X_{A D M}^{(\mu)} P_{A D M}^{(\nu)}-X_{A D M}^{(\nu)} P_{A D M}^{(\mu)}+S_{A D M}^{(\mu)(\nu)}$.

${ }^{38}$ They were obtained in Ref. [31] to ensure the differentiability of the Dirac Hamiltonian of metric gravity. They satisfy the parity conditions but in general they still contain odd supertranslations (direction-dependent translations, which are an obstacle to the definition of angular momentum in general relativity as shown in the review part of Ref. [6]). Let us remark that the restriction (2.19) on the lapse and shift functions of metric gravity is analogous to the one introduced for the gauge parameters of the gauge transformations generated by the Yang-Mills Gauss laws in gauge theories 17] (see Eq.(3.1) of Ref. [6]).
} 
$N_{(a s) \check{r}}$ equal to the lapse and shift functions associated with Minkowski hyperplanes $\$$.

This very strong assumption implies that one is selecting asymptotically at spatial infinity only coordinate systems in which the lapse and shift functions have behaviours similar to those of Minkowski spacelike hyperplanes, so that the allowed foliations of the $3+1$ splittings of the spacetime $M^{4}$ are restricted to have the leaves $\Sigma_{\tau}$ approaching these Minkowski hyperplanes at spatial infinity. But this is coherent with Dirac's choice of asymptotic Cartesian coordinates (modulo 3-pseudo-diffeomorphisms not changing the nature of the coordinates, namely tending to the identity at spatial infinity like in Ref. [32]). The request that the decomposition (2.19) holds in all the allowed coordinate systems of $M^{4}$ is also needed to eliminate coordinate transformations not becoming the identity at spatial infinity, which are not associated with the gravitational fields of isolated systems [33].

By replacing the ADM configuration variables $N(\tau, \vec{\sigma})$ and $N_{\check{r}}(\tau, \vec{\sigma})$ with the new ones $\tilde{\lambda}_{A}(\tau)=\left\{\tilde{\lambda}_{\tau}(\tau) ; \tilde{\lambda}_{\check{r}}(\tau)\right\}, \tilde{\lambda}_{A B}(\tau)=-\tilde{\lambda}_{B A}(\tau), n(\tau, \vec{\sigma}), n_{\check{r}}(\tau, \vec{\sigma})$ 四 inside the ADM Lagrangian, one only gets the replacement of the primary first class constraints of ADM metric gravity

$$
\tilde{\pi}^{N}(\tau, \vec{\sigma}) \approx 0, \quad \tilde{\pi}_{\vec{N}}^{\check{r}}(\tau, \vec{\sigma}) \approx 0,
$$

with the new first class constraints

$$
\tilde{\pi}^{n}(\tau, \vec{\sigma}) \approx 0, \quad \tilde{\pi}_{\vec{n}}^{\check{r}}(\tau, \vec{\sigma}) \approx 0, \quad \tilde{\pi}^{A}(\tau) \approx 0, \quad \tilde{\pi}^{A B}(\tau)=-\tilde{\pi}^{B A}(\tau) \approx 0,
$$

corresponding to the vanishing of the canonical momenta $\tilde{\pi}^{A}, \tilde{\pi}^{A B}$ conjugate to the new configuration variables $\boxplus$. The only change in the Dirac Hamiltonian of metric gravity $H_{(D) A D M}=H_{(c) A D M}+\int d^{3} \sigma\left[\lambda_{N} \tilde{\pi}^{N}+\lambda_{\tilde{r}}^{\vec{N}} \tilde{\pi}_{\vec{N}}^{\check{R}}\right](\tau, \vec{\sigma}), H_{(c) A D M}=\int d^{3} \sigma\left[N \tilde{\mathcal{H}}+N_{\check{r}} \tilde{\mathcal{H}}^{\check{r}}\right](\tau, \vec{\sigma})$ is

$$
\int d^{3} \sigma\left[\lambda_{N} \tilde{\pi}^{N}+\lambda_{\check{r}}^{\vec{N}} \tilde{\pi}_{\vec{N}}^{\check{r}}\right](\tau, \vec{\sigma}) \mapsto \zeta_{A}(\tau) \tilde{\pi}^{A}(\tau)+\zeta_{A B}(\tau) \tilde{\pi}^{A B}(\tau)+\int d^{3} \sigma\left[\lambda_{n} \tilde{\pi}^{n}+\lambda_{\check{r}}^{\vec{n}} \tilde{\pi}_{\vec{n}}^{\check{r}}\right](\tau, \vec{\sigma}),
$$

39

In general, there is the problem that in the gauges where $\tilde{\lambda}_{(\mu)(\nu)}(\tau)$ are different from zero the foliations with leaves $\Sigma_{\tau}$ associated to arbitrary $3+1$ splittings of Minkowski spacetime are geometrically ill-defined at spatial infinity so that the variational principle describing the isolated system could make sense only for those $3+1$ splittings having these part of the Dirac's multipliers vanishing. The problem is that, since on hyperplanes $i^{(\mu)}=0$ and $l^{(\mu)} b_{\breve{r}(\mu)}(\tau)=0$ imply $l^{(\mu)} \dot{b}_{\breve{r}(\mu)}(\tau)=0$, then the analogue of Eqs.(2.19) implies $\tilde{\lambda}_{\tau \tilde{r}}(\tau)=0$ (i.e. only three $\tilde{\lambda}_{(\mu)(\nu)}(\tau)$ independent) on spacelike hyperplane, because otherwise Lorentz boosts can create crossing of the leaves of the foliation. This points toward the necessity of making the reduction from arbitrary spacelike hypersurfaces either directly to the Wigner hyperplanes or to spacelike hypersurfaces approaching asymptotically Wigner hyperplanes.

${ }^{40} \mathrm{We}$ are using the notation $n, n_{\check{r}}$ for $m, m_{\check{r}}$, because after the elimination of supertranslation this the notation used in the rest of the paper.

${ }^{41}$ We assume the Poisson brackets $\left\{\tilde{\lambda}_{A}(\tau), \tilde{\pi}^{B}(\tau)\right\}=\delta_{A}^{B},\left\{\tilde{\lambda}_{A B}(\tau), \tilde{\pi}^{C D}(\tau)\right\}=\delta_{A}^{C} \delta_{B}^{D}-\delta_{A}^{D} \delta_{B}^{C}$. 
with $\zeta_{A}(\tau), \zeta_{A B}(\tau)$ Dirac's multipliers.

The presence of the terms $N_{(a s)}, N_{(a s) \check{r}}$ in Eq.(2.19) makes $H_{D}$ not differentiable. In Refs, 26, 31], following Refs. [35, 36, it is shown that the differentiability of the ADM canonical Hamiltonian requires the introduction of a surface term $H_{\infty}{ }^{42}\left[H_{(c) A D M} \rightarrow \hat{H}_{(c) A D M}+H_{\infty}\right]$. By putting $N=N_{(a s)}, N_{\check{r}}=N_{(a s) \check{r}}$ into these surface integrals, the added term $H_{\infty}$ becomes the linear combination $\tilde{\lambda}_{A}(\tau) P_{A D M}^{A}+\frac{1}{2} \tilde{\lambda}_{A B}(\tau) J_{A D M}^{A B}$ of the strong ADM Poincaré charges $P_{A D M}^{A}, J_{A D M}^{A B}$ [26,31] first identified in the linearized theory [5] (they are the analogue of the strong Yang-Mills non-Abelian charges [17]):

$$
\begin{aligned}
& P_{A D M}^{\tau}=\frac{\epsilon c^{3}}{16 \pi G} \int_{S_{\tau, \infty}^{2}} d^{2} \Sigma_{\check{u}}\left[\sqrt{\gamma}^{3} g^{\check{u} \check{v} 3} g^{\check{r} \check{s}}\left(\partial_{\check{r}}^{3} g_{\check{v} \check{s}}-\partial_{\tilde{v}}{ }^{3} g_{\check{r} \check{s}}\right)\right](\tau, \vec{\sigma}), \\
& P_{A D M}^{\check{r}}=-2 \int_{S_{\tau, \infty}^{2}} d^{2} \Sigma_{\check{u}}^{3} \tilde{\Pi}^{\check{r} \check{u}}(\tau, \vec{\sigma}), \\
& J_{A D M}^{\tau \check{r}}=\frac{\epsilon c^{3}}{16 \pi G} \int_{S_{\tau, \infty}^{2}} d^{2} \Sigma_{\check{u}} \sqrt{\gamma}^{3} g^{\check{u} \check{v} 3} g^{\check{n} \check{s}} . \\
& \text { - }\left[\sigma^{\check{r}}\left(\partial_{\check{n}}^{3} g_{\check{v} \check{s}}-\partial_{\check{v}}{ }^{3} g_{\check{n} \check{s}}\right)+\delta_{\check{v}}^{\check{r}}\left({ }^{3} g_{\check{n} \check{s}}-\delta_{\check{n} \check{s})}\right) \delta_{\check{n}}^{\check{r}}\left({ }^{3} g_{\check{s} \check{v}}-\delta_{\check{s} \check{v}}\right)\right](\tau, \vec{\sigma}), \\
& J_{A D M}^{\check{r} \breve{s}}=\int_{S_{\tau, \infty}^{2}} d^{2} \Sigma_{\breve{u}}\left[\sigma^{\check{r} 3} \tilde{\Pi}^{\check{s} \breve{u}}-\sigma^{\check{s} 3} \tilde{\Pi}^{\check{r} \check{u}}\right](\tau, \vec{\sigma}), \\
& P_{A D M}^{(\mu)}=l^{(\mu)} P_{A D M}^{\tau}+b_{(\infty) \check{r}}^{(\mu)}(\tau) P_{A D M}^{\check{r}}=b_{(\infty) A}^{(\mu)}(\tau) P_{A D M}^{A}, \\
& S_{A D M}^{(\mu)(\nu)}=\left[l_{(\infty)}^{(\mu)} b_{(\infty) \check{r}}^{(\nu)}(\tau)-l_{(\infty)}^{(\nu)} b_{(\infty) \check{r}}^{(\mu)}(\tau)\right] J_{A D M}^{\tau \check{r}}+ \\
& +\left[b_{(\infty) \check{r}}^{(\mu)}(\tau) b_{(\infty) \check{s}}^{(\nu)}(\tau)-b_{(\infty) \check{r}}^{(\nu)}(\tau) b_{(\infty) \check{s}}^{(\mu)}(\tau)\right] J_{A D M}^{\check{r} \breve{s}}= \\
& =\left[b_{(\infty) A}^{(\mu)}(\tau) b_{(\infty) B}^{(\nu)}(\tau)-b_{(\infty) A}^{(\nu)}(\tau) b_{(\infty) B}^{(\mu)}(\tau)\right] J_{A D M}^{A B} .
\end{aligned}
$$

Here $J_{A D M}^{\tau \check{r}}=-J_{A D M}^{\check{r} \tau}$ by definition and the inverse asymptotic tetrads are defined by $b_{(\infty)(\mu)}^{A} b_{(\infty) B}^{(\nu)}=\delta_{B}^{A}, b_{(\infty)(\mu)}^{A} b_{(\infty) A}^{(\nu)}=\delta_{(\mu)}^{(\nu)}$. As shown in Ref. 26, 31, the parity conditions of Ref. [31] are necessary to have a well defined and finite 3-angular-momentum $J_{A D M}^{\check{r} \breve{s}}$. Moreover in Ref. [31] it is noted that, with the boundary conditions of Refs. [26], a regularization of the boosts is needed.

In Ref. [6] it is shown that the surface term $H_{\infty}$ arises from a suitable splitting of the superhamiltonian and supermomentum constraints of metric gravity.

By using Eqs.(2.19) the modified canonical Hamiltonian becomes

$$
\begin{aligned}
\hat{H}_{(c) A D M} & =\int d^{3} \sigma\left[N \tilde{\mathcal{H}}+N_{\check{r}}^{3} \tilde{\mathcal{H}}^{\check{r}}\right](\tau, \vec{\sigma})= \\
& =\int d^{3} \sigma\left[\left(N_{(a s)}+m\right) \tilde{\mathcal{H}}+\left(N_{(a s) \check{r}}+m_{\check{r}}\right)^{3} \tilde{\mathcal{H}}^{\check{r}}\right](\tau, \vec{\sigma}) \mapsto \\
\mapsto \hat{H}_{(c) A D M}^{\prime} & =\hat{H}_{(c) A D M}^{\prime}\left[N, N^{\check{r}}\right]=\hat{H}_{(c) A D M}+H_{\infty}=
\end{aligned}
$$

\footnotetext{
${ }^{42}$ As shown in Ref. 34] $H_{\infty}$ contains the surface integrals neglected going from the Hilbert action to the ADM action and then from the ADM action to the ADM canonical Hamiltonian with the Legendre transformation.
} 


$$
\begin{aligned}
& =\int d^{3} \sigma\left[\left(N_{(a s)}+m\right) \tilde{\mathcal{H}}+\left(N_{(a s) \check{r}}+m_{\check{r}}\right)^{3} \tilde{\mathcal{H}}^{\check{r}}\right](\tau, \vec{\sigma})+ \\
& +\tilde{\lambda}_{(\mu)}(\tau) P_{A D M}^{(\mu)}+\tilde{\lambda}_{(\mu)(\nu)}(\tau) S_{A D M}^{(\mu)(\nu)}= \\
& =\int d^{3} \sigma\left[\left(N_{(a s)}+m\right) \tilde{\mathcal{H}}+\left(N_{(a s) \check{r}}+m_{\check{r}}\right)^{3} \tilde{\mathcal{H}}^{\check{r}}\right](\tau, \vec{\sigma})+ \\
& +\tilde{\lambda}_{A}(\tau) P_{A D M}^{A}+\frac{1}{2} \tilde{\lambda}_{A B}(\tau) J_{A D M}^{A B}= \\
& =\int d^{3} \sigma\left[m \tilde{\mathcal{H}}+m_{\check{r}}^{3} \tilde{\mathcal{H}}^{\check{r}}\right](\tau, \vec{\sigma})+\tilde{\lambda}_{(\mu)}(\tau) \hat{P}_{A D M}^{(\mu)}+\tilde{\lambda}_{(\mu)(\nu)}(\tau) \hat{S}_{A D M}^{(\mu)(\nu)}= \\
& =\int d^{3} \sigma\left[m \tilde{\mathcal{H}}+m_{\check{r}}{ }^{3} \tilde{\mathcal{H}}^{\check{r}}\right](\tau, \vec{\sigma})+\tilde{\lambda}_{A}(\tau) \hat{P}_{A D M}^{A}+\frac{1}{2} \tilde{\lambda}_{A B}(\tau) \hat{J}_{A D M}^{A B}, \\
\hat{H}_{(D) A D M}^{\prime} & =\hat{H}_{(c) A D M}^{\prime}\left[m, m^{\check{r}}\right]+ \\
& +\int d^{3} \sigma\left[\lambda_{n} \tilde{\pi}^{n}+\lambda_{r}^{\vec{n}} \tilde{\pi}_{\vec{n}}^{r}\right](\tau, \vec{\sigma})+\zeta_{A}(\tau) \tilde{\pi}^{A}(\tau)+\zeta_{A B}(\tau) \tilde{\pi}^{A B}(\tau) .
\end{aligned}
$$

In the last expression we introduced the weak conserved improper Poincaré charges $\hat{P}_{A D M}^{A}$, $\hat{J}_{A D M}^{A B} 43$

$$
\begin{aligned}
& \hat{P}_{A D M}^{\tau}=\int d^{3} \sigma \epsilon\left[\frac{c^{3}}{16 \pi G} \sqrt{\gamma}{ }^{3} g^{\check{r} \check{s}}\left({ }^{3} \Gamma_{\check{r} \check{v}}^{\check{u}}{ }^{3} \Gamma_{\check{s} \check{u}}^{\check{v}}-{ }^{3} \Gamma_{\check{r} \check{s}}^{\check{u}}{ }^{3} \Gamma_{\check{v} \check{u}}^{\check{v}}\right)-\right. \\
& \left.-\frac{8 \pi G}{c^{3} \sqrt{\gamma}}{ }^{3} G_{\check{r} \check{s} \check{u} \check{v}}{ }^{3} \tilde{\Pi}^{\check{r} \check{s} 3} \tilde{\Pi}^{\check{u} \check{v}}\right](\tau, \vec{\sigma}), \\
& \hat{P}_{A D M}^{\check{r}}=-2 \int d^{3} \sigma^{3} \Gamma_{\check{s} \check{u}}^{\check{r}}(\tau, \vec{\sigma})^{3} \tilde{\Pi}^{\check{s} \check{u}}(\tau, \vec{\sigma}), \\
& \hat{J}_{A D M}^{\tau \check{r}}=-\hat{J}_{A D M}^{\check{r} \tau}=\int d^{3} \sigma \epsilon\left\{\sigma^{\check{r}}\right.
\end{aligned}
$$

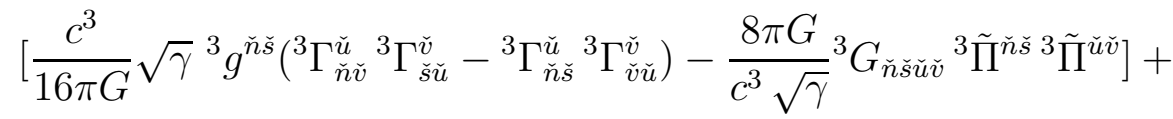

$$
\begin{aligned}
& \left.+\frac{c^{3}}{16 \pi G} \delta_{\breve{u}}^{\check{r}}\left({ }^{3} g_{\check{v} \check{s}}-\delta_{\check{v} \check{s}}\right) \partial_{\check{n}}\left[\sqrt{\gamma}\left({ }^{3} g^{\check{n} \check{s} 3} g^{\check{u} \check{v}}-{ }^{3} g^{\check{n} \check{u} 3} g^{\check{s} \check{v}}\right)\right]\right\}(\tau, \vec{\sigma}), \\
& \hat{J}_{A D M}^{\check{r} \check{s}}=\int d^{3} \sigma\left[\left(\sigma^{\check{r} 3} \Gamma_{\check{u} \check{v}}^{\check{s}}-\sigma^{\check{s} 3} \Gamma_{\check{u} \check{v}}^{\check{r}}\right)^{3} \tilde{\Pi}^{\check{u} \check{v}}\right](\tau, \vec{\sigma}), \\
& \hat{P}_{A D M}^{(\mu)}=l_{(\infty)}^{(\mu)} \hat{P}_{A D M}^{\tau}+b_{(\infty) \check{r}}^{(\mu)}(\tau) \hat{P}_{A D M}^{\check{r}}=b_{(\infty) A}^{(\mu)}(\tau) \hat{P}_{A D M}^{A}, \\
& \hat{S}_{A D M}^{(\mu)(\nu)}=\left[l_{(\infty)}^{(\mu)} b_{(\infty) \check{r}}^{(\nu)}(\tau)-l_{(\infty)}^{(\nu)} b_{(\infty) \check{r}}^{(\mu)}(\tau)\right] \hat{J}_{A D M}^{\tau \check{r}}+ \\
& +\left[b_{(\infty) \check{r}(}^{(\mu)}(\tau) b_{(\infty) \check{s}}^{(\nu)}(\tau)-b_{(\infty) \check{r}}^{(\nu)}(\tau) b_{(\infty) \check{s}}^{(\mu)}(\tau)\right] \hat{J}_{A D M}^{\check{r} \check{s}}= \\
& =\left[b_{(\infty) A}^{(\mu)} b_{(\infty) B}^{(\nu)}-b_{(\infty) A}^{(\nu)} b_{(\infty) B}^{(\mu)}\right](\tau) \hat{J}_{A D M}^{A B}, \\
& P_{A D M}^{\tau}=\hat{P}_{A D M}^{\tau}+\int d^{3} \sigma \tilde{\mathcal{H}}(\tau, \vec{\sigma}) \approx \hat{P}_{A D M}^{\tau},
\end{aligned}
$$

${ }^{43}$ These volume expressions (the analogue of the weak Yang-Mills non Abelian charges) for the ADM 4-momentum are used in Ref. [37] in the study of the positiviteness of the energy; the weak charges are Noether charges. 


$$
\begin{aligned}
& P_{A D M}^{\check{r}}=\hat{P}_{A D M}^{\check{r}}+\int d^{3} \sigma^{3} \tilde{\mathcal{H}}^{\check{r}}(\tau, \vec{\sigma}) \approx \hat{P}_{A D M}^{\check{r}}, \\
& J_{A D M}^{\tau \check{r}}=\hat{J}_{A D M}^{\tau \check{r}}+\frac{1}{2} \int d^{3} \sigma \sigma^{\check{r}} \tilde{\mathcal{H}}(\tau, \vec{\sigma}) \approx \hat{J}_{A D M}^{\tau \check{r}}, \\
& J_{A D M}^{\check{r} \check{s}}=\hat{J}_{A D M}^{\check{r} \check{s}}+\int d^{3} \sigma\left[\sigma^{\check{s}} \tilde{\mathcal{H}}^{\check{r}}(\tau, \vec{\sigma})-\sigma^{\check{r}} \tilde{\mathcal{H}}^{\check{s}}(\tau, \vec{\sigma})\right] \approx \hat{J}_{A D M}^{\check{r} \check{s}} .
\end{aligned}
$$

In both Refs. 26, 31] it is shown that the canonical Hamiltonian $\hat{H}_{(c) A D M}^{\prime}\left[N, N^{\check{r}}\right]$ of Eq.(2.24) with general $N, N^{\check{r}}={ }^{3} g^{\check{r} \check{s}} N_{\check{s}}$ like the ones of Eqs.(2.19) (their asymptotic parts are the parameters of improper gauge transformations), has the same Poisson brackets as in the case of proper gauge transformations with $N=m, N^{\check{r}}=m^{\check{r}}$ (see the third and fourth line of Eqs.(2.14) for the universal Dirac algebra of the superhamiltonian and supermomentum constraints).

This implies [31.6]:

i) the Poisson brackets of two proper gauge transformations $\left[\tilde{\lambda}_{i A}=\tilde{\lambda}_{i A B}=0, \mathrm{i}=1,2\right]$ is a proper gauge transformation $\left[\tilde{\lambda}_{3 A}=\tilde{\lambda}_{3 A B}=0\right]$;

ii) if $N_{2}=m_{2}, N_{2 \check{r}}=m_{2 \check{r}}\left[\tilde{\lambda}_{2 A}=\tilde{\lambda}_{2 A B}=0\right]$ correspond to a proper gauge transformation and $N_{1}, N_{1 \check{r}}\left[m_{1}=m_{1 \check{r}}=0\right]$ to an improper one, then we get a proper gauge transformation $\left[\tilde{\lambda}_{3 A}=\tilde{\lambda}_{3 A B}=0, m_{3} \neq 0, m_{3 \check{r}} \neq 0\right]$ and this may be interpreted as saying that the 10 Poincaré charges are gauge invariant and Noether constants of motion.

iii) the Poisson bracket of two improper gauge transformations $\left[m_{i}=m_{i \check{r}}=0\right.$, i=1,2] is an improper gauge transformation $\left[\tilde{\lambda}_{3 A} \neq 0, \tilde{\lambda}_{3 A B} \neq 0, m_{3} \neq 0, m_{3 \check{r}} \neq 0\right]$. This implies that the 10 strong Poincaré charges (and, therefore, also the weak ones) satisfy the Poincaré algebra modulo the first class constraints, namely modulo the Hamiltonian group of gauge transformations

$$
\begin{aligned}
&\left\{\hat{P}_{A D M}^{A}, \hat{P}_{A D M}^{B}\right\}= 0, \\
&\left\{\hat{P}_{A D M}^{A}, \hat{J}_{A D M}^{B C}\right\} \approx{ }^{4} \eta^{A C} \hat{P}_{A D M}^{B}-{ }^{4} \eta^{A B} \hat{P}_{A D M}^{C}, \\
&\left\{\hat{J}_{A D M}^{A B}, \hat{J}_{A D M}^{C D}\right\} \approx-C_{E F}^{A B C D} \hat{J}_{A D M}^{E F}, \\
& \Downarrow \\
&\left\{P_{A D M}^{A}, P_{A D M}^{B}\right\} \approx 0, \\
&\left\{P_{A D M}^{A}, J_{A D M}^{B C}\right\} \approx{ }^{4} \eta^{A C} P_{A D M}^{B}-{ }^{4} \eta^{A B} P_{A D M}^{C}, \\
&\left\{J_{A D M}^{A B}, J_{A D M}^{C D}\right\} \approx-C_{E F}^{A B C D} J_{A D M}^{E F},
\end{aligned}
$$

in accord with Eqs. (2.18).

As shown in Ref. [6], the requirement of absence of supertranslations, implying the existence of a well defined asymptotic Poincaré group, may be satisfied by restricting all the fields to have a direction-independent limit at spatial infinity. Let us call $n(\tau, \vec{\sigma}), n_{\check{r}}(\tau, \vec{\sigma})$ the lapse and shift functions $m(\tau, \vec{\sigma}), m_{\check{r}}(\tau, \vec{\sigma})$ with such a behaviour. In a suitable class $\mathcal{C}$ of coordinate systems for $M^{4}$ asymptotic to Minkowski coordinates and with the general

\footnotetext{
${ }^{44}$ Then transformed to coordinates adapted to the $3+1$ splitting of $M^{4}$ with an allowed foliation with spacelike leaves $\Sigma_{\tau}$, whose allowed coordinates systems are in the previously defined atlas $\mathcal{C}_{\tau}$.
} 
coordinate transformations suitably restricted at spatial infinity so that it is not possible to go out this class (they are proper gauge transformations which do not introduce asymptotic angle-dependence), we must assume the following direction-independent boundary conditions for the ADM variables for $r \rightarrow \infty[\epsilon>0]$

$$
\begin{aligned}
& { }^{3} g_{\check{r} \check{s}}(\tau, \vec{\sigma})=\left(1+\frac{M}{r}\right) \delta_{\check{r} \check{s}}+{ }^{3} h_{\check{r} \check{s}}(\tau, \vec{\sigma}), \quad{ }^{3} h_{\check{r} \check{s}}(\tau, \vec{\sigma}) \rightarrow_{r \rightarrow \infty} O\left(r^{-(1+\epsilon)}\right), \\
& { }^{3} \tilde{\Pi}^{\check{r} \check{s}}(\tau, \vec{\sigma}) \rightarrow_{r \rightarrow \infty} O\left(r^{-(2+\epsilon)}\right), \\
& N(\tau, \vec{\sigma})=N_{(a s)}(\tau, \vec{\sigma})+n(\tau, \vec{\sigma}), \quad n(\tau, \vec{\sigma}) \rightarrow_{r \rightarrow \infty} O\left(r^{-(2+\epsilon)}\right), \\
& N_{\check{r}}(\tau, \vec{\sigma})=N_{(a s) \check{r}}(\tau, \vec{\sigma})+n_{\check{r}}(\tau, \vec{\sigma}), \quad n_{\check{r}}(\tau, \vec{\sigma}) \rightarrow_{r \rightarrow \infty} O\left(r^{-\epsilon}\right), \\
& N_{(a s)}(\tau, \vec{\sigma})=-\tilde{\lambda}_{\tau}(\tau)-\frac{1}{2} \tilde{\lambda}_{\tau \check{s}}(\tau) \sigma^{\check{s}} \\
& N_{(a s) \check{r}}(\tau, \vec{\sigma})=-\tilde{\lambda}_{\check{r}}(\tau)-\frac{1}{2} \tilde{\lambda}_{\check{r} \check{s}}(\tau) \sigma^{\check{s}}, \\
& \Rightarrow \quad N_{(a s) A}(\tau, \vec{\sigma}) \stackrel{\text { def }}{=}\left(N_{(a s)} ; N_{(a s) \check{r}}\right)(\tau, \vec{\sigma})=-\tilde{\lambda}_{A}(\tau)-\frac{1}{2} \tilde{\lambda}_{A \check{s}}(\tau) \sigma^{\check{s}},
\end{aligned}
$$

in accord with Regge-Teitelboim [26] and Beig-O'Murchadha [31].

This implies the vanishing of the ADM momentum, $P_{A D M}^{\check{r}}=0$, so that the elimination of supertranslations is connected with a definition of rest frame in the asymptotic Dirac coordinates $z_{(\infty)}^{(\mu)}(\tau, \vec{\sigma})$. Therefore, the previous boundary conditions on ${ }^{3} g,{ }^{3} \tilde{\Pi}$, are compatible and can be replaced with the Christodoulou-Klainermann ones [19], but in general with non vanishing shift functions.

The vanishing of the strong ADM 3-momentum $P_{A D M}^{\check{r}}=0$ and Eq.(2.25) imply

$$
\begin{aligned}
& \hat{P}_{A D M}^{\check{r}} \approx 0 \\
& P_{A D M}^{(\mu)}=b_{(\infty) \tau}^{(\mu)} P_{A D M}^{\tau}=l_{(\infty)}^{(\mu)} P_{A D M}^{\tau}, \\
& \hat{P}_{A D M}^{(\mu)} \approx l_{(\infty)}^{(\mu)} \hat{P}_{A D M}^{\tau} .
\end{aligned}
$$

Therefore, the boundary conditions (2.27) require three first class constraints implying the vanishing of the weak ADM 3-momentum as a rest frame condition.

Therefore, to have a formulation of metric gravity in which all the fields and the gauge transformations have an angle-independent limit at spatial infinity we have to add 6 gauge fixings on the $b_{(\infty) A}^{(\mu)}(\tau)$ [see later on Eqs.(2.39)] like we do in parametrized Minkowski theory for going from arbitrary spacelike hyperplanes to the Wigner ones (orthogonal to $p_{s}^{(\mu)} \approx P_{\text {sys }}^{(\mu)}$, where $P_{\text {sys }}^{(\mu)}$ is the 4-momentum of the isolated system under study): only on them we get the constraints $\vec{P}_{\text {sys }} \approx 0$ giving the rest-frame conditions.

Let us call Wigner-Sen-Witten (WSW) the so selected allowed spacelike hypersurfaces $\Sigma_{\tau}^{(W S W)}$ (see Section XII of Ref. [6] and Section VII for the justification of the name) asymptotically orthogonal to the weak ADM 4-momentum. Since $b_{(\infty) A}^{(\mu)}=\frac{\partial z_{(\infty)}^{(\mu)}(\sigma)}{\partial \sigma^{A}}$, this is a strong restriction on the coordinate systems $x^{\mu}=z^{\mu}(\tau, \vec{\sigma}) \rightarrow \delta_{(\mu)}^{\mu} z_{(\infty)}^{(\mu)}(\tau, \vec{\sigma})$ of $M^{4}$, which can be 
reached from the $\Sigma_{\tau}^{(W S W)}$-adapted coordinates $\sigma^{A}=(\tau, \vec{\sigma})$ without introducing asymptotic angle dependence (namely supertranslations).

With these assumptions one has the following form of the line element (it becomes Minkowskian in cartesian coordinates at spatial infinity)

$$
\begin{aligned}
d s^{2} & =\epsilon\left(\left[N_{(a s)}+n\right]^{2}-\left[N_{(a s) \check{r}}+n_{\check{r}}\right]^{3} g^{\check{r} \check{s}}\left[N_{(a s) \check{s}}+n_{\check{s}}\right]\right)(d \tau)^{2}- \\
& \left.-2 \epsilon\left[N_{(a s) \check{r}}+n_{\check{r}}\right] d \tau d \sigma^{\check{r}}-\epsilon^{3} g_{\check{r} \check{s}} d \sigma^{\check{r}} d \sigma^{\check{s}}\right)= \\
& =\epsilon\left(\left[N_{(a s)}+n\right]^{2}(d \tau)^{2}-\right. \\
& \left.-{ }^{3} g^{\check{r} \check{s}}\left[{ }^{3} g_{\check{r} \check{u}} d \sigma^{\check{u}}+\left(N_{(a s) \check{r}}+n_{\check{r}}\right) d \tau\right]\left[{ }^{3} g_{\check{s} \check{v}} d \sigma^{\check{v}}+\left(N_{(a s) \check{s}}+n_{\check{s}}\right) d \tau\right]\right) .
\end{aligned}
$$

Since we have $\dot{x}_{(\infty)}^{(\mu)}(\tau) \stackrel{\circ}{=} b_{(\infty) A}^{(\mu)}(\tau) \tilde{\lambda}^{A}(\tau)$, it follows that for $\tilde{\lambda}_{\tau}(\tau)=\epsilon, \tilde{\lambda}_{r}(\tau)=0$, the point $\tilde{x}_{(\infty)}^{(\mu)}(\tau)$ moves with 4 -velocity $(\epsilon ; \overrightarrow{0})$ and has attached an accelerated rotating coordinate system [38, which becomes inertial when $\tilde{\lambda}_{A B}(\tau)=0$, namely when the foliations become geometrically well defined at spatial infinity.

As a consequence of what has been said and of Eqs.(2.27), in the allowed coordinate atlases $\mathcal{C}$ of $M^{4}$ and $\mathcal{C}_{\tau}$ of $\Sigma_{\tau}$ the function space $\mathcal{W}$ (an appropriate weighted Sobolev space as for Yang-Mills theory [17]) is needed for the field variables ${ }^{3} g_{\check{r} \check{s}}(\tau, \vec{\sigma}),{ }^{3} \tilde{\Pi}^{\check{r} \breve{s}}(\tau, \vec{\sigma}), n(\tau, \vec{\sigma})$, $n_{\check{r}}(\tau, \vec{\sigma})$ and for the parameters $\alpha(\tau, \vec{\sigma}), \alpha_{\check{r}}(\tau, \vec{\sigma})$ (of which $n(\tau, \vec{\sigma}), n_{\check{r}}(\tau, \vec{\sigma})$ are special cases) of allowed proper gauge transformations connected to the identity, should be defined by angle-independent boundary conditions for $r \rightarrow \infty$ of the following form:

$$
\begin{aligned}
& { }^{3} g_{\check{r} \check{s}}(\tau, \vec{\sigma}) \rightarrow_{r \rightarrow \infty}\left(1+\frac{M}{r}\right) \delta_{\check{r} \check{s}}+{ }^{3} h_{\check{r} \check{s}}(\tau, \vec{\sigma})=\left(1+\frac{M}{r}\right) \delta_{\check{r} \check{s}}+O\left(r^{-3 / 2}\right), \\
& { }^{3} \tilde{\Pi}^{\check{r} \check{s}}(\tau, \vec{\sigma}) \rightarrow_{r \rightarrow \infty}{ }^{3} k^{\check{r} \check{s}}(\tau, \vec{\sigma})=O\left(r^{-5 / 2}\right), \\
& n(\tau, \vec{\sigma}) \rightarrow_{r \rightarrow \infty} O\left(r^{-(2+\epsilon)}\right), \quad \epsilon>0, \\
& n_{\check{r}}(\tau, \vec{\sigma}) \rightarrow_{r \rightarrow \infty} O\left(r^{-\epsilon}\right), \quad \epsilon>0, \\
& \tilde{\pi}_{n}(\tau, \vec{\sigma}) \rightarrow_{r \rightarrow \infty} O\left(r^{-3}\right), \\
& \tilde{\pi}_{\vec{n}}^{\check{n}}(\tau, \vec{\sigma}) \rightarrow_{r \rightarrow \infty} O\left(r^{-3}\right), \\
& \lambda_{n}(\tau, \vec{\sigma}) \rightarrow_{r \rightarrow \infty} O\left(r^{-(3+\epsilon)}\right), \\
& \lambda_{\check{r}}^{\vec{n}}(\tau, \vec{\sigma}) \rightarrow_{r \rightarrow \infty} O\left(r^{-\epsilon}\right), \\
& \alpha(\tau, \vec{\sigma}) \rightarrow_{r \rightarrow \infty} O\left(r^{-(2+\epsilon)}\right), \\
& \alpha_{\check{r}}(\tau, \vec{\sigma}) \rightarrow_{r \rightarrow \infty} O\left(r^{-\epsilon}\right), \\
& \Downarrow \\
& \tilde{\mathcal{H}}(\tau, \vec{\sigma}) \rightarrow_{r \rightarrow \infty} O\left(r^{-3}\right), \\
& { }^{3} \tilde{\mathcal{H}} \tilde{r}(\tau, \vec{\sigma}) \rightarrow_{r \rightarrow \infty} O\left(r^{-3}\right) .
\end{aligned}
$$

With these boundary conditions we have $\partial_{\breve{u}}^{3} g_{\check{r} \check{s}}=O\left(r^{-2}\right)$ and not $O\left(r^{-(1+\epsilon)}\right)$; this is compatible with the definition of gravitational radiation given by Christodoulou and Klainermann [19], but not with the one of Ref. [39].

In this function space $\mathcal{W}$ supertranslations are not allowed by definition and proper gauge transformations generated by the secondary constraints map $\mathcal{W}$ into itself. A coordinateindependent characterization of $\mathcal{W}$ (see Ref. 40 for an attempt) should be given through 
an intrinsic definition of a minimal atlas of coordinate charts $\mathcal{C}_{\tau}$ of $\Sigma_{\tau}$ such that the lifts to 3-tensors on $\Sigma_{\tau}$ in $\mathcal{W}$ of the 3-diffeomorphisms in Diff $\Sigma_{\tau}$ maps them into them.

Therefore, a unique asymptotic Poincaré group, modulo gauge transformations, is selected. Moreover, in accord with Anderson [32 also Diff $M^{4}$ is restricted to Diff $\mathrm{f}^{4} \times$ $\mathcal{P}_{(\infty)}$. Now in Dif $f_{I} M^{4} \times \mathcal{P}_{(\infty)}$ the allowed proper diffeomorphisms $D$ if $f_{I} M^{4}$ are a normal subgroup (they go to the identity in an angle-independent way at spatial infinity), while the Poincaré group $\mathcal{P}_{(\infty)}$ describes the rigid improper gauge transformations (the non-rigid improper ones are assumed to be absent) as in Bergmann"s proposal [41]. Finally, following Marolf [42], the Poincaré group $\mathcal{P}_{(\infty)}$ is not interpreted as a group of improper gauge transformations but only as a source of superselection rules (like it happens for the vanishing of the color charges for the confinement of quarks), which however are consistent only in the rest frame $P_{A D M}^{\check{r}}=0$.

Since in Ref. [6] it was shown that the gauge transformations generated by the superhamiltonian constraint produce a change in the extrinsic curvature of the spacelike hypersurface $\Sigma_{\tau}$ transforming it in a different spacelike hypersurface, one has the indication that, in absence of supertranslations, the functions $N, \alpha, \lambda_{N}$, should go like $O\left(r^{-(2+\epsilon)}\right)$ and not like $O\left(r^{-\epsilon}\right)$ (in the case of proper gauge transformations).

The previous discussion points toward assuming the following Dirac Hamiltonian

$$
\begin{aligned}
\hat{H}_{(c) A D M}^{\prime \prime} & =\int d^{3} \sigma\left[\left(N_{(a s)}+n\right) \tilde{\mathcal{H}}+\left(N_{(a s) \check{r}}+n_{\check{r}}\right)^{3} \tilde{\mathcal{H}}^{\check{r}}\right](\tau, \vec{\sigma})+ \\
& +\tilde{\lambda}_{A}(\tau) P_{A D M}^{A}+\frac{1}{2} \tilde{\lambda}_{A B}(\tau) J_{A D M}^{A B}= \\
& =\int d^{3} \sigma\left[n \tilde{\mathcal{H}}+n_{\check{r}}^{3} \tilde{\mathcal{H}}^{\check{r}}\right](\tau, \vec{\sigma})+\tilde{\lambda}_{A}(\tau) \hat{P}_{A D M}^{A}+\frac{1}{2} \tilde{\lambda}_{A B}(\tau) \hat{J}_{A D M}^{A B}, \\
\hat{H}_{(D) A D M}^{\prime \prime} & =\hat{H}_{(c) A D M}^{\prime \prime}+\int d^{3} \sigma\left[\lambda_{n} \tilde{\pi}^{n}+\lambda_{r}^{\vec{n}} \tilde{\pi}_{\vec{n}}^{r}\right](\tau, \vec{\sigma})+\zeta_{A}(\tau) \tilde{\pi}^{A}(\tau)+\zeta_{A B}(\tau) \tilde{\pi}^{A B}(\tau) .
\end{aligned}
$$

However, the criticism of footnote 39 suggests that this Hamiltonian is well defined only in the gauges where $\tilde{\lambda}_{A B}(\tau)=0$.

After this modification of metric gravity at the canonical level two possible Hamiltonian scenarios can be imagined:

a) Consider as configurational variables

$$
n_{A}(\tau, \vec{\sigma})=\left(n ; n_{\check{r}}\right)(\tau, \vec{\sigma}), \quad \tilde{\lambda}_{A}(\tau), \quad \tilde{\lambda}_{A B}(\tau), \quad{ }^{3} g_{\check{r} \breve{s}}(\tau, \vec{\sigma}),
$$

with conjugate momentat5

$$
\tilde{\pi}_{n}^{A}(\tau, \vec{\sigma})=\left(\tilde{\pi}^{n} ; \tilde{\pi}_{\vec{n}}^{\check{r}}\right)(\tau, \vec{\sigma}) \approx 0, \quad \tilde{\pi}^{A}(\tau) \approx 0, \quad \tilde{\pi}^{A B}(\tau) \approx 0, \quad{ }^{3} \tilde{\Pi}^{\check{r} \check{s}}(\tau, \vec{\sigma}),
$$

and take the following Dirac Hamiltonian (it is finite and differentiable) as the defining Hamiltonian:

$$
\begin{aligned}
\hat{H}_{(D) A D M}^{(1)} & =\int d^{3} \sigma\left[n_{A} \tilde{\mathcal{H}}^{A}+\lambda_{n A} \tilde{\pi}_{n}^{A}\right](\tau, \vec{\sigma})+\tilde{\lambda}_{A}(\tau) \hat{P}_{A D M}^{A}+\frac{1}{2} \tilde{\lambda}_{A B}(\tau) \hat{J}_{A D M}^{A B}+ \\
& +\zeta_{A}(\tau) \tilde{\pi}^{A}(\tau)+\zeta_{A B}(\tau) \tilde{\pi}^{A B}(\tau),
\end{aligned}
$$

\footnotetext{
${ }^{45}$ The vanishing momenta are assumed to be the primary constraints.
} 
where $n_{A}=\left(n ; n_{\check{r}}\right), \tilde{\mathcal{H}}^{A}=\left(\tilde{\mathcal{H}} ;{ }^{3} \tilde{\mathcal{H}}^{\check{r}}\right)$ and where $\lambda_{n A}(\tau, \vec{\sigma})=\left(\lambda_{n} ; \lambda_{\check{r}}^{\vec{n}}\right)(\tau, \vec{\sigma}), \zeta_{A}(\tau), \zeta_{A B}(\tau)$, are Dirac multipliers associated with the primary constraints.

For $\tilde{\lambda}_{A B}(\tau)=0, \tilde{\lambda}_{A}(\tau)=\epsilon \delta_{A \tau}$, one has [35:

$$
\hat{H}_{(D) A D M}^{(1)} \approx-\epsilon \hat{P}_{A D M}^{\tau}
$$

The time constancy of the primary constraints implies the following secondary ones

$$
\begin{aligned}
& \tilde{\mathcal{H}}^{A}(\tau, \vec{\sigma}) \approx 0 \\
& \hat{P}_{A D M}^{A} \approx 0, \quad \hat{J}_{A D M}^{A B} \approx 0,
\end{aligned}
$$

all of which are constants of the motion. While the $\tilde{\mathcal{H}}^{A}$ 's are generators of proper gauge transformations, the other 10 constraints are either generators of improper gauge transformations (in this case 10 conjugate degrees of freedom in the 3-metric are extra gauge variables) or, following Marolf's proposal [42], defining a superselection sector. All the constraints are first class, so that:

i) $\tilde{\lambda}_{A}(\tau), \tilde{\lambda}_{A B}(\tau)$ are arbitrary gauge variables conjugate to $\tilde{\pi}^{A}(\tau) \approx 0, \tilde{\pi}^{A B}(\tau) \approx 0$ 物;

ii) the physical reduced phase space of canonical metric gravity is restricted to have zero asymptotic Poincaré charges so that there is no natural Hamiltonian for the evolution in $\tau$. This corresponds to the exceptional orbit $\hat{P}_{A D M}^{A}=0$ of the asymptotic Poincaré group.

This is the natural interpretation of ADM metric gravity which leads to the Wheeler-De Witt equation after quantization and, in a sense, it is a Machian formulation of an asymptotically flat noncompact (with boundary) spacetime $M^{4}$ in the same spirit of Barbour's approach 43] and of the closed (without boundary) Einstein-Wheeler universes. However, in this case there is no solution to the problem of deparametrization of metric gravity and no connection with parametrized Minkowski theories restricted to spacelike hyperplanes.

b) According to the suggestion of Dirac, modify ADM metric gravity by adding the 10 new canonical pairs $x_{(\infty)}^{(\mu)}(\tau), p_{(\infty)}^{(\mu)}, b_{(\infty) A}^{(\mu)}(\tau), S_{\infty}^{(\mu)(\nu)}$ to the metric gravity phase space with canonical basis $n_{A}(\tau, \vec{\sigma})=\left(n ; n_{\check{r}}\right)(\tau, \vec{\sigma}), \tilde{\pi}_{n}^{A}(\tau, \vec{\sigma})=\left(\tilde{\pi}^{n} ; \tilde{\pi}_{\vec{n}}^{\check{r}}\right) \approx 0$ (the primary constraints), ${ }^{3} g_{\check{r} \check{s}}(\tau, \vec{\sigma}),{ }^{3} \tilde{\Pi} \check{r} \check{s}(\tau, \vec{\sigma})$, and then:

i) add the 10 new primary constraints

$$
\begin{aligned}
\chi^{A}= & p_{(\infty)}^{A}-\hat{P}_{A D M}^{A}=b_{(\infty)(\mu)}^{A}(\tau)\left[p_{(\infty)}^{(\mu)}-b_{(\infty) B}^{(\mu)}(\tau) \hat{P}_{A D M}^{B}\right] \approx 0 \\
\chi^{A B}= & J_{(\infty)}^{A B}-\hat{J}_{A D M}^{A B}=b_{(\infty)(\mu)}^{A}(\tau) b_{(\infty)(\nu)}^{B}(\tau)\left[S_{(\infty)}^{(\mu)(\nu)}-b_{(\infty) C}^{(\mu)}(\tau) b_{(\infty) D}^{(\nu)}(\tau) \hat{J}_{A D M}^{C D}\right] \approx 0 \\
& \left\{\chi^{A}(\tau), \chi^{B C}(\tau)\right\} \approx{ }^{4} \eta^{A C} \chi^{B}(\tau)-{ }^{4} \eta^{A B} \chi^{C}(\tau) \approx 0, \quad\left\{\chi^{A}(\tau), \chi^{B}(\tau)\right\} \approx 0 \\
& \left\{\chi^{A B}(\tau), \chi^{C D}(\tau)\right\} \approx-C_{E F}^{A B C D} \chi^{E F}(\tau) \approx 0 \\
& \left\{\chi^{A}(\tau), \tilde{\pi}_{n}^{D}(\tau, \vec{\sigma})\right\}=\left\{\chi^{A B}(\tau), \tilde{\pi}_{n}^{D}(\tau, \vec{\sigma})\right\}=0 \\
& \left\{\chi^{A}(\tau), \tilde{\mathcal{H}}^{D}(\tau, \vec{\sigma})\right\} \approx 0, \quad\left\{\chi^{A B}(\tau), \tilde{\mathcal{H}}^{D}(\tau, \vec{\sigma})\right\} \approx 0
\end{aligned}
$$

\footnotetext{
${ }^{46} 6$ gauge fixings to the constraints $\hat{J}_{A D M}^{A B} \approx 0$ are needed to get the induced result $\tilde{\lambda}_{A B}(\tau)=0$ which ensures foliations well defined at spatial infinity.
} 
with $p_{(\infty)}^{A}, J_{(\infty)}^{A B}$ of Eqs.(2.17);

ii) consider $\tilde{\lambda}_{A}(\tau), \tilde{\lambda}_{A B}(\tau)$, as Dirac multipliers [like $\lambda_{n A}(\tau, \vec{\sigma})$ ] for these 10 new primary constraints, and not as configurational (arbitrary gauge) variables coming from the lapse and shift functions 7 , in the assumed Dirac Hamiltonian (it is finite and differentiable)

$$
\begin{aligned}
H_{(D) A D M} & =\int d^{3} \sigma\left[n_{A} \tilde{\mathcal{H}}^{A}+\lambda_{n A} \tilde{\pi}_{n}^{A}\right](\tau, \vec{\sigma})- \\
& -\tilde{\lambda}_{A}(\tau)\left[p_{(\infty)}^{A}-\hat{P}_{A D M}^{A}\right]-\frac{1}{2} \tilde{\lambda}_{A B}(\tau)\left[J_{(\infty)}^{A B}-\hat{J}_{A D M}^{A B}\right] \approx 0 .
\end{aligned}
$$

The reduced phase space is the ADM one and there is consistency with Marolf's proposal regarding superselection sectors: on the ADM variables there are only the secondary first class constraints $\tilde{\mathcal{H}}^{A}(\tau, \vec{\sigma}) \approx 0$ (generators of proper gauge transformations), because the other first class constraints $p_{(\infty)}^{A}-\hat{P}_{A D M}^{A} \approx 0, J_{(\infty)}^{A B}-\hat{J}_{A D M}^{A B} \approx 0$ do not generate improper gauge transformations but eliminate 10 of the extra 20 variables. One has an asymptotically flat at spatial infinity noncompact (with boundary $S_{\infty}$ ) spacetime $M^{4}$ with non-vanishing asymptotic Poincaré charges and the possibility to deparametrize metric gravity so to obtain the connection with parametrized Minkowski theories restricted to Wigner hyperplanes.

Scenario b) contains the rest-frame instant form of ADM metric gravity.

To go to the WSW hypersurfaces 8 one follows the procedure defined for Minkowski spacetime:

i) one restricts oneself to spacetimes with $\epsilon p_{(\infty)}^{2}={ }^{4} \eta_{(\mu)(\nu)} p_{(\infty)}^{(\mu)} p_{(\infty)}^{(\nu)}>0$ 弫

ii) one boosts at rest $b_{(\infty) A}^{(\mu)}(\tau)$ and $S_{(\infty)}^{(\mu)(\nu)}$ with the Wigner boost $L_{(\nu)}^{(\mu)}\left(p_{(\infty)}, \stackrel{\circ}{p}_{(\infty)}\right)$;

iii) one adds the gauge-fixings $\left(u^{(\mu)}\left(p_{(\infty)}\right)=p_{(\infty)}^{(\mu)} / \pm \sqrt{\epsilon p_{(\infty)}^{2}}\right)$

$$
\begin{aligned}
& b_{(\infty) A}^{(\mu)}(\tau) \approx L_{(\nu)=A}^{(\mu)}\left(p_{(\infty)}, \stackrel{\circ}{p}_{(\infty)}\right)=\epsilon_{A}^{(\mu)}\left(u\left(p_{(\infty)}\right)\right), \\
& \text { implying } \quad \tilde{\lambda}_{A B}(\tau)=0,
\end{aligned}
$$

to the constraints $\chi^{A B}(\tau) \approx 0$ and goes to Dirac brackets.

In this way one gets

\footnotetext{
${ }^{47}$ So that there are no conjugate momenta $\tilde{\pi}^{A}(\tau), \tilde{\pi}^{A B}(\tau)$ and no associated Dirac multipliers $\zeta_{A}(\tau), \zeta_{A B}(\tau)$.

${ }^{48}$ The analogue of the Minkowski Wigner hyperplanes with the asymptotic normal $l_{(\infty)}^{(\mu)}=l_{(\infty) \Sigma}^{(\mu)}$ parallel to $\hat{P}_{A D M}^{(\mu)}$ (i.e. $l_{(\infty)}^{(\mu)}=\hat{b}_{(\infty) l}^{(\mu)}=\hat{P}_{A D M}^{(\mu)} / \sqrt{\epsilon \hat{P}_{A D M}^{2}}$ ).

${ }^{49}$ This is possible, because the positivity theorems for the ADM energy imply that one has only timelike or light-like orbits of the asymptotic Poincaré group.
} 


$$
\begin{aligned}
S_{(\infty)}^{(\mu)(\nu)} & \equiv \epsilon_{C}^{(\mu)}\left(u\left(p_{(\infty)}\right)\right) \epsilon_{D}^{(\nu)}\left(u\left(p_{(\infty)}\right)\right) \hat{J}_{A D M}^{C D}=S_{A D M}^{(\mu)(\nu)} \\
z_{(\infty)}^{(\mu)}(\tau, \vec{\sigma}) & =x_{(\infty)}^{(\mu)}(\tau)+\epsilon_{r}^{(\mu)}\left(u\left(p_{(\infty)}\right)\right) \sigma^{r}
\end{aligned}
$$

so that $z_{(\infty)}^{(\mu)}(\tau, \vec{\sigma})$ becomes equal to the embedding identifying a Wigner hyperplane in Minkowski spacetime.

The origin $x_{(\infty)}^{(\mu)}$ is now replaced by the not covariant external center-of-mass canonical variable

$$
\tilde{x}_{(\infty)}^{(\mu)}=x_{(\infty)}^{(\mu)}+\frac{1}{2} \epsilon_{(\nu)}^{A}\left(u\left(p_{(\infty)}\right)\right) \eta_{A B} \frac{\partial \epsilon_{(\rho)}^{B}\left(u\left(p_{(\infty)}\right)\right)}{\partial p_{(\infty)(\mu)}} S_{(\infty)}^{(\nu)(\rho)}
$$

and one has

$$
J_{(\infty)}^{(\mu)(\nu)}=\tilde{x}_{(\infty)}^{(\mu)} p_{(\infty)}^{(\nu)}-\tilde{x}_{(\infty)}^{(\nu)} p_{(\infty)}^{(\mu)}+\tilde{S}_{(\infty)}^{(\mu)(\nu)}
$$

with $\tilde{S}_{(\infty)}^{(\mu)(\nu)}=S_{(\infty)}^{(\mu)(\nu)}-\frac{1}{2} \epsilon_{(\rho)}^{A}\left(u\left(p_{(\infty)}\right)\right) \eta_{A B}\left(\frac{\partial \epsilon_{(\sigma)}^{B}\left(u\left(p_{(\infty)}\right)\right)}{\partial p_{(\infty)(\mu)}} p_{(\infty)}^{(\nu)}-\frac{\partial \epsilon_{(\sigma)}^{B}\left(u\left(p_{(\infty)}\right)\right)}{\partial p_{(\infty)(\nu)}} p_{(\infty)}^{(\mu)}\right) S_{(\infty)}^{(\rho)(\sigma)}$

As in the Minkowski case one defines

$$
\bar{S}_{(\infty)}^{A B}=\epsilon_{(\mu)}^{A}\left(u\left(p_{(\infty)}\right)\right) \epsilon_{(\nu)}^{B}\left(u\left(p_{(\infty)}\right)\right) \tilde{S}_{(\infty)}^{(\mu)(\nu)},
$$

and one obtains at the level of Dirac brackets $\left[\epsilon_{(\infty)}=-\epsilon \sqrt{\epsilon p_{(\infty)}^{2}}\right]$

$$
\begin{aligned}
\bar{S}_{(\infty)}^{\check{r} \check{s}} & \equiv \hat{J}_{A D M}^{\check{r} \check{s}}, \quad \tilde{\lambda}_{A B}(\tau)=0, \\
-\tilde{\lambda}_{A}(\tau) \chi^{A} & =-\tilde{\lambda}_{A}(\tau) \epsilon_{(\mu)}^{A}\left(u\left(p_{(\infty)}\right)\right)\left[p_{(\infty)}^{(\mu)}-\epsilon_{B}^{(\mu)}\left(u\left(p_{(\infty)}\right)\right) \hat{P}_{A M}^{B}\right]= \\
& =-\tilde{\lambda}_{A}(\tau) \epsilon_{(\mu)}^{A}\left(u\left(p_{(\infty)}\right)\right)\left[u^{(\mu)}\left(p_{(\infty)}\right)\left(\epsilon_{(\infty)}-\hat{P}_{A D M}^{\tau}\right)-\epsilon_{\check{r}}^{(\mu)}\left(p_{(\infty)}\right) \hat{P}_{A D M}^{\check{r}}\right]= \\
& =-\tilde{\lambda}_{\tau}(\tau)\left[\epsilon_{(\infty)}-\hat{P}_{A D M}^{\tau}\right]+\tilde{\lambda}_{\check{r}}(\tau) \hat{P}_{A D M}^{\check{r}}, \\
\Rightarrow & \epsilon_{(\infty)}-\hat{P}_{A D M}^{\tau} \approx 0, \quad \hat{P}_{A D M}^{\check{r}} \approx 0, \\
H_{(D) A D M} & =\int d^{3} \sigma\left[n_{A} \mathcal{H}^{A}+\lambda_{n A} \tilde{\pi}_{n}^{A}\right](\tau, \vec{\sigma})-\tilde{\lambda}_{\tau}(\tau)\left[\epsilon_{(\infty)}-\hat{P}_{A D M}^{\tau}\right]+\tilde{\lambda}_{\check{r}}(\tau) \hat{P}_{A D M}^{\check{r}},
\end{aligned}
$$

in accord with Eq.(2.28).

Therefore, on the WSW hypersurfaces (whose 3-coordinates are denoted $\left\{\sigma^{r}\right\}$ ), which define the intrinsic asymptotic rest frame of the gravitational field, the remaining four extra constraints are:

$$
\begin{aligned}
& \hat{P}_{A D M}^{\check{r}} \approx 0 \\
& \epsilon_{(\infty)}=-\epsilon \sqrt{\epsilon p_{(\infty)}^{2}} \approx \hat{P}_{A D M}^{\tau} \approx-\epsilon M_{A D M}=-\epsilon \sqrt{\epsilon \hat{P}_{A M}^{2}}
\end{aligned}
$$

Now the spatial indices have become spin-1 Wigner indices (they transform with Wigner rotations under asymptotic Lorentz transformations). As for parametrized theories in 
Minkowski spacetime, in this special gauge 3 degrees of freedom of the gravitational field become gauge variables, while $\tilde{x}_{(\infty)}^{(\mu)}$ becomes a decoupled observer with his clock near spatial infinity. These 3 degrees of freedom represent an internal center-of-mass 3-variable $\vec{\sigma}_{A D M}\left[{ }^{3} g,{ }^{3} \tilde{\Pi}\right]$ inside the WSW hypersurface; $\sigma^{r}=\sigma_{A D M}^{r}$ is a variable representing the 3center of mass of the 3-metric of the slice $\Sigma_{\tau}$ of the asymptotically flat spacetime $M^{4}$ and is obtainable from the weak Poincaré charges with the group-theoretical methods of Ref. [44] as it is done in Ref. [28] for the Klein-Gordon field on the Wigner hyperplane. Due to $\widehat{P}_{A D M}^{r} \approx 0$ we have

$$
\begin{aligned}
\sigma_{A D M}^{r}= & -\frac{\hat{J}^{\tau r}}{\sqrt{\left(\hat{P}_{A D M}^{\tau}\right)^{2}-\left(\hat{\vec{P}}_{A D M}\right)^{2}}}+ \\
+ & \frac{\left(\hat{\vec{J}}_{A D M} \times \hat{\vec{P}}_{A D M}\right)^{r}}{\sqrt{\left(\hat{P}_{A D M}^{\tau}\right)^{2}-\left(\hat{\vec{P}}_{A D M}\right)^{2}}\left(\hat{P}_{A D M}^{\tau}+\sqrt{\left(\hat{P}_{A D M}^{\tau}\right)^{2}-\left(\hat{\vec{P}}_{A D M}\right)^{2}}\right)}+ \\
+ & \frac{\left(\hat{J}_{A D M}^{\tau s} \hat{P}_{A D M}^{s}\right) \hat{P}_{A D M}^{r}}{\hat{P}_{A D M}^{\tau} \sqrt{\left(\hat{P}_{A D M}^{\tau}\right)^{2}-\left(\hat{\vec{P}}_{A D M}\right)^{2}}\left(\hat{P}_{A D M}^{\tau}+\sqrt{\left(\hat{P}_{A D M}^{\tau}\right)^{2}-\left(\hat{\vec{P}}_{A D M}\right)^{2}}\right)} \approx \\
\approx & -\hat{J}_{A D M}^{\tau r} / \hat{P}_{A D M}^{\tau}, \quad\left\{\sigma_{A D M}^{r}, \hat{P}_{A D M}^{s}\right\}=\delta^{r s}, \\
& \left\{\sigma_{A D M}^{r}, \sigma_{A D M}^{s}\right\}=0, \quad
\end{aligned}
$$

so that $\vec{\sigma}_{A D M} \approx 0$ is equivalent to the requirement that the weak ADM boosts vanish: this is the way out from the boost problem in the framework of the rest-frame instant form.

When $\epsilon \hat{P}_{A D M}^{2}>0$, with the asymptotic Poincaré Casimirs $\hat{P}_{A D M}^{2}, \hat{W}_{A D M}^{2}$ one can build the Møller radius $\rho_{A M D}=\sqrt{-\epsilon \hat{W}_{A D M}^{2}} / \epsilon \hat{P}_{A D M}^{2} c$, which is an intrinsic classical unit of length like in parametrized Minkowski theories, to be used as an ultraviolet cutoff in a future attempt of quantization.

By going from $\tilde{x}_{(\infty)}^{(\mu)}$ and $p_{(\infty)}^{(\mu)}$ to the canonical basis [15]

$$
\begin{aligned}
& T_{(\infty)}=p_{(\infty)(\mu)} \tilde{x}_{(\infty)}^{(\mu)} / \epsilon_{(\infty)}=p_{(\infty)(\mu)} x_{(\infty)}^{(\mu)} / \epsilon_{(\infty)}, \\
& \epsilon_{(\infty)}, \\
& z_{(\infty)}^{(i)}=\epsilon_{(\infty)}\left(\tilde{x}_{(\infty)}^{(i)}-p_{(\infty)}^{(i)} \tilde{x}_{(\infty)}^{(o)} / p_{(\infty)}^{(o)}\right), \\
& k_{(\infty)}^{(i)}=p_{(\infty)}^{(i)} / \epsilon_{(\infty)}=u^{(i)}\left(p_{(\infty)}^{(\rho)}\right),
\end{aligned}
$$

one finds that the final reduction requires the gauge-fixings

$$
T_{(\infty)}-\tau \approx 0, \quad \sigma_{A D M}^{\check{r}} \approx 0 \quad\left(\text { or } \hat{J}_{A D M}^{\tau r} \approx 0\right)
$$

Since $\left\{T_{(\infty)}, \epsilon_{(\infty)}\right\}=-\epsilon$, with the gauge fixing $T_{(\infty)}-\tau \approx 0$ one gets $\tilde{\lambda}_{\tau}(\tau) \approx \epsilon, \epsilon_{(\infty)} \equiv$ $\hat{P}_{A D M}^{\tau}$ and $H_{(D) A D M}=\tilde{\lambda}_{\check{r}}(\tau) \hat{P}_{A D M}^{\check{r}}$. This is the frozen picture of the reduced phase space, like it happens in the standard Hamilton-Jacobi theory: there is no time evolution. To reintroduce an evolution in $T_{(\infty)} \equiv \tau$ we must use the energy $M_{A D M}=-\epsilon \hat{P}_{A D M}^{\tau}$ (the 
ADM mass of the universe) as the natural physical Hamiltonian. Therefore the final Dirac Hamiltonian is

$$
\begin{aligned}
H_{D} & =M_{A D M}+\tilde{\lambda}_{\check{r}}(\tau) \hat{P}_{A D M}^{\check{r}}+\int d^{3} \sigma\left[n_{A} \tilde{\mathcal{H}}^{A}+\lambda_{n A} \tilde{\pi}_{n}^{A}\right](\tau, \vec{\sigma}) \approx \\
& \approx M_{A D M}=-\epsilon \hat{P}_{A D M}^{\tau} .
\end{aligned}
$$

That $M_{A D M}$ is the correct Hamiltonian for getting a $\tau$-evolution equivalent to Einstein's equations in spacetimes asymptotically flat at spatial infinity is also shown in Ref. [45]. In the rest-frame the mathematical time is identified with the parameter $\tau$ labelling the leaves $\Sigma_{\tau}$ of the foliation of $M^{4}$.

The final gauge fixings $\sigma_{A D M}^{\check{r}} \approx 0\left[\right.$ or $\hat{J}_{A D M}^{\tau r} \approx 0$ ] imply $\tilde{\lambda}_{\check{r}}(\tau) \approx 0, H_{D}=M_{A D M}$ and a reduced theory with the external 3 -center-of-mass variables $z_{(\infty)}^{(i)}, k_{(\infty)}^{(i)}$ decoupled (therefore the choice of the origin $x_{(\infty)}^{(\mu)}$ becomes irrelevant) and playing the role of a point particle clock for the time $T_{(\infty)} \equiv \tau$. There would be a weak form of Mach's principle, because only relative degrees of freedom would be present.

The condition $\tilde{\lambda}_{A B}(\tau)=0$ with $\tilde{\lambda}_{\tau}(\tau)=\epsilon, \tilde{\lambda}_{r}(\tau)=0$ means that at spatial infinity there are no local (direction dependent) accelerations and/or rotations. The asymptotic line

element for $\overrightarrow{\tilde{\lambda}}(\tau)=0$ reduces to the line element of an inertial system near spatial infinity: it defines the preferred asymptotic inertial observers, for instance the fixed stars [46].

While the asymptotic internal realization of the Poincaré algebra has the weak Poincaré charges $\hat{P}_{A D M}^{\tau} \approx-\epsilon M_{A D M}, \hat{P}_{A D M}^{r} \approx 0, \hat{J}_{A D M}^{r s}, \hat{K}_{A D M}^{r}=\hat{J}_{A D M}^{r r} \approx 0$ as generators, the rest-frame instant form asymptotic external realization of the Poincaré generators becomes

$$
\begin{aligned}
& \epsilon_{(\infty)}=M_{A D M}, \\
& p_{(\infty)}^{(i)}, \\
& J_{(\infty)}^{(i)(j)}=\tilde{x}_{(\infty)}^{(i)} p_{(\infty)}^{(j)}-\tilde{x}_{(\infty)}^{(j)} p_{(\infty)}^{(i)}+\delta^{(i) \check{r}} \delta^{(j) \check{s}} \hat{J}_{A D M}^{\check{r} \check{s}}, \\
& J_{(\infty)}^{(o)(i)}=p_{(\infty)}^{(i)} \tilde{x}_{(\infty)}^{(o)}-\sqrt{M_{A D M}^{2}+\vec{p}_{(\infty)}^{2}} \tilde{x}_{(\infty)}^{(i)}-\frac{\delta^{(i) \check{r}} \hat{J}_{A D M}^{\check{r} \check{s}} \delta^{(\check{s}(j)} p_{(\infty)}^{(j)}}{M_{A D M}+\sqrt{M_{A D M}^{2}+\vec{p}_{(\infty)}^{2}}} .
\end{aligned}
$$

\section{Tetrad Gravity and its Rest-Frame Instant Form.}

Since we have used the ADM action of metric gravity in our formulation of tetrad gravity, all the discussion about the differentiability of the Hamiltonian, the definition of Poisson brackets, the definition of proper and improper gauge transformations can be directly reformulated in tetrad gravity. The only difference inside tetrad gravity in the Hamiltonian treatment of quantities depending upon ${ }^{3} g_{r s}(\tau, \vec{\sigma})={ }^{3} e_{(a) r}{ }^{3} e_{(a) s}$, ${ }^{3} \tilde{\Pi}^{r s}(\tau, \vec{\sigma})=\frac{1}{4}\left[{ }^{3} e_{(a)}^{r}{ }^{3} \tilde{\pi}_{(a)}^{s}+{ }^{3} e_{(a)}^{s}{ }^{3} \tilde{\pi}_{(a)}^{r}\right]$ (they are now derived quantities), is that now we have $\left\{{ }^{3} \tilde{\Pi}^{r s}(\tau, \vec{\sigma}),{ }^{3} \tilde{\Pi}^{u v}\left(\tau, \vec{\sigma}^{\prime}\right)\right\}=\delta^{3}\left(\vec{\sigma}, \vec{\sigma}^{\prime}\right) F_{(a)(b)}^{r s u v}(\tau, \vec{\sigma})^{3} \tilde{M}_{(a)(b)}(\tau, \vec{\sigma}) \approx 0$ (see Eqs.(4.14) of Ref. 四) and not $=0$. Therefore, constants of motion (functional $F\left[{ }^{3} g_{r s},{ }^{3} \tilde{\Pi}^{r s}\right]$ ) of metric gravity remain such in tetrad gravity, since they have weakly zero Poisson brackets with $\tilde{\mathcal{H}}(\tau, \vec{\sigma})$, ${ }^{3} \tilde{\mathcal{H}}^{r}(\tau, \vec{\sigma})$ [and, therefore, with ${ }^{3} \tilde{\Theta}_{r}(\tau, \vec{\sigma})$ and $\left.\hat{\tilde{\mathcal{H}}}_{(a)}(\tau, \vec{\sigma})\right]$ and also with the other first class constraints $\tilde{\pi}_{(a)}^{\vec{\varphi}}(\tau, \vec{\sigma}) \approx 0,{ }^{3} \tilde{M}_{(a)}(\tau, \vec{\sigma}) \approx 0$. 
As a consequence the weak and strong Poincaré charges are still constants of motion in tetrad gravity and their weak Poincaré algebra under Poisson brackets may only be modified by extra terms containing ${ }^{3} \tilde{M}_{(a)}(\tau, \vec{\sigma}) \approx 0$. In particular, after having added the gauge fixings to these constraints and after having gone to Dirac brackets, the weak and strong Poincaré algebras coincide with those of metric gravity. A more complete study of these properties would require the study of the quasi-invariances of the Lagrangian (2.8) of tetrad gravity under the gauge transformations generated by the 14 first class constraints of the theory 0 .

The only lacking ingredients are the definition of proper gauge transformations generated by the primary (without associated secondary) first class constraints $\tilde{\pi}_{(a)}^{\vec{\varphi}}(\tau, \vec{\sigma}) \approx 0$, ${ }^{3} \tilde{M}_{(a)}(\tau, \vec{\sigma}) \approx 0$, and the boundary conditions for cotriads ${ }^{3} e_{(a) r}(\tau, \vec{\sigma})$, because the lapse and shift functions $N(\tau, \vec{\sigma}), N_{(a)}(\tau, \vec{\sigma})={ }^{3} e_{(a)}^{r}(\tau, \vec{\sigma}) N_{r}(\tau, \vec{\sigma})$ are treated in the same way as in metric gravity, namely we assume the validity of Eqs. (2.19) in the form

$$
\begin{aligned}
N(\tau, \vec{\sigma}) & =N_{(a s)}(\tau, \vec{\sigma})+m(\tau, \vec{\sigma}), \\
N_{(a)}(\tau, \vec{\sigma}) & ={ }^{3} e_{(a)}^{\check{r}}(\tau, \vec{\sigma})\left[N_{(a s) \check{r}}(\tau, \vec{\sigma})+m_{\check{r}}(\tau, \vec{\sigma})\right]= \\
& ={ }^{3} e_{(a)}^{\check{r}}(\tau, \vec{\sigma}) N_{(a s) \check{r}}(\tau, \vec{\sigma})+m_{(a)}(\tau, \vec{\sigma}), \\
N_{(a s)}(\tau, \vec{\sigma}) & =-\tilde{\lambda}_{\tau}(\tau)-\frac{1}{2} \tilde{\lambda}_{\tau \check{s}}(\tau) \sigma^{\check{s}}, \\
N_{(a s) \check{r}}(\tau, \vec{\sigma}) & =-\tilde{\lambda}_{\check{r}}(\tau)-\frac{1}{2} \tilde{\lambda}_{\check{r} \check{s}}(\tau) \sigma^{\check{s}} .
\end{aligned}
$$

Therefore, we shall assume that there exist the same coordinate systems of $M^{4}$ and $\Sigma_{\tau}$ as in metric gravity and that the $\Sigma_{\tau}$-adapted tetrads of Eqs.(2.1), whose expression is ${ }_{(\Sigma)}^{4} \check{E}_{(\mu)}^{\mu}$ with円

$$
{ }_{(\Sigma)}^{4} \check{E}_{(o)}^{\mu}=l^{\mu}=\hat{b}_{l}^{\mu}=\frac{1}{N}\left[b_{\tau}^{\mu}-N^{\check{r}} b_{\check{r}}^{\mu}\right], \quad{ }_{(\Sigma)}^{4} \check{E}_{(a)}^{\mu}={ }^{3} e_{(a)}^{\check{s}} b_{\check{s}}^{\mu},
$$

have a well defined angle-independent limit ${ }_{(\Sigma)}^{4} \check{E}_{(\infty)(\mu)}^{\mu}$ at spatial infinity, such that

$$
\begin{aligned}
& { }_{(\Sigma)}^{4} \check{E}_{(\infty)(o)}^{\mu}=\delta_{(\mu)}^{\mu} l_{(\infty)}^{(\mu)}=\delta_{(\mu)}^{\mu} \hat{b}_{(\infty) l}^{(\mu)}=\delta_{(\mu)}^{\mu} \frac{1}{N_{(a s)}}\left(b_{(\infty) \tau}^{(\mu)}-N_{(a s)}^{\check{r}} b_{(\infty) \check{r})}^{(\mu)},\right. \\
& { }_{(\Sigma)}^{4} \check{E}_{(\infty)(a)}^{\mu}=\delta_{(a)}^{\check{s}} \delta_{(\mu)}^{\mu} b_{(\infty) \check{s}}^{(\mu)}(\tau),
\end{aligned}
$$

with the same asymptotic $b_{(\infty) A}^{(\mu)}(\tau)$ 's of Eq.(2.15).

Let us remark that the $\Sigma_{\tau}$-adapted tetrads in adapted coordinates of Eqs.(2.2), are ${ }_{(\Sigma)}^{4} \check{\tilde{E}}_{(\mu)}^{A}$ with

$$
\begin{aligned}
& { }_{(\Sigma)}^{4} \check{\tilde{E}}_{(o)}^{A}=\frac{1}{N}\left(1 ;-{ }^{3} e_{(a)}^{\check{r}} N_{(a)}\right), \\
& { }_{(\Sigma)}^{4} \check{\tilde{E}}_{(a)}^{A}=\left(0 ;{ }^{3} e_{(a)}^{\check{r}}\right) .
\end{aligned}
$$

\footnotetext{
${ }^{50}$ Using the second Noether theorem as it was done in Appendix A of III for metric gravity.

${ }^{51} b_{A}^{\mu}$ are the transformation coefficients to $\Sigma_{\tau}$-adapted coordinates.
} 
Due to the presence of the lapse function in the denominator which is linearly increasing in $\vec{\sigma}$ (to have the possibility of defining $J_{A D M}^{A B}$ ), these adapted tetrads exist without singularities at spatial infinity only if $\tilde{\lambda}_{A B}(\tau)=0$, i.e. on WSW hypersurfaces ${ }^{52}$. The same happens for the adapted cotetrads ${ }_{(\Sigma)}^{4} \underset{\tilde{E}}{\check{\tilde{E}}_{A}^{(\mu)}}$ with ${ }_{(\Sigma)}^{4} \check{\tilde{E}}_{A}^{(o)}=(N ; 0),{ }_{(\Sigma)}^{4} \check{\tilde{E}}_{A}^{(a)}=\left(N^{(a)}=N_{(a)} ;^{3} e_{r}^{(a)}=\right.$ $\left.{ }^{3} e_{(a) r}\right)$. Also the concept of proper time of Eulerian observers connected with the lapse function is divergent at spatial infinity. Therefore, tetrad gravity without supertranslations $\left[\tilde{\lambda}_{A B}(\tau)=0, m=n, m_{(a)}=n_{(a)}={ }^{3} e_{(a)}^{r} n_{r}\right]$ and with Poincaré charges, admits well defined adapted tetrads and cotetrads (with components in adapted holonomic coordinates) only after having been restricted to WSW hypersurfaces (rest frame), whose asymptotic normals $l_{(\infty)}^{(\mu)}=l_{(\infty) \Sigma}^{(\mu)}$, tangent to $S_{\infty}$, are parallel to $\hat{P}_{A D M}^{(\mu)}=b_{(\infty) A}^{(\mu)} \hat{P}_{A D M}^{A}$ with $\hat{P}_{A D M}^{r} \approx 0$ ए). This again implies the existence of an inertial system at spatial infinity when $\tilde{\lambda}_{A}(\tau)=(\epsilon ; \overrightarrow{0})$ and $\tilde{\lambda}_{A B}(\tau)=0$, namely the absence of accelerations and rotations there 04 .

In tetrad gravity we shall assume the following boundary conditions consistent with Eqs. 2.27) and (2.30) of metric gravity

$$
\begin{aligned}
& { }^{3} e_{(a) \check{r}}(\tau, \vec{\sigma}) \rightarrow_{r \rightarrow \infty}\left(1+\frac{M}{2 r}\right) \delta_{(a) \check{r}}+{ }^{3} w_{(a) \check{r}}(\tau, \vec{\sigma}), \quad{ }^{3} w_{(a) \check{r}}(\tau, \vec{\sigma})=O\left(r^{-3 / 2}\right), \\
& { }^{3} e_{(a)}^{\check{r}}(\tau, \vec{\sigma}) \rightarrow_{r \rightarrow \infty}\left(1-\frac{M}{2 r}\right) \delta_{(a)}^{\check{r}}+{ }^{3} w_{(a)}^{\check{r}}(\tau, \vec{\sigma}), \quad{ }^{3} w_{(a)}^{\check{r}}(\tau, \vec{\sigma})=O\left(r^{-3 / 2}\right), \\
& { }^{3} g_{\check{r} \check{s}}(\tau, \vec{\sigma})=\left[{ }^{3} e_{(a) \check{r}}{ }^{3} e_{(a) \check{s}}\right](\tau, \vec{\sigma}) \rightarrow_{r \rightarrow \infty}\left(1+\frac{M}{r}\right) \delta_{\check{r} \check{s}}+{ }^{3} h_{\check{r} \check{s}}(\tau, \vec{\sigma}), \\
& { }^{3} h_{\check{r} \check{s}}(\tau, \vec{\sigma})=\frac{1}{r}\left[\delta_{(a) \check{r}}{ }^{3} w_{(a s)(a) \check{s}}(\tau, \vec{\sigma})+{ }^{3} w_{(a s)(a) \check{r}}(\tau, \vec{\sigma}) \delta_{(a) \check{s}}\right]+O\left(r^{-2}\right)=O\left(r^{-3 / 2}\right), \\
& { }^{3} \tilde{\pi}_{(a)}^{\check{r}}(\tau, \vec{\sigma}) \rightarrow_{r \rightarrow \infty} O\left(r^{-5 / 2}\right), \\
& { }^{3} \tilde{\Pi}^{\check{r} \check{s}}(\tau, \vec{\sigma})=\frac{1}{4}\left[{ }^{3} e_{(a)}^{\check{r}}{ }^{3} \tilde{\pi}_{(a)}^{\check{s}}+{ }^{3} e_{(a)}^{\check{s}}{ }^{3} \tilde{\pi}_{(a)}^{\check{r}}\right](\tau, \vec{\sigma}) \rightarrow_{r \rightarrow \infty}{ }^{3} \tilde{k}^{\check{r} \check{s}}(\tau, \vec{\sigma})=O\left(r^{-5 / 2}\right), \\
& N(\tau, \vec{\sigma})=N_{(a s)}(\tau, \vec{\sigma})+n(\tau, \vec{\sigma}), \\
& n(\tau, \vec{\sigma}) \rightarrow_{r \rightarrow \infty} O\left(r^{-(2+\epsilon)}\right), \\
& N_{(a s)}(\tau, \vec{\sigma})=-\tilde{\lambda}_{\tau}(\tau)-\frac{1}{2} \tilde{\lambda}_{\tau \check{s}}(\tau) \sigma^{\check{s}}, \\
& N_{\check{r}}(\tau, \vec{\sigma})=N_{(a s) \check{r}}(\tau, \vec{\sigma})+n_{\check{r}}(\tau, \vec{\sigma}), \\
& n_{\check{r}}(\tau, \vec{\sigma}) \rightarrow_{r \rightarrow \infty} O\left(r^{-\epsilon}\right) \text {, }
\end{aligned}
$$

${ }^{52}$ This is connected with the criticism in footnote 39.

${ }^{53}$ Namely when one is inside the Christodoulou-Klainermann class of solutions [19], but in general with non vanishing shift functions.

${ }^{54}$ When $\tilde{\lambda}_{A}(\tau) \neq 0$ there is a direction independent global acceleration of the origin $x_{(\infty)}^{(\mu)}(\tau)$, since $\dot{x}_{(\infty)}^{(\mu)}(\tau)=b_{(\infty) A}^{(\mu)} \tilde{\lambda}_{A}(\tau)$. 


$$
\begin{aligned}
& N_{(a s) \check{r}}(\tau, \vec{\sigma})=-\tilde{\lambda}_{r}(\tau)-\frac{1}{2} \tilde{\lambda}_{\check{r} \check{s}}(\tau) \sigma^{\check{s}}, \\
& N_{(a)}(\tau, \vec{\sigma})={ }^{3} e_{(a)}^{\check{r}}(\tau, \vec{\sigma}) N_{\check{r}}(\tau, \vec{\sigma})=N_{(a s)(a)}(\tau, \vec{\sigma})+n_{(a)}(\tau, \vec{\sigma}), \\
& n_{(a)}(\tau, \vec{\sigma})=\left[{ }^{3} e_{(a)}^{\check{r}} n_{\check{r}}\right](\tau, \vec{\sigma}) \rightarrow_{r \rightarrow \infty} O\left(r^{-\epsilon}\right) \text {, } \\
& \tilde{\pi}^{n}(\tau, \vec{\sigma}) \rightarrow_{r \rightarrow \infty} O\left(r^{-3}\right), \\
& \tilde{\pi}_{\vec{n},(a)}(\tau, \vec{\sigma}) \rightarrow_{r \rightarrow \infty} O\left(r^{-3}\right) \text {, } \\
& \lambda_{n}(\tau, \vec{\sigma}) \rightarrow_{r \rightarrow \infty} O\left(r^{-(3+\epsilon)}\right), \\
& \lambda_{\vec{n},(a)}(\tau, \vec{\sigma}) \rightarrow_{r \rightarrow \infty} O\left(r^{-\epsilon}\right) \text {, } \\
& \beta(\tau, \vec{\sigma}) \rightarrow_{r \rightarrow \infty} O\left(r^{-(3+\epsilon)}\right), \\
& \beta^{\check{r}}(\tau, \vec{\sigma}) \rightarrow_{r \rightarrow \infty} O\left(r^{-\epsilon}\right) \text {, } \\
& \hat{\mathcal{H}}(\tau, \vec{\sigma}) \rightarrow_{r \rightarrow \infty} O\left(r^{-3}\right), \\
& { }^{3} \tilde{\Theta}_{\check{r}}(\tau, \vec{\sigma}) \rightarrow_{r \rightarrow \infty} O\left(r^{-3}\right), \\
& { }^{3} \tilde{M}_{(a)}(\tau, \vec{\sigma}) \rightarrow_{r \rightarrow \infty} O\left(r^{-6}\right), \\
& \alpha_{(a)}(\tau, \vec{\sigma}) \rightarrow_{r \rightarrow \infty} O\left(r^{-(1+\epsilon)}\right) \text {, } \\
& \hat{\mu}_{(a)}(\tau, \vec{\sigma}) \rightarrow_{r \rightarrow \infty} O\left(r^{-(1+\epsilon)}\right) \text {, } \\
& \varphi_{(a)}(\tau, \vec{\sigma}) \rightarrow_{r \rightarrow \infty} O\left(r^{-(1+\epsilon)}\right), \\
& \tilde{\pi}_{(a)}^{\vec{\varphi}}(\tau, \vec{\sigma}) \rightarrow_{r \rightarrow \infty} O\left(r^{-2}\right), \\
& \lambda_{(a)}^{\vec{\varphi}}(\tau, \vec{\sigma}) \rightarrow_{r \rightarrow \infty} O\left(r^{-(1+\epsilon)}\right),
\end{aligned}
$$

with the asymptotic line element

$$
\begin{aligned}
d s^{2} & =\epsilon\left(\left[N_{(a s)}+n\right]^{2}-\left[N_{(a s) \check{r}}+n_{\check{r}}\right]^{3} e_{(a)}^{\check{r}}{ }^{3} e_{(a)}^{\check{s}}\left[N_{(a s) \check{s}}+n_{\check{s}}\right]\right)(d \tau)^{2}- \\
& -2 \epsilon\left[N_{(a s) \check{r}}+n_{\check{r}}\right] d \tau d \sigma^{\check{r}}-\epsilon^{3} e_{(a) \check{r}}{ }^{3} e_{(a) \check{s}} d \sigma^{\check{r}} d \sigma^{\check{s}}= \\
& =\epsilon\left(\left[N_{(a s)}+n\right]^{2}(d \tau)^{2}-\left[{ }^{3} e_{(a) \check{r}} d \sigma^{\check{r}}+\left(N_{(a s)(a)}+n_{(a)}\right) d \tau\right]\left[{ }^{3} e_{(a) \check{s}} d \sigma^{\check{s}}+\left(N_{(a s)(a)}+n_{(a)}\right) d \tau\right]\right) .
\end{aligned}
$$

With these boundary conditions all proper gauge transformations go asymptotically to the identity.

Near spatial infinity there is a dynamical preferred observer with an associated asymp-

${ }^{55}$ Generated by $\tilde{\mathcal{H}}(\tau, \vec{\sigma})$ with parameter $\beta(\tau, \vec{\sigma}) \rightarrow O\left(r^{-(3+\epsilon)}\right), \tilde{\Theta}_{r}(\tau, \vec{\sigma})$ with $\beta^{\check{r}}(\tau, \vec{\sigma}) \rightarrow O\left(r^{-\epsilon}\right)$, ${ }^{3} \tilde{M}_{(a)}(\tau, \vec{\sigma})$ with $\alpha_{(a)}(\tau, \vec{\sigma}) \rightarrow O\left(r^{-(1+\epsilon)}\right), \tilde{\pi}_{(a)}^{\vec{\varphi}}(\tau, \vec{\sigma})$ with $\varphi_{(a)}(\tau, \vec{\sigma}) \rightarrow O\left(r^{-(1+\epsilon)}\right)$ for $r \rightarrow \infty$.

${ }^{56}$ Either the canonical non-covariant Newton-Wigner-like position $\tilde{x}_{(\infty)}^{(\mu)}(\tau)$ or the covariant noncanonical origin of asymptotic Cartesian coordinates $x_{(\infty)}^{(\mu)}(\tau)$. 
totic inertial Lorentz reference frame given by the asymptotic limit of the $\Sigma_{\tau}$-adapted tetrads of Eqs.(2.2): however, as said, these asymptotic tetrads are well defined only in absence of supertranslations on the rest-frame WSW hypersurfaces, where (modulo a rigid 3-rotation) we get

$$
\begin{aligned}
& { }_{(\infty \Sigma)}^{4} \stackrel{\tilde{E}}{(o)}_{(a)}^{A}=\left(\frac{1}{N_{(a s)}(\tau)}=-\epsilon ;-\frac{\delta_{(a)}^{r} N_{(a s)(a)}(\tau)}{N_{(a s)}(\tau)}=\overrightarrow{0}\right), \quad{ }_{(\infty \Sigma)}^{4} \check{\tilde{E}}_{A}^{(a)}=\left(0 ; \delta_{(a)}^{r}\right), \\
& { }_{(\infty \Sigma)}^{4} \check{\tilde{E}}_{A}^{(o)}=\left(N_{(a s)}=-\epsilon ; \overrightarrow{0}\right), \quad{ }_{(\infty \Sigma)}^{4} \check{\tilde{E}}_{A}^{(a)}=\left(N_{(a s)(a)}=0 ; \delta_{r}^{(a)}\right) .
\end{aligned}
$$

Then, following the scenario b), the differentiable and finite Dirac Hamiltonian [replacing the one of Eqs.(2.13)] is assumed to be

$$
\begin{aligned}
\hat{H}_{(D) A D M} & =\int d^{3} \sigma\left[n \hat{\mathcal{H}}+n_{(a)}^{3} e_{(a)}^{\check{r}^{3}} \tilde{\Theta}_{\check{r}}+\lambda_{(a)}^{\vec{\varphi}} \tilde{\pi}_{(a)}^{\vec{\varphi}}+\hat{\mu}_{(a)}{ }^{3} \tilde{M}_{(a)}+\right. \\
& \left.+\lambda_{n} \tilde{\pi}^{n}+\lambda_{(a)}^{\vec{n}} \tilde{\pi}_{(a)}^{\vec{n}}\right](\tau, \vec{\sigma})- \\
& -\tilde{\lambda}_{A}(\tau)\left[p_{(\infty)}^{A}-\hat{P}_{A D M}^{A}\right]-\frac{1}{2} \tilde{\lambda}_{A B}(\tau)\left[J_{(\infty)}^{A B}-\hat{J}_{A D M}^{A B}\right],
\end{aligned}
$$

with the same weak (and strong) Poincaré charges of metric gravity, Eqs. (2.23) [(2.25)], expressed in terms of cotriads ${ }^{3} e_{(a) r}$ and their conjugate momenta ${ }^{3} \tilde{\pi}_{(a)}^{r}$, by using ${ }^{3} g_{r s}=$ ${ }^{3} e_{(a) r}{ }^{3} e_{(a) s},{ }^{3} \tilde{\Pi}^{r s}=\frac{1}{4}\left[{ }^{3} e_{(a)}^{r}{ }^{3} \tilde{\pi}_{(a)}^{s}+{ }^{3} e_{(a)}^{s}{ }^{3} \tilde{\pi}_{(a)}^{r}\right]$. Let us remark that we have $n_{\check{r}}{ }^{3} \tilde{\mathcal{H}}^{\check{r}} \approx$ $-n_{(a)}{ }^{3} e_{(a)}^{\check{s}}{ }^{3} \tilde{\Theta}_{\check{s}} \approx-n^{\check{s} 3} \tilde{\Theta}_{\check{s}}=-n_{(a)}{ }^{3} e_{(a)}^{s}{ }^{3} \tilde{\Theta}_{s} \approx-n_{(a)}{ }^{3} e_{(a)}^{s} \frac{\partial \xi^{r}}{\partial \sigma^{s}} \tilde{\pi}_{r}^{\vec{\xi}}=-n_{u}{ }^{3} g^{u s} \frac{\partial \xi^{r}}{\partial \sigma^{s}} \tilde{\pi}_{r}^{\vec{\xi}}=-\tilde{n}^{r} \tilde{\pi}_{r}^{\vec{\xi}}$.

However, as already said, we must restrict ourselves to gauges with $\tilde{\lambda}_{A B}(\tau)=0$, namely to WSW foliations, to avoid inconsistencies at spatial infinity.

The rest-frame instant form of tetrad gravity on WSW hypersurfaces is defined by Eqs. (2.39)-(2.49). In this gauge the final Hamiltonian for the evolution in $\tau \equiv T_{s}$ is weakly $M_{A D M}=-\epsilon \hat{P}_{A D M}^{\tau}$ and we also have $\tilde{\lambda}_{A}(\tau)=(\epsilon ; \overrightarrow{0}), N=-\epsilon+n, N_{r}=n_{r}\left[n_{(a)}={ }^{3} e_{(a)}^{r} n_{r}\right]$.

With the Dirac Hamiltonian (2.58) the Hamilton equations on WSW hypersurfaces are

$$
\begin{aligned}
& \partial_{\tau} n(\tau, \vec{\sigma})\left.\stackrel{\circ}{=} n(\tau, \vec{\sigma}), \hat{H}_{(D) A D M}\right\}=\lambda_{n}(\tau, \vec{\sigma}), \\
& \partial_{\tau} n_{(a)}(\tau, \vec{\sigma}) \stackrel{\circ}{=}\left\{n_{(a)}(\tau, \vec{\sigma}), \hat{H}_{(D) A D M}\right\}=\lambda_{(a)}^{\vec{n}}(\tau, \vec{\sigma}), \\
& \partial_{\tau} \varphi_{(a)}(\tau, \vec{\sigma}) \stackrel{\circ}{=}\left\{\varphi_{(a)}(\tau, \vec{\sigma}), \hat{H}_{(D) A D M}\right\}=\lambda_{(a)}^{\vec{\varphi}}(\tau, \vec{\sigma}), \\
& \partial_{\tau}{ }^{3} e_{(a) r}(\tau, \vec{\sigma}) \stackrel{\circ}{=}-\frac{\epsilon 4 \pi G}{c^{3}}\left[\frac{n}{3}{ }^{3} G_{o(a)(b)(c)(d)}{ }^{3} e_{(b) r}{ }^{3} e_{(c) s}{ }^{3} \tilde{\pi}_{(d)}^{s}\right](\tau, \vec{\sigma})+ \\
&+\left[n_{(b)}{ }^{3} e_{(b)}^{s} \frac{\partial^{3} e_{(a) r}}{\partial \sigma^{s}}+{ }^{3} e_{(a) s} \frac{\partial}{\partial \sigma^{r}}\left(n_{(b)}{ }^{3} e_{(b)}^{s}\right)\right](\tau, \vec{\sigma})+ \\
&+\epsilon_{(a)(b)(c)} \hat{\mu}_{(b)}(\tau, \vec{\sigma})^{3} e_{(c) r}(\tau, \vec{\sigma})+ \\
&+\tilde{\lambda}_{A}(\tau)\left\{{ }^{3} e_{(a) r}(\tau, \vec{\sigma}), \hat{P}_{A D M}^{A}\right\}, \\
& \partial_{\tau}{ }^{3} \tilde{\pi}_{(a)}^{r}(\tau, \vec{\sigma}) \stackrel{\circ}{=} \frac{\epsilon c^{3}}{8 \pi G}\left[{ }^{3} e n\left({ }^{3} R^{r s}-\frac{1^{3}}{2} g^{r s}{ }^{3} R\right)^{3} e_{(a) s}+{ }^{3} e\left(n^{|r| s}-{ }^{3} g^{r s} n^{\mid u}{ }_{\mid u}\right)^{3} e_{(a) s}\right](\tau, \vec{\sigma})- \\
&-\epsilon \frac{2 \pi G{ }^{n}(\tau, \vec{\sigma})}{c^{3}}\left[\frac{1}{3}{ }^{3} G_{o(a)(b)(c)(d)}{ }^{3} \tilde{\pi}_{(b)}^{r}{ }^{3} e_{(c) s}{ }^{3} \tilde{\pi}_{(d)}^{s}-\right. \\
&\left.-\frac{2}{3} e{ }^{3} e_{(a)}^{r}{ }^{3} G_{o(b)(c)(d)(e)}{ }^{3} e_{(b) u}{ }^{3} \tilde{\pi}_{(c)}^{u}{ }^{3} e_{(d) v}{ }^{3} \tilde{\pi}_{(e)}^{v}\right](\tau, \vec{\sigma})+
\end{aligned}
$$




$$
\begin{aligned}
+ & \frac{\partial}{\partial \sigma^{s}}\left[n_{(b)}{ }^{3} e_{(b)}^{s}{ }^{3} \tilde{\pi}_{(a)}^{r}\right](\tau, \vec{\sigma})-{ }^{3} \tilde{\pi}_{(a)}^{u}(\tau, \vec{\sigma}) \frac{\partial}{\partial \sigma^{u}}\left[n_{(b)}{ }^{3} e_{(b)}^{r}\right](\tau, \vec{\sigma})+ \\
+ & \epsilon_{(a)(b)(c)} \hat{\mu}_{(b)}(\tau, \vec{\sigma})^{3} \tilde{\pi}_{(c)}^{r}(\tau, \vec{\sigma})+ \\
+ & \tilde{\lambda}_{A}(\tau)\left\{{ }^{3} \tilde{\pi}_{(a)}^{r}(\tau, \vec{\sigma}), \hat{P}_{A D M}^{A}\right\}, \\
& \text { with } \\
\hat{P}_{A D M}^{\tau}= & \epsilon \int d^{3} \sigma\left[\frac{c^{3}}{16 \pi G}{ }^{3} e^{3} e_{(a)}^{r}{ }^{3} e_{(a)}^{s}\left({ }^{3} \Gamma_{r v}^{u}{ }^{3} \Gamma_{s u}^{v}-{ }^{3} \Gamma_{r s}^{u}{ }^{3} \Gamma_{v u}^{v}\right)-\right. \\
- & \left.\frac{2 \pi G}{c^{3}{ }^{3} e}{ }^{3} G_{o(a)(b)(c)(d)}{ }^{3} e_{(a) r}{ }^{3} \tilde{\pi}_{(b)}^{r}{ }^{3} e_{(c) s}{ }^{3} \tilde{\pi}_{(d)}^{s}\right](\tau, \vec{\sigma}), \\
\hat{P}_{A D M}^{r}= & -\int d^{3} \sigma\left[{ }^{3} \Gamma_{u v}^{r}{ }^{3} e_{(a)}^{u}{ }^{3} \tilde{\pi}_{(a)}^{v}\right](\tau, \vec{\sigma}) .
\end{aligned}
$$

In $\hat{P}_{A D M}^{A}$ the 3-Christoffel coefficients must be expressed in terms of the cotriads, see Eq.(A1) of Appendix A.

Let us remark that, since we are using the ADM expression for the energy $\hat{P}_{A D M}^{\tau}$, we have not to show that it is definite positive, because the ADM canonical approach to metric gravity is contained in the one to tetrad gravity. 


\section{THE HAMILTONIAN GROUP OF GAUGE TRANSFORMATIONS.}

In this Section we shall study the Hamiltonian group of gauge transformations in the framework of scenario b) of the previous Section following the scheme outlined in Ref. [6] for metric gravity. The generators of the infinitesimal Hamiltonian gauge transformations connected with the identity are the first class constraints. As shown in Appendix A of III for metric gravity, the ADM action is quasi-invariant under the pull-back at the Lagrangian level of the gauge transformations generated by the Hamiltonian group of gauge transformations. As shown in Subsection B of Section IX of Ref. [6] it is only on the solutions of Einstein equations that the Hamiltonian group of gauge transformations agrees with the spacetime diffeomorphisms in Diff $M^{4}$, under which the Hilbert action is invariant. Instead, outside the solutions the Hamiltonian gauge group connects different 4-geometries (4-metric modulo Diff $\left.M^{4}\right)$.

In the 32-dimensional functional phase space $T^{*} \mathcal{C}$ spanned by the 16 field variables $n(\tau, \vec{\sigma}), n_{(a)}(\tau, \vec{\sigma}), \varphi_{(a)}(\tau, \vec{\sigma}),{ }^{3} e_{(a) r}(\tau, \vec{\sigma})$ of the Lagrangian configuration space $\mathcal{C}$ and by their 16 conjugate momenta, we have 14 first class constraints $\tilde{\pi}^{n}(\tau, \vec{\sigma}) \approx$ $0, \quad \tilde{\pi}_{(a)}^{\vec{n}}(\tau, \vec{\sigma}) \approx 0, \quad \tilde{\pi}_{(a)}^{\vec{\varphi}}(\tau, \vec{\sigma}) \approx 0,{ }^{3} \tilde{M}_{(a)}(\tau, \vec{\sigma}) \approx 0, \hat{\mathcal{H}}(\tau, \vec{\sigma}) \approx 0$ and either ${ }^{3} \tilde{\Theta}_{r}(\tau, \vec{\sigma}) \approx 0$ or $\hat{\mathcal{H}}_{(a)}(\tau, \vec{\sigma}) \approx 0$. Seven pairs of conjugate canonical variables, $\left\{n(\tau, \vec{\sigma}), \tilde{\pi}^{n}(\tau, \vec{\sigma}) ; n_{(a)}(\tau, \vec{\sigma}), \tilde{\pi}_{(a)}^{\vec{n}}(\tau, \vec{\sigma}) ; \varphi_{(a)}(\tau, \vec{\sigma}), \tilde{\pi}_{(a)}^{\vec{\varphi}}(\tau, \vec{\sigma})\right\}$, are already decoupled from the 18-dimensional subspace spanned by $\left\{{ }^{3} e_{(a) r}(\tau, \vec{\sigma}) ;{ }^{3} \tilde{\pi}_{(a)}^{r}(\tau, \vec{\sigma})\right\}$. The variables in $\mathcal{C}_{g}=$ $\left\{n(\tau, \vec{\sigma}), n_{(a)}(\tau, \vec{\sigma}), \varphi_{(a)}(\tau, \vec{\sigma})\right\}$ are gauge variables, but due to the decoupling there is no need to introduce gauge-fixing constraints to eliminate them explicitly, at least at this stage. Therefore, let us concentrate on the reduced 9-dimensional configuration function space $\mathcal{C}_{e}=\left\{{ }^{3} e_{(a) r}(\tau, \vec{\sigma})\right\}\left[\mathcal{C}=\mathcal{C}_{g}+\mathcal{C}_{e}, T^{*} \mathcal{C}=T^{*} \mathcal{C}_{g}+T^{*} \mathcal{C}_{e}\right]$ and on the 18-dimensional function phase space $T^{*} \mathcal{C}_{e}=\left\{{ }^{3} e_{(a) r}(\tau, \vec{\sigma}),{ }^{3} \tilde{\pi}_{(a)}^{r}(\tau, \vec{\sigma})\right\}$, on which we have the seven first class constraints ${ }^{3} \tilde{M}_{(a)}(\tau, \vec{\sigma}) \approx 0,{ }^{3} \tilde{\Theta}_{r}(\tau, \vec{\sigma}) \approx 0, \hat{\mathcal{H}}(\tau, \vec{\sigma}) \approx 0$, whose Poisson brackets, defining an algebra $\bar{g}$, are given in Eqs. 2.14).

\section{A. Hamiltonian Gauge Transformations.}

Let us call $\overline{\mathcal{G}}$ the (component connected to the identity of the) gauge group obtained from successions of gauge transformations generated by the previous seven first class constraints. Since ${ }^{3} \tilde{M}_{(a)}(\tau, \vec{\sigma}) \widetilde{\square}$ and ${ }^{3} \tilde{\Theta}_{r}(\tau, \vec{\sigma})$ Ð form a Lie subalgebra $\bar{g}_{R}$ of $\bar{g}$ (the algebra of $\overline{\mathcal{G}}$ ), let $\overline{\mathcal{G}}_{R}$ be the gauge group without the superhamiltonian constraint and $\overline{\mathcal{G}}_{R O T}$ its invariant subgroup containing only $\mathrm{SO}(3)$ rotations. The addition to $\bar{g}_{R}$ of the superhamiltonian $\hat{\mathcal{H}}(\tau, \vec{\sigma})$ introduces structure functions [the last of Eqs.(2.14)] as in the ADM Hamiltonian formulation of metric gravity, so that $\bar{g}$ is not a Lie algebra.

\footnotetext{
${ }^{57}$ The generators of the inner gauge $\mathrm{SO}(3)$-rotations.

${ }^{58}$ The generators of space pseudo-diffeomorphisms (passive diffeomorphisms) in Diff $\Sigma_{\tau}$ extended to cotriads.
} 
The gauge group $\overline{\mathcal{G}}_{R}$ may be identified with the automorphism group Aut $L \Sigma_{\tau}$ of the trivial principal $\mathrm{SO}(3)$-bundle $L \Sigma_{\tau} \approx \Sigma_{\tau} \times S O(3)$ of orthonormal coframes, whose properties are studied in Ref. [47]. The automorphism group Aut $L \Sigma_{\tau}$ contains the structure group $\mathrm{SO}(3)$ of $L \Sigma_{\tau}$ as a subgroup, and, moreover, Aut $L \Sigma_{\tau}$ is itself a principal bundle with base Diff $\Sigma_{\tau}$ (which acts on the base $\Sigma_{\tau}$ of $L \Sigma_{\tau}$ ) and structure group the group of gauge transformations O of the principal bundle $L \Sigma_{\tau}$ : therefore, locally Aut $L \Sigma_{\tau}$ has the trivialization $\left[U \subset \operatorname{Diff} \Sigma_{\tau}\right] \times S O(3)$ and we have

$$
\begin{aligned}
& \text { Aut } L \Sigma_{\tau} \rightarrow L \Sigma_{\tau} \approx \Sigma_{\tau} \times S O(3) \\
& \downarrow \\
& \text { Diff } \Sigma_{\tau} \rightarrow \Sigma_{\tau}
\end{aligned}
$$

Let us concentrate on the study of the non-Abelian Lie algebra $\bar{g}_{R}$ and of the associated group of gauge transformations $\overline{\mathcal{G}}_{R}$. Since $\overline{\mathcal{G}}_{R}$ contains the group of space pseudodiffeomorphisms Diff $\Sigma_{\tau}$ (or better its action on the cotriads), it is not a Hilbert-Lie group, at least in standard sense [48,47] [07; therefore, the standard technology from the theory of Lie groups used for Yang-Mills theory 1 is not directly available. However this technology can be used for the invariant subgroup of gauge $\mathrm{SO}(3)$-rotations. The main problem is that it is not clear how to parametrize the group manifold of $\operatorname{Diff} \Sigma_{\tau}$ : one only knows that its algebra (the infinitesimal space diffeomorphisms) is isomorphic to the tangent bundle $T \Sigma_{\tau}$ 48].

Moreover, while in a Lie (and also in a Hilbert-Lie) group the basic tool is the grouptheoretical exponential map, associated with the one-parameter subgroups, which coincides with the geodesic exponential map when the group manifold of a compact semisimple Lie group is regarded as a symmetric Riemann manifold [50], in Diff $\Sigma_{\tau}$ this map does not produce a diffeomorphism between a neighbourhood of zero in the algebra and a neighbourhood of the identity in Diff $\Sigma_{\tau}$ [48,47]. Therefore, to study the Riemannian 3-manifold $\Sigma_{\tau}$ we have to use the geodesic exponential map as the main tool [21, 21], even if it is not clear its relationship with the differential structure of Diff $\Sigma_{\tau}$. The geodesic exponential map at $p \in M^{4}$ sends each vector ${ }^{4} V_{p}={ }^{4} V_{p}^{\mu} \partial_{\mu} \in T_{p} M^{4}$ at $\mathrm{p}$ to the point of unit parameter distance along the unique geodesic through $\mathrm{p}$ with tangent vector ${ }^{4} V_{p}$ at $\mathrm{p}$; in a small neighbourhood $\mathrm{U}$ of $\mathrm{p}$ the exponential map has an inverse: $q \in U \subset M^{4} \Rightarrow q=\operatorname{Exp}^{4} V_{p}$ for some ${ }^{4} V_{p} \in T_{p} M^{4}$. Then, ${ }^{4} V_{p}^{\mu}$ are the normal coordinates $x_{2}^{\mu}$ of $q$ and $\mathrm{U}$ is a normal neighbourhood. Let us remark that in this way one defines an inertial observer in free fall at $q$ in general relativity.

In Yang-Mills theory with trivial principal bundles $P(M, G)=M \times G$ [17], the abstract object behind the configuration space is the connection 1-form $\omega$ on $P(M, G)=M \times G$ [2]; instead Yang-Mills configuration space contains the gauge potentials over the base $\mathrm{M}$,

\footnotetext{
${ }^{59} \mathrm{Gau} L \Sigma_{\tau}$; see Ref. [17] for a review of the notations.

${ }^{60}$ Its differential structure is defined in an inductive way.

${ }^{61}$ See Ref. 17] and the Appendix of Ref. 49.

${ }^{62} \mathrm{G}$ is a compact, semisimple, connected, simply connected Lie group with compact, semisimple
} 
${ }^{\sigma} A^{(\omega)}=\sigma^{*} \omega$, i.e. the pull-backs to $\mathrm{M}$ of the connection 1-form through global cross sections $\sigma: M \rightarrow P$. The group $\mathcal{G}$ of gauge transformations (its component connected to the identity) acting on the gauge potentials on $\mathrm{M}$ is interpreted in a passive sense as a change of global cross section at fixed connection $\omega,{ }^{\sigma_{U}} A^{(\omega)}=U^{-1} \sigma A^{(\omega)} U+U^{-1} d U$ os: this formula describes the gauge orbit associated with the given $\omega$. In this case, the group manifold of $\mathcal{G}$ may be considered the principal bundle $P(M, G)=M \times G$ itself parametrized with a special connection-dependent family of global cross sections, after having chosen canonical coordinates of first kind on a reference fiber (a copy of the group manifold of G) and having parallel (with respect to the given connection) transported them to the other fibers (see the next Section). In this way we avoid the overparametrization of $\mathcal{G}$ by means of the infinitedimensional space of all possible local and global cross sections from M to $\mathrm{P}$ (this would be the standard description of $\mathcal{G}$ ). The infinitesimal gauge transformations 0 in phase space are generated by the first class constraints giving the Gauss laws $\Gamma_{a} \approx 0$. By Legendre pullback to configuration space, we find

$$
\begin{aligned}
{ }^{\sigma+\delta \sigma} A^{(\omega)} & ={ }^{\sigma} A^{(\omega)}+\delta_{o}{ }^{\sigma} A^{(\omega)}={ }^{\sigma} A^{(\omega)}+U^{-1}\left(d U+\left[{ }^{\sigma} A^{(\omega)}, U\right]\right)={ }^{\sigma} A^{(\omega)}+\hat{D}^{(A)} \alpha= \\
& ={ }^{\sigma} A^{(\omega)}+\left\{{ }^{\sigma} A^{(\omega)}, \int \alpha_{a} \Gamma_{a}\right\}, \quad \text { if } U=I+\alpha .
\end{aligned}
$$

In our formulation of tetrad gravity the relevant configuration variables are globally defined cotriads ${ }^{3} e_{(a) r}(\tau, \vec{\sigma})$ on the hypersurface $\Sigma_{\tau} \approx R^{3}$, which is a parallelizable Riemannian 3-manifold $\left(\Sigma_{\tau},{ }^{3} g_{r s}={ }^{3} e_{(a) r}{ }^{3} e_{(a) s}\right)$ assumed asymptotically flat (therefore noncompact) at spatial infinity and geodesically complete; with these hypotheses we have $T \Sigma_{\tau} \approx \Sigma_{\tau} \times R^{3}$ and the coframe orthogonal principal affine $\mathrm{SO}(3)$-bundle is also trivial $L \Sigma_{\tau} \approx \Sigma_{\tau} \times S O(3)$ 0. In the phase space of tetrad gravity the rotations of the gauge group $\mathrm{SO}(3)$ are generated by the first class constraints ${ }^{3} \tilde{M}_{(a)}(\tau, \vec{\sigma}) \approx 0$. Therefore, in this case the abstract object behind the configuration space is the so(3)-valued soldering 1-form ${ }^{3} \theta=\hat{R}^{(a)}{ }^{3} \theta_{(a)}$ ๆ. This shows that to identify the global cotriads ${ }^{3} e_{(a) r}(\tau, \vec{\sigma})$ we have to choose an atlas of coordinate charts on $\Sigma_{\tau}$, so that in each chart ${ }^{3} \theta \mapsto \hat{R}^{(a)}{ }^{3} e_{(a) r}(\tau, \vec{\sigma}) d \sigma^{r}$. Since $\Sigma_{\tau}$ is assumed diffeomorphic to $R^{3}$, global coordinate systems exist.

The general coordinate transformations or space pseudo-diffeomorphisms of Diff $\Sigma_{\tau}$ are denoted as $\vec{\sigma} \mapsto \vec{\sigma}^{\prime}(\vec{\sigma})=\vec{\xi}(\vec{\sigma})=\vec{\sigma}+\hat{\vec{\xi}}(\vec{\sigma})$; for infinitesimal pseudo-diffeomorphisms, $\hat{\vec{\xi}}(\vec{\sigma})=\delta \vec{\sigma}(\vec{\sigma})$ is an infinitesimal quantity and the inverse infinitesimal pseudo-diffeomorphism

real Lie algebra $g$.

${ }^{63} \sigma_{U}=\sigma \cdot U$ with $U: M \rightarrow G$.

${ }^{64}$ It is the space of the cross sections of the principal bundle $P(M, G)$.

${ }^{65}$ The Lie algebra $g_{\mathcal{G}}$ of $\mathcal{G}$ : it is a vector bundle whose standard fiber is the Lie algebra $g$.

${ }^{66}$ Its points are the abstract coframes ${ }^{3} \theta_{(a)}\left(={ }^{3} e_{(a) r} d \sigma^{r}\right.$ in global $\Sigma_{\tau^{-}}$adapted coordinates $)$.

${ }^{67} \hat{R}^{(a)}$ are the generators of the Lie algebra $\mathrm{so}(3)$. 
is $\vec{\sigma}\left(\vec{\sigma}^{\prime}\right)=\vec{\sigma}^{\prime}-\delta \vec{\sigma}\left(\vec{\sigma}^{\prime}\right)=\vec{\sigma}^{\prime}-\hat{\vec{\xi}}\left(\vec{\sigma}^{\prime}\right)$. The cotriads ${ }^{3} e_{(a) r}(\tau, \vec{\sigma})$ and the 3 -metric ${ }^{3} g_{r s}(\tau, \vec{\sigma})=$ ${ }^{3} e_{(a) r}(\tau, \vec{\sigma})^{3} e_{(a) s}(\tau, \vec{\sigma})$ transform as $\vec{b}^{8}\left[\mathcal{L}_{X}\right.$ is the Lie derivative along the vector field $\left.X\right]$

$$
\begin{aligned}
& { }^{3} e_{(a) r}(\tau, \vec{\sigma}) \mapsto{ }^{3} e_{(a) r}^{\prime}\left(\tau, \vec{\sigma}^{\prime}(\vec{\sigma})\right)=\frac{\partial \sigma^{s}}{\partial \sigma^{\prime r}}{ }^{3} e_{(a) s}(\tau, \vec{\sigma}), \\
& \Rightarrow{ }^{3} e_{(a) r}(\tau, \vec{\sigma})=\frac{\partial \xi^{s}(\vec{\sigma})}{\partial \sigma^{r}}{ }^{3} e_{(a) s}^{\prime}(\tau, \vec{\xi}(\vec{\sigma}))=\frac{\partial \xi^{s}(\vec{\sigma})}{\partial \sigma^{r}} \hat{V}(\vec{\xi}(\vec{\sigma}))^{3} e_{(a) s}^{\prime}(\tau, \vec{\sigma}), \\
& { }^{3} g_{r s}(\tau, \vec{\sigma}) \mapsto{ }^{3} g_{r s}^{\prime}\left(\tau, \vec{\sigma}^{\prime}(\vec{\sigma})\right)=\frac{\partial \sigma^{u}}{\partial \sigma^{\prime r}} \frac{\partial \sigma^{v}}{\partial \sigma^{\prime s}}{ }^{3} g_{u v}(\tau, \vec{\sigma}), \\
& \delta^{3} e_{(a) r}(\tau, \vec{\sigma})={ }^{3} e_{(a) r}^{\prime}\left(\tau, \vec{\sigma}^{\prime}(\vec{\sigma})\right)-{ }^{3} e_{(a) r}(\tau, \vec{\sigma})=\delta_{o}{ }^{3} e_{(a) r}(\tau, \vec{\sigma})+\hat{\xi}^{s}(\vec{\sigma}) \partial_{s}{ }^{3} e_{(a) r}(\tau, \vec{\sigma})= \\
& =\frac{\partial \sigma^{s}}{\partial \sigma^{\prime r}} e_{(a) s}(\tau, \vec{\sigma})-{ }^{3} e_{(a) r}(\tau, \vec{\sigma})=-\partial_{r} \hat{\xi}^{s}(\vec{\sigma})^{3} e_{(a) s}(\tau, \vec{\sigma}) \\
& \delta_{o}^{3} e_{(a) r}(\tau, \vec{\sigma})={ }^{3} e_{(a) r}^{\prime}(\vec{\sigma})-{ }^{3} e_{(a) r}(\vec{\sigma})=-\left[\partial_{r} \hat{\xi}^{s}(\vec{\sigma})+\delta_{r}^{s} \hat{\xi}^{u}(\vec{\sigma}) \partial_{u}\right]^{3} e_{(a) s}(\tau, \vec{\sigma})= \\
& =\left[\mathcal{L}_{-\hat{\xi}^{s} \partial_{s}}{ }^{3} e_{(a) u}(\tau, \vec{\sigma}) d \sigma^{u}\right]_{r}=-\left\{{ }^{3} e_{(a) r}(\tau, \vec{\sigma}), \int d^{3} \sigma_{1} \hat{\xi}^{s}\left(\vec{\sigma}_{1}\right)^{3} \tilde{\Theta}_{s}\left(\tau, \vec{\sigma}_{1}\right)\right\}, \\
& \delta^{3} g_{r s}(\tau, \vec{\sigma})={ }^{3} g_{r s}^{\prime}\left(\tau, \vec{\sigma}^{\prime}(\vec{\sigma})\right)-{ }^{3} g_{r s}(\tau, \vec{\sigma})=\delta_{o}{ }^{3} g_{r s}(\tau, \vec{\sigma})+\hat{\xi}^{u}(\vec{\sigma}) \partial_{u}{ }^{3} g_{r s}(\tau, \vec{\sigma})= \\
& =\frac{\partial \sigma^{u}}{\partial \sigma^{\prime r}} \frac{\partial \sigma^{v}}{\partial \sigma^{\prime} s}{ }^{3} g_{u v}(\tau, \vec{\sigma})-{ }^{3} g_{r s}(\tau, \vec{\sigma})=-\left[\delta_{r}^{u} \partial_{s} \hat{\xi}^{v}(\vec{\sigma})+\delta_{s}^{v} \partial_{r} \hat{\xi}^{u}(\vec{\sigma})\right]^{3} g_{u v}(\tau, \vec{\sigma}), \\
& \delta_{o}{ }^{3} g_{r s}(\tau, \vec{\sigma})={ }^{3} g_{r s}^{\prime}(\tau, \vec{\sigma})-{ }^{3} g_{r s}(\tau, \vec{\sigma})= \\
& =-\left[\delta_{r}^{u} \partial_{s} \hat{\xi}^{v}(\vec{\sigma})+\delta_{s}^{v} \partial_{r} \hat{\xi}^{u}(\vec{\sigma})+\delta_{r}^{u} \delta_{s}^{v} \hat{\xi}^{w}(\vec{\sigma}) \partial_{w}\right]^{3} g_{u v}(\tau, \vec{\sigma})= \\
& =\left[\mathcal{L}_{-\hat{\xi}^{w} \partial_{w}}{ }^{3} g_{u v}(\tau, \vec{\sigma}) d \sigma^{u} \otimes d \sigma^{v}\right]_{r s}=-\left\{{ }^{3} g_{r s}(\tau, \vec{\sigma}), \int d^{3} \sigma_{1} \hat{\xi}^{s}\left(\vec{\sigma}_{1}\right)^{3} \tilde{\Theta}_{s}\left(\tau, \vec{\sigma}_{1}\right)\right\} .
\end{aligned}
$$

Instead the action of finite and infinitesimal gauge rotations of angles $\alpha_{(c)}(\vec{\sigma})$ and $\delta \alpha_{(c)}(\vec{\sigma})$ is respectively

$$
\begin{aligned}
{ }^{3} e_{(a) r}(\tau, \vec{\sigma}) & \mapsto{ }^{3} R_{(a)(b)}\left(\alpha_{(c)}(\vec{\sigma})\right)^{3} e_{(b) r}(\tau, \vec{\sigma}), \\
\delta_{o}{ }^{3} e_{(a) r}(\tau, \vec{\sigma}) & =\left\{{ }^{3} e_{(a) r}(\tau, \vec{\sigma}), \int d^{3} \sigma_{1} \delta \alpha_{(c)}\left(\vec{\sigma}_{1}\right)^{3} \tilde{M}_{(c)}\left(\tau, \vec{\sigma}_{1}\right)\right\}= \\
& =\epsilon_{(a)(b)(c)} \delta \alpha_{(b)}(\vec{\sigma})^{3} e_{(c) r}(\tau, \vec{\sigma}) .
\end{aligned}
$$

To identify the algebra $\bar{g}_{R}$ of $\overline{\mathcal{G}}_{R}$, let us study its symplectic action on $T^{*} \mathcal{C}_{e}$, i.e. the infinitesimal canonical transformations generated by the first class constraints ${ }^{3} \tilde{M}_{(a)}(\tau, \vec{\sigma})$, ${ }^{3} \tilde{\Theta}_{r}(\tau, \vec{\sigma})$. Let us define the vector fields

$$
\begin{aligned}
& X_{(a)}(\tau, \vec{\sigma})=-\left\{.,{ }^{3} \tilde{M}_{(a)}(\tau, \vec{\sigma})\right\} \\
& Y_{r}(\tau, \vec{\sigma})=-\left\{.,{ }^{3} \tilde{\Theta}_{r}(\tau, \vec{\sigma})\right\} .
\end{aligned}
$$

Due to Eqs. 2.14) they close the algebra

${ }^{68} \hat{V}(\vec{\xi}(\vec{\sigma}))$ is the operator whose action on functions is $\hat{V}(\vec{\xi}(\vec{\sigma})) f(\vec{\sigma})=f(\vec{\xi}(\vec{\sigma}))$ 


$$
\begin{aligned}
& {\left[X_{(a)}(\tau, \vec{\sigma}), X_{(b)}\left(\tau, \vec{\sigma}^{\prime}\right)\right]=\delta^{3}\left(\vec{\sigma}, \vec{\sigma}^{\prime}\right) \epsilon_{(a)(b)(c)} X_{(c)}(\tau, \vec{\sigma})} \\
& {\left[X_{(a)}(\tau, \vec{\sigma}), Y_{r}\left(\tau, \vec{\sigma}^{\prime}\right)\right]=-\frac{\partial \delta^{3}\left(\vec{\sigma}, \vec{\sigma}^{\prime}\right)}{\partial \sigma^{\prime r}} X_{(a)}\left(\tau, \vec{\sigma}^{\prime}\right)} \\
& {\left[Y_{r}(\tau, \vec{\sigma}), Y_{s}\left(\tau, \vec{\sigma}^{\prime}\right)\right]=-\frac{\partial \delta^{3}\left(\vec{\sigma}, \vec{\sigma}^{\prime}\right)}{\partial \sigma^{\prime} s} Y_{r}\left(\tau, \vec{\sigma}^{\prime}\right)-\frac{\partial \delta^{3}\left(\vec{\sigma}, \vec{\sigma}^{\prime}\right)}{\partial \sigma^{\prime} r} Y_{s}(\tau, \vec{\sigma}) .}
\end{aligned}
$$

These six vector fields describe the symplectic action of rotation and space pseudodiffeomorphism gauge transformations on the subspace of phase space containing cotriads ${ }^{3} e_{(a) r}(\tau, \vec{\sigma})$ and their conjugate momenta ${ }^{3} \tilde{\pi}_{(a)}^{r}(\tau, \vec{\sigma})$. The non commutativity of rotations and space pseudo-diffeomorphisms means that the action of a space pseudo-diffeomorphism on a rotated cotriad produces a cotriad which differ by a rotation with modified angles from the action of the space pseudo-diffeomorphism on the original cotriad: if $\vec{\sigma} \rightarrow \vec{\sigma}^{\prime}(\vec{\sigma})$ is a space pseudo-diffeomorphism and ${ }^{3} R_{(a)(b)}\left(\alpha_{(c)}(\vec{\sigma})\right)$ is a rotation matrix parametrized with angles $\alpha_{(c)}(\vec{\sigma})$, then

$$
\begin{aligned}
{ }^{3} e_{(a) r}(\tau, \vec{\sigma}) & \mapsto{ }^{3} e_{(a) r}^{\prime}\left(\tau, \vec{\sigma}^{\prime}(\vec{\sigma})\right)=\frac{\partial \sigma^{s}}{\partial \sigma^{\prime r}}{ }^{3} e_{(a) s}(\tau, \vec{\sigma}), \\
{ }^{3} R_{(a)(b)}\left(\alpha_{(c)}(\vec{\sigma})\right) & { }^{3} e_{(b) r}(\tau, \vec{\sigma}) \mapsto{ }^{3} R_{(a)(b)}\left(\alpha_{(c)}^{\prime}\left(\vec{\sigma}^{\prime}(\vec{\sigma})\right)\right)^{3} e_{(b) r}^{\prime}\left(\tau, \vec{\sigma}^{\prime}(\vec{\sigma})\right)= \\
= & \frac{\partial \sigma^{s}}{\partial \sigma^{\prime r}}\left[{ }^{3} R_{(a)(b)}\left(\alpha_{(c)}(\vec{\sigma})\right)^{3} e_{(b) s}(\tau, \vec{\sigma})\right]= \\
= & { }^{3} R_{(a)(b)}\left(\alpha_{(c)}(\vec{\sigma})\right)^{3} e_{(b) r}^{\prime}\left(\vec{\sigma}^{\prime}(\vec{\sigma})\right), \\
\Rightarrow \quad & \alpha_{(c)}^{\prime}\left(\vec{\sigma}^{\prime}(\vec{\sigma})\right)=\alpha_{(c)}(\vec{\sigma}),
\end{aligned}
$$

i.e. the rotation matrices, namely the angles $\alpha_{(c)}(\vec{\sigma})$, behave as scalar fields under space pseudo-diffeomorphisms. Under infinitesimal rotations ${ }^{3} R_{(a)(b)}\left(\delta \alpha_{(c)}(\vec{\sigma})\right)=\delta_{(a)(b)}+$ $\delta \alpha_{(c)}(\vec{\sigma})\left(\hat{R}^{(c)}\right)_{(a)(b)}=\delta_{(a)(b)}+\epsilon_{(a)(b)(c)} \delta \alpha_{(c)}(\vec{\sigma})$ and space pseudo-diffeomorphisms $\vec{\sigma}^{\prime}(\vec{\sigma})=$ $\vec{\sigma}+\delta \vec{\sigma}(\vec{\sigma})$ [9 , we have

$$
\begin{aligned}
\int d^{3} \sigma_{1} d^{3} \sigma_{2} \quad & \delta \sigma^{s}\left(\vec{\sigma}_{2}\right) \delta \alpha_{(c)}\left(\vec{\sigma}_{1}\right)\left[Y_{s}\left(\tau, \vec{\sigma}_{2}\right), X_{(c)}\left(\tau, \vec{\sigma}_{1}\right)\right]^{3} e_{(a) r}(\tau, \vec{\sigma})= \\
= & \int d^{3} \sigma_{2} \delta \beta_{(c)}\left(\vec{\sigma}_{2}\right) X_{(c)}\left(\tau, \vec{\sigma}_{2}\right)^{3} e_{(a) r}(\tau, \vec{\sigma}) \\
\delta \beta_{(c)}(\vec{\sigma})= & \delta \sigma^{s}(\vec{\sigma}) \frac{\partial \alpha_{(c)}(\vec{\sigma})}{\partial \sigma^{s}} \\
\Rightarrow \quad & \alpha_{(c)}^{\prime}(\vec{\sigma})=\alpha_{(c)}(\vec{\sigma}-\delta \vec{\sigma}(\vec{\sigma}))=\alpha_{(c)}(\vec{\sigma})-\delta \beta_{(c)}(\vec{\sigma}) \Rightarrow \delta_{o} \alpha_{(c)}(\vec{\sigma})=-\delta \beta_{(c)}(\vec{\sigma}) .
\end{aligned}
$$

\section{B. What is Known on the Group Manifold of Gauge Transformations.}

The group manifold of the group $\overline{\mathcal{G}}_{R}$ of gauge transformations (isomorphic to Aut $L \Sigma_{\tau}$ ) is locally parametrized by three parameters $\vec{\xi}(\vec{\sigma})$ and by three angles $\alpha_{(c)}(\vec{\sigma})$ (which are

${ }^{69} \hat{R}^{(c)}$ are the $\mathrm{SO}(3)$ generators in the adjoint representation; $\delta \alpha_{(c)}(\vec{\sigma}), \delta \vec{\sigma}(\vec{\sigma})$ are infinitesimal variations. 
also functions of $\tau$ ), which are scalar fields under pseudo-diffeomorphisms, and contains an

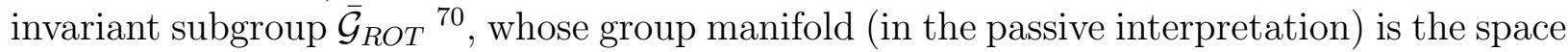
of the cross sections of the trivial principal bundle $\Sigma_{\tau} \times S O(3) \approx L \Sigma_{\tau}$ over $\Sigma_{\tau}$, like in $\mathrm{SO}(3)$ Yang-Mills theory [17], if $\Sigma_{\tau}$ is topologically trivial (its homotopy groups $\pi_{k}\left(\Sigma_{\tau}\right)$ all vanish); therefore, it may be parametrized as said above. As affine function space of connections on this principal $\mathrm{SO}(3)$-bundle we shall take the space of spin connection 1-forms ${ }^{3} \omega_{(a)}$, whose pullback to $\Sigma_{\tau}$ by means of cross sections $\sigma: \Sigma_{\tau} \rightarrow \Sigma_{\tau} \times S O(3)$ are the (Levi-Civita) spin connections (or Ricci rotation coefficients) ${ }^{3} \omega_{r(a)}(\tau, \vec{\sigma}) d \sigma^{r}=\sigma^{* 3} \omega_{(a)}$ built with cotriads ${ }^{3} e_{(a) r}(\tau, \vec{\sigma}) \square$ such that ${ }^{3} g_{r s}={ }^{3} e_{(a) r}{ }^{3} e_{(a) s}$.

Due to our hypotheses on $\Sigma_{\tau}$ (parallelizable, asymptotically flat, topologically trivial, geodesically complete), the Hopf-Rinow theorem [21] implies the existence of (at least) one point $p \in \Sigma_{\tau}$ which can be chosen as reference point and can be connected to every other point $q \in \Sigma_{\tau}$ with a minimizing geodesic segment $\gamma_{p q}$; moreover, the theorem says that there exists a point $p \in \Sigma_{\tau}$ from which $\Sigma_{\tau}$ is geodesically complete and that the geodesic exponential map $\operatorname{Exp}_{p}$ is defined on all $T_{p} \Sigma_{\tau}$. If $\Sigma_{\tau}$ is further restricted to have sectional curvature ${ }^{3} K_{p}(\Pi) \leq 0$ for each $p \in \Sigma_{\tau}$ and each tangent plane $\Pi \subset T_{p} \Sigma_{\tau}$, the Hadamard theorem [21] says that for each $p \in \Sigma_{\tau}$ the geodesic exponential map $\operatorname{Exp}_{p}: T_{p} \Sigma_{\tau} \rightarrow \Sigma_{\tau}$ is a diffeomorphism: therefore, there is a unique geodesic joining any pair of points $p, q \in \Sigma_{\tau}$ and $\Sigma_{\tau}$ is diffeomorphic to $R^{3}$ as we have assumed.

In absence of rotations, the group $\overline{\mathcal{G}}_{R}$ is reduced to the group Diff $\Sigma_{\tau}$ of space pseudodiffeomorphisms. In the active point of view, diffeomorphisms are smooth mappings (with smooth inverse) $\Sigma_{\tau} \rightarrow \Sigma_{\tau}$ : under Diff $\Sigma_{\tau}$ a point $p \in \Sigma_{\tau}$ is sent (in many ways) in every point of $\Sigma_{\tau}$. In the passive point of view, the action of the elements of Diff $\Sigma_{\tau}$, called pseudo-diffeomorphisms, on a neighbourhood of a point $p \in \Sigma_{\tau}$ is equivalent to all the possible coordinatizations of the subsets of the neighbourhood of $\mathrm{p}^{2}$.

A coordinate system (or chart) $(U, \sigma)$ in $\Sigma_{\tau}$ is a homeomorphism (which is also a diffeomorphism) $\sigma$ of an open set $U \subset \Sigma_{\tau}$ onto an open set $\sigma(U)$ of $R^{3}$ : if $\sigma: U \rightarrow \sigma(U)$ and $p \in U$, then $\sigma(p)=\left(\sigma^{r}(p)\right)$, where the functions $\sigma^{r}$ are called the coordinate functions of $\sigma$. An atlas on $\Sigma_{\tau}$ is a collection of charts in $\Sigma_{\tau}$ such that: i) each point $p \in \Sigma_{\tau}$ is contained in the domain of some chart; ii) any two charts overlap smoothly. Let $\mathcal{A}=\left\{\left(U_{\alpha}, \sigma_{\alpha}\right)\right\}$ be the unique complete atlas on $\Sigma_{\tau}$, i.e. an atlas by definition containing each coordinate system $\left(U_{\alpha}, \sigma_{\alpha}\right)$ in $\Sigma_{\tau}$ that overlaps smoothly with every coordinate system in $\mathcal{A}$.

Given an active diffeomorphism $\phi: \Sigma_{\tau} \rightarrow \Sigma_{\tau}$ (i.e. a smooth mapping with smooth inverse) and any chart $(U, \sigma)$ in $\mathcal{A}$, then $\left(\phi(U), \sigma_{\phi}=\sigma \circ \phi\right)$ is another chart in $\mathcal{A}$ (the draggedalong chart) with $\sigma_{\phi}(p) \stackrel{\text { def }}{=} \sigma(\phi(p))$ and, if $p \in U$. Therefore, to each active diffeomorphism $\phi: \Sigma_{\tau} \rightarrow \Sigma_{\tau}$ we can associate a mapping $\phi_{\mathcal{A}}: \mathcal{A} \rightarrow \mathcal{A}$, i.e. a pseudo-diffeomorphism. If we consider a point $p \in \Sigma_{\tau}$ and the set $\mathcal{A}_{p}=\left\{\left(U_{\beta}^{p}, \sigma_{\beta}^{p}\right)\right\}$ of all charts in $\mathcal{A}$ containing

\footnotetext{
${ }^{70}$ The group of gauge transformations of the coframe bundle $L \Sigma_{\tau}$; it is a splitting normal Lie subgroup of Aut $L \Sigma_{\tau}$ [47 .

${ }^{71}$ They and not the spin connections are the independent variables of tetrad gravity.

${ }^{72}$ I.e. to all possible changes of coordinate charts containing p.
} 
$\mathrm{p}$, then for each diffeomorphism $\phi: \Sigma_{\tau} \rightarrow \Sigma_{\tau}$ we will have the pseudo-diffeomorphism $\phi_{\mathcal{A}}: \mathcal{A}_{p} \rightarrow \mathcal{A}_{p}$. This suggests that a local parametrization of Diff $\Sigma_{\tau}$ around a point $p \in \Sigma_{\tau}$ may be done by choosing an arbitrary chart $\left(U_{o}^{p}, \sigma_{o}^{p}\right)$ as the local identity of pseudo-diffeomorphisms $[\vec{\xi}(\vec{\sigma})=\vec{\sigma}]$ and associating with every nontrivial diffeomorphism $\phi: \Sigma_{\tau} \rightarrow \Sigma_{\tau}, \vec{\sigma} \mapsto \vec{\sigma}^{\prime}(\vec{\sigma})=\vec{\xi}(\vec{\sigma})$, the chart $\left(U_{\beta}^{p}=\phi\left(U_{o}^{p}\right), \sigma_{\beta}^{p}=\sigma_{o}^{p} \circ \phi\right)$. Since $\Sigma_{\tau} \approx R^{3}$ admits global charts $\Xi$, then the group manifold of $\operatorname{Diff} \Sigma_{\tau}$ may be tentatively parametrized (in a nonredundant way) with the space of smooth global cross sections (global coordinate systems) in a fibration $\Sigma_{\tau} \times \Sigma_{\tau} \rightarrow \Sigma_{\tau}$ : this is analogous to the parametrization of the gauge group of Yang-Mills theory with a family of global cross sections of the trivial principal bundle $P(M, G)=M \times G$. The infinitesimal pseudo-diffeomorphisms $\square$ would correctly correspond to the cross sections of the fibration $\Sigma_{\tau} \times T \Sigma_{\tau} \rightarrow \Sigma_{\tau}$. With more general $\Sigma_{\tau}$ the previous description would hold only locally.

By remembering Eq.(3.1), the following picture emerges:

i) Choose a global coordinate system $\Xi$ on $\Sigma_{\tau} \approx R^{3}$ (for instance 3-orthogonal coordinates).

ii) In the description of Diff $\Sigma_{\tau}$ as $\Sigma_{\tau} \times \Sigma_{\tau} \rightarrow \Sigma_{\tau}$ this corresponds to the choice of a global cross section $\sigma_{\Xi}$ in $\Sigma_{\tau} \times \Sigma_{\tau}$, chosen as conventional origin of the pseudo-diffeomorphisms parametrized as $\vec{\sigma} \mapsto \vec{\xi}(\vec{\sigma})$.

iii) This procedure identifies a cross section $\tilde{\sigma}_{\Xi}$ of the principal bundle Aut $L \Sigma_{\tau} \rightarrow$ Diff $\Sigma_{\tau}$, whose action on $L \Sigma_{\tau}$ will be the $\mathrm{SO}(3)$ gauge rotations in the chosen coordinate system $\Xi$ on $\Sigma_{\tau}$.

iv) This will induce a $\Xi$-dependent trivialization of $L \Sigma_{\tau}$ to $\Sigma_{\tau}^{(\Xi)} \times S O(3)$, in which $\Sigma_{\tau}$ has $\Xi$ as coordinate system and the identity cross section $\sigma_{I}^{(\Xi)}$ of $\Sigma_{\tau}^{(\Xi)} \times S O(3)$ corresponds to the origin of rotations in the coordinate system $\Xi \square$.

v) As we will see in the next Section, it is possible to define new vector fields $\tilde{Y}_{r}(\tau, \vec{\sigma})$ which commute with the rotations $\left(\left[X_{(a)}(\tau, \vec{\sigma}), \tilde{Y}_{r}\left(\tau, \vec{\sigma}^{\prime}\right)\right]=0\right)$ and still satisfy the last line of Eqs.(3.6). In this way the algebra $\bar{g}_{R}$ of the group $\overline{\mathcal{G}}_{R}$ is replaced (at least locally) by a new algebra $\bar{g}_{R}^{\prime}$, which defines a group $\overline{\mathcal{G}}_{R}^{\prime}$, which is a (local) trivialization of Aut $L \Sigma_{\tau}$. It is at this level that the rotations in $\overline{\mathcal{G}}_{R O T}$ may be parametrized with a special family of cross sections of the trivial orthogonal coframe bundle $\Sigma_{\tau}^{(\Xi)} \times S O(3) \approx L \Sigma_{\tau}$, as for $\operatorname{SO}(3)$ Yang-Mills theory, as said in iv).

We do not know whether these steps can be implemented rigorously in a global way for $\Sigma_{\tau} \approx R^{3}$; if this is possible, then the quasi-Shanmugadhasan canonical transformation of Section $\mathrm{V}$ can be defined globally for global coordinate systems on $\Sigma_{\tau}$.

Both to study the singularity structure of De Witt superspace [36,52,53] for the Rieman-

\footnotetext{
${ }^{73}$ I.e. local pseudo-diffeomorphisms defined on the open sets containing p.

${ }^{74}$ Each global cross section of this fibration is a copy $\Sigma_{\tau}^{(\Xi)}$ of $\Sigma_{\tau}$ with the given coordinate system $\Xi$.

${ }^{75}$ The algebra $T \Sigma_{\tau}$ of $\operatorname{Diff} \Sigma_{\tau}$ 48; its generators in its symplectic action on $T^{*} \mathcal{C}_{e}$ are the vector fields $Y_{r}(\tau, \vec{\sigma})$.

${ }^{76}$ Remember that the angles are scalar fields under pseudo-diffeomorphisms in Diff $\Sigma_{\tau}$.
} 
nian 3-manifolds $\Sigma_{\tau}$ (the space of 3-metrics ${ }^{3} g$ modulo Diff $\Sigma_{\tau}$ ), for instance the cone over cone singularities of Ref. [54], and the analogous phenomenon (called in this case Gribov ambiguity) for the group $\overline{\mathcal{G}}_{R O T}$ of $\mathrm{SO}(3)$ gauge transformations, we have to analyze the stability subgroups of the group $\overline{\mathcal{G}}_{R}$ of gauge transformations for special $\operatorname{cotriads}{ }^{3} e_{(a) r}(\tau, \vec{\sigma})$, the basic variables in tetrad gravity. In metric gravity, where the metric is the basic variable and pseudo-diffeomorphisms are the only gauge transformations (we are ignoring the superhamiltonian constraint at this stage), it is known that if the 3 -metric ${ }^{3} g$ over a noncompact 3-manifold like $\Sigma_{\tau}$ satisfies boundary conditions compatible with being a function in a Sobolev space $W^{2, s}$ with $s>3 / 2$, then there exist special metrics admitting isometries. The group Iso $\left(\Sigma_{\tau},{ }^{3} g\right)$ of isometries of a 3-metric of a Riemann 3-manifold $\left(\Sigma_{\tau},{ }^{3} g\right)$ is the subgroup of Diff $\Sigma_{\tau}$ which leaves the functional form of the 3 -metric ${ }^{3} g_{r s}(\tau, \vec{\sigma})$ invariant (its Lie algebra is spanned by the Killing vector fields): the pseudo-diffeomorphism $\vec{\sigma} \mapsto \vec{\sigma}^{\prime}(\vec{\sigma})=\vec{\xi}(\vec{\sigma})$ in Diff $\Sigma_{\tau}$ is an isometry in $\operatorname{Iso}\left(\Sigma_{\tau},{ }^{3} g\right)$ if

$$
{ }^{3} g_{r s}\left(\tau, \vec{\sigma}^{\prime}(\vec{\sigma})\right)={ }^{3} g_{r s}^{\prime}\left(\tau, \vec{\sigma}^{\prime}(\vec{\sigma})\right)=\frac{\partial \sigma^{u}}{\partial \sigma^{\prime r}} \frac{\partial \sigma^{v}}{\partial \sigma^{\prime s}}{ }^{3} g_{u v}(\tau, \vec{\sigma}) .
$$

In such a case the function space of 3-metrics turns out to be a stratified manifold with singularities [52]. Each stratum contains all metrics ${ }^{3} g$ with the same subgroup $I s o\left(\Sigma_{\tau},{ }^{3} g\right) \subset$ Diff $\Sigma_{\tau}{ }^{77}$; each point in a stratum with $\mathrm{n}$ Killing vectors is the vertex of a cone, which is a stratum with n-1 Killing vectors (the cone over cone structure of singularities [54]).

From 18

$$
\begin{aligned}
{ }^{3} g_{r s}\left(\tau, \vec{\sigma}^{\prime}(\vec{\sigma})\right) & ={ }^{3} g_{r s}^{\prime}\left(\tau, \vec{\sigma}^{\prime}(\vec{\sigma})\right)={ }^{3} e_{(a) r}^{\prime}\left(\tau, \vec{\sigma}^{\prime}(\vec{\sigma})\right)^{3} e_{(a) s}^{\prime}\left(\tau, \vec{\sigma}^{\prime}(\vec{\sigma})\right)= \\
& =\frac{\partial \sigma^{u}}{\partial \sigma^{\prime r}} \frac{\partial \sigma^{v}}{\partial \sigma^{\prime}}{ }^{3} g_{u v}(\tau, \vec{\sigma})=\frac{\partial \sigma^{u}}{\partial \sigma^{\prime r}} \frac{\partial \sigma^{v}}{\partial \sigma^{\prime s}}{ }^{3} e_{(a) r}(\tau, \vec{\sigma})^{3} e_{(a) s}(\tau, \vec{\sigma}), \\
{ }^{3} e_{(a) r}^{\prime}\left(\tau, \vec{\sigma}^{\prime}(\vec{\sigma})\right) & =R_{(a)(b)}\left(\gamma\left(\tau, \vec{\sigma}^{\prime}(\vec{\sigma})\right)\right) \frac{\partial \sigma^{u}}{\partial \sigma^{\prime r}}{ }^{3} e_{(b) u}(\tau, \vec{\sigma}),
\end{aligned}
$$

it follows that also the functional form of the associated cotriads is invariant under $\operatorname{Iso}\left(\Sigma_{\tau},{ }^{3} g\right)$

$$
{ }^{3} e_{(a) r}\left(\tau, \vec{\sigma}^{\prime}(\vec{\sigma})\right)={ }^{3} e_{(a) r}^{\prime}\left(\tau, \vec{\sigma}^{\prime}(\vec{\sigma})\right)=R_{(a)(b)}\left(\gamma\left(\tau, \vec{\sigma}^{\prime}(\vec{\sigma})\right)\right) \frac{\partial \sigma^{s}}{\partial \sigma^{\prime r}}{ }^{3} e_{(b) s}(\tau, \vec{\sigma})
$$

Moreover, ${ }^{3} g_{r s}\left(\tau, \vec{\sigma}^{\prime}(\vec{\sigma})\right)={ }^{3} g_{r s}^{\prime}\left(\tau, \vec{\sigma}^{\prime}(\vec{\sigma})\right)$ implies ${ }^{3} \Gamma_{r s}^{\prime} u\left(\tau, \vec{\sigma}^{\prime}(\vec{\sigma})\right)={ }^{3} \Gamma_{r s}^{u}\left(\tau, \vec{\sigma}^{\prime}(\vec{\sigma})\right)$ and ${ }^{3} R^{\prime u}{ }_{r s t}\left(\tau, \vec{\sigma}^{\prime}(\vec{\sigma})\right)={ }^{3} R_{r s t}^{u}\left(\tau, \vec{\sigma}^{\prime}(\vec{\sigma})\right)$, so that $I s o\left(\Sigma_{\tau},{ }^{3} g\right)$ is also the stability group for the associated Christoffel symbols and Riemann tensor

$$
\begin{aligned}
& { }^{3} \Gamma_{r s}^{u}\left(\tau, \vec{\sigma}^{\prime}(\vec{\sigma})\right)={ }^{3} \Gamma_{r s}^{\prime u}\left(\tau, \vec{\sigma}^{\prime}(\vec{\sigma})\right)= \\
& =\frac{\partial \sigma^{\prime} u}{\partial \sigma^{v}} \frac{\partial \sigma^{m}}{\partial \sigma^{\prime} r} \frac{\partial \sigma^{n}}{\partial \sigma^{\prime} s}{ }^{3} \Gamma_{m n}^{v}(\tau, \vec{\sigma})+\frac{\partial^{2} \sigma^{v}}{\partial \sigma^{\prime} r \partial \sigma^{\prime} s} \frac{\partial \sigma^{\prime} u}{\partial \sigma^{v}},
\end{aligned}
$$

\footnotetext{
${ }^{77}$ Isomorphic but not equivalent subgroups of $\operatorname{Diff} \Sigma_{\tau}$ produce different strata of 3-metrics.

${ }^{78}$ At the level of cotriads a pseudo-diffeomorphism-dependent rotation is allowed.
} 


$$
\begin{aligned}
{ }^{3} R_{r s t}^{u}\left(\tau, \vec{\sigma}^{\prime}(\vec{\sigma})\right) & ={ }^{3} R^{\prime u}{ }_{r s t}\left(\tau, \vec{\sigma}^{\prime}(\vec{\sigma})\right)= \\
& =\frac{\partial \sigma^{\prime} u}{\partial \sigma^{v}} \frac{\partial \sigma^{l}}{\partial \sigma^{\prime r}} \frac{\partial \sigma^{m}}{\partial \sigma^{\prime}} \frac{\partial \sigma^{n}}{\partial \sigma^{\prime t}}{ }^{3} R^{v}{ }_{l m n}(\tau, \vec{\sigma}) .
\end{aligned}
$$

Let us remark that in the Yang-Mills case (see Ref. [17] and the end of this Section) the field strengths have generically a larger stability group (the gauge copies problem) than the gauge potentials (the gauge symmetry problem). Here, one expects that Riemann tensors (the field strengths) should have a stability group $\mathcal{S}_{R}\left(\Sigma_{\tau},{ }^{3} \mathrm{~g}\right)$ generically larger of the one of the Christoffel symbols (the connection) $\mathcal{S}_{\Gamma}\left(\Sigma_{\tau},{ }^{3} \mathrm{~g}\right)$, which in turn should be larger of the isometry group of the metric: $\mathcal{S}_{R}\left(\Sigma_{\tau},{ }^{3} g\right) \supseteq \mathcal{S}_{\Gamma}\left(\Sigma_{\tau},{ }^{3} g\right) \supseteq I s o\left(\Sigma_{\tau},{ }^{3} g\right)$. However, these stability groups do not seem to have been explored in the literature.

Since the most general transformation in $\overline{\mathcal{G}}_{R}$ for cotriads ${ }^{3} e_{(a) r}(\tau, \vec{\sigma})$, spin connections $\hat{R}^{(a)}{ }^{3} \omega_{r(a)}(\tau, \vec{\sigma})$ and field strengths $\hat{R}^{(a)}{ }^{3} \Omega_{r s(a)}(\tau, \vec{\sigma})$ is $\square$

$$
\begin{aligned}
& { }^{3} e_{(a) r}^{\prime R}\left(\tau, \vec{\sigma}^{\prime}(\vec{\sigma})\right)={ }^{3} R_{(a)(b)}\left(\alpha_{(c)}(\tau, \vec{\sigma})\right) \frac{\partial \sigma^{s}}{\partial \sigma^{\prime r}}{ }^{3} e_{(b) s}(\tau, \vec{\sigma}), \\
& \hat{R}^{(a){ }^{3} \omega_{r(a)}^{\prime} R}\left(\tau, \vec{\sigma}^{\prime}(\vec{\sigma})=\frac{\partial \sigma^{u}}{\partial \sigma^{\prime} r}\left[{ }^{3} R^{-1}\left(\alpha_{(e)}(\tau, \vec{\sigma})\right) \hat{R}^{(a)}{ }^{3} \omega_{u(a)}(\tau, \vec{\sigma}){ }^{3} R\left(\alpha_{(e)}(\tau, \vec{\sigma})\right)+\right.\right. \\
& \left.+{ }^{3} R^{-1}\left(\alpha_{(e)}(\tau, \vec{\sigma})\right) \partial_{u}{ }^{3} R\left(\alpha_{(e)}(\tau, \vec{\sigma})\right)\right]= \\
& =\frac{\partial \sigma^{u}}{\partial \sigma^{\prime r}}\left[\hat{R}^{(a) 3} \omega_{u(a)}(\tau, \vec{\sigma})+{ }^{3} R^{-1}\left(\alpha_{(e)}(\tau, \vec{\sigma})\right) \hat{D}_{u}^{(\omega)}{ }^{3} R\left(\alpha_{(e)}(\tau, \vec{\sigma})\right)\right]= \\
& =\hat{R}^{(a) 3} \omega_{r(a)}^{\prime}\left(\tau, \vec{\sigma}^{\prime}(\vec{\sigma})\right)+{ }^{3} R^{-1}\left(\alpha_{(e)}(\tau, \vec{\sigma})\right) \hat{D}_{r}^{\left(\omega^{\prime}\right) 3} R\left(\alpha_{(e)}^{\prime}\left(\tau, \vec{\sigma}^{\prime}(\vec{\sigma})\right)\right),
\end{aligned}
$$

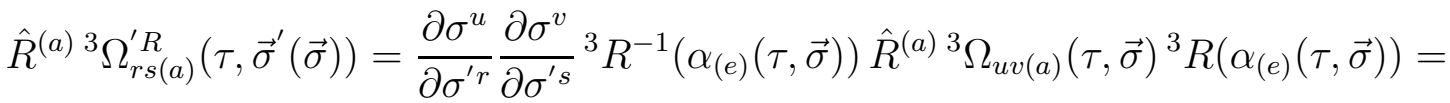

$$
\begin{aligned}
& =\frac{\partial \sigma^{u}}{\partial \sigma^{\prime r}} \frac{\partial \sigma^{v}}{\partial \sigma^{\prime}}\left(\hat{R}^{(a) 3} \Omega_{u v(a)}(\tau, \vec{\sigma})+\right. \\
& \left.+{ }^{3} R^{-1}\left(\alpha_{(e)}(\tau, \vec{\sigma})\right)\left[\hat{R}^{(a) 3} \Omega_{u v(a)}(\tau, \vec{\sigma}),{ }^{3} R\left(\alpha_{(e)}(\tau, \vec{\sigma})\right)\right]\right)= \\
& =\hat{R}^{(a) 3} \Omega_{r s(a)}^{\prime}\left(\tau, \vec{\sigma}^{\prime}(\vec{\sigma})\right)+ \\
& +{ }^{3} R^{-1}\left(\alpha_{(e)}^{\prime}\left(\tau, \vec{\sigma}^{\prime}(\vec{\sigma})\right)\right)\left[\hat{R}^{(a) 3} \Omega_{r s(a)}^{\prime}\left(\tau, \vec{\sigma}^{\prime}(\vec{\sigma})\right),{ }^{3} R\left(\alpha_{(e)}^{\prime}\left(\tau, \vec{\sigma}^{\prime}(\vec{\sigma})\right)\right)\right] .
\end{aligned}
$$

where $\left(\hat{D}_{r}^{(\omega)}\right)_{(a)(b)}=\hat{D}_{(a)(b) r}^{(\omega)}(\tau, \vec{\sigma})=\delta_{(a)(b)} \partial_{r}+\epsilon_{(a)(c)(b)}{ }^{3} \omega_{r(c)}(\tau, \vec{\sigma})$ and ${ }^{3} R\left(\alpha_{(e)}\right)$ are $3 \times 3$ rotation matrices, the behaviour of spin connections and field strengths under isometries can be studied.

\section{The Gribov Ambiguity.}

Let us now briefly review the Gribov ambiguity for the spin connections and the field strengths following Ref. [17]. All spin connections are invariant under gauge transformations

\footnotetext{
${ }^{79}$ We conform with the notations of Ref. [17].
} 
belonging to the center $Z_{3}$ of $\mathrm{SO}(3):{ }^{3} R \in Z_{3} \Rightarrow{ }^{3} \omega_{r(a)}^{R}={ }^{3} \omega_{r(a)}$.

As shown in Ref. [17], there can be special spin connections ${ }^{3} \omega_{r(a)}(\tau, \vec{\sigma})$, which admit a stability subgroup $\overline{\mathcal{G}}_{R O T}^{\omega}$ (gauge symmetries) of $\overline{\mathcal{G}}_{R O T}$, leaving them fixed

$$
{ }^{3} R\left(\alpha_{(e)}(\tau, \vec{\sigma})\right) \in \overline{\mathcal{G}}_{R}^{\omega} \Rightarrow \hat{D}_{r}^{(\omega)}{ }^{3} R\left(\alpha_{(e)}(\tau, \vec{\sigma})\right)=0 \Rightarrow{ }^{3} \omega_{r(a)}^{R}(\tau, \vec{\sigma})={ }^{3} \omega_{r(a)}(\tau, \vec{\sigma}) .
$$

From Eq.(3.11), it follows that under an isometry in $I s o\left(\Sigma_{\tau},{ }^{3} g\right)$ we have ${ }^{3} \omega_{r(a)}^{\prime}\left(\tau, \vec{\sigma}^{\prime}(\vec{\sigma})\right)=$ ${ }^{3} \omega_{r(a)}\left(\tau, \vec{\sigma}^{\prime}(\vec{\sigma})\right)$, namely the rotations ${ }^{3} R\left(\gamma\left(\tau, \vec{\sigma}^{\prime}(\vec{\sigma})\right)\right)$ are gauge symmetries.

When there are gauge symmetries, the spin connection is reducible: its holonomy group $\Phi^{\omega}$ is a subgroup of $\mathrm{SO}(3)\left[\Phi^{\omega} \subset S O(3)\right]$ and $\overline{\mathcal{G}}_{R O T}^{\omega} \nabla$ satisfies $\overline{\mathcal{G}}_{R O T}^{\omega}=Z_{S O(3)}\left(\Phi^{\omega}\right) \supset Z_{3}$.

Moreover, there can be special field strengths ${ }^{3} \Omega_{r s(a)}$ which admit a stability subgroup $\overline{\mathcal{G}}_{R O T}^{\Omega}$ of $\overline{\mathcal{G}}_{R O T}$ leaving them fixed

$$
\begin{gathered}
{ }^{3} R\left(\alpha _ { ( e ) } ( \tau , \vec { \sigma } ) \in \overline { \mathcal { G } } _ { R } ^ { \Omega } \Rightarrow \left[\hat{R}^{(a)}{ }^{3} \Omega_{r s(a)}(\tau, \vec{\sigma}),{ }^{3} R\left(\alpha_{(e)}(\tau, \vec{\sigma})\right]=0\right.\right. \\
\Rightarrow{ }^{3} \Omega_{r s(a)}^{R}(\tau, \vec{\sigma})={ }^{3} \Omega_{r s(a)}(\tau, \vec{\sigma}) .
\end{gathered}
$$

We have $\overline{\mathcal{G}}_{R O T}^{\Omega} \supseteq \overline{\mathcal{G}}_{R O T}^{\omega}=Z_{S O(3)}\left(\Phi^{\omega}\right) \supset Z_{3}$ and there is the problem of gauge copies: there exist different spin connections ${ }^{3} \omega_{r(a)}(\tau, \vec{\sigma})$ giving rise to the same field strength ${ }^{3} \Omega_{r s(a)}(\tau, \vec{\sigma})$.

A spin connection is irreducible, when its holonomy group $\Phi^{\omega}$ is a not closed irreducible matrix subgroup of $\mathrm{SO}(3)$. In this case we have $\overline{\mathcal{G}}_{R O T}^{\Omega} \supset \overline{\mathcal{G}}_{R O T}^{\omega}=Z_{S O(3)}\left(\Phi^{\omega}\right)=Z_{3}$ and there are gauge copies, but not gauge symmetries.

Finally, a spin connection ${ }^{3} \omega_{r(a)}(\tau, \vec{\sigma})$ is fully irreducible if $\Phi^{\omega}=S O(3)$ : in this case there are neither gauge symmetries nor gauge copies $\left(\overline{\mathcal{G}}_{R O T}^{\Omega}=\overline{\mathcal{G}}_{R O T}^{\omega}=Z_{3}\right)$ and the holonomy bundle $P^{\omega}(p)$ of every point $p \in \Sigma_{\tau} \times S O(3)$ coincides with $\Sigma_{\tau} \times S O(3)$ itself, so that every two points in $\Sigma_{\tau} \times S O(3)$ can be joined by a $\omega$-horizontal curve. Only in this case the covariant divergence is an elliptic operator without zero modes (this requires the use of special weighted Sobolev spaces for the spin connections to exclude the irreducible and reducible ones) and its Green function can be globally defined (absence of Gribov ambiguities).

In conclusion, the following diagram

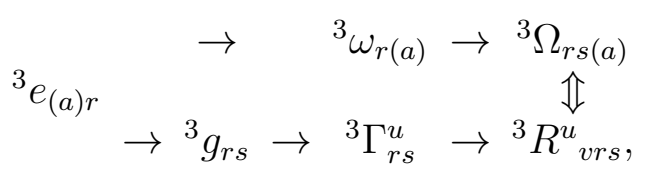

together with Eqs. 3.11), (3.13), implies that, to avoid any kind of pathology associated with stability subgroups of gauge transformations, one has to work with cotriads belonging to a function space such that: i) there is no subgroup of isometries in the action of Diff $\Sigma_{\tau}$ on the cotriads (no cone over cone structure of singularities in the lower branch of the diagram); ii) all the spin connections associated with the cotriads are fully irreducible (no type of Gribov ambiguity in the upper branch of the diagram). Both these requirements point towards the use of special weighted Sobolev spaces like in Yang-Mills theory 17,55.

It would be useful to make a systematic study of the relationships between the stability groups $\mathcal{S}_{R}\left(\Sigma_{\tau},{ }^{3} g\right) \supseteq \mathcal{S}_{\Gamma}\left(\Sigma_{\tau},{ }^{3} g\right) \supseteq I s o\left(\Sigma_{\tau},{ }^{3} g\right)$ and the stability groups $\overline{\mathcal{G}}_{R O T}^{\Omega} \supseteq \overline{\mathcal{G}}_{R O T}^{\omega}$ and

\footnotetext{
${ }^{80} \mathrm{It}$ is always equal to the centralizer of the holonomy group in $\mathrm{SO}(3), Z_{S O(3)}\left(\Phi^{\omega}\right) \dot{i}$
} 
to show rigorously that the presence of isometries (Gribov ambiguity) in the lower (upper) branch of the diagram implies the existence of Gribov ambiguity (isometries) in the upper (lower) branch.

Let us make a comment on the global Gribov ambiguity. Both in Yang-Mills theory and in tetrad gravity with angle-dependent boundary conditions on noncompact spacelike Cauchy surfaces we cannot make an one-point compactification of these surfaces: therefore, the relevant principal fiber bundles remain trivial and have the global identity cross section needed for the evaluation of Dirac's observables, but at the price of having the local Gribov ambiguities described in this Section. In the limit of angle-independent boundary conditions in suitable weighted Sobolev spaces (only completely irreducible connections; absence of local Gribov problem; well defined color charges in Yang-Mills theory and absence of supertranslations in tetrad gravity) we will go on to consider trivial principal fiber bundle: even if now it would be possible to make the one-point compactification, we are not doing it not to loose the global identity cross section. Otherwise our construction of Dirac's observables in the following Sections would become local.

In the more complex case in which magnetic monopoles or other objects which require the use of a non-trivial principal bundle from the beginning are assumed to exist, we remark that strictly speaking action principles depending on gauge potentials on the base manifold do not exist and one should reformulate the gauge theory starting from an action principle defined on the principal bundle manifold and depending on the connections on it [56]. Then one would have many more gauge degrees of freedom (the vertical vector fields) and the search of Dirac's observables should be reformulated in this framework.

See Ref. [57] for a treatment of large diffeomorphisms, the analogous of the large gauge transformations (due to winding number) of Yang-Mills theory [17], not connected to the identity.

The requirement of absence of isometries for every Riemann 3-manifold $\Sigma_{\tau}$ (the Cauchy surfaces) in the foliation of the spacetime $M^{4}$, associated with its allowed $3+1$ splittings, should not be an obstruction to the existence of 4-isometries of the pseudo-Riemannian 4manifold $M^{4}$. For instance Minkowski spacetime has ten 4-isometries (the Killing vectors are associated to the kinematical Poincaré group) and can be foliated with foliations whose spacelike leaves admit no 3-isometry of their intrinsic Riemannian structure.

\section{The Superhamiltonian Constraint as a Generator of Gauge Transformations.}

Let us now consider the gauge transformations generated by the superhamiltonian constraint, whose meaning has never been completely clarified in the literature (see for instance Refs. [58,59]). Here we shall repeat what has been already said in Ref. [6] regarding metric gravity. Since in tetrad gravity the superhamiltonian constraint is the same as in metric gravity [1]], only re-expressed in terms of the cotriads and their momenta, the interpretation of the gauge transformations generated by this constraint is the same in the two theories.

In Ref. 60] the superhamiltonian and supermomentum constraints of ADM metric grav-

ity are interpreted as the generators of the change of the canonical data ${ }^{3} g_{r s},{ }^{3} \tilde{\Pi}^{r s}$, under the normal and tangent deformations of the spacelike hypersurface $\Sigma_{\tau}$ which generate $\Sigma_{\tau+d \tau}$ 
๒. Therefore, the algebra of supermomentum and superhamiltonian constraints reflects the embeddability of $\Sigma_{\tau}$ into $M^{4}$ (see also Ref. [59]).

As a consequence of this geometrical property in the case of compact spacetimes without boundary the superhamiltonian constraint is interpreted as a time-dependent Hamiltonian for general relativity in some internal time variable defined in terms of the canonical variables (see for instance Ref. [62] and the so called internal intrinsic many-fingered time [63]).

The two main proposals for an internal time are:

i) The intrinsic internal time: it is the conformal factor $q(\tau, \vec{\sigma})=\frac{1}{6} \ln \operatorname{det}^{3} g_{r s}$ or $\phi(\tau, \vec{\sigma})=$ $e^{\frac{1}{2} q(\tau, \vec{\sigma})}=\left({ }^{3} g\right)^{1 / 12}>0$ of the 3 -metric. It is not a scalar and is proportional to Misner's time $\Omega=-\frac{1}{3} \ln \sqrt{\hat{\gamma}}$ [64] for asymptotically flat spacetimes (see Appendix C of II): $q=-\frac{1}{2} \Omega$.

ii) York's extrinsic internal time $\mathcal{T}=-{\frac{\epsilon c^{3}}{12 \pi G}}^{3} K=\frac{2}{3 \sqrt{\gamma}}^{3} \tilde{\Pi} \square$.

There are two interpretations of the superhamiltonian constraint in this framework

a) Either as a generator of time evolution (being a time-dependent Hamiltonian) like in the commonly accepted viewpoint based on the Klein-Gordon interpretation of the quantized superhamiltonian constraint, i.e. the Wheeler-DeWitt equation ${ }^{3}$.

b) or as a quantum Hamilton-Jacobi equation without any time (one can introduce a concept of evolution, somehow connected with an effective time, only in a WKB sense [72]).

A related problem is the validity of the full or thick sandwich conjecture [69, [0] of the thin sandwich conjecture 阿: see Ref. [73] (and also Ref. [74]) for the non validity of the full case and for the restricted validity (and its connection with constraint theory) of the thin case.

Since the superhamiltonian constraint is quadratic in the momenta, one is naturally driven to make a comparison with the free scalar relativistic particle described by the

\begin{abstract}
${ }^{81}$ One thinks to $\Sigma_{\tau}$ as determined by a cloud of observers, one per space point; the idea of bifurcation and re-encounter of the observers is expressed by saying that the data on $\Sigma_{\tau}$ (where the bifurcation took place) are propagated to some final $\Sigma_{\tau+d \tau}$ (where the re-encounter arises) along different intermediate paths, each path being a monoparametric family of surfaces that fills the sandwich in between the two surfaces; embeddability of $\Sigma_{\tau}$ in $M^{4}$ becomes the synonymous with path independence; see also Ref. [61] for the connection with the theorema egregium of Gauss.
\end{abstract}

${ }^{82}$ See Ref. [62] for a review of the known results with York's extrinsic internal time, Ref. [65] for York cosmic time versus proper time and Refs. [66, 67] for more general reviews about the problem of time in general relativity.

${ }^{83}$ See Kuchar in Ref. [68] and Wheeler's evolution of 3-geometries in superspace in Ref. [69,70] ; see Ref. [71] for the cosmological implications.

${ }^{84}$ Given two nearby 3-metrics on Cauchy surfaces $\Sigma_{\tau_{1}}$ and $\Sigma_{\tau_{2}}$, there is a unique spacetime $M^{4}$, satisfying Einstein's equations, with these 3-metrics on those Cauchy surfaces.

${ }^{85}$ Given ${ }^{3} g$ and $\partial_{\tau}{ }^{3} g$ on $\Sigma_{\tau}$, there is a unique spacetime $M^{4}$ with these initial data satisfying Einstein's equations; doing so the constraints become equations for the lapse and shift functions against the logic of the Hamiltonian presymplectic theory. 
first class constraint $p^{2}-\epsilon m^{2} \approx 0$. As shown in Refs. 15, 75, the constraint manifold in phase space has 1-dimensional gauge orbits (the two disjointed branches of the masshyperboloid); the $\tau$-evolution generated by the Dirac Hamiltonian $H_{D}=\lambda(\tau)\left(p^{2}-\epsilon m^{2}\right)$ gives the parametrized solution $x^{\mu}(\tau)$. Instead, if we go to the reduced phase space by adding the non-covariant gauge-fixing $x^{o}-\tau \approx 0$ and eliminating the pair of canonical variables $x^{o} \approx \tau, p^{o} \approx \pm \sqrt{\vec{p}^{2}+m^{2}}$, we get a frozen Jacobi description in terms of independent Cauchy data, in which the same Minkowski trajectory of the particle can be recovered in the non-covariant form $\vec{x}\left(x^{o}\right)$ by introducing as Hamiltonian the energy generator $\pm \sqrt{\vec{p}^{2}+m^{2}}$ of the Poincaré group

This comparison would suggest to solve the superhamiltonian constraint in one component of the ADM canonical momenta ${ }^{3} \tilde{\Pi}^{r s}$, namely in one component of the extrinsic curvature.

But, differently from the scalar particle, the solution of the superhamiltonian constraint does not define the weak ADM energy, which, instead, is connected with an integral over 3-space of that part of the superhamiltonian constraint dictated by the associated Gauss law, see Eqs.(5.4) of Ref. [6]. Indeed, the superhamiltonian constraint, being a secondary first class constraint of a field theory, has an associated Gauss law like the supermomentum constraints. In every Gauss law, the piece of the secondary first class constraint corresponding to a divergence and giving the strong form of the conserved charge (the strong ADM energy in this case) as the flux through the surface at infinity of a corresponding density depends on the variable which has to be eliminated in the canonical reduction by using the constraint (the conjugate variable is the gauge variable): once the constraint is solved in this variable, it can be put inside the volume expression of the weak form of the conserved charge to obtain its expression in the reduced phase space; the strong ADM energy is the only known charge, associated with a constraint bilinear in the momenta, depending only on the coordinates and not on the momenta, so that this implies that the superhamiltonian constraint has to be solved in one of the components of the 3-metric.

This shows that the right approach to the superhamiltonian constraint is the one of Lichnerowicz [76] leading to the conformal approach to the reduction of ADM metric gravity 77,78,65,79 87. In this approach the superhamiltonian constraint supplemented with the gauge fixing ${ }^{3} K(\tau, \vec{\sigma}) \approx 0$ (or $\approx$ const.; it is a condition on the internal extrinsic York time), named maximal slicing condition, is considered as an elliptic equation (the Lichnerowicz equation) to be solved in the conformal factor $\phi(\tau, \vec{\sigma})=e^{\frac{1}{2} q(\tau, \vec{\sigma})}>0$ of the 3-metric $\square$ rather

${ }^{86}$ With the variables of Ref. [24], one adds the covariant gauge-fixing $p \cdot x / \sqrt{p^{2}}-\tau \approx 0$ and eliminates the pair $T=p \cdot x / \sqrt{p^{2}}, \epsilon=\eta \sqrt{p^{2}} \approx \pm m$; now, since the invariant mass is constant, $\pm m$, the non-covariant Jacobi data $\vec{z}=\epsilon\left(\vec{x}-\vec{p} x^{o} / p^{o}\right), \vec{k}=\vec{p} / \epsilon$ cannot be made to evolve.

${ }^{87}$ See Appendix C of II for its review and for some notions on mean extrinsic curvature slices, for the TT (transverse traceless)-decomposition and for more comments about internal intrinsic and extrinsic times.

${ }^{88}$ Namely in its determinant ${ }^{3} g=\phi^{12}\left({ }^{3} g=\left({ }^{3} e\right)^{2}\right.$ with ${ }^{3} e=\operatorname{det}\left({ }^{3} e_{(a) r}\right)$ in tetrad gravity), which can be extracted from it in a 3 -covariant way. 
than in its conjugate momentum. Lichnerowicz has shown that the superhamiltonian and supermomentum constraints plus the maximal slicing condition of ADM metric gravity form a system of 5 elliptic differential equations which can be shown to have one and only one solution; moreover, with this condition Schoen and Yau [80] have shown that the ADM 4-momentum is timelike (i.e. the ADM energy is positive or zero for Minkowski spacetime). Moreover, Schoen-Yau have shown in their last proof of the positivity of the ADM energy that one can relax the maximal slicing condition. See the reviews [78,62] with their rich bibliography.

In the conformal approach one put ${ }^{3} g_{r s}=\phi^{4}{ }^{3} \sigma_{r s}\left[\operatorname{det}^{3} \sigma_{r s}=1\right]$ and ${ }^{3} \tilde{\Pi}^{r s}=\phi^{-10}{ }^{3} \tilde{\Pi}_{A}^{r s}+$ $\frac{1}{3}^{3} g^{r s}{ }^{3} \tilde{\Pi}\left[{ }^{3} g_{r s}{ }^{3} \tilde{\Pi}_{A}^{r s}=0\right]$. Then, one makes the TT-decomposition ${ }^{3} \tilde{\Pi}_{A}^{r s}={ }^{3} \tilde{\Pi}_{T T}^{r s}+{ }^{3} \tilde{\Pi}_{L}^{r s}$ (the TT-part is the conformally rescaled distortion tensor) with ${ }^{3} \tilde{\Pi}_{L}^{r s}=\left(L W_{\pi}\right)^{r s}=W_{\pi}^{r \mid s}+W_{\pi}^{s \mid r}-$ $\frac{2}{3}{ }^{3} g^{r s} W_{\pi \mid u}^{u}$, where $W_{\pi}^{r}$ is York gravitomagnetic vector potential. The superhamiltonian and supermomentum constraints are interpreted as coupled quasilinear elliptic equations for $\phi$ and $W_{\pi}^{r}$ (the four conjugate variables are free gauge variables), which decouple with the maximal slicing condition ${ }^{3} K=0$; the two physical degrees of freedom are hidden in ${ }^{3} \tilde{\Pi}_{T T}^{r s}$ (and in two conjugate variables).

In Ref. 81] it is shown that given the non-canonical basis [see the end of Appendix $\mathrm{C}$ of II, in particular its Eq.(C7)] $\mathcal{T}=-{\frac{\epsilon c^{3}}{12 \pi G}}^{3} K=\frac{2}{3 \sqrt{\gamma}}^{3} \tilde{\Pi}, \mathcal{P}_{\mathcal{T}}=-\operatorname{det}^{3} g_{\text {rs }}=-\phi^{12}$, ${ }^{3} \sigma_{r s}={ }^{3} g_{r s} /\left(\operatorname{det}^{3} g\right)^{1 / 3},{ }^{3} \tilde{\Pi}_{A}^{r s}$, there exists a canonical basis hidden in the variables ${ }^{3} \sigma_{r s},{ }^{3} \tilde{\Pi}_{A}^{r s}$ (but it has never been found explicitly) and that one can define the reduced phase space (the conformal superspace) $\tilde{\mathcal{S}} \boldsymbol{\nabla}$, in which one has gone to the quotient with respect to the space diffeomorphisms and to the conformal rescalings. It is also shown that one can define a York map from this reduced phase space to the subset of the standard phase superspace (90) defined by the condition ${ }^{3} K=$ const..

In the conformal approach one uses York's TT-variables [77, because most of the work on the Cauchy problem for Einstein's equations in metric gravity is done by using spacelike hypersurfaces $\Sigma$ of constant mean extrinsic curvature (CMC surfaces) in the compact case (see Refs. [78, 83, 84]) and with the maximal slicing condition $\mathcal{T}(\tau, \vec{\sigma})=0$. It may be extended

\footnotetext{
${ }^{89}$ The conformal superspace $\tilde{\mathcal{S}}$ may be defined as the space of conformal 3-geometries on closed manifolds and can be identified in a natural way with the space of conformal 3-metrics (the quotient of superspace by the group $W e y l \Sigma_{\tau}$ of conformal Weyl rescalings) modulo space diffeomorphisms, or, equivalently, with the space of Riemannian 3-metrics modulo space diffeomorphisms and conformal transformations of the form ${ }^{3} g_{r s} \mapsto \phi^{4}{ }^{3} g_{r s}, \phi>0$. Instead, the ordinary superspace $\mathcal{S}$ is the space of Lorentzian 4-metrics modulo spacetime diffeomorphisms. In this way a bridge is built towards the phase superspace, which is mathematically connected with the Moncrief splitting theorem [82,78] valid for closed $\Sigma_{\tau}$. See however Ref. [78] for what is known in the asymptotically flat case by using weighted Sobolev spaces.

${ }^{90}$ Quotient of the ADM phase space with respect to the space diffeomorphisms plus the gauge transformations generated by the superhamiltonian constraint; it is the phase space of the superspace, the configuration space obtained from the 3-metrics going to the quotient with respect to the space- and time- diffeomorphisms of the ADM formalism.
} 
to non constant $\mathcal{T}$ ) in the asymptotically free case 97 .

Let us remark that in Minkowski spacetime ${ }^{3} K(\tau, \vec{\sigma})=0$ are the hyperplanes, while ${ }^{3} K(\tau, \vec{\sigma})=$ const. are the mass hyperboloids, corresponding to the instant and point form of the dynamics according to Dirac [87] respectively (see Refs. [88] for other types of foliations).

Instead in asymptotically free spacetimes there exists a time evolution in the mathematical time parametrizing the leaves $\Sigma_{\tau}$ of the $3+1$ splitting of $M^{4}$ governed by the weak ADM energy [45] as we have seen with the rest-frame instant form of gravity. The superhamiltonian constraint is not connected with time evolution: the strong and weak ADM energies are only integrals of parts of this constraint. Instead it is the generator of Hamiltonian gauge transformations.

As a constraint it determines the non-scalar conformal factor (the determinant) of the 3 -metric as a functional of ${ }^{3} \sigma_{r s}$ and ${ }^{3} \tilde{\Pi}^{r s}\left[\right.$ of ${ }^{3} e_{(a) r},{ }^{3} \tilde{\pi}_{(a)}^{r}$ in tetrad gravity]. But this means that the associated gauge variable is the canonical momentum conjugate to the conformal factor. This variable, and not York time, parametrizes the normal deformation of the embeddable spacelike hypersurfaces $\Sigma_{\tau}$. Now, since different $\Sigma_{\tau}$ corresponds to different $3+1$ splittings of $M^{4}$, in the class of the allowed ones going in an angle-independent way to Minkowski spacelike hyperplanes, we get that the gauge transformations generated by the superhamiltonian constraint correspond to the transition from an allowed $3+1$ splitting to another one (this is the gauge orbit in the phase space over superspace). Therefore the theory is independent from the choice of the $3+1$ splitting like parametrized Minkowski theories.

Since the solution of the Lichnerowicz equation gives the conformal factor $\phi=e^{q / 2}=$ $\left({ }^{3} \mathrm{~g}\right)^{1 / 12}$ as a function of its conjugate momentum and of the remaining canonical variables as in the compact case, also in the asymptotically free case only the conformal 3-geometries contain the physical degrees of freedom, whose functional form depends on the other gauge fixings, in particular on the choice of the 3-coordinates.

A gauge fixing to the superhamiltonian constraint is a choice of a particular $3+1$ splitting and this is done by fixing the momentum conjugate to the conformal factor $\%$.

Therefore it is important to study the Shanmugadhasan canonical bases of both metric and tetrad gravity, in which the conformal factor of the 3-metric 33 is one of the configurational canonical variables (see Section VI). One of these bases should correspond to the extension of the York map to asymptotically flat spacetimes: in it the momentum conjugate to the conformal factor is just York time and one can add the maximal slicing condition as a gauge fixing.

This leads to the conclusion that neither York's internal extrinsic time nor Misner's in-

\footnotetext{
${ }^{91}$ See also Ref. 850 for recent work in the compact case with non constant $\mathcal{T}$ and Ref. 86 for solutions of Einstein's equations in presence of matter which do not admit constant mean extrinsic curvature slices.

${ }^{92} \mathrm{~A}$ non-local information on the extrinsic curvature of $\Sigma_{\tau}$, which becomes the York time, or the maximal slicing condition, only with the special canonical basis identified by the York map.

${ }^{93}$ Or better, if one succeeds in doing it, an Abelianized form of the superhamiltonian constraint having zero Poisson bracket with itself.
} 
ternal intrinsic time are to be used as time parameters: Misner's time (the conformal factor) is determined by the Lichnerowicz equation while York's time (the trace of the extrinsic curvature) by the gauge-fixing. As said in Section XI of Ref. [6], the rest-frame instant form of metric and tetrad gravity uses a mathematical time identified before quantization: the parameter $\tau \equiv T_{(\infty)}$ labelling the WSW hypersurfaces and coinciding with the rest-frame time of the external decoupled center of mass of the universe considered as a point particle clock. We refer to Ref. [6] for further details and for the connection to the either proper or coordinate time of physical clocks. 


\section{MULTITEMPORAL EQUATIONS AND THEIR SOLUTION.}

In this Chapter we study the multitemporal equations [89] (or generalized Lie equations [90]) associated with the gauge transformations in $\overline{\mathcal{G}}_{R}$, to find a local parametrization of the cotriads ${ }^{3} e_{(a) r}(\tau, \vec{\sigma})$ in terms of the parameters $\xi_{r}(\tau, \vec{\sigma})$ and $\alpha_{(a)}(\tau, \vec{\sigma})$ of $\overline{\mathcal{G}}_{R}$. We shall assume to have chosen a global coordinate system $\Xi$ on $\Sigma_{\tau} \approx R^{3}$ to conform with the discussion of the previous Section.

\section{A. The Multi-Temporal Equations for the Rotations.}

Let us start with the invariant subalgebra $\bar{g}_{R O T}$ (the algebra of $\overline{\mathcal{G}}_{R O T}$ ) of rotations, whose generators are the vector fields $X_{(a)}(\tau, \vec{\sigma})$ of Eqs.(3.5). Since the group manifold of $\overline{\mathcal{G}}_{R O T}$ is a trivial principal bundle $\Sigma_{\tau}^{(\Xi)} \times S O(3) \approx L \Sigma_{\tau}$ over $\Sigma_{\tau}$, endowed with the coordinate system $\Xi$, with structure group $\mathrm{SO}(3)$, we can use the results of Ref. [17 for the case of $\mathrm{SO}(3)$ Yang-Mills theory.

Let $\alpha_{(a)}$ be canonical coordinates of first kind on the group manifold of $\mathrm{SO}(3)$. If $r^{(a)}$ are the generators of $\operatorname{so}(3),\left[r^{(a)}, r^{(b)}\right]=\epsilon_{(a)(b)(c)} r^{(c)}$ [4 and if $\gamma_{\alpha}(s)=\exp _{S O(3)}\left(s \alpha_{(a)} r^{(a)}\right)$ is a one-parameter subgroup of $\mathrm{SO}(3)$ with tangent vector $\alpha_{(a)} r^{(a)}$ at the identity $I \in S O(3)$, then the group element $\gamma_{\alpha}(1)=\exp _{S O(3)}\left(\alpha_{(a)} r^{(a)}\right) \in N_{I} \subset S O(3)$ 诃 is given coordinates $\left\{\alpha_{(a)}\right\}$. If $\tilde{Y}_{(a)}$ and $\tilde{\theta}_{(a)}$ are dual bases $\left(i_{\tilde{Y}_{(a)}} \tilde{\theta}_{(b)}=\delta_{(a)(b)}\right)$ of left invariant vector fields and left invariant (or Maurer-Cartan) 1-forms on $\mathrm{SO}(3)$, we have the standard Maurer-Cartan structure equations

$$
\begin{aligned}
& {\left[\tilde{Y}_{(a)}, \tilde{Y}_{(b)}\right]=\epsilon_{(a)(b)(c)} \tilde{Y}_{(c)}, \quad\left[\left.\tilde{Y}_{(a)}\right|_{I}=r^{(a)} \in s o(3)\right],} \\
& d \tilde{\theta}_{(a)}=-\frac{1}{2} \epsilon_{(a)(b)(c)} \tilde{\theta}_{(b)} \wedge \tilde{\theta}_{(c)}, \quad\left[\left.\tilde{\theta}_{(a)}\right|_{I}=r_{(a)} \in s o(3)^{*}\right] .
\end{aligned}
$$

Then, from Lie theorems, on the group manifold we have

$$
\tilde{Y}_{(a)}=B_{(b)(a)}(\alpha) \frac{\partial}{\partial \alpha_{(b)}}, \quad \tilde{\theta}_{(a)}=A_{(a)(b)}(\alpha) d \alpha_{(b)}, \quad A(\alpha)=B^{-1}(\alpha), \quad A(0)=B(0)=1,
$$

and the Maurer-Cartan equations become

$$
\begin{aligned}
& \frac{\partial A_{(a)(c)}(\alpha)}{\partial \alpha_{(b)}}-\frac{\partial A_{(a)(b)}(\alpha)}{\partial \alpha_{(c)}}=-\epsilon_{(a)(u)(v)} A_{(u)(b)}(\alpha) A_{(v)(c)}(\alpha), \\
& \tilde{Y}_{(b)} B_{(a)(c)}(\alpha)-\tilde{Y}_{(c)} B_{(a)(b)}(\alpha)=B_{(u)(b)}(\alpha) \frac{\partial B_{(a)(c)}(\alpha)}{\partial \alpha_{(u)}}-B_{(u)(c)}(\alpha) \frac{\partial B_{(a)(b)}(\alpha)}{\partial \alpha_{(u)}}= \\
& =B_{(a)(u)}(\alpha) \epsilon_{(u)(b)(c)} .
\end{aligned}
$$

\footnotetext{
${ }^{94}$ Instead $\hat{R}^{(a)}$ are the generators in the adjoint representation, $\left(\hat{R}^{(a)}\right)_{(b)(c)}=\epsilon_{(a)(b)(c)}$.

${ }^{95} N_{I}$ is a neighbourhood of the identity such that $\exp _{S O(3)}$ is a diffeomorphism from a neighbourhood of $0 \in s o(3)$ to $N_{I}$.

${ }^{96} s o(3) *$ is the dual Lie algebra; $T S O(3) \approx s o(3), T^{*} S O(3) \approx s o(3)^{*}$.
} 
By definition these coordinates are said canonical of first kind and satisfy $A_{(a)(b)}(\alpha) \alpha_{(b)}=$ $\alpha_{(a)}$, so that we get $A(\alpha)=\left(e^{R \alpha}-1\right) / R \alpha$ with $(R \alpha)_{(a)(b)}=\left(\hat{R}^{(c)}\right)_{(a)(b)} \alpha_{(c)}=\epsilon_{(a)(b)(c)} \alpha_{(c)}$. The canonical 1-form on $\mathrm{SO}(3)$ is $\tilde{\omega}_{S O(3)}=\tilde{\theta}_{(a)} r^{(a)}=A_{(a)(b)}(\alpha) d \alpha_{(b)} r^{(a)}$ 甲). Due to the Maurer-Cartan structure equations the 1-forms $\tilde{\theta}_{(a)}$ are not integrable on $\mathrm{SO}(3)$; however in the neighbourhood $N_{I} \subset S O(3)$ we can integrate them along the preferred defining line $\gamma_{\alpha}(s)$ defining the canonical coordinates of first kind to get the phases

$$
\Omega_{(a)}^{\gamma_{\alpha}}(\alpha(s))=\left.{ }_{\gamma_{\alpha}} \int_{I}^{\gamma_{\alpha}(s)} \tilde{\theta}_{(a)}\right|_{\gamma_{\alpha}}={ }_{\gamma_{\alpha}} \int_{0}^{\alpha(s)} A_{(a)(b)}(\bar{\alpha}) d \bar{\alpha}_{(b)} .
$$

If $d_{\gamma_{\alpha}}=\left.d s \frac{d \alpha_{(a)}(s)}{d s} \frac{\partial}{\partial \alpha_{(a)}}\right|_{\alpha=\alpha(s)}=\left.d_{S O(3)}\right|_{\gamma_{\alpha}(s)}$ is the directional derivative along $\gamma_{\alpha}$, on $\gamma_{\alpha}$ we have $d_{\gamma_{\alpha}} \Omega_{(a)}^{\gamma_{\alpha}}(\alpha(s))=\tilde{\theta}_{(a)}(\alpha(s))$ and $d_{\gamma_{\alpha}} \tilde{\theta}_{(a)}(\alpha(s))=0 \Rightarrow d_{\gamma_{\alpha}}^{2}=0$. The analytic atlas $\mathcal{N}$ for the group manifold of $\mathrm{SO}(3)$ is built by starting from the neighbourhood $N_{I}$ of the identity with canonical coordinates of first kind by left multiplication by elements of $\mathrm{SO}(3)$ : $\mathcal{N}=\cup_{a \in S O(3)}\left\{a \cdot N_{I}\right\}$.

As shown in Ref. [17] for $R^{3} \times S O(3)$, in a tubular neighbourhood of the identity cross section $\sigma_{I}$ of the trivial principal bundle $R^{3} \times S O(3)$, in which each fiber is a copy of the $\mathrm{SO}(3)$ group manifold, we can define generalized canonical coordinates of first kind on each fiber so to build a coordinatization of $R^{3} \times S O(3)$. We now extend this construction from the flat Riemannian manifold $\left(R^{3}, \delta_{r s}\right)$ to a Riemannian manifold $\left(\Sigma_{\tau},{ }^{3} g_{r s}\right)$ satisfying our hypotheses, especially the Hadamard theorem, so that the 3 -manifold $\Sigma_{\tau}$, diffeomorphic to $R^{3}$, admits global charts.

Let us consider the fiber $\mathrm{SO}(3)$ over a point $p \in \Sigma_{\tau}$, chosen as origin $\vec{\sigma}=0$ of the global chart $\Xi$ on $\Sigma_{\tau}$, with canonical coordinates of first kind $\alpha_{(a)}=\alpha_{(a)}(\tau, \overrightarrow{0})$ on it. For a given spin connection ${ }^{3} \omega_{(a)}$ on $\Sigma_{\tau}^{(\Xi)} \times S O(3)$ let us consider the ${ }^{3} \omega$-horizontal lifts through each point of the fiber $\mathrm{SO}(3)$ of the star of geodesics of the Riemann 3-manifold $\left(\Sigma_{\tau},{ }^{3} g_{r s}={ }^{3} e_{(a) r}{ }^{3} e_{(a) s}\right)$ emanating from $p \in \Sigma_{\tau}$. If the spin connection ${ }^{3} \omega_{(a)}$ is fully irreducible, $\Sigma_{\tau} \times S O(3)$ is in this way foliated by a connection-dependent family of global cross sections defined by the ${ }^{3} \omega$-horizontal lifts of the star of geodesics 8 . The canonical coordinates of first kind on the reference $\mathrm{SO}$ (3) fiber may then be parallel- (with respect to ${ }^{3} \omega_{(a)}$ ) transported to all the other fibers along these ${ }^{3} \omega$-dependent global cross sections. If $\tilde{p}=\left(p ; \alpha_{(a)}\right)=\left(\tau, \overrightarrow{0} ; \alpha_{(a)}(\tau, \overrightarrow{0})\right)$ is a point in $\Sigma_{\tau} \times S O(3)$ over $p \in \Sigma_{\tau}$, if $\sigma_{(\tilde{p})}: \Sigma_{\tau} \rightarrow \Sigma_{\tau} \times S O(3)$ is the ${ }^{3} \omega$-dependent cross section through $\tilde{p}$ and if ${ }^{3} \omega_{r(a)}^{(\tilde{p})}(\tau, \vec{\sigma}) d \sigma^{r}=\sigma_{(\tilde{p})}^{*}{ }^{3} \omega_{(a)}$, then the coordinates of the point intersected by $\sigma_{(\tilde{p})}$ on the $\mathrm{SO}(3)$ fiber over the point $p^{\prime}$ of $\Sigma_{\tau}$ with coordinates $(\tau, \vec{\sigma})$ are

$$
\begin{aligned}
\alpha_{(a)}(\tau, \vec{\sigma}) & =\alpha_{(b)}(\tau, \overrightarrow{0}) \zeta_{(b)(a)}^{\left(\omega_{(a)}^{(\vec{p})}\right)}(\vec{\sigma}, \overrightarrow{0} ; \tau)= \\
& =\alpha_{(b)}(\tau, \overrightarrow{0})\left(P_{\gamma_{p p^{\prime}}} e^{\int_{\overrightarrow{0}}^{\vec{\sigma}} d z^{r} \hat{R}^{(c) 3} \omega_{r(c)}^{(\vec{p})}(\tau, \vec{z})}\right)_{(b)(a)},
\end{aligned}
$$

$$
{ }^{97}=a^{-1}(\alpha) d_{S O(3)} a(\alpha), a(\alpha) \in S O(3) ; d_{S O(3)} \text { is the exterior derivative on } \mathrm{SO}(3) \text {. }
$$

${ }^{98}$ They are not ${ }^{3} \omega$-horizontal cross sections, as it was erroneously written in Ref. [17], since such cross sections do not exist when the holonomy groups in each point of $\Sigma_{\tau} \times S O(3)$ are not trivial. 
where $\zeta_{(b)(a)}^{(\omega)}(\vec{\sigma}, \overrightarrow{0} ; \tau)$ is the Wu-Yang non-integrable phase with the path ordering evaluated along the geodesic $\gamma_{p p^{\prime}}$ from $\mathrm{p}$ to $p^{\prime}$. The infinitesimal form is

$$
\begin{aligned}
\alpha_{(a)}(\tau, d \vec{\sigma}) & \approx \alpha_{(a)}(\tau, \overrightarrow{0})+\left.\frac{\partial \alpha_{(a)}(\tau, \vec{\sigma})}{\partial \sigma^{r}}\right|_{\vec{\sigma}=0} d \sigma^{r} \approx \\
& \approx \alpha_{(b)}(\tau, \overrightarrow{0})\left[\delta_{(b)(a)}+\left(\hat{R}^{(c)}\right)_{(b)(a)}{ }^{3} \omega_{r(c)}(\tau, \overrightarrow{0}) d \sigma^{r}\right]
\end{aligned}
$$

implying that the identity cross section $\sigma_{I}$ of $\Sigma_{\tau}^{(\Xi)} \times S O(3)\left[\alpha_{(a)}=\alpha_{(a)}(\tau, \overrightarrow{0})=0\right]$ is the origin for all $\mathrm{SO}(3)$ fibers: $\left.\alpha_{(a)}(\tau, \vec{\sigma})\right|_{\sigma_{I}}=0$. As shown in Ref. [17], on $\sigma_{I}$ we also have $\left.\partial_{r} \alpha_{(a)}(\tau, \vec{\sigma})\right|_{\sigma_{I}}=0$. With this coordinatization the difference between the coordinates $\alpha_{(a)}(\tau, \overrightarrow{0})$ of a point $\tilde{p}$ in the fiber over the reference point $p \in \Sigma_{\tau}, \sigma_{p}^{A}=(\tau, \overrightarrow{0})$, and the point $\tilde{p}^{\prime}$ on the neighbouring fiber over $p^{\prime} \in \Sigma_{\tau}, \sigma_{p^{\prime}}^{A}=(\tau, d \vec{\sigma})$, joined to $\tilde{p}$ by the lift of the geodesic joining $p$ and $p^{\prime}$, is numerically equal to the horizontal infinitesimal increment $\left.\partial_{r} \alpha_{(a)}(\tau, \vec{\sigma}) d \sigma^{r}\right|_{\vec{\sigma}=\overrightarrow{0}}$ in going from $\vec{\sigma}=\overrightarrow{0}$ to $\overrightarrow{0}+d \vec{\sigma}$ in $\Sigma_{\tau}$ of a function $\alpha_{(a)}(\tau, \vec{\sigma})$

$$
\left.d \alpha_{(a)}\right|_{\alpha=\alpha(\tau, \vec{\sigma})}=d \alpha_{(a)}(\tau, \vec{\sigma})=\partial_{r} \alpha_{(a)}(\tau, \vec{\sigma}) d \sigma^{r} .
$$

These new coordinates of $\tilde{p}^{\prime}$ differ from the natural canonical coordinates of first kind existing on the fiber to which $\tilde{p}^{\prime}$ belong by just this quantity, which then assumes the meaning of a vertical infinitesimal increment added to the natural coordinates.

With this coordinatization of $\Sigma_{\tau}^{(\Xi)} \times S O(3)$, in the chosen global coordinate system $\Xi$ for $\Sigma_{\tau}$ in which the identity cross section $\sigma_{I}$ is chosen as the origin of the angles, as in Ref. [17] we have the following realization for the vector fields $X_{(a)}(\tau, \vec{\sigma})$ of Eqs.(3.5)

$$
X_{(a)}(\tau, \vec{\sigma})=B_{(b)(a)}\left(\alpha_{(e)}(\tau, \vec{\sigma})\right) \frac{\tilde{\delta}}{\delta \alpha_{(b)}(\tau, \vec{\sigma})} \Rightarrow \frac{\tilde{\delta}}{\delta \alpha_{(a)}(\tau, \vec{\sigma})}=A_{(b)(a)}\left(\alpha_{(e)}(\tau, \vec{\sigma})\right) X_{(b)}(\tau, \vec{\sigma}),
$$

where the functional derivative is the directional functional derivative along the path $\gamma_{\alpha(\tau, \vec{\sigma})}(s)$ in $\Sigma_{\tau}^{(\Xi)} \times S O(3)$ originating at the identity cross section $\sigma_{I}$ (the origin of all $\mathrm{SO}(3)$ fibers) in the $\mathrm{SO}(3)$ fiber over the point $p \in \Sigma_{\tau}$ with coordinates $(\tau, \vec{\sigma})$, corresponding in the above construction to the path $\gamma_{\alpha}(s)$ defining the canonical coordinates of first kind in the reference $\mathrm{SO}(3)$ fiber. It satisfies the commutator in Eq.(3.6) due to the generalized Maurer-Cartan equations for $\Sigma_{\tau} \times S O(3)\left[A=B^{-1}\right]$

$$
\begin{gathered}
B_{(u)(a)}\left(\alpha_{(e)}(\tau, \vec{\sigma})\right)= \\
\left.\quad \frac{\partial B_{(v)(b)}\left(\alpha_{(e)}\right)}{\partial \alpha_{(u)}}\right|_{\alpha=\alpha(\tau, \vec{\sigma})}-\left.B_{(u)(b)}\left(\alpha_{(e)}(\tau, \vec{\sigma})\right) \frac{\partial B_{(v)(a)}\left(\alpha_{(e)}\right)}{\partial \alpha_{(u)}}\right|_{\alpha=\alpha(\tau, \vec{\sigma})}= \\
\left.\frac{\partial A_{(a)(c)}\left(\alpha_{(e)}\right)}{\partial \alpha_{(b)}}\right|_{\alpha=\alpha(\tau, \vec{\sigma})}-\left.\frac{\partial A_{(a)(b)}\left(\alpha_{(e)}\right)}{\partial \alpha_{(c)}}\right|_{\alpha=\alpha(\tau, \vec{\sigma})}= \\
=\quad \epsilon_{(a)(u)(v)} A_{(u)(b)}\left(\alpha_{(e)}(\tau, \vec{\sigma})\right) A_{(v)(c)}\left(\alpha_{(e)}(\tau, \vec{\sigma})\right),
\end{gathered}
$$

holding pointwise on each fiber of $\Sigma_{\tau}^{(\Xi)} \times S O(3)$ over $(\tau, \vec{\sigma})$ in a suitable tubular neighbourhood of the identity cross section.

By defining a generalized canonical 1-form for $\overline{\mathcal{G}}_{R O T}$, 


$$
\begin{gathered}
\tilde{\omega}=\hat{R}^{(a)} \tilde{\theta}_{(a)}(\tau, \vec{\sigma})=H_{(a)}\left(\alpha_{(e)}(\tau, \vec{\sigma})\right) d \alpha_{(a)}(\tau, \vec{\sigma}), \\
\text { where } \\
\tilde{\theta}_{(a)}(\tau, \vec{\sigma})=\hat{\theta}_{(a)}\left(\alpha_{(e)}(\tau, \vec{\sigma}), \partial_{r} \alpha_{(e)}(\tau, \vec{\sigma})\right)=\tilde{\theta}_{(a) r}(\tau, \vec{\sigma}) d \sigma^{r}=A_{(a)(b)}\left(\alpha_{(e)}(\tau, \vec{\sigma})\right) d \alpha_{(b)}(\tau, \vec{\sigma}),
\end{gathered}
$$

are the generalized Maurer-Cartan 1-forms on the Lie algebra $\bar{g}_{R O T}$ of $\overline{\mathcal{G}}_{R O T}$ and where we defined the matrices $H_{(a)}\left(\alpha_{(e)}(\tau, \vec{\sigma})\right)=\hat{R}^{(b)} A_{(b)(a)}\left(\alpha_{(e)}(\tau, \vec{\sigma})\right)$, the previous equations can be rewritten in the form of a zero curvature condition

$$
\left.\frac{\partial H_{(a)}\left(\alpha_{(e)}\right)}{\partial \alpha_{(b)}}\right|_{\alpha=\alpha(\tau, \vec{\sigma})}-\left.\frac{\partial H_{(b)}\left(\alpha_{(e)}\right)}{\partial \alpha_{(a)}}\right|_{\alpha=\alpha(\tau, \vec{\sigma})}+\left[H_{(a)}\left(\alpha_{(e)}(\tau, \vec{\sigma})\right), H_{(b)}\left(\alpha_{(e)}(\tau, \vec{\sigma})\right)\right]=0
$$

Eq.(3.19) of Ref. [1] and Eqs.(3.5) give the following multitemporal equations for the dependence of the cotriad ${ }^{3} e_{(a) r}(\tau, \vec{\sigma})$ on the 3 gauge angles $\alpha_{(a)}(\tau, \vec{\sigma})$

$$
\begin{aligned}
X_{(b)}\left(\tau, \vec{\sigma}^{\prime}\right)^{3} e_{(a) r}(\tau, \vec{\sigma}) & =B_{(c)(b)}\left(\alpha_{(e)}\left(\tau, \vec{\sigma}^{\prime}\right) \frac{\tilde{\delta}^{3} e_{(a) r}(\tau, \vec{\sigma})}{\delta \alpha_{(c)}\left(\tau, \vec{\sigma}^{\prime}\right)}=\right. \\
& =-\left\{{ }^{3} e_{(a) r}(\tau, \vec{\sigma}),{ }^{3} \tilde{M}_{(b)}\left(\tau, \vec{\sigma}^{\prime}\right)\right\}=-\epsilon_{(a)(b)(c)}{ }^{3} e_{(c) r}(\tau, \vec{\sigma}) \delta^{3}\left(\vec{\sigma}, \vec{\sigma}^{\prime}\right), \\
\Rightarrow & \frac{\tilde{\delta}^{3} e_{(a) r}(\tau, \vec{\sigma})}{\delta \alpha_{(b)}\left(\tau, \vec{\sigma}^{\prime}\right)}=-\epsilon_{(a)(c)(d)} A_{(c)(b)}\left(\alpha_{(e)}(\tau, \vec{\sigma})\right)^{3} e_{(a) r}(\tau, \vec{\sigma}) \delta^{3}\left(\vec{\sigma}, \vec{\sigma}^{\prime}\right)= \\
& =\left[\hat{R}^{(c)} A_{(c)(b)}\left(\alpha_{(e)}(\tau, \vec{\sigma})\right)\right]_{(a)(d)}{ }^{3} e_{(d) r}(\tau, \vec{\sigma}) \delta^{3}\left(\vec{\sigma}, \vec{\sigma}^{\prime}\right)= \\
& =\left[H_{(b)}\left(\alpha_{(e)}(\tau, \vec{\sigma})\right)\right]_{(a)(d)}{ }^{3} e_{(d) r}(\tau, \vec{\sigma}) \delta^{3}\left(\vec{\sigma}, \vec{\sigma}^{\prime}\right) .
\end{aligned}
$$

These equations are a functional multitemporal generalization of the matrix equation $\frac{d}{d t} U\left(t, t_{o}\right)=h U\left(t, t_{o}\right), U\left(t_{o}, t_{o}\right)=1$, generating the concept of time-ordering. They are integrable (i.e. their solution is path independent) due to Eqs.(4.11) and their solution is

$$
{ }^{3} e_{(a) r}(\tau, \vec{\sigma})={ }^{3} R_{(a)(b)}\left(\alpha_{(e)}(\tau, \vec{\sigma})\right)^{3} \bar{e}_{(b) r}(\tau, \vec{\sigma}),
$$

where Py

$$
\begin{aligned}
& { }^{3} R_{(a)(b)}\left(\alpha_{(e)}(\tau, \vec{\sigma})\right)=\left(P e^{(l)} \int_{0}^{\alpha(e)(\tau, \vec{\sigma})} H_{(c)}\left(\bar{\alpha}_{(e)}\right) \mathcal{D} \bar{\alpha}_{(c)}\right)_{(a)(b)}=
\end{aligned}
$$

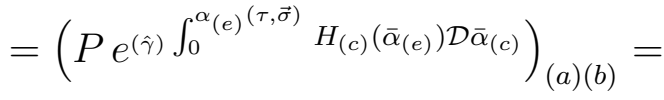

$$
\begin{aligned}
& =\left(P e^{\Omega^{\hat{\gamma}\left(\alpha_{(e)}(\tau, \vec{\sigma})\right)}}\right)_{(a)(b)},
\end{aligned}
$$

\footnotetext{
${ }^{99} l$ is an arbitrary path originating at the identity cross section of $\Sigma_{\tau}^{(\Xi)} \times S O(3)$; due to the path independence it can be replaced with the defining path $\gamma_{\alpha(\tau, \vec{\sigma})}(s)=\hat{\gamma}(\tau, \vec{\sigma} ; s)$.
} 
is a point dependent rotation matrix $\left[{ }^{3} R_{(a)(b)}^{T}(\alpha)={ }^{3} R_{(a)(b)}^{-1}(\alpha)\right.$ since $\left.\hat{R}^{(a) \dagger}=-\hat{R}^{(a)}\right]$.

In Eq.(4.14) we introduced the generalized phase obtained by functional integration along the defining path in $\Sigma_{\tau}^{(\Xi)} \times S O(3)$ of the generalized Maurer-Cartan 1-forms

$$
\begin{aligned}
\Omega^{\hat{\gamma}}\left(\alpha_{(e)}(\tau, \vec{\sigma} ; s)\right) & =\left.{ }_{(\hat{\gamma})} \int_{I}^{\gamma_{\alpha(\tau, \vec{\sigma} ; s)}} \hat{R}^{(a)} \tilde{\theta}_{(a)}\right|_{\gamma_{\alpha(\tau, \vec{\sigma} ; s)}}= \\
& ={ }_{(\hat{\gamma})} \int_{0}^{\alpha_{(e)}(\tau, \vec{\sigma} ; s)} H_{(a)}\left(\bar{\alpha}_{(e)}\right) \mathcal{D} \bar{\alpha}_{(a)}= \\
& ={ }_{(\hat{\gamma})} \int_{0}^{\alpha_{(e)}(\tau, \vec{\sigma} ; s)} \hat{R}^{(a)} A_{(a)(b)}\left(\bar{\alpha}_{(e)}\right) \mathcal{D} \bar{\alpha}_{(b)} .
\end{aligned}
$$

As shown in Ref. [17], we have

$$
d_{\hat{\gamma}} \Omega^{\hat{\gamma}}\left(\alpha_{(e)}(\tau, \vec{\sigma} ; s)\right)=\hat{R}^{(a)} \hat{\theta}_{(a)}\left(\alpha_{(e)}(\tau, \vec{\sigma} ; s), \partial_{r} \alpha_{(e)}(\tau, \vec{\sigma} ; s)\right),
$$

where $d_{\hat{\gamma}}$ is the restriction of the fiber or vertical derivative $d_{V}$ on $\Sigma_{\tau}^{(\Xi)} \times S O(3)$ (the BRST operator) to the defining path, satisfying $d_{\hat{\gamma}}^{2}=0$ due to the generalized Maurer-Cartan equations.

In Eq.(4.13),${ }^{3} \bar{e}_{(a) r}(\tau, \vec{\sigma})$ are the cotriads evaluated at $\alpha_{(a)}(\tau, \vec{\sigma})=0$, i.e. on the identity cross section. Being Cauchy data of Eqs.(4.12), they are independent from the angles $\alpha_{(a)}(\tau, \vec{\sigma})$, satisfy $\left\{{ }^{3} \bar{e}_{(a) r}(\tau, \vec{\sigma}),{ }^{3} \tilde{M}_{(b)}\left(\tau, \vec{\sigma}^{\prime}\right)\right\}=0$ and depend only on 6 independent functions 100. We have not found 3 specific conditions on cotriads implying their independency from the angles $\alpha_{(a)}$.

Since Eq.(A24) of Ref. [1] gives the law ${ }^{3} \omega_{r} \mapsto R^{3} \omega_{r} R^{T}-R \partial_{r} R^{T}$ for the spin connection under local $\mathrm{SO}(3)$ rotations, under infinitesimal rotations we get ${ }^{\mathrm{TO}}$

$$
\begin{aligned}
X_{(b)}\left(\tau, \vec{\sigma}^{\prime}\right)^{3} \omega_{r(a)}(\tau, \vec{\sigma}) & =B_{(c)(b)}\left(\alpha_{(e)}\left(\tau, \vec{\sigma}^{\prime}\right)\right) \frac{\tilde{\delta}^{3} \omega_{r(a)}(\tau, \vec{\sigma})}{\delta \alpha_{(c)}\left(\tau, \vec{\sigma}^{\prime}\right)}= \\
& =-\left\{{ }^{3} \omega_{r(a)}(\tau, \vec{\sigma}),{ }^{3} \tilde{M}_{(b)}\left(\tau, \vec{\sigma}^{\prime}\right)\right\}= \\
& =\left[\delta_{(a)(b)} \partial_{r}+\epsilon_{(a)(c)(b)}{ }^{3} \omega_{r(c)}(\tau, \vec{\sigma})\right] \delta^{3}\left(\vec{\sigma}, \vec{\sigma}^{\prime}\right)= \\
& =\left[\delta_{(a)(b)} \partial_{r}-\left(\hat{R}^{(c)}{ }^{3} \omega_{r(c)}(\tau, \vec{\sigma})\right)_{(a)(b)}\right] \delta^{3}\left(\vec{\sigma}, \vec{\sigma}^{\prime}\right)=\hat{D}_{(a)(b) r}^{(\omega)}(\tau, \vec{\sigma}) \delta^{3}\left(\vec{\sigma}, \vec{\sigma}^{\prime}\right),
\end{aligned}
$$

which is the same result as for the gauge potential of the $\mathrm{SO}(3)$ Yang-Mills theory. We can use the results of Ref. [17] to write the solution of Eq.(4.17)

$$
\begin{gathered}
{ }^{3} \omega_{r(a)}(\tau, \vec{\sigma})=A_{(a)(b)}\left(\alpha_{(e)}(\tau, \vec{\sigma})\right) \partial_{r} \alpha_{(b)}(\tau, \vec{\sigma})+{ }^{3} \omega_{r(a)}^{(T)}\left(\tau, \vec{\sigma}, \alpha_{(e)}(\tau, \vec{\sigma})\right), \\
\text { with } \\
\left.\frac{\partial^{3} \omega_{r(a)}^{(T)}\left(\tau, \vec{\sigma}, \alpha_{(e)}\right)}{\partial \alpha_{(b)}} \quad\right|_{\alpha=\alpha(\tau, \vec{\sigma})}=-\epsilon_{(a)(d)(c)} A_{(d)(b)}\left(\alpha_{(e)}(\tau, \vec{\sigma})\right)^{3} \omega_{r(c)}^{(T)}\left(\tau, \vec{\sigma}, \alpha_{(e)}(\tau, \vec{\sigma})\right) .
\end{gathered}
$$

${ }^{100}$ The $\alpha_{(a)}(\tau, \vec{\sigma})$ are the 3 rotational gauge degrees of freedom hidden in the 9 variables ${ }^{3} e_{(a) r}(\tau, \vec{\sigma})$. ${ }^{101} \hat{D}_{(a)(b) r}^{(\omega)}(\tau, \vec{\sigma})$ is the $\mathrm{SO}(3)$ covariant derivative in the adjoint representation. 
In ${ }^{3} \omega_{r(a)}(\tau, \vec{\sigma}) d \sigma^{r}=\tilde{\theta}_{(a)}(\tau, \vec{\sigma})+{ }^{3} \omega_{r(a)}^{(T)}\left(\tau, \vec{\sigma}, \alpha_{(e)}(\tau, \vec{\sigma})\right) d \sigma^{r}$, the first term is a pure gauge spin connection (the BRST ghost), while the second one is the source of the field strength: ${ }^{3} \Omega_{r s(a)}=\partial_{r}{ }^{3} \omega_{s(a)}^{(T)}-\partial_{s}{ }^{3} \omega_{r(a)}^{(T)}-\epsilon_{(a)(b)(c)}{ }^{3} \omega_{r(b)}^{(T)}{ }^{3} \omega_{s(c)}^{(T)}$. Moreover, the Hodge decomposition theorem (in the functional spaces where the spin connections are fully irreducible) implies that ${ }^{3} \omega_{r(a)}^{(\perp)}(\tau, \vec{\sigma}) \stackrel{\text { def }}{=} \omega_{r(a)}^{(T)}\left(\tau, \vec{\sigma}, \alpha_{(e)}(\tau, \vec{\sigma})\right)$ satisfies ${ }^{3} \nabla^{r}{ }^{3} \omega_{r(a)}^{(\perp)}=0$.

Since we have $X_{(b)}\left(\tau, \vec{\sigma}^{\prime}\right){ }^{3} \omega_{r(a)}^{(\perp)}(\tau, \vec{\sigma})=-\epsilon_{(a)(c)(b)}{ }^{3} \omega_{r(c)}^{(\perp)}(\tau, \vec{\sigma}) \delta^{3}\left(\vec{\sigma}, \vec{\sigma}^{\prime}\right)$, we get

$$
\begin{aligned}
& \frac{\tilde{\delta}^{3} \omega_{r(a)}^{(\perp)}(\tau, \vec{\sigma})}{\delta \alpha_{(b)}\left(\tau, \vec{\sigma}^{\prime}\right)}= {\left[H_{(b)}\left(\alpha_{(e)}(\tau, \vec{\sigma})\right)\right]_{(a)(c)}{ }^{3} \omega_{r(c)}^{(\perp)}(\tau, \vec{\sigma}) \delta^{3}\left(\vec{\sigma}, \vec{\sigma}^{\prime}\right), } \\
& \Rightarrow \quad{ }^{3} \omega_{r(a)}^{(\perp)}(\tau, \vec{\sigma})=\left(P e^{\Omega^{\hat{\gamma}}\left(\alpha_{(e)}(\tau, \vec{\sigma})\right)}\right)_{(a)(b)}{ }^{3} \bar{\omega}_{r(b)}^{(\perp)}(\tau, \vec{\sigma}), \\
&{ }^{3} \nabla^{r 3} \bar{\omega}_{r(a)}^{(\perp)}(\tau, \vec{\sigma})=0 .
\end{aligned}
$$

The transverse spin connection ${ }^{3} \bar{\omega}_{r(a)}^{(\perp)}(\tau, \vec{\sigma})$ is independent from the gauge angles $\alpha_{(a)}(\tau, \vec{\sigma})$ and is the source of the field strength ${ }^{3} \bar{\Omega}_{r s(a)}=\partial_{r}{ }^{3} \bar{\omega}_{s(a)}^{(\perp)}-\partial_{s}{ }^{3} \bar{\omega}_{r(a)}^{(\perp)}-\epsilon_{(a)(b)(c)}{ }^{3} \bar{\omega}_{r(b)}^{(\perp)}{ }^{3} \bar{\omega}_{s(c)}^{(\perp)}$ invariant under the rotation gauge transformations. Clearly, ${ }^{3} \bar{\omega}_{r(a)}^{(\perp)}$ is built with the reduced cotriads ${ }^{3} \bar{e}_{(a) r}$.

Let us remark that for ${ }^{3} \omega_{r(a)}^{F}(\tau, \vec{\sigma}) d \sigma^{r}=\tilde{\theta}_{(a)}(\tau, \vec{\sigma})$ we get ${ }^{3} \Omega_{r s(a)}(\tau, \vec{\sigma})=0$ and then ${ }^{3} R_{\text {rsuv }}=0$ : in this case the Riemannian manifold $\left(\Sigma_{\tau},{ }^{3} g_{r s}={ }^{3} e_{(a) r}{ }^{3} e_{(a) s}\right)$ becomes the Euclidean manifold $\left(R^{3},{ }^{3} g_{r s}^{F}\right)$ with ${ }^{3} g_{r s}^{F}$ the flat 3 -metric in curvilinear coordinates. Since Eq.(4.13) implies that ${ }^{3} g_{r s}={ }^{3} \bar{e}_{(a) r}{ }^{3} \bar{e}_{(a) s}$ and since ${ }^{3} g_{r s}^{F}(\tau, \vec{\sigma})=\frac{\partial \tilde{\sigma}^{u}}{\partial \sigma^{r}} \frac{\partial \tilde{\sigma}^{v}}{\partial \sigma^{s}} \delta_{u v}={ }^{3} g_{r s}^{F}(\vec{\sigma})$, if $\tilde{\sigma}^{u}(\vec{\sigma})$ are Cartesian coordinates, we get

$$
{ }^{3} \Gamma_{r s}^{F u}=\frac{\partial \sigma^{u}}{\partial \tilde{\sigma}^{n}} \frac{\partial^{2} \tilde{\sigma}^{n}}{\partial \sigma^{r} \partial \sigma^{s}}={ }^{3} e_{(a)}^{u} \partial_{r}{ }^{3} e_{(a) s}={ }^{3} \triangle_{r s}^{u},
$$

(see the remark after Eqs.(A19) of Ref. [1] ). This implies that for an arbitrary ${ }^{3} g$ we have the decomposition ${ }^{3} \Gamma_{r s}^{u}={ }^{3} \triangle_{r s}^{u}+{ }^{3} \bar{\Gamma}_{r s}^{u}$ with ${ }^{3} \bar{\Gamma}_{r s}^{u}={ }^{3} e_{(a)}^{u}{ }^{3} e_{(b) s}{ }^{3} \omega_{r(a)(b)}$ the source of the Riemann tensor. This implies that

$$
3 \bar{e}_{(a) r}^{F}(\tau, \vec{\sigma})=\frac{\partial \tilde{\sigma}^{u}}{\partial \sigma^{r}} 3 \tilde{\bar{e}}_{(a) u}^{F}(\tau, \overrightarrow{\tilde{\sigma}})=\delta_{(a) u} \frac{\partial \tilde{\sigma}^{u}(\vec{\sigma})}{\partial \sigma^{r}}
$$

Therefore, a flat cotriad on $R^{3}$ has the form

$$
{ }^{3} e_{(a) r}^{F}(\tau, \vec{\sigma})={ }^{3} R_{(a)(b)}\left(\alpha_{(e)}(\tau, \vec{\sigma})\right) \delta_{(b) u} \frac{\partial \tilde{\sigma}^{u}(\vec{\sigma})}{\partial \sigma^{r}} .
$$

Eqs.(3.19) of Ref. [1] imply the following multitemporal equations for the momenta

$$
\begin{aligned}
X_{(b)}\left(\tau, \vec{\sigma}^{\prime}\right)^{3} \tilde{\pi}_{(a)}^{r}(\tau, \vec{\sigma}) & =B_{(c)(b)}\left(\alpha_{(e)}\left(\tau, \vec{\sigma}^{\prime}\right) \frac{\tilde{\delta}^{3} \tilde{\pi}_{(a)}^{r}(\tau, \vec{\sigma})}{\delta \alpha_{(c)}\left(\tau, \vec{\sigma}^{\prime}\right)}=\right. \\
& =-\epsilon_{(a)(b)(c)}{ }^{3} \tilde{\pi}_{(c)}^{r}(\tau, \vec{\sigma}) \delta^{3}\left(\vec{\sigma}, \vec{\sigma}^{\prime}\right)
\end{aligned}
$$

whose solution is $\left[{ }^{3} \overline{\tilde{\pi}}_{(a)}^{r}(\tau, \vec{\sigma})\right.$ depends only on 6 independent functions] 


$$
{ }^{3} \tilde{\pi}_{(a)}^{r}(\tau, \vec{\sigma})={ }^{3} R_{(a)(b)}\left(\alpha_{(e)}(\tau, \vec{\sigma})\right)^{3} \overline{\tilde{\pi}}_{(b)}^{r}(\tau, \vec{\sigma})
$$

With the definition of $\mathrm{SO}(3)$ covariant derivative given in Eq.(4.17), the constraints $\hat{\mathcal{H}}_{(a)}(\tau, \vec{\sigma}) \approx 0$ of Eqs. 2.11 ), may be written as

$$
\begin{aligned}
\hat{\mathcal{H}}_{(a)}(\tau, \vec{\sigma}) & =-{ }^{3} e_{(a)}^{r}(\tau, \vec{\sigma})\left[{ }^{3} \tilde{\Theta}_{r}+{ }^{3} \omega_{r(b)}{ }^{3} \tilde{M}_{(b)}\right](\tau, \vec{\sigma})= \\
& =\hat{D}_{(a)(b) r}^{(\omega)}(\tau, \vec{\sigma})^{3} \tilde{\pi}_{(b)}^{r}(\tau, \vec{\sigma}) \approx 0
\end{aligned}
$$

so that we have $\left({ }^{3} \tilde{\pi}_{(a)}^{(T) r}(\tau, \vec{\sigma})\right.$ is a field with zero $\mathrm{SO}(3)$ covariant divergence)

$$
\begin{aligned}
{ }^{3} \tilde{\pi}_{(a)}^{r}(\tau, \vec{\sigma})= & { }^{3} \tilde{\pi}_{(a)}^{(T) r}(\tau, \vec{\sigma})-\int d^{3} \sigma^{\prime} \zeta_{(a)(b)}^{(\omega) r}\left(\vec{\sigma}, \vec{\sigma}^{\prime} ; \tau\right) \hat{\mathcal{H}}_{(b)}\left(\tau, \vec{\sigma}^{\prime}\right) \\
& \hat{D}_{(a)(b) r}^{(\omega)}(\tau, \vec{\sigma})^{3} \tilde{\pi}_{(b)}^{(T) r}(\tau, \vec{\sigma}) \equiv 0 \\
& \text { with } \\
{ }^{3} \tilde{\pi}_{(a)}^{(T) r}(\tau, \vec{\sigma})= & \int d^{3} \sigma_{1}\left[\delta_{s}^{r} \delta_{(a)(b)} \delta^{3}\left(\vec{\sigma}, \vec{\sigma}_{1}\right)+\zeta_{(a)(c)}^{(\omega) r}\left(\vec{\sigma}, \vec{\sigma}_{1} ; \tau\right) \hat{D}_{(c)(b) s}^{(\omega)}\left(\tau, \vec{\sigma}_{1}\right)\right]^{3} \tilde{\pi}_{(b)}^{s}\left(\tau, \vec{\sigma}_{1}\right) .
\end{aligned}
$$

In this equation, we introduced the Green function of the $\mathrm{SO}(3)$ covariant divergence, defined by

$$
\hat{D}_{(a)(b) r}^{(\omega)}(\tau, \vec{\sigma}) \zeta_{(b)(c)}^{(\omega) r}\left(\vec{\sigma}, \vec{\sigma}^{\prime} ; \tau\right)=-\delta_{(a)(c)} \delta^{3}\left(\vec{\sigma}, \vec{\sigma}^{\prime}\right) .
$$

In Ref. [17], this Green function was evaluated for $\Sigma_{\tau}=R^{3}$, the flat Euclidean space, by using the Green function $\vec{c}\left(\vec{\sigma}-\vec{\sigma}^{\prime}\right)$ of the flat ordinary divergence $\left[\triangle=\vec{\partial}_{\sigma}^{2}\right]$ in Cartesian coordinates

$$
\begin{aligned}
\vec{c}\left(\vec{\sigma}-\vec{\sigma}^{\prime}\right) & =\vec{\partial}_{\sigma} c\left(\vec{\sigma}-\vec{\sigma}^{\prime}\right)=-\frac{\vec{\partial}_{\sigma}}{\triangle} \delta^{3}\left(\vec{\sigma}-\vec{\sigma}^{\prime}\right)=\frac{\vec{\sigma}-\vec{\sigma}^{\prime}}{4 \pi\left|\vec{\sigma}-\vec{\sigma}^{\prime}\right|^{3}}=\frac{\vec{n}\left(\vec{\sigma}-\vec{\sigma}^{\prime}\right)}{4 \pi\left(\vec{\sigma}-\vec{\sigma}^{\prime}\right)^{2}} \\
\vec{\partial}_{\sigma} \cdot \vec{c}\left(\vec{\sigma}-\vec{\sigma}^{\prime}\right) & =-\delta^{3}\left(\vec{\sigma}-\vec{\sigma}^{\prime}\right),
\end{aligned}
$$

where $\vec{n}\left(\vec{\sigma}-\vec{\sigma}^{\prime}\right)$ is the tangent to the flat geodesic (straight line segment) joining the point of coordinates $\vec{\sigma}$ and $\vec{\sigma}^{\prime}$, so that $\vec{n}\left(\vec{\sigma}-\vec{\sigma}^{\prime}\right) \cdot \vec{\partial}_{\sigma}$ is the directional derivative along the flat geodesic.

With our special family of Riemannian 3-manifolds $\left(\Sigma_{\tau},{ }^{3} g\right)$, we would use Eq.(4.28) in the special global normal chart in which the star of geodesics originating from the reference point $\mathrm{p}$ becomes a star of straight lines. In non normal coordinates, the Green function $\vec{c}\left(\vec{\sigma}-\vec{\sigma}^{\prime}\right)$ will be replaced with the gradient of the Synge world function [91] or De Witt geodesic interval bitensor 36] $\sigma_{D W}\left(\vec{\sigma}, \vec{\sigma}^{\prime}\right)$ (giving the arc length of the geodesic from $\vec{\sigma}$ to $\left.\vec{\sigma}^{\prime}\right)$ adapted from the Lorentzian spacetime $M^{4}$ to the Riemannian 3 -manifold $\left(\Sigma_{\tau},{ }^{3} g\right)$, i.e.

$$
d_{\gamma_{p p^{\prime}}}^{r}\left(\vec{\sigma}, \vec{\sigma}^{\prime}\right)=\frac{1}{3} \sigma_{D W}^{r}\left(\vec{\sigma}, \vec{\sigma}^{\prime}\right)=\frac{1}{3}{ }^{3} \nabla_{\sigma}^{r} \sigma_{D W}\left(\vec{\sigma}, \vec{\sigma}^{\prime}\right)=\frac{1}{3} \partial_{\sigma}^{r} \sigma_{D W}\left(\vec{\sigma}, \vec{\sigma}^{\prime}\right),
$$


giving in each point $\vec{\sigma}$ the tangent to the geodesic $\gamma_{p p^{\prime}}$ joining the points $\mathrm{p}$ and $p^{\prime}$ of coordinates $\vec{\sigma}$ and $\vec{\sigma}^{\prime}$ in the direction from $p^{\prime}$ to $\mathrm{p}$. Therefore, the Green function is ए2

$$
\zeta_{(a)(b)}^{(\omega) r}\left(\vec{\sigma}, \vec{\sigma}^{\prime} ; \tau\right)=d_{\gamma_{p p^{\prime}}}^{r}\left(\vec{\sigma}, \vec{\sigma}^{\prime}\right)\left(P_{\gamma_{p p^{\prime}}} e^{\int_{\vec{\sigma}^{\prime}}^{\vec{\sigma}} d \sigma_{1}^{s} \hat{R}^{(c) 3} \omega_{s(c)}\left(\tau, \vec{\sigma}_{1}\right)}\right)_{(a)(b)},
$$

with the path ordering done along the geodesic $\gamma_{p p^{\prime}}$. This path ordering ( Wu-Yang nonintegrable phase or geodesic Wilson line) is defined on all $\Sigma_{\tau} \times S O(3)$ only if the spin connection is fully irreducible; it is just the parallel transporter of Eq.(4.5).

Eqs.(4.13) show the dependence of the cotriad on the 3 angles $\alpha_{(a)}(\tau, \vec{\sigma})$, which therefore must be expressible only in terms of the cotriad itself and satisfy $\left\{\alpha_{(a)}(\tau, \vec{\sigma}), \alpha_{(b)}\left(\tau, \vec{\sigma}^{\prime}\right)\right\}=$ 0 . They are the rotational gauge variables, canonically conjugate to Abelianized rotation constraints $\tilde{\pi}_{(a)}^{\vec{\alpha}}(\tau, \vec{\sigma}) \approx 0$. From Eqs. (4.8), since the functional derivatives commute, we see that we have the following expression for the Abelianized constraints 17,92

$$
\begin{aligned}
\tilde{\pi}_{(a)}^{\vec{\alpha}}(\tau, \vec{\sigma}) & =-{ }^{3} \tilde{M}_{(b)}(\tau, \vec{\sigma}) A_{(b)(a)}\left(\alpha_{(e)}(\tau, \vec{\sigma})\right) \approx 0, \\
\left\{\tilde{\pi}_{(a)}^{\vec{\alpha}}(\tau, \vec{\sigma}), \tilde{\pi}_{(b)}^{\vec{\alpha}}\left(\tau, \vec{\sigma}^{\prime}\right)\right\} & =0, \\
\left\{\alpha_{(a)}(\tau, \vec{\sigma}), \tilde{\pi}_{(b)}^{\vec{\alpha}}\left(\tau, \vec{\sigma}^{\prime}\right)\right\} & =A_{(c)(b)}\left(\alpha_{(e)}\left(\tau, \vec{\sigma}^{\prime}\right)\right) X_{(c)}\left(\tau, \vec{\sigma}^{\prime}\right) \alpha_{(a)}(\tau, \vec{\sigma})= \\
& =\delta_{(a)(b)} \delta^{3}\left(\vec{\sigma}, \vec{\sigma}^{\prime}\right) .
\end{aligned}
$$

The functional equation determining $\alpha_{(a)}(\tau, \vec{\sigma})$ in terms of ${ }^{3} e_{(a) r}(\tau, \vec{\sigma})$ is

$$
\begin{aligned}
-\delta_{(a)(b)} \delta^{3}\left(\vec{\sigma}, \vec{\sigma}^{\prime}\right)= & \left\{\alpha_{(a)}(\tau, \vec{\sigma}),{ }^{3} \tilde{M}_{(c)}\left(\tau, \vec{\sigma}^{\prime}\right)\right\} A_{(c)(b)}\left(\alpha_{(e)}\left(\tau, \vec{\sigma}^{\prime}\right)=\right. \\
= & \epsilon_{(c)(u)(v)}{ }^{3} e_{(u) r}\left(\tau, \vec{\sigma}^{\prime}\right)\left\{\alpha_{(a)}(\tau, \vec{\sigma}),{ }^{3} \tilde{\pi}_{(v)}^{r}\left(\tau, \vec{\sigma}^{\prime}\right)\right\} A_{(c)(b)}\left(\alpha_{(e)}\left(\tau, \vec{\sigma}^{\prime}\right)\right)= \\
= & \epsilon_{(c)(u)(v)} A_{(c)(b)}\left(\alpha_{(e)}\left(\tau, \vec{\sigma}^{\prime}\right)\right)^{3} e_{(u) r}\left(\tau, \vec{\sigma}^{\prime}\right) \frac{\delta \alpha_{(a)}(\tau, \vec{\sigma})}{\delta^{3} e_{(v) r}\left(\tau, \vec{\sigma}^{\prime}\right)}, \\
\Rightarrow \quad & \epsilon_{(b)(u)(v)}{ }^{3} e_{(u) r}\left(\tau, \vec{\sigma}^{\prime}\right) \frac{\delta \alpha_{(a)}(\tau, \vec{\sigma})}{\delta^{3} e_{(v) r}\left(\tau, \vec{\sigma}^{\prime}\right)}=-B_{(a)(b)}\left(\alpha_{(e)}(\tau, \vec{\sigma})\right) \delta^{3}\left(\vec{\sigma}, \vec{\sigma}^{\prime}\right), \\
& \epsilon_{(b)(u)(v)}{ }^{3} e_{(u) r}\left(\tau, \vec{\sigma}^{\prime}\right)\left[A_{(a)(c)}\left(\alpha_{(e)}(\tau, \vec{\sigma})\right) \frac{\delta \alpha_{(a)}(\tau, \vec{\sigma})}{\delta^{3} e_{(v) r}\left(\tau, \vec{\sigma}^{\prime}\right)}\right]=-\delta_{(a)(b)} \delta^{3}\left(\vec{\sigma}, \vec{\sigma}^{\prime}\right), \\
\Rightarrow \quad & \epsilon_{(b)(u)(v)}{ }^{3} e_{(u) r}\left(\tau, \vec{\sigma}^{\prime}\right) \frac{\delta \Omega_{(a)}^{\hat{\gamma}}\left(\alpha_{(e)}(\tau, \vec{\sigma})\right)}{\delta^{3} e_{(v) r}\left(\tau, \vec{\sigma}^{\prime}\right)}=-\delta_{(a)(b)} \delta^{3}\left(\vec{\sigma}, \vec{\sigma}^{\prime}\right), \\
& \frac{\delta \Omega_{(a)}^{\hat{\gamma}}\left(\alpha_{(e)}(\tau, \vec{\sigma})\right)}{\delta^{3} e_{(v) r}\left(\tau, \vec{\sigma}^{\prime}\right)}=-\frac{1}{3} \delta_{(a)(b)}{ }^{3} e_{(u)}^{r}\left(\tau, \vec{\sigma}^{\prime}\right) \delta^{3}\left(\vec{\sigma}, \vec{\sigma}^{\prime}\right), \\
& \quad \delta \Omega_{(a)}^{\hat{\gamma}}\left(\alpha_{(e)}(\tau, \vec{\sigma})\right) \\
\delta^{3} e_{(b) r}\left(\tau, \vec{\sigma}^{\prime}\right) & \frac{1}{6}\left(\hat{R}^{(u)}\right)_{(a)(b)}{ }^{3} e_{(u)}^{r}(\tau, \vec{\sigma}) \delta^{3}\left(\vec{\sigma}, \vec{\sigma}^{\prime}\right) .
\end{aligned}
$$

${ }^{102} \partial_{r} d_{\gamma_{p p^{\prime}}}^{r}\left(\vec{\sigma}, \vec{\sigma}^{\prime}\right)=-\delta^{3}\left(\vec{\sigma}, \vec{\sigma}^{\prime}\right) ; d_{\gamma_{p p^{\prime}}}^{r}\left(\vec{\sigma}, \vec{\sigma}^{\prime}\right) \partial_{r}$ is the directional derivative along the geodesic $\gamma_{p p^{\prime}}$ at $p$ of coordinates $\vec{\sigma}$. 
This equation is not integrable like the corresponding one in the Yang-Mills case [17]. Having chosen a global coordinate system $\Xi$ on $\Sigma_{\tau}$ as the conventional origin of pseudodiffeomorphisms, the discussion in the previous Section allows to define the trivialization $\Sigma_{\tau}^{(\Xi)} \times S O(3)$ of the coframe bundle $L \Sigma_{\tau}$. If:

i) $\sigma_{I}^{(\Xi)}$ is the identity cross section of $\Sigma_{\tau}^{(\Xi)} \times S O(3)$, corresponding to the coframe ${ }^{3} \theta_{(a)}^{I}=$ ${ }^{3} e_{(a) r}^{I} d \sigma^{r}$ in $L \Sigma_{\tau}\left(\sigma^{r}\right.$ are the coordinate functions of $\left.\Xi\right)$;

ii) $\sigma^{(\Xi)}$ is an arbitrary global cross section of $\Sigma_{\tau}^{(\Xi)} \times S O(3)$, corresponding to a coframe ${ }^{3} \theta_{(a)}={ }^{3} e_{(a) r} d \sigma^{r}$ in $L \Sigma_{\tau}$, in a tubular neighbourhood of the identity cross section where the generalized canonical coordinates of first kind on the fibers of $\Sigma_{\tau}^{(\Xi)} \times S O(3)$ are defined;

iii) $\sigma^{(\Xi)}(s)$ is the family of global cross sections of $\Sigma_{\tau}^{(\Xi)} \times S O(3)$ connecting $\Sigma_{I}^{(\Xi)}=\sigma^{(\Xi)}(s=0)$ and $\Sigma^{(\Xi)}=\sigma^{(\Xi)}(s=1)$ so that on each fiber the point on $\sigma_{I}^{(\Xi)}$ is connected with the point on $\Sigma^{(\Xi)}$ by the defining path $\hat{\gamma}$ of canonical coordinates of first kind;

then the formal solution of the previous equation is

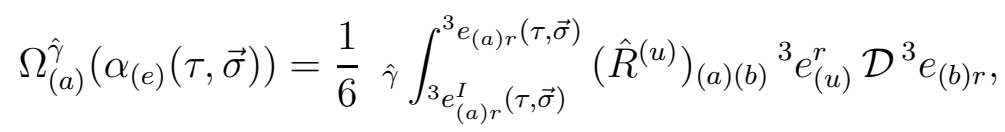

where the path integral is made along the path of coframes connecting ${ }^{3} \theta_{(a)}^{I}$ with ${ }^{3} \theta_{(a)}$ just described. As in Ref. [17], to get the angles $\alpha_{(a)}(\tau, \vec{\sigma})$ from $\Omega_{(a)}^{\hat{\gamma}}\left(\alpha_{(e)}(\tau, \vec{\sigma})\right)$, we essentially have to invert the equation $\Omega_{(a)}^{\hat{\gamma}}\left(\alpha_{(e)}\right)={ }_{\hat{\gamma}} \int_{0}^{\alpha_{(e)}} A_{(a)(b)}(\bar{\alpha}) d \bar{\alpha}_{(b)}$ with $A=\left(e^{R \alpha}-1\right) / R \alpha$.

\section{B. The Multi-Temporal Equations for the Pseudo-Diffeomorphisms.}

Let us now study the multitemporal equations associated with pseudo-diffeomorphisms to find the dependence of ${ }^{3} e_{(a) r}(\tau, \vec{\sigma})$ on the parameters $\xi^{r}(\tau, \vec{\sigma})$. Disregarding momentarily

rotations, let us look for a realization of vector fields $\tilde{Y}_{r}(\tau, \vec{\sigma})$ satisfying the last line of Eqs.(3.6). If we put

$$
\tilde{Y}_{r}(\tau, \vec{\sigma})=-\frac{\partial \xi^{s}(\tau, \vec{\sigma})}{\partial \sigma^{r}} \frac{\delta}{\delta \xi^{s}(\tau, \vec{\sigma})}
$$

we find

$$
\begin{aligned}
{\left[\tilde{Y}_{r}(\tau, \vec{\sigma}), \tilde{Y}_{s}\left(\tau, \vec{\sigma}^{\prime}\right)\right] } & =\left[\frac{\partial \xi^{u}(\tau, \vec{\sigma})}{\partial \sigma^{r}} \frac{\delta}{\delta \xi^{u}(\tau, \vec{\sigma})}, \frac{\partial \xi^{v}\left(\tau, \vec{\sigma}^{\prime}\right)}{\partial \sigma^{\prime}} \frac{\delta}{\delta \xi^{v}\left(\tau, \vec{\sigma}^{\prime}\right)}\right]= \\
& =\frac{\partial \xi^{u}(\tau, \vec{\sigma})}{\partial \sigma^{r}} \frac{\partial \delta^{3}\left(\vec{\sigma}, \vec{\sigma}^{\prime}\right)}{\partial \sigma^{\prime} s} \frac{\delta}{\delta \xi^{u}\left(\tau, \vec{\sigma}^{\prime}\right)}-\frac{\partial \xi^{u}\left(\tau, \vec{\sigma}^{\prime}\right)}{\left.\partial \sigma^{\prime}\right)} \frac{\partial \delta^{3}\left(\vec{\sigma}, \vec{\sigma}^{\prime}\right)}{\partial \sigma^{r}} \frac{\delta}{\delta \xi^{u}(\tau, \vec{\sigma})}= \\
& =-\frac{\partial \xi^{u}(\tau, \vec{\sigma})}{\partial \sigma^{r}} \frac{\partial \delta^{3}\left(\vec{\sigma}, \vec{\sigma}^{\prime}\right)}{\partial \sigma^{s}} \frac{\delta}{\delta \xi^{u}\left(\tau, \vec{\sigma}^{\prime}\right)}+\frac{\partial \xi^{u}\left(\tau, \vec{\sigma}^{\prime}\right)}{\partial \sigma^{\prime}} \frac{\partial \delta^{3}\left(\vec{\sigma}, \vec{\sigma}^{\prime}\right)}{\partial \sigma^{\prime r}} \frac{\delta}{\delta \xi^{u}(\tau, \vec{\sigma})}= \\
& =\left[-\frac{\partial}{\partial \sigma^{s}}\left(\frac{\partial \xi^{u}(\tau, \vec{\sigma})}{\partial \sigma^{r}} \delta^{3}\left(\vec{\sigma}, \vec{\sigma}^{\prime}\right)\right)+\frac{\partial^{2} \xi^{u}(\tau, \vec{\sigma})}{\partial \sigma^{r} \partial \sigma^{s}} \delta^{3}\left(\vec{\sigma}, \vec{\sigma}^{\prime}\right)\right] \frac{\delta}{\delta \xi^{u}\left(\tau, \vec{\sigma}^{\prime}\right)}+ \\
& +\left[\frac{\partial}{\partial \sigma^{\prime r}}\left(\frac{\partial \xi^{u}\left(\tau, \vec{\sigma}^{\prime}\right)}{\partial \sigma^{\prime} s} \delta^{3}\left(\vec{\sigma}, \vec{\sigma}^{\prime}\right)\right)-\frac{\partial^{2} \xi^{u}\left(\tau, \vec{\sigma}^{\prime}\right)}{\partial \sigma^{\prime r} \partial \sigma^{\prime} s} \delta^{3}\left(\vec{\sigma}, \vec{\sigma}^{\prime}\right)\right] \frac{\delta}{\delta \xi^{u}(\tau, \vec{\sigma})}=
\end{aligned}
$$




$$
\begin{aligned}
& =-\frac{\partial \delta^{3}\left(\vec{\sigma}, \vec{\sigma}^{\prime}\right)}{\partial \sigma^{s}} \frac{\partial \xi^{u}\left(\tau, \vec{\sigma}^{\prime}\right)}{\partial \sigma^{\prime r}} \frac{\delta}{\delta \xi^{u}\left(\tau, \vec{\sigma}^{\prime}\right)}+\frac{\partial \delta^{3}\left(\vec{\sigma}, \vec{\sigma}^{\prime}\right)}{\partial \sigma^{\prime r}} \frac{\partial \xi^{u}(\tau, \vec{\sigma})}{\partial \sigma^{s}} \frac{\delta}{\delta \xi^{u}(\tau, \vec{\sigma})}= \\
& =-\frac{\partial \delta^{3}\left(\vec{\sigma}, \vec{\sigma}^{\prime}\right)}{\partial \sigma^{\prime s}} \tilde{Y}_{r}\left(\tau, \vec{\sigma}^{\prime}\right)-\frac{\partial \delta^{3}\left(\vec{\sigma}, \vec{\sigma}^{\prime}\right)}{\partial \sigma^{\prime r}} \tilde{Y}_{s}(\tau, \vec{\sigma}),
\end{aligned}
$$

in accord with the last of Eqs.(3.6). Therefore, the role of the Maurer-Cartan matrix B for rotations is taken by minus the Jacobian matrix of the pseudo-diffeomorphism $\vec{\sigma} \mapsto \vec{\xi}(\vec{\sigma})$. To take into account the noncommutativity of rotations and pseudo-diffeomorphisms [the second line of Eqs.(3.6)], we need the definition

$$
Y_{r}(\tau, \vec{\sigma})=-\left\{.,{ }^{3} \tilde{\Theta}_{r}(\tau, \vec{\sigma})\right\}=-\frac{\partial \xi^{s}(\tau, \vec{\sigma})}{\partial \sigma^{r}} \frac{\delta}{\delta \xi^{s}(\tau, \vec{\sigma})}-\frac{\partial \alpha_{(a)}(\tau, \vec{\sigma})}{\partial \sigma^{r}} \frac{\tilde{\delta}}{\delta \alpha_{(a)}(\tau, \vec{\sigma})} .
$$

Clearly the last line of Eqs.(3.6) is satisfied, while regarding the second line we have consistently

$$
\begin{aligned}
{\left[X_{(a)}(\tau, \vec{\sigma}), Y_{r}\left(\tau, \vec{\sigma}^{\prime}\right)\right] } & =-\left[B_{(b)(a)}\left(\alpha_{(e)}(\tau, \vec{\sigma})\right) \frac{\tilde{\delta}}{\delta \alpha_{(b)}(\tau, \vec{\sigma})}, \frac{\partial \alpha_{(c)}\left(\tau, \vec{\sigma}^{\prime}\right)}{\partial \sigma^{\prime r}} \frac{\tilde{\delta}}{\delta \alpha_{(c)}\left(\tau, \vec{\sigma}^{\prime}\right)}\right]= \\
& =-B_{(b)(a)}\left(\alpha_{(e)}(\tau, \vec{\sigma})\right) \frac{\partial \delta^{3}\left(\vec{\sigma}, \vec{\sigma}^{\prime}\right)}{\partial \sigma^{\prime r}} \frac{\tilde{\delta}}{\delta \alpha_{(b)}\left(\tau, \vec{\sigma}^{\prime}\right)}+ \\
& +\left.\frac{\partial \alpha_{(c)}(\tau, \vec{\sigma})}{\partial \sigma^{r}} \delta^{3}\left(\vec{\sigma}, \vec{\sigma}^{\prime}\right) \frac{\partial B_{(b)(a)}\left(\alpha_{(e)}\right)}{\partial \alpha_{(c)}}\right|_{\alpha=\alpha(\tau, \vec{\sigma})} \frac{\tilde{\delta}}{\delta \alpha_{(b)}(\tau, \vec{\sigma})}= \\
& =-\frac{\partial \delta^{3}\left(\vec{\sigma}, \vec{\sigma}^{\prime}\right)}{\partial \sigma^{\prime r}} X_{(a)}\left(\tau, \vec{\sigma}^{\prime}\right) .
\end{aligned}
$$

From Eqs.(4.36) and (4.8) we get

$$
\begin{aligned}
\frac{\delta}{\delta \xi^{r}(\tau, \vec{\sigma})}= & -\left.\frac{\partial \sigma^{s}(\vec{\xi})}{\partial \xi^{r}}\right|_{\vec{\xi}=\vec{\xi}(\tau, \vec{\sigma})}\left[Y_{s}(\tau, \vec{\sigma})+A_{(a)(b)}\left(\alpha_{(e)}(\tau, \vec{\sigma})\right) \frac{\partial \alpha_{(b)}(\tau, \vec{\sigma})}{\partial \sigma^{s}} X_{(a)}(\tau, \vec{\sigma})\right]= \\
= & \left.\frac{\partial \sigma^{s}(\vec{\xi})}{\partial \xi^{r}}\right|_{\vec{\xi}=\vec{\xi}(\tau, \vec{\sigma})}\left[\left\{., \tilde{\Theta}^{3}(\tau, \vec{\sigma})\right\}+\tilde{\theta}_{(a) s}(\tau, \vec{\sigma})\left\{.,{ }^{3} \tilde{M}_{(a)}(\tau, \vec{\sigma})\right\}\right] \stackrel{\text { def }}{=} \\
& \stackrel{\text { def }}{=}\left\{., \tilde{\pi}_{r}^{\xi}(\tau, \vec{\sigma})\right\}, \\
\Rightarrow \quad & \left\{\xi^{r}(\tau, \vec{\sigma}), \tilde{\pi}_{s}^{\vec{\xi}}\left(\tau, \vec{\sigma}^{\prime}\right)\right\}=\delta_{s}^{r} \delta^{3}\left(\vec{\sigma}, \vec{\sigma}^{\prime}\right), \\
& \left\{\tilde{\pi}_{r}^{\xi}(\tau, \vec{\sigma}), \tilde{\pi}_{s}^{\vec{\xi}}\left(\tau, \vec{\sigma}^{\prime}\right)\right\}=0,
\end{aligned}
$$

where $\tilde{\pi}_{r}^{\vec{\xi}}(\tau, \vec{\sigma})$ is the momentum conjugate to the 3 gauge variables $\xi^{r}(\tau, \vec{\sigma})$, which will be functions only of the cotriads. On the space of cotriads the Abelianized form of the pseudo-diffeomorphism constraints is

$$
\begin{aligned}
\tilde{\pi}_{r}^{\xi}(\tau, \vec{\sigma}) & =\left.\frac{\partial \sigma^{s}(\vec{\xi})}{\partial \xi^{r}}\right|_{\vec{\xi}=\vec{\xi}(\tau, \vec{\sigma})}\left[{ }^{3} \tilde{\Theta}_{s}(\tau, \vec{\sigma})+\hat{\tilde{\theta}}_{(a) s}\left(\alpha_{(e)}(\tau, \vec{\sigma}), \partial_{u} \alpha_{(e)}(\tau, \vec{\sigma})\right)^{3} \tilde{M}_{(a)}(\tau, \vec{\sigma})\right]= \\
& =\left.\frac{\partial \sigma^{s}(\vec{\xi})}{\partial \xi^{r}}\right|_{\vec{\xi}=\vec{\xi}(\tau, \vec{\sigma})}\left[{ }^{3} \tilde{\Theta}_{s}-\frac{\partial \alpha_{(a)}}{\partial \sigma^{s}} \tilde{\pi}_{(a)}^{\vec{\alpha}}\right](\tau, \vec{\sigma}) \approx 0,
\end{aligned}
$$


and both $\xi^{r}(\tau, \vec{\sigma})$ and $\tilde{\pi}_{r}^{\vec{\xi}}(\tau, \vec{\sigma})$ have zero Poisson bracket with $\alpha_{(a)}(\tau, \vec{\sigma}), \tilde{\pi}_{(a)}^{\vec{\alpha}}(\tau, \vec{\sigma})$.

Therefore, the 6 gauge variables $\xi^{r}(\tau, \vec{\sigma})$ and $\alpha_{(a)}(\tau, \vec{\sigma})$ and their conjugate momenta form 6 canonical pairs of a new canonical basis adapted to the rotation and pseudodiffeomorphisms constraints and replacing 6 of the 9 conjugate pairs ${ }^{3} e_{(a) r}(\tau, \vec{\sigma}),{ }^{3} \tilde{\pi}_{(a)}^{r}(\tau, \vec{\sigma})$.

From Eqs.(3.19) of Ref. [1] and from Eqs.4.36) and (4.13), we get

$$
\begin{aligned}
Y_{s}\left(\tau, \vec{\sigma}^{\prime}\right)^{3} e_{(a) r}(\tau, \vec{\sigma}) & =-\left(\frac{\partial \xi^{u}\left(\tau, \vec{\sigma}^{\prime}\right)}{\partial \sigma^{\prime} s} \frac{\delta}{\delta \xi^{u}\left(\tau, \vec{\sigma}^{\prime}\right)}+\frac{\partial \alpha_{(c)}\left(\tau, \vec{\sigma}^{\prime}\right)}{\partial \sigma^{\prime r}} \frac{\tilde{\delta}}{\delta \alpha_{(c)}\left(\tau, \vec{\sigma}^{\prime}\right)}\right) \\
& \cdot\left[{ }^{3} R_{(a)(b)}\left(\alpha_{(e)}(\tau, \vec{\sigma})\right)^{3} \bar{e}_{(b) r}(\tau, \vec{\sigma})\right]= \\
& =-{ }^{3} R_{(a)(b)}\left(\alpha_{(e)}(\tau, \vec{\sigma})\right) \frac{\partial \xi^{u}\left(\tau, \vec{\sigma}^{\prime}\right)}{\partial \sigma^{\prime} s} \frac{\delta^{3} \bar{e}_{(b) r}(\tau, \vec{\sigma})}{\delta \xi^{u}\left(\tau, \vec{\sigma}^{\prime}\right)}- \\
& -\frac{\partial \alpha_{(c)}\left(\tau, \vec{\sigma}^{\prime}\right)}{\partial \sigma^{\prime} s} \frac{\tilde{\delta}^{3} R_{(a)(b)}\left(\alpha_{(e)}(\tau, \vec{\sigma})\right)}{\delta \alpha_{(c)}\left(\tau, \vec{\sigma}^{\prime}\right)}{ }^{3} \bar{e}_{(b) r}(\tau, \vec{\sigma})= \\
& =-{ }^{3} R_{(a)(b)}\left(\alpha_{(e)}(\tau, \vec{\sigma})\right) \frac{\partial \xi^{u}\left(\tau, \vec{\sigma}^{\prime}\right)}{\left.\partial \sigma^{\prime}\right)} \frac{\delta^{3} \bar{e}_{(b) r}(\tau, \vec{\sigma})}{\delta \xi^{u}\left(\tau, \vec{\sigma}^{\prime}\right)}- \\
& -\frac{\partial^{3} R_{(a)(b)}\left(\alpha_{(e)}(\tau, \vec{\sigma})\right)}{\partial \sigma^{s}} \delta^{3}\left(\vec{\sigma}, \vec{\sigma}^{\prime}\right){ }^{3} \bar{e}_{(b) r}(\tau, \vec{\sigma})= \\
& =-\left\{{ }^{3} e_{(a) r}(\tau, \vec{\sigma}),{ }^{3} \tilde{\Theta}_{s}\left(\tau, \vec{\sigma}^{\prime}\right)\right\}= \\
& =-\frac{\partial^{3} e_{(a) r}(\tau, \vec{\sigma})}{\partial \sigma^{s}} \delta^{3}\left(\vec{\sigma}, \vec{\sigma}^{\prime}\right)+{ }^{3} e_{(a) s}(\tau, \vec{\sigma}) \frac{\partial \delta^{3}\left(\vec{\sigma}, \vec{\sigma}^{\prime}\right)}{\partial \sigma^{r}}= \\
& =-{ }^{3} R_{(a)(b)}\left(\alpha_{(e)}(\tau, \vec{\sigma})\right) \frac{\partial^{3} \bar{e}_{(b) r}(\tau, \vec{\sigma})}{\partial \sigma^{s}} \delta^{3}\left(\vec{\sigma}, \vec{\sigma}^{\prime}\right)- \\
& -\frac{\partial^{3} R_{(a)(b)}\left(\alpha_{(e)}(\tau, \vec{\sigma})\right)}{\partial \sigma^{s}}{ }^{3} \bar{e}_{(b) r}(\tau, \vec{\sigma}) \delta^{3}\left(\vec{\sigma}, \vec{\sigma}^{\prime}\right)+ \\
& +{ }^{3} R_{(a)(b)}\left(\alpha_{(e)}(\tau, \vec{\sigma})\right){ }^{3} \bar{e}_{(b) r}(\tau, \vec{\sigma}) \frac{\partial \delta^{3}\left(\vec{\sigma}, \vec{\sigma}^{\prime}\right)}{\partial \sigma^{r}} \\
&
\end{aligned}
$$

so that the pseudo-diffeomorphism multitemporal equations for ${ }^{3} \bar{e}_{(a) r}(\tau, \vec{\sigma})$ are

$$
\begin{aligned}
-\tilde{Y}_{s}\left(\tau, \vec{\sigma}^{\prime}\right){ }^{3} \bar{e}_{(a) r}(\tau, \vec{\sigma}) & =\frac{\partial \xi^{u}\left(\tau, \vec{\sigma}^{\prime}\right)}{\partial \sigma^{\prime} s} \frac{\delta^{3} \bar{e}_{(a) r}(\tau, \vec{\sigma})}{\delta \xi^{u}\left(\tau, \vec{\sigma}^{\prime}\right)}= \\
& =\frac{\partial^{3} \bar{e}_{(a) r}(\tau, \vec{\sigma})}{\partial \sigma^{s}} \delta^{3}\left(\vec{\sigma}, \vec{\sigma}^{\prime}\right)-{ }^{3} \bar{e}_{(a) s}(\tau, \vec{\sigma}) \frac{\partial \delta^{3}\left(\vec{\sigma}, \vec{\sigma}^{\prime}\right)}{\partial \sigma^{\prime r}}
\end{aligned}
$$

Analogously, from Eqs.(3.19) of Ref. [1] and Eqs.(4.36) and (4.24) we have

$$
\begin{aligned}
Y_{s}\left(\tau, \vec{\sigma}^{\prime}\right)^{3} \tilde{\pi}_{(a)}^{r}(\tau, \vec{\sigma}) & =-\left(\frac{\partial \xi^{u}\left(\tau, \vec{\sigma}^{\prime}\right)}{\partial \sigma^{\prime} s} \frac{\delta}{\delta \xi^{u}\left(\tau, \vec{\sigma}^{\prime}\right)}+\frac{\partial \alpha_{(c)}\left(\tau, \vec{\sigma}^{\prime}\right)}{\partial \sigma^{\prime r}} \frac{\tilde{\delta}}{\delta \alpha_{(c)}\left(\tau, \vec{\sigma}^{\prime}\right)}\right) \cdot \\
& \cdot\left[{ }^{3} R_{(a)(b)}\left(\alpha_{(e)}(\tau, \vec{\sigma})\right)^{3} \overline{\tilde{\pi}}_{(b)}^{r}(\tau, \vec{\sigma})\right]= \\
& =-\left[{ }^{3} R_{(a)(b)}\left(\alpha_{(e)}\left(\tau, \vec{\sigma}^{\prime}\right)\right)^{3} \overline{\tilde{\pi}}_{(b)}^{r}\left(\tau, \vec{\sigma}^{\prime}\right)\right] \frac{\partial \delta^{3}\left(\vec{\sigma}, \vec{\sigma}^{\prime}\right)}{\partial \sigma^{\prime} s}+ \\
& +\delta_{s}^{r 3} R_{(a)(b)}\left(\alpha_{(e)}(\tau, \vec{\sigma})\right)^{3} \overline{\tilde{\pi}}_{(b)}^{u}(\tau, \vec{\sigma}) \frac{\partial \delta^{3}\left(\vec{\sigma}, \vec{\sigma}^{\prime}\right)}{\partial \sigma^{\prime u}}
\end{aligned}
$$


and we get the pseudo-diffeomorphism multitemporal equation for ${ }^{3} \overline{\tilde{\pi}}_{(a)}^{r}(\tau, \vec{\sigma})$

$$
\begin{aligned}
-\tilde{Y}_{s}\left(\tau, \vec{\sigma}^{\prime}\right)^{3} \overline{\tilde{\pi}}_{(a)}^{r}(\tau, \vec{\sigma}) & =\frac{\partial \xi^{u}\left(\tau, \vec{\sigma}^{\prime}\right)}{\partial \sigma^{\prime s}} \frac{\delta^{3} \overline{\tilde{\pi}}_{(a)}^{r}(\tau, \vec{\sigma})}{\delta \xi^{u}\left(\tau, \vec{\sigma}^{\prime}\right)}= \\
& =-{ }^{3} \overline{\tilde{\pi}}_{(a)}^{r}\left(\tau, \vec{\sigma}^{\prime}\right) \frac{\partial \delta^{3}\left(\vec{\sigma}, \vec{\sigma}^{\prime}\right)}{\partial \sigma^{\prime} s}-\delta_{s}^{r}{ }^{3} \bar{\pi}_{(a)}^{u}(\tau, \vec{\sigma}) \frac{\partial \delta^{3}\left(\vec{\sigma}, \vec{\sigma}^{\prime}\right)}{\partial \sigma^{\prime u}} .
\end{aligned}
$$

Let us remark that the Jacobian matrix satisfies an equation like (4.41)

$$
\begin{aligned}
-\tilde{Y}_{s}\left(\tau, \vec{\sigma}^{\prime}\right) & \frac{\partial \xi^{u}(\tau, \vec{\sigma})}{\partial \sigma^{r}}=\frac{\partial \xi^{v}\left(\tau, \vec{\sigma}^{\prime}\right)}{\partial \sigma^{\prime s}} \frac{\delta}{\delta \xi^{v}\left(\tau, \vec{\sigma}^{\prime}\right)} \frac{\partial \xi^{u}(\tau, \vec{\sigma})}{\partial \sigma^{r}}= \\
= & \frac{\partial \xi^{u}\left(\tau, \vec{\sigma}^{\prime}\right)}{\partial \sigma^{\prime}} \frac{\partial \delta^{3}\left(\vec{\sigma}, \vec{\sigma}^{\prime}\right)}{\partial \sigma^{r}}=-\frac{\partial^{u}\left(\tau, \vec{\sigma}^{\prime}\right)}{\partial \sigma^{\prime}} \frac{\partial \delta^{3}\left(\vec{\sigma}, \vec{\sigma}^{\prime}\right)}{\partial \sigma^{\prime r}}= \\
= & -\frac{\partial \xi^{u}(\tau, \vec{\sigma})}{\partial \sigma^{s}} \frac{\partial \delta^{3}\left(\vec{\sigma}, \vec{\sigma}^{\prime}\right)}{\partial \sigma^{\prime r}}+\frac{\partial^{2} \xi^{u}(\tau, \vec{\sigma})}{\partial \sigma^{r} \partial \sigma^{s}} \delta^{3}\left(\vec{\sigma}, \vec{\sigma}^{\prime}\right)= \\
= & \frac{\partial}{\partial \sigma^{s}}\left(\frac{\partial \xi^{u}(\tau, \vec{\sigma})}{\partial \sigma^{r}}\right) \delta^{3}\left(\vec{\sigma}, \vec{\sigma}^{\prime}\right)-\frac{\partial \xi^{u}(\tau, \vec{\sigma})}{\partial \sigma^{s}} \frac{\partial \delta^{3}\left(\vec{\sigma}, \vec{\sigma}^{\prime}\right)}{\partial \sigma^{\prime r}} .
\end{aligned}
$$

so that the identity $\frac{\partial \xi^{u}\left(\tau, \vec{\sigma}^{\prime}\right)}{\partial \sigma^{\prime} s} \frac{\delta f(\tau, \vec{\xi}(\tau, \vec{\sigma}))}{\delta \xi^{u}\left(\tau, \vec{\sigma}^{\prime}\right)}=\frac{\partial f(\tau, \vec{\xi}(\tau, \vec{\sigma}))}{\partial \sigma^{s}} \delta^{3}\left(\vec{\sigma}, \vec{\sigma}^{\prime}\right)$, implies the following solutions of the multitemporal equations 103

$$
\begin{aligned}
{ }^{3} \bar{e}_{(a) r}(\tau, \vec{\sigma})= & \frac{\partial \xi^{s}(\tau, \vec{\sigma})}{\partial \sigma^{r}}{ }^{3} \hat{e}_{(a) s}(\tau, \vec{\xi}(\tau, \vec{\sigma}))=\frac{\partial \xi^{s}(\tau, \vec{\sigma})}{\partial \sigma^{r}} \hat{V}(\vec{\xi}(\tau, \vec{\sigma}))^{3} \hat{e}_{(a) s}(\tau, \vec{\sigma}), \\
& \frac{\delta^{3} \hat{e}_{(a) r}(\tau, \vec{\sigma})}{\delta \xi^{s}\left(\tau, \vec{\sigma}^{\prime}\right)}=0, \\
{ }^{3} e_{(a) r}(\tau, \vec{\sigma})= & { }^{3} R_{(a)(b)}\left(\alpha_{(e)}(\tau, \vec{\sigma})\right) \frac{\partial \xi^{s}(\tau, \vec{\sigma})}{\partial \sigma^{r}}{ }^{3} \hat{e}_{(b) s}(\tau, \vec{\xi}(\tau, \vec{\sigma}))= \\
= & \frac{\partial \xi^{s}(\tau, \vec{\sigma})}{\partial \sigma^{r}}{ }^{3} R_{(a)(b)}\left(\alpha_{(e)}^{\prime}(\tau, \vec{\xi}(\tau, \vec{\sigma}))\right)^{3} \hat{e}_{(b) s}(\tau, \vec{\xi}(\tau, \vec{\sigma})), \\
{ }^{3} g_{r s}(\tau, \vec{\sigma})= & \frac{\partial \xi^{u}(\tau, \vec{\sigma})}{\partial \sigma^{r}} \frac{\partial \xi^{v}(\tau, \vec{\sigma})}{\partial \sigma^{s}}{ }^{3} \hat{e}_{(a) u}(\tau, \vec{\xi}(\tau, \vec{\sigma}))^{3} \hat{e}_{(a) v}(\tau, \vec{\xi}(\tau, \vec{\sigma})) .
\end{aligned}
$$

Here the cotriads ${ }^{3} \hat{e}_{(a) r}(\tau, \vec{\sigma})$ depend only on 3 degrees of freedom and are Dirac observables with respect to both Abelianized rotations and pseudo-diffeomorphisms. Again, like in the case of rotations, we have not found 3 specific conditions on the cotriads implying this final reduction. This is due to the fact that, even if one has a trivial coframe bundle, one does not know the group manifold of $\operatorname{Diff} \Sigma_{\tau}$ and there is no canonical identity for pseudo-diffeomorphisms and therefore also for rotations inside the gauge group $\overline{\mathcal{G}}_{R}$.

Eqs.(4.45) are the counterpart in tetrad gravity of the solutions of the 3 elliptic equations for the gravitomagnetic vector potential $\check{W}^{r}$ of the conformal approach [79] (see the end of Appendix C of II).

${ }^{103}$ Again $\hat{V}(\vec{\xi}(\tau, \vec{\sigma}))$ is the operator with the action $\hat{V}(\vec{\xi}(\tau, \vec{\sigma})) f(\tau, \vec{\sigma})=f(\tau, \vec{\xi}(\tau, \vec{\sigma}))$; and Eqs. 3.7) is used. 
If $\left.\frac{\partial \sigma^{r}(\vec{\xi})}{\partial \xi^{s}}\right|_{\vec{\xi}=\vec{\xi}(\tau, \vec{\sigma})}$ is the inverse Jacobian matrix and $\left|\frac{\partial \xi(\tau, \vec{\sigma})}{\partial \sigma}\right|$ the determinant of the Jacobian matrix, the following identities

$$
\begin{aligned}
\delta_{s}^{r}= & \left.\frac{\partial \sigma^{r}(\vec{\xi})}{\partial \xi^{u}}\right|_{\vec{\xi}=\vec{\xi}(\tau, \vec{\sigma})} \frac{\partial \xi^{u}(\tau, \vec{\sigma})}{\partial \sigma^{s}} \\
\Rightarrow & \left.\frac{\partial}{\partial \sigma^{v}} \frac{\partial \sigma^{r}(\vec{\xi})}{\partial \xi^{u}}\right|_{\vec{\xi}=\vec{\xi}(\tau, \vec{\sigma})}=-\left.\left.\frac{\partial \sigma^{s}(\vec{\xi})}{\partial \xi^{u}}\right|_{\vec{\xi}=\vec{\xi}(\tau, \vec{\sigma})} \frac{\partial \sigma^{r}(\vec{\xi})}{\partial \xi^{w}}\right|_{\vec{\xi}=\vec{\xi}(\tau, \vec{\sigma})} \frac{\partial^{2} \xi^{w}(\tau, \vec{\sigma})}{\partial \sigma^{v} \partial \sigma^{s}} \\
\Rightarrow \quad & \left.\frac{\delta}{\delta \xi^{v}\left(\tau, \vec{\sigma}^{\prime}\right)} \frac{\partial \sigma^{r}(\vec{\xi})}{\partial \xi^{u}}\right|_{\vec{\xi}=\vec{\xi}(\tau, \vec{\sigma})}=-\left.\left.\frac{\partial \sigma^{s}(\vec{\xi})}{\partial \xi^{u}}\right|_{\vec{\xi}=\vec{\xi}(\tau, \vec{\sigma})} \frac{\partial \sigma^{r}(\vec{\xi})}{\partial \xi^{s}}\right|_{\vec{\xi}=\vec{\xi}(\tau, \vec{\sigma})} \frac{\partial \delta^{3}\left(\vec{\sigma}, \vec{\sigma}^{\prime}\right)}{\partial \sigma^{v}} \\
-\tilde{Y}_{s}\left(\tau, \vec{\sigma}^{\prime}\right) \quad & \left.\frac{\partial \sigma^{r}(\vec{\xi})}{\partial \xi^{u}}\right|_{\vec{\xi}=\vec{\xi}(\tau, \vec{\sigma})}= \\
= & \frac{\partial}{\partial \sigma^{s}}\left(\left.\frac{\partial \sigma^{r}(\vec{\xi})}{\partial \xi^{u}}\right|_{\vec{\xi}=\vec{\xi}(\tau, \vec{\sigma})}\right) \delta^{3}\left(\vec{\sigma}, \vec{\sigma}^{\prime}\right)+\left.\delta_{s}^{r} \frac{\partial \sigma^{v}(\vec{\xi})}{\partial \xi^{u}}\right|_{\vec{\xi}-\vec{\xi}(\tau, \vec{\sigma})} \frac{\partial \delta^{3}\left(\vec{\sigma}, \vec{\sigma}^{\prime}\right)}{\partial \sigma^{\prime v}}
\end{aligned}
$$

and [use is done of $\delta \ln \operatorname{det} M=\operatorname{Tr}\left(M^{-1} \delta M\right)$ ]

$$
\begin{aligned}
\frac{\partial}{\partial \sigma^{r}}\left|\frac{\partial \xi(\tau, \vec{\sigma})}{\partial \sigma}\right| & =\left.\left|\frac{\partial \xi(\tau, \vec{\sigma})}{\partial \sigma}\right| \frac{\partial \sigma^{s}(\vec{\xi})}{\partial \xi^{u}}\right|_{\vec{\xi}=\vec{\xi}(\tau, \vec{\sigma})} \frac{\partial^{2} \xi^{u}(\tau, \vec{\sigma})}{\partial \sigma^{r} \partial \sigma^{s}} \\
\frac{\delta}{\delta \xi^{r}\left(\tau, \vec{\sigma}^{\prime}\right)}\left|\frac{\partial \xi(\tau, \vec{\sigma})}{\partial \sigma}\right| & =\left.\left|\frac{\partial \xi(\tau, \vec{\sigma})}{\partial \sigma}\right| \frac{\partial \sigma^{s}(\vec{\xi})}{\partial \xi^{r}}\right|_{\vec{\xi}=\vec{\xi}(\tau, \vec{\sigma})} \frac{\partial \delta^{3}\left(\vec{\sigma}, \vec{\sigma}^{\prime}\right)}{\partial \sigma^{s}} \\
\downarrow & \\
-\tilde{Y}_{s}\left(\tau, \vec{\sigma}^{\prime}\right)\left|\frac{\partial \xi(\tau, \vec{\sigma})}{\partial \sigma}\right| & =-\left|\frac{\partial \xi(\tau, \vec{\sigma})}{\partial \sigma}\right| \frac{\partial \delta^{3}\left(\vec{\sigma}, \vec{\sigma}^{\prime}\right)}{\partial \sigma^{\prime} s}
\end{aligned}
$$

allow to get

$$
\begin{aligned}
{ }^{3} \tilde{\pi}_{(a)}^{r}(\tau, \vec{\sigma}) & ={ }^{3} R_{(a)(b)}\left(\alpha_{(a)}(\tau, \vec{\sigma})\right)^{3} \overline{\tilde{\pi}}_{(b)}^{r}(\tau, \vec{\sigma})= \\
& =\left.{ }^{3} R_{(a)(b)}\left(\alpha_{(e)}(\tau, \vec{\sigma})\right)\left|\frac{\partial \xi(\tau, \vec{\sigma})}{\partial \sigma}\right| \frac{\partial \sigma^{r}(\vec{\xi})}{\partial \xi^{s}}\right|_{\vec{\xi}=\vec{\xi}(\tau, \vec{\sigma})}{ }^{3} \hat{\tilde{\pi}}_{(b)}^{s}(\tau, \vec{\xi}(\tau, \vec{\sigma}))= \\
& =\left.{ }^{3} R_{(a)(b)}\left(\alpha_{(e)}(\tau . \vec{\sigma})\right)\left|\frac{\partial \xi(\tau, \vec{\sigma})}{\partial \sigma}\right| \frac{\partial \sigma^{r}(\vec{\xi})}{\partial \xi^{s}}\right|_{\vec{\xi}=\vec{\xi}(\tau, \vec{\sigma})} \hat{V}(\vec{\xi}(\tau, \vec{\sigma}))^{3} \hat{\tilde{\pi}}_{(b)}^{s}(\tau . \vec{\sigma}),
\end{aligned}
$$

where ${ }^{3} \hat{\tilde{\pi}}_{(a)}^{r}(\tau, \vec{\sigma})$ are Dirac observables with respect to both Abelianized rotations and pseudo-diffeomorphisms. In a similar way we get

$$
{ }^{3} e_{(a)}^{r}(\tau, \vec{\sigma})=\left.{ }^{3} R_{(a)(b)}\left(\alpha_{(e)}(\tau, \vec{\sigma})\right) \frac{\partial \sigma^{r}(\vec{\xi})}{\partial \xi^{s}}\right|_{\vec{\xi}=\vec{\xi}(\tau, \vec{\sigma})}{ }^{3} \hat{e}_{(b)}^{r}(\tau, \vec{\xi}(\tau, \vec{\sigma})),
$$

with ${ }^{3} \hat{e}_{(a)}^{r}(\tau, \vec{\sigma})$ the Dirac observables for triads dual to ${ }^{3} e_{(a) r}(\tau, \vec{\sigma})$. The line element becomes

$$
d s^{2}=\epsilon\left(\left[N_{(a s)}+n\right]^{2}-\left[N_{(a s) r}+n_{r}\right] \frac{\partial \sigma^{r}(\vec{\xi})}{\partial \xi^{u}}{ }^{3} \hat{e}_{(a)}^{u}(\vec{\xi})^{3} \hat{e}_{(a)}^{v}(\vec{\xi}) \frac{\partial \sigma^{s}(\vec{\xi})}{\partial \xi^{v}}\left[N_{(a s) s}+n_{s}\right]\right)(d \tau)^{2}-
$$




$$
\begin{aligned}
- & 2 \epsilon\left[N_{(a s) r}+n_{r}\right] d \tau d \sigma^{r}-\epsilon{\frac{\partial \xi^{u}}{\partial \sigma^{r}}}^{3} \hat{e}_{(a) u}(\vec{\xi})^{3} \hat{e}_{(a) v}(\vec{\xi}) \frac{\partial \xi^{v}}{\partial \sigma^{s}} d \sigma^{r} d \sigma^{s}= \\
= & \epsilon\left(\left[N_{(a s)}+n\right]^{2}(d \tau)^{2}-\left[{ }^{3} \hat{e}_{(a) u}(\vec{\xi}) \frac{\partial \xi^{u}}{\partial \sigma^{r}} d \sigma^{r}+{ }^{3} \hat{e}_{(a)}^{u}(\vec{\xi}) \frac{\partial \sigma^{r}(\vec{\xi})}{\partial \xi^{u}}\left(N_{(a s) r}+n_{r}\right) d \tau\right]\right. \\
& {\left.\left[{ }^{3} \hat{e}_{(a) v}(\vec{\xi}) \frac{\partial \xi^{v}}{\partial \sigma^{s}} d \sigma^{s}+{ }^{3} \hat{e}_{(a)}^{v}(\vec{\xi}) \frac{\partial \sigma^{s}(\vec{\xi})}{\partial \xi^{v}}\left(N_{(a s) s}+n_{s}\right) d \tau\right]\right) . }
\end{aligned}
$$

To get $\xi^{r}(\tau, \vec{\sigma})$ in terms of the cotriads we have to solve the equations 104

$$
\begin{aligned}
\delta_{s}^{r} \delta^{3}\left(\vec{\sigma}, \vec{\sigma}^{\prime}\right)= & \left\{\xi^{r}(\tau, \vec{\sigma}), \tilde{\pi}_{s}^{\vec{\xi}}\left(\tau, \vec{\sigma}^{\prime}\right)\right\}= \\
= & \left.\frac{\partial \sigma^{u}(\vec{\xi})}{\partial \xi^{s}}\right|_{\vec{\xi}=\vec{\xi}\left(\tau, \vec{\sigma}^{\prime}\right)}\left\{\xi^{r}(\tau, \vec{\sigma}),{ }^{3} \tilde{\Theta}_{u}\left(\tau, \vec{\sigma}^{\prime}\right)\right\}= \\
= & \left.\frac{\partial \sigma^{u}(\vec{\xi})}{\partial \xi^{s}}\right|_{\vec{\xi}=\vec{\xi}\left(\tau, \vec{\sigma}^{\prime}\right)}\left[\left(\frac{\partial^{3} e_{(a) v}\left(\tau, \vec{\sigma}^{\prime}\right)}{\partial \sigma^{\prime u}}-\frac{\partial^{3} e_{(a) u}\left(\tau, \vec{\sigma}^{\prime}\right)}{\partial \sigma^{\prime} v}\right) \frac{\delta \xi^{r}(\tau, \vec{\sigma})}{\delta^{3} e_{(a) v}\left(\tau, \vec{\sigma}^{\prime}\right)}-\right. \\
- & \left.{ }^{3} e_{(a) u}\left(\tau, \vec{\sigma}^{\prime}\right) \frac{\partial}{\partial \sigma^{\prime} v} \frac{\delta \xi^{r}(\tau, \vec{\sigma})}{\delta^{3} e_{(a) v}\left(\tau, \vec{\sigma}^{\prime}\right)}\right], \\
& \Downarrow \\
\left(\left[\delta_{(a)(b)} \partial_{v}^{\prime}-\right.\right. & \left.{ }^{3} e_{(a)}^{u}\left(\partial_{u}^{\prime}{ }^{3} e_{(b) v}-\partial_{v}^{\prime}{ }^{3} e_{(b) u}\right)\right]\left(\tau, \vec{\sigma}^{\prime}\right) \frac{\delta}{\delta^{3} e_{(b) v}\left(\tau, \vec{\sigma}^{\prime}\right)}+ \\
+ & \left.\delta^{3}\left(\vec{\sigma}, \vec{\sigma}^{\prime}\right)^{3} e_{(a)}^{u}(\tau, \vec{\sigma}) \frac{\partial}{\partial \sigma^{u}}\right) \xi^{r}(\tau, \vec{\sigma})=0 .
\end{aligned}
$$

We do not know how to solve these equations along some privileged path in the group manifold of Diff $\Sigma_{\tau}$ after having chosen a global coordinate system $\Xi$ as a conventional origin of pseudo-diffeomorphisms ए0, due to the poor understanding of the geometry and differential structure of this group manifold. Presumably, since the fibers of $\Sigma_{\tau} \times \Sigma_{\tau}$ are also copies of $\Sigma_{\tau}$, on each one of them one can try to define an analogue of canonical coordinates of first kind by using the geodesic exponential map:

i) choose a reference fiber $\Sigma_{\tau, 0}$ in $\Sigma_{\tau} \times \Sigma_{\tau}$ over a point $p=(\tau, \overrightarrow{0})$ chosen as origin in the base ए06;

ii) if $q_{o}$ is the point in $\Sigma_{\tau} \times \Sigma_{\tau}$ at the intersection of $\Sigma_{\tau, 0}$ with the conventional identity cross section $\Sigma_{\tau}^{(\Xi)}$ and $q_{1}$ the point where $\Sigma_{\tau, 0}$ intersects a nearby global cross section $\Sigma_{\tau}^{\left(\Xi^{\prime}\right)}\left(\Xi^{\prime}\right.$ is another global coordinate system on $\Sigma_{\tau}$ ), we can consider the geodesic $\gamma_{q_{o} q_{1}}$ on $\Sigma_{\tau, 0}$; iii) use the geodesic exponential map along the geodesic $\gamma_{q_{o} q_{1}}$ to define pseudo-diffeomorphism

\footnotetext{
${ }^{104}$ Use is done of Eq.(4.38), of (2.11) and of $\left\{\xi^{r}(\tau, \vec{\sigma}),{ }^{3} \tilde{M}_{(a)}\left(\tau, \vec{\sigma}^{\prime}\right)\right\}=0$.

${ }^{105}$ This identifies a conventional identity cross section $\Sigma_{\tau}^{(\Xi)}$ in the proposed description of Diff $\Sigma_{\tau}$ with the fibration $\Sigma_{\tau} \times \Sigma_{\tau} \rightarrow \Sigma_{\tau}$ for the case $\Sigma_{\tau} \approx R^{3}$.

${ }^{106}$ Then connected to all the points in base with geodesics; for $\Sigma_{\tau} \approx R^{3}$ this is well defined; the global cross sections corresponding to global coordinate systems should be horizontal lifts of this geodesic star with respect to some notion of connection on the fibration.
} 
coordinates $\vec{\xi}(\tau, \overrightarrow{0})$ describing the transition from the global coordinate system $\Xi$ to $\Xi^{\prime}$ over the base point $p=(\tau, \overrightarrow{0})$;

iv) parallel transport these coordinates on the fiber $\Sigma_{\tau, 0}$ to the other fibers along the geodesics of the cross sections $\Sigma_{\tau}^{\left(\Xi^{\prime}\right)}$.

If this coordinatization of the group manifold of Diff $\Sigma_{\tau}$ for $\Sigma_{\tau} \approx R^{3}$ can be justified, then one could try to solve the previous equations.

Instead, we are able to give a formal expression for the operator $\hat{V}(\vec{\xi}(\vec{\sigma}))$ एण), whose action on functions $f(\vec{\sigma})$ is $\hat{V}(\vec{\xi}(\vec{\sigma})) f(\vec{\sigma})=f(\vec{\xi}(\vec{\sigma}))$. We have

$$
\hat{V}(\vec{\xi}(\vec{\sigma}))=P_{\gamma} e^{\left(\int_{\vec{\sigma}}^{\vec{\xi}(\vec{\sigma})} \frac{\partial \sigma^{r}(u)}{\partial u^{s}} \mathcal{D} u^{s}\right) \frac{\partial}{\partial \sigma^{r}},}
$$

where the path ordering is along the geodesic $\gamma$ in $\Sigma_{\tau}$ joining the points with coordinates $\vec{\sigma}$ and $\vec{\sigma}^{\prime}=\vec{\xi}(\vec{\sigma})$. For infinitesimal pseudo-diffeomorphisms $\vec{\sigma} \mapsto \vec{\sigma}^{\prime}(\vec{\sigma})=\vec{\xi}(\vec{\sigma})=\vec{\sigma}+\delta \vec{\sigma}(\vec{\sigma})$ [with inverse $\vec{\sigma}^{\prime}=\vec{\xi} \mapsto \vec{\sigma}(\vec{\xi})=\vec{\xi}-\delta \vec{\sigma}(\vec{\xi})$ ], we have

$$
\begin{gathered}
\hat{V}(\vec{\sigma}+\delta \vec{\sigma}) \approx 1+\left[\left.\delta \sigma^{s}(\vec{\sigma}) \frac{\partial \sigma^{r}(\vec{\xi})}{\partial \xi^{s}}\right|_{\vec{\xi}(\vec{\sigma})-\delta \vec{\sigma}(\vec{\xi}(\vec{\sigma}))} \frac{\partial}{\partial \sigma^{r}} \approx 1+\delta \sigma^{s}(\vec{\sigma}) \frac{\partial}{\partial \sigma^{s}}:\right. \\
: f(\vec{\sigma}) \mapsto f(\vec{\sigma})+\delta \sigma^{s}(\vec{\sigma}) \frac{\partial f(\vec{\sigma})}{\partial \sigma^{s}} \approx f(\vec{\sigma}+\delta \vec{\sigma}(\vec{\sigma})) .
\end{gathered}
$$

Formally we have

$$
\begin{aligned}
\frac{\delta}{\delta \xi^{r}\left(\vec{\sigma}^{\prime}\right)}[\hat{V}(\vec{\xi}(\vec{\sigma})) f(\vec{\sigma})] & =\left.\delta^{3}\left(\vec{\sigma}, \vec{\sigma}^{\prime}\right) \frac{\partial \sigma^{s}(\vec{\xi})}{\partial \xi^{r}}\right|_{\vec{\xi}=\vec{\xi}(\vec{\sigma})} \frac{\partial}{\partial \sigma^{s}}[\hat{V}(\vec{\xi}(\vec{\sigma})) f(\vec{\sigma})]= \\
& =\left.\delta^{3}\left(\vec{\sigma}, \vec{\sigma}^{\prime}\right) \frac{\partial \sigma^{s}(\vec{\xi})}{\partial \xi^{r}}\right|_{\vec{\xi}=\vec{\xi}(\vec{\sigma})} \frac{\partial f(\vec{\xi}(\vec{\sigma}))}{\partial \sigma^{s}}=\left.\delta^{3}\left(\vec{\sigma}, \vec{\sigma}^{\prime}\right) \frac{\partial f(\vec{\xi})}{\partial \xi^{r}}\right|_{\vec{\xi}=\vec{\xi}(\vec{\sigma})}= \\
& =\frac{\delta f(\vec{\xi}(\vec{\sigma}))}{\delta \xi^{r}\left(\vec{\sigma}^{\prime}\right)}
\end{aligned}
$$

By using Eqs.(4.27) and (3.19) of Ref. [1], we get

$$
\begin{aligned}
\hat{D}_{(a)(b) r}^{(\omega)}(\tau, \vec{\sigma}) \quad & \left\{\zeta_{(b)(c)}^{(\omega) r}\left(\vec{\sigma}, \vec{\sigma}_{1} ; \tau\right),{ }^{3} \tilde{M}_{(g)}\left(\tau, \vec{\sigma}_{2}\right)\right\}= \\
= & -\epsilon_{(a)(d)(b)}\left\{{ }^{3} \omega_{s(d)}(\tau, \vec{\sigma}),{ }^{3} \tilde{M}_{(g)}\left(\tau, \vec{\sigma}_{2}\right)\right\} \zeta_{(b)(c)}^{(\omega) s}\left(\vec{\sigma}, \vec{\sigma}_{1} ; \tau\right), \\
\hat{D}_{(a)(b) r}^{(\omega)}(\tau, \vec{\sigma}) \quad & \left\{\zeta_{(b)(c)}^{(\omega) r}\left(\vec{\sigma}, \vec{\sigma}_{1} ; \tau\right),{ }^{3} \tilde{\Theta}_{u}\left(\tau, \vec{\sigma}_{2}\right)\right\}= \\
= & -\epsilon_{(a)(d)(f)}\left\{{ }^{3} \omega_{s(d)}(\tau, \vec{\sigma}),{ }^{3} \tilde{\Theta}_{u}\left(\tau, \vec{\sigma}_{2}\right)\right\} \zeta_{(f)(c)}^{(\omega) s}\left(\vec{\sigma}, \vec{\sigma}_{1} ; \tau\right) .
\end{aligned}
$$

Then Eqs.(3.19) of Ref. [1], (4.17) and (4.27) imply the following transformation properties under rotations and space pseudo-diffeomorphisms of the Green function of the SO(3) covariant divergence (which we do not know how to verify explicitly due to the path-ordering contained in it)

\footnotetext{
${ }^{107}$ For the sake of simplicity we do not consider the $\tau$-dependence.

${ }^{108}$ If $\delta / \delta \xi^{r}(\vec{\sigma})$ is interpreted as the directional functional derivative along $\gamma$.
} 


$$
\begin{aligned}
\left\{\zeta_{(a)(b)}^{(\omega) r}\left(\vec{\sigma}, \vec{\sigma}_{1} ; \tau\right),\right. & \left.\quad{ }^{3} \tilde{M}_{(g)}\left(\tau, \vec{\sigma}_{2}\right)\right\}=\frac{\partial}{\partial \sigma_{2}^{s}}\left[\zeta_{(a)(e)}^{(\omega) r}\left(\vec{\sigma}, \vec{\sigma}_{2} ; \tau\right) \epsilon_{(e)(g)(f)} \zeta_{(f)(b)}^{(\omega) s}\left(\vec{\sigma}_{2}, \vec{\sigma}_{1} ; \tau\right)\right]+ \\
+ & \zeta_{(a)(e) r}^{(\omega) r}\left(\vec{\sigma}, \vec{\sigma}_{2}\right){ }^{3} \omega_{s(e)}\left(\tau, \vec{\sigma}_{2}\right) \zeta_{(g)(b)}^{(\omega) s}\left(\vec{\sigma}_{2}, \vec{\sigma}_{1} ; \tau\right)- \\
& -\zeta_{(a)(g)}^{(\omega)}\left(\vec{\sigma}, \vec{\sigma}_{2} ; \tau\right){ }^{3} \omega_{s(f)}\left(\tau, \vec{\sigma}_{2}\right) \zeta_{(f)(b)}^{(\omega) s}\left(\vec{\sigma}_{2}, \vec{\sigma}_{1} ; \tau\right), \\
\left\{\zeta_{(a)(b)}^{(\omega) r}\left(\vec{\sigma}, \vec{\sigma}_{1} ; \tau\right), \quad\right. & \left.{ }^{3} \tilde{\Theta}_{u}\left(\tau, \vec{\sigma}_{2}\right)\right\}= \\
= & \int d^{3} \sigma_{3} \zeta_{(a)(e)}^{(\omega) r}\left(\vec{\sigma}, \vec{\sigma}_{3} ; \tau\right) \epsilon_{(e)(d)(f)}\left\{{ }^{3} \omega_{s(d)}\left(\tau, \vec{\sigma}_{3}\right),{ }^{3} \tilde{\Theta}_{u}\left(\tau, \vec{\sigma}_{2}\right)\right\} \zeta_{(f)(b)}^{(\omega) s}\left(\vec{\sigma}_{3}, \vec{\sigma}_{1} ; \tau\right) .
\end{aligned}
$$

Collecting all previous results, we obtain the following form for the Dirac Hamiltonian (2.58) of scenario b) on WSW hypersurfaces with $\tilde{\lambda}_{A B}(\tau)=0{ }^{109}$

$$
\begin{aligned}
& \hat{H}_{(D) A D M}=\int d^{3} \sigma\left[n \hat{\mathcal{H}}+n_{(a)}{ }^{3} e_{(a)}^{r}{ }^{3} \tilde{\Theta}_{r}+\right. \\
& \left.+\lambda_{n} \tilde{\pi}^{n}+\lambda_{(a)}^{\vec{n}} \tilde{\pi}_{(a)}^{\vec{n}}+\lambda_{(a)}^{\vec{\varphi}} \tilde{\pi}_{(a)}^{\vec{\varphi}}+\hat{\mu}_{(a)}{ }^{3} \tilde{M}_{(a)}\right](\tau, \vec{\sigma})- \\
& -\tilde{\lambda}_{\tau}(\tau)\left[\epsilon_{(\infty)}-\hat{P}_{A D M}^{\tau}\right]+\tilde{\lambda}_{\check{r}}(\tau) \hat{P}_{A D M}^{\check{r}}= \\
& =\int d^{3} \sigma\left[n \hat{\mathcal{H}}+n_{(a)}{ }^{3} e_{(a)}^{r} \frac{\partial \xi^{s}}{\partial \sigma^{r}} \tilde{\pi}_{s}^{\vec{\xi}}+\lambda_{n} \tilde{\pi}^{n}+\lambda_{(a)}^{\vec{n}} \tilde{\pi}_{(a)}^{\vec{n}}+\right. \\
& \left.+\lambda_{(a)}^{\vec{\varphi}} \tilde{\pi}_{(a)}^{\vec{\varphi}}-\left(\hat{\mu}_{(b)} B_{(b)(a)}\left(\alpha_{(e)}\right)+n_{(b)}{ }^{3} e_{(b)}^{r} \frac{\partial \alpha_{(a)}}{\partial \sigma^{r}}\right) \tilde{\pi}_{(a)}^{\vec{\alpha}}\right](\tau, \vec{\sigma})- \\
& -\tilde{\lambda}_{\tau}(\tau)\left[\epsilon_{(\infty)}-\hat{P}_{A D M}^{\tau}\right]+\tilde{\lambda}_{\check{r}}(\tau) \hat{P}_{A D M}^{\check{r}}= \\
& =\int d^{3} \sigma\left[n \hat{\mathcal{H}}+n_{(a)} e^{r} e_{(a)}^{r} \frac{\partial \xi^{s}}{\partial \sigma^{r}} \tilde{\pi}_{s}^{\vec{\xi}}+\right. \\
& \left.+\lambda_{n} \tilde{\pi}^{n}+\lambda_{(a)}^{\vec{n}} \tilde{\pi}_{(a)}^{\vec{n}}+\lambda_{(a)}^{\vec{\varphi}} \tilde{\pi}_{(a)}^{\vec{\varphi}}+\tilde{\mu}_{(a)} \tilde{\pi}_{(a)}^{\vec{\alpha}}\right](\tau, \vec{\sigma})- \\
& -\tilde{\lambda}_{\tau}(\tau)\left[\epsilon_{(\infty)}-\hat{P}_{A D M}^{\tau}\right]+\tilde{\lambda}_{\tilde{r}}(\tau) \hat{P}_{A D M}^{\check{r}},
\end{aligned}
$$

where $\tilde{\mu}_{(a)}$ are new Dirac multipliers.

The phase space action, which usually is incorrectly written without the primary constraints, is

$$
\begin{aligned}
\bar{S} & =\int d \tau d^{3} \sigma\left[{ }^{3} \tilde{\pi}_{(a)}^{r} \partial_{\tau}{ }^{3} e_{(a) r}-n \hat{\mathcal{H}}+n_{(a)} \mathcal{H}_{(a)}-\right. \\
& \left.-\lambda_{n} \tilde{\pi}^{n}-\lambda_{(a)}^{\vec{n}} \tilde{\pi}_{(a)}^{\vec{n}}-\lambda_{(a)}^{\vec{\varphi}} \tilde{\pi}_{(a)}^{\vec{\varphi}}-\mu_{(a)}{ }^{3} \tilde{M}_{(a)}\right](\tau, \vec{\sigma})+ \\
& +\tilde{\lambda}_{\tau}(\tau)\left[\epsilon_{(\infty)}-\hat{P}_{A D M}^{\tau}\right]-\tilde{\lambda}_{\tilde{r}}(\tau) \hat{P}_{A D M}^{r}= \\
& =\int d \tau d^{3} \sigma\left[{ }^{3} \tilde{\pi}_{(a)}^{r} \partial_{\tau}{ }^{3} e_{(a) r}-n \hat{\mathcal{H}}-n_{(a)}{ }^{3} e_{(a)}^{r}{ }^{3} \tilde{\Theta}_{r}-\right. \\
& \left.-\lambda_{n} \tilde{\pi}^{n}-\lambda_{(a)}^{\vec{n}} \tilde{\pi}_{(a)}^{\vec{n}}-\lambda_{(a)}^{\vec{\varphi}} \tilde{\pi}_{(a)}^{\vec{\varphi}}-\hat{\mu}_{(a)}{ }^{3} \tilde{M}_{(a)}\right](\tau, \vec{\sigma})+
\end{aligned}
$$

${ }^{109}$ We have $-n_{(a)} \mathcal{H}_{(a)} \approx n_{(a)}{ }^{3} e_{(a)}^{r}{ }^{3} \tilde{\Theta}_{r} \approx \tilde{n}^{r} \tilde{\pi}_{r}^{\vec{\xi}}$ modulo ${ }^{3} \tilde{M}_{(a)} \approx 0$, see Eqs.(2.11) and after Eq. 2.58). 


$$
\begin{aligned}
& +\tilde{\lambda}_{\tau}(\tau)\left[\epsilon_{(\infty)}-\hat{P}_{A D M}^{\tau}\right]-\tilde{\lambda}_{\check{r}}(\tau) \hat{P}_{A D M}^{\check{r}}= \\
& =\int d \tau d^{3} \sigma{ }^{3} \tilde{\pi}_{(a)}^{r} \partial_{\tau}^{3} e_{(a) r}-n \hat{\mathcal{H}}-n_{(a)}{ }^{3} e_{(a)}^{r} \frac{\partial \xi^{s}}{\partial \sigma^{r}} \tilde{\pi}_{s}^{\vec{\xi}}- \\
& \left.-\lambda_{n} \tilde{\pi}^{n}-\lambda_{(a)}^{\vec{n}} \tilde{\pi}_{(a)}^{\vec{n}}-\lambda_{(a)}^{\vec{\varphi}} \tilde{\pi}_{(a)}^{\vec{\varphi}}-\tilde{\mu}_{(a)} \tilde{\pi}_{(a)}^{\vec{\alpha}}\right](\tau, \vec{\sigma})+ \\
& +\tilde{\lambda}_{\tau}(\tau)\left[\epsilon_{(\infty)}-\hat{P}_{A D M}^{\tau}\right]-\tilde{\lambda}_{\breve{r}}(\tau) \hat{P}_{A D M}^{\check{r}} .
\end{aligned}
$$

In conclusion the 18-dimensional phase space spanned by ${ }^{3} e_{(a) r}$ and ${ }^{3} \tilde{\pi}_{(a)}^{r}$ has a global (since $\Sigma_{\tau} \approx R^{3}$ ) canonical basis, in which 12 variables are $\alpha_{(a)}, \tilde{\pi}_{(a)}^{\vec{\alpha}} \approx 0, \xi^{r}, \tilde{\pi}_{r}^{\vec{\xi}} \approx 0$. The remaining 6 variables, hidden in the reduced quantities ${ }^{3} \hat{e}_{(a) r},{ }^{3} \hat{\tilde{\pi}}_{(a)}^{r}$, are 3 pairs of conjugate Dirac's observables with respect to the gauge transformations in $\overline{\mathcal{G}}_{R}$, namely they are invariant under Abelianized rotations and space pseudo-diffeomorphisms tro connected with the identity and obtainable as a succession of infinitesimal gauge transformations. However, since space pseudo-diffeomorphisms connect different charts in the atlas of $\Sigma_{\tau}$ and since $\xi^{r}(\tau, \vec{\sigma})=\sigma^{r}$ means to choose as origin of space pseudo-diffeomorphisms an arbitrary chart, the functional form of the Dirac's observables will depend on the chart chosen as origin. This will reflect itself in the freedom of how to parametrize the reduced $\operatorname{cotriad}^{3} \hat{e}_{(a) r}(\tau, \vec{\sigma})$ in terms of only 3 independent functions: in each chart 'c' they will be denoted $Q_{r}^{(c)}(\tau, \vec{\sigma})$ and, if ' $\mathrm{c}+\mathrm{dc}$ ' is a new chart connected to ' $\mathrm{c}$ ' by an infinitesimal space pseudo-diffeomorphism of parameters $\vec{\xi}(\tau, \vec{\sigma})$, then we will have $Q_{r}^{(c+d c)}(\tau, \vec{\sigma})=\frac{\partial \xi^{s}(\tau, \vec{\sigma})}{\partial \sigma^{r}} Q_{s}^{(c)}(\tau, \vec{\xi}(\tau, \vec{\sigma}))$.

The invariants under pseudo-diffeomorphisms of a Riemannian 3-manifold $\left(\Sigma_{\tau},{ }^{3} g\right)$ (no explicit basis is known for them), can be expressed in every chart ' $c$ ' as functionals of the 3 independent functions $Q_{r}^{(c)}(\tau, \vec{\sigma})$. Therefore, these 3 functions give a local coordinatization of the space of 3-geometries (superspace or moduli space) Riem $\Sigma_{\tau} /$ Diff $\Sigma_{\tau}$ [35.52].

By using Eqs. (4.45) and (4.48) in the Hamiltonian expressions of the 4-tensors, we could get the most important 4-tensors on the pseudo-Riemannian 4-manifold $\left(M^{4},{ }^{4} g\right)$ expressed in terms of $\tilde{\lambda}_{A}, n, \tilde{\pi}^{n} \approx 0, n_{(a)}, \tilde{\pi}_{(a)}^{\vec{n}} \approx 0, \alpha_{(a)}, \tilde{\pi}_{(a)}^{\vec{\alpha}} \approx 0, \xi^{r}, \tilde{\pi}_{r}^{\vec{\xi}} \approx 0$, and of the (non canonically conjugate) Dirac's observables with respect to the action of $\overline{\mathcal{G}}_{R}$, i.e. ${ }^{3} \hat{e}_{(a) r},{ }^{3} \hat{\tilde{\pi}}_{(a)}^{r}$ in the rest-frame instant form of tetrad gravity. If we could extract from ${ }^{3} \hat{e}_{(a) r},{ }^{3} \hat{\tilde{\pi}}_{(a)}^{r}$, the Dirac observables with respect to the gauge transformations generated by the superhamiltonian constraint $\hat{\mathcal{H}}(\tau, \vec{\sigma}) \approx 0$, then we could express all 4-tensors in terms of these final Dirac observables (the independent Cauchy data of tetrad gravity), of the gauge variables $n, n_{(a)}$, $\alpha_{(a)}, \xi^{r}$ and of the gauge variable associated with $\hat{\mathcal{H}}(\tau, \vec{\sigma}) \approx 0$, when all the constraints are satisfied. Therefore, we would get not only a chart-dependent expression of the 4-metrics ${ }^{4} g \in$ Riem $M^{4}$, but also of the 4-geometries in Riem $M^{4} / \operatorname{Diff} M^{4}$.

In the next Section we shall study the simplest charts of the atlas of $\Sigma_{\tau}$, namely the 3 -orthogonal ones.

${ }^{110}$ And, therefore, weakly invariant under the original rotations and space pseudodiffeomorphisms. 


\section{QUASI-SHANMUGADHASAN CANONICAL TRANSFORMATION TO THE 3-ORTHOGONAL GAUGES.}

In this Section we shall identify a Shanmugadhasan canonical transformation to a canonical basis adapted to 13 of the 14 first class constraints ${ }^{111}$. This canonical basis will then be specialized to 3-orthogonal coordinates for $\Sigma_{\tau}$.

The quasi-Shanmugadhasan canonical transformation [1] [1] Abelianizing the rotation and pseudo-diffeomorphism constraints ${ }^{3} \tilde{M}_{(a)}(\tau, \vec{\sigma}) \approx 0,{ }^{3} \widetilde{\Theta}_{r}(\tau, \vec{\sigma}) \approx 0$, will send the canonical basis ${ }^{3} e_{(a) r}(\tau, \vec{\sigma}),{ }^{3} \tilde{\pi}_{(a)}^{r}(\tau, \vec{\sigma})$, of $T^{*} \mathcal{C}_{e}$ in a new basis whose conjugate pairs are $\left(\alpha_{(a)}(\tau, \vec{\sigma}), \tilde{\pi}_{(a)}^{\vec{\alpha}}(\tau, \vec{\sigma}) \approx 0\right),\left(\xi^{r}(\tau, \vec{\sigma}), \tilde{\pi}_{r}^{\vec{\xi}_{(}}(\tau, \vec{\sigma}) \approx 0\right)$ for the gauge sector and $\left(Q_{r}(\tau, \vec{\sigma}), \tilde{\Pi}^{r}(\tau, \vec{\sigma})\right)$ for the sector of Dirac observables.

Therefore, we must parametrize the Dirac observables ${ }^{3} \hat{e}_{(a) r}(\tau, \vec{\sigma})$ in terms of three functions $Q_{r}(\tau, \vec{\sigma}),{ }^{3} \hat{e}_{(a) r}(\tau, \vec{\sigma})={ }^{3} \hat{e}_{(a) r}\left[\tau, \vec{\sigma}, Q_{s}(\tau, \vec{\sigma})\right]$, and then find how the Dirac observables $3 \hat{\tilde{\pi}}_{(a)}^{r}(\tau, \vec{\sigma})$ are expressible in terms of $Q_{r}(\tau, \vec{\sigma}), \tilde{\Pi}^{r}(\tau, \vec{\sigma}), \tilde{\pi}_{r}^{\vec{\xi}}(\tau, \vec{\sigma}), \tilde{\pi}_{(a)}^{\vec{\alpha}}(\tau, \vec{\sigma}) \amalg$. Since from Eqs.4.45) we get

$$
\begin{aligned}
{ }^{3} g_{r s}(\tau, \vec{\sigma})= & { }^{3} e_{(a) r}(\tau, \vec{\sigma}){ }^{3} e_{(a) s}(\tau, \vec{\sigma})={ }^{3} \bar{e}_{(a) r}(\tau, \vec{\sigma})^{3} \bar{e}_{(a) s}(\tau, \vec{\sigma})= \\
= & \frac{\partial \xi^{u}(\tau, \vec{\sigma})}{\partial \sigma^{r}} \frac{\partial \xi^{v}(\tau, \vec{\sigma})}{\partial \sigma^{s}}{ }^{3} \hat{e}_{(a) u}\left[\tau, \vec{\xi}(\tau, \vec{\sigma}), Q_{w}(\tau, \vec{\xi}(\tau, \vec{\sigma}))\right] \\
& { }^{3} \hat{e}_{(a) v}\left[\tau, \vec{\xi}(\tau, \vec{\sigma}), Q_{w}(\tau, \vec{\xi}(\tau, \vec{\sigma}))\right]= \\
= & \frac{\partial \xi^{u}(\tau, \vec{\sigma})}{\partial \sigma^{r}} \frac{\partial \xi^{v}(\tau, \vec{\sigma})}{\partial \sigma^{s}}{ }^{3} \hat{g}_{u v}\left[\tau, \vec{\xi}(\tau, \vec{\sigma}), Q_{w}(\tau, \vec{\xi}(\tau, \vec{\sigma}))\right],
\end{aligned}
$$

the new metric ${ }^{3} \hat{g}_{u v}(\tau, \vec{\xi})$ must depend only on the functions $Q_{w}(\tau, \vec{\xi})$. This shows that the parametrization of ${ }^{3} \hat{e}_{(a) r}(\tau, \vec{\sigma})$ will depend on the chosen system of coordinates, which will be declared the origin $\vec{\xi}(\tau, \vec{\sigma})=\vec{\sigma}$ of pseudo-diffeomorphisms from the given chart. Therefore, each Dirac observable 3-metric ${ }^{3} \hat{g}_{u v}$ is an element of De Witt superspace 36] for Riemannian 3-manifolds: it defines a 3-geometry on $\Sigma_{\tau}$.

The simplest global systems of coordinates on $\Sigma_{\tau} \approx R^{3}$, where to learn how to construct the quasi-Shanmugadhasan canonical transformation, are the 3-orthogonal ones, in which ${ }^{3} \hat{g}_{u v}$ is diagonal. In it we have the parametrization

$$
\begin{aligned}
&{ }^{3} \hat{e}_{(a) r}(\tau, \vec{\sigma})= \delta_{(a) r} Q_{r}(\tau, \vec{\sigma}) \\
& \Rightarrow \quad{ }^{3} \hat{e}_{(a)}^{r}(\tau, \vec{\sigma})=\frac{\delta_{(a)}^{r}}{Q_{r}(\tau, \vec{\sigma})}, \\
& \Rightarrow \quad{ }^{3} \hat{g}_{r s}(\tau, \vec{\sigma})=\delta_{r s} Q_{r}^{2}(\tau, \vec{\sigma}),
\end{aligned}
$$

\footnotetext{
${ }^{111}$ In paper II there was a preliminary attempt to find this canonical transformation, which was subsequently realized to be wrong.

${ }^{112}$ Quasi- because we are not including the superhamiltonian constraint $\hat{\mathcal{H}}(\tau, \vec{\sigma}) \approx 0$.

${ }^{113}$ They cannot depend on $\alpha_{(a)}(\tau, \vec{\sigma}), \xi_{r}(\tau, \vec{\sigma})$, because they are Dirac observables.
} 


$$
\begin{aligned}
d s^{2}= & \epsilon\left(\left[N_{(a s)}+n\right]^{2}-\left[N_{(a s) r}+n_{r}\right] \sum_{u} \frac{\partial \sigma^{r}(\vec{\xi})}{\partial \xi^{u}} \frac{1}{Q_{u}^{2}(\vec{\xi})} \frac{\partial \sigma^{s}(\vec{\xi})}{\partial \xi^{u}}\left[N_{(a s) s}+n_{s}\right]\right)(d \tau)^{2}- \\
- & 2 \epsilon\left[N_{(a s) r}+n_{r}\right] d \tau d \sigma^{r}-\epsilon \sum_{u} \frac{\partial \xi^{u}}{\partial \sigma^{r}} Q_{u}^{2}(\vec{\xi}) \frac{\partial \xi^{u}}{\partial \sigma^{s}} d \sigma^{r} d \sigma^{s}= \\
= & \epsilon\left(\left[N_{(a s)}+n\right]^{2}(d \tau)^{2}-\delta_{u v}\left[Q_{u} \frac{\partial \xi^{u}}{\partial \sigma^{r}} d \sigma^{r}+\frac{1}{Q_{u}} \frac{\partial \sigma^{r}(\vec{\xi})}{\partial \xi^{u}}\left(N_{(a s) r}+n_{r}\right) d \tau\right]\right. \\
& {\left[Q_{v} \frac{\partial \xi^{v}}{\partial \sigma^{s}} d \sigma^{s}+\frac{1}{Q_{v}} \frac{\partial \sigma^{s}(\vec{\xi})}{\partial \xi^{v}}\left(N_{(a s) s}+n_{s}\right) d \tau\right] }
\end{aligned}
$$

with $Q_{r}(\tau, \vec{\sigma})=1+h_{r}(\tau, \vec{\sigma})>0$ to avoid singularities. The 3 functions $Q_{r}^{2}(\tau, \vec{\sigma})$ give a local parametrization of superspace; the presence of singularities in superspace depends on the boundary conditions for $Q_{r}(\tau, \vec{\sigma})$, i.e. on the possible existence of stability subgroups (isometries) of the group $\overline{\mathcal{G}}$ of gauge transformations, which we assume to be absent if a suitable weighted Sobolev space is chosen for cotriads.

The choice of the parametrization of ${ }^{3} \hat{e}_{(a) r}$ is equivalent to the coordinate conditions of Refs. 93,94 114 110.

\footnotetext{
${ }^{114}$ The 3-orthogonal gauges could be implemented with three gauge-fixing constraints of the type ${ }^{3} g_{r s}(\tau, \vec{\sigma})={ }^{3} e_{(a) r}(\tau, \vec{\sigma})^{3} e_{(a) s}(\tau, \vec{\sigma}) \approx 0$ for $r \neq s$. They would replace our Shanmugadhasanoriented constraints $\xi^{r}(\tau, \vec{\sigma})-\vec{\sigma} \approx 0$, but the information about the parametrization would remain implicit.
}

${ }^{115}$ For instance one would like to have a parametrization of the cotriads ${ }^{3} \hat{e}_{(a) r}$ corresponding to 3-normal coordinates around the point $\{\tau, \vec{\sigma}=0\} \in \Sigma_{\tau}$. Cartan 95.96 showed that, given Riemann normal coordinates $y^{\mu}$ at $p \in M^{4}\left[\left.y^{\mu}\right|_{p}=0\right]$, one can choose adapted orthonormal frames and coframes ${ }^{4} E_{(\alpha)}^{(N)}={ }^{4} E_{(\alpha)}^{(N) \mu}(y) \partial / \partial y^{\mu},{ }^{4} \theta^{(N)(\alpha)}={ }^{4} E_{\mu}^{(N)(\alpha)}(y) d y^{\mu}$, obtained from $\left.{ }^{4} E_{(\alpha)}^{(N)}\right|_{p}=$ $\delta_{(\alpha)}^{\mu} \partial / \partial y^{\mu},\left.{ }^{4} \theta^{(N)(\alpha)}\right|_{p}=\delta_{\mu}^{(\alpha)} d y^{\mu}$, by parallel transport along the geodesic arcs originating at $\mathrm{p}$. Then one has the following properties

$$
\begin{aligned}
{ }^{4} E_{\mu}^{(N)(\alpha)}(y) y^{\mu}= & \delta_{\mu}^{(\alpha)} y^{\mu} \\
{ }^{4} \theta^{(N)(\alpha)}= & \delta_{\mu}^{(\alpha)}\left[d y^{\mu}+y^{\rho} y^{\sigma} N^{\mu}{ }_{\rho \sigma \lambda}(y) d y^{\lambda}\right], \\
& N_{\mu \rho \sigma \lambda}=-N_{\rho \mu \sigma \lambda}=-N_{\mu \rho \lambda \sigma} .
\end{aligned}
$$

These 4-coordinates are important for the free fall observer description of phenomena, but are difficult to identify in the canonical approach, which privileges 3-coordinates and rebuilds 4-coordinates only a posteriori after a complete fixation of the Hamiltonian gauge and restriction to the solutions of Einstein equations.

Since normal coordinates are the most natural from a differential geometric point of view, let us look for a parametrization, in such a system of 3-coordinates, of the Dirac observables ${ }^{3} \hat{e}_{(a) r}(\tau, \vec{\sigma})$ on $\Sigma_{\tau}$ in terms of 3 real functions $\hat{Q}_{r}(\tau, \vec{\sigma})$, whose conjugate momenta will be denoted $\hat{\tilde{\Pi}}^{r}(\tau, \vec{\sigma})$. The previous equation gives the Cartan definition of orthonormal tetrads adapted to normal coordinates 
Eqs.(4.45), rewritten in the form

$$
{ }^{3} e_{(a) r}(\tau, \vec{\sigma})={ }^{3} R_{(a)(b)}\left(\alpha_{(e)}(\tau, \vec{\sigma})\right) \frac{\partial \xi^{s}(\tau, \vec{\sigma})}{\partial \sigma^{r}}{ }^{3} \hat{e}_{(b) s}\left(\tau, \vec{\xi}(\tau, \vec{\sigma}), Q_{u}(\tau, \vec{\xi}(\tau, \vec{\sigma})],\right.
$$

define a point canonical trasformation, in which the configuration variables transform in the following way

$$
\begin{array}{|l|l|l|l|}
{ }^{3} e_{(a) r} & \alpha_{(a)} & \xi^{r} & Q_{r} \\
\hline
\end{array}
$$

We have now to find the second half of this canonical transformation, namely

$$
\begin{array}{|l|l|l|l|}
\hline{ }^{3} \tilde{\pi}_{(a)}^{r} & \tilde{\pi}_{(a)}^{\vec{\alpha}}(\approx 0) & \tilde{\pi}_{r}^{\tilde{\xi}}(\approx 0) & \tilde{\Pi}^{r} \\
\hline
\end{array}
$$

For the cotriad canonical momentum we have [see Eq.(4.48)]

$$
\begin{aligned}
{ }^{3} \tilde{\pi}_{(a)}^{r}(\tau, \vec{\sigma}) & =\left.{ }^{3} R_{(a)(b)}\left(\alpha_{(e)}(\tau, \vec{\sigma})\right)\left|\frac{\partial \xi(\tau, \vec{\sigma})}{\partial \sigma}\right| \frac{\partial \sigma^{r}(\vec{\xi})}{\partial \xi^{s}}\right|_{\vec{\xi}=\vec{\xi}(\tau, \vec{\sigma}))} 3 \hat{\tilde{\pi}}_{(b)}^{s}(\tau, \vec{\xi}(\tau, \vec{\sigma}))= \\
& ={ }^{3} \tilde{\pi}_{(a)}^{r}\left(\tau, \vec{\sigma} \mid \alpha_{(e)}, \xi^{s}, Q_{s}, \tilde{\pi}_{(e)}^{\vec{\alpha}}, \tilde{\pi}_{s}^{\vec{\xi}}, \tilde{\Pi}^{s}\right] \equiv \\
& \equiv{ }^{3} \check{\tilde{\pi}}_{(a)}^{r}\left(\tau, \vec{\sigma} \mid \alpha_{(e)}, \xi^{s}, Q_{s}, \tilde{\Pi}^{s}\right]+ \\
& +\int d^{3} \sigma_{1} \tilde{F}_{(a)(b)}^{r}\left(\vec{\sigma}, \vec{\sigma}_{1} ; \tau \mid \alpha_{(e)}, \xi^{s}, Q_{s}, \tilde{\Pi}^{s}\right] \tilde{\pi}_{(b)}^{\alpha}\left(\tau, \vec{\sigma}_{1}\right)+ \\
& +\sum_{u} \int d^{3} \sigma_{1} \tilde{G}_{(a)}^{r u}\left(\vec{\sigma}, \vec{\sigma}_{1} ; \tau \mid \alpha_{(e)}, \xi^{s}, Q_{s}, \tilde{\Pi}^{s}\right] \tilde{\pi}_{u}^{\vec{\xi}}\left(\tau, \vec{\sigma}_{1}\right),
\end{aligned}
$$

with

for Lorentzian 4-manifolds. This suggests that for Riemannian 3-manifolds like $\Sigma_{\tau}$, to get 3-normal coordinates geodesic at the point $\vec{\sigma}=0$ we have to parametrize the reduced cotriads ${ }^{3} \hat{e}_{(a) r}(\tau, \vec{\sigma})$ as follows

$$
\begin{aligned}
{ }^{3} \hat{e}_{(a) r}(\tau, \vec{\sigma}) & =\delta_{(a)}^{s}\left[\delta_{r s}+\sum_{n} \epsilon_{r u n} \epsilon_{s v n} \sigma^{u} \sigma^{v} \hat{Q}_{n}(\tau, \vec{\sigma})\right] \\
& \Rightarrow{ }^{3} \hat{e}_{(a) r}(\tau, \vec{\sigma}) \sigma^{r}=\delta_{(a) r} \sigma^{r}
\end{aligned}
$$

with $N_{\text {surv }}(\tau, \vec{\sigma})=\sum_{n} \epsilon_{\text {sun }} \epsilon_{r v n} \hat{Q}_{n}(\tau, \vec{\sigma})=-N_{\text {usrv }}(\tau, \vec{\sigma})=-N_{\text {suvr }}(\tau, \vec{\sigma})=N_{\text {rvsu }}(\tau, \vec{\sigma})$. Then one gets

$$
\begin{aligned}
{ }^{3} \hat{g}_{r s}(\tau, \vec{\sigma}) & ={ }^{3} \hat{e}_{(a) r}(\tau, \vec{\sigma})^{3} \hat{e}_{(a) s}(\tau, \vec{\sigma})=\delta_{r s}+ \\
& +\sigma^{u} \sigma^{v}\left[\sum_{n} \epsilon_{r u n} \epsilon_{s v n}\left(2+\vec{\sigma}^{2} \hat{Q}_{n}(\tau, \vec{\sigma})\right) \hat{Q}_{n}(\tau, \vec{\sigma})-\sum_{n m} \epsilon_{r u n} \epsilon_{s v m} \sigma^{n} \sigma^{m} \hat{Q}_{n}(\tau, \vec{\sigma}) \hat{Q}_{m}(\tau, \vec{\sigma})\right] .
\end{aligned}
$$




$$
\begin{aligned}
3 \tilde{\tilde{\pi}}_{(a)}^{r}(\tau, \vec{\sigma}) & =\left.{ }^{3} \tilde{\pi}_{(a)}^{r}(\tau, \vec{\sigma})\right|_{\tilde{\pi}_{(e)}^{\vec{\alpha}}=\tilde{\pi}_{u}^{\vec{\xi}}=0}, \\
\tilde{F}_{(a)(b)}^{r}\left(\vec{\sigma}, \vec{\sigma}_{1} ; \tau \mid \alpha_{(e)}, \xi^{s}, Q_{s}, \tilde{\Pi}^{s}\right] & =\left.\frac{\delta^{3} \tilde{\pi}_{(a)}^{r}(\tau, \vec{\sigma})}{\delta \tilde{\pi}_{(b)}^{\vec{\alpha}}\left(\tau, \vec{\sigma}_{1}\right)}\right|_{\tilde{\pi}_{(e)}^{\vec{\alpha}}=\tilde{\pi}_{u}^{\vec{\xi}}=0}, \\
\tilde{G}_{(a)}^{r u}\left(\vec{\sigma}, \vec{\sigma}_{1} ; \tau \mid \alpha_{(e)}, \xi^{s}, Q_{s}, \tilde{\Pi}^{s}\right] & =\left.\frac{\delta^{3} \tilde{\pi}_{(a)}^{r}(\tau, \vec{\sigma})}{\delta \tilde{\pi}_{u}^{\vec{\xi}}\left(\tau, \vec{\sigma}_{1}\right)}\right|_{\tilde{\pi}_{(e)}^{\vec{\alpha}}=\tilde{\pi}_{u}^{\vec{\xi}}=0} .
\end{aligned}
$$

The last equality in Eq.(5.6) is a priori a strong equality in the sense of Dirac: powers of the constraints are ineffective near the constraint hypersurface. However, since the canonical transformation is a point one, the old momenta depend linearly upon the new ones, so that the strong equality sign may be replaced with an ordinary equality sign. Indeed, if in a phase space with canonical Darboux basis $q^{i}, p_{i}$, we make a point canonical transformation $q^{i}, p_{i} \mapsto$ $Q^{i}, P_{i}$ with $q^{i}=q^{i}(Q)$ [whose inverse is $Q^{i}=Q^{i}(q)$ ] and $p_{i}=p_{i}(Q, P)$, then the canonicity conditions $\delta_{j}^{i}=\left\{q^{i}, p_{j}\right\}_{q p}=\left\{q^{i}(Q), p_{j}(Q, P)\right\}_{Q P}=\sum_{k} \frac{\partial q^{i}(Q)}{\partial Q^{k}} \frac{\partial p_{j}(Q, P)}{\partial P_{k}}$ imply $p_{j}(Q, P)=$ $\left.\sum_{k} \frac{\partial Q^{k}(q)}{\partial q^{j}}\right|_{q=q(Q)} P_{k}+F_{j}(Q)$; moreover, from $0=\left\{p_{i}, p_{j}\right\}_{q, p}=\left\{p_{i}(Q, P), p_{j}(Q, P)\right\}_{Q, P}$ we get $F_{i}(Q)=\left.\frac{\partial F(Q(q))}{\partial q^{i}}\right|_{q=q(Q)}$. In what follows we shall put $F(Q)=0$, since this corresponds to a so called trivial phase canonical transformation.

Let us see what we can say about the dependence of the momenta ${ }^{3} \tilde{\pi}_{(a)}^{r}(\tau, \vec{\sigma})$ upon the Abelianized constraints $\tilde{\pi}_{(a)}^{\vec{\alpha}}(\tau, \vec{\sigma}) \approx 0, \tilde{\pi}_{r}^{\vec{\xi}}(\tau, \vec{\sigma}) \approx 0$.

Since the rotation constraints ${ }^{3} \tilde{M}_{(a)}=\epsilon_{(a)(b)(c)}{ }^{3} e_{(b) r}{ }^{3} \tilde{\pi}_{(c)}^{r}=\frac{1}{2} \epsilon_{(a)(b)(c)}{ }^{3} \tilde{M}_{(b)(c)}$ may be written as

$$
{ }^{3} \tilde{M}_{(a)(b)}={ }^{3} e_{(a) r}{ }^{3} \tilde{\pi}_{(b)}^{r}-{ }^{3} e_{(b) r}{ }^{3} \tilde{\pi}_{(a)}^{r}=\epsilon_{(a)(b)(c)}{ }^{3} \tilde{M}_{(c)}=-\epsilon_{(a)(b)(c)} \tilde{\pi}_{(d)}^{\vec{\alpha}} B_{(d)(c)}\left(\alpha_{(e)}\right) \approx 0,
$$

due to Eqs.(4.31), we may extract the following dependence of ${ }^{3} \tilde{\pi}_{(a)}^{r}(\tau, \vec{\sigma})$ on $\tilde{\pi}_{(a)}^{\vec{\alpha}}(\tau, \vec{\sigma})$

$$
\begin{aligned}
{ }^{3} \tilde{\pi}_{(a)}^{r} & ={ }^{3} e_{(b)}^{r}{ }^{3} e_{(b) s}{ }^{3} \tilde{\pi}_{(a)}^{s}= \\
& =\frac{1}{2}{ }^{3} e_{(b)}^{r}\left({ }^{3} e_{(b) s}{ }^{3} \tilde{\pi}_{(a)}^{s}+{ }^{3} e_{(a) s}{ }^{3} \tilde{\pi}_{(b)}^{s}\right)+\frac{1}{2}{ }^{3} e_{(b)}^{r}\left({ }^{3} e_{(b) s}{ }^{3} \tilde{\pi}_{(a)}^{s}-{ }^{3} e_{(a) s}{ }^{3} \tilde{\pi}_{(b)}^{s}\right)= \\
& =\frac{1}{2}{ }^{3} e_{(b)}^{r}\left({ }^{3} e_{(b) s}{ }^{3} \tilde{\pi}_{(a)}^{s}+{ }^{3} e_{(a) s}{ }^{3} \tilde{\pi}_{(b)}^{s}\right)- \\
& -\frac{1}{2}{ }^{3} e_{(b)}^{r} \epsilon_{(a)(b)(c)} \tilde{\pi}_{(d)}^{\vec{\alpha}} B_{(d)(c)}\left(\alpha_{(e)}\right) \stackrel{\text { def }}{=}, \\
& \stackrel{\text { def }}{=} \tilde{\pi}_{(a)}^{(M) r}-\frac{1}{2}{ }^{3} e_{(b)}^{r} \epsilon_{(a)(b)(c)} \tilde{\pi}_{(d)}^{\vec{\alpha}} B_{(d)(c)}\left(\alpha_{(e)}\right),
\end{aligned}
$$

with

$$
\begin{aligned}
{ }^{3} \tilde{\pi}_{(a)}^{(M)} & =\frac{1}{2} Z_{(a)(b)}{ }^{3} e_{(b)}^{r}=\sum_{s} P_{(a)(b) s}^{(M) r}{ }^{3} \tilde{\pi}_{(b)}^{s}, \\
Z_{(a)(b)} & =Z_{(b)(a)}={ }^{3} e_{(a) s}{ }^{3} \tilde{\pi}_{(b)}^{s}+{ }^{3} e_{(b) s}{ }^{3} \tilde{\pi}_{(a)}^{s}, \\
P_{(a)(b) s}^{(M) r} & =\frac{1}{2}{ }^{3} e_{(c)}^{r}\left({ }^{3} e_{(a) s} \delta_{(c)(b)}+{ }^{3} e_{(c) s} \delta_{(a)(b)}\right), \quad P_{(a)(c) u}^{(M) r} P_{(c)(b) s}^{(M) u}=P_{(a)(b) s}^{(M) r} .
\end{aligned}
$$


Let us note that, due to the projector $P_{(a)(b) s}^{(M) r}(\tau, \vec{\sigma}),{ }^{3} \tilde{\pi}_{(a)}^{(M) r}(\tau, \vec{\sigma})$ is a solution of the rotation constraints ${ }^{3} \tilde{M}_{(a)}(\tau, \vec{\sigma}) \approx 0$. However, it is not known the dependence of ${ }^{3} \tilde{\pi}_{(a)}^{(M) r}(\tau, \vec{\sigma})$ upon $\tilde{\pi}_{r}^{\vec{\xi}}(\tau, \vec{\sigma})$.

To extract the dependence of ${ }^{3} \tilde{\pi}_{(a)}^{r}(\tau, \vec{\sigma})$ on $\tilde{\pi}_{r}^{\vec{\xi}}(\tau, \vec{\sigma})$, let us recall Eqs.(2.11), (4.31), (4.39) and 4.26$)$

$$
\begin{aligned}
& \hat{\mathcal{H}}_{(a)}(\tau, \vec{\sigma})= \hat{D}_{(a)(b) r}^{(\omega)}(\tau, \vec{\sigma})^{3} \tilde{\pi}_{(b)}^{r}(\tau, \vec{\sigma})= \\
&=-{ }^{3} e_{(a)}^{r}(\tau, \vec{\sigma})\left[{ }^{3} \tilde{\Theta}_{r}+{ }^{3} \omega_{r(b)}{ }^{3} \tilde{M}_{(b)}\right](\tau, \vec{\sigma})= \\
&=-{ }^{3} e_{(a)}^{r}(\tau, \vec{\sigma})\left[\frac{\partial \xi^{s}}{\partial \sigma^{r}} \tilde{\pi}_{s}^{\vec{\xi}}-\left(B_{(b)(c)}\left(\alpha_{(e)}\right)^{3} \omega_{r(c)}-\frac{\partial \alpha_{(b)}}{\partial \sigma^{r}}\right) \tilde{\pi}_{(b)}^{\vec{\alpha}}\right](\tau, \vec{\sigma}) \approx 0, \\
&{ }^{3} \tilde{\pi}_{(a)}^{r}(\tau, \vec{\sigma})={ }^{3} \tilde{\pi}_{(a)}^{(T) r}(\tau, \vec{\sigma})-\int d^{3} \sigma_{1} \zeta_{(a)(b)}^{(\omega) r}\left(\vec{\sigma}, \vec{\sigma}_{1} ; \tau\right) \hat{\mathcal{H}}_{(b)}\left(\tau, \vec{\sigma}_{1}\right)= \\
&={ }^{3} \tilde{\pi}_{(a)}^{(T) r}(\tau, \vec{\sigma})+\int d^{3} \sigma_{1} \zeta_{(a)(b)}^{(\omega) r}\left(\vec{\sigma}, \vec{\sigma}_{1} ; \tau\right) \\
&{ }^{3} e_{(b)}^{s}\left(\tau, \vec{\sigma}_{1}\right)\left[\frac{\partial \xi^{u}}{\partial \sigma_{1}^{s}} \tilde{\pi}_{u}^{\vec{\xi}}-\left(B_{(d)(c)}\left(\alpha_{(e)}\right)^{3} \omega_{s(c)}-\frac{\partial \alpha_{(d)}}{\partial \sigma_{1}^{s}}\right) \tilde{\pi}_{(d)}^{\alpha}\right]\left(\tau, \vec{\sigma}_{1}\right), \\
&{ }^{3} \tilde{\pi}_{(a)}^{(T) r}(\tau, \vec{\sigma})= \int d^{3} \sigma_{1}\left[\delta_{s}^{r} \delta_{(a)(b)} \delta^{3}\left(\vec{\sigma}, \vec{\sigma}_{1}\right)+\zeta_{(a)(c)}^{(\omega) r}\left(\vec{\sigma}, \vec{\sigma}_{1}, \tau\right) \hat{D}_{(c)(b) s}^{(\omega)}\left(\tau, \vec{\sigma}_{1}\right)\right]{ }^{3} \tilde{\pi}_{(b)}^{s}\left(\tau, \vec{\sigma}_{1}\right)= \\
& \stackrel{d e f}{=} \int d^{3} \sigma_{1} P_{(a)(b) s}^{(T) r}\left(\vec{\sigma}, \vec{\sigma}_{1}, \tau\right)^{3} \tilde{\pi}_{(b)}^{s}\left(\tau, \vec{\sigma}_{1}\right), \\
& \\
& \hat{D}_{(a)(b) r}^{(\omega)}(\tau, \vec{\sigma}){ }^{3} \tilde{\pi}_{(b)}^{(T) r}(\tau, \vec{\sigma})=0 .
\end{aligned}
$$

We have introduced the projector $P_{(a)(b) s}^{(T) r}\left(\vec{\sigma}, \vec{\sigma}_{1} ; \tau\right)$, satisfying $\int d^{3} \sigma_{1} P_{(a)(b) u}^{(T) r}\left(\vec{\sigma}, \vec{\sigma}_{1} ; \tau\right) P_{(b)(c) s}^{(T) u}\left(\vec{\sigma}_{1}, \vec{\sigma}_{2} ; \tau\right)=P_{(a)(c) s}^{(T) r}\left(\vec{\sigma}, \vec{\sigma}_{2} ; \tau\right)$. In this second presentation we have privileged the solution ${ }^{3} \tilde{\pi}_{(a)}^{(T) r}(\tau, \vec{\sigma})$ of the constraints $\hat{\mathcal{H}}_{(a)}(\tau, \vec{\sigma}) \approx 0$. However, it is not known how ${ }^{3} \tilde{\pi}_{(a)}^{(T) r}(\tau, \vec{\sigma})$ depends upon $\tilde{\pi}_{(a)}^{\vec{\alpha}}(\tau, \vec{\sigma})$.

Eqs.(5.9) and (5.10) show: i) that the dependence of the old momenta upon the Abelianized constraints is linear; ii) but also that the non-commutativity of the two projectors $P_{(a)(b) s}^{(M) r}(\tau, \vec{\sigma}), P_{(a)(b) s}^{(T) r}(\tau, \vec{\sigma})$ is an obstruction to the determination of the kernels $F_{(a)(b)}^{r}, G_{(a)}^{r s}$ of Eqs.(5.6) starting from these equations.

Therefore, let us come back to Eqs.(5.7) and let us use the point nature of the canonical transformation

$$
\begin{aligned}
& \begin{array}{|l|l|l|l|}
\hline{ }^{3} e_{(a) r} \\
\hline{ }^{3} \tilde{\pi}_{(a)}^{r} \\
\hline
\end{array} \quad \longrightarrow \quad \begin{array}{l|l|l|}
\hline \alpha_{(a)} & \xi^{r} & Q_{r} \\
\hline \tilde{\pi}_{(a)}^{\vec{\alpha}} & \tilde{\pi}_{r}^{\tilde{\xi}} & \tilde{\Pi}^{r} \\
\hline
\end{array} \\
& \left\{\alpha_{(a)}(\tau, \vec{\sigma}), \tilde{\pi}_{(b)}^{\vec{\alpha}}\left(\tau, \vec{\sigma}^{\prime}\right)\right\}=\delta_{(a)(b)} \delta^{3}\left(\vec{\sigma}, \vec{\sigma}^{\prime}\right), \\
& \left\{\xi^{r}(\tau, \vec{\sigma}), \tilde{\pi}_{s}^{\vec{\xi}}\left(\tau, \vec{\sigma}^{\prime}\right)\right\}=\left\{Q_{s}(\tau, \vec{\sigma}), \tilde{\Pi}^{r}\left(\tau, \vec{\sigma}^{\prime}\right)\right\}=\delta_{s}^{r} \delta^{3}\left(\vec{\sigma}, \vec{\sigma}^{\prime}\right), \\
& \left\{{ }^{3} e_{(a) r}(\tau, \vec{\sigma}), \alpha_{(b)}\left(\tau, \vec{\sigma}^{\prime}\right)\right\}=\left\{{ }^{3} e_{(a) r}(\tau, \vec{\sigma}), \xi_{s}\left(\tau, \vec{\sigma}^{\prime}\right)\right\}=
\end{aligned}
$$




$$
\begin{aligned}
& =\left\{{ }^{3} e_{(a) r}(\tau, \vec{\sigma}), Q_{s}\left(\tau, \vec{\sigma}^{\prime}\right)\right\}=0, \\
\delta_{r}^{s} \delta_{(a)(b)} \delta^{3}\left(\vec{\sigma}, \vec{\sigma}^{\prime}\right)= & \left\{{ }^{3} e_{(a) r}(\tau, \vec{\sigma}),{ }^{3} \tilde{\pi}_{(b)}^{s}\left(\tau, \vec{\sigma}^{\prime}\right)\right\}= \\
= & \int d^{3} \sigma_{1}\left[\left\{{ }^{3} e_{(a) r}(\tau, \vec{\sigma}), \tilde{\pi}_{(c)}^{\vec{\alpha}}\left(\tau, \vec{\sigma}_{1}\right)\right\}\left\{\alpha_{(c)}\left(\tau, \vec{\sigma}_{1}\right),{ }^{3} \tilde{\pi}_{(b)}^{s}\left(\tau, \vec{\sigma}^{\prime}\right)\right\}+\right. \\
& +\left\{{ }^{3} e_{(a) r}(\tau, \vec{\sigma}), \tilde{\pi}_{u}^{\xi}\left(\tau, \vec{\sigma}_{1}\right)\right\}\left\{\xi^{u}\left(\tau, \vec{\sigma}_{1}\right),{ }^{3} \tilde{\pi}_{(b)}^{s}\left(\tau, \vec{\sigma}^{\prime}\right)\right\}+ \\
& \left.+\left\{{ }^{3} e_{(a) r}(\tau, \vec{\sigma}), \tilde{\Pi}^{u}\left(\tau, \vec{\sigma}_{1}\right)\right\}\left\{Q_{u}\left(\tau, \vec{\sigma}_{1}\right),{ }^{3} \tilde{\pi}_{(b)}^{s}\left(\tau, \vec{\sigma}^{\prime}\right)\right\}\right]= \\
= & \int d^{3} \sigma_{1}\left[\frac{\tilde{\delta}^{3} e_{(a) r}(\tau, \vec{\sigma})}{\delta \alpha_{(c)}\left(\tau, \vec{\sigma}_{1}\right)} \frac{\delta^{3} \tilde{\pi}_{(b)}^{s}\left(\tau, \vec{\sigma}^{\prime}\right)}{\delta \tilde{\pi}_{(c)}^{\vec{\alpha}}\left(\tau, \vec{\sigma}_{1}\right)}+\frac{\delta^{3} e_{(a) r}(\tau, \vec{\sigma})}{\delta \xi^{u}\left(\tau, \vec{\sigma}_{1}\right)} \frac{\delta^{3} \tilde{\pi}_{(b)}^{s}\left(\tau, \vec{\sigma}^{\prime}\right)}{\delta \tilde{\pi}_{u}\left(\tau, \vec{\sigma}_{1}\right)}+\right. \\
& \left.+\frac{\delta^{3} e_{(a) r}(\tau, \vec{\sigma})}{\delta Q_{u}\left(\tau, \vec{\sigma}_{1}\right)} \frac{\delta^{3} \tilde{\pi}_{(b)}^{s}\left(\tau, \vec{\sigma}^{\prime}\right)}{\delta \tilde{\Pi}^{u}\left(\tau, \vec{\sigma}_{1}\right)}\right] .
\end{aligned}
$$

The two equations defining the kernels may be rewritten in the following form

$$
\begin{aligned}
\tilde{F}_{(a)(b)}^{r}\left(\vec{\sigma}, \vec{\sigma}_{1} ; \tau \mid \alpha_{(e)}, \xi^{s}, Q_{s}, \tilde{\Pi}^{s}\right] & =\left.\frac{\delta^{3} \tilde{\pi}_{(a)}^{r}(\tau, \vec{\sigma})}{\delta \tilde{\pi}_{(b)}^{\vec{\alpha}}\left(\tau, \vec{\sigma}_{1}\right)}\right|_{\tilde{\pi}_{(e)}^{\vec{\alpha}}=\tilde{\pi}_{u}^{\vec{\xi}}=0}= \\
& =\left.\left\{\alpha_{(b)}\left(\tau, \vec{\sigma}_{1}\right),{ }^{3} \tilde{\pi}_{(a)}^{r}(\tau, \vec{\sigma})\right\}\right|_{\tilde{\pi}_{(e)}^{\vec{\alpha}}=\tilde{\pi}_{u}^{\vec{\xi}}=0}=\frac{\delta \alpha_{(b)}\left(\tau, \vec{\sigma}_{1}\right)}{\delta^{3} e_{(a) r}(\tau, \vec{\sigma})} \\
\tilde{G}_{(a)}^{r u}\left(\vec{\sigma}, \vec{\sigma}_{1} ; \tau \mid \alpha_{(e)}, \xi^{s}, Q_{s}, \tilde{\Pi}^{s}\right] & =\left.\frac{\delta^{3} \tilde{\pi}_{(a)}^{r}(\tau, \vec{\sigma})}{\delta \tilde{\pi}_{u}^{\vec{\xi}}\left(\tau, \vec{\sigma}_{1}\right)}\right|_{\tilde{\pi}_{(e)}^{\vec{\alpha}}=\tilde{\pi}_{u}^{\vec{\xi}}=0}= \\
& =\left.\left\{\xi^{u}\left(\tau, \vec{\sigma}_{1}\right),{ }^{3} \tilde{\pi}_{(a)}^{r}(\tau, \vec{\sigma})\right\}\right|_{\tilde{\pi}_{(e)}^{\vec{\alpha}}=\tilde{\pi}_{u}^{\vec{\xi}}=0}=\frac{\delta \xi^{u}\left(\tau, \vec{\sigma}_{1}\right)}{\delta^{3} e_{(a) r}(\tau, \vec{\sigma})},
\end{aligned}
$$

where in both the final expressions there is no more the restriction $\tilde{\pi}_{(a)}^{\vec{\alpha}}(\tau, \vec{\sigma})=\tilde{\pi}_{r}^{\vec{\xi}}(\tau, \vec{\sigma})=0$ due to the point nature of the canonical transformation. Therefore, Eq. (5.6) becomes

$$
\begin{aligned}
{ }^{3} \tilde{\pi}_{(a)}^{r}(\tau, \vec{\sigma})= & { }^{3} \tilde{\tilde{\pi}}_{(a)}^{r}\left(\tau, \vec{\sigma} \mid \alpha_{(e)}, \xi^{s}, Q_{s}, \tilde{\Pi}^{s}\right]+ \\
+ & \int d^{3} \sigma_{1} F_{(a)(b)}^{r}\left(\vec{\sigma}, \vec{\sigma}_{1} ; \tau\right) \tilde{\pi}_{(b)}^{\vec{\alpha}}\left(\tau, \vec{\sigma}_{1}\right)+ \\
+ & \sum_{u} \int d^{3} \sigma_{1} G_{(a)}^{r u}\left(\vec{\sigma}, \vec{\sigma}_{1} ; \tau\right) \tilde{\pi}_{u}^{\xi}\left(\tau, \vec{\sigma}_{1}\right), \\
& \text { with } \\
F_{(a)(b)}^{r}\left(\vec{\sigma}, \vec{\sigma}_{1} ; \tau\right)= & \tilde{F}_{(a)(b)}^{r}\left(\vec{\sigma}, \vec{\sigma}_{1} ; \tau \mid \alpha_{(e)}, \xi^{s}, Q_{s}\right], \\
G_{(a)}^{r u}\left(\vec{\sigma}, \vec{\sigma}_{1} ; \tau\right)= & \tilde{G}_{(a)}^{r u}\left(\vec{\sigma}, \vec{\sigma}_{1} ; \tau \mid \alpha_{(e)}, \xi^{s}, Q_{s}\right] .
\end{aligned}
$$

This equation and the point nature of the canonical transformation imply

$$
\frac{\delta^{3} \check{\tilde{\pi}}_{(a)}^{r}(\tau, \vec{\sigma})}{\delta \tilde{\Pi}^{u}\left(\tau, \vec{\sigma}_{1}\right)}=\frac{\delta^{3} \tilde{\pi}_{(a)}^{r}(\tau, \vec{\sigma})}{\delta \tilde{\Pi}^{u}\left(\tau, \vec{\sigma}_{1}\right)}=
$$




$$
\begin{gathered}
=\left\{Q_{u}\left(\tau, \vec{\sigma}_{1}\right),{ }^{3} \tilde{\pi}_{(a)}^{r}(\tau, \vec{\sigma})\right\}=\frac{\delta Q_{u}\left(\tau, \vec{\sigma}_{1}\right)}{\delta^{3} e_{(a) r}(\tau, \vec{\sigma})} \stackrel{\text { def }}{=} \\
\stackrel{\text { def }}{=} \tilde{\mathcal{K}}_{(a) u}^{r}\left(\vec{\sigma}, \vec{\sigma}_{1} ; \tau \mid \alpha_{(e)}, \xi^{s}, Q_{s}\right]=\mathcal{K}_{(a) u}^{r}\left(\vec{\sigma}, \vec{\sigma}_{1} ; \tau\right) .
\end{gathered}
$$

In conclusion the quasi-Shanmugadhasan canonical transformation is defined by

$$
\begin{aligned}
& \begin{array}{|l|}
{ }^{3} e_{(a) r} \\
\hline{ }^{3} \tilde{\pi}_{(a)}^{r} \\
\hline
\end{array} \\
& \longrightarrow \begin{array}{|c|c|c|}
\hline \alpha_{(a)} & \xi^{r} & Q_{r} \\
\hline \tilde{\pi}_{(a)}^{\vec{\alpha}} & \tilde{\pi}_{r}^{\vec{\xi}} & \tilde{\Pi}^{r} \\
\hline
\end{array} \\
& { }^{3} e_{(a) r}(\tau, \vec{\sigma})={ }^{3} R_{(a)(b)}\left(\alpha_{(e)}(\tau, \vec{\sigma})\right) \frac{\partial \xi^{s}(\tau, \vec{\sigma})}{\partial \sigma^{r}}{ }^{3} \hat{e}_{(b) s}\left(\tau, \vec{\xi}(\tau, \vec{\sigma}), Q_{u}(\tau, \vec{\xi}(\tau, \vec{\sigma})]\right. \\
& { }^{3} \tilde{\pi}_{(a)}^{r}(\tau, \vec{\sigma})=\sum_{u} \int d^{3} \sigma_{1} \mathcal{K}_{(a) u}^{r}\left(\vec{\sigma}, \vec{\sigma}_{1} ; \tau\right) \tilde{\Pi}^{u}\left(\tau, \vec{\sigma}_{1}\right)+ \\
& +\int d^{3} \sigma_{1} F_{(a)(b)}^{r}\left(\vec{\sigma}, \vec{\sigma}_{1} ; \tau\right) \tilde{\pi}_{(b)}^{\vec{\alpha}}\left(\tau, \vec{\sigma}_{1}\right)+ \\
& +\sum_{u} \int d^{3} \sigma_{1} G_{(a)}^{r u}\left(\vec{\sigma}, \vec{\sigma}_{1} ; \tau\right) \tilde{\pi}_{u}^{\vec{\xi}}\left(\tau, \vec{\sigma}_{1}\right),
\end{aligned}
$$

where the kernels

$$
\begin{gathered}
F_{(a)(b)}^{r}\left(\vec{\sigma}, \vec{\sigma}_{1} ; \tau\right)=\tilde{F}_{(a)(b)}^{r}\left(\vec{\sigma}, \vec{\sigma}_{1} ; \tau \mid \alpha_{(e)}, \xi^{s}, Q_{s}\right]=\frac{\delta \alpha_{(b)}\left(\tau, \vec{\sigma}_{1}\right)}{\delta^{3} e_{(a) r}(\tau, \vec{\sigma})}, \\
G_{(a)}^{r u}\left(\vec{\sigma}, \vec{\sigma}_{1} ; \tau\right)=\tilde{G}_{(a)}^{r u}\left(\vec{\sigma}, \vec{\sigma}_{1} ; \tau \mid \alpha_{(e)}, \xi^{s}, Q_{s}\right]=\frac{\delta \xi^{u}\left(\tau, \vec{\sigma}_{1}\right)}{\delta^{3} e_{(a) r}(\tau, \vec{\sigma})}, \\
\mathcal{K}_{(a) u}^{r}\left(\vec{\sigma}, \vec{\sigma}_{1} ; \tau\right)=\tilde{\mathcal{K}}_{(a) u}^{r}\left(\vec{\sigma}, \vec{\sigma}_{1} ; \tau \mid \alpha_{(e)}, \xi^{s}, Q_{s}\right]=\frac{\delta Q_{u}\left(\tau, \vec{\sigma}_{1}\right)}{\delta^{3} e_{(a) r}(\tau, \vec{\sigma})},
\end{gathered}
$$

are the matrix elements of the inverse of the Jacobian matrix of the point canonical transformation

$$
\begin{aligned}
\left|\frac{\partial\left({ }^{3} e_{(a) r}\right)}{\partial\left(\alpha_{(b)}, \xi^{s}, Q_{u}\right)}\right|\left(\vec{\sigma}, \vec{\sigma}_{1} ; \tau\right)= & \left(\frac{\delta^{3} e_{(a) r}(\tau, \vec{\sigma})}{\delta \alpha_{(b)}\left(\tau, \vec{\sigma}_{1}\right)}, \frac{\delta^{3} e_{(a) r}(\tau, \vec{\sigma})}{\delta \xi^{s}\left(\tau, \vec{\sigma}_{1}\right)}, \frac{\delta^{3} e_{(a) r}(\tau, \vec{\sigma})}{\delta Q_{u}\left(\tau, \vec{\sigma}_{1}\right)}\right), \\
\frac{\tilde{\delta}^{3} e_{(a) r}(\tau, \vec{\sigma})}{\delta \alpha_{(c)}\left(\tau, \vec{\sigma}_{1}\right)}= & \delta^{3}\left(\vec{\sigma}, \vec{\sigma}_{1}\right)\left[H_{(c)}\left(\alpha_{(e)}(\tau, \vec{\sigma})\right)^{3} R\left(\alpha_{(e)}(\tau, \vec{\sigma})\right)\right]_{(a)(b)} \\
& \sum_{u} \frac{\partial \xi^{u}(\tau, \vec{\sigma})}{\partial \sigma^{r}} \delta_{(b) u} Q_{u}(\tau, \vec{\xi}(\tau, \vec{\sigma}))= \\
& \delta^{3}\left(\vec{\sigma}, \vec{\sigma}_{1}\right) \epsilon_{(a)(n)(d)} A_{(d)(c)}\left(\alpha_{(e)}(\tau, \vec{\sigma})\right)^{3} R_{(n)(m)}\left(\alpha_{(e)}(\tau, \vec{\sigma})\right) . \\
\frac{\delta^{3} e_{(a) r}(\tau, \vec{\sigma})}{\delta \xi^{u}\left(\tau, \vec{\sigma}_{1}\right)}= & \sum_{u} \frac{\partial \xi^{u}(\tau, \vec{\sigma})}{\partial \sigma^{r}} \delta_{(m) u} Q_{u}(\tau, \vec{\xi}(\tau, \vec{\sigma}))
\end{aligned}
$$




$$
\begin{aligned}
& \sum_{v} \delta_{(n) v}\left[\left.\frac{\partial \xi^{v}(\tau, \vec{\sigma})}{\partial \sigma^{r}} \frac{\partial Q_{v}(\tau, \vec{\xi})}{\partial \xi^{u}}\right|_{\vec{\xi}=\vec{\xi}(\tau, \vec{\sigma})} \delta^{3}\left(\vec{\sigma}, \vec{\sigma}_{1}\right)+\right. \\
+ & \left.\delta_{u}^{v} Q_{v}(\tau, \vec{\xi}(\tau, \vec{\sigma})) \frac{\partial \delta^{3}\left(\vec{\sigma}, \vec{\sigma}_{1}\right)}{\partial \sigma^{r}}\right] \\
\frac{\delta^{3} e_{(a) r}(\tau, \vec{\sigma})}{\delta Q_{u}\left(\tau, \vec{\sigma}_{1}\right)}= & { }^{3} R_{(a)(n)}\left(\alpha_{(e)}(\tau, \vec{\sigma})\right) \sum_{v} \frac{\partial \xi^{v}(\tau, \vec{\sigma})}{\partial \sigma^{r}} \delta_{(n) v} \delta_{v}^{u} \delta^{3}\left(\vec{\xi}(\tau, \vec{\sigma}), \vec{\sigma}_{1}\right), \\
& \operatorname{with} \\
\delta^{3}\left(\vec{\sigma}, \vec{\sigma}_{1}\right) \delta_{(a)(b)} \delta_{r}^{s}= & \frac{\delta^{3} e_{(a) r}(\tau, \vec{\sigma})}{\delta^{3} e_{(b) s}\left(\tau, \vec{\sigma}_{1}\right)}= \\
= & \int d^{3} \sigma_{2}\left(\frac{\delta^{3} e_{(a) r}(\tau, \vec{\sigma})}{\delta \alpha_{(e)}\left(\tau, \vec{\sigma}_{2}\right)} F_{(b)(e)}^{s}\left(\vec{\sigma}_{1}, \vec{\sigma}_{2} ; \tau\right)+\right. \\
+ & \left.\frac{\delta^{3} e_{(a) r}(\tau, \vec{\sigma})}{\delta \xi^{u}\left(\tau, \vec{\sigma}_{2}\right)} G_{(b)}^{s u}\left(\vec{\sigma}_{1}, \vec{\sigma}_{2} ; \tau\right)+\frac{\delta^{3} e_{(a) r}(\tau, \vec{\sigma})}{\delta Q_{v}\left(\tau, \vec{\sigma}_{2}\right)} \mathcal{K}_{(b) v}^{s}\left(\vec{\sigma}_{1}, \vec{\sigma}_{2} ; \tau\right)\right) .
\end{aligned}
$$

Even if Eqs.(5.9) and (5.10) give the solution of the constraints ${ }^{3} \tilde{M}_{(a)}(\tau, \vec{\sigma}) \approx 0$ and $\hat{\mathcal{H}}_{(a)}(\tau, \vec{\sigma}) \approx 0$ respectively, their non-zero Poisson brackets ${ }^{116}$ imply the necessity to solve these equations for the kernels to find the connection of the old momenta with the Abelianized ones.

By comparing Eq.(5.15) with Eqs. (5.8), (5.9) and (5.10) we get

$$
\begin{aligned}
& 3 \check{\tilde{\pi}}_{(a)}^{r}(\tau, \vec{\sigma})=\sum_{u} \int d^{3} \sigma_{1} \mathcal{K}_{(a) u}^{r}\left(\vec{\sigma}, \vec{\sigma}_{1} ; \tau\right) \tilde{\Pi}^{u}\left(\tau, \vec{\sigma}_{1}\right) \approx \\
& { }^{3} \tilde{\pi}_{(a)}^{(M) r}(\tau, \vec{\sigma})=P_{(a)(b) s}^{(M) r}(\tau, \vec{\sigma})^{3} \tilde{\pi}_{(b)}^{s}(\tau, \vec{\sigma}) \approx \\
& \approx^{3} \tilde{\pi}_{(a)}^{(T) r}(\tau, \vec{\sigma})=\int d^{3} \sigma_{1} P_{(a)(b) s}^{(T) r}\left(\vec{\sigma}, \vec{\sigma}_{1} ; \tau\right)^{3} \tilde{\pi}_{(b)}^{s}\left(\tau, \vec{\sigma}_{1}\right), \\
& \Downarrow \\
& \sum_{u} \int d^{3} \sigma_{1}\left[{ }^{3} e_{(a) r}(\tau, \vec{\sigma}) \mathcal{K}_{(b) u}^{r}-{ }^{3} e_{(b) r}(\tau, \vec{\sigma}) \mathcal{K}_{(a) u}^{r}\right]\left(\vec{\sigma}, \vec{\sigma}_{1} ; \tau\right) \tilde{\Pi}^{u}\left(\tau, \vec{\sigma}_{1}\right)= \\
& =-\epsilon_{(a)(b)(c)}\left[\tilde{\pi}_{(d)}^{\vec{\alpha}} B_{(d)(c)}\left(\alpha_{(e)}\right)\right](\tau, \vec{\sigma})- \\
& -\int d^{3} \sigma_{1}\left[{ }^{3} e_{(a) r}(\tau, \vec{\sigma}) F_{(b)(c)}^{r}-{ }^{3} e_{(b) r}(\tau, \vec{\sigma}) F_{(a)(c)}^{r}\right]\left(\vec{\sigma}, \vec{\sigma}_{1} ; \tau\right) \tilde{\pi}_{(c)}^{\vec{\alpha}}\left(\tau, \vec{\sigma}_{1}\right)- \\
& -\sum_{u} \int d^{3} \sigma_{1}\left[{ }^{3} e_{(a) r}(\tau, \vec{\sigma}) G_{(b)}^{r u}-{ }^{3} e_{(b) r}(\tau, \vec{\sigma}) G_{(a)}^{r u}\right]\left(\vec{\sigma}, \vec{\sigma}_{1} ; \tau\right) \tilde{\pi}_{u}^{\vec{\xi}}\left(\tau, \vec{\sigma}_{1}\right) \approx \\
& \approx 0 \text {, }
\end{aligned}
$$

${ }^{116}$ See Eqs. 2.14) for the Poisson bracket of ${ }^{3} \tilde{M}_{(a)}$ and ${ }^{3} \tilde{\Theta}_{r}$ : from it we can deduce the quoted Poisson brackets. 


$$
\begin{aligned}
\sum_{u} & \int d^{3} \sigma_{1} \hat{D}_{(a)(b) r}^{(\omega)}(\tau, \vec{\sigma}) \mathcal{K}_{(b) u}^{r}\left(\vec{\sigma}, \vec{\sigma}_{1} ; \tau\right) \tilde{\Pi}^{u}\left(\tau, \vec{\sigma}_{1}\right)= \\
= & -{ }^{3} e_{(a)}^{r}(\tau, \vec{\sigma})\left[\frac{\partial \xi^{s}}{\partial \sigma^{r}} \tilde{\pi}_{s}^{\vec{\xi}}-\left(B_{(b)(c)}\left(\alpha_{(e)}\right)^{3} \omega_{r(c)}-\frac{\partial \alpha_{(b)}}{\partial \sigma^{r}}\right) \tilde{\pi}_{(b)}^{\vec{\alpha}}\right](\tau, \vec{\sigma})- \\
& -\int d^{3} \sigma_{1} \hat{D}_{(a)(b) r}^{(\omega)}(\tau, \vec{\sigma}) F_{(b)(c)}^{r}\left(\vec{\sigma}, \vec{\sigma}_{1} ; \tau\right) \tilde{\pi}_{(c)}^{\vec{\alpha}}\left(\tau, \vec{\sigma}_{1}\right)- \\
& -\sum_{u} \int d^{3} \sigma_{1} \hat{D}_{(a)(b) r}^{(\omega)}(\tau, \vec{\sigma}) G_{(b)}^{r u}\left(\vec{\sigma}, \vec{\sigma}_{1} ; \tau\right) \tilde{\pi}_{u}^{\vec{\xi}}\left(\tau, \vec{\sigma}_{1}\right) \approx 0,
\end{aligned}
$$

namely that the momenta ${ }^{3} \tilde{\pi}_{(a)}^{r}(\tau, \vec{\sigma})$ are simultaneously weak solutions of the constraints ${ }^{3} \tilde{M}_{(a)}(\tau, \vec{\sigma}) \approx 0$ and $\hat{\mathcal{H}}_{(a)}(\tau, \vec{\sigma}) \approx 0$ and, then, also of ${ }^{3} \tilde{\Theta}_{r}(\tau, \vec{\sigma}) \approx 0$. Therefore, the Einstein equations ${ }^{4} \bar{G}_{l r}(\tau, \vec{\sigma}) \stackrel{\circ}{=}$ (see after Eq.(A10) of Ref. [四) are satisfied, if the kernels $\mathcal{K}_{(a) u}^{r}$, solutions of the last line of Eqs.(5.17), also satisfy these equations. Since in every gauge (like the 3-orthogonal ones) Eqs.(5.17) will turn out to be linear homogeneous and inhomogeneous partial differential equations (see Eqs.(5.23) for the 3-orthogonal gauges), their solution will depend on arbitrary functions: the solutions of the homogeneous equations associated to the inhomogeneous ones. Eqs.(5.18) are restrictions on these arbitrary functions.

The class of 3-orthogonal gauges is defined by putting $\tilde{\pi}_{(a)}^{\vec{\alpha}}(\tau, \vec{\sigma})=\tilde{\pi}_{r}^{\vec{\xi}}(\tau, \vec{\sigma})=0$, by adding the gauge fixings $\xi^{r}(\tau, \vec{\sigma})-\sigma^{r}=0, \alpha_{(a)}(\tau, \vec{\sigma})=0$ and by parametrizing the reduced cotriad ${ }^{3} \hat{e}_{(a) r}\left(\tau, \vec{\sigma} \mid Q_{u}\right]$ as in Eq. (5.2).

In these gauges Eq.(5.17) becomes

$$
\begin{aligned}
& \left.\frac{\delta^{3} e_{(a) r}(\tau, \vec{\sigma})}{\delta \alpha_{(b)}\left(\tau, \vec{\sigma}_{1}\right)}\right|_{3-0}=\epsilon_{(a)(c)(b)} \delta_{(c) r} Q_{r}(\tau, \vec{\sigma}) \delta^{3}\left(\vec{\sigma}, \vec{\sigma}_{1}\right) \\
& \left.\frac{\delta^{3} e_{(a) r}(\tau, \vec{\sigma})}{\delta \xi^{u}\left(\tau, \vec{\sigma}_{1}\right)}\right|_{3-0}=\delta_{(a) r} \frac{\partial Q_{r}(\tau, \vec{\sigma})}{\partial \sigma^{u}} \delta^{3}\left(\vec{\sigma}, \vec{\sigma}_{1}\right)+\delta_{(a) u} Q_{u}(\tau, \vec{\sigma}) \frac{\partial \delta^{3}\left(\vec{\sigma}, \vec{\sigma}_{1}\right)}{\partial \sigma^{r}} \\
& \left.\frac{\delta^{3} e_{(a) r}(\tau, \vec{\sigma})}{\delta Q_{u}\left(\tau, \vec{\sigma}_{1}\right)}\right|_{3-0}=\delta_{(a) r} \delta_{r u} \delta^{3}\left(\vec{\sigma}, \vec{\sigma}_{1}\right)
\end{aligned}
$$

and we have to solve the following equations for the kernels restricted to these gauges

$$
\begin{aligned}
\delta^{3}\left(\vec{\sigma}, \vec{\sigma}_{1}\right) \delta_{(a)(b)} \delta_{r}^{s} & =\epsilon_{(a)(d)(c)} \delta_{(d) r} Q_{r}(\tau, \vec{\sigma}) F_{(b)(c)}^{s}\left(\vec{\sigma}_{1}, \vec{\sigma} ; \tau\right)+ \\
& +\delta_{(a) r} \mathcal{K}_{(b) r}^{s}\left(\vec{\sigma}_{1}, \vec{\sigma} ; \tau\right)+ \\
& +\delta_{(a) r} \sum_{u} \frac{\partial Q_{r}(\tau, \vec{\sigma})}{\partial \sigma^{u}} G_{(b)}^{s u}\left(\vec{\sigma}_{1}, \vec{\sigma} ; \tau\right)+\sum_{u} \delta_{(a) u} Q_{u}(\tau, \vec{\sigma}) \frac{\partial}{\partial \sigma^{r}} G_{(b)}^{s u}\left(\vec{\sigma}_{1}, \vec{\sigma} ; \tau\right) \\
F_{(a)(b)}^{r}\left(\vec{\sigma}, \vec{\sigma}_{1} ; \tau\right) & =\tilde{F}_{(a)(b) 3-0}^{r}\left(\vec{\sigma}, \vec{\sigma}_{1} ; \tau \mid Q_{v}\right] \\
G_{(a)}^{r u}\left(\vec{\sigma}, \vec{\sigma}_{1} ; \tau\right) & =\tilde{G}_{(a) 3-0}^{r u}\left(\vec{\sigma}, \vec{\sigma}_{1} ; \tau \mid Q_{v}\right] \\
\mathcal{K}_{(a) u}^{r}\left(\vec{\sigma}, \vec{\sigma}_{1} ; \tau\right) & =\tilde{\mathcal{K}}_{(a) u 3-0}^{r}\left(\vec{\sigma}, \vec{\sigma}_{1} ; \tau \mid Q_{v}\right]
\end{aligned}
$$

In Eqs.(5.20) we must separate the cases $a=r$ and $a \neq r$.

To select $a=r$ let us multiply Eqs.(5.20) by $\delta_{(a) r}$ and then let us sum over $(a)$. If we make the substitutions $s \mapsto r, r \mapsto u, b \mapsto a$ in the final expression, we get 


$$
\begin{aligned}
\mathcal{K}_{(a) u}^{r}\left(\vec{\sigma}_{1}, \vec{\sigma} ; \tau\right) & =\delta_{(a)}^{r} \delta_{(a) u} \delta^{3}\left(\vec{\sigma}_{1}, \vec{\sigma}\right)- \\
& -Q_{u}(\tau, \vec{\sigma}) \frac{\partial G_{(a)}^{r u}\left(\vec{\sigma}_{1}, \vec{\sigma} ; \tau\right)}{\partial \sigma^{u}}-\sum_{v} \frac{\partial Q_{u}(\tau, \vec{\sigma})}{\partial \sigma^{v}} G_{(a)}^{r v}\left(\vec{\sigma}_{1}, \vec{\sigma} ; \tau\right)
\end{aligned}
$$

To select $a \neq r$, with the antisymmetry $a \leftrightarrow r$, let us multiply Eqs. (5.20) by $\sum_{(m)} \epsilon_{(a)(m)(d)} \delta_{(m) r}$ and then let us sum over $(a)$. If we make the substitutions $b \mapsto a$, $d \mapsto b$ at the end of the calculations, we get the result that each $F_{(a)(b)}^{s}$ can be expressed in two different ways in terms of the $G$ 's ${ }^{117}$

$$
\begin{aligned}
\left(1-\delta_{(b) r}\right) F_{(a)(b)}^{s}\left(\vec{\sigma}_{1}, \vec{\sigma} ; \tau\right)= & \epsilon_{(a)(r)(b)} \delta_{r}^{s} \frac{\delta^{3}\left(\vec{\sigma}_{1}, \vec{\sigma}\right)}{Q_{r}(\tau, \vec{\sigma})}- \\
& -\sum_{u} \epsilon_{(u)(r)(b)} \frac{Q_{u}(\tau, \vec{\sigma})}{Q_{r}(\tau, \vec{\sigma})} \frac{\partial G_{(a)}^{s u}\left(\vec{\sigma}_{1}, \vec{\sigma} ; \tau\right)}{\partial \sigma^{r}} \\
& \Downarrow \quad \sum_{r}, \\
2 F_{(a)(b)}^{s}\left(\vec{\sigma}_{1}, \vec{\sigma} ; \tau\right)= & \epsilon_{(a)(s)(b)} \frac{\delta^{3}\left(\vec{\sigma}_{1}, \vec{\sigma}\right)}{Q_{s}(\tau, \vec{\sigma})}- \\
- & \sum_{u, r} \epsilon_{(u)(r)(b)} \frac{Q_{u}(\tau, \vec{\sigma})}{Q_{r}(\tau, \vec{\sigma})} \frac{\partial G_{(a)}^{s u}\left(\vec{\sigma}_{1}, \vec{\sigma} ; \tau\right)}{\partial \sigma^{r}}
\end{aligned}
$$

As a consequence the $G_{(a)}^{r u}$ 's are determined by the following linear partial differential equations ${ }^{118}$

$$
\begin{aligned}
& \epsilon_{(a)\left(r_{1}\right)(b)} \delta_{r_{1}}^{s} \frac{\delta^{3}\left(\vec{\sigma}_{1}, \vec{\sigma}\right)}{Q_{r_{1}}(\tau, \vec{\sigma})}-\sum_{u} \epsilon_{(u)\left(r_{1}\right)(b)} \frac{Q_{u}(\tau, \vec{\sigma})}{Q_{r_{1}}(\tau, \vec{\sigma})} \frac{\partial G_{(a)}^{s u}\left(\vec{\sigma}_{1}, \vec{\sigma} ; \tau\right)}{\partial \sigma^{r_{1}}}= \\
= & \epsilon_{(a)\left(r_{2}\right)(b)} \delta_{r_{2}}^{s} \frac{\delta^{3}\left(\vec{\sigma}_{1}, \vec{\sigma}\right)}{Q_{r_{2}}(\tau, \vec{\sigma})}-\sum_{u} \epsilon_{(u)\left(r_{2}\right)(b)} \frac{Q_{u}(\tau, \vec{\sigma})}{Q_{r_{2}}(\tau, \vec{\sigma})} \frac{\partial G_{(a)}^{s u}\left(\vec{\sigma}_{1}, \vec{\sigma} ; \tau\right)}{\partial \sigma^{r_{2}}}, \\
& \Downarrow \\
& \frac{1}{Q_{r_{1}}^{2}(\tau, \vec{\sigma})} \frac{\partial G_{(a)}^{s r_{2}}\left(\vec{\sigma}_{1}, \vec{\sigma} ; \tau\right)}{\partial \sigma^{r_{1}}}+\frac{1}{Q_{r_{2}}^{2}(\tau, \vec{\sigma})} \frac{\partial G_{(a)}^{s r_{1}}\left(\vec{\sigma}_{1}, \vec{\sigma} ; \tau\right)}{\partial \sigma^{r_{2}}}= \\
= & {\left[\frac{\delta_{(a) r_{1}} \delta_{r_{2}}^{s} \delta_{r_{1}}^{s}}{Q_{r_{1}}(\tau, \vec{\sigma}) Q_{r_{2}}^{2}(\tau, \vec{\sigma})}+\frac{\delta_{r_{1}}^{2}(\tau, \vec{\sigma}) Q_{r_{2}}(\tau, \vec{\sigma})}{Q_{r_{1}}^{2}}\right] \delta^{3}\left(\vec{\sigma}_{1}, \vec{\sigma}\right), } \\
& \Downarrow
\end{aligned}
$$

${ }^{117}$ For $b=r$ the following equations give $0=0$.

${ }^{118} r_{1}, r_{2} \neq b, r_{1} \neq r_{2} ; \epsilon_{(a)\left(r_{1}\right)(b)}=-\delta_{(a) r_{2}} \epsilon_{\left(r_{1}\right)\left(r_{2}\right)(b)}, \epsilon_{(a)\left(r_{2}\right)(b)}=\delta_{(a) r_{1}} \epsilon_{\left(r_{1}\right)\left(r_{2}\right)(b)}, \epsilon_{(u)\left(r_{1}\right)(b)}=$
$-\delta_{(u) r_{2}} \epsilon_{\left(r_{1}\right)\left(r_{2}\right)(b)}, \epsilon_{(u)\left(r_{2}\right)(b)}=\delta_{(u) r_{1}} \epsilon_{\left(r_{1}\right)\left(r_{2}\right)(b)} ; Q_{r}=\sqrt{3 \hat{g}_{r r} .}$ 
1) $s=a$ homogeneous equations :

$$
\begin{aligned}
& \frac{1}{Q_{1}^{2}(\tau, \vec{\sigma})} \frac{\partial G_{(a)}^{a 2}\left(\vec{\sigma}_{1}, \vec{\sigma} ; \tau\right)}{\partial \sigma^{1}}+\frac{1}{Q_{2}^{2}(\tau, \vec{\sigma})} \frac{\partial G_{(a)}^{a 1}\left(\vec{\sigma}_{1}, \vec{\sigma} ; \tau\right)}{\partial \sigma^{2}}= \\
& =\frac{1}{Q_{2}^{2}(\tau, \vec{\sigma})} \frac{\partial G_{(a)}^{a 3}\left(\vec{\sigma}_{1}, \vec{\sigma} ; \tau\right)}{\partial \sigma^{2}}+\frac{1}{Q_{3}^{2}(\tau, \vec{\sigma})} \frac{\partial G_{(a)}^{a 2}\left(\vec{\sigma}_{1}, \vec{\sigma} ; \tau\right)}{\partial \sigma^{3}}= \\
& =\frac{1}{Q_{3}^{2}(\tau, \vec{\sigma})} \frac{\partial G_{(a)}^{a 1}\left(\vec{\sigma}_{1}, \vec{\sigma} ; \tau\right)}{\partial \sigma^{3}}+\frac{1}{Q_{1}^{2}(\tau, \vec{\sigma})} \frac{\partial G_{(a)}^{a 3}\left(\vec{\sigma}_{1}, \vec{\sigma} ; \tau\right)}{\partial \sigma^{1}}=0, \quad a=1,2,3 ;
\end{aligned}
$$

2) $s \neq a[s \neq r, r \neq a]$ in-homogeneous equations :

$$
\begin{aligned}
& \frac{1}{Q_{s}^{2}(\tau, \vec{\sigma})} \frac{\partial G_{(a)}^{s r}\left(\vec{\sigma}_{1}, \vec{\sigma} ; \tau\right)}{\partial \sigma^{s}}+\frac{1}{Q_{r}^{2}(\tau, \vec{\sigma})} \frac{\partial G_{(a)}^{s s}\left(\vec{\sigma}_{1}, \vec{\sigma} ; \tau\right)}{\partial \sigma^{r}}= \\
& =\frac{1}{Q_{r}^{2}(\tau, \vec{\sigma})} \frac{\partial G_{(a)}^{s a}\left(\vec{\sigma}_{1}, \vec{\sigma} ; \tau\right)}{\partial \sigma^{r}}+\frac{1}{Q_{a}^{2}(\tau, \vec{\sigma})} \frac{\partial G_{(a)}^{s r}\left(\vec{\sigma}_{1}, \vec{\sigma} ; \tau\right)}{\partial \sigma^{a}}=0 \\
& \frac{1}{Q_{a}^{2}(\tau, \vec{\sigma})} \frac{\partial G_{(a)}^{s s}\left(\vec{\sigma}_{1}, \vec{\sigma} ; \tau\right)}{\partial \sigma^{a}}+\frac{1}{Q_{s}^{2}(\tau, \vec{\sigma})} \frac{\partial G_{(a)}^{s a}\left(\vec{\sigma}_{1}, \vec{\sigma} ; \tau\right)}{\partial \sigma^{s}}=\frac{\delta^{3}\left(\vec{\sigma}_{1} ; \vec{\sigma}\right)}{Q_{a}(\tau, \vec{\sigma}) Q_{s}^{2}(\tau, \vec{\sigma})} .
\end{aligned}
$$

Each set of homogeneous equations, considered as equations for functions of $\vec{\sigma}$ and disregarding the $\tau$-dependence, is of the form $a_{2}(\vec{\sigma}) \partial_{2} u_{(1)}(\vec{\sigma})+a_{1}(\vec{\sigma}) \partial_{1} u_{(2)}(\vec{\sigma})=a_{3}(\vec{\sigma}) \partial_{3} u_{(2)}(\vec{\sigma})+$ $a_{2}(\vec{\sigma}) \partial_{2} u_{(3)}(\vec{\sigma})=a_{1}(\vec{\sigma}) \partial_{1} u_{(3)}(\vec{\sigma})+a_{3}(\vec{\sigma}) \partial_{3} u_{(1)}(\vec{\sigma})=0\left[a_{i}=Q_{i}^{-2}\right]$. This is a system of three linear partial differential equations for the three unknown functions $u_{(i)}(\vec{\sigma})$ of elliptic type, since the determinant of its characteristic matrix 97 is $2 a_{1}(\vec{\sigma}) a_{2}(\vec{\sigma}) a_{3}(\vec{\sigma}) \xi_{1} \xi_{2} \xi_{3} \neq 0$. Moreover it is integrable, since $u_{(r)}(\vec{\sigma})=f_{(r)}\left(\sigma^{r}\right)$ with arbitrary $f_{(r)}$ are solutions of the system. We do not know whether they exhaust all the possible solutions. Therefore, $G_{(a)}^{a r}\left(\vec{\sigma}_{1}, \vec{\sigma} ; \tau\right)=h_{(a)}^{a r}\left(\vec{\sigma}_{1}, \sigma^{r} ; \tau\right)$, with $h_{(a)}^{a r}$ arbitrary functions, are solutions of the homogeneous equations.

As a consequence, if $\bar{G}_{(a)}^{r u}\left(\vec{\sigma}_{1}, \vec{\sigma} ; \tau\right), r \neq a$, is a particular solution of each set of inhomogeneous equations (5.23), then the general solution is $G_{(a)}^{r u}\left(\vec{\sigma}_{1}, \vec{\sigma} ; \tau\right)=$ $\bar{G}_{(a)}^{r u}\left(\vec{\sigma}_{1}, \vec{\sigma} ; \tau\right)+g_{(a)}^{r u}\left(\vec{\sigma}_{1}, \vec{\sigma} ; \tau\right), \quad r \neq a$, with the $g_{(a)}^{r u}$ 's arbitrary homogeneous solutions (again with $g_{(a)}^{r u}\left(\vec{\sigma}_{1}, \sigma^{r} ; \tau\right)$, if this is the most general solution of the associated homogeneous equations). A way to find a particular solution may be to define $G_{f(a)}^{r u}(\vec{\sigma} ; \tau)=\int d^{3} \sigma_{1} f\left(\vec{\sigma}_{1}\right) G_{(a)}^{r u}\left(\vec{\sigma}_{1}, \vec{\sigma} ; \tau\right)$ : then, disregarding the $\tau$-dependence, we get the system of elliptic linear partial differential equations $\left[a_{s}=Q_{s}^{-2}, s \neq r, a\right.$, $r \neq a] \quad a_{s}(\vec{\sigma}) \partial_{s} G_{f(a)}^{s r}(\vec{\sigma})+a_{r}(\vec{\sigma}) \partial_{r} G_{f(a)}^{s s}(\vec{\sigma})=a_{r}(\vec{\sigma}) \partial_{r} G_{f(a)}^{s a}(\vec{\sigma})+a_{a}(\vec{\sigma}) \partial_{a} G_{f(a)}^{s r}(\vec{\sigma})=0$, $a_{a}(\vec{\sigma}) \partial_{a} G_{f(a)}^{s s}(\vec{\sigma})+a_{s}(\vec{\sigma}) \partial_{s} G_{f(a)}^{s a}(\vec{\sigma})=f(\vec{\sigma}) / \sqrt{a_{a}(\vec{\sigma})} a_{s}(\vec{\sigma})$. Each particular solution of this system which is a functional linear in $f(\vec{\sigma})$ will allow to find a particular solution $\bar{G}_{(a)}^{r u}$.

Therefore the kernels $G_{(a)}^{r u}\left(\vec{\sigma}_{1}, \vec{\sigma} ; \tau\right)$ solutions of Eqs. (5.23) can be written in the following form

$$
G_{(a)}^{r u}\left(\vec{\sigma}_{1}, \vec{\sigma} ; \tau\right)=\delta_{(a)}^{r} h_{(a)}^{a u}\left(\vec{\sigma}_{1}, \vec{\sigma} ; \tau\right)+\left(1-\delta_{(a)}^{r}\right)\left[\bar{G}_{(a)}^{r u}\left(\vec{\sigma}_{1}, \vec{\sigma} ; \tau\right)+g_{(a)}^{r u}\left(\vec{\sigma}_{1}, \vec{\sigma} ; \tau\right)\right],
$$

with arbitrary $h_{(a)}^{a u}$ 's and $g_{(a)}^{r u}$ 's. Then, $\mathrm{Eq}(5.21)$ gives the following expression for the kernels $\mathcal{K}_{(a) u}^{r}{ }^{\text {'S }}$ 


$$
\begin{aligned}
& \mathcal{K}_{(a) u}^{r}\left(\vec{\sigma}_{1}, \vec{\sigma}\right)=\delta_{(a)}^{r} \delta_{(a) u} \delta^{3}\left(\vec{\sigma}_{1}, \vec{\sigma}\right)- \\
& -Q_{u}(\tau, \vec{\sigma})\left(\delta_{(a)}^{r} \frac{\partial h_{(a)}^{a u}\left(\vec{\sigma}_{1}, \vec{\sigma} ; \tau\right)}{\partial \sigma^{u}}+\left(1-\delta_{(a)}^{r}\right)\left[\frac{\partial \bar{G}_{(a)}^{r u}\left(\vec{\sigma}_{1}, \vec{\sigma} ; \tau\right)}{\partial \sigma^{u}}+\frac{\partial g_{(a)}^{r u}\left(\vec{\sigma}_{1}, \vec{\sigma} ; \tau\right)}{\partial \sigma^{u}}\right]\right)- \\
& -\sum_{v} \frac{\partial Q_{u}(\tau, \vec{\sigma})}{\partial \sigma^{v}}\left(\delta_{(a)}^{r} h_{(a)}^{a v}\left(\vec{\sigma}_{1}, \vec{\sigma} ; \tau\right)+\left(1-\delta_{(a)}^{r}\right)\left[\bar{G}_{(a)}^{r v}\left(\vec{\sigma}_{1}, \vec{\sigma} ; \tau\right)+g_{(a)}^{r v}\left(\vec{\sigma}_{1}, \vec{\sigma} ; \tau\right)\right]\right) .
\end{aligned}
$$

The solutions of Eqs.(5.23) for the $G_{(a)}^{r u}$ 's are restricted by the requirement that the $\mathcal{K}_{(a) u}^{r}$ 's of Eqs.(5.21) satisfy Eqs.(5.18), which in the 3-orthogonal gauges become ${ }^{119}$

$$
\begin{aligned}
& \sum_{r} Q_{r}(\tau, \vec{\sigma})\left[\delta_{(a) r} \mathcal{K}_{(b) u}^{r}-\delta_{(b) r} \mathcal{K}_{(a) u}^{r}\right]\left(\vec{\sigma}, \vec{\sigma}_{1} ; \tau\right)= \\
& =Q_{u}\left(\tau, \vec{\sigma}_{1}\right)\left[Q_{a}(\tau, \vec{\sigma}) \frac{\partial G_{(b)}^{a u}\left(\vec{\sigma}, \vec{\sigma}_{1} ; \tau\right)}{\partial \sigma_{1}^{u}}-Q_{b}(\tau, \vec{\sigma}) \frac{\partial G_{(a)}^{b u}\left(\vec{\sigma}, \vec{\sigma}_{1} ; \tau\right)}{\partial \sigma_{1}^{u}}\right]+ \\
& +\sum_{v} \frac{\partial Q_{u}\left(\tau, \vec{\sigma}_{1}\right)}{\partial \sigma_{1}^{v}}\left[Q_{a}(\tau, \vec{\sigma}) G_{(b)}^{a v}\left(\vec{\sigma}, \vec{\sigma}_{1} ; \tau\right)-Q_{b}(\tau, \vec{\sigma}) G_{(a)}^{b v}\left(\vec{\sigma}, \vec{\sigma}_{1} ; \tau\right)\right]= \\
& =0, \quad a \neq b \\
& \hat{D}_{(a)(b) r}^{(\hat{\omega})}(\tau, \vec{\sigma}) \mathcal{K}_{(b) u}^{r}\left(\vec{\sigma}, \vec{\sigma}_{1} ; \tau\right)= \\
& =\left(\delta_{(a)(b)} \partial_{r}+\epsilon_{(a)(b)(c)} \hat{\omega}_{r(c)}(\tau, \vec{\sigma})\right) \mathcal{K}_{(b) u}^{r}\left(\vec{\sigma}, \vec{\sigma}_{1} ; \tau\right)= \\
& =\left(\delta_{(a)(b)} \partial_{r}+\sum_{u}\left(\delta_{(a) r} \delta_{(b) u}-\delta_{(a) u} \delta_{(b) r}\right) \frac{\partial_{u} Q_{r}(\tau, \vec{\sigma})}{Q_{u}(\tau, \vec{\sigma})}\right)\left[\delta_{(b)}^{r} \delta_{(b) u} \delta^{3}\left(\vec{\sigma}, \vec{\sigma}_{1}\right)-\right. \\
& \left.-Q_{u}\left(\tau, \vec{\sigma}_{1}\right) \frac{\partial G_{(b)}^{r u}\left(\vec{\sigma}, \vec{\sigma}_{1} ; \tau\right)}{\partial \sigma_{1}^{u}}-\sum_{v} \frac{\partial Q_{u}\left(\tau, \vec{\sigma}_{1}\right)}{\partial \sigma_{1}^{v}} G_{(b)}^{r v}\left(\vec{\sigma}, \vec{\sigma}_{1} ; \tau\right)\right]=0 \\
& { }^{3} \hat{\omega}_{r(a)}(\tau, \vec{\sigma})=\sum_{u} \epsilon_{(a)(m)(n)} \delta_{(m) r} \delta_{(n) u} \frac{\partial_{u} Q_{r}(\tau, \vec{\sigma})}{Q_{u}(\tau, \vec{\sigma})}
\end{aligned}
$$

The first set of Eqs. (5.26) becomes the following set of three linear partial differential equations to get the $g_{(a)}^{r u}$ 's with $a \neq b$ in terms of the $\bar{G}_{(a)}^{r u}$ 's

$$
\begin{aligned}
& Q_{a}\left(\tau, \vec{\sigma}_{1}\right) \frac{\partial g_{(b)}^{a u}\left(\vec{\sigma}_{1}, \vec{\sigma} ; \tau\right)}{\partial \sigma^{u}}-Q_{b}\left(\tau, \vec{\sigma}_{1}\right) \frac{\partial g_{(a)}^{b u}\left(\vec{\sigma}_{1}, \vec{\sigma} ; \tau\right)}{\partial \sigma^{u}}+ \\
&+ \sum_{v} \frac{\partial \ln Q_{u}(\tau, \vec{\sigma})}{\partial \sigma^{v}}\left[Q_{a}\left(\tau, \vec{\sigma}_{1}\right) g_{(b)}^{a v}\left(\vec{\sigma}_{1}, \vec{\sigma} ; \tau\right)-Q_{b}\left(\tau, \vec{\sigma}_{1}\right) g_{(a)}^{b v}\left(\vec{\sigma}_{1}, \vec{\sigma} ; \tau\right)\right]= \\
&=-\left[Q_{a}\left(\tau, \vec{\sigma}_{1}\right) \frac{\partial \bar{G}_{(b)}^{a u}\left(\vec{\sigma}_{1}, \vec{\sigma} ; \tau\right)}{\partial \sigma^{u}}-Q_{b}\left(\tau, \vec{\sigma}_{1}\right) \frac{\partial \bar{G}_{(a)}^{b u}\left(\vec{\sigma}_{1}, \vec{\sigma} ; \tau\right)}{\partial \sigma^{u}}+\right. \\
&+\left.\sum_{v} \frac{\partial \ln Q_{u}(\tau, \vec{\sigma})}{\partial \sigma^{v}}\left[Q_{a}\left(\tau, \vec{\sigma}_{1}\right) \bar{G}_{(b)}^{a v}\left(\vec{\sigma}_{1}, \vec{\sigma} ; \tau\right)-Q_{b}\left(\tau, \vec{\sigma}_{1}\right) \bar{G}_{(a)}^{b v}\left(\vec{\sigma}_{1}, \vec{\sigma} ; \tau\right)\right]\right]= \\
& \stackrel{d e f}{=} m_{a b}^{u}\left(\vec{\sigma}_{1}, \vec{\sigma} ; \tau\right),
\end{aligned}
$$

${ }^{119}$ In the last line we give ${ }^{3} \hat{\omega}_{r(a)}(\tau, \vec{\sigma})$ in the 3 -orthogonal gauges, see Eq.(6.14) of next Section. 
or

$$
\frac{\partial f_{a b}^{u}\left(\vec{\sigma}_{1}, \vec{\sigma} ; \tau\right)}{\partial \sigma^{u}}+\sum_{v} \frac{\partial \ln Q_{u}(\tau, \vec{\sigma})}{\partial \sigma^{v}} f_{a b}^{v}\left(\vec{\sigma}_{1}, \vec{\sigma} ; \tau\right)=m_{a b}^{u}\left(\vec{\sigma}_{1}, \vec{\sigma} ; \tau\right)
$$

with

$$
f_{a b}^{u}\left(\vec{\sigma}_{1}, \vec{\sigma} ; \tau\right)=Q_{a}\left(\tau, \vec{\sigma}_{1}\right) g_{(b)}^{a u}\left(\vec{\sigma}_{1}, \vec{\sigma} ; \tau\right)-Q_{b}\left(\tau, \vec{\sigma}_{1}\right) g_{(a)}^{b u}\left(\vec{\sigma}_{1}, \vec{\sigma} ; \tau\right)
$$

For each pair $a \neq b$, this is a system of three elliptic linear partial differential equations for the $f_{a b}^{u}$. Each choice of the $g_{(b)}^{a u}$ 's, $a \neq b$, which gives a solution of this system, implies that the associated kernels $\mathcal{K}_{(a) u}^{r}$ 's satisfy the rotation constraints.

Having found a solution for the $g_{(b)}^{a u}$ 's, $a \neq b$, the second set of Eqs. (5.26) becomes the following set of equations for the $h_{(a)}^{a u}$ 's in terms of the $\bar{G}_{(a)}^{r u}$ 's and $g_{(a)}^{r u}$ 's

$$
\begin{aligned}
& \sum_{r,(b)} \hat{D}_{(a)(b) r}^{(\omega)}\left(\tau, \vec{\sigma}_{1}\right) \delta_{(b)}^{r}\left[q_{u}(\tau, \vec{\sigma}) \frac{\partial h_{(b)}^{b u}\left(\vec{\sigma}_{1}, \vec{\sigma} ; \tau\right)}{\partial \sigma^{u}}-\sum_{v} \frac{\partial Q_{u}(\tau, \vec{\sigma})}{\partial \sigma^{v}} h_{(b)}^{b v}\left(\vec{\sigma}_{1}, \vec{\sigma} ; \tau\right)\right]= \\
& =\sum_{r,(b)} \hat{D}_{(a)(b) r}^{(\omega)}\left(\tau, \vec{\sigma}_{1}\right)\left[\delta_{(b)}^{r} \delta_{(b) u} \delta^{3}\left(\vec{\sigma}_{1}, \vec{\sigma}\right)-\right. \\
& -\left(1-\delta_{(b)}^{r}\right)\left(q_{u}(\tau, \vec{\sigma})\left[\frac{\partial \bar{G}_{(b)}^{r u}\left(\vec{\sigma}_{1}, \vec{\sigma} ; \tau\right)}{\partial \sigma^{u}}+\frac{\partial g_{(b)}^{r u}\left(\vec{\sigma}_{1}, \vec{\sigma} ; \tau\right)}{\partial \sigma^{u}}\right]+\right. \\
& \left.\left.+\sum_{v} \frac{\partial Q_{u}(\tau, \vec{\sigma})}{\partial \sigma^{v}}\left[\bar{G}_{(b)}^{r v}\left(\vec{\sigma}_{1}, \vec{\sigma} ; \tau\right)+g_{(b)}^{r v}\left(\vec{\sigma}_{1}, \vec{\sigma} ; \tau\right)\right]\right)\right] .
\end{aligned}
$$

By using the Green function of Eqs.(4.27), (4.30), we get $\left(f_{(T)}^{s u}\right.$ are solutions of the homogeneous equation)

$$
\begin{aligned}
& \frac{\partial h_{(s)}^{s u}\left(\vec{\sigma}_{1}, \vec{\sigma} ; \tau\right)}{\partial \sigma^{u}}+\sum_{v} \frac{\partial \ln Q_{u}(\tau, \vec{\sigma})}{\partial \sigma^{v}} h_{(s)}^{s v}\left(\vec{\sigma}_{1}, \vec{\sigma} ; \tau\right)= \\
& =f_{(T)}^{s u}\left(\vec{\sigma}_{1}, \vec{\sigma} ; \tau\right)- \\
& -\int d^{3} \sigma_{2} \sum_{(a)} \zeta_{(s)(a)}^{(\omega) s}\left(\vec{\sigma}_{1}, \vec{\sigma}_{2} ; \tau\right) \sum_{r,(b)} \hat{D}_{(a)(b) r}^{(\omega)}\left(\tau, \vec{\sigma}_{2}\right)\left[\frac{\delta_{(b)}^{r} \delta_{(b) u}}{Q_{u}(\tau, \vec{\sigma})} \delta^{3}\left(\vec{\sigma}_{2}, \vec{\sigma}\right)-\right. \\
& -\left(1-\delta_{(b)}^{r}\right)\left(Q_{u}(\tau, \vec{\sigma})\left[\frac{\partial \bar{G}_{(b)}^{r u}\left(\vec{\sigma}_{2}, \vec{\sigma} ; \tau\right)}{\partial \sigma^{u}}+\frac{\partial g_{(b)}^{r u}\left(\vec{\sigma}_{2}, \vec{\sigma} ; \tau\right)}{\partial \sigma^{u}}\right]+\right. \\
& \left.\left.+\sum_{v} \frac{\partial \ln Q_{u}(\tau, \vec{\sigma})}{\partial \sigma^{v}}\left[\bar{G}_{(b)}^{r v}\left(\vec{\sigma}_{2}, \vec{\sigma} ; \tau\right)+g_{(b)}^{r v}\left(\vec{\sigma}_{2}, \vec{\sigma} ; \tau\right)\right]\right)\right] .
\end{aligned}
$$

Again this is a system of elliptic linear partial differential equations for the $h_{(a)}^{a u}$ 's with fixed $a$.

Finally, we have to find the conditions imposed on the kernels by the vanishing of the Poisson brackets of the old momenta thought as functions of the new variables through 
Eq. (5.15): $\left\{{ }^{3} \tilde{\pi}_{(a)}^{r}(\tau, \vec{\sigma}),{ }^{3} \tilde{\pi}_{(c)}^{s}\left(\tau, \vec{\sigma}^{\prime}\right)\right\}=0$. It turns out that we get the following quasi-linear partial differential equations for the remaining arbitrariness in the homogeneous solutions [the kernels $F_{(a)(b)}^{r}$ 's are given in Eqs.(5.22)]:

$$
\begin{aligned}
& \sum_{v}\left(\mathcal{K}_{(c) v}^{s}\left(\vec{\sigma}^{\prime}, \vec{\sigma}_{2} ; \tau\right) \frac{\delta \mathcal{K}_{(a) u}^{r}\left(\vec{\sigma}, \vec{\sigma}_{1} ; \tau\right)}{\delta Q_{v}\left(\tau, \vec{\sigma}_{2}\right)}-\mathcal{K}_{(a) v}^{r}\left(\vec{\sigma}, \vec{\sigma}_{2} ; \tau\right) \frac{\delta \mathcal{K}_{(c) u}^{s}\left(\vec{\sigma}^{\prime}, \vec{\sigma}_{1} ; \tau\right)}{\delta Q_{v}\left(\tau, \vec{\sigma}_{2}\right)}\right)+ \\
& +\left(F_{(c)(d)}^{s}\left(\vec{\sigma}^{\prime}, \vec{\sigma}_{2} ; \tau\right) \frac{\delta \mathcal{K}_{(a) u}^{r}\left(\vec{\sigma}, \vec{\sigma}_{1}\right)}{\delta \alpha_{(d)}\left(\tau, \vec{\sigma}_{2}\right)}-F_{(a)(d)}^{r}\left(\vec{\sigma}, \vec{\sigma}_{2} ; \tau\right) \frac{\delta \mathcal{K}_{(c) u}^{s}\left(\vec{\sigma}^{\prime}, \vec{\sigma}_{1}\right)}{\delta \alpha_{(d)}\left(\tau, \vec{\sigma}_{2}\right)}\right)+ \\
& +\sum_{v}\left(G_{(c)}^{s v}\left(\vec{\sigma}^{\prime}, \vec{\sigma}_{2} ; \tau\right) \frac{\delta \mathcal{K}_{(a) u}^{r}\left(\vec{\sigma}, \vec{\sigma}_{1} ; \tau\right)}{\delta \xi^{v}\left(\tau, \vec{\sigma}_{2}\right)}-G_{(a)}^{r v}\left(\vec{\sigma}, \vec{\sigma}_{2} ; \tau\right) \frac{\delta \mathcal{K}_{(c) u}^{s}\left(\vec{\sigma}^{\prime}, \vec{\sigma}_{1} ; \tau\right)}{\delta \xi^{v}\left(\tau, \vec{\sigma}_{2}\right)}\right)=0, \\
& \sum_{v}\left(\mathcal{K}_{(c) v}^{s}\left(\vec{\sigma}^{\prime}, \vec{\sigma}_{2} ; \tau\right) \frac{\delta F_{(a)(b)}^{r}\left(\vec{\sigma}, \vec{\sigma}_{1} ; \tau\right)}{\delta Q_{v}\left(\tau, \vec{\sigma}_{2}\right)}-\mathcal{K}_{(a) v}^{r}\left(\vec{\sigma}, \vec{\sigma}_{2} ; \tau\right) \frac{\delta F_{(c)(b)}^{s}\left(\vec{\sigma}^{\prime}, \vec{\sigma}_{1} ; \tau\right)}{\delta Q_{v}\left(\tau, \vec{\sigma}_{2}\right)}\right)+ \\
& +\left(F_{(c)(d)}^{s}\left(\vec{\sigma}^{\prime}, \vec{\sigma}_{2} ; \tau\right) \frac{\delta F_{(a)(b)}^{r}\left(\vec{\sigma}, \vec{\sigma}_{1} ; \tau\right)}{\delta \alpha_{(d)}\left(\tau, \vec{\sigma}_{2}\right)}-F_{(a)(d)}^{r}\left(\vec{\sigma}, \vec{\sigma}_{2} ; \tau\right) \frac{\delta F_{(c)(b)}^{s}\left(\vec{\sigma}^{\prime}, \vec{\sigma}_{1} ; \tau\right)}{\delta \alpha_{(d)}\left(\tau, \vec{\sigma}_{2}\right)}\right)+ \\
& +\sum_{v}\left(G_{(c)}^{s v}\left(\vec{\sigma}^{\prime}, \vec{\sigma}_{2} ; \tau\right) \frac{\delta F_{(a)(b)}^{r}\left(\vec{\sigma}, \vec{\sigma}_{1} ; \tau\right)}{\delta \xi^{v}\left(\tau, \vec{\sigma}_{2}\right)}-G_{(a)}^{r v}\left(\vec{\sigma}, \vec{\sigma}_{2} ; \tau\right) \frac{\delta F_{(c)(b)}^{s}\left(\vec{\sigma}^{\prime}, \vec{\sigma}_{1} ; \tau\right)}{\delta \xi^{v}\left(\tau, \vec{\sigma}_{2}\right)}\right)=0 \\
& \sum_{v}\left(\mathcal{K}_{(c) v}^{s}\left(\vec{\sigma}^{\prime}, \vec{\sigma}_{2} ; \tau\right) \frac{\delta G_{(a)}^{r u}\left(\vec{\sigma}, \vec{\sigma}_{1} ; \tau\right)}{\delta Q_{v}\left(\tau, \vec{\sigma}_{2}\right)}-\mathcal{K}_{(a) v}^{r}\left(\vec{\sigma}, \vec{\sigma}_{2} ; \tau\right) \frac{\delta G_{(c)}^{s u}\left(\vec{\sigma}^{\prime}, \vec{\sigma}_{1} ; \tau\right)}{\delta Q_{v}\left(\tau, \vec{\sigma}_{2}\right)}\right)+ \\
& +\left(F_{(c)(d)}^{s}\left(\vec{\sigma}^{\prime}, \vec{\sigma}_{2} ; \tau\right) \frac{\delta G_{(a)}^{r u}\left(\vec{\sigma}, \vec{\sigma}_{1} ; \tau\right)}{\delta \alpha_{(d)}\left(\tau, \vec{\sigma}_{2}\right)}-F_{(a)(d)}^{r}\left(\vec{\sigma}, \vec{\sigma}_{2} ; \tau\right) \frac{\delta G_{(c)}^{s u}\left(\vec{\sigma}^{\prime}, \vec{\sigma}_{1} ; \tau\right)}{\delta \alpha_{(d)}\left(\tau, \vec{\sigma}_{2}\right)}\right)+ \\
& +\sum_{v}\left(G_{(c)}^{s v}\left(\vec{\sigma}^{\prime}, \vec{\sigma}_{2} ; \tau\right) \frac{\delta G_{(a)}^{r u}\left(\vec{\sigma}, \vec{\sigma}_{1} ; \tau\right)}{\delta \xi^{v}\left(\tau, \vec{\sigma}_{2}\right)}-G_{(a)}^{r v}\left(\vec{\sigma}, \vec{\sigma}_{2} ; \tau\right) \frac{\delta G_{(c)}^{s u}\left(\vec{\sigma}^{\prime}, \vec{\sigma}_{1} ; \tau\right)}{\delta \xi^{v}\left(\tau, \vec{\sigma}_{2}\right)}\right)=0,
\end{aligned}
$$

$\Downarrow$ in 3-orthogonal gauges

$$
\begin{aligned}
& \sum_{v}\left(\mathcal{K}_{(c) v}^{s}\left(\vec{\sigma}^{\prime}, \vec{\sigma}_{2} ; \tau\right) \frac{\delta \mathcal{K}_{(a) u}^{r}\left(\vec{\sigma}, \vec{\sigma}_{1} ; \tau\right)}{\delta Q_{v}\left(\tau, \vec{\sigma}_{2}\right)}-\mathcal{K}_{(a) v}^{r}\left(\vec{\sigma}, \vec{\sigma}_{2} ; \tau\right) \frac{\delta \mathcal{K}_{(c) u}^{s}\left(\vec{\sigma}^{\prime}, \vec{\sigma}_{1} ; \tau\right)}{\delta Q_{v}\left(\tau, \vec{\sigma}_{2}\right)}\right)=0 \\
& \sum_{v}\left(\mathcal{K}_{(c) v}^{s}\left(\vec{\sigma}^{\prime}, \vec{\sigma}_{2} ; \tau\right) \frac{\delta F_{(a)(b)}^{r}\left(\vec{\sigma}, \vec{\sigma}_{1} ; \tau\right)}{\delta Q_{v}\left(\tau, \vec{\sigma}_{2}\right)}-\mathcal{K}_{(a) v}^{r}\left(\vec{\sigma}, \vec{\sigma}_{2} ; \tau\right) \frac{\delta F_{(c)(b)}^{s}\left(\vec{\sigma}^{\prime}, \vec{\sigma}_{1} ; \tau\right)}{\delta Q_{v}\left(\tau, \vec{\sigma}_{2}\right)}\right)=0 \\
& \sum_{v}\left(\mathcal{K}_{(c) v}^{s}\left(\vec{\sigma}^{\prime}, \vec{\sigma}_{2} ; \tau\right) \frac{\delta G_{(a)}^{r u}\left(\vec{\sigma}, \vec{\sigma}_{1} ; \tau\right)}{\delta Q_{v}\left(\tau, \vec{\sigma}_{2}\right)}-\mathcal{K}_{(a) v}^{r}\left(\vec{\sigma}, \vec{\sigma}_{2} ; \tau\right) \frac{\delta G_{(c)}^{s u}\left(\vec{\sigma}^{\prime}, \vec{\sigma}_{1} ; \tau\right)}{\delta Q_{v}\left(\tau, \vec{\sigma}_{2}\right)}\right)=0 .
\end{aligned}
$$

After having found the general solutions for the kernels $G_{(a)}^{r u}$ 's, $\mathcal{K}_{(a) u}^{r}$ 's and $F_{(a)(b)}^{r}$ 's, every remaining arbitrariness will be fixed by the boundary conditions at spatial infinity, which must be the ones given in Eqs.(2.25) for the momenta ${ }^{3} \tilde{\pi}_{(a)}^{r}(\tau, \vec{\sigma})$, as implied by Eqs.(5.15). The final solutions are equivalent not only to the solution of the rotation and diffeomorphisms constraints, but also to their Abelianization in the 3-orthogonal gauges with $\alpha_{(a)}(\tau, \vec{\sigma})=0$. 
Even if we do not know explicitly the kernels $\mathcal{K}_{(a) u}^{r}{ }^{120}$, we are able to get the following decomposition of the original variables in the 3 -orthogonal gauges

$$
\begin{aligned}
\left.{ }^{3} e_{(a) r}(\tau, \vec{\sigma})\right|_{3-0} & =\delta_{(a) r} Q_{r}(\tau, \vec{\sigma}), \\
\left.{ }^{3} \tilde{\pi}_{(a)}^{r}(\tau, \vec{\sigma})\right|_{3-0} & =\sum_{u} \int d^{3} \sigma_{1} \tilde{\mathcal{K}}_{(a) u}^{r}\left(\vec{\sigma}, \vec{\sigma}_{1} ; \tau \mid Q_{v}\right] \tilde{\Pi}^{u}\left(\tau, \vec{\sigma}_{1}\right)= \\
& =\sum_{u} \int d^{3} \sigma_{1} \mathcal{K}_{(a) u}^{r}\left(\vec{\sigma}, \vec{\sigma}_{1} ; \tau\right) \tilde{\Pi}^{u}\left(\tau, \vec{\sigma}_{1}\right) .
\end{aligned}
$$

From Eqs.(2.12) we get $\left[{ }^{3} \hat{e}=\sqrt{\hat{\gamma}}=Q_{1} Q_{2} Q_{3}\right]$

$$
\begin{aligned}
{ }^{3} \hat{K}_{r s} & =\left.\sum_{u} \frac{\epsilon 4 \pi G Q_{r} Q_{s} Q_{u}}{c^{3} Q_{1} Q_{2} Q_{3}}{ }^{3} G_{o(a)(b)(c)(d)} \delta_{(\bar{a}) r} \delta_{(\bar{b}) s} \delta_{(\bar{c}) u}{ }^{3} \tilde{\pi}_{(\bar{d})}^{u}\right|_{3-O}, \\
{ }^{3} \hat{K} & =-\frac{\epsilon 8 \pi G}{c^{3} Q_{1} Q_{2} Q_{3}}{ }^{3} \hat{\tilde{\Pi}}=-\left.\frac{\epsilon 4 \pi G}{c^{3} Q_{1} Q_{2} Q_{3}} \sum_{r} \delta_{(a) r} Q_{r}{ }^{3} \tilde{\pi}_{(a)}^{r}\right|_{3-O}, \\
{ }^{3} \hat{\tilde{\Pi}}^{r s} & =\frac{\epsilon c^{3}}{16 \pi G} Q_{1} Q_{2} Q_{3}\left({ }^{3} \hat{K}^{r s}-Q_{r}^{2} \delta^{r s} 3 \hat{K}\right)=\frac{1}{4}\left[\frac{\left.\delta_{(a)}^{r}{ }^{3} \tilde{\pi}_{(a)}^{s}\right|_{3-O}}{Q_{r}}+\frac{\left.\delta_{(a)}^{s}{ }^{3} \tilde{\pi}_{(a)}^{r}\right|_{3-O}}{Q_{s}}\right] .
\end{aligned}
$$

The first line allows to get $\partial_{\tau}{ }^{3} \hat{g}_{r s}(\tau, \vec{\sigma})$ in terms of lapse, shift functions and the final variables by using Eq.(2.12).

With the canonical transformation (5.11) the functions ${ }^{3} \Gamma_{r s}^{u},{ }^{3} R_{r s u v},{ }^{3} \omega_{r(a)},{ }^{3} \Omega_{r s(a)}$ and, by using Eqs.(2.12), ${ }^{3} K_{r s}$ (and also the metric ADM momentum ${ }^{3} \tilde{\Pi}^{r s}$ of Eq.(2.12)) may now be expressed in terms of $\alpha_{(a)}, \tilde{\pi}_{(a)}^{\vec{\alpha}}, \xi^{r}, \tilde{\pi}_{r}^{\vec{\xi}}, Q_{r}, \tilde{\Pi}^{r}$.

Instead the inverse canonical transformation cannot be computed explicitly till when one does not understand how to solve Eqs.(4.51). If the solution $\xi^{r}\left[{ }^{3} e_{(c) s}\right]$ of this equation would be known, then with $\alpha_{(a)}\left[{ }^{3} e_{(c) s}\right]$, given by Eq.44.33), we implicitly would get from Eqs.(5.3), (5.2) with the 3-orthogonal parametrization of the reduced cotriad [2]

$$
Q_{r}(\tau, \vec{\sigma})=\left(\left({ }^{3} R^{-1}\right)_{(b)(a)}\left(\alpha_{(e)}\right)\left[\left(\frac{\partial \xi}{\partial \sigma}\right)^{-1}\right]_{r}^{s}{ }^{3} e_{(a) s}\right)\left(\tau, \vec{\sigma}\left(\tau, \xi^{u}\right)\right)
$$

Then Eqs.(5.15) could be inverted to get $\tilde{\Pi}^{u}\left[{ }^{3} e_{(a) r},{ }^{3} \tilde{\pi}_{(a)}^{r}\right]$.

Let us remark that if instead of the parametrization (5.2) corresponding to the choice of 3-orthogonal coordinates for $\Sigma_{\tau}^{(W S W)}$ we had made a different choice of 3-coordinates (for instance the 3-normal ones), we would have obtained a different quasi-Shanmugadhasan canonical transformation

${ }^{120}$ The solution of Eqs. 5.23 ), (5.26) is equivalent to solve the three Einstein equations corresponding to the supermomentum constraints of metric gravity; the separate solution of the rotation and diffeomorphism constraints of tetrad gravity is not enough to find their simultaneous Abelianization: this implies Eqs.(5.26).

${ }^{121} \vec{\sigma}\left(\tau, \xi^{u}\right)$ is the inverse of $\xi^{r}=\xi^{r}(\tau, \vec{\sigma})$. 


$$
\begin{array}{|l|l|l|l|}
\hline{ }^{3} e_{(a) r}^{\prime} \\
\hline{ }^{3} \tilde{\pi}_{(a)}^{r} & \xi^{\prime} r & Q_{r}^{\prime} \\
\hline \tilde{\pi}_{(a)}^{\vec{\alpha}} & \tilde{\pi}_{r}^{\vec{\xi}} & \tilde{\Pi}^{\prime} r \\
\hline
\end{array}
$$

with ${ }^{3} \tilde{\pi}_{(a)}^{r}$ given by Eq.(5.15) with different kernels.

Since there is no canonical identity for pseudo-diffeomorphisms and since the rotations do not have zero Poisson brackets with them, in general the point canonical transformation connecting Eqs.(5.11) and (5.34) will require a redefinition of both the parameters of pseudodiffeomorphisms and of the angles:

$$
\begin{aligned}
\alpha_{(a)}^{\prime} & =\alpha_{(a)}^{\prime}\left[\alpha_{(b)}, \xi^{s}, Q^{s}\right], \\
\xi^{\prime r} & =\xi^{\prime}\left[\alpha_{(b)}, \xi^{s}, Q^{s}\right], \\
Q^{\prime r} & =Q^{\prime r}\left[\alpha_{(b)}, \xi^{s}, Q^{s}\right] .
\end{aligned}
$$

From Eqs.(2.55) and (5.2) the asymptotic behaviour at spatial infinity of the canonical variables $Q_{r}, \tilde{\Pi}^{r}$ parametrizing the phase space over superspace (the space of 3-geometries) is

$$
\begin{aligned}
& Q_{r}(\tau, \vec{\sigma}) \rightarrow_{r \rightarrow \infty} 1+\frac{M}{2 r}+O\left(r^{-3 / 2}\right), \\
& \tilde{\Pi}^{r}(\tau, \vec{\sigma}) \rightarrow_{r \rightarrow \infty} O\left(r^{-2}\right)
\end{aligned}
$$

since $\alpha_{(a)}(\tau, \vec{\sigma}) \rightarrow_{r \rightarrow \infty} O\left(r^{-(1+\epsilon)}\right)$.

The only non-Abelianized constraint is now the superhamiltonian one given in Eq.(2.11). Its expression in the 3-orthogonal gauges is

$$
\begin{aligned}
\hat{\mathcal{H}}(\tau, \vec{\sigma})= & \epsilon\left[\frac{c^{3}}{16 \pi G}{ }^{3} e^{3} R-\frac{2 \pi G}{c^{3}{ }^{3} e}{ }^{3} G_{o(a)(b)(c)(d)}{ }^{3} e_{(a) r}{ }^{3} \tilde{\pi}_{(b)}^{r}{ }^{3} e_{(c) s}{ }^{3} \tilde{\pi}_{(d)}^{s}\right](\tau, \vec{\sigma})= \\
= & \frac{\epsilon c^{3}}{16 \pi G}\left[Q_{1} Q_{2} Q_{3}{ }^{3} R\right](\tau, \vec{\sigma})-\frac{\epsilon 2 \pi G}{c^{3}\left[Q_{1} Q_{2} Q_{3}\right](\tau, \vec{\sigma})} \sum_{r s}\left[Q_{r} Q_{s}\right](\tau, \vec{\sigma}) \\
& \delta_{(a) r}\left[\sum_{u} \int d^{3} \sigma_{1} \mathcal{K}_{(b) u}^{r}\left(\vec{\sigma}, \vec{\sigma}_{1} ; \tau\right) \tilde{\Pi}^{u}\left(\tau, \vec{\sigma}_{1}\right)+\right. \\
+ & \int d^{3} \sigma_{1} F_{(b)(m)}^{r}\left(\vec{\sigma}, \vec{\sigma}_{1} ; \tau\right) \tilde{\pi}_{(m)}^{\vec{\alpha}}\left(\tau, \vec{\sigma}_{1}\right)+ \\
+ & \left.\sum_{u} \int d^{3} \sigma_{1} G_{(b)}^{r u}\left(\vec{\sigma}, \vec{\sigma}_{1} ; \tau\right) \tilde{\pi}_{u}^{\vec{\xi}}\left(\tau, \vec{\sigma}_{1}\right)\right] \\
& \delta_{(c) s}\left[\sum_{v} \int d^{3} \sigma_{2} \mathcal{K}_{(d) v}^{s}\left(\vec{\sigma}, \vec{\sigma}_{2} ; \tau\right) \tilde{\Pi}^{u}\left(\tau, \vec{\sigma}_{2}\right)+\right. \\
+\quad & \int d^{3} \sigma_{2} F_{(d)(n)}^{s}\left(\vec{\sigma}, \vec{\sigma}_{2} ; \tau\right) \tilde{\pi}_{(n)}^{\vec{\alpha}}\left(\tau, \vec{\sigma}_{2}\right)+ \\
+\quad & \left.\sum_{v} \int d^{3} \sigma_{2} G_{(d)}^{s v}\left(\vec{\sigma}, \vec{\sigma}_{2} ; \tau\right) \tilde{\pi}_{v}^{\xi}\left(\tau, \vec{\sigma}_{2}\right)\right] \\
3-\text { orth.gauge } \hat{\mathcal{H}}_{R}(\tau, \vec{\sigma})= & \frac{\epsilon c^{3}}{16 \pi G}\left[Q_{1} Q_{2} Q_{3}{ }^{3} \hat{R}\right](\tau, \vec{\sigma})-\frac{\epsilon 2 \pi G}{c^{3}\left[Q_{1} Q_{2} Q_{3}\right](\tau, \vec{\sigma})}{ }^{3} G_{o(a)(b)(c)(d)} \sum_{r s}\left[Q_{r} Q_{s}\right](\tau, \vec{\sigma}) \\
= &
\end{aligned}
$$




$$
\sum_{u v} \delta_{(a) r} \delta_{(c) s} \int d^{3} \sigma_{1} \mathcal{K}_{(b) u}^{r}\left(\vec{\sigma}, \vec{\sigma}_{1} ; \tau\right) \tilde{\Pi}^{u}\left(\tau, \vec{\sigma}_{1}\right) \int d^{3} \sigma_{2} \mathcal{K}_{(d) v}^{s}\left(\vec{\sigma}, \vec{\sigma}_{2} ; \tau\right) \tilde{\Pi}^{v}\left(\tau, \vec{\sigma}_{2}\right)
$$




\section{A NEW CANONICAL BASIS AND THE SUPERHAMILTONIAN CONSTRAINT AS THE LICHNEROWICZ EQUATION IN THE 3-ORTHOGONAL GAUGES.}

We shall now look at tetrad gravity in the quasi-Shanmugadhasan canonical basis of Eq.(5.11) associated with 3-orthogonal coordinates on the WSW hypersurfaces $\Sigma_{\tau}^{(W S W)}$, namely in the 3-orthogonal gauges of Eq.(5.2). We shall study in more detail the six gauge fixings on the gauge variables $\alpha_{(a)}(\tau, \vec{\sigma}), \xi^{r}(\tau, \vec{\sigma})$ needed to get this gauge. Then we shall give a more convenient canonical basis for the superspace sector and finally we shall show how the superhamiltonian constraint becomes the reduced Lichnerowicz equation. Also the gauge fixing on the last gauge variable, replacing the maximal slicing condition of the conformal approach, will be studied.

\section{A. The Gauge-Fixings for the 3-Orthogonal Gauges.}

Let us study the phase space spanned by the canonical coordinates $n, \tilde{\pi}^{n} \approx 0, n_{(a)}$, $\tilde{\pi}_{(a)}^{\vec{n}} \approx 0, \varphi_{(a)}, \tilde{\pi}_{(a)}^{\vec{\varphi}} \approx 0$ (for the spacetime description), and $Q_{r}, \tilde{\Pi}^{r}$ (for the superspace of 3-geometries).

Let us add the gauge-fixing constraints on the boost parameters

$$
\begin{aligned}
\varphi_{(a)}(\tau, \vec{\sigma}) & \approx 0, \\
\partial_{\tau} \varphi_{(a)}(\tau, \vec{\sigma}) & \stackrel{\risingdotseq}{=}\left\{\varphi_{(a)}(\tau, \vec{\sigma}), \hat{H}_{(D) A D M}\right\}=\lambda_{(a)}^{\vec{\varphi}}(\tau, \vec{\sigma}) \approx 0,
\end{aligned}
$$

namely let us restrict to the surface-forming timelike congruence of the Eulerian observers at rest on $\Sigma_{\tau}^{(W S W)}$.

Since there is no canonical origin in the group of pseudo-diffeomorphisms and since rotations do not have zero Poisson bracket with them, we shall make the convention that in the canonical basis (5.11) the 3-orthogonal gauges corresponding to Eqs.(5.2) are identified by the gauge fixings

$$
\begin{aligned}
\xi^{r}(\tau, \vec{\sigma})-\sigma^{r} & \approx 0 \\
\alpha_{(a)}(\tau, \vec{\sigma}) & \approx 0
\end{aligned}
$$

The gauge fixings $\xi^{r}(\tau, \vec{\sigma})-\sigma^{r} \approx 0$ are equivalent to the statement that a 3-orthogonal coordinate system $\vec{\sigma}$ is chosen as reference origin for the pseudo-diffeomorphisms parametrized by the $\xi^{r}$ 's, while the gauge fixings $\alpha_{(a)}(\tau, \vec{\sigma}) \approx 0$ say that in the associated $\mathrm{SO}(3)$-principal frame bundle over $\Sigma_{\tau}^{(W S W)}$ with these coordinates we choose the identity cross-section as an origin for the rotations parametrized by the $\alpha_{(a)}$ 's 122 . A different 3-orthogonal gauge can be defined by changing the convention (6.2) to $\xi^{r}(\tau, \vec{\sigma})-f^{r}(\tau, \vec{\sigma}) \approx 0, \alpha_{(a)}(\tau, \vec{\sigma})-g_{(a)}(\tau, \vec{\sigma}) \approx 0$ for given functions $f^{r}$ and $g_{(a)}$. But this would only correspond to make a point canonical transformation from the basis (5.11) to a new basis with the same $Q_{r}$ and with

${ }^{122}$ Definition of the standard of non rotation of the coordinates in each point. 
$\alpha_{(a)} \mapsto \alpha_{(a)}^{\prime}=\alpha_{(a)}-g_{(a)}, \xi^{r} \mapsto \xi^{\prime}=\xi^{r}-f^{r}$ (in this way one could obtain rotating 3-orthogonal gauges).

The meaning of the previous gauge fixings is to restrict the Cauchy data of cotriads on $\Sigma_{\tau}^{(W S W)}$ by eliminating the gauge degrees of freedom of boosts, rotations and space pseudodiffeomorphisms, i.e. by restricting ourselves to 3-orthogonal coordinates on $\Sigma_{\tau}^{(W S W)}$ and by having made the choice of the $\Sigma_{\tau}^{(W S W)}$-adapted tetrads ${ }_{\left(\Sigma_{\tau}\right)}^{4} \check{\tilde{E}}_{(\alpha)}^{A}{ }^{A 23}$ as the reference nongeodesic congruence of timelike Eulerian nonrotating observers with 4-velocity field $l^{A}(\tau, \vec{\sigma})$.

By remembering Eqs.(4.31), (4.38) and (5.11), the Dirac brackets are strongly equal to

$$
\begin{aligned}
& \left\{A(\tau, \vec{\sigma}), B\left(\tau, \vec{\sigma}^{\prime}\right)\right\}^{*}=\left\{A(\tau, \vec{\sigma}), B\left(\tau, \vec{\sigma}^{\prime}\right)\right\}+ \\
& +\int d^{3} \sigma_{1}\left[\left\{A(\tau, \vec{\sigma}), \alpha_{(a)}\left(\tau, \vec{\sigma}_{1}\right)\right\}\left\{\tilde{\pi}_{(a)}^{\vec{\alpha}}\left(\tau, \vec{\sigma}_{1}\right), B\left(\tau, \vec{\sigma}^{\prime}\right)\right\}-\right. \\
& -\left\{A(\tau, \vec{\sigma}), \tilde{\pi}_{(a)}^{\vec{\alpha}}\left(\tau, \vec{\sigma}_{1}\right)\right\}\left\{\alpha_{(a)}\left(\tau, \vec{\sigma}_{1}\right), B\left(\tau, \vec{\sigma}^{\prime}\right)\right\}+ \\
& +\left\{A(\tau, \vec{\sigma}), \xi^{r}\left(\tau, \vec{\sigma}_{1}\right)\right\}\left\{\tilde{\pi}_{r}^{\vec{\xi}}\left(\tau, \vec{\sigma}_{1}\right), B\left(\tau, \vec{\sigma}^{\prime}\right)\right\}- \\
& \left.-\left\{A(\tau, \vec{\sigma}), \tilde{\pi}_{r}^{\vec{\xi}}\left(\tau, \vec{\sigma}_{1}\right)\right\}\left\{\xi^{r}\left(\tau, \vec{\sigma}_{1}\right), B\left(\tau, \vec{\sigma}^{\prime}\right)\right\}\right] \equiv \\
& \equiv\left\{A(\tau, \vec{\sigma}), B\left(\tau, \vec{\sigma}^{\prime}\right)\right\}+ \\
& +\int d^{3} \sigma_{1}\left(\left[\left\{A(\tau, \vec{\sigma}), \alpha_{(a)}\left(\tau, \vec{\sigma}_{1}\right)\right\}\left\{{ }^{3} \tilde{M}_{(b)}\left(\tau, \vec{\sigma}_{1}\right), B\left(\tau, \vec{\sigma}^{\prime}\right)\right\}-\right.\right. \\
& \left.-\left\{A(\tau, \vec{\sigma}),{ }^{3} \tilde{M}_{(b)}\left(\tau, \vec{\sigma}_{1}\right)\right\}\left\{\alpha_{(a)}\left(\tau, \vec{\sigma}_{1}\right), B\left(\tau, \vec{\sigma}^{\prime}\right)\right\}\right] . \\
& \text { - } A_{(b)(a)}\left(\alpha_{(e)}\left(\tau, \vec{\sigma}_{1}\right)\right)+\left.\frac{\partial \sigma_{1}^{s}(\vec{\xi})}{\partial \xi^{r}}\right|_{\vec{\xi}=\vec{\xi}\left(\tau, \vec{\sigma}_{1}\right)} \text {. } \\
& \cdot\left[\left\{A(\tau, \vec{\sigma}), \xi^{r}\left(\tau, \vec{\sigma}_{1}\right)\right\}\left\{{ }^{3} \tilde{\Theta}_{s}\left(\tau, \vec{\sigma}_{1}\right), B\left(\tau, \vec{\sigma}^{\prime}\right)\right\}-\right. \\
& \left.-\left\{A(\tau, \vec{\sigma}),{ }^{3} \tilde{\Theta}_{s}\left(\tau, \vec{\sigma}_{1}\right)\right\}\left\{\xi^{r}\left(\tau, \vec{\sigma}_{1}\right), B\left(\tau, \vec{\sigma}^{\prime}\right)\right\}\right]+ \\
& +\left.\frac{\partial \sigma_{1}^{s}(\vec{\xi})}{\partial \xi^{r}}\right|_{\vec{\xi}=\vec{\xi}\left(\tau, \vec{\sigma}_{1}\right)} A_{(b)(a)}\left(\alpha_{(e)}\left(\tau, \vec{\sigma}_{1}\right)\right) \frac{\partial \alpha_{(a)}\left(\tau, \vec{\sigma}_{1}\right)}{\partial \sigma_{1}^{s}} . \\
& \text { - }\left[\left\{A(\tau, \vec{\sigma}), \xi^{r}\left(\tau, \vec{\sigma}_{1}\right)\right\}\left\{{ }^{3} \tilde{M}_{(b)}\left(\tau, \vec{\sigma}_{1}\right), B\left(\tau, \vec{\sigma}^{\prime}\right)\right\}-\right. \\
& \left.\left.-\left\{A(\tau, \vec{\sigma}),{ }^{3} \tilde{M}_{(b)}\left(\tau, \vec{\sigma}_{1}\right)\right\}\left\{\xi^{r}\left(\tau, \vec{\sigma}_{1}\right), B\left(\tau, \vec{\sigma}^{\prime}\right)\right\}\right]\right) \text {. }
\end{aligned}
$$

Since the variables $\alpha_{(a)}(\tau, \vec{\sigma}), \xi^{r}(\tau, \vec{\sigma})$, are not known as explicit functions of the cotriads, these Dirac brackets can be used only implicitly. We must have $\alpha_{(a)}(\tau, \vec{\sigma}) \rightarrow O\left(r^{-(1+\epsilon)}\right)$ and $\xi^{r}(\tau, \vec{\sigma}) \rightarrow \sigma^{r}+O\left(r^{-\epsilon}\right)$ for $r \rightarrow \infty$ due to Eqs. 2.55).

We have seen in Section IV that the differential geometric description for rotations already showed that the restriction to the identity cross section $\alpha_{(a)}(\tau, \vec{\sigma})=0$ implied also $\partial_{r} \alpha_{(a)}(\tau, \vec{\sigma})=0$; we also have $\left.A_{(a)(b)}\left(\alpha_{(e)}(\tau, \vec{\sigma})\right)\right|_{\alpha=0}=0$. When we add the gauge-fixings $\alpha_{(a)}(\tau, \vec{\sigma}) \approx 0$, the derivatives of all orders of $\alpha_{(a)}(\tau, \vec{\sigma})$ weakly vanish at $\alpha_{(a)}(\tau, \vec{\sigma})=0$. Similarly, it can be shown that, if we have the pseudo-diffeomorphism $\vec{\xi}(\tau, \vec{\sigma})=\vec{\sigma}+\hat{\vec{\xi}}(\tau, \vec{\sigma})$, so that for $\vec{\xi}(\tau, \vec{\sigma}) \rightarrow \vec{\sigma}$ we have $\hat{\vec{\xi}}(\tau, \vec{\sigma}) \rightarrow \delta \vec{\sigma}(\tau, \vec{\sigma})$, then the quantities ${ }^{3} e_{(a) r}(\tau, \vec{\sigma}), \partial_{r}{ }^{3} e_{(a) s}(\tau, \vec{\sigma})$,

\footnotetext{
${ }^{123}$ See Eqs.(2.1), (2.2) rewritten in terms of the Dirac observables ${ }^{3} \hat{e}_{(a)}^{r}$ dual to ${ }^{3} \hat{e}_{(a) r}$.
} 
${ }^{3} \omega_{r(a)}(\tau, \vec{\sigma}),{ }^{3} \Omega_{r s(a)}(\tau, \vec{\sigma})$, become functions only of $Q_{r}(\tau, \vec{\sigma})$ and $\tilde{\Pi}^{r}(\tau, \vec{\sigma})$ for $\vec{\xi}(\tau, \vec{\sigma}) \rightarrow \vec{\sigma}$ and $\alpha_{(a)}(\tau, \vec{\sigma}) \rightarrow 0$ only if we have the following behaviour of the parameters $\xi^{r}(\tau, \vec{\sigma})$

$$
\begin{aligned}
& \left.\frac{\partial \delta \sigma^{r}(\tau, \vec{\sigma})}{\partial \sigma^{s}}\right|_{\vec{\xi}=\vec{\sigma}}=\left.0 \Rightarrow \frac{\partial \xi^{r}(\tau, \vec{\sigma})}{\partial \sigma^{s}}\right|_{\vec{\xi}=\vec{\sigma}}=\delta_{s}^{r},\left.\quad \frac{\partial^{2} \xi^{r}(\tau, \vec{\sigma})}{\partial \sigma^{s} \partial \sigma^{u}}\right|_{\vec{\xi}=\vec{\sigma}}=0, \\
& \left.\frac{\partial^{2} \delta \sigma^{r}(\tau, \vec{\sigma})}{\partial \sigma^{u} \partial \sigma^{v}}\right|_{\vec{\xi}=\vec{\sigma}}=\left.0 \Rightarrow\left[\left.\frac{\partial}{\partial \sigma^{u}} \frac{\partial \sigma^{r}(\tau, \vec{\sigma})}{\partial \xi^{v}}\right|_{\vec{\xi}=\vec{\xi}(\tau, \vec{\sigma})}\right]\right|_{\vec{\xi}=\vec{\sigma}}=0, \\
& \left.\frac{\partial^{3} \delta \sigma^{r}(\tau, \vec{\sigma})}{\partial \sigma^{s} \partial \sigma^{u} \partial \sigma^{v}}\right|_{\vec{\xi}=\vec{\sigma}}=\left.0 \Rightarrow\left[\left.\frac{\partial^{2}}{\partial \sigma^{u} \partial \sigma^{v}} \frac{\partial \sigma^{r}(\vec{\xi})}{\partial \xi^{s}}\right|_{\vec{\xi}=\vec{\xi}(\tau, \vec{\sigma})}\right]\right|_{\vec{\xi}=\vec{\sigma}}=0 .
\end{aligned}
$$

These conditions should be satisfied by the parameters of pseudo-diffeomorphisms near the identity, i.e. near the chart chosen as reference chart (the 3-orthogonal one in this case). With the gauge-fixings $\xi^{r}(\tau, \vec{\sigma}) \approx \vec{\sigma}$ all these properties are satisfied.

Let us remember the Dirac Hamiltonian (4.57) valid on WSW hypersurfaces $\Sigma_{\tau}^{(W S W)}$ before going to the rest-frame instant form with $\hat{\lambda}_{\tau}(\tau)=\epsilon, \tilde{\lambda}_{r}(\tau)=0$

$$
\begin{aligned}
\hat{H}_{(D) A D M} & =\int d^{3} \sigma\left[n \hat{\mathcal{H}}+\tilde{n}^{r} \tilde{\pi}_{r}^{\vec{\xi}}+\lambda_{n} \tilde{\pi}^{n}+\lambda_{(a)}^{\vec{n}} \tilde{\pi}_{(a)}^{\vec{n}}+\lambda_{(a)}^{\vec{\varphi}} \tilde{\pi}_{(a)}^{\vec{\varphi}}+\tilde{\mu}_{(a)} \tilde{\pi}_{(a)}^{\vec{\alpha}}\right](\tau, \vec{\sigma})- \\
& -\tilde{\lambda}_{\tau}(\tau)\left[\epsilon_{(\infty)}-\hat{P}_{A D M}^{\tau}\right]+\tilde{\lambda}_{r}(\tau) \hat{P}_{A D M}^{r},
\end{aligned}
$$

with $\tilde{n}^{r}=n_{(a)}^{3} e_{(a)}^{s} \frac{\partial \xi^{r}}{\partial \sigma^{s}}=n_{u}^{3} g^{u v} \frac{\partial \xi^{r}}{\partial \sigma^{v}}=n^{v} \frac{\partial \xi^{r}}{\partial \sigma^{v}}$.

The time constancy of the gauge fixings $\alpha_{(a)}(\tau, \vec{\sigma}) \approx 0, \xi^{r}(\tau, \vec{\sigma})-\sigma^{r} \approx 0$ [implying $\left.\tilde{n}^{r} \approx n^{r}\right]$ gives $^{124}$

$$
\begin{gathered}
\partial_{\tau} \alpha_{(a)}(\tau, \vec{\sigma}) \stackrel{\circ}{=}\left\{\alpha_{(a)}(\tau, \vec{\sigma}), \hat{H}_{(D) A D M}\right\}=\tilde{\mu}_{(a)}(\tau, \vec{\sigma}) \approx 0, \\
\rightarrow \quad \tilde{\mu}_{(a)}(\tau, \vec{\sigma}) \quad \text { determined } \\
\partial_{\tau}\left[\xi^{r}(\tau, \vec{\sigma})-\sigma^{r}\right] \stackrel{\circ}{=}\left\{\xi^{r}(\tau, \vec{\sigma}), \hat{H}_{(D) A D M}\right\} \approx n^{s}(\tau, \vec{\sigma})+ \\
+\int d^{3} \sigma_{1} n\left(\tau, \vec{\sigma}_{1}\right)\left\{\xi^{r}(\tau, \vec{\sigma}), \hat{\mathcal{H}}\left(\tau, \vec{\sigma}_{1}\right)\right\}-\tilde{\lambda}_{A}(\tau)\left\{\xi^{r}(\tau, \vec{\sigma}), \hat{P}_{A D M}^{A}\right\} \approx 0, \\
\Rightarrow \quad n_{r}(\tau, \vec{\sigma})-\hat{n}_{r}\left(\tau, \vec{\sigma} \mid r_{\bar{a}}, \pi_{\bar{a}}, \tilde{\lambda}_{A}\right] \approx 0, \\
\hat{n}_{r}\left(\tau, \vec{\sigma} \mid r_{\bar{a}}, \pi_{\bar{a}}, \tilde{\lambda}_{A}\right]={ }^{3} g_{r s}(\tau, \vec{\sigma})\left[\tilde{\lambda}_{A}(\tau) \frac{\delta \hat{P}_{A D M}^{A}}{\delta \tilde{\pi}_{s}^{\vec{\xi}}(\tau, \vec{\sigma})}-\right.
\end{gathered}
$$

${ }^{124}$ As shown in Ref. 99, when one has a chain of a primary and a secondary first class constraint, both of them appear in the Dirac Hamiltonin: the primary constraint with in front the arbitrary Dirac multiplier and the secondary one with in front some function of the canonical variables not determined by the Hamilton equations. To make the gauge fixing one has to follow Ref. [98]: i) add a gauge fixing $\chi_{1} \approx 0$ to the secondary constraint; ii) find the implied gauge fixing $\chi_{2} \approx 0$ for the primary constraint by requiring the time constancy of $\chi_{1} \approx 0$; iii) determine the Dirac multiplier by requiring the time constancy of $\chi_{2} \approx 0$. 


$$
\begin{aligned}
& \left.-\int d^{3} \sigma_{1} n\left(\tau, \vec{\sigma}_{1}\right) \frac{\delta \hat{\mathcal{H}}\left(\tau, \vec{\sigma}_{1}\right)}{\delta \tilde{\pi}_{s}^{(}(\tau, \vec{\sigma})}\right], \\
& \Downarrow \\
& \partial_{\tau}\left[n_{r}(\tau, \vec{\sigma}) \quad-\hat{n}_{r}\left(\tau, \vec{\sigma} \mid r_{\bar{a}}, \pi_{\bar{a}}, \tilde{\lambda}_{A}\right]\right] \approx \\
& \approx\left[\lambda_{(a)}^{\vec{n}} 3 \hat{e}_{(a) r}\right](\tau, \vec{\sigma})-\left\{\hat{n}_{r}\left(\tau, \vec{\sigma} \mid r_{\bar{a}}, \pi_{\bar{a}}, \tilde{\lambda}_{A}\right], \hat{H}_{(D) A D M}\right\} \approx 0, \\
& \Rightarrow \quad \lambda_{(a)}^{\vec{n}}(\tau, \vec{\sigma}) \quad \text { determined. }
\end{aligned}
$$

Therefore, the shift functions do not vanish in the 3-orthogonal gauges avoiding the synchronous coordinates of $M^{4}$ with their tendency to develop coordinate singularities in short times 64,100. This shows the presence of gravitomagnetism and the non-validity of Einstein simultaneity convention in these gauges, since ${ }^{4} g_{\tau r} \neq 0$. See Ref. 65 for the problems of the fixation of the lapse and shift functions in ADM metric gravity (coordinate conditions to rebuild spacetime) and for the origin of the coordinates used in numerical gravity (see Ref. [101] for a recent review of it). Let us remark that in Refs. [102 the Einstein's equations corresponding to the supermomentum constraints of metric gravity are thought as elliptic equations for the shift functions in the framework either of a new conformal thin sandwich formulation or of an enlargement of Einstein's equations to get a manifestly hyperbolic system of equations: this approach, even if mathematically legitimate, is completely innatural from the Hamiltonian point of view with its interpretation based on constraint theory.

In the rest-frame instant form of tetrad gravity with $\tilde{\lambda}_{A}(\tau)=(\epsilon ; \overrightarrow{0})$ we get $[25$

$$
\begin{gathered}
n_{r}(\tau, \vec{\sigma}) \approx{ }^{3} e_{(a) r}(\tau, \vec{\sigma})^{3} e_{(a) s}(\tau, \vec{\sigma}) \int d^{3} \sigma_{1}\left[\epsilon-n\left(\tau, \vec{\sigma}_{1}\right)\right] \frac{\delta \hat{\mathcal{H}}\left(\tau, \vec{\sigma}_{1}\right)}{\delta \tilde{\pi}_{s}^{\vec{\xi}}(\tau, \vec{\sigma})} \\
\left.\stackrel{{ }^{3-\text { orth gauge }}}{\rightarrow} Q_{r}^{2}(\tau, \vec{\sigma}) \int d^{3} \sigma_{1}\left[\epsilon-n\left(\tau, \vec{\sigma}_{1}\right)\right] \frac{\delta \hat{\mathcal{H}}\left(\tau, \vec{\sigma}_{1}\right)}{\delta \tilde{\pi}_{r}^{\vec{\xi}}(\tau, \vec{\sigma})}\right|_{3-0},
\end{gathered}
$$

with

$$
\begin{gathered}
\frac{\delta \hat{\mathcal{H}}\left(\tau, \vec{\sigma}_{1}\right)}{\delta \tilde{\pi}_{s}^{\vec{\xi}}(\tau, \vec{\sigma})}=\int d^{3} \sigma_{2} \frac{\delta \hat{\mathcal{H}}\left(\tau, \vec{\sigma}_{1}\right)}{\delta \tilde{\pi}_{(d)}^{u}\left(\tau, \vec{\sigma}_{2}\right)} \frac{\delta \tilde{\pi}_{(d)}^{u}\left(\tau, \vec{\sigma}_{2}\right)}{\delta \tilde{\pi}_{s}^{\vec{\xi}}(\tau, \vec{\sigma})}= \\
=\quad-\frac{\epsilon 4 \pi G}{c^{3}{ }^{3} e\left(\tau, \vec{\sigma}_{1}\right)}{ }^{3} G_{o(a)(b)(c)(d)}\left[{ }^{3} e_{(a) v}{ }^{3} \tilde{\pi}_{(b)}^{v}{ }^{3} e_{(c) u}\right]\left(\tau, \vec{\sigma}_{1}\right) G_{(d)}^{u s}\left(\vec{\sigma}_{1}, \vec{\sigma} ; \tau\right) \\
\stackrel{\epsilon-\text { orth gauge }}{\rightarrow}-\frac{\epsilon 2 \pi G}{c^{3}\left[Q_{1} Q_{2} Q_{3}\right]\left(\tau, \vec{\sigma}_{1}\right)}{ }^{3} G_{o(a)(b)(c)(d)} \\
\sum_{w u}\left[Q_{w} Q_{u}\right]\left(\tau, \vec{\sigma}_{1}\right) \delta_{(a) w} \sum_{v} \int d^{3} \sigma_{2} \mathcal{K}_{(b) v}^{w}\left(\vec{\sigma}_{1}, \vec{\sigma}_{2} ; \tau\right) \tilde{\Pi}^{v}\left(\tau, \vec{\sigma}_{2}\right) \delta_{(c) u} G_{(d)}^{u s}\left(\vec{\sigma}_{1}, \vec{\sigma} ; \tau\right),
\end{gathered}
$$

${ }^{125}$ From Eqs.(2.25), (2.11) and from ${ }^{3} \tilde{\Pi}^{r s}=\frac{1}{4}\left({ }^{3} e_{(a)}^{r}{ }^{3} \tilde{\pi}_{(a)}^{s}+{ }^{3} e_{(a)}^{s}{ }^{3} \tilde{\pi}_{(a)}^{r}\right)$ we get $\frac{\delta \hat{P}_{A D M}^{\tau}}{\delta \tilde{\pi}_{s}^{\xi}(\tau, \vec{\sigma})}=$ $\int d^{3} \sigma_{1} \frac{\delta \hat{\mathcal{H}}\left(\tau, \vec{\sigma}_{1}\right)}{\delta \tilde{\pi}_{s}^{\xi}(\tau, \vec{\sigma})}$. 
where we used Eqs.2.11) and (5.12). We see that the shift functions in the 3-orthogonal gauges depend from the not yet determined lapse function $n(\tau, \vec{\sigma})$. Only after having added the gauge fixing to the superhamiltonian constraint, namely only after having selected a completely fixed 3-orthogonal gauge, the lapse and shift functions will be determined. This is a feature common to all the completely fixed gauges.

The Dirac Hamiltonian in the 3-orthogonal gauges, but not yet in the rest-frame instant form, is

$$
\hat{H}_{(D) A D M, R}=\int d^{3} \sigma\left[n \hat{\mathcal{H}}_{R}+\lambda_{n} \tilde{\pi}^{n}\right](\tau, \vec{\sigma})-\tilde{\lambda}_{\tau}(\tau)\left[\epsilon_{(\infty)}-\hat{P}_{A D M}^{\tau}\right]+\tilde{\lambda}_{r}(\tau) \hat{P}_{A D M}^{r},
$$

where $\hat{\mathcal{H}}_{R}$ is the reduced superhamiltonian constraint.

\section{B. A New Canonical Basis for Superspace in the 3-Orthogonal Gauges.}

Let us now consider a new canonical transformation from the basis $Q_{u}(\tau, \vec{\sigma}), \tilde{\Pi}^{u}(\tau, \vec{\sigma})^{126}$ to a new basis $q_{u}(\tau, \vec{\sigma}), \rho_{u}(\tau, \vec{\sigma})$ defined in the following way

$$
\begin{aligned}
q_{u}(\tau, \vec{\sigma})= & \ln Q_{u}(\tau, \vec{\sigma}), \quad Q_{u}(\tau, \vec{\sigma})=e^{q_{u}(\tau, \vec{\sigma})}, \\
\rho_{u}(\tau, \vec{\sigma})= & Q_{u}(\tau, \vec{\sigma}) \tilde{\Pi}^{u}(\tau, \vec{\sigma}), \quad \tilde{\Pi}^{u}(\tau, \vec{\sigma})=e^{-q_{u}(\tau, \vec{\sigma})} \rho_{u}(\tau, \vec{\sigma}), \\
& \left\{q_{u}(\tau, \vec{\sigma}), \rho_{v}\left(\tau, \vec{\sigma}^{\prime}\right)\right\}=\delta_{u v} \delta^{3}\left(\vec{\sigma}, \vec{\sigma}^{\prime}\right) .
\end{aligned}
$$

It is convenient to make one more canonical transformation, like for the determination of the center of mass of a particle system [15], to the following new canonical basis

$$
\begin{aligned}
& q(\tau, \vec{\sigma})=\frac{1}{3} \sum_{u} q_{u}(\tau, \vec{\sigma})=\frac{1}{3} \sum_{u} \ln Q_{u}(\tau, \vec{\sigma}) \rightarrow_{r \rightarrow \infty} \frac{M}{2 r}+O\left(r^{-3 / 2}\right), \\
& r_{\bar{a}}(\tau, \vec{\sigma})=\sqrt{3} \sum_{u} \gamma_{\bar{a} u} q_{u}(\tau, \vec{\sigma})=\sqrt{3} \sum_{u} \gamma_{\bar{a} u} \ln Q_{u}(\tau, \vec{\sigma}) \rightarrow_{r \rightarrow \infty} O\left(r^{-3 / 2}\right) \text {, } \\
& \bar{a}=1,2, \\
& \rho(\tau, \vec{\sigma})=\sum_{u} \rho_{u}(\tau, \vec{\sigma})=\sum_{u}\left[Q_{u} \tilde{\Pi}^{u}\right](\tau, \vec{\sigma}) \rightarrow_{r \rightarrow \infty} O\left(r^{-2}\right), \\
& \pi_{\bar{a}}(\tau, \vec{\sigma})=\frac{1}{\sqrt{3}} \sum_{u} \gamma_{\bar{a} u} \rho_{u}(\tau, \vec{\sigma})=\frac{1}{\sqrt{3}} \sum_{u} \gamma_{\bar{a} u}\left[Q_{u} \tilde{\Pi}^{u}\right](\tau, \vec{\sigma}) \rightarrow_{r \rightarrow \infty} O\left(r^{-2}\right), \\
& \bar{a}=1,2, \\
& \left\{q(\tau, \vec{\sigma}), \rho\left(\tau, \vec{\sigma}^{\prime}\right)\right\}=\delta^{3}\left(\vec{\sigma}, \vec{\sigma}^{\prime}\right), \quad\left\{r_{\bar{a}}(\tau, \vec{\sigma}), \pi_{\bar{b}}\left(\tau, \vec{\sigma}^{\prime}\right)\right\}=\delta_{\bar{a} \bar{b}} \delta^{3}\left(\vec{\sigma}, \vec{\sigma}^{\prime}\right), \\
& q_{u}(\tau, \vec{\sigma})=q(\tau, \vec{\sigma})+\frac{1}{\sqrt{3}} \sum_{\bar{a}} \gamma_{\bar{a} u} r_{\bar{a}}(\tau, \vec{\sigma}), \quad Q_{u}(\tau, \vec{\sigma})=e^{q_{u}(\tau, \vec{\sigma})},
\end{aligned}
$$

\footnotetext{
${ }^{126}$ Where ${ }^{3} \hat{e}_{(a) r}(\tau, \vec{\sigma})=\delta_{(a) r} Q_{r}(\tau, \vec{\sigma})$, see Eq. (5.2).
} 


$$
\begin{aligned}
& \rho_{u}(\tau, \vec{\sigma})=\frac{1}{3} \rho(\tau, \vec{\sigma})+\sqrt{3} \sum_{\bar{a}} \gamma_{\bar{a} u} \pi_{\bar{a}}(\tau, \vec{\sigma}), \quad \tilde{\Pi}^{u}(\tau, \vec{\sigma})=\left[e^{-q_{u}} \rho_{u}\right](\tau, \vec{\sigma}),
\end{aligned}
$$

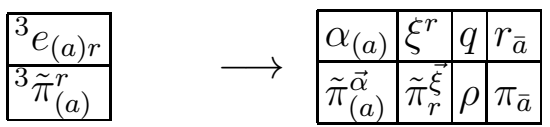

$$
\begin{aligned}
& \longrightarrow \begin{array}{|l|l|l|l|}
\hline \alpha_{(a)} & \xi^{r} & \phi & r_{\bar{a}} \\
\hline \tilde{\pi}_{(a)}^{\vec{\alpha}} & \tilde{\pi}_{r}^{\tilde{\xi}} & \pi_{\phi} & \pi_{\bar{a}} \\
\hline
\end{array} \\
& \phi(\tau, \vec{\sigma})=e^{\frac{1}{2} q(\tau, \vec{\sigma})} \rightarrow_{r \rightarrow \infty} 1+\frac{M}{4 r}+O\left(r^{-3 / 2}\right), \quad \pi_{\phi}(\tau, \vec{\sigma})=2 e^{\frac{1}{2} q(\tau, \vec{\sigma})} \rho(\tau, \vec{\sigma}), \\
& \left\{\phi(\tau, \vec{\sigma}), \pi_{\phi}\left(\tau, \vec{\sigma}^{\prime}\right)\right\}=\delta^{3}\left(\vec{\sigma}, \vec{\sigma}^{\prime}\right), \\
& Q_{u}(\tau, \vec{\sigma})=\left(e^{q+\frac{1}{\sqrt{3}} \sum_{\bar{a}} \gamma_{\bar{a} u} r_{\bar{a}}}\right)(\tau, \vec{\sigma})=\phi^{2}(\tau, \vec{\sigma}) e^{\frac{1}{\sqrt{3}} \sum_{\bar{a}} \gamma_{\bar{a} u} r_{\bar{a}}(\tau, \vec{\sigma})}, \\
& \tilde{\Pi}^{u}(\tau, \vec{\sigma})=e^{-q(\tau, \vec{\sigma})} e^{-\frac{1}{\sqrt{3}} \sum_{\bar{a}} \gamma_{\bar{a} u} r_{\bar{a}}(\tau, \vec{\sigma})}\left[\frac{1}{3} \rho+\sqrt{3} \sum_{\bar{b}} \gamma_{\bar{b} u} \pi_{\bar{b}}\right](\tau, \vec{\sigma})= \\
& =\phi^{-2}(\tau, \vec{\sigma}) e^{-\frac{1}{\sqrt{3}} \sum_{\bar{a}} \gamma_{\bar{a} u} r_{\bar{a}}(\tau, \vec{\sigma})}\left[\frac{1}{6} \phi^{-1} \pi_{\phi}+\sqrt{3} \sum_{\bar{b}} \gamma_{\bar{b} u} \pi_{\bar{b}}\right](\tau, \vec{\sigma}) .
\end{aligned}
$$

where $\gamma_{\bar{a} u}$ are numerical constants satisfying [15]

$$
\sum_{u} \gamma_{\bar{a} u}=0, \quad \sum_{u} \gamma_{\bar{a} u} \gamma_{\bar{b} u}=\delta_{\bar{a} \bar{b}}, \quad \sum_{\bar{a}} \gamma_{\bar{a} u} \gamma_{\bar{a} v}=\delta_{u v}-\frac{1}{3} .
$$

Therefore, Eqs.(6.11) define a one-parameter family of canonical transformations, one for each solution of Eqs.(6.12).

In Eq.(6.10) we have also shown the canonical transformation from the canonical pair $q, \rho$ to the canonical pair $\phi, \pi_{\phi}$ consisting in the conformal factor of the 3 -metric and its conjugate momentum.

In terms of these variables we have $\left[N_{(a s)}=-\tilde{\lambda}_{\tau}(\tau), N_{(a s) r}=-\tilde{\lambda}_{r}(\tau)\right]$

$$
\begin{aligned}
& { }^{3} \hat{g}_{r s}=e^{2 q}\left(\begin{array}{ccc}
e^{\frac{2}{\sqrt{3}} \sum_{\bar{a}} \gamma_{\bar{a} 1} r_{\bar{a}}} & 0 & 0 \\
0 & e^{\frac{2}{\sqrt{3}} \sum_{\bar{a}} \gamma_{\bar{a} 2} r_{\bar{a}}} & 0 \\
0 & 0 & e^{\frac{2}{\sqrt{3}} \sum_{\bar{a}} \gamma_{\bar{a} 3} r_{\bar{a}}}
\end{array}\right)=\phi^{4} \hat{g}_{r s}^{\text {diag }} \\
& \hat{\gamma}={ }^{3} \hat{g}={ }^{3} \hat{e}^{2}=e^{6 q}=\phi^{12}, \quad \operatorname{det}\left|\hat{g}_{r s}^{\text {diag }}\right|=1, \\
& d \hat{s}^{2}=\epsilon\left(\left[N_{(a s)}+n\right]^{2}-e^{-2 q} \sum_{u} e^{-\frac{2}{\sqrt{3}} \sum_{\bar{a}} \gamma_{\bar{a} u} r_{\bar{a}}}\left[N_{(a s) u}+n_{u}\right]^{2}\right)(d \tau)^{2}- \\
& -2 \epsilon\left[N_{(a s) r}+n_{r}\right] d \tau d \sigma^{r}-\epsilon e^{2 q} \sum_{u} e^{\frac{2}{\sqrt{3}} \sum_{\bar{a}} \gamma \bar{a} u r_{\bar{a}}}\left(d \sigma^{u}\right)^{2}= \\
& =\epsilon\left(\left[N_{(a s)}+n\right]^{2}(d \tau)^{2}-\right. \\
& -\delta_{u v}\left[\phi^{2} e^{\frac{2}{\sqrt{3}} \sum_{\bar{a}} \gamma_{\bar{a} u} r_{\bar{a}}} d \sigma^{u}+\phi^{-2} e^{-\frac{2}{\sqrt{3}} \sum_{\bar{a}} \gamma_{\bar{a} u} r_{\bar{a}}}\left(N_{(a s) u}+n_{u}\right) d \tau\right]
\end{aligned}
$$




$$
\begin{aligned}
& {\left.\left[\phi^{2} e^{\frac{2}{\sqrt{3}} \sum_{\bar{a}} \gamma_{\bar{a} v} r_{\bar{a}}} d \sigma^{v}+\phi^{-2} e^{-\frac{2}{\sqrt{3}} \sum_{\bar{a}} \gamma_{\bar{a} v} r_{\bar{a}}}\left(N_{(a s) v}+n_{v}\right) d \tau\right]\right), } \\
q= & 2 \ln \phi=\frac{1}{6} \ln ^{3} \hat{g} \\
r_{\bar{a}}= & \frac{\sqrt{3}}{2} \sum_{r} \gamma_{\bar{a} r} \ln \frac{{ }^{3} \hat{g}_{r r}}{3 \hat{g}} .
\end{aligned}
$$

We see that the freedom in the choice of the solutions of Eqs.(6.12) allows to put two of the diagonal elements of ${ }^{3} \hat{g}_{r s}^{\text {diag }}$ equal but only in one point $\vec{\sigma}$. To select which diagonal elements is convenient to make equal, we should need an intrinsic $2+1$ splitting of the WSW hypersurfaces. In Ref. [19] there is the statement that the independent degrees of freedom of the gravitational field are described by symmetric trace-free 2-tensors on 2-planes. On the other hand, the tangent plane in each point of the WSW hypersurfaces is naturally decomposed in the gauge direction identified by the shift functions $n_{r}(\tau, \vec{\sigma})$ in that point and in the orthogonal 2-plane. However, this has to be done after the addition of the gauge fixing to the superhamiltonian constraint, so that the lapse and shift functions are determined. Moreover, Eqs. 6.11) and (6.12) should be generalized so that the $\gamma_{\bar{a} u}$ 's become point dependent. This will be studied in a future paper, because the accomplishment of this result would identify the tensoriality quoted in Ref. [19] for the Dirac observables $r_{\bar{a}}(\tau, \vec{\sigma})$, which, in each point, would be functions only of the physical 2-plane in that point. Let us remark that this type of $3+1 \rightarrow 2+1+1$ splitting is reminiscent of the $2+2$ splittings of Refs. 99.

Another possible canonical basis could be obtained by the point canonical transformation $\phi, r_{\bar{a}} \mapsto \phi, \lambda_{i}^{C Y}(i=1,2)$, where the $\lambda_{i}^{C Y}$ are two independent eigenvalues of the CottonYork 3-tensor defined in Appendix A (this tensor has only two independent components and vanishes for $r_{\bar{a}} \rightarrow \infty$, namely when the hypersurfaces are 3-conformally flat).

In terms of the variables $q=2 \ln \phi, r_{\bar{a}}$, we have the following results ${ }^{127}\left[{ }^{128}\right.$

$$
\begin{aligned}
3 \hat{e}_{(a) r}= & \delta_{(a) r} e^{q_{r}}=\delta_{(a) r} e^{q+\frac{1}{\sqrt{3}} \sum_{\bar{a}} \gamma_{\bar{a} r} r_{\bar{a}}}=\delta_{(a) r} \phi^{2} e^{\frac{1}{\sqrt{3}} \sum_{\bar{a}} \gamma_{\bar{a} r} r_{\bar{a}}} \\
& \rightarrow_{r_{\bar{a}} \rightarrow 0} \delta_{(a) r} e^{q}=\delta_{(a) r} \phi^{2} \\
& \rightarrow_{q, r_{\bar{a}} \rightarrow 0} \delta_{(a) r} \\
& \rightarrow_{q \rightarrow 0} \delta_{(a) r} e^{\frac{1}{\sqrt{3}} \sum_{\bar{a}} \gamma_{\bar{a} r} r_{\bar{a}}}, \\
{ }^{3} \hat{e}_{(a)}^{r}= & \delta_{(a)}^{r} e^{-q_{r}}=\delta_{(a)}^{r} e^{-q-\frac{1}{\sqrt{3}} \sum_{\bar{a}} \gamma_{\bar{a} r} r_{\bar{a}}}=\delta_{(a)}^{r} \phi^{-2} e^{-\frac{1}{\sqrt{3}} \sum_{\bar{a}} \gamma_{\bar{a} r} r_{\bar{a}}} \\
& \rightarrow_{r_{\bar{a}} \rightarrow 0} \delta_{(a)}^{r} e^{-q}=\delta_{(a)}^{r} \phi^{-2} \\
& \rightarrow_{q, r_{\bar{a}} \rightarrow 0} \delta_{(a)}^{r}, \\
& \rightarrow_{q \rightarrow 0} \delta_{(a)}^{r} e^{-\frac{1}{\sqrt{3}} \sum_{\bar{a}} \gamma_{\bar{a} r} r_{\bar{a}}},
\end{aligned}
$$

${ }^{127}$ Use is done of Eqs.(A25) of Ref. [1]]; see also Appendix A.

${ }^{128} \gamma_{P P_{1}}$ is the geodesic between $\mathrm{P}$ and $P_{1}$ for the 3 -metric; $\phi=e^{q / 2}, \rho=\pi_{\phi} / 2 \phi$. 


$$
\begin{aligned}
& { }^{3} \hat{g}_{r s}=\delta_{r s} e^{2 q_{r}}=\delta_{r s} e^{2 q+\frac{2}{\sqrt{3}} \sum_{\bar{a}} \gamma_{\bar{a} r} r_{\bar{a}}}=\delta_{r s} \phi^{4} e^{\frac{2}{\sqrt{3}} \sum_{\bar{a}} \gamma_{\bar{a} r} r_{\bar{a}}} \\
& \rightarrow_{r_{\bar{a}} \rightarrow 0} \delta_{r s} e^{2 q}=\delta_{r s} \phi^{4} \rightarrow_{q \rightarrow 0} \delta_{r s}, \quad \rightarrow_{q \rightarrow 0} \delta_{r s} e^{\frac{2}{\sqrt{3}} \sum_{\bar{a}} \gamma_{\bar{a} r} r_{\bar{a}}}, \\
& { }^{3} \hat{g}^{r s}=\delta^{r s} e^{-2 q_{r}}=\delta^{r s} e^{-2 q-\frac{2}{\sqrt{3}} \sum_{\bar{a}} \gamma_{\bar{a} r} r_{\bar{a}}}=\delta^{r s} \phi^{-4} e^{-\frac{2}{\sqrt{3}} \sum_{\bar{a}} \gamma_{\bar{a}} r r_{\bar{a}}} \\
& \rightarrow_{r_{\bar{a}} \rightarrow 0} \delta^{r s} e^{-2 q}=\delta^{r s} \phi^{-4} \rightarrow_{q \rightarrow 0} \delta^{r s}, \quad \rightarrow_{q \rightarrow 0} \delta^{r s} e^{-\frac{2}{\sqrt{3}} \sum_{\bar{a}} \gamma_{\bar{a} r} r_{\bar{a}}} \\
& { }^{3} \hat{e}=\sqrt{\hat{\gamma}}=e^{\sum_{r} q_{r}}=e^{3 q}=\phi^{6} \rightarrow_{q \rightarrow 0} 1, \\
& { }^{3} \hat{\Gamma}_{u v}^{r}=-\delta_{u v} \sum_{s} \delta_{s}^{r} e^{2\left(q_{u}-q_{s}\right)} \partial_{s} q_{u}+\delta_{u}^{r} \partial_{v} q_{u}+\delta_{v}^{r} \partial_{u} q_{v}= \\
& =-\delta_{u v} \sum_{s} \delta_{s}^{r} e^{\frac{2}{\sqrt{3}} \sum_{\bar{a}}\left(\gamma_{\bar{a} u}-\gamma_{\bar{a}}\right) r_{\bar{a}}}\left[2 \partial_{s} \ln \phi+\frac{1}{\sqrt{3}} \sum_{\bar{b}} \gamma_{\bar{b} u} \partial_{s} r_{\bar{b}}\right]+ \\
& +\delta_{u}^{r}\left[2 \partial_{v} \ln \phi+\frac{1}{\sqrt{3}} \sum_{\bar{a}} \gamma_{\bar{a} u} \partial_{v} r_{\bar{a}}\right]+\delta_{v}^{r}\left[2 \partial_{u} \ln \phi+\frac{1}{\sqrt{3}} \sum_{\bar{a}} \gamma_{\bar{a} v} \partial_{u} r_{\bar{a}}\right] \\
& \rightarrow_{r_{\bar{a}} \rightarrow 0} 2\left[-\delta_{u v} \sum_{s} \delta_{s}^{r} \partial_{s} \ln \phi+\delta_{u}^{r} \partial_{v} \ln \phi+\delta_{v}^{r} \partial_{u} \ln \phi \rightarrow_{q \rightarrow 0} 0,\right. \\
& \rightarrow_{q \rightarrow 0} \frac{1}{\sqrt{3}}\left(-\delta_{u v} \sum_{s} \delta_{s}^{r} e^{\frac{2}{\sqrt{3}} \sum_{\bar{a}}\left(\gamma_{\bar{a} u}-\gamma_{\bar{a} s}\right) r_{\bar{a}}} \sum_{\bar{b}} \gamma_{\bar{b} u} \partial_{s} r_{\bar{b}}+\right. \\
& \left.+\sum_{\bar{a}}\left[\delta_{u}^{r} \gamma_{\bar{a} u} \partial_{v} r_{\bar{a}}+\delta_{v}^{r} \gamma_{\bar{a} v} \partial_{u} r_{\bar{a}}\right]\right) \text {, } \\
& \sum_{u}{ }^{3} \hat{\Gamma}_{u v}^{u}=\partial_{v} \sum_{u} q_{u}=3 \partial_{v} q=6 \partial_{v} \ln \phi, \\
& { }^{3} \hat{\omega}_{r(a)}=\epsilon_{(a)(b)(c)} \delta_{(b) r} \delta_{(c) u} e^{q_{r}-q_{u}} \partial_{u} q_{r}= \\
& \left.=\epsilon_{(a)(b)(c)} \delta_{(b) r} \delta_{(c) u} e^{\frac{1}{\sqrt{3}} \sum_{\bar{a}}\left(\gamma_{\bar{a} r}-\gamma_{\bar{a} u}\right) r_{\bar{a}}}\left[2 \partial_{u} \ln \phi+\frac{1}{\sqrt{3}} \sum_{\bar{b}} \gamma_{\bar{b} r} \partial_{u} r_{\bar{b}}\right]\right] \\
& \rightarrow_{r_{\bar{a}} \rightarrow 0} \epsilon_{(a)(b)(c)} \delta_{(b) r} \delta_{(c) u} 2 \partial_{u} \ln \phi \rightarrow_{q \rightarrow 0} 0 \\
& \rightarrow_{q \rightarrow 0} \frac{1}{\sqrt{3}} \epsilon_{(a)(b)(c)} \sum_{u} \delta_{(b) r} \delta_{(c) u} e^{\frac{1}{\sqrt{3}} \sum_{\bar{a}}\left(\gamma_{\bar{a} r}-\gamma_{\bar{a} u}\right) r_{\bar{a}}} \sum_{\bar{b}} \gamma_{\bar{b} r} \partial_{u} r_{\bar{b}}, \\
& \zeta_{(a)(b)}^{(\hat{\omega}) r}\left(\vec{\sigma}, \vec{\sigma}_{1} ; \tau\right)=d_{\gamma_{P P_{1}}}^{r}\left(\vec{\sigma}, \vec{\sigma}_{1}\right)\left(P_{\gamma_{P P_{1}}} e^{\int_{\vec{\sigma}_{1}}^{\vec{\sigma}} d \sigma_{2}^{w 3} \hat{\omega}_{w(c)}\left(\tau, \vec{\sigma}_{2}\right) \hat{R}^{(c)}}\right)_{(a)(b)} \\
& \rightarrow_{q, r_{\bar{a}} \rightarrow 0} \quad \zeta_{(a)(b)}^{(o) r}\left(\vec{\sigma}, \vec{\sigma}_{1}\right)=-\frac{\sigma^{r}-\sigma_{1}^{r}}{4 \pi\left|\vec{\sigma}-\vec{\sigma}_{1}\right|^{3}} \delta_{(a)(b)}=-\delta_{(a)(b)} \frac{\partial}{\partial \sigma_{1}^{r}} \frac{1}{4 \pi\left|\vec{\sigma}-\vec{\sigma}_{1}\right|} .
\end{aligned}
$$

See Appendix A for the expression of ${ }^{3} \Omega_{r s(a)},{ }^{3} R_{r s u v},{ }^{3} R_{r s},{ }^{3} R$ [29 and of other 3-tensors.

Moreover from Eqs.(5.15)-(5.20), by choosing the representation of the cotriad momentum satisfying $\hat{\mathcal{H}}_{(a)}(\tau, \vec{\sigma})=0$, we find

${ }^{129}$ We find that ${ }^{3} \omega_{r(a)}$ goes as $O\left(r^{-2}\right)$, while ${ }^{3} \hat{R}_{r s u v}(\tau, \vec{\sigma})$ and ${ }^{3} \hat{\Omega}_{r s(a)}(\tau, \vec{\sigma})$ go as $O\left(r^{-3}\right)$ for $r \rightarrow \infty$. 


$$
\begin{aligned}
& 3 \hat{\tilde{\pi}}_{(a)}^{r}(\tau, \vec{\sigma})=\left.{ }^{3} \tilde{\pi}_{(a)}^{r}(\tau, \vec{\sigma})\right|_{\alpha_{(a)}=0, \xi^{r}=\sigma^{r}, \tilde{\pi}_{(a)}^{\vec{\alpha}}=\tilde{\pi}_{r}^{\vec{\xi}}=0}= \\
&=\left.{ }^{3} \tilde{\pi}_{(a)}^{r}(\tau, \vec{\sigma})\right|_{\left((a)=0, \xi^{r}=\sigma^{r}\right.}= \\
&= \sum_{s} \int d^{3} \sigma_{1} \mathcal{K}_{(a) s}^{r}\left(\vec{\sigma}, \vec{\sigma}_{1} ; \tau\right) \tilde{\Pi}^{s}\left(\tau, \vec{\sigma}_{1}\right)= \\
&= \sum_{s} \int d^{3} \sigma_{1} \mathcal{K}_{(a) s}^{r}\left(\vec{\sigma}, \vec{\sigma}_{1} ; \tau\right)\left(\phi^{-2} e^{-\frac{1}{\sqrt{3}} \sum_{\bar{a}} \gamma_{\bar{a} s} r_{\bar{a}}}\right)\left(\tau, \vec{\sigma}_{1}\right) \\
& {\left[\frac{1}{3} \rho+\sqrt{3} \sum_{\bar{b}} \gamma_{\bar{b} s} \pi_{\bar{b}}\right]\left(\tau, \vec{\sigma}_{1}\right) \quad \rho=\pi_{\phi} / 2 \phi } \\
& \rightarrow_{\rho \rightarrow 0} \quad \sqrt{3} \sum_{s, \bar{b}} \gamma_{\bar{b} s} \int d^{3} \sigma_{1} \mathcal{K}_{(a) s}^{r}\left(\vec{\sigma}, \vec{\sigma}_{1} ; \tau \mid \phi, r_{\bar{a}}\right]\left(\phi^{-2} e^{-\frac{1}{\sqrt{3}} \sum_{\bar{a}} \gamma_{\bar{a} s} r_{\bar{a}}} \pi_{\bar{b}}\right)\left(\tau, \vec{\sigma}_{1}\right),
\end{aligned}
$$

with the kernel

$$
\begin{aligned}
\mathcal{K}_{(a) s}^{r}\left(\vec{\sigma}, \vec{\sigma}_{1}, \tau\right) & =\tilde{\mathcal{K}}_{(a) s}^{r}\left(\vec{\sigma}, \vec{\sigma}_{1}, \tau \mid \phi, r_{\bar{a}}\right] \stackrel{\text { def }}{=} \delta_{(a)}^{r} \delta_{s}^{r} \delta^{3}\left(\vec{\sigma}, \vec{\sigma}_{1}\right)+\mathcal{T}_{(a) s}^{r}\left(\vec{\sigma}, \vec{\sigma}_{1}, \tau\right), \\
\mathcal{T}_{(a) s}^{r}\left(\vec{\sigma}, \vec{\sigma}_{1}, \tau\right) & =\tilde{\mathcal{T}}_{(a) s}^{r}\left(\vec{\sigma}, \vec{\sigma}_{1}, \tau \mid \phi, r_{\bar{a}}\right]= \\
& =-Q_{s}\left(\tau, \vec{\sigma}_{1}\right) \frac{\partial G_{(a)}^{r s}\left(\vec{\sigma}, \vec{\sigma}_{1} ; \tau \mid \phi, r_{\bar{a}}\right]}{\partial \sigma_{1}^{u}}-\sum_{v} \frac{\partial Q_{s}\left(\tau, \vec{\sigma}_{1}\right)}{\partial \sigma_{1}^{v}} G_{(a)}^{r v}\left(\vec{\sigma}, \vec{\sigma}_{1} ; \tau \mid \phi, r_{\bar{a}}\right],
\end{aligned}
$$

where the splitting of $\mathcal{K}_{(a) u}^{r}$ follows from Eqs.(5.21).

From Eqs.(3.12) and (4.2)of Ref. [1] in the 3-orthogonal gauges the extrinsic curvature of $\Sigma_{\tau}^{(W S W)}$, the ADM momentum and the ADM Wheeler-DeWitt supermetric become

$$
\begin{aligned}
{ }^{3} \hat{K}_{r s}(\tau, \vec{\sigma})= & \frac{\epsilon 4 \pi G}{c^{3}}\left[e^{\frac{1}{\sqrt{3}} \sum_{\bar{c}}\left(\gamma_{\bar{c} r}+\gamma_{\bar{c} s}\right) r_{\bar{c}}} \sum_{u}\left(\delta_{r u} \delta_{(a) s}+\delta_{s u} \delta_{(a) r}-\delta_{r s} \delta_{(a) u}\right)\right. \\
& \left.e^{\frac{1}{\sqrt{3}} \sum_{\bar{c}} \gamma_{\bar{c} u} r_{\bar{c}} 3} \hat{\tilde{\pi}}_{(a)}^{u}\right](\tau, \vec{\sigma}), \\
{ }^{3} \hat{K}(\tau, \vec{\sigma})= & -\frac{\epsilon 4 \pi G}{c^{3}}\left[\phi^{-4} \sum_{u} \delta_{(a) u} e^{\frac{1}{\sqrt{3}} \sum_{\bar{c}} \gamma_{\bar{c} u} r_{\bar{c}}} 3 \hat{\tilde{\pi}}_{(a)}^{u}\right](\tau, \vec{\sigma})=\quad\left[\rho=\pi_{\phi} / 2 \phi\right] \\
= & -\frac{\epsilon 4 \pi G}{c^{3}} \phi^{-4}(\tau, \vec{\sigma}) \sum_{u} \delta_{(a) u} e^{\frac{1}{\sqrt{3}} \sum_{\bar{c}} \gamma_{\bar{c} u} r_{\bar{c}}(\tau, \vec{\sigma})} \\
& \sum_{s} \int d^{3} \sigma_{1} \mathcal{K}_{(a) s}^{u}\left(\vec{\sigma}, \vec{\sigma}_{1} ; \tau \mid \phi, r_{\bar{a}}\right]\left(\phi^{-2} e^{-\frac{1}{\sqrt{3}} \sum_{\bar{a}} \gamma_{\bar{a} s} r_{\bar{a}}}\right)\left(\tau, \vec{\sigma}_{1}\right) \\
& {\left[\frac{1}{3} \rho+\sqrt{3} \sum_{\bar{b}} \gamma_{\bar{b} s} \pi_{\bar{b}}\right]\left(\tau, \vec{\sigma}_{1}\right) } \\
\rightarrow{ }_{\rho \rightarrow 0} & -\frac{\epsilon 4 \sqrt{3} \pi G}{c^{3}} \phi^{-4}(\tau, \vec{\sigma}) \sum_{u} \delta_{(a) u} e^{\frac{1}{\sqrt{3}} \sum_{\bar{c}} \gamma_{\bar{c} u} r_{\bar{c}}(\tau, \vec{\sigma})} \\
& \sum_{s} \sum_{\bar{b}} \gamma_{\bar{b} s} \int d^{3} \sigma_{1} \mathcal{K}_{(a) s}^{r}\left(\vec{\sigma}, \vec{\sigma}_{1} ; \tau \mid \phi, r_{\bar{a}}\right]\left(\phi^{-2} e^{-\frac{1}{\sqrt{3}} \sum_{\bar{a}} \gamma_{\bar{a} s} r_{\bar{a}}} \pi_{\bar{b}}\right)\left(\tau, \vec{\sigma}_{1}\right),
\end{aligned}
$$




$$
\begin{aligned}
{ }^{3} \hat{\tilde{\Pi}}^{r s}(\tau, \vec{\sigma})= & \left.\frac{1}{4}{ }^{3} \hat{e}_{(a)}^{r}{ }^{3} \hat{\tilde{\pi}}_{(a)}^{s}+{ }^{3} \hat{e}_{(a)}^{s}{ }^{3} \hat{\tilde{\pi}}_{(a)}^{r}\right](\tau, \vec{\sigma})= \\
= & \frac{1}{4} \phi^{-2}(\tau, \vec{\sigma})\left[e^{-\frac{1}{\sqrt{3}} \sum_{\bar{a}} \gamma_{\bar{a} r} r_{\bar{a}}} \delta_{(a)}^{r} 3 \hat{\tilde{\pi}}_{(a)}^{s}+\right. \\
+ & \left.e^{-\frac{1}{\sqrt{3}} \sum_{\bar{a}} \gamma_{\bar{a} s} r_{\bar{a}}} \delta_{(a)}^{s}{ }^{3} \hat{\tilde{\pi}}_{(a)}^{r}\right](\tau, \vec{\sigma})= \\
= & \frac{1}{4} \phi^{-2}(\tau, \vec{\sigma})\left[e^{-\frac{1}{\sqrt{3}} \sum_{\bar{a}} \gamma_{\bar{a} r} r_{\bar{a}}} \delta_{(a)}^{r}\right. \\
& \sum_{u} \int d^{3} \sigma_{1} \mathcal{K}_{(a) u}^{r}\left(\vec{\sigma}, \vec{\sigma}_{1}, \tau \mid \phi, r_{\bar{a}}, \tilde{\Pi}\right]+ \\
+ & \left.e^{-\frac{1}{\sqrt{3}} \sum_{\bar{a}} \gamma_{\bar{a} s} r_{\bar{a}}} \delta_{(a)}^{s} \sum_{u} \int d^{3} \sigma_{1} \mathcal{K}_{(a) u}^{s}\left(\vec{\sigma}, \vec{\sigma}_{1}, \tau \mid \phi, r_{\bar{a}}, \tilde{\Pi}\right]\right] \\
& \left(\phi^{-2} e^{-\frac{1}{\sqrt{3}} \sum_{\bar{a}} \gamma_{\bar{a} u} r_{\bar{a}}}\right)\left(\tau, \vec{\sigma}_{1}\right)\left[\frac{1}{3} \rho+\sqrt{3} \sum_{\bar{b}} \gamma_{\bar{b} u} \pi_{\bar{b}}\right]\left(\tau, \vec{\sigma}_{1}\right), \\
{ }^{3} \hat{G}_{r s u v}(\tau, \vec{\sigma})= & {\left[\hat{g}_{r u}^{3} \hat{g}_{s v}+{ }^{3} \hat{g}_{r v}{ }^{3} \hat{g}_{s u}-{ }^{3} \hat{g}_{r s}{ }^{3} \hat{g}_{u v}\right](\tau, \vec{\sigma})=} \\
= & \phi^{8}(\tau, \vec{\sigma})\left[e^{\frac{2}{\sqrt{3}} \sum_{\bar{a}}\left(\gamma_{\bar{a} r}+\gamma_{\bar{a} s}\right) r_{\bar{a}}}\left(\delta_{r u} \delta_{s v}+\delta_{r v} \delta_{s u}\right)-\right. \\
- & \left.e^{\frac{2}{\sqrt{3}} \sum_{\bar{a}}\left(\gamma_{\bar{a} r}+\gamma_{\bar{a} u}\right) r_{\bar{a}}} \delta_{r s} \delta_{u v}\right](\tau, \vec{\sigma}) .
\end{aligned}
$$

The momenta ${ }^{3} \hat{\tilde{\pi}}_{(a)}^{r}$ and ${ }^{3} \hat{\tilde{\Pi}}^{r s}$ and the mean extrinsic curvature ${ }^{3} \hat{K}$ are linear functions of the new momenta $\rho$ and $\pi_{\bar{c}}$, but with a coordinate- and momentum-dependent integral kernel. The determination of the gravitomagnetic potential $W_{\pi}^{r}(\tau, \vec{\sigma})$, see Appendix C of II, by solving the elliptic equations associated with the supermomentum constraints in the conformal approach to metric gravity, has been replaced here by the determination of the kernel $\mathcal{K}_{(a) s}^{r}\left(\vec{\sigma}, \vec{\sigma}^{\prime} ; \tau\right)$ connecting the old momenta ${ }^{3} \hat{\tilde{\pi}}_{(a)}^{r}(\tau, \vec{\sigma})$ to the new canonical ones $\tilde{\Pi}^{r}(\tau, \vec{\sigma})$, and therefore to the solution of the linear partial differential equations (5.23) and (5.26).

Let us remark that at the level of the Dirac brackets for the 3-orthogonal gauges we have the strong vanishing of the ADM supermomentum constraints ${ }^{3} \tilde{\Pi}^{r s}(\tau, \vec{\sigma})_{\mid s} \equiv 0$ of Eqs.(4.16) of Ref. [1], so that (see Appendix C of II) the ADM momentum ${ }^{3} \tilde{\Pi}^{r s}(\tau, \vec{\sigma})$ of metric gravity becomes transverse and is the sum of a TT-term and of a trace term [77, 65, 79, The determination of ${ }^{3} \tilde{\Pi}_{T T}^{r s}(\tau, \vec{\sigma})$ can be done once one has found the solution of Eqs.(5.23), (5.26).

The variables $\rho$ and $\pi_{\bar{a}}$ replace ${ }^{3} \hat{K}$ and ${ }^{3} \hat{K}_{T T}^{r s} \quad$ or ${ }^{3} \hat{\tilde{\Pi}}$ and ${ }^{3} \hat{\tilde{\Pi}}_{T T}^{r s}$ of the conformal approach respectively (see Appendix $\mathrm{C}$ of II) after the solution of the supermomentum constraints (i.e. after the determination of the gravitomagnetic potential) in the 3-orthogonal gauges. It would be important to find the expression of $\rho$ and $\pi_{\bar{a}}$ in terms of ${ }^{3} \hat{g}_{r s}$ and ${ }^{3} \hat{K}_{r s}$ [or $\left.{ }^{3} \hat{\tilde{\Pi}}^{r s}\right]$. The equation for ${ }^{3} \hat{K}$ can be read as an integral equation to get $\rho(\tau, \vec{\sigma})$, the momentum conjugate to the conformal factor, in terms of ${ }^{3} \hat{K}(\tau, \vec{\sigma}), q(\tau, \vec{\sigma})=\frac{1}{6} \ln ^{3} \hat{g}(\tau, \vec{\sigma})$ and $r_{\bar{a}}(\tau, \vec{\sigma})=$ $\frac{\sqrt{3}}{2} \sum_{r} \gamma_{\bar{a} r} \ln \left[{ }^{3} \hat{g}_{r r} /{ }^{3} \hat{g}\right](\tau, \vec{\sigma})$ [see Eqs.6.13)], in the 3-orthogonal gauges.

Let us remark that if we would have added only the gauge-fixing $\alpha_{(a)}(\tau, \vec{\sigma}) \approx 0$ ए30,

\footnotetext{
${ }^{130}$ So that the associated Dirac brackets would coincide with the ADM Poisson brackets for metric gravity.
} 
the four variables $\vec{\xi}(\tau, \vec{\sigma}), q(\tau, \vec{\sigma}){ }^{131}$ of the canonical basis (6.11) would correspond to the variables used in Ref. [66 to label the points of the spacetime $M^{4}$ (assumed compact), following the suggestion of Ref. [69], if $q(\tau, \vec{\sigma})$ is interpreted as a time variable. However, we do not follow this interpretation.

In Appendix B there is the expression of the weak and strong Poincare charges of Eqs. 2.25) and (2.23) in the new canonical basis in 3-orthogonal gauges.

The shift functions $n_{r}(\tau, \vec{\sigma})$ of Eq. (6.8) in the rest-frame instant form on the WSW hypersurfaces and in 3-orthogonal gauges become

$$
\begin{aligned}
n_{r}(\tau, \vec{\sigma}) \approx & -\frac{\epsilon 4 \pi G}{c^{3}}\left[\phi^{4} e^{\frac{2}{\sqrt{3}} \sum_{\bar{a}} \gamma_{\bar{a} r} r_{\bar{a}}}\right](\tau, \vec{\sigma}) \int d^{3} \sigma_{1}\left[\epsilon-n\left(\tau, \vec{\sigma}_{1}\right)\right] \\
& \phi^{-2}\left(\tau, \vec{\sigma}_{1}\right) \sum_{w u} e^{\frac{1}{\sqrt{3}} \sum_{\bar{a}}\left(\gamma_{\bar{a} w}+\gamma_{\bar{a} u}\right) r_{\bar{a}} 3} G_{o(a)(b)(c)(d)} \\
& \delta_{(a) w} \sum_{v} \int d^{3} \sigma_{2} \mathcal{K}_{(b) v}^{w}\left(\vec{\sigma}_{1}, \vec{\sigma}_{2} ; \tau\right)\left[\phi^{-2} e^{-\frac{1}{\sqrt{3}} \sum_{\bar{a}} \gamma_{\bar{a} v} r_{\bar{a}}}\right]\left(\tau, \vec{\sigma}_{2}\right) \\
& {\left[\frac{1}{3} \rho+\sqrt{3} \sum_{\bar{b}} \gamma_{\bar{b} v} \pi_{\bar{b}}\right]\left(\tau, \vec{\sigma}_{2}\right) \delta_{(c) u} G_{(d)}^{u r}\left(\vec{\sigma}_{1}, \vec{\sigma} ; \tau\right), } \\
\rightarrow \rho_{\rho \rightarrow 0} \quad & -\frac{\epsilon 4 \sqrt{3} \pi G}{c^{3}}\left[\phi^{2} e^{\frac{1}{\sqrt{3}} \sum_{\bar{a}} \gamma_{\bar{a}} r_{\bar{a}}}\right](\tau, \vec{\sigma}) \int d^{3} \sigma_{1}\left[\epsilon-n\left(\tau, \vec{\sigma}_{1}\right)\right] \phi^{-2}\left(\tau, \vec{\sigma}_{1}\right) \\
& \sum_{w u} e^{\frac{1}{\sqrt{3}} \sum_{\bar{a}}\left(\gamma_{\bar{a} w}+\gamma_{\bar{a} u}\right) r_{\bar{a}}\left(\tau, \vec{\sigma}_{1}\right)}\left(\delta_{w u} \delta_{(b)(d)}+\delta_{(b) u} \delta_{(d) w}-\delta_{(b) w} \delta_{(d) u}\right) \\
& \sum_{v} \int d^{3} \sigma_{2} \mathcal{K}_{(b) v}^{w}\left(\vec{\sigma}_{1}, \vec{\sigma}_{2} ; \tau\right)\left[\phi^{-2} e^{\left.-\frac{1}{\sqrt{3}} \sum_{\bar{a}} \gamma_{\bar{a} u} r_{\bar{a}}\right]}\right]\left(\tau, \vec{\sigma}_{2}\right) \\
& \sum_{\bar{b}} \gamma_{\bar{b} v} \pi_{\bar{b}}\left(\tau, \vec{\sigma}_{2}\right) G_{(d)}^{u r}\left(\vec{\sigma}_{1}, \vec{\sigma} ; \tau\right) .
\end{aligned}
$$

\section{The Superhamiltonian Constraint as the Reduced Lichnerowicz Equation.}

By using the new canonical basis and Eq. (6.15) the superhamiltonian constraint (2.11), (5.33) restricted to 3 -orthogonal gauges becomes $\left[\rho=\pi_{\phi} / 2 \phi\right]$

$$
\begin{aligned}
\hat{\mathcal{H}}_{R}(\tau, \vec{\sigma})= & -\epsilon \frac{2 \pi G \phi^{-2}(\tau, \vec{\sigma})}{c^{3}}\left[\left(\phi^{-4}\left[6 \sum_{\bar{a}} \pi_{\bar{a}}^{2}-\frac{1}{3} \rho^{2}\right]\right)(\tau, \vec{\sigma})+\right. \\
+ & 2\left(\phi^{-2} \sum_{u} e^{\frac{1}{\sqrt{3}} \sum_{\bar{a}} \gamma_{\bar{a} u} r_{\bar{a}}}\left[2 \sqrt{3} \sum_{\bar{b}} \gamma_{\bar{b} u} \pi_{\bar{b}}-\frac{1}{3} \rho\right]\right)(\tau, \vec{\sigma}) \times \\
& \int d^{3} \sigma_{1} \sum_{r} \delta_{(a)}^{u} \mathcal{T}_{(a) r}^{u}\left(\vec{\sigma}, \vec{\sigma}_{1}, \tau\right)\left(\phi^{-2} e^{-\frac{1}{\sqrt{3}} \sum_{\bar{a}} \gamma_{\bar{a} r} r_{\bar{a}}}\left[\frac{\rho}{3}+\sqrt{3} \sum_{\bar{b}} \gamma_{\bar{b} r} \pi_{\bar{b}}\right]\right)\left(\tau, \vec{\sigma}_{1}\right)+ \\
+ & \int d^{3} \sigma_{1} d^{3} \sigma_{2}\left(\sum_{u} e^{\frac{2}{\sqrt{3}} \sum_{\bar{a}} \gamma_{\bar{a} u} r_{\bar{a}}(\tau, \vec{\sigma})} \times\right.
\end{aligned}
$$

${ }^{131}$ With conjugate momenta $\tilde{\pi}_{r}^{\vec{\xi}}(\tau, \vec{\sigma}) \approx 0, \rho(\tau, \vec{\sigma})$. 


$$
\begin{aligned}
& \sum_{r} \mathcal{T}_{(a) r}^{u}\left(\vec{\sigma}, \vec{\sigma}_{1}, \tau\right)\left(\phi^{-2} e^{-\frac{1}{\sqrt{3}} \sum_{\bar{a}} \gamma_{\bar{a}} r r_{\bar{a}}}\left[\frac{\rho}{3}+\sqrt{3} \sum_{\bar{b}} \gamma_{\bar{b} r} \pi_{\bar{b}}\right]\right)\left(\tau, \vec{\sigma}_{1}\right) \times \\
& \sum_{s} \mathcal{T}_{(a) s}^{u}\left(\vec{\sigma}, \vec{\sigma}_{2}, \tau\right)\left(\phi^{-2} e^{-\frac{1}{\sqrt{3}} \sum_{\bar{a}} \gamma_{\bar{a} s} r_{\bar{a}}}\left[\frac{\rho}{3}+\sqrt{3} \sum_{\bar{c}} \gamma_{\bar{c} s} \pi_{\bar{c}}\right]\right)\left(\tau, \vec{\sigma}_{2}\right)+ \\
+ & \sum_{u v} e^{\frac{1}{\sqrt{3}} \sum_{\bar{a}}\left(\gamma_{\bar{a} u}+\gamma_{\bar{a} v}\right) r_{\bar{a}}(\tau, \vec{\sigma})}\left(\delta_{(b)}^{u} \delta_{(a)}^{v}-\delta_{(a)}^{u} \delta_{(b)}^{v}\right) \times \\
& \sum_{r} \mathcal{T}_{(a) r}^{u}\left(\vec{\sigma}, \vec{\sigma}_{1}, \tau\right)\left(\phi^{-2} e^{-\frac{1}{\sqrt{3}} \sum_{\bar{a}} \gamma_{\bar{a}} r r_{\bar{a}}}\left[\frac{\rho}{3}+\sqrt{3} \sum_{\bar{b}} \gamma_{\bar{b} r} \pi_{\bar{b}}\right]\right)\left(\tau, \vec{\sigma}_{1}\right) \\
& \left.\left.\sum_{s} \mathcal{T}_{(b) s}^{v}\left(\vec{\sigma}, \vec{\sigma}_{2}, \tau\right)\left(\phi^{-2} e^{-\frac{1}{\sqrt{3}} \sum_{\bar{a}} \gamma_{\bar{a} s} r_{\bar{a}}}\left[\frac{\rho}{3}+\sqrt{3} \sum_{\bar{c}} \gamma_{\bar{c} s} \pi_{\bar{c}}\right]\right)\left(\tau, \vec{\sigma}_{2}\right)\right)\right]+ \\
+ & \frac{\epsilon c^{3}}{16 \pi G} \sum_{r, s}\left[\phi^{2} e^{-\frac{1}{\sqrt{3}} \sum_{\bar{a}}\left(\gamma_{\bar{a}} r \gamma_{\bar{a} s}\right) r_{\bar{a}}}\right](\tau, \vec{\sigma}) \epsilon_{(a)(b)(c)} \delta_{(a) r} \delta_{(b) s}{ }^{3} \hat{\Omega}_{r s(c)}\left[\phi, r_{\bar{c}}\right](\tau, \vec{\sigma}) \approx 0 \\
& \left\{\hat{\mathcal{H}}_{R}(\tau, \vec{\sigma}), \hat{\mathcal{H}}_{R}\left(\tau, \vec{\sigma}^{\prime}\right)\right\}^{*} \equiv\left\{\hat{\mathcal{H}}(\tau, \vec{\sigma}), \hat{\mathcal{H}}\left(\tau, \vec{\sigma}^{\prime}\right)\right\}^{*} \equiv \\
& \equiv\left(-\frac{\partial}{\partial \sigma^{s}}\left[\left\{\xi^{r}(\tau, \vec{\sigma}), \hat{\mathcal{H}}\left(\tau, \vec{\sigma}^{\prime}\right)\right\} \hat{\mathcal{H}}_{R}(\tau, \vec{\sigma})\right]+\right. \\
& \left.+\frac{\partial}{\partial \sigma^{\prime} s}\left[\left\{\xi^{r}\left(\tau, \vec{\sigma}^{\prime}\right), \hat{\mathcal{H}}(\tau, \vec{\sigma})\right\} \hat{\mathcal{H}}_{R}\left(\tau, \vec{\sigma}^{\prime}\right)\right]\right)_{\xi^{r}=\sigma^{r}} \approx 0
\end{aligned}
$$

The last line of $\hat{\mathcal{H}}_{R}$ is equal to $\frac{\epsilon c^{3}}{16 \pi G} \phi^{6} \hat{R}\left[\phi, r_{\bar{a}}\right]$.

The constraint is no more an algebraic relation among the final variables, but rather an integro-differential equation, the reduced Lichnerowicz equation, whose unknown is the conformal factor $\phi(\tau, \vec{\sigma})=e^{\frac{1}{2} q(\tau, \vec{\sigma})}$ as said in Subsection D of Section III for the case of asymptotically flat spacetimes. Its solution in 3-orthogonal coordinates gives $\phi=e^{q / 2}$ as a functional $e^{F\left[r_{\bar{a}}, \pi_{\bar{a}}, \pi_{\phi}\right]}$ of the canonical variables $r_{\bar{a}}(\tau, \vec{\sigma}), \pi_{\bar{a}}(\tau, \vec{\sigma})$, and of the last gauge variable: the momentum $\pi_{\phi}(\tau, \vec{\sigma})=2 e^{-q(\tau \vec{\sigma}) / 2} \rho(\tau, \vec{\sigma})$ conjugate to the conformal factor.

In the 3-orthogonal gauges the functions $r_{\bar{a}}(\tau, \vec{\sigma}), \bar{a}=1,2$, give a parametrization of the Hamiltonian physical degrees of freedom of the gravitational field and of the space of conformal 3-geometries [33: it turns out that a point (a conformal 3-geometry) in this space, i.e. a ${ }^{3} \hat{g}_{r s}^{\text {diag }}$ [3], is an equivalence class of conformally related 3-metrics (conformal gauge orbit).

The solution $\phi=e^{q / 2} \approx e^{F\left[r_{\bar{a}}, \pi_{\bar{a}}, \pi_{\phi}\right]}$ of the reduced Lichnerowicz equation in these gauges just determines an equivalence class of 3-geometries (i.e. a conformal 3-geometry) parametrized by the gauge variable $\rho(\tau, \vec{\sigma})=2\left[\phi^{-1} \pi_{\phi}\right](\tau, \vec{\sigma})$ (it is the coordinate in the conformal gauge orbit); the natural representative of an equivalence class is obtained with the gauge-fixing $\rho(\tau, \vec{\sigma}) \approx 0:{ }^{3} \hat{g}_{r s}=e^{4 F\left[r_{\bar{a}}, \pi_{\bar{a}}, 0\right]}{ }^{3} \hat{g}_{r s}^{\text {diag }}\left[r_{\bar{a}}, \pi_{\bar{a}}\right]$.

When we add the natural gauge-fixing $\rho(\tau, \vec{\sigma}) \approx 0$ to the reduced superhamiltonian constraint in 3-orthogonal coordinates on $\Sigma_{\tau}^{(W S W)}$ and go to Dirac brackets eliminating

\footnotetext{
${ }^{132}$ The quotient of superspace by the group $W e y l \Sigma_{\tau}$, if by varying $\rho$ the solution $\phi=e^{q / 2} \approx$ $e^{F\left[r_{\bar{a}}, \pi_{\bar{a}}, \rho\right]}$ of the reduced Lichnerowicz equation spans all the Weyl rescalings.

${ }^{133}$ It is simultaneously the York 77 reduced metric and the Misner's one 64 in 3-orthogonal coordinates.
} 
the conjugate variables $q(\tau, \vec{\sigma}), \rho(\tau, \vec{\sigma})$ [or $\left.\phi, \pi_{\phi}\right]$, the functions $r_{\bar{a}}(\tau, \vec{\sigma})$ and $\pi_{\bar{a}}(\tau, \vec{\sigma})$ become the physical canonical variables for the gravitational field in this special 3-orthogonal gauge, because they have vanishing Poisson brackets with $\rho$. This does not happens with the gauge-fixing (maximal slicing condition) ${ }^{3} K(\tau, \vec{\sigma}) \approx 0$ (or const.) when we use 3-orthogonal coordinates.

Let us remark that usually (see for instance Ref. [106]) in metric gravity one introduces four gauge-fixing constraints on ${ }^{3} g_{r s},{ }^{3} \tilde{\Pi}^{r s}$ (they can be used also in tetrad gravity) instead of our gauge fixings $\vec{\xi}(\tau, \vec{\sigma})-\vec{\sigma} \approx 0, \rho(\tau, \vec{\sigma}) \approx 0$, whose functional form is oriented to the parametrizations needed for the Shanmugadhasan canonical transformations.

The evolution in $\tau$ (the time parameter labelling the leaves $\Sigma_{\tau}^{(W S W)}$ of the foliation associated with the $3+1$ splitting of $M^{4}$ ) is instead generated by the weak ADM energy $\hat{P}_{A D M}^{\tau}$, absent in closed spacetimes. The ADM energy, which, in this special gauge, depends only on $r_{\bar{a}}, \pi_{\bar{a}}$ is the Hamiltonian generating the $\tau$-evolution of the physical (non covariant) gravitational field degrees of freedom ${ }^{134}$.

However, since a closed form of the conformal factor in terms of $r_{\bar{a}}, \pi_{\bar{a}}$ as a solution of the superhamiltonian constraint (after having put $\rho(\tau, \vec{\sigma})=0$ in it) is not known, the ADM energy 135 cannot be explicitly expressed in terms of the physical degrees of freedom of the gravitational field in 3-orthogonal coordinates.

It seems quite difficult to be able to implement the last step of the program, namely to find the final Shanmugadhasan canonical transformation $\phi, \pi_{\phi}, r_{\bar{a}}, \pi_{\bar{a}} \mapsto \hat{\mathcal{H}}_{R}^{\prime}, \pi_{\phi}^{\prime}, r_{\bar{a}}^{\prime}, \pi_{\bar{a}}^{\prime}$ एँ, so that all the first class constraints of tetrad gravity appear in the final canonical basis in Abelianized form (this would implement Kuchar's program defined in Refs. [103],67]). Indeed, if $e^{F\left[r_{\bar{a}}, \pi_{\bar{a}}, \pi_{\phi}\right]}$ is the solution of the reduced Lichnerowicz equation, a functional form of such a $\hat{\mathcal{H}}_{R}^{\prime}$ is $\hat{\mathcal{H}}_{R}^{\prime}=\phi-e^{F\left[r_{\bar{a}}, \pi_{\bar{a}}, \pi_{\phi}\right]} \approx 0$. The existence of this final canonical transformation is connected to the integrability problem of Einstein equations: in Ref. [104 it is reported that they could admit chaotic solutions and, according to Newman, this would be an obstruction to the existence of Dirac observables in the final canonical basis.

Equally difficult is to find the analogue of the York map 81 in the 3-orthogonal gauges: $\phi, \pi_{\phi}, r_{\bar{a}}, \pi_{\bar{a}} \mapsto \mathcal{T}=-\frac{c^{3}}{12 \pi G} \epsilon^{3} \hat{K}, \mathcal{P}_{\mathcal{T}}=-\phi^{12}, r_{\bar{a}}^{(K)}, \pi_{\bar{a}}^{(K)}$.

To transform the superhamiltonian constraint in the reduced Lichnerowicz equation for the conformal factor, we shall use the canonical variable $\phi=e^{q / 2}$ but we shall go on to use the notation $\rho$ for $\frac{1}{2} \phi \pi_{\phi}$ for notational simplicity. By using Eq. A2 of Appendix A we get

$$
\begin{aligned}
{ }^{3} \hat{g}_{r s}= & \phi^{4} e^{\frac{2}{\sqrt{3}} \sum_{\bar{a}} \gamma_{\bar{a} r} r_{\bar{a}}} \delta_{r s} \equiv \phi^{43} \tilde{g}_{r s}, \quad{ }^{3} \tilde{g}_{r s}=e^{\frac{2}{\sqrt{3}} \sum_{\bar{a}} \gamma_{\bar{a} r} r_{\bar{a}}} \delta_{r s}, \\
& \Rightarrow \sum_{r}{ }^{3} \tilde{\Gamma}_{r s}^{r}=0, \\
{ }^{3} \hat{R}= & \phi^{-4}\left[-8^{3} \tilde{g}^{r s} \partial_{r} \partial_{s} \ln \phi-8^{3} \tilde{g}^{r s} \partial_{r} \ln \phi \partial_{s} \ln \phi-8 \partial_{r}{ }^{3} \tilde{g}^{r s} \partial_{s} \ln \phi+{ }^{3} \tilde{R}\left[r_{\bar{a}}\right]\right]=
\end{aligned}
$$

\footnotetext{
${ }^{134}$ This corresponds to the two dynamical equations contained in the 10 Einstein equations in this gauge.
}

${ }^{135}$ Weakly coinciding with the ADM invariant mass in the rest-frame instant form.

${ }^{136} \hat{\mathcal{H}}_{R}^{\prime}(\tau, \vec{\sigma}) \approx 0$ equivalent to $\hat{\mathcal{H}}_{R}(\tau, \vec{\sigma}) \approx 0$ but with $\left\{\hat{\mathcal{H}}_{R}^{\prime}(\tau, \vec{\sigma}), \hat{\mathcal{H}}_{R}^{\prime}\left(\tau, \vec{\sigma}^{\prime}\right)\right\}=0$. 


$$
\begin{aligned}
& =\phi^{-4}\left[\phi^{-1}\left(-8 \tilde{\triangle}\left[r_{\bar{a}}\right] \phi+8{ }^{3} \tilde{\Gamma}_{r s}^{r}{ }^{3} \tilde{g}^{s u} \partial_{u} \phi\right)+{ }^{3} \tilde{R}\left[r_{\bar{a}}\right]\right]= \\
& =\phi^{-5}\left[-8 \tilde{\triangle}\left[r_{\bar{a}}\right] \phi+{ }^{3} \tilde{R}\left[r_{\bar{a}}\right] \phi\right],
\end{aligned}
$$

where ${ }^{3} \tilde{R}={ }^{3} \tilde{R}\left[r_{\bar{a}}\right]$ and $\tilde{\triangle}=\tilde{\triangle}\left[r_{\bar{a}}\right]=\partial_{r}\left({ }^{3} \tilde{g}^{r s} \partial_{s}\right)$ are the scalar curvature and the LaplaceBeltrami operator associated with the 3 -metric ${ }^{3} \tilde{g}_{r s}$ respectively [37. From Eqs.(A3) of Appendix A, we have $\left[\tilde{\gamma}=\left.\operatorname{det}\right|^{3} \tilde{g}_{r s} \mid=1\right]$

$$
\begin{aligned}
& { }^{3} \hat{R}\left[\phi, r_{\bar{a}}\right]=-\sum_{u v}\left\{\left(2 \partial_{v} \ln \phi+\frac{1}{\sqrt{3}} \sum_{\bar{a}} \gamma_{\bar{a} u} \partial_{v} r_{\bar{a}}\right)\left(4 \partial_{v} \ln \phi-\frac{1}{\sqrt{3}} \sum_{\bar{b}} \gamma_{\bar{b} u} \partial_{v} r_{\bar{b}}\right)+\right. \\
& +\phi^{-4} e^{\frac{2}{\sqrt{3}} \sum_{\bar{c}} \gamma_{\bar{c} v} r_{\bar{c}}}\left[2 \partial_{v}^{2} \ln \phi+\frac{1}{\sqrt{3}} \sum_{\bar{a}} \gamma_{\bar{a} u} \partial_{v}^{2} r_{\bar{a}}+\right. \\
& +\frac{2}{\sqrt{3}}\left(2 \partial_{v} \ln \phi+\frac{1}{\sqrt{3}} \sum_{\bar{a}} \gamma_{\bar{a} u} \partial_{v} r_{\bar{a}}\right) \sum_{\bar{b}}\left(\gamma_{\bar{b} u}-\gamma_{\bar{b} v}\right) \partial_{v} r_{\bar{b}}- \\
& \left.-\left(2 \partial_{v} \ln \phi+\frac{1}{\sqrt{3}} \sum_{\bar{a}} \gamma_{\bar{a} v} \partial_{v} r_{\bar{a}}\right)\left(2 \partial_{v} \ln \phi+\frac{1}{\sqrt{3}} \sum_{\bar{b}} \gamma_{\bar{b} u} \partial_{v} r_{\bar{b}}\right]\right\}+ \\
& +\phi^{-4} \sum_{u} e^{\frac{2}{\sqrt{3}} \sum_{\bar{c}} \gamma_{\bar{c} u} r_{\bar{c}}}\left[-2 \partial_{u}^{2} \ln \phi+\frac{2}{\sqrt{3}} \sum_{\bar{a}} \gamma_{\bar{a} u} \partial_{u}^{2} r_{\bar{a}}+\right. \\
& \left.+\left(2 \partial_{u} \ln \phi+\frac{1}{\sqrt{3}} \sum_{\bar{a}} \gamma_{\bar{a} u} \partial_{u} r_{\bar{a}}\right)\left(2 \partial_{u} \ln \phi-\frac{2}{\sqrt{3}} \sum_{\bar{b}} \gamma_{\bar{b} u} \partial_{u} r_{\bar{b}}\right)\right] \\
& {\left[\rightarrow_{r_{\bar{a}} \rightarrow 0}-24 \sum_{u}\left(\partial_{u} \ln \phi\right)^{2}-8 \phi^{-4} \sum_{u}\left[\partial_{u}^{2} \ln \phi-2\left(\partial_{u} \ln \phi\right)^{2}\right] \rightarrow_{q \rightarrow 0} 0\right] \text {, }} \\
& \rightarrow_{q \rightarrow 0} \quad{ }^{3} \tilde{R}\left[r_{\bar{a}}\right]= \\
& =-\frac{1}{\sqrt{3}} \sum_{u v}\left\{-\frac{1}{\sqrt{3}} \sum_{\bar{a} \bar{b}} \gamma_{\bar{a} u} \gamma_{\bar{b} u} \partial_{v} r_{\bar{a}} \partial_{v} r_{\bar{b}}+e^{-\frac{2}{\sqrt{3}} \sum_{\bar{c}} \gamma_{\bar{c} v} r_{\bar{c}}} \sum_{\bar{a}} \gamma_{\bar{a} u} .\right. \\
& \left.\left[\partial_{v}^{2} r_{\bar{a}}+\frac{2}{\sqrt{3}} \sum_{\bar{b}}\left(\gamma_{\bar{b} u}-\gamma_{\bar{b} v}\right) \partial_{v} r_{\bar{a}} \partial_{v} r_{\bar{b}}-\frac{1}{\sqrt{3}} \sum_{\bar{b}} \gamma_{\bar{b} v} \partial_{v} r_{\bar{a}} \partial_{v} r_{\bar{b}}\right]\right\}+ \\
& +\frac{2}{\sqrt{3}} \sum_{u} e^{-\frac{2}{\sqrt{3}} \sum_{\bar{c}} \gamma_{\bar{c} u} r_{\bar{c}}} \sum_{\bar{a}} \gamma_{\bar{a} u}\left[\partial_{u}^{2} r_{\bar{a}}+\frac{1}{\sqrt{3}} \sum_{\bar{b}} \gamma_{\bar{b} u} \partial_{u} r_{\bar{a}} \partial_{u} r_{\bar{b}}\right]= \\
& =\frac{1}{3} \sum_{u}\left(1-2 e^{-\frac{2}{\sqrt{3}} \sum_{\bar{a}} \gamma_{\bar{a} u} r_{\bar{a}}}\right) \sum_{\bar{b}}\left(\partial_{u} r_{\bar{b}}\right)^{2}+ \\
& +\frac{2}{\sqrt{3}} \sum_{u} e^{-\frac{2}{\sqrt{3}} \sum_{\bar{c}} \gamma_{\bar{c} u} r_{\bar{c}}} \sum_{\bar{a}} \gamma_{\bar{a} u}\left[\partial_{u}^{2} r_{\bar{a}}+\frac{1}{\sqrt{3}} \sum_{\bar{b}} \gamma_{\bar{b} u} \partial_{u} r_{\bar{a}} \partial_{u} r_{\bar{b}}\right] \\
& \tilde{\triangle}\left[r_{\bar{a}}\right]=\partial_{r}\left[\tilde{g}^{r s} \partial_{s}\right]={ }^{3} \tilde{g}^{r s} 3 \tilde{\nabla}_{r}{ }^{3} \tilde{\nabla}_{s}= \\
& =\sum_{r} e^{-\frac{2}{\sqrt{3}} \sum_{\bar{a}} \gamma_{\bar{a} r} r_{\bar{a}}}\left[\partial_{r}^{2}-\frac{2}{\sqrt{3}} \sum_{\bar{b}} \gamma_{\bar{b} r} \partial_{r} r_{\bar{b}} \partial_{r}\right] .
\end{aligned}
$$

Using Eq.(6.18), the reduced superhamiltonian constraint becomes the following reduced Lichnerowicz equation

$137 \tilde{\triangle}-\frac{1}{8}{ }^{3} \tilde{R}$ is a conformally invariant operator 76 . 


$$
\begin{aligned}
& \tilde{\mathcal{H}}_{R}(\tau, \vec{\sigma})=\epsilon\left[\frac{c^{3}}{16 \pi G} \phi^{63} \hat{R}-\frac{2 \pi G \phi^{-6}}{c^{3}}{ }^{3} G_{o(a)(b)(c)(d)}{ }^{3} \hat{e}_{((a) r}{ }^{3} \hat{\tilde{\pi}}_{(b))}^{r}{ }^{3} \hat{e}_{((c) s}{ }^{3} \hat{\tilde{\pi}}_{(d))}^{s}\right](\tau, \vec{\sigma})= \\
& =\epsilon \phi(\tau, \vec{\sigma})\left[\frac{c^{3}}{16 \pi G}\left(-8 \tilde{\triangle}\left[r_{\bar{a}}\right]+{ }^{3} \tilde{R}\left[r_{\bar{a}}\right]\right) \phi-\right. \\
& \left.-\frac{2 \pi G \phi^{-7}}{c^{3}}{ }^{3} G_{o(a)(b)(c)(d)}{ }^{3} \hat{e}_{((a) r}{ }^{3} \hat{\tilde{\pi}}_{(b))}^{r} 3 \hat{e}_{((c) s} 3 \hat{\tilde{\pi}}_{(d))}^{s}\right](\tau, \vec{\sigma})= \\
& =\epsilon \phi(\tau, \vec{\sigma})\left[\frac{c^{3}}{16 \pi G}\left(-8 \tilde{\triangle}\left[r_{\bar{a}}\right]+{ }^{3} \tilde{R}\left[r_{\bar{a}}\right]\right) \phi-\right. \\
& -\frac{2 \pi G}{c^{3}}\left[\left(\phi^{-7}\left(6 \sum_{\bar{a}} \pi_{\bar{a}}^{2}-\frac{1}{3} \rho^{2}\right)\right)(\tau, \vec{\sigma})+\right. \\
& +2\left(\phi^{-5} \sum_{u} e^{\frac{1}{\sqrt{3}} \sum_{\bar{a}} \gamma_{\bar{a} u} r_{\bar{a}}}\left[2 \sqrt{3} \sum_{\bar{b}} \gamma_{\bar{b} u} \pi_{\bar{b}}-\frac{1}{3} \rho\right]\right)(\tau, \vec{\sigma}) \times \\
& \int d^{3} \sigma_{1} \sum_{r} \delta_{(a)}^{u} \mathcal{T}_{(a) r}^{u}\left(\vec{\sigma}, \vec{\sigma}_{1}, \tau\right)\left(\phi^{-2} e^{-\frac{1}{\sqrt{3}} \sum_{\bar{a}} \gamma_{\bar{a} r} r_{\bar{a}}}\left[\frac{\rho}{3}+\sqrt{3} \sum_{\bar{b}} \gamma_{\bar{b} r} \pi_{\bar{b}}\right]\right)\left(\tau, \vec{\sigma}_{1}\right)+ \\
& +\quad \phi^{-3}(\tau, \vec{\sigma}) \int d^{3} \sigma_{1} d^{3} \sigma_{2}\left(\sum_{u} e^{\frac{2}{\sqrt{3}} \sum_{\bar{a}} \gamma_{\bar{a} u} r_{\bar{a}}(\tau, \vec{\sigma})} \times\right. \\
& \sum_{r} \mathcal{T}_{(a) r}^{u}\left(\vec{\sigma}, \vec{\sigma}_{1}, \tau\right)\left(\phi^{-2} e^{-\frac{1}{\sqrt{3}} \sum_{\bar{a}} \gamma_{\bar{a} r} r_{\bar{a}}}\left[\frac{\rho}{3}+\sqrt{3} \sum_{\bar{b}} \gamma_{\bar{b} r} \pi_{\bar{b}}\right]\right)\left(\tau, \vec{\sigma}_{1}\right) \times \\
& \sum_{s} \mathcal{T}_{(a) s}^{u}\left(\vec{\sigma}, \vec{\sigma}_{2}, \tau\right)\left(\phi^{-2} e^{-\frac{1}{\sqrt{3}} \sum_{\bar{a}} \gamma_{\bar{a} s} r_{\bar{a}}}\left[\frac{\rho}{3}+\sqrt{3} \sum_{\bar{c}} \gamma_{\bar{c} s} \pi_{\bar{c}}\right]\right)\left(\tau, \vec{\sigma}_{2}\right)+ \\
& +\sum_{u v} e^{\frac{1}{\sqrt{3}} \sum_{\bar{a}}\left(\gamma_{\bar{a} u}+\gamma_{\bar{a} v}\right) r_{\bar{a}}(\tau, \vec{\sigma})}\left(\delta_{(b)}^{u} \delta_{(a)}^{v}-\delta_{(a)}^{u} \delta_{(b)}^{v}\right) \times \\
& \sum_{r} \mathcal{T}_{(a) r}^{u}\left(\vec{\sigma}, \vec{\sigma}_{1}, \tau\right)\left(\phi^{-2} e^{-\frac{1}{\sqrt{3}} \sum_{\bar{a}} \gamma_{\bar{a} r} r_{\bar{a}}}\left[\frac{\rho}{3}+\sqrt{3} \sum_{\bar{b}} \gamma_{\bar{b} r} \pi_{\bar{b}}\right]\right)\left(\tau, \vec{\sigma}_{1}\right) \\
& \left.\left.\left.\sum_{s} \mathcal{T}_{(b) s}^{v}\left(\vec{\sigma}, \vec{\sigma}_{2}, \tau\right)\left(\phi^{-2} e^{-\frac{1}{\sqrt{3}} \sum_{\bar{a}} \gamma_{\bar{a} s} r_{\bar{a}}}\left[\frac{\rho}{3}+\sqrt{3} \sum_{\bar{c}} \gamma_{\bar{c} s} \pi_{\bar{c}}\right]\right)\left(\tau, \vec{\sigma}_{2}\right)\right)\right]\right] \approx 0 \\
& \tilde{\mathcal{H}}_{R}(\tau, \vec{\sigma}) \rightarrow_{\rho \rightarrow 0} \epsilon \phi(\tau, \vec{\sigma})\left[\frac{c^{3}}{16 \pi G}\left(-8 \tilde{\triangle}\left[r_{\bar{a}}\right]+{ }^{3} \tilde{R}\left[r_{\bar{a}}\right]\right) \phi-\right. \\
& -\frac{6 \pi G}{c^{3}}\left[\left(2 \phi^{-7} \sum_{\bar{a}} \pi_{\bar{a}}^{2}\right)(\tau, \vec{\sigma})+\right. \\
& +4\left(\phi^{-5} \sum_{u} e^{\frac{1}{\sqrt{3}} \sum_{\bar{a}} \gamma_{\bar{a} u} r_{\bar{a}}} \sum_{\bar{b}} \gamma_{\bar{b} u} \pi_{\bar{b}}\right)(\tau, \vec{\sigma}) \times \\
& \int d^{3} \sigma_{1} \sum_{r} \delta_{(a)}^{u} \mathcal{T}_{(a) r}^{u}\left(\vec{\sigma}, \vec{\sigma}_{1}, \tau\right)\left(\phi^{-2} e^{-\frac{1}{\sqrt{3}} \sum_{\bar{a}} \gamma_{\bar{a} r} r_{\bar{a}}} \sum_{\bar{b}} \gamma_{\bar{b} r} \pi_{\bar{b}}\right)\left(\tau, \vec{\sigma}_{1}\right)+ \\
& +\quad \phi^{-3}(\tau, \vec{\sigma}) \int d^{3} \sigma_{1} d^{3} \sigma_{2}\left(\sum_{u} e^{\frac{2}{\sqrt{3}} \sum_{\bar{a}} \gamma_{\bar{a} u} r_{\bar{a}}(\tau, \vec{\sigma})} \times\right. \\
& \sum_{r} \mathcal{T}_{(a) r}^{u}\left(\vec{\sigma}, \vec{\sigma}_{1}, \tau\right)\left(\phi^{-2} e^{-\frac{1}{\sqrt{3}} \sum_{\bar{a}} \gamma_{\bar{a} r} r_{\bar{a}}} \sum_{\bar{b}} \gamma_{\bar{b} r} \pi_{\bar{b}}\right)\left(\tau, \vec{\sigma}_{1}\right) \times \\
& \sum_{s} \mathcal{T}_{(a) s}^{u}\left(\vec{\sigma}, \vec{\sigma}_{2}, \tau\right)\left(\phi^{-2} e^{-\frac{1}{\sqrt{3}} \sum_{\bar{a}} \gamma_{\bar{a} s} r_{\bar{a}}} \sum_{\bar{c}} \gamma_{\bar{c} s} \pi_{\bar{c}}\right)\left(\tau, \vec{\sigma}_{2}\right)+ \\
& +\sum_{u v} e^{\frac{1}{\sqrt{3}} \sum_{\bar{a}}\left(\gamma_{\bar{a} u}+\gamma_{\bar{a} v}\right) r_{\bar{a}}(\tau, \vec{\sigma})}\left(\delta_{(b)}^{u} \delta_{(a)}^{v}-\delta_{(a)}^{u} \delta_{(b)}^{v}\right) \times
\end{aligned}
$$




$$
\begin{aligned}
& \sum_{r} \mathcal{T}_{(a) r}^{u}\left(\vec{\sigma}, \vec{\sigma}_{1}, \tau\right)\left(\phi^{-2} e^{-\frac{1}{\sqrt{3}} \sum_{\bar{a}} \gamma_{\bar{a} r} r_{\bar{a}}} \sum_{\bar{b}} \gamma_{\bar{b} r} \pi_{\bar{b}}\right)\left(\tau, \vec{\sigma}_{1}\right) \\
& \left.\left.\left.\sum_{s} \mathcal{T}_{(b) s}^{v}\left(\vec{\sigma}, \vec{\sigma}_{2}, \tau\right)\left(\phi^{-2} e^{-\frac{1}{\sqrt{3}} \sum_{\bar{a}} \gamma_{\bar{a} s} r_{\bar{a}}} \sum_{\bar{c}} \gamma_{\bar{c} s} \pi_{\bar{c}}\right)\left(\tau, \vec{\sigma}_{2}\right)\right)\right]\right] \approx 0
\end{aligned}
$$

\section{The Natural Gauge Replacing the Maximal Slicing Condition in 3-Orthogonal Gauges.}

As already said the canonical basis (6.11) suggests that in the 3-orthogonal gauges the natural gauge fixing to the superhamiltonian constraint is $\rho(\tau, \vec{\sigma}) \approx 0$ and not the maximal

slicing condition ${ }^{3} K(\tau, \vec{\sigma}) \approx 0$. This gauge fixing selects a well defined $3+1$ splitting whose leaves are a well defined family of WSW hypersurfaces $\Sigma_{\tau}^{(W S W) \rho=0}$. For $\rho(\tau, \vec{\sigma}) \approx 0$ we get from Eqs. 6.16)

$$
\begin{aligned}
{ }^{3} \hat{K}(\tau, \vec{\sigma})= & -\frac{\epsilon 4 \sqrt{3} \pi G}{c^{3}} \phi^{-4}(\tau, \vec{\sigma}) \sum_{u} \delta_{(a) u} \\
& \sum_{s} \sum_{\bar{b}} \gamma_{\bar{b} s} \int d^{3} \sigma_{1} \mathcal{K}_{(a) s}^{r}\left(\vec{\sigma}, \vec{\sigma}_{1} ; \tau\right)\left(\phi^{-2} e^{-\frac{1}{\sqrt{3}} \sum_{\bar{a}} \gamma_{\bar{a} s} r_{\bar{a}}} \pi_{\bar{b}}\right)\left(\tau, \vec{\sigma}_{1}\right) .
\end{aligned}
$$

From Eq. 6.20) the final reduced form of the Lichnerowicz equation in the special 3orthogonal gauge identified by the natural gauge fixing $\rho(\tau, \vec{\sigma}) \approx 0$ is

$$
\begin{aligned}
\left(-\tilde{\triangle}\left[r_{\bar{a}}\right]+\right. & \left.\frac{1}{8} \tilde{R}\left[r_{\bar{a}}\right]\right)(\tau, \vec{\sigma}) \phi(\tau, \vec{\sigma})=\frac{12 \pi^{2} G^{2}}{c^{6}}\left[2\left(\phi^{-7} \sum_{\bar{a}} \pi_{\bar{a}}^{2}\right)(\tau, \vec{\sigma})+\right. \\
+ & 4\left(\phi^{-5} \sum_{u} e^{\frac{1}{\sqrt{3}} \sum_{\bar{a}} \gamma_{\bar{a} u} r_{\bar{a}}} \sum_{\bar{b}} \gamma_{\bar{b} u} \pi_{\bar{b}}\right)(\tau, \vec{\sigma}) \times \\
& \quad d^{3} \sigma_{1} \sum_{r} \delta_{(a)}^{u} \mathcal{T}_{(a) r}^{u}\left(\vec{\sigma}, \vec{\sigma}_{1}, \tau\right)\left(\phi^{-2} e^{-\frac{1}{\sqrt{3}} \sum_{\bar{a}} \gamma_{\bar{a} r} r_{\bar{a}}} \sum_{\bar{b}} \gamma_{\bar{b} r} \pi_{\bar{b}}\right)\left(\tau, \vec{\sigma}_{1}\right)+ \\
+ & \phi^{-3}(\tau, \vec{\sigma}) \int d^{3} \sigma_{1} d^{3} \sigma_{2}\left(\sum_{u} e^{\frac{2}{\sqrt{3}} \sum_{\bar{a}} \gamma_{\bar{a} u} r_{\bar{a}}(\tau, \vec{\sigma})} \times\right. \\
& \sum_{r} \mathcal{T}_{(a) r}^{u}\left(\vec{\sigma}, \vec{\sigma}_{1}, \tau\right)\left(\phi^{-2} e^{-\frac{1}{\sqrt{3}} \sum_{\bar{a}} \gamma_{\bar{a} r} r_{\bar{a}}} \sum_{\bar{b}} \gamma_{\bar{b} r} \pi_{\bar{b}}\right)\left(\tau, \vec{\sigma}_{1}\right) \times \\
& \sum_{s} \mathcal{T}_{(a) s}^{u}\left(\vec{\sigma}, \vec{\sigma}_{2}, \tau\right)\left(\phi^{-2} e^{-\frac{1}{\sqrt{3}} \sum_{\bar{a}} \gamma_{\bar{a} s} r_{\bar{a}}} \sum_{\bar{c}} \gamma_{\bar{c} s} \pi_{\bar{c}}\right)\left(\tau, \vec{\sigma}_{2}\right)+ \\
+ & \sum_{u v} e^{\frac{1}{\sqrt{3}} \sum_{\bar{a}}\left(\gamma_{\bar{a} u}+\gamma_{\bar{a} v}\right) r_{\bar{a}}(\tau, \vec{\sigma})}\left(\delta_{(b)}^{u} \delta_{(a)}^{v}-\delta_{(a)}^{u} \delta_{(b)}^{v}\right) \times \\
& \sum_{r} \mathcal{T}_{(a) r}^{u}\left(\vec{\sigma}, \vec{\sigma}_{1}, \tau\right)\left(\phi^{-2} e^{-\frac{1}{\sqrt{3}} \sum_{\bar{a}} \gamma_{\bar{a} r} r_{\bar{a}}} \sum_{\bar{b}} \gamma_{\bar{b} r} \pi_{\bar{b}}\right)\left(\tau, \vec{\sigma}_{1}\right) \\
& \left.\left.\sum_{s} \mathcal{T}_{(b) s}^{v}\left(\vec{\sigma}, \vec{\sigma}_{2}, \tau\right)\left(\phi^{-2} e^{-\frac{1}{\sqrt{3}} \sum_{\bar{a}} \gamma_{\bar{a} s} r_{\bar{a}}} \sum_{\bar{c}} \gamma_{\bar{c} s} \pi_{\bar{c}}\right)\left(\tau, \vec{\sigma}_{2}\right)\right)\right]
\end{aligned}
$$

Let us remark that, if this integro-differential equation for $\phi(\tau, \vec{\sigma})=e^{\frac{1}{2} q(\tau, \vec{\sigma})}>0$ would admit different solutions $\phi_{1}\left[r_{\bar{a}}, \pi_{\bar{a}}\right], \phi_{2}\left[r_{\bar{a}}, \pi_{\bar{a}}\right], \ldots$, they would correspond to inequivalent gravitational fields in vacuum (there are no more gauge transformations for correlating them) 
evolving according to the associated ADM energies. But it is hoped that Lichnerowicz's results in the case of maximal slicing imply the unicity of the solution [38] also in tetrad gravity with 3 -orthogonal coordinates and with the natural gauge fixing $\rho(\tau, \vec{\sigma}) \approx 0$.

If we add the natural gauge-fixing $\rho(\tau, \vec{\sigma})=\frac{1}{2} \phi(\tau, \vec{\sigma}) \pi_{\phi}(\tau, \vec{\sigma}) \approx 0$ to $\hat{\mathcal{H}}_{R}(\tau, \vec{\sigma}) \approx 0$, its time constancy implies ए5:

$$
\begin{aligned}
& \partial_{\tau} \rho(\tau, \vec{\sigma}) \stackrel{\circ}{=}\left\{\rho(\tau, \vec{\sigma}), \hat{H}_{(D) A D M, R}\right\}=\int d^{3} \sigma_{1} n\left(\tau, \vec{\sigma}_{1}\right)\left\{\rho(\tau, \vec{\sigma}), \hat{\mathcal{H}}_{R}\left(\tau, \vec{\sigma}_{1}\right)\right\}+ \\
&+\tilde{\lambda}_{\tau}(\tau)\left\{\rho(\tau, \vec{\sigma}), \hat{P}_{A D M, R}^{\tau}\right\}+\tilde{\lambda}_{r}(\tau)\left\{\rho(\tau, \vec{\sigma}), \hat{P}_{A D M, R}^{r}\right\} \approx \\
& \approx-\frac{1}{2} \phi(\tau, \vec{\sigma})\left[\int d^{3} \sigma_{1} n\left(\tau, \vec{\sigma}_{1}\right) \frac{\delta \hat{\mathcal{H}}_{R}\left(\tau, \vec{\sigma}_{1}\right)}{\delta \phi(\tau, \vec{\sigma})}+\right. \\
&\left.+\tilde{\lambda}_{\tau} \frac{\delta \hat{P}_{A D M, R}^{\tau}}{\delta \phi(\tau, \vec{\sigma})}+\tilde{\lambda}_{r}(\tau) \frac{\delta \hat{P}_{A D M, R}^{r}}{\delta \phi(\tau, \vec{\sigma})}\right] \approx 0, \\
& \Rightarrow \quad n(\tau, \vec{\sigma})-\hat{n}\left(\tau, \vec{\sigma} \mid r_{\bar{a}}, \pi_{\bar{a}}, \tilde{\lambda}_{A}\right] \approx 0, \\
& \partial_{\tau} \quad\left[n(\tau, \vec{\sigma})-\hat{n}\left(\tau, \vec{\sigma} \mid r_{\bar{a}}, \pi_{\bar{a}}, \tilde{\lambda}_{A}\right]\right]= \\
&=\lambda_{n}(\tau, \vec{\sigma})-\left\{\hat{n}\left(\tau, \vec{\sigma} \mid r_{\bar{a}}, \pi_{\bar{a}}, \tilde{\lambda}_{A}\right], \hat{H}_{(D) A D M, R}^{(W S W)}\right\} 0, \\
& \Rightarrow \quad \lambda_{n}(\tau, \vec{\sigma}) \quad \text { determined; }
\end{aligned}
$$

the rest-frame instant form expression of this equation is

$$
\int d^{3} \sigma_{1} \quad n\left(\tau, \vec{\sigma}_{1}\right) \frac{\delta \hat{\mathcal{H}}_{R}\left(\tau, \vec{\sigma}_{1}\right)}{\delta \phi(\tau, \vec{\sigma})}=-\epsilon \frac{\delta \hat{P}_{A D M, R}^{\tau}}{\delta \phi(\tau, \vec{\sigma})} .
$$

Therefore we find an integral equation for the lapse function $n(\tau, \vec{\sigma})$ implying its being different from zero (this avoids a finite time breakdown), even in the rest-frame instant form where $\tilde{\lambda}_{r}(\tau)=0, \tilde{\lambda}_{\tau}(\tau)=\epsilon$. It is hoped that the boundary condition $n(\tau, \vec{\sigma}) \rightarrow_{r \rightarrow \infty} 0+$ $O\left(r^{-(2+\epsilon)}\right)$ in a direction-independent way [see Eq.(2.55)] implies a unique solution of this integral equation. Then, Eq. 6.17) would imply a unique determination of the shift functions.

If we now go to the final Dirac brackets with respect to the second class constraints $\rho(\tau, \vec{\sigma}) \approx 0, \hat{\mathcal{H}}_{R}(\tau, \vec{\sigma}) \approx 0, n(\tau, \vec{\sigma})-\hat{n}\left(\tau, \vec{\sigma} \mid r_{\bar{a}}, \pi_{\bar{a}}, \tilde{\lambda}_{A}\right] \approx 0, \tilde{\pi}^{n}(\tau, \vec{\sigma}) \approx 0$, on the WSW hypersurfaces $\Sigma_{\tau}^{(W S W) \rho=0}$, asymptotically orthogonal to $\hat{P}_{A D M, R}^{(\mu)}$ at spatial infinity, we remain only with the canonical variables $r_{\bar{a}}, \pi_{\bar{a}}$ and with the following form of the Dirac-Hamiltonian and of the remaining four first class constraints

${ }^{138}$ With the boundary condition $\phi(\tau, \vec{\sigma}) \rightarrow_{r \rightarrow \infty} 1+\frac{M}{4 r}+O\left(r^{-3 / 2}\right)$ of Eqs.(6.11).

${ }^{139}$ With $\hat{H}_{(D) A D M, R}$ from Eq.(6.9), $\hat{P}_{A D M, R}^{A}$ from Eq.(B2) of Appendix B and $\hat{\mathcal{H}}_{R}(\tau, \vec{\sigma})$ from Eq. 6.22. 


$$
\begin{aligned}
\hat{H}_{(D) A D M, R \rho=0}^{(W S W)}= & -\tilde{\lambda}_{\tau}(\tau)\left(\epsilon_{(\infty)}-\hat{P}_{A D M, R}^{\tau}\left[r_{\bar{a}}, \pi_{\bar{a}}, \phi\left(r_{\bar{a}}, \pi_{\bar{a}}\right)\right]\right)- \\
- & \tilde{\lambda}_{r}(\tau) \hat{P}_{A D M, R}^{r}\left[r_{\bar{a}}, \pi_{\bar{a}}, \phi\left(r_{\bar{a}}, \pi_{\bar{a}}\right)\right] \\
& \epsilon_{(\infty)}-\hat{P}_{A D M, R}^{\tau}\left[r_{\bar{a}}, \pi_{\bar{a}}, \phi\left(r_{\bar{a}}, \pi_{\bar{a}}\right)\right] \approx 0, \\
& \hat{P}_{A D M, R}^{r}\left[r_{\bar{a}}, \pi_{\bar{a}}, \phi\left(r_{\bar{a}}, \pi_{\bar{a}}\right)\right] \approx 0 .
\end{aligned}
$$

where $\phi\left(r_{\bar{a}}, \pi_{\bar{a}}\right)$ is the solution of the reduced Lichnerowicz equation $\left.\hat{\mathcal{H}}_{R}(\tau, \vec{\sigma})\right|_{\rho(\tau, \vec{\sigma})=0}=0$ and the weak ADM energy is given in Eq.(B3) of Appendix B.

After the gauge-fixing $T_{(\infty)}-\tau \approx 0$, one gets $\tilde{\lambda}_{\tau}(\tau)=\epsilon$ and Eq.(2.49) imply

$$
\begin{aligned}
\hat{H}_{(D) A D M}^{(W S W)} & =-\epsilon \hat{P}_{A D M, R}^{\tau}\left[r_{\bar{a}}, \pi_{\bar{a}}, \phi\left(r_{\bar{a}}, \pi_{\bar{a}}\right)\right]+ \\
& +\tilde{\lambda}_{r}(\tau) \hat{P}_{A D M, R}^{r}\left[r_{\bar{a}}, \pi_{\bar{a}}, \phi\left(r_{\bar{a}}, \pi_{\bar{a}}\right)\right], \\
\hat{P}_{A D M, R}^{r}\left[r_{\bar{a}}, \pi_{\bar{a}}, \phi\left(r_{\bar{a}}, \pi_{\bar{a}}\right)\right] & \approx 0 .
\end{aligned}
$$

In the gauge $\tilde{\lambda}_{r}(\tau)=0$, implied by the gauge fixings $\hat{J}_{A D M, R}^{\tau r}\left[r_{\bar{a}}, \pi_{\bar{a}}, \phi\left(r_{\bar{a}}, \pi_{\bar{a}}\right)\right] \approx 0$ [see Eq.(B3)] on the internal 3-center-of-mass, we get the final Dirac Hamiltonian in the asymptotic rest-frame instant form of dynamics for tetrad gravity.

$$
\hat{H}_{(D) A D M}^{(W S W)^{\prime}}=-\epsilon \hat{P}_{A D M, R}^{\tau}
$$

and that the Hamilton equations imply the following normal form (namely solved in the accelerations) of the two dynamical Einstein equations for the gravitational field Dirac observables in the 3 -orthogonal gauge with $\rho(\tau, \vec{\sigma}) \approx 0$ and in the rest frame

$$
\begin{aligned}
& \partial_{\tau} r_{\bar{a}}(\tau, \vec{\sigma}) \stackrel{\circ}{=}\left\{r_{\bar{a}}(\tau, \vec{\sigma}),-\epsilon \hat{P}_{A D M, R}^{\tau}\left[r_{\bar{b}}, \pi_{\bar{b}}, \phi\left(r_{\bar{b}}, \pi_{\bar{b}}\right)\right]\right\}, \\
& \partial_{\tau} \pi_{\bar{a}}(\tau, \vec{\sigma}) \stackrel{\circ}{=}\left\{\pi_{\bar{a}}(\tau, \vec{\sigma}),-\epsilon \hat{P}_{A D M, R}^{\tau}\left[r_{\bar{b}}, \pi_{\bar{b}}, \phi\left(r_{\bar{b}}, \pi_{\bar{b}}\right)\right]\right\}, \\
& \hat{P}_{A D M, R}^{r}\left[r_{\bar{a}}, \pi_{\bar{a}}, \phi\left(r_{\bar{a}}, \pi_{\bar{a}}\right)\right] \approx 0, \\
& \hat{J}_{A D M, R}^{\tau r}\left[r_{\bar{a}}, \pi_{\bar{a}}, \phi\left(r_{\bar{a}}, \pi_{\bar{a}}\right)\right] \approx 0 .
\end{aligned}
$$

The ADM Hamilton equations of metric gravity, equivalent to the spatial Einstein equations, are [回] $\partial_{\tau}{ }^{3} g_{r s}(\tau, \vec{\sigma}) \stackrel{\circ}{=}\left[n_{r \mid s}+n_{s \mid r}-2 N^{3} K_{r s}\right](\tau, \vec{\sigma})$ and $\partial_{\tau}{ }^{3} K_{r s}(\tau, \vec{\sigma}) \stackrel{\circ}{=}\left(N\left[{ }^{3} R_{r s}+\right.\right.$ $\left.\left.{ }^{3} K^{3} K_{r s}-2^{3} K_{r u}{ }^{3} K^{u}{ }_{s}\right]-n_{|s| r}+n_{\mid s}^{u} K_{u r}+N^{u}{ }^{3} K_{r s \mid u}\right)(\tau, \vec{\sigma})$ : their restriction to our completely fixed gauge is satisfied due to the Hamilton equations (6.28).

The 4-metric and the line element in adapted coordinates $\sigma^{A}$ on the WSW hypersurfaces are

$$
\begin{aligned}
{ }^{4} g_{\tau \tau} & =\epsilon\left[\left(-\epsilon+n\left[r_{\bar{a}}, \pi_{\bar{a}}, \vec{\lambda}\right]\right)^{2}-\phi^{-4}\left[r_{\bar{a}}, \pi_{\bar{a}}\right] \sum_{r} e^{-\frac{2}{\sqrt{3}} \sum_{\bar{a}} \gamma_{\bar{a} r} r_{\bar{a}}}\left(\lambda_{r}(\tau)-n_{r}\left[r_{\bar{a}}, \pi_{\bar{a}}, \vec{\lambda}\right]\right)^{2}\right] \\
& \rightarrow \vec{\lambda}(\tau, \vec{\sigma})=0 \\
\epsilon & {\left[\left(-\epsilon+n\left[r_{\bar{a}}, \pi_{\bar{a}}, 0\right]\right)^{2}-\phi^{-4}\left[r_{\bar{a}}, \pi_{\bar{a}}\right] \sum_{r} e^{-\frac{2}{\sqrt{3}} \sum_{\bar{a}} \gamma_{\bar{a} r} r_{\bar{a}}} n_{r}^{2}\left[r_{\bar{a}}, \pi_{\bar{a}}, 0\right]\right] }
\end{aligned}
$$




$$
\begin{aligned}
{ }^{4} g_{\tau r} & =\epsilon\left[-\lambda_{r}(\tau)+n_{r}\left[r_{\bar{a}}, \pi_{\bar{a}}, \vec{\lambda}\right]\right] \\
& \rightarrow \vec{\lambda}(\tau, \vec{\sigma})=0 \\
{ }^{4} g_{r s} & =-\epsilon n_{r}\left[r_{\bar{a}}, \pi_{\bar{a}}, 0\right] \\
& {\left[r_{\bar{a}}, \pi_{\bar{a}}\right] e^{\frac{2}{\sqrt{3}} \sum_{\bar{a}} \gamma_{\bar{a} r} r_{\bar{a}}} \delta_{r s}, } \\
d s^{2} & ={ }^{4} \hat{g}_{\tau \tau}(d \tau)^{2}+2{ }^{4} \hat{g}_{\tau r} d \tau d \sigma^{r}+\sum_{r}{ }^{4} \hat{g}_{r r}\left(d \sigma^{r}\right)^{2} .
\end{aligned}
$$

Even for $\lambda_{r}(\tau)=0$ we do not get vanishing shift functions (synchronous coordinates), like instead it is assumed by Christodoulou and Klainermann for their singularity-free solutions.

Let us remark that Eqs. (6.17) and (6.24) imply that both $n$ and $n_{r}$ depend on $G / c^{3}$ and $c^{3} / G$ simultaneously, so that both their post-Newtonian (expansion in $1 / c$ ) and postMinkowskian (formal expansion in powers of $\mathrm{G}$ ) may be non trivial after having done the gauge fixings. From Eq. (6.23) it is clear that $\phi\left[r_{\bar{a}}, \pi_{\bar{a}}\right]$ depends on $G^{2} / c^{6}$, while the previous equation implies that $\hat{H}_{(D) A D M}^{(W S W)^{\prime}}=\hat{P}_{A D M, R}^{\tau}$ depends a priori on both $G / c^{3}$ and $c^{3} / G$. 


\section{THE EMBEDDING INTO SPACETIME OF THE WIGNER-SEN-WITTEN HYPERSURFACES.}

We will see in this Section that the special WSW spacelike hypersurfaces $\Sigma_{\tau}^{(W S W)}$, needed for the rest-frame instant form of tetrad gravity in our class of spacetimes, asymptotically flat at spatial infinity and without supertranslations, and corresponding to the Wigner hyperplanes orthogonal to the 4-momentum of an isolated system, can be defined by special embeddings $z^{\mu}(\tau, \vec{\sigma})$ 140.

Moreover, we will see that they enjoy the same formal properties of spacelike hyperplanes in Minkowski spacetime, namely that, given an origin on each one of them and an adapted tetrad at this origin, there is a natural parallel transport so that one can uniquely define the adapted tetrads in all points of the hyperplane starting from the given adapted one at the origin. Namely due to the property of tending asymptotically to Minkowski Wigner spacelike hyperplanes in a direction-independent way at spatial infinity, the WSW spacelike hypersurfaces allow the definition of asymptotic (angle-independent) adapted tetrads with the timelike component parallel to the weak ADM 4-momentum. Then an adaptation to tensors of the Sen-Witten spinorial equation [107 111] based on the Sen connection 44 allows to define preferred adapted tetrads in each point of $\Sigma_{\tau}^{(W S W)}$ tending to the given ones at spatial infinity: this can be reinterpreted as a special form of parallel transport generalizing the trivial Euclidean one on Minkowski spacelike hyperplanes.

In Ref. [113] Frauendiener, exploiting the fact that there is a unique 2-1 (up to a global sign) correspondence between a $\mathrm{SU}(2)$ spinor and a triad on a spacelike hypersurface, derives the necessary and sufficient conditions that have to be satisfied by a triad in order to correspond to a spinor that satisfies the Sen-Witten equation. In this way it is possible to eliminate completely any reference to spinors and to speak only of triads ${ }^{3} e_{(a)}^{(W S W) r}$ on $\Sigma_{\tau}^{(W S W)}$ and $\Sigma_{\tau}^{(W S W)}$-adapted tetrads on $M^{4}$.

These triads ${ }^{3} e^{(W S W) r}(a)$ are built in terms of the $\mathrm{SU}(2)$ spinors solutions of the SenWitten equation and, as a consequence of this equation, they are shown [113] to satisfy the following equations

$$
\begin{aligned}
& { }^{3} \nabla_{r}{ }^{3} e_{(1)}^{(W S W) r}={ }^{3} \nabla_{r}{ }^{3} e_{(2)}^{(W S W) r}=0 \\
& { }^{3} \nabla_{r}{ }^{3} e_{(3)}^{(W S W) r}=-\alpha^{3} K \\
& { }^{3} e_{(1)}^{(W S W) r}{ }^{3} e_{(3)}^{(W S W) s}{ }^{3} \nabla_{r}{ }^{3} e_{(2) s}^{(W S W)}+{ }^{3} e_{(3)}^{(W S W) r}{ }^{3} e_{(2)}^{(W S W) s}{ }^{3} \nabla_{r}{ }^{3} e_{(1) s}^{(W S W)}+ \\
& +{ }^{3} e_{(2)}^{(W S W) r}{ }^{3} e_{(1)}^{(W S W) s}{ }^{3} \nabla_{r}{ }^{3} e_{(3) s}^{(W S W)}=0 .
\end{aligned}
$$

Here for ${ }^{3} K$ one uses Eq.(6.17) in the 3 -orthogonal gauge or $(6.23)$ when $\rho(\tau, \vec{\sigma}) \approx 0$.

\footnotetext{
${ }^{140}$ Generalizing the embeddings $z^{(\mu)}(\tau, \vec{\sigma})=x^{(\mu)}(\tau)+\epsilon_{r}^{(\mu)}\left(u\left(p_{s}\right)\right) \sigma^{r}$ for Minkowski Wigner spacelike hyperplanes.

${ }^{141}$ See Ref. [112 for the existence of solutions on noncompact spacetimes including the Christodoulou-Klinermann ones.
} 
Therefore, these triads are formed by 3 vector fields with the properties: i) two vector fields are divergence free; ii) the third one has a non-vanishing divergence proportional to the trace of the extrinsic curvature of $\Sigma_{\tau}^{(W S W)}$ (on a maximal slicing hypersurface $\left({ }^{3} K=0\right.$ ) all three vectors would be divergence free); iii) the vectors satisfy a cyclic condition.

In Ref. [113] it is shown: 1) these triads do not exist for compact $\left.\Sigma_{\tau} ; 2\right)$ with nontrivial topology for $\Sigma_{\tau}$ there can be less than 4 real solutions and the triads cannot be build; 3) the triads exist for asymptotically null surfaces, but the corresponding tetrad will be degenerate in the limit of null infinity.

Moreover, in Ref. [113], using the results of Ref. [114], it is noted that the Einstein energy-momentum pseudo-tensor [115] is a canonical object only in the frame bundle over $M^{4}$, where it coincides with the Sparling 3-form. In order to bring this 3-form back to a 3 -form (and then to an energy-momentum tensor) over the spacetime $M^{4}$, one needs a section (i.e. a tetrad) in the frame bundle. Only with the $3+1$ decomposition of $M^{4}$ with WSW foliations one gets that (after imposition of Einstein's equations together with the local energy condition) one has a preferred (geometrical and dynamical) adapted tetrad on the initial surface $\Sigma_{\tau}^{(W S W)}$.

A triad satisfying Eqs.(7.1) is unique up to global frame rotations and homotheties. But Eqs.(2.55) imply that we must select the solutions of Eqs.(7.1) with the same asymptotic behaviour of ordinary triads ${ }^{3} e_{(a)}^{r}$, i.e. ${ }^{3} e_{(\infty)}^{(W S W) r}(a)={ }^{3} e_{(\infty)(a)}^{r}=\delta_{(a)}^{r}$ and ${ }^{3} e^{(W S W) r}(a)(\tau, \vec{\sigma}) \rightarrow_{r \rightarrow \infty}\left(1-\frac{M}{2 r}\right) \delta_{(a)}^{r}+O\left(r^{-3 / 2}\right)$. In this sense, the geometry of an initial data set uniquely determines a triad on $\Sigma_{\tau}^{(W S W)}$ (called geometrical in Ref. [113) and hence together with the normal an adapted tetrad ${ }_{(\Sigma)}^{4} \check{E}_{A}^{(W)(\mu)}$ in spacetime according to Eq.(2.28).

Therefore, we can define the $\Sigma_{\tau}^{(W S W)}$-adapted preferred tetrads of the rest-frame instant form

$$
\begin{aligned}
& { }_{(\Sigma)}^{4} \check{\tilde{E}}_{(o)}^{(W S W)}{ }_{(o)}=\frac{1}{-\epsilon+n}\left(1 ;-n^{r}\right), \\
& { }_{(\Sigma)}^{4} \tilde{\tilde{E}}_{(a)}^{(W S W)} \underset{A}{A}=\left(0 ;{ }^{3} e^{(W S W) r}(a)\right), \\
& { }_{(\Sigma)}^{4} \check{E}_{(o)}^{(W S W) \mu}=l^{\mu}, \\
& { }_{(\Sigma)}^{4} \check{E}_{(a)}^{\mu}=b_{s}^{\mu}{ }^{3} e^{(W S W) s}{ }_{(a)} .
\end{aligned}
$$

Since the WSW hypersurfaces and the 3-metric on them are dynamically determined 442 , one has neither a static background on system-independent hyperplanes like in parametrized Newton theories nor a static one on the system-dependent Wigner hyperplanes like in parametrized Minkowski theories. Now both the WSW hyperplanes and the metric on it are system dependent.

These preferred tetrads correspond to the non-flat preferred observers of Bergmann [41: they are a set of privileged observers (privileged tetrads adapted to $\Sigma_{\tau}^{(W S W)}$ ) of geometrical

\footnotetext{
${ }^{142}$ The solution of Einstein equations is needed to find the physical 3-metric, the allowed WSW hypersurfaces and the Sen connection.
} 
nature ${ }^{143}$ and not of static nature like in the approaches of Møller [116, Pirani [117 and Goldberg 118. These privileged observers are associated with the existence of the asymptotic Poincaré charges, since their asymptotic 4-velocity is determined by the weak ADM 4-momentum. A posteriori, namely after having solved Einstein's equations, one could try to use these geometrical and dynamical privileged observers in the same way as, in metric gravity, are used the bimetric theories, like the one of Rosen [119], with a set of privileged static non-flat background metrics. This congruence of timelike preferred observers ${ }^{140}$ is a non-Machian element of these noncompact spacetimes. The asymptotic worldlines of the congruence may replace the static concept of fixed stars in the study of the precessional effects of gravitomagnetism on gyroscopes (dragging of inertial frames) and seem to be naturally connected with the definition of post-Newtonian coordinates [70,46].

With the asymptotic triads ${ }^{3} e_{(\infty)(a)}^{r}={ }^{3} e_{(\infty)}^{(W S W)_{(a)}}=\delta_{(a)}^{r}$ as boundary conditions at spatial infinity for the Frauendiener equations, their solution defines a set of preferred triads ${ }^{3} e^{(W S W) r}(a)(\tau, \vec{\sigma})$ in each point of $\Sigma_{\tau}^{(W S W)}$ (Sen-Witten parallel transport of the asymptotic triads), which will be connected by a rotation of angle $\alpha_{(a)}^{(W S W)}(\tau, \vec{\sigma})$ to the ordinary triads (analogous formulas are valid for cotriads)

$$
{ }^{3} e_{(a)}^{r}(\tau, \vec{\sigma})=R_{(a)(b)}\left(\alpha_{(c)}^{(W S W)}(\tau, \vec{\sigma})\right)^{3} e_{(b)}^{(W S W) r}(\tau, \vec{\sigma}) .
$$

The asymptotic transition functions from arbitrary coordinates on $M^{4}$ to WSW hypersurfaces $\Sigma_{\tau}^{(W S W)}$ are [see Eqs.(2.39)-(2.45)]

$$
\begin{aligned}
& \hat{b}_{(\infty) l}^{(\mu)} \approx \epsilon l_{(\infty)}^{(\mu)} \approx \frac{b_{(\infty) \tau}^{(\mu)}}{-\epsilon+n}, \\
& \hat{b}_{(\infty) r}^{(\mu)}=b_{(\infty) r}^{(\mu)}=\epsilon_{r}^{(\mu)}\left(u\left(p_{(\infty)}\right)\right), \\
& \hat{b}_{(\infty)(\mu)}^{l}=l_{(\infty)(\mu)}=-\epsilon b_{(\infty)(\mu)}^{\tau}, \\
& \hat{b}_{(\infty)(\mu)}^{s}=b_{(\infty)(\mu)}^{s}-\tilde{\lambda}_{s}(\tau) b_{(\infty)(\mu)}^{\tau} \approx b_{(\infty)(\mu)}^{s}, \\
& w i t h \\
& b_{(\infty) A}^{(\mu)}(\tau) \equiv L_{(\nu)=A}^{(\mu)}\left(p_{(\infty)}, \stackrel{\circ}{(\infty)}_{(\infty)}\right)=\epsilon_{A}^{(\mu)}\left(u\left(p_{(\infty)}\right)\right), \\
& \epsilon l_{(\infty)}^{(\mu)}= \epsilon_{o}^{(\mu)}\left(u\left(p_{(\infty)}\right)\right)=u^{(\mu)}\left(p_{(\infty)}\right) \approx \frac{\hat{P}_{A D M}^{(\mu)}}{\epsilon_{(\infty)}}, \\
& \epsilon_{(\infty)} \approx M_{A D M}= \sqrt{\epsilon \hat{P}_{A D M}^{2}}, \quad S_{(\infty)}^{(\mu)(\nu)} \equiv \hat{S}_{A D M}^{(\mu)(\nu)} .
\end{aligned}
$$

${ }^{143}$ Since they depend on the intrinsic and extrinsic geometry of $\Sigma_{\tau}^{(W S W)}$; on the solutions of Einstein's equations they also acquire a dynamical nature depending on the configuration of the gravitational field itself.

${ }^{144}$ Privileged non-holonomic coordinate systems replacing the rectangular Minkowski coordinates of the flat case.

${ }^{145}$ With asymptotic inertial observers in the rest-frame instant form with $\tilde{\lambda}_{A}(\tau)=(\epsilon ; \overrightarrow{0})$ and $\tilde{\lambda}_{A B}(\tau)=0$. 
Given the previous boundary conditions on the triads $\left[{ }^{3} e_{(a)}^{r} \rightarrow{ }^{3} e_{(\infty)(a)}^{r}={ }^{3} e_{(\infty)}^{(W S W)_{(a)}}=\right.$ $\left.\delta_{(a)}^{r}\right]$ and cotriads $\left[{ }^{3} e_{(a) r} \rightarrow{ }^{3} e_{(\infty)(a) r}={ }^{3} e_{(\infty)(a) r}^{(W S W)}=\delta_{(a) r}\right]$, we have the following associated asymptotic tetrads on $\Sigma_{\tau}^{(W S W)}$ (we assumed $\varphi_{(a)}(\tau, \vec{\sigma}) \rightarrow_{r \rightarrow \infty} 0$ in Eq.(2.55) as a standard of asymptotic inertiality)

$$
\begin{aligned}
& { }^{4} E_{(\infty)}^{(W S W)(\mu)}{ }_{(o)}^{(\mu)} \delta_{(\mu)}^{\mu}={ }_{(\Sigma)}^{4} \check{E}_{(\infty)}^{(W S W)(\mu)}{ }_{(o)} \delta_{(\mu)}^{\mu}=\delta_{(\mu)}^{\mu} l_{(\infty)}^{(\mu)}=\delta_{(\mu)}^{\mu} \hat{b}_{(\infty) l}^{(\mu)} \equiv \delta_{(\mu)}^{\mu} b_{(\infty) \tau}^{(\mu)}=\delta_{(\mu)}^{\mu} u^{(\mu)}\left(p_{(\infty)}\right), \\
& { }^{4} E_{(\infty)}^{(W S W)(\mu)}{ }_{(a)}^{(\mu)} \delta_{(\mu)}^{\mu}={ }_{(\Sigma)}^{4} \check{E}_{(\infty)}^{(W S W)(\mu)}{ }_{(a)}^{(\mu)} \delta_{(\mu)}^{\mu}=\delta_{(\mu)}^{\mu} \hat{b}_{(\infty) s}^{(\mu)}{ }^{3} e_{(\infty)}^{(W S W)_{(a)}} \equiv \delta_{(\mu)}^{\mu} b_{(\infty) s}^{(\mu)}{ }^{3} e_{(\infty)}^{(W S W)_{(a)}}= \\
& =\delta_{(\mu)}^{\mu} b_{(\infty) s}^{(\mu)} \delta_{(a)}^{s}=\delta_{(\mu)}^{\mu} \epsilon_{s}^{(\mu)}\left(u\left(p_{(\infty)}\right)\right) \delta_{(a)}^{s}, \\
& { }^{4} E_{(\infty)}^{(W S W) \underset{(o)}{A}}={ }_{(\Sigma)}^{4} \check{\tilde{E}}_{(\infty)}^{(W S W)}{ }_{(o)}^{A}=(-\epsilon ; 0), \\
& { }^{4} E_{(\infty)}^{(W S W) a}(a)={ }_{(\Sigma)}^{4} \check{\tilde{E}}_{(\infty)}^{(W S W)} \underset{(a)}{A}=\left(0 ; \delta_{(a)}^{r}\right), \\
& { }^{4} E_{(\infty)}^{(W S W)(o)}{ }_{(\mu)}^{(\mu)} \delta_{\mu}^{(\mu)}={ }_{(\Sigma)}^{4} \check{E}_{(\infty)}^{(W S W)(o)}{ }_{(\mu)} \delta_{\mu}^{(\mu)}=\delta_{\mu}^{(\mu)} l_{(\infty)(\mu)}=\delta_{\mu}^{(\mu)} \hat{b}_{(\infty)(\mu)}^{l}=-\epsilon \delta_{\mu}^{(\mu)} b_{(\infty)(\mu)}^{\tau}, \\
& { }^{4} E_{(\infty)}^{(W S W)(a)}{ }_{(\mu)}^{(a)} \delta_{\mu}^{(\mu)}={ }_{(\Sigma)}^{4} \check{E}_{(\infty)}^{(W S W)(a)}{ }_{(\mu)}^{(\mu)} \delta_{\mu}^{(\mu)}=\delta_{\mu}^{(\mu)} \hat{b}_{(\infty)(\mu)}^{s}{ }^{3} e_{(\infty)}^{(W S W)(a)}{ }_{s}^{(a)} \equiv \delta_{\mu}^{(\mu)} b_{(\infty)(\mu)}^{s} \delta_{s}^{(a)}, \\
& { }^{4} E_{(\infty)}^{(W S W) \stackrel{(o)}{A}}={ }_{(\Sigma)}^{4} \check{\tilde{E}}_{(\infty)}^{(W S W)} \stackrel{(o)}{A} \equiv(-\epsilon ; 0), \\
& \left.{ }^{4} E_{(\infty)}^{(W S W)(a)}{ }_{A}={ }_{(\Sigma)}^{4} \check{\tilde{E}}_{(\infty)}^{(W S W)}{ }_{A} a\right) \equiv\left(0 ;{ }^{3} e_{(\infty)}^{(W S W)}{ }_{(a) r}=\delta_{(a) r}\right) .
\end{aligned}
$$

The final form of arbitrary tetrads and cotetrads obtained starting from the adapted ones of Eqs.(7.2) is [see Eqs.(2.2), (2.4)]

$$
\begin{aligned}
& { }^{4} E_{(o)}^{\mu}=\sqrt{1+\sum_{(c)} \varphi_{(c)}^{2}} l^{\mu}+\epsilon \sum_{(b)} \varphi_{(b)}{ }^{3} e_{(b)}^{s} b_{s}^{\mu}= \\
& =\sqrt{1+\sum_{(c)} \varphi_{(c)}^{2}} l^{\mu}+\epsilon \sum_{(b),(c)} \varphi_{(b)} R_{(b)(c)}\left(\alpha_{(a)}^{(W S W)}\right)^{3} e_{(c)}^{(W S W) s} b_{s}^{\mu}, \\
& \left.{ }^{4} E_{(a)}^{\mu}=\epsilon \varphi_{(a)}\right)^{\mu}+\sum_{(b)}\left[\delta_{(a)(b)}+\frac{\varphi_{(a)} \varphi_{(b)}}{1+\sqrt{1+\sum_{(c)} \varphi_{(c)}^{2}}}\right]^{3} e_{(b)}^{s} b_{s}^{\mu}= \\
& =\epsilon \varphi_{(a)} l^{\mu}+\sum_{(b),(c)}\left[\delta_{(a)(b)}+\frac{\varphi_{(a)} \varphi_{(b)}}{1+\sqrt{1+\sum_{(c)} \varphi_{(c)}^{2}}}\right] R_{(b)(c)}\left(\alpha_{(a)}^{(W S W)}\right)^{3} e^{(W S W) s}(b) b b_{s}^{\mu}, \\
& { }^{4} E_{(o)}^{A} \equiv\left(\frac{\sqrt{1+\sum_{(c)} \varphi_{(c)}^{2}}}{-\epsilon+n} ;-\sqrt{1+\sum_{(c)} \varphi_{(c)}^{2}} \frac{n^{r}}{-\epsilon+n}+\epsilon \sum_{(b)} \varphi_{(b)}{ }^{3} e_{(b)}^{r}=\right. \\
& \left.=-\sqrt{1+\sum_{(c)} \varphi_{(c)}^{2}} \frac{n^{r}}{-\epsilon+n}+\epsilon \sum_{(b),(c)} \varphi_{(b)} R_{(b)(c)}\left(\alpha_{(a)}^{(W S W)}\right)^{3} e_{(c)}^{(W S W) r}\right), \\
& { }^{4} E_{(a)}^{A} \equiv\left(\frac{\epsilon \varphi_{(a)}}{-\epsilon+n} ;-\epsilon \varphi_{(a)} \frac{n^{r}}{-\epsilon+n}+\sum_{(b)}\left[\delta_{(a)(b)}+\frac{\varphi_{(a)} \varphi_{(b)}}{1+\sqrt{1+\sum_{(c)} \varphi_{(c)}^{2}}}\right]^{3} e_{(b)}^{r}=\right. \\
& \left.=-\epsilon \varphi_{(a)} \frac{n^{r}}{-\epsilon+n}+\sum_{(b),(c)}\left[\delta_{(a)(b)}+\frac{\varphi_{(a)} \varphi_{(b)}}{1+\sqrt{1+\sum_{(c)} \varphi_{(c)}^{2}}}\right] R_{(b)(c)}\left(\alpha_{(a)}^{(W S W)}\right)^{3} e_{(c)}^{(W S W) r}\right) \text {, }
\end{aligned}
$$




$$
\begin{aligned}
& { }^{4} E_{\mu}^{(o)}=\epsilon \sqrt{1+\sum_{(c)} \varphi_{(c)}^{2}} l_{\mu}-\epsilon \sum_{(b)} \varphi_{(b)}{ }^{3} e_{(b) s} b_{\mu}^{s}= \\
& =\epsilon \sqrt{1+\sum_{(c)} \varphi_{(c)}^{2}} l_{\mu}-\epsilon \sum_{(b),(c)} \varphi_{(b)} R_{(b)(c)}\left(\alpha_{(a)}^{(W S W)}\right)^{3} e^{(W S W)}{ }_{(b) s} b_{\mu}^{s}, \\
& { }^{4} E_{\mu}^{(a)}=-\varphi_{(a)} l_{\mu}=\sum_{(b)}\left[\delta_{(a)(b)}+\frac{\varphi_{(a)} \varphi_{(b)}}{1+\sqrt{1+\sum_{(c)} \varphi_{(c)}^{2}}}\right]^{3} e_{(b) s} b_{\mu}^{s}= \\
& =-\varphi_{(a)} l_{\mu}=\sum_{(b),(c)}\left[\delta_{(a)(b)}+\frac{\varphi_{(a)} \varphi_{(b)}}{1+\sqrt{1+\sum_{(c)} \varphi_{(c)}^{2}}}\right] R_{(b)(c)}\left(\alpha_{(a)}^{(W S W)}\right)^{3} e^{(W S W)}{ }_{(b) s} b_{\mu}^{s}, \\
& { }^{4} E_{A}^{(o)}=\left(\sqrt{1+\sum_{(c)} \varphi_{(c)}^{2}}(-\epsilon+n)-\epsilon \sum_{(a)} \varphi_{(a)} n_{(a)}\right. \\
& \left.-\epsilon \sum_{(a)} \varphi_{(a)}{ }^{3} e_{(a) r}=-\epsilon \sum_{(a),(b)} \varphi_{(a)} R_{(a)(b)}\left(\alpha_{(c)}^{(W S W)}\right)^{3} e^{(W S W)}{ }_{(b) r}\right), \\
& { }^{4} E_{A}^{(a)}=\left(-\epsilon(-\epsilon+n) \varphi_{(a)}+\sum_{(b)}\left[\delta_{(a)(b)}+\frac{\varphi_{(a)} \varphi_{(b)}}{1+\sqrt{1+\sum_{(c)} \varphi_{(c)}^{2}}}\right] n_{(b)} ;\right. \\
& \sum_{(b)}\left[\delta_{(a)(b)}+\frac{\varphi_{(a)} \varphi_{(b)}}{1+\sqrt{1+\sum_{(c)} \varphi_{(c)}^{2}}}\right]^{3} e_{(b) r}= \\
& \left.=\sum_{(b),(c)}\left[\delta_{(a)(b)}+\frac{\varphi_{(a)} \varphi_{(b)}}{1+\sqrt{1+\sum_{(c)} \varphi_{(c)}^{2}}}\right] R_{(b)(c)}\left(\alpha_{(a)}^{(W S W)}\right)^{3} e^{(W S W)}(c) r\right) \text {. }
\end{aligned}
$$

Since ${ }^{4} \check{E}_{\mu}^{(\alpha)} d z^{\mu}={ }^{4} \theta^{(\alpha)}$ are non-holonomic coframes, there are not coordinate hypersurfaces and lines for the associated non-holonomic coordinates $z^{(\alpha)} \| 120$ on $M^{4}$; as shown in Ref. [121] for them we have ${ }^{4} \theta^{(\alpha)}=d z^{(\alpha)}+z^{(\beta)}\left[{ }^{4} \check{E}_{\mu}^{(\alpha)} \frac{\partial^{4} \check{E}_{(\beta)}^{\mu}}{\partial z^{(\gamma)}}\right] d z^{(\gamma)}$.

The embeddings $z^{\mu}(\tau, \vec{\sigma})$ of $R^{3}$ into $M^{4}$ associated with WSW spacelike hypersurfaces $\Sigma_{\tau}^{(W S W)}$ in the rest-frame instant form of tetrad gravity are restricted to assume the same form at spatial infinity of those in Minkowski spacetime identifying the Wigner hyperplanes in the rest-frame instant form

$$
\begin{aligned}
& z^{\mu}(\tau, \vec{\sigma}) \rightarrow_{r \rightarrow \infty} \delta_{(\mu)}^{\mu} z_{(\infty)}^{(\mu)}(\tau, \vec{\sigma}) \\
& z_{(\infty)}^{(\mu)}(\tau, \vec{\sigma})=x_{(\infty)}^{(\mu)}(\tau)+\epsilon_{r}^{(\mu)}\left(u\left(p_{(\infty)}\right)\right) \sigma^{r}= \\
&=x_{(\infty)}^{(\mu)}(0)+u^{(\mu)}\left(p_{(\infty)}\right) \tau+\epsilon_{r}^{(\mu)}\left(u\left(p_{(\infty)}\right)\right) \sigma^{r} .
\end{aligned}
$$

By using the notation

$$
\begin{aligned}
& l^{\mu}=\epsilon \hat{b}_{l}^{\mu}=\frac{\epsilon}{-\epsilon+n}\left[b_{\tau}^{\mu}-n^{r} b_{r}^{\mu}\right]=\frac{1}{\sqrt{{ }^{3} g}} \epsilon_{\alpha \beta \gamma(\Sigma)}^{\mu} \stackrel{4}{4} \check{E}_{(1)(\Sigma)}^{(W S W) \alpha \underset{(2)}{4} \check{E}_{(\Sigma)}^{(W S W) \beta}} \underset{(4)}{4} \check{E}_{(3 S W) \gamma}^{(W)}, \\
& \epsilon_{r}^{\mu}=b_{s}^{\mu 3} e_{(a)}^{(W S W) s} \delta_{(a) r} \rightarrow \delta_{(\mu)}^{\mu} b_{(\infty) s}^{(\mu)} \delta_{(a)}^{s} \delta_{(a) r}=\delta_{(\mu)}^{\mu} b_{(\infty) r}^{(\mu)}, \\
& \hat{b}_{r}^{\mu}=b_{r}^{\mu},
\end{aligned}
$$




$$
\begin{aligned}
& \hat{b}_{\mu}^{l}=l_{\mu}=(-\epsilon+n) b_{\mu}^{\tau}=(-\epsilon+n) \partial_{\mu} \tau(z), \\
& \hat{b}_{\mu}^{r}=b_{\mu}^{r}+n^{r} b_{\mu}^{\tau},
\end{aligned}
$$

we get the following expression for the embedding

$$
\begin{aligned}
z_{(W S W)}^{\mu}(\tau, \vec{\sigma})= & \delta_{(\mu)}^{\mu} x_{(\infty)}^{(\mu)}(0)+l^{\mu}(\tau, \vec{\sigma}) \tau+\epsilon_{r}^{\mu}(\tau, \vec{\sigma}) \sigma^{r}= \\
= & x_{(\infty)}^{\mu}(0)+l^{\mu}(\tau, \vec{\sigma}) \tau+b_{s}^{\mu}(\tau, \vec{\sigma}){ }^{3} e^{(W S W) s}(\tau) \\
= & x_{(\infty)}^{\mu}(0)+b_{A}^{\mu}(\tau, \vec{\sigma}) \delta_{(a) r} F^{r}(\tau, \vec{\sigma}), \\
& F^{\tau}(\tau, \vec{\sigma})=\frac{\tau}{-\epsilon+n(\tau, \vec{\sigma})}, \\
& F^{s}(\tau, \vec{\sigma})={ }^{3} e^{(W S W) \underset{(a)}{s}(\tau, \vec{\sigma}) \delta_{(a) r} \sigma^{r}-\frac{n^{s}(\tau, \vec{\sigma})}{-\epsilon+n(\tau, \vec{\sigma})} \tau,}
\end{aligned}
$$

with $x_{(\infty)}^{(\mu)}(0)$ arbitrary 140. See Ref. 122 and its interpretation of the center of mass in general relativity [47: $x_{(\infty)}^{(\mu)}(\tau)$ may be interpreted as the arbitrary reference (or central) timelike worldline of this paper.

From Eqs.(7.9) we can find the equations for determining the transition coefficients $b_{A}^{\mu}(\tau, \vec{\sigma})=\frac{\partial z_{(W S W)}^{\mu}(\tau, \vec{\sigma})}{\partial \sigma^{A}}$ and therefore the coordinate transformation $x^{\mu} \mapsto \sigma^{A}$ from general 4-coordinates to adapted 4-coordinates

$$
\begin{gathered}
b_{A}^{\mu}=\frac{\partial z_{(W S W)}^{\mu}}{\partial \sigma^{A}}=b_{B}^{\mu} \frac{\partial F^{B}}{\partial \sigma^{A}}+\frac{\partial b_{B}^{\mu}}{\partial \sigma^{A}} F^{B}, \\
A_{A}^{B}=\delta_{A}^{B}-\frac{\partial F^{B}}{\partial \sigma^{A}}, \\
F^{B} \frac{\partial b_{B}^{\mu}}{\partial \sigma^{A}}=A_{A}^{B} b_{B}^{\mu}, \\
\text { or } \quad b_{b}^{\mu}=\left(A^{-1}\right)_{B}{ }^{a} F^{C} \frac{\partial b_{C}^{\mu}}{\partial \sigma^{A}} .
\end{gathered}
$$

The coordinates $\sigma^{A}$ (our special 3-orthogonal coordinates) for the $3+1$ splitting of $M^{4}$ with leaves $\Sigma_{\tau}^{(W S W)}$ replace the standard $P N$ coordinates $\left(x^{\mu}(o)\right.$ is the arbitrary origin) and should tend to them in the Post-Newtonian approximation!

Moreover, from the equation $\partial_{\mu} \tau(z)=l_{\mu}(z) /[-\epsilon+n(z)]$ we could determine the function $\tau(z)$ associated with this class of globally hyperbolic spacetimes. The WSW hypersurface $\Sigma_{\tau}^{(W S W)}$ associated with the given solution is the set of points $z^{\mu}(\tau, \vec{\sigma})$ such that $\tau(z)=\tau$.

In conclusion, there are preferred $A D M$ geometrical and dynamical Eulerian observers

\footnotetext{
${ }^{146}$ It reflects the arbitrariness of the absolute location of the origin of asymptotic coordinates (and, therefore, also of the external center of mass $\left.\tilde{x}_{(\infty)}^{(\mu)}(0)\right)$ near spatial infinity.

${ }^{147}$ This paper contains the main references on the problem starting from Dixon's definition [123],
} 


$$
\begin{aligned}
& { }_{(\Sigma)}^{4} \check{E}_{(\alpha)}^{(W S W) \mu}=\left(l^{\mu} ; b_{s}^{\mu 3} e^{(W S W){ }_{(a)}}\right), \\
& \underset{(\Sigma)}{4} \check{\tilde{E}}_{(\alpha)}^{A}=\left(\frac{1}{-\epsilon+n}\left(1 ;-n^{r}\right) ;\left(0 ;{ }^{3} e_{(a)}^{(W S W) r}\right)\right) .
\end{aligned}
$$

They should be used as conventional celestial reference system (CCRS) $S_{I}$ based on an extragalactic radio-source catalogue system [148] : 124] this is a conventional definition of inertiality with respect to rotations ${ }^{149}$. Here the Fermi-Walker transport is replaced by the $\tau$-evolution of the WSW preferred tetrads. In this way one construct a kinematical reference frame in our special 3-orthogonal coordinates 50 . Let us remember that, given a reference (coordinate) system (one gives the form of the 4-metric), to construct a reference frame is to prescribe (materialization) some definite values of coordinates for reference astronomical objects.

Let us remark that in presence of matter Eq.(7.9) can be used to reformulate Dixon's theory of multipoles for extended objects [125,123] on WSW hypersurfaces by using the results of Refs. [126, 122].

\footnotetext{
${ }^{148}$ Or , somewhat less accurate, by a star catalogue system such as the FK5; or else by using the cosmic microwave background.

${ }^{149}$ The tabulated right ascensions and declinations and, in the case of a star catalogue, the proper motions (ephemerides) define the reference axes of CCRS; the axes are chosen in such a way that at a basic epoch they coincide in optimal approximation with the mean equatorial frame defined by the mean celestial pole and the mean dynamical equinox; these are non-relativistic definitions which can be applied to the asymptotic triads; in the relativistic case one considers the proper reference frame of a single observer, represented as a tetrad propagated along the worldline of the observer by Fermi-Walker transport: the time axis of the tetrad is the timelike worldline of the observer, while the three space axes are spacelike geodesics (Fermi normal coordinates).
}

${ }^{150}$ It is dynamical if referred to the ephemerides of some body in the solar system. 


\section{VOID SPACETIMES IN THE 3-ORTHOGONAL GAUGES.}

Let us remark that Minkowski spacetime in Cartesian coordinates is a solution of Einstein equations, which in the 3-orthogonal gauges corresponds to $q=\rho=r_{\bar{a}}=\pi_{\bar{a}}=0\left[\phi=e^{q / 2}=\right.$ 1] and $n=n_{r}=N_{(a s) r}=0, N_{(a s)}=-\epsilon$. For $q=\rho=r_{\bar{a}}=0$ Eq.(6.15) implies ${ }^{3} \hat{\tilde{\pi}}_{(a)}^{r}$ proportional to $\pi_{\bar{a}}$; the condition $\Sigma_{\tau}=R^{3}$ implies ${ }^{3} K_{r s}=0$ and then $\pi_{\bar{a}}=0$ as it will be shown in Eq. (8.6).

Therefore, it is consistent with Einstein equations to add by hand the two pairs of second class constraints $r_{\bar{a}}(\tau, \vec{\sigma}) \approx 0, \pi_{\bar{a}}(\tau, \vec{\sigma}) \approx 0$, to the Dirac Hamiltonian (6.5) in the 3-orthogonal gauges with arbitrary multipliers,

$$
H_{(D) A D M, R}^{\prime}=H_{(D) A D M, R}+\int d^{3} \sigma\left[\sum_{\bar{a}}\left(\mu_{\bar{a}} r_{\bar{a}}+\nu_{\bar{a}} \pi_{\bar{a}}\right)\right](\tau, \vec{\sigma}) .
$$

The time constancy of these second class constraints determines the multipliers

$$
\begin{aligned}
& \partial_{\tau} r_{\bar{a}}(\tau, \vec{\sigma}) \stackrel{\circ}{=} \nu_{\bar{a}}(\tau, \vec{\sigma})+\int d^{3} \sigma_{1} n\left(\tau, \vec{\sigma}_{1}\right)\left\{r_{\bar{a}}(\tau, \vec{\sigma}), \hat{\mathcal{H}}_{R}\left(\tau, \vec{\sigma}_{1}\right)\right\}^{*} \approx 0, \\
& \partial_{\tau} \pi_{\bar{a}}(\tau, \vec{\sigma}) \stackrel{\circ}{=}-\mu_{\bar{a}}(\tau, \vec{\sigma})+\int d^{3} \sigma_{1} n\left(\tau, \vec{\sigma}_{1}\right)\left\{\pi_{\bar{a}}(\tau, \vec{\sigma}), \hat{\mathcal{H}}_{R}\left(\tau, \vec{\sigma}_{1}\right)\right\}^{*} \approx 0 .
\end{aligned}
$$

By going to new Dirac brackets, we remain with the only conjugate pair $q(\tau, \vec{\sigma}), \rho(\tau, \vec{\sigma})$, constrained by the first class constraint $\left.\hat{\mathcal{H}}_{R}(\tau, \vec{\sigma})\right|_{r_{\bar{a}=\pi_{\bar{a}}=0}} \approx 0$. In this way we get the description of a family of gauge equivalent spacetimes $M^{4}$ without gravitational field (see III), which could be called void spacetimes, with 3-orthogonal coordinates for $\Sigma_{\tau}$. They turn out to be 3-conformally flat because ${ }^{3} \hat{g}_{r s}=\phi^{4} \delta_{r s}$, but with the conformal factor determined by the Lichnerowicz equation as a function of $\rho$ (therefore it is gauge dependent). Now, the line of Eqs.(6.16) giving ${ }^{3} K$ (with $r_{\bar{a}}=\pi_{\bar{a}}=0$ ) is an integral equation to get $\rho$ in terms of ${ }^{3} \hat{\tilde{\Pi}}\left(\right.$ or $\left.{ }^{3} \hat{K}\right)$ and $q=2 \ln \phi=\frac{1}{6} \ln { }^{3} \hat{g}$.

As we shall see, if we add the extra gauge-fixing $\rho(\tau, \vec{\sigma}) \approx 0$, we get the 3 -Euclidean metric $\delta_{r s}$ on $\Sigma_{\tau}$, since the superhamiltonian constraint (6.21) has $q(\tau, \vec{\sigma}) \approx 0[\phi(\tau, \vec{\sigma}) \approx 1]$ as a solution in absence of matter. The time constancy of $\rho(\tau, \vec{\sigma}) \approx 0$ implies $n(\tau, \vec{\sigma}) \approx 0$. Indeed, for the reduction to Minkowski spacetime, besides the solution $q(\tau, \vec{\sigma}) \approx 0$ of the superhamiltonian constraint (vanishing of the so called internal intrinsic (many-fingered) time [63]), we also need the gauge-fixings $N_{(a s)}(\tau, \vec{\sigma}) \approx \epsilon, N_{(a s) r}(\tau, \vec{\sigma}) \approx 0, n_{r}=0$.

The members of the equivalence class of void spacetimes represent flat Minkowski spacetimes in the most arbitrary coordinates compatible with Einstein theory with the associated inertial effects: they should correspond to the relativistic generalization (in absence of matter) of the class of Galilean non inertial frames (with their inertial forces) obtainable from an inertial frame of the nonrelativistic Galileo spacetime [5]. Therefore, they seem to represent the most general pure acceleration effects without gravitational field (i.e. without tidal

\footnotetext{
${ }^{151}$ For example the (maybe time-dependent) pseudo-diffeomorphisms in Diff $\Sigma_{\tau}$ replace the Galilean coordinate transformations generating the inertial forces.
} 
effects) but with a control on the boundary conditions compatible with Einstein's general relativity for globally hyperbolic, asymptotically flat at spatial infinity spacetimes 5 .52.

The concept of void spacetime implements the viewpoint of Synge [91] that, due to tidal (i.e. curvature) effects, there is a difference between true gravitational fields and accelerated motions, even if, as shown in Ref. [127], Einstein arrived at general relativity through the intermediate step of showing the equivalence of uniform acceleration with special homogeneous gravitational fields. It is only in the not generally covariant Hamiltonian approach that one is able to identify the genuine physical degrees of freedom of the gravitational field.

Since in void spacetimes without matter there are no physical degrees of freedom of the gravitational field but only gauge degrees of freedom, we expect that this equivalence class of spacetimes is not described by scenario b) of Chapter IV, but that it corresponds to scenario a) with vanishing Poincaré charges (the exceptional Poincaré orbit). Indeed, in this way Minkowski spacetime (and its gauge copies) would be selected as the static background for special relativity with zero energy (after the regularization of Ref. [34] which however influences only the ADM boosts), starting point for parametrized Minkowski theories where the special relativistic energy would be generated only by the added matter (and/or fields). Tetrad gravity with matter is described by scenario b) (with the WSW hypersurfaces, defined by the gravitational field and by matter, corresponding to Wigner hyperplanes, defined by matter) and in the limit $G \rightarrow 0$ the weak ADM energy [present in scenario b) but not in scenario a)] tends to the special relativistic energy of that matter system with no trace left of the gravitational field energy. However, starting from parametrized Minkowski theories on the background given by Minkowski spacetime in Cartesian coordinates, we can introduce much more allowed $3+1$ splittings (much more types of inertial forces) than in general relativity, because in special relativity there is no restriction on the spacelike hypersurfaces like being conformally flat.

To define void spacetimes independently from the 3-orthogonal gauge, let us remark that, since the conditions $r_{\bar{a}}(\tau, \vec{\sigma})=0$ imply the vanishing of the 3-conformal Cotton-York tensor (see Appendix A after Eq. (A3) for the definition of this tensor and Eq.(A4) for its vanishing). Therefore, the general theory of void spacetimes could be reformulated in arbitrary gauges by adding with Lagrange multipliers the two independent components of the Cotton-York tensor ${ }^{3} \mathcal{Y}_{r s}(\tau, \vec{\sigma})$ (which is a function only of cotriads) to the tetrad ADM Lagrangian of Eq.(2.8) for tetrad gravity. In this way one should get two extra holonomic constraints equivalent to $r_{\bar{a}}(\tau, \vec{\sigma}) \approx 0$. Their time constancy should produce two secondary (momentum dependent) constraints equivalent to $\pi_{\bar{a}}(\tau, \vec{\sigma}) \approx 0$.

\footnotetext{
${ }^{152}$ See also the discussion on general covariance and on the various formulations of the equivalence principle (homogeneous gravitational fields $=$ absence of tidal effects) in Norton's papers 127. See also Refs. 128] for the definition of inertial forces in general relativity: the criticism to this approach saying that the separation of inertial forces from the gravitational field is arbitrary due to the equivalence principle, is solved in our approach by the Shanmugadhasan canonical transformation which separates the conformal factor from the Dirac observables for the gravitational field. In this way the inertial forces become gauge quantities as expected, to be determined by the gauge fixing to the superhamiltonian constraint, which determines a unique $3+1$ splitting of spacetime. These forces depend on the embedding $z^{\mu}(\tau, \vec{\sigma})$ of the leaves $\Sigma_{\tau}$.
} 
Deferring to a future paper the study of the general case, let us explore the properties of void spacetimes in the 3 -orthogonal gauges.

Void spacetimes depend only on the following variables:

i) $\phi(\tau, \vec{\sigma})=e^{q(\tau, \vec{\sigma}) / 2}$, to be determined by the reduced Lichnerowicz equation;

ii) $\pi_{\phi}(\tau, \vec{\sigma})=2 \phi^{-1}(\tau, \vec{\sigma}) \rho(\tau, \vec{\sigma})$, the conjugate gauge variable.

We have

$$
\begin{aligned}
& r_{\bar{a}}=\pi_{\bar{a}}=0, \quad \phi=e^{q / 2} \quad[\text { or } q=2 \ln \phi], \\
& { }^{3} \hat{e}_{(a) r}=\phi^{2} \delta_{(a) r}, \\
& { }^{3} \hat{e}_{(a)}^{r}=\phi^{-2} \delta_{(a)}^{r}, \\
& { }^{3} \hat{g}_{r s}=\phi^{4} \delta_{r s}, \quad{ }^{3} \tilde{g}_{r s}\left[r_{\bar{a}}=0\right]=\delta_{r s}, \\
& \tilde{\triangle}\left[r_{\bar{a}}=0\right]=\triangle_{F L A T}, \quad{ }^{3} \tilde{R}\left[r_{\bar{a}}=0\right]=0 .
\end{aligned}
$$

From Eqs.(6.14), 6.15), (6.16) and (6.21) one has in void spacetimes (before putting $\left.\rho=\frac{1}{2} \phi \pi_{\phi}=0\right)$

$$
\begin{aligned}
{ }^{3} \hat{g}_{r s}(\tau, \vec{\sigma}) & =\phi^{4}(\tau, \vec{\sigma}) \delta_{r s}, \\
{ }^{3} \hat{\tilde{\pi}}_{(a)}^{r}(\tau, \vec{\sigma}) & =\frac{1}{3} \int d^{3} \sigma_{1} \tilde{\mathcal{K}}_{(a) s}^{r}\left(\vec{\sigma}, \vec{\sigma}_{1} ; \tau \mid \phi, 0\right] \rho\left(\tau, \vec{\sigma}_{1}\right),
\end{aligned}
$$

and

$$
\begin{aligned}
{ }^{3} \hat{K}_{r s}(\tau, \vec{\sigma})= & \frac{\epsilon \pi G}{c^{3}}\left[\sum_{u}\left(\delta_{r u} \delta_{(a) s}+\delta_{s u} \delta_{(a) r}-\delta_{r s} \delta_{(a) u}\right)^{3} \hat{\tilde{\pi}}_{(a)}^{u}\right](\tau, \vec{\sigma}), \\
{ }^{3} \hat{K}(\tau, \vec{\sigma})= & -\frac{\epsilon 4 \pi G}{c^{3}}\left[\phi^{-4} \sum_{u} \delta_{(a) u}{ }^{3} \hat{\tilde{\pi}}_{(a)}^{u}\right](\tau, \vec{\sigma})= \\
= & -\frac{\epsilon 4 \pi G}{c^{3}} \phi^{-6}(\tau, \vec{\sigma})\left\{\rho(\tau, \vec{\sigma})+\frac{1}{3} \phi^{2}(\tau, \vec{\sigma}) \sum_{u} \int d^{3} \sigma_{1} \delta_{(a) u}\right. \\
& \left.\sum_{s} \mathcal{T}_{(a) s}^{u}\left(\vec{\sigma}, \vec{\sigma}_{1} ; \tau \mid \phi, 0\right] \phi^{-2}\left(\tau, \vec{\sigma}_{1}\right) \rho\left(\tau, \vec{\sigma}_{1}\right)\right\} \\
\hat{\mathcal{H}}_{R}^{\prime}(\tau, \vec{\sigma})= & \left.\hat{\mathcal{H}}_{R}(\tau, \vec{\sigma})\right|_{r_{\bar{a}}=\pi_{\bar{a}}=0}= \\
= & \epsilon \phi(\tau, \vec{\sigma})\left(-\frac{c^{3}}{2 \pi G} \triangle_{F L A T} \phi(\tau, \vec{\sigma})+\frac{2 \pi G}{3 c^{3}}\left[\left(\phi^{-7} \rho\right)(\tau, \vec{\sigma})+\right.\right. \\
+ & \frac{2}{3}\left(\phi^{-5} \rho\right)(\tau, \vec{\sigma}) \int d^{3} \sigma_{1} \sum_{r} \delta_{(a)}^{u} \mathcal{T}_{(a) r}^{u}\left(\vec{\sigma}, \vec{\sigma}_{1} ; \tau \mid \phi, 0\right]\left(\phi^{-2} \rho\right)\left(\tau, \vec{\sigma}_{1}\right)- \\
- & \frac{1}{3} \phi^{-3}(\tau, \vec{\sigma}) \int d^{3} \sigma_{1} d^{3} \sigma_{2}\left(\sum_{u}\right. \\
& \sum_{r} \mathcal{T}_{(a) r}^{u}\left(\vec{\sigma}, \vec{\sigma}_{1} ; \tau \mid \phi, 0\right]\left(\phi^{-2} \rho\right)\left(\tau, \vec{\sigma}_{1}\right) \\
& \sum_{s} \mathcal{T}_{(a) s}^{u}\left(\vec{\sigma}, \vec{\sigma}_{2} ; \tau \mid \phi, 0\right]\left(\phi^{-2} \rho\right)\left(\tau, \vec{\sigma}_{2}\right)+ \\
+ & \sum_{u v}\left(\delta_{(b)}^{u} \delta_{(a)}^{v}-\delta_{(a)}^{u} \delta_{(b)}^{v}\right) \\
& \sum_{r} \mathcal{T}_{(a) r}^{u}\left(\vec{\sigma}, \vec{\sigma}_{1} ; \tau \mid \phi, 0\right]\left(\phi^{-2} \rho\right)\left(\tau, \vec{\sigma}_{1}\right) \\
& \left.\left.\left.\sum_{s} \mathcal{T}_{(a) s}^{v}\left(\vec{\sigma}, \vec{\sigma}_{2} ; \tau \mid \phi, 0\right]\left(\phi^{-2} \rho\right)\left(\tau, \vec{\sigma}_{2}\right)\right)\right]\right) \approx 0 .
\end{aligned}
$$


With the natural gauge $\rho(\tau, \vec{\sigma}) \approx 0$, one has

$$
\begin{aligned}
& { }^{3} \hat{\tilde{\pi}}_{(a)}^{r}(\tau, \vec{\sigma}) \approx 0, \\
& { }^{3} \hat{K}_{r s}(\tau, \vec{\sigma}) \approx 0, \quad \Rightarrow{ }^{3} \hat{K}(\tau, \vec{\sigma}) \approx 0,
\end{aligned}
$$

and the reduced superhamiltonian constraint becomes the reduced Lichnerowicz equation $\left[\triangle_{F L A T}=\vec{\partial}^{2}\right]$

$$
\triangle_{F L A T} \phi(\tau, \vec{\sigma}) \approx 0, \quad \Rightarrow \phi(\tau, \vec{\sigma})=1, \Rightarrow{ }^{3} \hat{g}_{r s}=\delta_{r s}
$$

where we have shown the solution corresponding to the boundary condition of Eq.(6.11).

In scenario a) with the Dirac Hamiltonian (2.22) we add the gauge fixing constraints $\tilde{\lambda}_{\tau}(\tau) \approx \epsilon, \tilde{\lambda}_{r}(\tau) \approx 0, \tilde{\lambda}_{A B}(\tau) \approx 0$ to the primary constraints $\tilde{\pi}^{A}(\tau) \approx 0, \tilde{\pi}^{A B}(\tau) \approx 0$. This implies $\zeta_{A}(\tau) \approx 0, \zeta_{A B}(\tau)=0$ and $N_{(a s)}(\tau, \vec{\sigma}) \approx-\epsilon, N_{(a s) r}(\tau, \vec{\sigma}) \approx 0$. Then we add the gauge fixings $\alpha_{(a)}(\tau, \vec{\sigma}) \approx 0, \varphi_{(a)}(\tau, \vec{\sigma}) \approx 0$ to the rotation and boost primary constraints (they imply $\left.\lambda_{(a)}^{\vec{\varphi}}(\tau, \vec{\sigma})=\hat{\mu}_{(a)}(\tau, \vec{\sigma})=0\right)$. Then we add the second class constraints $r_{\bar{a}}(\tau, \vec{\sigma}) \approx 0, \pi_{\bar{a}}(\tau, \vec{\sigma}) \approx 0$ by hand. We choose the 3 -orthogonal gauges for parametrizing the cotriads and we add the gauge fixings $\xi^{r}(\tau, \vec{\sigma})-\vec{\sigma} \approx 0$ : this determines the shift functions $N_{r}(\tau, \vec{\sigma})=n_{r}(\tau, \vec{\sigma})$ and the Dirac multipliers $\lambda_{r}^{\vec{n}}(\tau, \vec{\sigma})$. We have $N(\tau, \vec{\sigma})=-\epsilon+n(\tau, \vec{\sigma})$.

The Dirac Hamiltonian becomes

$$
H_{(D) A D M}^{(1)}=\int d^{3} \sigma\left[n \hat{\mathcal{H}}_{R}^{\prime}+\lambda_{n} \tilde{\pi}^{n}\right](\tau, \vec{\sigma})-\epsilon \hat{P}_{A D M}^{\tau^{\prime}},
$$

with $\hat{\mathcal{H}}_{R}^{\prime}=\left.\hat{\mathcal{H}}_{R}\right|_{r_{\bar{a}}=\pi_{\bar{a}}=0}, \hat{P}_{A D M}^{\tau^{\prime}}=\left.\hat{P}_{A D M}^{\tau}\right|_{r_{\bar{a}}=\pi_{\bar{a}}=0}$.

The natural gauge fixing $\rho(\tau, \vec{\sigma}) \approx 0$ implies

$$
\partial_{\tau} \rho(\tau, \vec{\sigma}) \stackrel{\circ}{=} \int d^{3} \sigma_{1} n\left(\tau, \vec{\sigma}_{1}\right)\left\{\rho(\tau, \vec{\sigma}), \hat{\mathcal{H}}_{R}^{\prime}\left(\tau, \vec{\sigma}_{1}\right)\right\}-\epsilon\left\{\rho(\tau, \vec{\sigma}), \hat{P}_{A D N}^{\tau^{\prime}}\right\}
$$

but from Eq.(2.25) and from ${ }^{3} \tilde{\Pi}^{r s} \approx 0$ we see that only the term bilinear in the Christoffel symbols contributes to $\left\{\rho(\tau, \vec{\sigma}), \hat{P}_{A D N}^{\tau^{\prime}}\right\}$ for $\rho(\tau, \vec{\sigma}) \approx 0$. Now from Eq.(6.16) we get ${ }^{3} \hat{\Gamma}_{u v}^{r}=$ $2 \phi^{-1}\left[\delta_{u v} \partial_{r} \phi+\delta_{r u} \partial_{v} \phi+\delta_{r v} \partial_{u} \phi\right] \rightarrow_{\phi \rightarrow \text { const. }} 0$. Since $\phi(\tau, \vec{\sigma})=1$ is the solution of the reduced Lichnerowicz equation for $\rho(\tau, \vec{\sigma}) \approx 0$, we get $\left\{\rho(\tau, \vec{\sigma}), \hat{P}_{A D N}^{\tau^{\prime}}\right\} \approx 0$ and then $n(\tau, \vec{\sigma}) \approx 0$ and $\lambda_{n}(\tau, \vec{\sigma}) \approx 0$. Therefore, at the end the lapse function is $N(\tau, \vec{\sigma}) \approx-\epsilon$.

Since, as we shall see, $\hat{P}_{A D N}^{\tau^{\prime}}$ vanishes for $\rho(\tau, \vec{\sigma}) \approx 0, \phi(\tau, \vec{\sigma})=1$, the final Dirac Hamiltonian vanishes: $H_{(D) A D M}^{(1)} \approx 0$, and the final 4-metric becomes

$$
{ }^{4} \hat{g}_{A B}(\tau, \vec{\sigma})=\epsilon\left(\begin{array}{cc}
1 & 0 \\
0 & -\delta_{r s}
\end{array}\right)
$$

In void spacetimes the two gauge-fixings $\rho(\tau, \vec{\sigma}) \approx 0$ and ${ }^{3} \hat{K}(\tau, \vec{\sigma}) \approx 0$ are equivalent and one has $\phi(\tau, \vec{\sigma})=1$ (i.e. $q(\tau, \vec{\sigma})=0$ ); in this gauge one has ${ }^{3} \hat{R}=0$ for the 3 -hypersurfaces $\Sigma_{\tau}$ (they have both the scalar curvature and the trace of the extrinsic one vanishing), but in other gauges the 3-curvature and the trace of the extrinsic one may be not vanishing because the solution $\phi(\tau, \vec{\sigma})$ of the reduced Lichnerowicz equation may become nontrivial.

In Appendix B there are the weak and strong Poincaré charges for void spacetimes. It is shown in Eqs.(B4) that at the level of Dirac brackets with respect to the natural gauge 
fixing $\rho(\tau, \vec{\sigma}) \approx 0$ (i.e. with respect to the pair of second class constraints $\rho \approx 0, \phi-1 \approx 0$ ) the ten weak and strong Poincaré charges vanish for the solution $\phi(\tau, \vec{\sigma})=1$ selected by the boundary conditions (6.11) (so that they must vanish in all the others gauges connected with this solution, being conserved gauge invariant quantities). In connection with this result let us remark that only the boosts depend on the regularization. The result is consistent with parametrized Minkowski theories whose Poincaré charges are not defined in absence of matter.

As said void spacetimes describe pure acceleration effects without dynamical gravitational field (no tidal effects), allowed in flat spacetimes as the relativistic generalization of Galilean non inertial observers, they cannot be used to describe test matter in flat spacetimes in some post-Minkowskian approximation.

As we shall see in a future paper, where we shall study the action-at-a- distance instantaneous effects on scalar particles implied by Einstein theory in the ideal limit of a negligible gravitational field [5], in presence of matter, in the static ideal limit $\left|r_{\bar{a}}(\tau, \vec{\sigma})\right|<<1$, $\left|\pi_{\bar{a}}(\tau, \vec{\sigma})\right|<<1$, Eq.(8.7) becomes the Poisson equation $-\triangle_{F L A T} \phi=\rho_{\text {matter }}+O\left(r_{\bar{a}}, \pi_{\bar{a}}\right)$, showing that $\phi(\tau, \vec{\sigma})$ is the general relativistic generalization of the Newton potential [see also the term $\mathrm{M}$ in the boundary conditions (6.11)]. But now the Poincaré charges are not vanishing so that we cannot use void spacetimes as approximations of spacetimes $M^{4}$ for extremely weak gravitational fields: already this kind of post-Minkowskian approximation lives in non trivial spacetimes (with non trivial WSW hypersurfaces) not gauge equivalent to Minkowski spacetime in Cartesian coordinates. This is contrary to the expectation that for weak gravitational fields a spacetime can be approximated by a void spacetime with test matter, but it is consistent with parametrized Minkowski theory for that test matter: its arbitrary spacelike hypersurfaces embedded in Minkowski spacetime describe a family of accelerated observers much bigger of the one allowed in tetrad gravity (namely the WSW hypersurfaces). However, as noted in footnote 39 of Section II, to avoid $3+1$ splittings geometrically ill-defined at spatial infinity one has to make the restriction $\tilde{\lambda}_{A B}(\tau)=0$ also in Minkowski spacetime, and this is a strong restriction on the allowed accelerated observers. The implications of this fact, which have still to be investigated, are that most of the possible accelerated reference systems of Minkowski spacetime (which are the starting point for the classical basis of the Unruh effect [129]) are unrelated with general relativity, at least with its canonical ADM formulation presented in this paper. Also, more study will be needed to clarify the conceptual difference between a test particle following a geodesic of an external gravitational field and a dynamical particle plus the gravitational field (such a particle will not follow a priori a geodesic of the resulting dynamical gravitational field).

\footnotetext{
${ }^{153}$ A more realistic situation with tidal effects will be possible only after having studied the linearization of tetrad gravity in 3-orthogonal gauges.
} 


\section{CONCLUSIONS.}

In this paper we have defined the rest-frame instant form and have studied the Hamiltonian group of gauge transformations of tetrad gravity, following what had been done in Ref. [6] for metric gravity, for a class of spacetimes of the Christodoulou-Klainermann type. We have clarified the meaning of the gauge transformations generated by the 14 first class constraints of tetrad gravity. In particular, like in metric gravity, the action of the superhamiltonian constraint is to transform the leaves of the foliation associated with an allowed $3+1$ splitting of spacetime into the leaves of another allowed $3+1$ splitting. Since in Ref. [6] there is also the distinction between Hamiltonian kinematical and dynamical gravitational fields(the second ones are solutions of the Einstein equations), which is needed because at the Hamiltonian level the Dirac observables are determined before solving the Hamilton equations, we have not touched this point here.

We have then found a quasi-Shanmugadhasan canonical transformation adapted to 3orthogonal gauges and identified the Hamiltonian (kinematical) Dirac observables for the gravitational field in a special 3-orthogonal gauge defined by the vanishing of the momentum conjugate to the conformal factor of the 3-metric, which in turn is determined by the reduced Lichnerowicz equation, even if we are not able to solve this equation. The evolution in the mathematical time labelling the WSW spacelike hypersurfaces of the rest-frame instant form, namely the leaves of the foliation in this special 3-orthogonal gauge, is governed by the weak ADM energy restricted to this gauge. We have also shown that the WSW hypersurfaces imply the existence of asymptotic preferred geometrical and dynamical inertial observers to be identified with the fixed stars.

Therefore for the first time we can visualize the (non covariant) physical degrees of freedom of the gravitational field in a completely fixed gauge. This allows to define special spacetimes, void spacetimes, in which there is no gravitational field but only inertial effects like in the non-inertial frames in Newtonian gravity. Minkowski spacetime without matter, with arbitrary coordinates and with the allowed $3+1$ splittings restricted to those whose leaves are 3-conformally flat (with the conformal factor determined by the Lichnerowicz equation) seems to be the only void spacetime: it is the background with vanishing Poincaré charges where parametrized Minkowski theories live [54.

The results of this paper seems to be the best which can be obtained with the existing technology.

Referring to the Conclusions of Ref. [6] for some comments on the quantization of gravity in a completely fixed gauge, we list here the main open problem to be studied:

1) To define the linearized theory: to find a solution of a linearization of the Lichnerowicz equation, which put inside a linearization of the weak ADM energy to a quadratic expression in $r_{\bar{a}}$ and $\pi_{\bar{a}}$ will imply a linear equation for the physical canonical variables $r_{\bar{a}}(\tau, \vec{\sigma})$ describing the gravitational field, so to make contact with the theory of gravitational waves.

\footnotetext{
${ }^{154}$ Parametrized Minkowski theories considered as autonomous special relativistic theories and not as a limiting case of general relativity have no restriction on the allowed $3+1$ splittings. Therefore the class of their allowed inertial forces is much bigger, since the leaves are not restricted to be 3-conformally flat.
} 
Let us remark that such a linearized theory is not a spin-2 theory on Minkowski background but an approximate linearized Einstein spacetime with non-vanishing shift functions and gravitomagnetic effects.

2) To study other 3-coordinate gauges like the 3-normal coordinates with respect to a point or systems of 3 -coordinates implying the reduction of the $3+1$ splitting to an effective $2+2$ splitting. It could be that the Lichnerowicz equation has some simplification in these gauges.

3) To add matter (either scalar particles or perfect fluids [130]) and extract the actionat-a-distance potential hidden in gravity in a post-Minkowskian approximation.

4) To add the electromagnetic field and to study the canonical reduction in its presence as a first step in the direction of unifying tetrad gravity with the standard $\mathrm{SU}(3) \mathrm{xSU}(2) \mathrm{xU}(1)$ elementary particle model.

5) To understand better the observables the non-tensorial Dirac observables versus the diffeomorphism-invariant ones), the time problem and the physical identification of the points of spacetime in general relativity (see Ref. [6]). In particular to make contact with measurements in a completely fixed gauge of tidal effects: which kind of observables are selected by them?

6) To study tetrad gravity as the dynamical theory of a congruence of timelike observers: reformulate gravitomagnetism, accelerated observers and their simultaneity surface and similar problems in the tetrad gravity language. 


\section{APPENDIX A: 3-TENSORS IN THE SPECIAL 3-ORTHOGONAL GAUGE.}

The 3-dimensional spin connection 1 -form ${ }^{3} \omega_{r(b)}^{(a)} d \sigma^{r}$, Christoffel coefficients ${ }^{3} \Gamma_{u v}^{r}$, field strength ${ }^{3} \Omega_{r s(a)}$ and the curvature tensor ${ }^{3} R_{\text {suv }}^{r}$ of the Riemannian 3 -manifold $\left(\Sigma_{\tau},{ }^{3} g_{r s}=\right.$ $\left.{ }^{3} e_{(a) r}{ }^{3} e_{(a) s}\right)$ have the following expression in terms of the cotriads ${ }^{3} e_{(a) r}$ (see Ref. [1] for the definitions)

$$
\begin{aligned}
& { }^{3} \omega_{r(b)}^{(a)}={ }^{3} \omega_{(c)(b)}^{(a)}{ }^{3} e_{r}^{(c)}={ }^{3} e_{s}^{(a) 3} \nabla_{r}{ }^{3} e_{(b)}^{s}= \\
& ={ }^{3} e_{s}^{(a) 3} e_{(b) \mid r}^{s}={ }^{3} e_{s}^{(a)}\left[\partial_{r}{ }^{3} e_{(b)}^{s}+{ }^{3} \Gamma_{r u}^{s}{ }^{3} e_{(b)}^{u}\right], \\
& { }^{3} \omega_{(a)(b)}=\delta_{(a)(c)}{ }^{3} \omega_{r(b)}^{(c)} d \sigma^{r}=-{ }^{3} \omega_{(b)(a)}, \quad{ }^{3} \omega_{r(a)}=\frac{1}{2} \epsilon_{(a)(b)(c)}{ }^{3} \omega_{r(b)(c)}, \\
& { }^{3} \omega_{r(a)(b)}=\epsilon_{(a)(b)(c)}{ }^{3} \omega_{r(c)}=\left[\hat{R}^{(c) 3} \omega_{r(c)}\right]_{(a)(b)}=\left[{ }^{3} \omega_{r}\right]_{(a)(b)}, \\
& { }^{3} \Gamma_{r s}^{u}={ }^{3} \Gamma_{s r}^{u}=\frac{1}{2}{ }^{3} e_{(a)}^{u}\left[\partial_{r}{ }^{3} e_{(a) s}+\partial_{s}{ }^{3} e_{(a) r}+\right. \\
& \left.+{ }^{3} e_{(a)}^{v}\left({ }^{3} e_{(b) r}\left(\partial_{s}{ }^{3} e_{(b) v}-\partial_{v}{ }^{3} e_{(b) s}\right)+{ }^{3} e_{(b) s}\left(\partial_{r}{ }^{3} e_{(b) v}-\partial_{v}{ }^{3} e_{(b) r}\right)\right)\right]= \\
& =\frac{1}{2} \epsilon_{(a)(b)(c)}{ }^{3} e_{(a)}^{u}\left({ }^{3} e_{(b) r}{ }^{3} \omega_{s(c)}+{ }^{3} e_{(b) s}{ }^{3} \omega_{r(c)}\right)-\frac{1}{2}\left({ }^{3} e_{(a) r} \partial_{s}{ }^{3} e_{(a)}^{u}+{ }^{3} e_{(a) s} \partial_{r}{ }^{3} e_{(a)}^{u}\right), \\
& { }^{3} \omega_{r(a)(b)}=-{ }^{3} \omega_{r(b)(a)}=\frac{1}{2}\left[{ }^{3} e_{(a)}^{s}\left(\partial_{r}{ }^{3} e_{(b) s}-\partial_{s}{ }^{3} e_{(b) r}\right)+\right. \\
& \left.+{ }^{3} e_{(b)}^{s}\left(\partial_{s}{ }^{3} e_{(a) r}-\partial_{r}{ }^{3} e_{(a) s}\right)+{ }^{3} e_{(a)}^{u}{ }^{3} e_{(b)}^{v}{ }^{3} e_{(c) r}\left(\partial_{v}{ }^{3} e_{(c) u}-\partial_{u}{ }^{3} e_{(c) v}\right)\right]= \\
& =\frac{1}{2}\left[{ }^{3} e_{(a) u} \partial_{r}{ }^{3} e_{(b)}^{u}-{ }^{3} e_{(b) u} \partial_{r}{ }^{3} e_{(a)}^{u}+{ }^{3} \Gamma_{r s}^{u}\left({ }^{3} e_{(a) u}{ }^{3} e_{(b)}^{s}-{ }^{3} e_{(b) u}{ }^{3} e_{(a)}^{s}\right)\right], \\
& { }^{3} \omega_{r(a)}=\frac{1}{2} \epsilon_{(a)(b)(c)}\left[{ }^{3} e_{(b)}^{u}\left(\partial_{r}{ }^{3} e_{(c) u}-\partial_{u}{ }^{3} e_{(c) r}\right)+\right. \\
& \left.+\frac{1}{2}{ }^{3} e_{(b)}^{u}{ }^{3} e_{(c)}^{v}{ }^{3} e_{(d) r}\left(\partial_{v}{ }^{3} e_{(d) u}-\partial_{u}{ }^{3} e_{(d) v}\right)\right], \\
& {\left[{ }^{3} e_{(a)},{ }^{3} e_{(b)}\right]=\left({ }^{3} \omega_{(a)(b)}^{(c)}-{ }^{3} \omega_{(b)(a)}^{(c)}\right)^{3} e_{(c)},} \\
& { }^{3} \Omega^{(a)}{ }_{(b)(c)(d)}={ }^{3} e_{(c)}\left({ }^{3} \omega_{(d)(b)}^{(a)}\right)-{ }^{3} e_{(d)}\left({ }^{3} \omega_{(c)(b)}^{(a)}\right)+ \\
& +{ }^{3} \omega_{(d)(b)}^{(n)}{ }^{3} \omega_{(c)(n)}^{(a)}-{ }^{3} \omega_{(c)(b)}^{(n)}{ }^{3} \omega_{(d)(n)}^{(a)}-\left({ }^{3} \omega_{(c)(d)}^{(n)}-{ }^{3} \omega_{(d)(c)}^{(n)}\right)^{3} \omega_{(a)(b)}^{(a)}= \\
& ={ }^{3} e_{r}^{(a){ }^{3}} R^{r}{ }_{s t w}{ }^{3} e_{(b)}^{s}{ }^{3} e_{(c)}^{t}{ }^{3} e_{(d)}^{w}, \\
& { }^{3} \Omega_{r s}{ }^{(a)}{ }_{(b)}={ }^{3} e_{r}^{(c) 3} e_{s}^{(d) 3}{ }^{3}{ }^{(a)}{ }_{(b)(c)(d)}={ }^{3} R^{t}{ }_{w r s}{ }^{3} e_{t}^{(a)} 3 e_{(b)}^{w}= \\
& =\partial_{r}{ }^{3} \omega_{s(b)}^{(a)}-\partial_{s}{ }^{3} \omega_{r(b)}^{(a)}+{ }^{3} \omega_{r(c)}^{(a)}{ }^{3} \omega_{s(b)}^{(c)}-{ }^{3} \omega_{s(c)}^{(a)}{ }^{3} \omega_{r(b)}^{(c)}= \\
& =\delta^{(a)(c){ }^{3} \Omega_{r s(c)(b)}}=\delta^{(a)(c)} \epsilon_{(c)(b)(d)}{ }^{3} \Omega_{r s(d)}, \\
& { }^{3} \Omega_{r s(a)}=\frac{1}{2} \epsilon_{(a)(b)(c)}{ }^{3} \Omega_{r s(b)(c)}=\partial_{r}{ }^{3} \omega_{s(a)}-\partial_{s}{ }^{3} \omega_{r(a)}-\epsilon_{(a)(b)(c)}{ }^{3} \omega_{r(b)}{ }^{3} \omega_{s(c)}=
\end{aligned}
$$




$$
\begin{aligned}
& =\frac{1}{2} \epsilon_{(a)(b)(c)}\left[\partial_{r}{ }^{3} e_{(b)}^{u} \partial_{s}{ }^{3} e_{(c) u}-\partial_{s}{ }^{3} e_{(b)}^{u} \partial_{r}{ }^{3} e_{(c) u}+\right. \\
& +{ }^{3} e_{(b)}^{u}\left(\partial_{u} \partial_{s}{ }^{3} e_{(c) r}-\partial_{u} \partial_{r}{ }^{3} e_{(c) s}\right)+ \\
& +\frac{1}{2}\left({ }^{3} e_{(b)}^{u}{ }^{3} e_{(c)}^{v}\left(\partial_{r}{ }^{3} e_{(d) s}-\partial_{s}{ }^{3} e_{(d) r}\right)\left(\partial_{v}{ }^{3} e_{(d) u}-\partial_{u}{ }^{3} e_{(d) v}\right)+\right. \\
& \left.\left.+\left({ }^{3} e_{(d) s} \partial_{r}-{ }^{3} e_{(d) r} \partial_{s}\right)\left[{ }^{3} e_{(b)}^{u}{ }^{3} e_{(c)}^{v}\left(\partial_{v}{ }^{3} e_{(d) u}-\partial_{u}{ }^{3} e_{(d) v}\right)\right]\right)\right]- \\
& -\frac{1}{8}\left[\delta_{(a)\left(b_{1}\right)} \epsilon_{\left(c_{1}\right)\left(c_{2}\right)\left(b_{2}\right)}+\delta_{(a)\left(b_{2}\right)} \epsilon_{\left(c_{1}\right)\left(c_{2}\right)\left(b_{1}\right)}+\delta_{(a)\left(c_{1}\right)} \epsilon_{\left(b_{1}\right)\left(b_{2}\right)\left(c_{2}\right)}+\delta_{(a)\left(c_{2}\right)} \epsilon_{\left(b_{1}\right)\left(b_{2}\right)\left(c_{1}\right)}\right] \times \\
& { }^{3} e_{\left(b_{1}\right)}^{u_{1}}{ }^{3} e_{\left(b_{2}\right)}^{u_{2}}\left[\left(\partial_{r}{ }^{3} e_{\left(c_{1}\right) u_{1}}-\partial_{u_{1}}{ }^{3} e_{\left(c_{1}\right) r}\right)\left(\partial_{s}{ }^{3} e_{\left(c_{2}\right) u_{2}}-\partial_{u_{2}}{ }^{3} e_{\left(c_{2}\right) s}\right)+\right. \\
& +\frac{1}{2}\left({ }^{3} e_{\left(c_{2}\right)}^{v_{2}}{ }^{3} e_{(d) s}\left(\partial_{r}{ }^{3} e_{\left(c_{1}\right) u_{1}}-\partial_{u_{1}}{ }^{3} e_{\left(c_{1}\right) r}\right)\left(\partial_{v_{2}}{ }^{3} e_{(d) u_{2}}-\partial_{u_{2}}{ }^{3} e_{(d) v_{2}}\right)+\right. \\
& \left.+{ }^{3} e_{\left(c_{1}\right)}^{v_{1}} e_{(d) r}\left(\partial_{s}{ }^{3} e_{\left(c_{2}\right) u_{2}}-\partial_{u_{2}}{ }^{3} e_{\left(c_{2}\right) s}\right)\left(\partial_{v_{1}}{ }^{3} e_{(d) u_{1}}-\partial_{u_{1}}{ }^{3} e_{(d) v_{1}}\right)\right)+ \\
& \left.+\frac{1}{4}{ }^{3} e_{\left(c_{1}\right)}^{v_{1}}{ }^{3} e_{\left(c_{2}\right)}^{v_{2}}{ }^{3} e_{\left(d_{1}\right) r}{ }^{3} e_{\left(d_{2}\right) s}\left(\partial_{v_{1}}{ }^{3} e_{\left(d_{1}\right) u_{1}}-\partial_{u_{1}}{ }^{3} e_{\left(d_{1}\right) v_{1}}\right)\left(\partial_{v_{2}}{ }^{3} e_{\left(d_{2}\right) u_{2}}-\partial_{u_{2}}{ }^{3} e_{\left(d_{2}\right) v_{2}}\right)\right] \text {, } \\
& { }^{3} \Omega_{r s(a)(b)}=\epsilon_{(a)(b)(c)}{ }^{3} \Omega_{r s(c)}, \\
& { }^{3} R_{r s u v}=\epsilon_{(a)(b)(c)}{ }^{3} e_{(a) r}{ }^{3} e_{(b) s}{ }^{3} \Omega_{u v(c)}, \\
& { }^{3} R_{r s}=\frac{1}{2} \epsilon_{(a)(b)(c)}{ }^{3} e_{(a)}^{u}\left[{ }^{3} e_{(b) r}{ }^{3} \Omega_{u s(c)}+{ }^{3} e_{(b) s}{ }^{3} \Omega_{u r(c)}\right], \\
& { }^{3} R=\epsilon_{(a)(b)(c)}{ }^{3} e_{(a)}^{r}{ }^{3} e_{(b)}^{s}{ }^{3} \Omega_{r s(c)} .
\end{aligned}
$$

where $\epsilon_{(a)(b)(c)}$ is the standard Euclidean antisymmetric tensor and $\left(\hat{R}^{(c)}\right)_{(a)(b)}=\epsilon_{(a)(b)(c)}$ is the adjoint representation of $\mathrm{SO}(3)$ generators. The first Bianchi identity ${ }^{3} R_{r s u}^{t}+{ }^{3} R_{\text {sur }}^{t}+$ ${ }^{3} R_{\text {urs }}^{t} \equiv 0$ implies the cyclic identity ${ }^{3} \Omega_{r s(a)}{ }^{3} e_{(a)}^{s} \equiv 0$. Under local $\mathrm{SO}(3)$ rotations $\mathrm{R}$ $\left[R^{-1}=R^{t}\right]$ we have ${ }^{3} \omega_{r(b)}^{(a)} \mapsto\left[R^{3} \omega_{r} R^{T}-R \partial_{r} R^{T}\right]^{(a)}{ }_{(b)},{ }^{3} \Omega_{r s}{ }^{(a)}{ }_{(b)} \mapsto\left[R^{3} \Omega_{r s} R^{T}\right]^{(a)}{ }_{(b)}$.

For the conformal transformations in 3 -manifolds one has $\left({ }^{3} \nabla_{r}\right.$ is the covariant derivative associated with the 3 -metric $\left.{ }^{3} g_{r s}\right)$ :

$$
\begin{aligned}
&{ }^{3} g_{r s} \mapsto{ }^{3} \hat{g}_{r s}=\phi^{43} g_{r s}, \\
&{ }^{3} \Gamma_{r s}^{u} \mapsto{ }^{3} \hat{\Gamma}_{r s}^{u}={ }^{3} \Gamma_{r s}^{u}+2 \phi^{-1}\left(\delta_{r}^{u 3} \nabla_{s} \phi+\delta_{s}^{u}{ }^{3} \nabla_{r} \phi-{ }^{3} g_{r s}{ }^{3} g^{u v}{ }^{3} \nabla_{v} \phi\right), \\
&{ }^{3} R \mapsto{ }^{3} \hat{R}=\phi^{-43} R-8 \phi^{-5}\left({ }^{3} g^{u v}{ }^{3} \nabla_{u}{ }^{3} \nabla_{v}\right) \phi .
\end{aligned}
$$

Eqs.(6.14) imply the following expressions for the field strengths and curvature tensors of $\left(\Sigma_{\tau},{ }^{3} g\right)$ given in Eqs.(A1) (we also give their limits for $r_{\bar{a}} \rightarrow 0$ and $q=2 \ln \phi \rightarrow 0$ )

$$
\begin{aligned}
{ }^{3} \hat{\Omega}_{r s(a)}= & \epsilon_{(a)(b)(c)} \sum_{u} \delta_{(c) u} \\
& \left(\delta _ { ( b ) s } e ^ { \frac { 1 } { \sqrt { 3 } } \sum _ { \overline { a } } ( \gamma _ { \overline { a } s } - \gamma _ { \overline { a } u } ) r _ { \overline { a } } } \left[\frac{1}{\sqrt{3}}\left(2 \partial_{u} \ln \phi+\frac{1}{\sqrt{3}} \sum_{\bar{b}} \gamma_{\bar{b} s} \partial_{u} r_{\bar{b}}\right) \sum_{\bar{c}}\left(\gamma_{\bar{c} s}-\gamma_{\bar{c} u}\right) \partial_{r} r_{\bar{c}}+\right.\right. \\
& \left.+2 \partial_{u} \partial_{r} \ln \phi+\frac{1}{\sqrt{3}} \sum_{\bar{b}} \gamma_{\bar{b} s} \partial_{u} \partial_{r} r_{\bar{b}}\right]- \\
& -\delta_{(b) r} e^{\frac{1}{\sqrt{3}} \sum_{\bar{a}}\left(\gamma_{\bar{a} r}-\gamma_{\bar{a} u}\right) r_{\bar{a}}}\left[\frac{1}{\sqrt{3}}\left(2 \partial_{u} \ln \phi+\frac{1}{\sqrt{3}} \sum_{\bar{b}} \gamma_{\bar{b} r} \partial_{u} r_{\bar{b}}\right) \sum_{\bar{c}}\left(\gamma_{\bar{c} r}-\gamma_{\bar{c} u}\right) \partial_{s} r_{\bar{c}}+\right.
\end{aligned}
$$




$$
\begin{aligned}
& \left.\left.+2 \partial_{u} \partial_{s} \ln \phi+\frac{1}{\sqrt{3}} \sum_{\bar{b}} \gamma_{\bar{b} r} \partial_{u} \partial_{s} r_{\bar{b}}\right]\right)+ \\
& +\frac{1}{2} \sum_{u v}\left[\delta_{(a)(b)} \epsilon_{(c)(d)(e)}-\delta_{(a)(c)} \epsilon_{(b)(d)(e)}+\delta_{(a)(d)} \epsilon_{(e)(c)(b)}-\delta_{(a)(e)} \epsilon_{(d)(c)(b)}\right] \\
& e^{\frac{1}{\sqrt{3}} \sum_{\bar{a}}\left(\gamma_{\bar{a} r}+\gamma_{\bar{a} s}-\gamma_{\bar{a} u}-\gamma_{\bar{a} v}\right) r_{\bar{a}}}\left(2 \partial_{u} \ln \phi+\frac{1}{\sqrt{3}} \sum_{\bar{b}} \gamma_{\bar{b} r} \partial_{u} r_{\bar{b}}\right)\left(2 \partial_{v} \ln \phi+\frac{1}{\sqrt{3}} \sum_{\bar{c}} \gamma_{\bar{c} s} \partial_{v} r_{\bar{c}}\right) \\
& \rightarrow_{r_{\bar{a}} \rightarrow 0} \quad 2 \epsilon_{(a)(b)(c)} \sum_{u} \delta_{(c) u}\left[\delta_{(b) s} \partial_{u} \partial_{r} \ln \phi-\delta_{(b) r} \partial_{u} \partial_{s} \ln \phi\right]+ \\
& +2\left[\delta_{(a)(b)} \epsilon_{(c)(d)(e)}-\delta_{(a)(c)} \epsilon_{(b)(d)(e)}+\delta_{(a)(d)} \epsilon_{(e)(c)(b)}-\delta_{(a)(e)} \epsilon_{(d)(c)(b)}\right] \\
& \partial_{u} \ln \phi \partial_{v} \ln \phi \rightarrow_{q \rightarrow 0} 0 \text {, } \\
& \rightarrow_{q \rightarrow 0} \quad \frac{1}{\sqrt{3}} \epsilon_{(a)(b)(c)} \sum_{u} \delta_{(c) u}\left(\delta_{(b) s} e^{\frac{1}{\sqrt{3}} \sum_{\bar{a}}\left(\gamma_{\bar{a} s}-\gamma_{\bar{a} u}\right) r_{\bar{a}}} .\right. \\
& \sum_{\bar{b}} \gamma_{\bar{b} s}\left[\partial_{r} \partial_{u} r_{\bar{b}}+\frac{1}{\sqrt{3}} \partial_{u} r_{\bar{b}} \sum_{\bar{c}}\left(\gamma_{\bar{c} s}-\gamma_{\bar{c} u}\right) \partial_{r} r_{\bar{c}}\right]- \\
& \left.-\delta_{(b) r} e^{\frac{1}{\sqrt{3}} \sum_{\bar{a}}\left(\gamma_{\bar{a} r}-\gamma_{\bar{a} u}\right) r_{\bar{a}}} \sum_{\bar{b}} \gamma_{\bar{b} r}\left[\partial_{s} \partial_{u} r_{\bar{b}}+\frac{1}{\sqrt{3}} \partial_{u} r_{\bar{b}} \sum_{\bar{c}}\left(\gamma_{\bar{c} r}-\gamma_{\bar{c} u}\right) \partial_{s} r_{\bar{c}}\right]\right)+ \\
& +\frac{1}{6} \sum_{u v}\left[\delta_{(a)(b)} \epsilon_{(c)(d)(e)}-\delta_{(a)(c)} \epsilon_{(b)(d)(e)}+\delta_{(a)(d)} \epsilon_{(e)(c)(b)}-\delta_{(a)(e)} \epsilon_{(d)(c)(b)}\right] \\
& e^{\frac{1}{\sqrt{3}} \sum_{\bar{a}}\left(\gamma_{\bar{a} r}+\gamma_{\bar{a} s}-\gamma_{\bar{a} u}+\gamma_{\bar{a} v}\right) r_{\bar{a}}} \sum_{\bar{b}} \gamma_{\bar{b} r} \partial_{u} r_{\bar{b}} \sum_{\bar{c}} \gamma_{\bar{c} s} \partial_{v} r_{\bar{c}} \\
& { }^{3} \hat{R}_{r u s v}=\left(\delta_{r v} \delta_{s u}-\delta_{r s} \delta_{u v}\right) \phi^{8} e^{\frac{2}{\sqrt{3}} \sum_{\bar{c}}\left(\gamma_{\bar{c} r}+\gamma_{\bar{c} u}\right) r_{\bar{c}}}
\end{aligned}
$$$$
\sum_{n}\left(2 \partial_{n} \ln \phi+\frac{1}{\sqrt{3}} \sum_{\bar{a}} \gamma_{\bar{a} r} \partial_{n} r_{\bar{a}}\right)\left(2 \partial_{n} \ln \phi+\frac{1}{\sqrt{3}} \sum_{\bar{b}} \gamma_{\bar{b} u} \partial_{n} r_{\bar{b}}\right)+
$$$$
+\phi^{4} e^{\frac{2}{\sqrt{3}} \sum_{\bar{c}} \gamma_{\bar{c} r} r_{\bar{c}}}\left(\delta _ { r v } \left[2 \partial_{s} \partial_{u} \ln \phi+\frac{1}{\sqrt{3}} \sum_{\bar{a}} \gamma_{\bar{a} r} \partial_{s} \partial_{u} r_{\bar{a}}+\right.\right.
$$$$
+\frac{1}{\sqrt{3}}\left(2 \partial_{u} \ln \phi+\frac{1}{\sqrt{3}} \sum_{\bar{a}} \gamma_{\bar{a} r} \partial_{u} r_{\bar{a}}\right) \sum_{\bar{b}}\left(\gamma_{\bar{b} r}-\gamma_{\bar{b} u}\right) \partial_{s} r_{\bar{b}}-
$$$$
\left.-\left(2 \partial_{u} \ln \phi+\frac{1}{\sqrt{3}} \sum_{\bar{a}} \gamma_{\bar{a} s} \partial_{u} r_{\bar{a}}\right)\left(2 \partial_{s} \ln \phi+\frac{1}{\sqrt{3}} \sum_{\bar{b}} \gamma_{\bar{b} r} \partial_{s} r_{\bar{b}}\right)\right]-
$$$$
-\delta_{r s}\left[2 \partial_{v} \partial_{u} \ln \phi+\frac{1}{\sqrt{3}} \sum_{\bar{a} r} \partial_{v} \partial_{u} r_{\bar{a}}+\right.
$$$$
+\frac{1}{\sqrt{3}}\left(2 \partial_{u} \ln \phi+\frac{1}{\sqrt{3}} \sum_{\bar{a}} \gamma_{\bar{a} r} \partial_{u} r_{\bar{a}}\right) \sum_{\bar{b}}\left(\gamma_{\bar{b} r}-\gamma_{\bar{b} u}\right) \partial_{v} r_{\bar{b}}-
$$$$
\left.\left.-\left(2 \partial_{u} \ln \phi+\frac{1}{\sqrt{3}} \sum_{\bar{a}} \gamma_{\bar{a} v} \partial_{u} r_{\bar{a}}\right)\left(2 \partial_{v} \ln \phi+\frac{1}{\sqrt{3}} \sum_{\bar{b}} \gamma_{\bar{b} r} \partial_{v} r_{\bar{b}}\right)\right]\right)+
$$$$
+\phi^{4} e^{\frac{2}{\sqrt{3}} \sum_{\bar{c}} \gamma_{\bar{c} u} r_{\bar{c}}}\left(\delta _ { s u } \left[2 \partial_{v} \partial_{r} \ln \phi+\frac{1}{\sqrt{3}} \sum_{\bar{a}} \gamma_{\bar{a} u} \partial_{v} \partial_{r} r_{\bar{a}}+\frac{1}{\sqrt{3}}\left(2 \partial_{r} \ln \phi+\right.\right.\right.
$$$$
\left.+\frac{1}{\sqrt{3}} \sum_{\bar{a}} \gamma_{\bar{a} u} \partial_{r} r_{\bar{a}}\right) \sum_{\bar{b}}\left(\gamma_{\bar{b} u}-\gamma_{\bar{b} r}\right) \partial_{v} r_{\bar{b}}-
$$ 


$$
\begin{aligned}
& \left.-\left(2 \partial_{r} \ln \phi+\frac{1}{\sqrt{3}} \sum_{\bar{a}} \gamma_{\bar{a} r} \partial_{r} r_{\bar{a}}\right)\left(2 \partial_{v} \ln \phi+\frac{1}{\sqrt{3}} \sum_{\bar{b}} \gamma_{\bar{b} u} \partial_{v} r_{\bar{b}}\right)\right]- \\
& -\delta_{u v}\left[2 \partial_{s} \partial_{r} \ln \phi+\frac{1}{\sqrt{3}} \sum_{\bar{a}} \gamma_{\bar{a} u} \partial_{s} \partial_{r} r_{\bar{a}}+\right. \\
& +\frac{1}{\sqrt{3}}\left(2 \partial_{r} \ln \phi+\frac{1}{\sqrt{3}} \sum_{\bar{a}} \gamma_{\bar{a} u} \partial_{r} r_{\bar{a}}\right) \sum_{\bar{b}}\left(\gamma_{\bar{b} u}-\gamma_{\bar{b} r}\right) \partial_{s} r_{\bar{b}}- \\
& \left.\left.-\left(2 \partial_{r} \ln \phi+\frac{1}{\sqrt{3}} \sum_{\bar{a}} \gamma_{\bar{a} s} \partial_{r} r_{\bar{a}}\right)\left(2 \partial_{s} \ln \phi+\frac{1}{\sqrt{3}} \sum_{\bar{b}} \gamma_{\bar{b} u} \partial_{s} r_{\bar{b}}\right)\right]\right) \\
& \rightarrow_{r_{\bar{a}} \rightarrow 0} \quad 4\left(\delta_{r v} \delta_{s u}-\delta_{r s} \delta_{u v}\right) \phi^{8} \sum_{n}\left(\partial_{n} \ln \phi\right)^{2}+ \\
& +2 \phi^{4}\left(\delta_{r v}\left[\partial_{s} \partial_{u} \ln \phi-2 \partial_{s} \ln \phi \partial_{u} \ln \phi\right]-\delta_{r s}\left[\partial_{v} \partial_{u} \ln \phi-2 \partial_{v} \ln \phi \partial_{u} \ln \phi\right]+\right. \\
& \left.+\delta_{s u}\left[\partial_{v} \partial_{r} \ln \phi-2 \partial_{v} \ln \phi \partial_{r} \ln \phi\right]-\delta_{u v}\left[\partial_{s} \partial_{r} \ln \phi-2 \partial_{s} \ln \phi \partial_{r} \ln \phi\right]\right) \rightarrow_{q \rightarrow 0} 0 \text {, } \\
& \rightarrow_{q \rightarrow 0} \quad \frac{1}{3}\left(\delta_{r v} \delta_{s u}-\delta_{r s} \delta_{u v}\right) e^{\frac{2}{\sqrt{3}} \sum_{\bar{c}}\left(\gamma_{\bar{c} r}+\gamma_{\bar{c} u}\right) r_{\bar{c}}} \sum_{n} \sum_{\bar{a} \bar{b}} \gamma_{\bar{a} r} \gamma_{\bar{b} u} \partial_{n} r_{\bar{a}} \partial_{n} r_{\bar{b}}+ \\
& +\frac{1}{\sqrt{3}} e^{\frac{2}{\sqrt{3}} \sum_{\bar{c}} \gamma_{\bar{c} r} r_{\bar{c}}} \sum_{\bar{a}} \gamma_{\bar{a} r}\left(\delta _ { r v } \left[\partial_{s} \partial_{u} r_{\bar{a}}+\right.\right. \\
& \left.+\frac{1}{\sqrt{3}} \sum_{\bar{b}}\left(\gamma_{\bar{b} r}-\gamma_{\bar{b} u}\right) \partial_{u} r_{\bar{a}} \partial_{s} r_{\bar{b}}-\frac{1}{\sqrt{3}} \sum_{\bar{b}} \gamma_{\bar{b} s} \partial_{s} r_{\bar{a}} \partial_{u} r_{\bar{b}}\right]- \\
& \left.-\delta_{r s}\left[\partial_{v} \partial_{u} r_{\bar{a}}+\frac{1}{\sqrt{3}} \sum_{\bar{b}}\left(\gamma_{\bar{b} r}-\gamma_{b a r b u}\right) \partial_{u} r_{\bar{a}} \partial_{v} r_{\bar{b}}-\frac{1}{\sqrt{3}} \sum_{\bar{b}} \gamma_{\bar{b} v} \partial_{v} r_{\bar{a}} \partial_{u} r_{\bar{b}}\right]\right)+ \\
& +\frac{1}{\sqrt{3}} e^{\frac{2}{\sqrt{3}} \sum_{\bar{c}} \gamma_{\bar{c} u} r_{\bar{c}}} \sum_{\bar{a}} \gamma_{\bar{a} u}\left(\delta _ { s u } \left[\partial_{v} \partial_{r} r_{\bar{a}}+\right.\right. \\
& \left.+\frac{1}{\sqrt{3}} \sum_{\bar{b}}\left(\gamma_{\bar{b} u}-\gamma_{\bar{b} r}\right) \partial_{r} r_{\bar{a}} \partial_{v} r_{\bar{b}}-\frac{1}{\sqrt{3}} \sum_{\bar{b}} \gamma_{\bar{b} v} \partial_{v} r_{\bar{a}} \partial_{r} r_{\bar{b}}\right]- \\
& \left.-\delta_{u v}\left[\partial_{s} \partial_{r} r_{\bar{a}}+\frac{1}{\sqrt{3}} \sum_{\bar{b}}\left(\gamma_{\bar{b} u}-\gamma_{\bar{b} r}\right) \partial_{r} r_{\bar{a}} \partial_{s} r_{\bar{b}}-\frac{1}{\sqrt{3}} \sum_{\bar{b}} \gamma_{\bar{b} s} \partial_{s} r_{\bar{a}} \partial_{r} r_{\bar{b}}\right]\right), \\
& { }^{3} \hat{R}_{u v}=-2 \partial_{u} \partial_{v} \ln \phi+\frac{1}{\sqrt{3}} \sum_{\bar{a}}\left(\gamma_{\bar{a} u}+\gamma_{\bar{a} v}\right) \partial_{u} \partial_{v} r_{\bar{a}}+ \\
& +\frac{1}{2}\left[\left(2 \partial_{u} \ln \phi+\frac{1}{\sqrt{3}} \sum_{\bar{a}} \gamma_{\bar{a} v} \partial_{u} r_{\bar{a}}\right)\left(2 \partial_{v} \ln \phi-\frac{2}{\sqrt{3}} \sum_{\bar{b}} \gamma_{\bar{b} u} \partial_{v} r_{\bar{b}}\right)+\right. \\
& \left.+\left(2 \partial_{v} \ln \phi+\frac{1}{\sqrt{3}} \sum_{\bar{a}} \gamma_{\bar{u}} \partial_{v} r_{\bar{a}}\right)\left(2 \partial_{u} \ln \phi-\frac{2}{\sqrt{3}} \sum_{\bar{b}} \gamma_{\bar{b} v} r_{\bar{b}}\right)\right]- \\
& -\frac{1}{2 \sqrt{3}} \sum_{n}\left[\left(2 \partial_{u} \ln \phi+\frac{1}{\sqrt{3}} \sum_{\bar{a}} \gamma_{\bar{a} r} \partial_{y} r_{\bar{a}}\right) \sum_{\bar{b}}\left(\gamma_{\bar{b} n}-\gamma_{b a r b u}\right) \partial_{v} r_{\bar{b}}+\right. \\
& \left.+\left(2 \partial_{v} \ln \phi+\frac{1}{\sqrt{3}} \sum_{\bar{a}} \gamma_{\bar{a} r} \partial_{v} r_{\bar{a}}\right) \sum_{\bar{b}}\left(\gamma_{\bar{b} n}-\gamma_{\bar{b} v}\right) \partial_{u} r_{\bar{b}}\right]-\delta_{u v} \phi^{4} e^{\frac{2}{\sqrt{3}} \sum_{\bar{c}} \gamma_{\bar{c} u} r_{\bar{c}}} \\
& \sum_{n}\left(\left(2 \partial_{n} \ln \phi+\frac{1}{\sqrt{3}} \sum_{\bar{a}} \gamma_{\bar{a} u} \partial_{n} r_{\bar{a}}\right)\left(4 \partial_{n} \ln \phi-\frac{1}{\sqrt{3}} \sum_{\bar{b}} \gamma_{\bar{b} u} \partial_{n} r_{\bar{b}}\right)+\right.
\end{aligned}
$$




$$
\begin{aligned}
& +\phi^{-4} e^{-\frac{2}{\sqrt{3}} \sum_{\bar{c}} \gamma_{\bar{c} n} r_{\bar{c}}}\left[2 \partial_{n}^{2} \ln \phi+\right. \\
& \left.\left.+\frac{1}{\sqrt{3}} \sum_{\bar{a}} \gamma_{\bar{a} u} \partial_{n}^{2} r_{\bar{a}}+\frac{1}{\sqrt{3}}\left(2 \partial_{n} \ln \phi+\frac{1}{\sqrt{3}} \sum_{\bar{a} u} \partial_{n} r_{\bar{a}}\right) \sum_{\bar{b}}\left(\gamma_{\bar{b} u}-2 \gamma_{\bar{b} n}\right) \partial_{n} r_{\bar{b}}\right]\right) \\
& \rightarrow_{r_{\bar{a}} \rightarrow 0} \quad-2 \partial_{u} \partial_{v} \ln \phi+4 \partial_{u} \ln \phi \partial_{v} \ln \phi- \\
& -2 \delta_{u v} \phi^{4} \sum_{n}\left[4 \phi^{4}\left(\partial_{n} \ln \phi\right)^{2}+\partial_{n}^{2} \ln \phi-2\left(\partial_{n} \ln \phi\right)^{2}\right] \rightarrow_{q \rightarrow 0} 0, \\
& \rightarrow_{q \rightarrow 0} \quad \frac{1}{\sqrt{3}} \sum_{\bar{a}}\left(\gamma_{\bar{a} u}+\gamma_{\bar{a} v}\right) \partial_{u} \partial_{v} r_{\bar{a}}-\frac{2}{3} \sum_{\bar{a} \bar{b}} \gamma_{\bar{a} u} \gamma_{\bar{b} v} \partial_{v} r_{\bar{a}} \partial_{u} r_{\bar{b}}- \\
& -\frac{1}{6} \sum_{n} \sum_{\bar{a} \bar{b}} \gamma_{\bar{a} n}\left[\left(\gamma_{\bar{b} r}-\gamma_{\bar{b} u}\right) \partial_{u} r_{\bar{a}} \partial_{v} r_{\bar{b}}+\left(\gamma_{\bar{b} n}-\gamma_{\bar{b} v}\right) \partial_{v} r_{\bar{a}} \partial_{u} r_{\bar{b}}\right]+ \\
& +\frac{1}{\sqrt{3}} \delta_{u v} e^{\frac{2}{\sqrt{3}} \sum_{\bar{c}} \gamma_{\bar{c} u} r_{\bar{c}}} \sum_{n}\left(\frac{1}{\sqrt{3}} \sum_{\bar{a} \bar{b}} \gamma_{\bar{a} u} \gamma_{\bar{b} u} \partial_{n} r_{\bar{a}} \partial_{n} r_{\bar{b}}-\right. \\
& \left.-e^{-\frac{2}{\sqrt{3}} \sum_{\bar{c}} \gamma_{\bar{c} n} r_{\bar{c}}} \sum_{\bar{a}} \gamma_{\bar{a} u}\left[\partial_{n}^{2} r_{\bar{a}}+\frac{1}{\sqrt{3}} \sum_{\bar{b}}\left(\gamma_{\bar{b} u}-2 \gamma_{\bar{b} n}\right) \partial_{n} r_{\bar{a}} \partial_{n} r_{\bar{b}}\right]\right) \\
& { }^{3} \hat{R}=-\sum_{u v}\left(\left(2 \partial_{v} \ln \phi+\frac{1}{\sqrt{3}} \sum_{\bar{a}} \gamma_{\bar{a} u} \partial_{v} r_{\bar{a}}\right)\left(4 \partial_{v} \ln \phi-\frac{1}{\sqrt{3}} \sum_{\bar{b}} \gamma_{\bar{b} u} \partial_{v} r_{\bar{b}}\right)+\right. \\
& +\phi^{-4} e^{-\frac{2}{\sqrt{3}} \sum_{\bar{c}} \gamma_{\bar{c} v} r_{\bar{c}}}\left[2 \partial_{v}^{2} \ln \phi+\frac{1}{\sqrt{3}} \sum_{\bar{a}} \gamma_{\bar{a} u} \partial_{v}^{2} r_{\bar{a}}+\right. \\
& +\frac{2}{\sqrt{3}}\left(2 \partial_{v} \ln \phi+\frac{1}{\sqrt{3}} \sum_{\bar{a}} \gamma_{\bar{a} u} \partial_{v} r_{\bar{a}}\right) \sum_{\bar{b}}\left(\gamma_{\bar{b} u}-\gamma_{\bar{b} v}\right) \partial_{v} r_{\bar{b}}- \\
& \left.-\left(2 \partial_{v} \ln \phi+\frac{1}{\sqrt{3}} \sum_{\bar{a}} \gamma_{\bar{a} v} \partial_{v} r_{\bar{a}}\right)\left(2 \partial_{v} \ln \phi+\frac{1}{\sqrt{3}} \sum_{\bar{b}} \gamma_{\bar{b} u} \partial_{v} r_{\bar{b}}\right]\right)+ \\
& +\phi^{-4} \sum_{u} e^{-\frac{2}{\sqrt{3}} \sum_{\bar{c}} \gamma_{\bar{c} u} r_{\bar{c}}}\left[-2 \partial_{u}^{2} \ln \phi+\frac{2}{\sqrt{3}} \sum_{\bar{a}} \gamma_{\bar{a} u} \partial_{u}^{2} r_{\bar{a}}+\right. \\
& \left.+\left(2 \partial_{u} \ln \phi+\frac{1}{\sqrt{3}} \sum_{\bar{a}} \gamma_{\bar{a} u} \partial_{u} r_{\bar{a}}\right)\left(2 \partial_{u} \ln \phi-\frac{2}{\sqrt{3}} \sum_{\bar{b}} \gamma_{\bar{b} u} \partial_{u} r_{\bar{b}}\right)\right] \\
& \rightarrow_{r_{\bar{a}} \rightarrow 0} \quad-24 \sum_{u}\left(\partial_{u} \ln \phi\right)^{2}-8 \phi^{-4} \sum_{u}\left[\partial_{u}^{2} \ln \phi-2\left(\partial_{u} \ln \phi\right)^{2}\right] \rightarrow_{q \rightarrow 0} 0 \text {, } \\
& \rightarrow_{q \rightarrow 0} \quad-\frac{1}{\sqrt{3}} \sum_{u v}\left(-\frac{1}{\sqrt{3}} \sum_{\bar{a} \bar{b}} \gamma_{\bar{a} u} \gamma_{\bar{b} u} \partial_{v} r_{\bar{a}} \partial_{v} r_{\bar{b}}+e^{-\frac{2}{\sqrt{3}} \sum_{\bar{c}} \gamma_{\bar{c} v} r_{\bar{c}}} \sum_{\bar{a}} \gamma_{\bar{a} u} .\right. \\
& \left.\left[\partial_{v}^{2} r_{\bar{a}}+\frac{2}{\sqrt{3}} \sum_{\bar{b}}\left(\gamma_{\bar{b} u}-\gamma_{\bar{b} v}\right) \partial_{v} r_{\bar{a}} \partial_{v} r_{\bar{b}}-\frac{1}{\sqrt{3}} \sum_{\bar{b}} \gamma_{\bar{b} v} \partial_{v} r_{\bar{a}} \partial_{v} r_{\bar{b}}\right]\right)+ \\
& +\frac{2}{\sqrt{3}} \sum_{u} e^{-\frac{2}{\sqrt{3}} \sum_{\bar{c}} \gamma_{\bar{c} u} t_{\bar{c}}} \sum_{\bar{a}} \gamma_{\bar{a} u}\left[\partial_{u}^{2} r_{\bar{a}}+\frac{1}{\sqrt{3}} \sum_{\bar{b}} \gamma_{\bar{b} u} \partial_{u} r_{\bar{a}} \partial_{u} r_{\bar{b}}\right] .
\end{aligned}
$$

The Weyl-Schouten tensor ${ }^{3} C_{r s u}={ }^{3} \nabla_{u}{ }^{3} R_{r s}-{ }^{3} \nabla_{s}{ }^{3} R_{r u}-\frac{1}{4}\left({ }^{3} g_{r s} \partial_{u}{ }^{3} R-{ }^{3} g_{r u} \partial_{s}{ }^{3} R\right)$ of the 3-manifold $\left(\Sigma_{\tau},{ }^{3} g\right.$ ) (see Ref. [20]) satisfies ${ }^{3} C_{r u}^{r}=0,{ }^{3} C_{r s u}=-{ }^{3} C_{r u s},{ }^{3} C_{r s u}+{ }^{3} C_{u r s}+$ ${ }^{3} C_{\text {sur }}=0$ and has 5 independent components. The related York's conformal tensor [77,70] 
${ }^{3} Y^{r s}=\gamma^{1 / 3} \epsilon^{r u v}\left({ }^{3} R_{v}{ }^{s}-\frac{1}{4} \delta_{v}^{s} R\right)_{\mid u}=-\frac{1}{2} \gamma^{1 / 3} \epsilon^{r u v}{ }^{3} g^{s m 3} C_{m u v}{ }^{155}$ is symmetric $\left[{ }^{3} Y^{r s}={ }^{3} Y^{s r}\right]$, traceless $\left[{ }^{3} Y_{r}^{r}=0\right]$ and transverse $\left[{ }^{3} Y^{r s}{ }_{\mid s}=0\right]$ besides being invariant under 3-conformal transformations; therefore, it has only 2 independent components 500 and provides what York calls the pure spin-two representation of the 3-geometry intrinsic to $\Sigma_{\tau}$. Its explicitly symmetric form is the Cotton-York tensor given by ${ }^{3} \mathcal{Y}^{r s}=\frac{1}{2}\left({ }^{3} Y^{r s}+{ }^{3} Y^{s r}\right)=\frac{1}{2} \gamma^{1 / 3}\left(\epsilon^{r u v}{ }^{3} g^{s c}+\right.$ $\left.\epsilon^{\text {suv } 3} g^{r c}\right)^{3} R_{v c \mid u}=-\frac{1}{4} \gamma^{1 / 3}\left(\epsilon^{r u v} 3 g^{s m}+\epsilon^{\text {suv } 3} g^{r m}\right)^{3} C_{m u v}$.

A 3-manifold is conformally flat if and only if either the Weyl-Schouten or the CottonYork tensor vanishes [70,77,20]. We have

$$
\begin{aligned}
{ }^{3} C_{r s u}= & { }^{3} \nabla_{u}{ }^{3} R_{r s}-{ }^{3} \nabla_{s}{ }^{3} R_{r u}-\frac{1}{4}\left({ }^{3} g_{r s} \partial_{u}{ }^{3} R-{ }^{3} g_{r u} \partial_{s}{ }^{3} R\right) \mapsto \\
& \mapsto{ }^{3} \hat{C}_{r s u}={ }^{3} \hat{R}_{r s \mid u}-{ }^{3} \hat{R}_{r u \mid s}-\frac{1}{4} e^{2 q_{r}}\left(\delta_{r s} \partial_{u}{ }^{3} \hat{R}-\delta_{r u} \partial_{s}{ }^{3} \hat{R}\right) \\
{ }^{3} \mathcal{Y}_{m n}= & \frac{1}{2} \gamma^{1 / 3} \sum_{r s u}\left(\epsilon_{m u r}{ }^{3} g_{n s}+\epsilon_{n u r}{ }^{3} g_{m s}\right)^{3} R_{r s \mid u} \mapsto \\
& \mapsto{ }^{3} \hat{\mathcal{Y}}_{m n}=\frac{1}{2} e^{\frac{2}{3} \sum_{v} q_{v}} \sum_{r s u} e^{-2 q_{s}}\left(\epsilon_{m u r} \delta_{n s}+\epsilon_{n u r} \delta_{m s}\right)^{3} \hat{R}_{r s \mid u}= \\
& =\frac{1}{2} e^{2 q} \sum_{r s u} e^{-2 q_{s}}\left(\epsilon_{m u r} \delta_{n s}+\epsilon_{n u r} \delta_{m s}\right) \cdot \\
& \left(\partial_{u} \partial_{r} \partial_{s}\left(q_{r}+q_{s}-\sum_{t} q_{t}\right)-\partial_{u}\left(q_{r}+q_{s}\right) \partial_{r} \partial_{s}\left(q_{r}+q_{s}-\sum_{t} q_{t}\right)-\right. \\
& -\partial_{r} q_{u} \partial_{u} \partial_{s}\left(q_{u}+q_{s}-\sum_{t} q_{t}\right)-\partial_{s} q_{u} \partial_{u} \partial_{r}\left(q_{u}+q_{r}-\sum_{t} q_{t}\right)- \\
& -\frac{1}{2} \partial_{u}\left[\partial_{r} q_{s} \partial_{s}\left(2 q_{r}-\sum_{t} q_{t}\right)+\partial_{s} q_{r} \partial_{r}\left(2 q_{s}-\sum_{t} q_{t}\right)\right]- \\
& -\frac{1}{2} \sum_{n} \partial_{u}\left[\partial_{r} q_{n} \partial_{s}\left(q_{n}-q_{r}\right)+\partial_{s} q_{n} \partial_{r}\left(q_{n}-q_{s}\right)\right]+ \\
& +\frac{1}{2} \partial_{u}\left(q_{r}+q_{s}\right)\left[\partial_{r} q_{s} \partial_{s}\left(2 q_{r}-\sum_{t} q_{t}\right)+\partial_{s} q_{r} \partial_{r}\left(2 q_{s}-\sum_{t} q_{t}\right)\right]+ \\
& +\frac{1}{2} \partial_{r} q_{u}\left[\partial_{u} q_{s} \partial_{s}\left(2 q_{u}-\sum_{t} q_{t}\right)+\partial_{s} q_{u} \partial_{u}\left(2 q_{s}-\sum_{t} q_{t}\right)\right]+ \\
& +\frac{1}{2} \partial_{s} q_{u}\left[\partial_{u} q_{r} \partial_{r}\left(2 q_{u}-\sum_{t} q_{t}\right)+\partial_{r} q_{u} \partial_{u}\left(2 q_{r}-\sum_{t} q_{t}\right)\right]+ \\
& +\frac{1}{2} \partial_{u}\left(q_{r}+q_{s}\right) \sum_{n}\left[\partial_{r} q_{n} \partial_{s}\left(q_{n}-q_{r}\right)+\partial_{s} q_{n} \partial_{r}\left(q_{n}-q_{s}\right)\right]+ \\
& \left.q_{n} \partial_{s}\left(q_{n}-q_{u}\right)+\partial_{s} q_{n} \partial_{u}\left(q_{n}-q_{s}\right)\right]+ \\
& \\
& \\
&
\end{aligned}
$$

${ }^{155}$ It is a tensor density of weight $5 / 3$ and involves the third derivatives of the metric.

${ }^{156} Y^{r s}=Y_{T T}^{r s}$ according to York's decomposition of Appendix C of II. 


$$
\begin{aligned}
& +\frac{1}{2} \partial_{s} q_{u} \sum_{n}\left[\partial_{u} q_{n} \partial_{r}\left(q_{n}-q_{u}\right)+\partial_{r} q_{n} \partial_{u}\left(q_{n}-q_{r}\right)\right]+ \\
& +\delta_{r s} e^{2 q_{r}} \sum_{n}\left[2 \partial_{u} q_{r}\left[\partial_{n} q_{r} \partial_{n}\left(q_{r}-\sum_{t} q_{t}\right)-e^{-2 q_{n}}\left(\partial_{n}^{2} q_{r}+\partial_{n} q_{r} \partial_{n}\left(q_{r}-2 q_{n}\right)\right)\right]+\right. \\
& +\partial_{u}\left[\partial_{n} q_{r} \partial_{n}\left(q_{r}-\sum_{t} q_{t}\right)\right]+e^{-2 q_{n}}\left[2 \partial_{u} q_{n}\left(\partial_{n}^{2} q_{r}+\partial_{n} q_{r} \partial_{n}\left(q_{r}-2 q_{n}\right)\right)-\right. \\
& \left.-\partial_{u}\left(\partial_{n}^{2} q_{r}+\partial_{n} q_{r} \partial_{n}\left(q_{r}-2 q_{n}\right)\right)\right]- \\
& \left.-2 \partial_{u} q_{r}\left[\partial_{n} q_{r} \partial_{n}\left(q_{r}-\sum_{t} q_{t}\right)-e^{-2 q_{n}}\left(\partial_{n}^{2} q_{r}+\partial_{n} q_{r} \partial_{n}\left(q_{r}-2 q_{n}\right)\right)\right]\right]+ \\
& +\delta_{r u} e^{2 q_{u}}\left[\sum _ { v } e ^ { - 2 q _ { v } } \partial _ { v } q _ { u } \left(\partial_{v} \partial_{s}\left(q_{v}+q_{s}-\sum_{t} q_{t}\right)-\right.\right. \\
& -\frac{1}{2}\left[\partial_{v} q_{s} \partial_{s}\left(2 q_{v}-\sum_{t} q_{t}\right)+\partial_{s} q_{v} \partial_{v}\left(2 q_{s}-\sum_{t} q_{t}\right)\right]- \\
& \left.-\frac{1}{2} \sum_{n}\left[\partial_{v} q_{n} \partial_{s}\left(q_{n}-q_{v}\right)+\partial_{s} q_{n} \partial_{v}\left(q_{n}-q_{s}\right)\right]\right)+ \\
& +\sum_{n}\left(\partial_{s} q_{u}\left[\partial_{n} q_{s} \partial_{n}\left(q_{s}-\sum_{t} q_{t}\right)-e^{-2 q_{n}}\left(\partial_{n}^{2} q_{s}+\partial_{n} q_{s} \partial_{n}\left(q_{s}-2 q_{n}\right)\right)\right]-\right. \\
& \left.\left.-\partial_{s} q_{u}\left[\partial_{n} q_{r} \partial_{n}\left(q_{r}-\sum_{t} q_{t}\right)-e^{-2 q_{n}}\left(\partial_{n}^{2} q_{r}+\partial_{n} q_{r} \partial_{n}\left(q_{r}-2 q_{n}\right)\right)\right]\right)\right]+ \\
& +\delta_{s u} e^{2 q_{u}}\left[\sum _ { v } e ^ { - 2 q _ { v } } \partial _ { v } q _ { u } \left(\partial_{v} \partial_{r}\left(q_{v}+q_{r}-\sum_{t} q_{t}\right)-\right.\right. \\
& -\frac{1}{2}\left[\partial_{v} q_{r} \partial_{r}\left(2 q_{v}-\sum_{t} q_{t}\right)+\partial_{r} q_{v} \partial_{v}\left(2 q_{r}-\sum_{t} q_{t}\right)\right]- \\
& \left.-\frac{1}{2} \sum_{n}\left[\partial_{v} q_{n} \partial_{r}\left(q_{n}-q_{v}\right)+\partial_{r} q_{n} \partial_{v}\left(q_{n}-q_{r}\right)\right]\right)+ \\
& +\sum_{n}\left(\partial _ { r } q _ { u } \left[\partial_{n} q_{r} \partial_{n}\left(q_{r}-\sum_{t} q_{t}\right)-e^{-2 q_{n}}\left(\partial_{n}^{2} q_{r}+\partial_{n} q_{r} \partial_{n}\left(q_{r}-2 q_{n}\right)\right]-\right.\right. \\
& \left.\left.\left.-\partial_{r} q_{u}\left[\partial_{n} q_{s} \partial_{n}\left(q_{s}-\sum_{t} q_{t}\right)-e^{-2 q_{n}}\left(\partial_{n}^{2} q_{s}+\partial_{n} q_{s} \partial_{n}\left(q_{s}-2 q_{n}\right)\right)\right]\right)\right]\right) \\
& \rightarrow_{r_{\bar{a}} \rightarrow 0} \quad \frac{1}{2} \sum_{r s u}\left(\epsilon_{m u r} \delta_{n s}+\epsilon_{n u r} \delta_{m s}\right) \cdot \\
& -\partial_{u} \partial_{r} \partial_{s} q+2\left(\partial_{u} q \partial_{r} \partial_{s} q+\partial_{r} q \partial_{s} \partial_{u} q+\partial_{s} q \partial_{u} \partial_{r} q\right)-4 \partial_{u} q \partial_{r} q \partial_{s} q+ \\
& +\delta_{r s} \sum_{n}\left(\partial_{u}\left[\partial_{n}^{2} q-\left(\partial_{n} q\right)^{2}\right]+2 \partial_{u} q\left[\partial_{n}^{2} q-\left(\partial_{n} q\right)^{2}\right]-2 e^{2 q} \partial_{u}\left(\partial_{n} q\right)^{2}\right)- \\
& -\delta_{r u} \sum_{v} \partial_{v} q\left(\partial_{v} \partial_{s} q-\partial_{v} q \partial_{s} q\right)-\delta_{s u} \sum_{v} \partial_{v} q\left(\partial_{v} \partial_{r} q-\partial_{v} q \partial_{r} q\right)=0 .
\end{aligned}
$$

Since in the 3-orthogonal gauges the condition $r_{\bar{a}}=0$ corresponds to conformally flat 3manifolds $\Sigma_{\tau}$, the Cotton-York conformal tensor vanishes in the limit $r_{a} \rightarrow 0$ in these gauges. 


\section{APPENDIX B: THE STRONG AND WEAK ADM POINCARÉ CHARGES IN THE SPECIAL 3-ORTHOGONAL GAUGE.}

In the canonical basis of Section VI for the 3-orthogonal gauge, by using Eqs.(6.14) and (6.16) the weak Poincaré charges of Eqs.(2.25) take the form

$$
\begin{aligned}
& \hat{P}_{A D M, R}^{\tau}=\epsilon \int d^{3} \sigma\left(\frac { c ^ { 3 } } { 1 6 \pi G } \left[\phi^{2} \sum_{r} e^{-\frac{2}{\sqrt{3}} \sum_{\bar{a}} \gamma_{\bar{a} r} r_{\bar{a}}} \times\right.\right. \\
& \left(8\left(\partial_{r} \ln \phi\right)^{2}-\frac{1}{3} \sum_{\bar{b}}\left(\partial_{r} r_{\bar{b}}\right)^{2}-\right. \\
& \left.\left.-\frac{4}{\sqrt{3}} \partial_{r} \ln \phi \sum_{\bar{b}} \gamma_{\bar{b} r} \partial_{r} r_{\bar{b}}+\frac{2}{3}\left(\sum_{\bar{b}} \gamma_{\bar{b} r} \partial_{r} r_{\bar{b}}\right)^{2}\right)\right](\tau, \vec{\sigma})- \\
& -\frac{2 \pi G}{c^{3}} \phi^{-2}(\tau, \vec{\sigma})\left[\left(\phi^{-4}\left[6 \sum_{\bar{a}} \pi_{\bar{a}}^{2}-\frac{1}{3} \rho^{2}\right]\right)(\tau, \vec{\sigma})+\right. \\
& +2\left(\phi^{-2} \sum_{u} e^{\frac{1}{\sqrt{3}} \sum_{\bar{a}} \gamma_{\bar{a} u} r_{\bar{a}}}\left[2 \sqrt{3} \sum_{\bar{b}} \gamma_{\bar{b} u} \pi_{\bar{b}}-\frac{1}{3} \rho\right]\right)(\tau, \vec{\sigma}) \times \\
& \int d^{3} \sigma_{1} \sum_{r} \delta_{(a)}^{u} \tilde{\mathcal{T}}_{(a) r}^{u}\left(\vec{\sigma}, \vec{\sigma}_{1}, \tau \mid \phi, r_{\bar{a}}\right]\left(\phi^{-2} e^{-\frac{1}{\sqrt{3}} \sum_{\bar{a}} \gamma_{\bar{a} r} r_{\bar{a}}}\left[\frac{\rho}{3}+\sqrt{3} \sum_{\bar{b}} \gamma_{\bar{b} r} \pi_{\bar{b}}\right]\right)\left(\tau, \vec{\sigma}_{1}\right)+ \\
& +\int d^{3} \sigma_{1} d^{3} \sigma_{2}\left(\sum_{u} e^{\frac{2}{\sqrt{3}} \sum_{\bar{a}} \gamma_{\bar{a} u} r_{\bar{a}}(\tau, \vec{\sigma})} \times\right. \\
& \sum_{r} \tilde{\mathcal{T}}_{(a) r}^{u}\left(\vec{\sigma}, \vec{\sigma}_{1}, \tau \mid \phi, r_{\bar{a}}\right]\left(\phi^{-2} e^{-\frac{1}{\sqrt{3}} \sum_{\bar{a}} \gamma_{\bar{a}} r r_{\bar{a}}}\left[\frac{\rho}{3}+\sqrt{3} \sum_{\bar{b}} \gamma_{\bar{b} r} \pi_{\bar{b}}\right]\right)\left(\tau, \vec{\sigma}_{1}\right) \times \\
& \sum_{s} \tilde{\mathcal{T}}_{(a) s}^{u}\left(\vec{\sigma}, \vec{\sigma}_{2}, \tau \mid \phi, r_{\bar{a}}\right]\left(\phi^{-2} e^{-\frac{1}{\sqrt{3}} \sum_{\bar{a}} \gamma_{\bar{a} s} r_{\bar{a}}}\left[\frac{\rho}{3}+\sqrt{3} \sum_{\bar{c}} \gamma_{\bar{c} s} \pi_{\bar{c}}\right]\right)\left(\tau, \vec{\sigma}_{2}\right)+ \\
& +\sum_{u v} e^{\frac{1}{\sqrt{3}} \sum_{\bar{a}}\left(\gamma_{\bar{a} u}+\gamma_{\bar{a} v}\right) r_{\bar{a}}(\tau, \vec{\sigma})}\left(\delta_{(b)}^{u} \delta_{(a)}^{v}-\delta_{(a)}^{u} \delta_{(b)}^{v}\right) \times \\
& \sum_{r} \tilde{\mathcal{T}}_{(a) r}^{u}\left(\vec{\sigma}, \vec{\sigma}_{1}, \tau \mid \phi, r_{\bar{a}}\right]\left(\phi^{-2} e^{-\frac{1}{\sqrt{3}} \sum_{\bar{a}} \gamma_{\bar{a} r} r_{\bar{a}}}\left[\frac{\rho}{3}+\sqrt{3} \sum_{\bar{b}} \gamma_{\bar{b} r} \pi_{\bar{b}}\right]\right)\left(\tau, \vec{\sigma}_{1}\right) \\
& \left.\left.\left.\sum_{s} \tilde{\mathcal{T}}_{(b) s}^{v}\left(\vec{\sigma}, \vec{\sigma}_{2}, \tau \mid \phi, r_{\bar{a}}\right]\left(\phi^{-2} e^{-\frac{1}{\sqrt{3}} \sum_{\bar{a}} \gamma_{\bar{a} s} r_{\bar{a}}}\left[\frac{\rho}{3}+\sqrt{3} \sum_{\bar{c}} \gamma_{\bar{c} s} \pi_{\bar{c}}\right]\right)\left(\tau, \vec{\sigma}_{2}\right)\right)\right]\right), \\
& \hat{P}_{A D M, R}^{r}=-\int d^{3} \sigma \phi^{-2}(\tau, \vec{\sigma})\left(\phi^{-2}(\tau, \vec{\sigma})\right. \\
& {\left[e^{-\frac{2}{\sqrt{3}} \sum_{\bar{a}} \gamma_{\bar{a} r} r_{\bar{a}}}\left(2 \partial_{r} \ln \phi \rho+\sum_{\bar{b}} \partial_{r} r_{\bar{b}} \pi_{\bar{b}}\right)+\right.} \\
& +2 \sum_{u} e^{-\frac{2}{\sqrt{3}} \sum_{\bar{a}} \gamma_{\bar{a} u} r_{\bar{a}}} \delta_{u}^{r}\left(2 \partial_{u} \ln \phi+\frac{1}{\sqrt{3}} \sum_{\bar{b}} \gamma_{\bar{b} u} \partial_{u} r_{\bar{b}}\right) \\
& \left.\left(\frac{\rho}{3}+\sqrt{3} \sum_{\bar{c}} \gamma_{\bar{c} u} \pi_{\bar{c}}\right)\right](\tau, \vec{\sigma})+ \\
& +\int d^{3} \sigma_{1} \sum_{s}\left[-\sum_{u}\left(e^{\frac{1}{\sqrt{3}} \sum_{\bar{a}}\left(\gamma_{\bar{a} u}-2 \gamma_{\bar{a} r}\right) r_{\bar{a}}}\left(2 \partial_{r} \ln \phi+\frac{1}{\sqrt{3}} \sum_{\bar{b}} \gamma_{\bar{b} u} \partial_{r} r_{\bar{b}}\right)\right)(\tau, \vec{\sigma})\right. \\
& \delta_{(a)}^{u} \tilde{\mathcal{T}}_{(a) s}^{u}\left(\vec{\sigma}, \vec{\sigma}_{1}, \tau \mid \phi, r_{\bar{a}}\right]
\end{aligned}
$$




$$
\begin{aligned}
& +\sum_{u v}\left(e ^ { - \frac { 1 } { \sqrt { 3 } } \sum _ { \overline { a } } \gamma _ { \overline { a } u } r _ { \overline { a } } } \left(\delta_{u}^{r}\left(2 \partial_{v} \ln \phi+\frac{1}{\sqrt{3}} \sum_{\bar{b}} \gamma_{\bar{b} u} \partial_{v} r_{\bar{b}}\right)+\right.\right. \\
& \left.\left.+\delta_{v}^{r}\left(2 \partial_{u} \ln \phi+\frac{1}{\sqrt{3}} \sum_{\bar{b}} \gamma_{\bar{b} v} \partial_{u} r_{\bar{b}}\right)\right)\right)(\tau, \vec{\sigma}) \\
& \left.\left.\delta_{(a)}^{u} \tilde{\mathcal{T}}_{(a) s}^{v}\left(\vec{\sigma}, \vec{\sigma}_{1}, \tau \mid \phi, r_{\bar{a}}\right]\right]\left(\phi^{-2} e^{-\frac{1}{\sqrt{3}} \sum_{\bar{a}} \gamma_{\bar{a} s} r_{\bar{a}}}\left[\frac{\rho}{3}+\sqrt{3} \sum_{\bar{c}} \gamma_{\bar{c} s} \pi_{\bar{c}}\right]\right)\left(\tau, \vec{\sigma}_{1}\right)\right), \\
& \hat{J}_{A D M, R}^{r s}=\int d^{3} \sigma \phi^{-2}(\tau, \vec{\sigma})\left(\phi^{-2}(\tau, \vec{\sigma})\right. \\
& {\left[e ^ { - \frac { 2 } { \sqrt { 3 } } \sum _ { \overline { a } } \gamma _ { \overline { a } } r r _ { \overline { a } } } \left(\sigma^{r}\left(2 \partial_{s} \ln \phi \rho+\sum_{\bar{b}} \partial_{s} r_{\bar{b}} \pi_{\bar{b}}\right)-\right.\right.} \\
& \left.-\sigma^{s}\left(2 \partial_{r} \ln \phi \rho+\sum_{\bar{b}} \partial_{r} r_{\bar{b}} \pi_{\bar{b}}\right)\right)+ \\
& +2 \sum_{u} e^{-\frac{2}{\sqrt{3}} \sum_{\bar{a}} \gamma_{\bar{a} u} r_{\bar{a}}}\left(\sigma^{r} \delta_{u}^{s}-\sigma^{s} \delta_{u}^{r}\right)\left(2 \partial_{u} \ln \phi+\frac{1}{\sqrt{3}} \sum_{\bar{b}} \gamma_{\bar{b} u} \partial_{u} r_{\bar{b}}\right) \\
& \left.\left(\frac{\rho}{3}+\sqrt{3} \sum_{\bar{c}} \gamma_{\bar{c} u} \pi_{\bar{c}}\right)\right](\tau, \vec{\sigma})- \\
& -\int d^{3} \sigma_{1} \sum_{w}\left[\left(-\sigma^{r} \sum_{u}\left(e^{\frac{1}{\sqrt{3}} \sum_{\bar{a}}\left(\gamma_{\bar{a} u}-2 \gamma_{\bar{a} s}\right) r_{\bar{a}}}\left(2 \partial_{s} \ln \phi+\frac{1}{\sqrt{3}} \sum_{\bar{b}} \gamma_{\bar{b} u} \partial_{s} r_{\bar{b}}\right)\right)(\tau, \vec{\sigma})+\right.\right. \\
& \left.+\sigma^{s} \sum_{u}\left(e^{\frac{1}{\sqrt{3}} \sum_{\bar{a}}\left(\gamma_{\bar{a} u}-2 \gamma_{\bar{a} r}\right) r_{\bar{a}}}\left(2 \partial_{r} \ln \phi+\frac{1}{\sqrt{3}} \sum_{\bar{b}} \gamma_{\bar{b} u} \partial_{r} r_{\bar{b}}\right)\right)(\tau, \vec{\sigma})\right)+ \\
& \delta_{(a)}^{u} \tilde{\mathcal{T}}_{(a) w}^{u}\left(\vec{\sigma}, \vec{\sigma}_{1}, \tau \mid \phi, r_{\bar{a}}\right] \\
& +\sum_{u v}\left(e ^ { - \frac { 1 } { \sqrt { 3 } } \sum _ { \overline { a } } \gamma _ { \overline { a } u } r _ { \overline { a } } } \left(\left(\sigma^{r} \delta_{u}^{s}-\sigma^{s} \delta_{u}^{r}\right)\left(2 \partial_{v} \ln \phi+\frac{1}{\sqrt{3}} \sum_{\bar{b}} \gamma_{\bar{b} u} \partial_{v} r_{\bar{b}}\right)+\right.\right. \\
& \left.\left.+\left(\sigma^{r} \delta_{v}^{s}-\sigma^{s} \delta_{v}^{r}\right)\left(2 \partial_{u} \ln \phi+\frac{1}{\sqrt{3}} \sum_{\bar{b}} \gamma_{\bar{b} v} \partial_{u} r_{\bar{b}}\right)\right)\right)(\tau, \vec{\sigma}) \\
& \left.\left.\delta_{(a)}^{u} \tilde{\mathcal{T}}_{(a) w}^{v}\left(\vec{\sigma}, \vec{\sigma}_{1}, \tau \mid \phi, r_{\bar{a}}\right]\right]\left(\phi^{-2} e^{-\frac{1}{\sqrt{3}} \sum_{\bar{a}} \gamma_{\bar{a} s} r_{\bar{a}}}\left[\frac{\rho}{3}+\sqrt{3} \sum_{\bar{c}} \gamma_{\bar{c} s} \pi_{\bar{c}}\right]\right)\left(\tau, \vec{\sigma}_{1}\right)\right), \\
& \hat{J}_{A D M, R}^{\tau r}=\epsilon \int d^{3} \sigma \sigma^{r}\left(\frac { c ^ { 3 } } { 1 6 \pi G } \left[\phi^{2} \sum_{r} e^{-\frac{2}{\sqrt{3}} \sum_{\bar{a}} \gamma_{\bar{a} r} r_{\bar{a}}} \times\right.\right. \\
& \left(8\left(\partial_{r} \ln \phi\right)^{2}-\frac{1}{3} \sum_{\bar{b}}\left(\partial_{r} r_{\bar{b}}\right)^{2}-\right. \\
& \left.\left.-\frac{4}{\sqrt{3}} \partial_{r} \ln \phi \sum_{\bar{b}} \gamma_{\bar{b} r} \partial_{r} r_{\bar{b}}+\frac{2}{3}\left(\sum_{\bar{b}} \gamma_{\bar{b} r} \partial_{r} r_{\bar{b}}\right)^{2}\right)\right](\tau, \vec{\sigma})- \\
& -\frac{\phi^{-2}(\tau, \vec{\sigma})}{8 k}\left[\left(\phi^{-4}\left[6 \sum_{\bar{a}} \pi_{\bar{a}}^{2}-\frac{1}{3} \rho^{2}\right]\right)(\tau, \vec{\sigma})+\right. \\
& +2\left(\phi^{-2} \sum_{u} e^{\frac{1}{\sqrt{3}} \sum_{\bar{a}} \gamma_{\bar{a} u} u \bar{a}}\left[2 \sqrt{3} \sum_{\bar{b}} \gamma_{\bar{b} u} \pi_{\bar{b}}-\frac{1}{3} \rho\right]\right)(\tau, \vec{\sigma}) \times \\
& \int d^{3} \sigma_{1} \sum_{r} \delta_{(a)}^{u} \tilde{\mathcal{T}}_{(a) r}^{u}\left(\vec{\sigma}, \vec{\sigma}_{1}, \tau \mid \phi, r_{\bar{a}}\right]\left(\phi^{-2} e^{-\frac{1}{\sqrt{3}} \sum_{\bar{a}} \gamma_{\bar{a}} r r_{\bar{a}}}\left[\frac{\rho}{3}+\sqrt{3} \sum_{\bar{b}} \gamma_{\bar{b} r} \pi_{\bar{b}}\right]\right)\left(\tau, \vec{\sigma}_{1}\right)+
\end{aligned}
$$




$$
\begin{aligned}
& +\int d^{3} \sigma_{1} d^{3} \sigma_{2}\left(\sum_{u} e^{\frac{2}{\sqrt{3}} \sum_{\bar{a}} \gamma_{\bar{a} u} r_{\bar{a}}(\tau, \vec{\sigma})} \times\right. \\
& \sum_{r} \tilde{\mathcal{T}}_{(a) r}^{u}\left(\vec{\sigma}, \vec{\sigma}_{1}, \tau \mid \phi, r_{\bar{a}}\right]\left(\phi^{-2} e^{-\frac{1}{\sqrt{3}} \sum_{\bar{a}} \gamma_{\bar{a} r} r_{\bar{a}}}\left[\frac{\rho}{3}+\sqrt{3} \sum_{\bar{b}} \gamma_{\bar{b} r} \pi_{\bar{b}}\right]\right)\left(\tau, \vec{\sigma}_{1}\right) \times \\
& \sum_{s} \tilde{\mathcal{T}}_{(a) s}^{u}\left(\vec{\sigma}, \vec{\sigma}_{2}, \tau \mid \phi, r_{\bar{a}}\right]\left(\phi^{-2} e^{-\frac{1}{\sqrt{3}} \sum_{\bar{a}} \gamma_{\bar{a} s} r_{\bar{a}}}\left[\frac{\rho}{3}+\sqrt{3} \sum_{\bar{c}} \gamma_{\bar{c} s} \pi_{\bar{c}}\right]\right)\left(\tau, \vec{\sigma}_{2}\right)+ \\
& +\sum_{u v} e^{\frac{1}{\sqrt{3}} \sum_{\bar{a}}\left(\gamma_{\bar{a} u}+\gamma_{\bar{a} v}\right) r_{\bar{a}}(\tau, \vec{\sigma})}\left(\delta_{(b)}^{u} \delta_{(a)}^{v}-\delta_{(a)}^{u} \delta_{(b)}^{v}\right) \times \\
& \sum_{r} \tilde{\mathcal{T}}_{(a) r}^{u}\left(\vec{\sigma}, \vec{\sigma}_{1}, \tau \mid \phi, r_{\bar{a}}\right]\left(\phi^{-2} e^{-\frac{1}{\sqrt{3}} \sum_{\bar{a}} \gamma_{\bar{a} r} r_{\bar{a}}}\left[\frac{\rho}{3}+\sqrt{3} \sum_{\bar{b}} \gamma_{\bar{b} r} \pi_{\bar{b}}\right]\right)\left(\tau, \vec{\sigma}_{1}\right) \\
& \left.\left.\left.\sum_{s} \tilde{\mathcal{T}}_{(b) s}^{v}\left(\vec{\sigma}, \vec{\sigma}_{2}, \tau \mid \phi, r_{\bar{a}}\right]\left(\phi^{-2} e^{-\frac{1}{\sqrt{3}} \sum_{\bar{a}} \gamma_{\bar{a} s} r_{\bar{a}}}\left[\frac{\rho}{3}+\sqrt{3} \sum_{\bar{c}} \gamma_{\bar{c} s} \pi_{\bar{c}}\right]\right)\left(\tau, \vec{\sigma}_{2}\right)\right)\right]\right)- \\
& -\frac{\epsilon c^{3}}{8 \pi G} \int d^{3} \sigma\left[\phi^{-2} \sum_{u v} \delta_{u}^{r}\left(\delta_{u v}-1\right)\left(\phi^{4} e^{\frac{2}{\sqrt{3}} \sum_{\bar{a}} \gamma_{\bar{a} u} r_{\bar{a}}}-1\right)\right. \\
& \left.e^{-\frac{2}{\sqrt{3}} \sum_{\bar{a}}\left(\gamma_{\bar{a} v}-\gamma_{\bar{a} u}\right) r_{\bar{a}}}\left(\partial_{u} \ln \phi+\frac{1}{\sqrt{3}} \sum_{\bar{b}}\left(\gamma_{\bar{b} v}-\gamma_{\bar{b} u}\right) \partial_{u} r_{\bar{b}}\right)\right](\tau, \vec{\sigma}),
\end{aligned}
$$

while the strong Poincaré charges of Eq.(2.23) are

$$
\begin{aligned}
& P_{A D M, R}^{\tau}=\hat{P}_{A D M, R}^{\tau}+\int d^{3} \sigma \hat{\mathcal{H}}_{R}(\tau, \vec{\sigma})= \\
& =\frac{\epsilon c^{3}}{8 \pi G} \sum_{u} \int_{S_{\tau, \infty}^{2}} d^{2} \Sigma_{u}\left(\phi^{-2} e^{-\frac{2}{\sqrt{3}} \sum_{\bar{a}} \gamma_{\bar{a} u} r_{\bar{a}}}\left[-4 \partial_{u} \ln \phi+\frac{1}{\sqrt{3}} \sum_{\bar{b}} \gamma_{\bar{b} u} \partial_{u} r_{\bar{b}}\right]\right)(\tau, \vec{\sigma}), \\
& P_{A D M, R}^{r}=\hat{P}_{A D M, R}^{r}+\int d^{3} \sigma \hat{\mathcal{H}}^{r}(\tau, \vec{\sigma}) \equiv \hat{P}_{A D M, R}^{r}= \\
& =-\int_{S_{\tau, \infty}^{2}} d^{2} \Sigma_{r}\left[\phi^{-4} e^{-\frac{2}{\sqrt{3}} \sum_{\bar{a}} \gamma_{\bar{a} r} r_{\bar{a}}}\left[\frac{\rho}{3}+\sqrt{3} \sum_{\bar{c}} \gamma_{\bar{c} r} \pi_{\bar{c}}\right]\right](\tau, \vec{\sigma})- \\
& -\frac{1}{2} \sum_{u} \int_{S_{\tau, \infty}^{2}} d^{2} \Sigma_{u} \phi(\tau, \vec{\sigma}) \sum_{v} \int d^{3} \sigma_{1}\left(e^{-\frac{1}{\sqrt{3}} \sum_{\bar{a}} \gamma_{\bar{a} r} r_{\bar{a}}(\tau, \vec{\sigma})} \delta_{(a)}^{r} \tilde{\mathcal{T}}_{(a) v}^{u}+\right. \\
& \left.+e^{-\frac{1}{\sqrt{3}} \sum_{\bar{a}} \gamma_{\bar{a} u} r_{\bar{a}}(\tau, \vec{\sigma})} \delta_{(a)}^{u} \tilde{\mathcal{T}}_{(a) v}^{r}\right)\left(\vec{\sigma}, \vec{\sigma}_{1}, \tau \mid \phi, r_{\bar{a}}\right] \\
& \left(\phi^{-2} e^{-\frac{1}{\sqrt{3}} \sum_{\bar{a}} \gamma_{\bar{a} v} r_{\bar{a}}}\left[\frac{\rho}{3}+\sqrt{3} \sum_{\bar{c}} \gamma_{\bar{c} v} \pi_{\bar{c}}\right]\right)\left(\tau, \vec{\sigma}_{1}\right), \\
& J_{A D M, R}^{r s}=\hat{J}_{A D M, R}^{r s}+\frac{1}{4} \int d^{3} \sigma\left[\sigma^{s} \hat{\mathcal{H}}^{r}-\sigma^{r} \hat{\mathcal{H}}^{s}\right](\tau, \vec{\sigma})= \\
& =-\frac{1}{2} \sum_{u} \int_{S_{\tau, \infty}^{2}} d^{2} \Sigma_{u} \phi^{-4}(\tau, \vec{\sigma}) \\
& \left(\delta_{u}^{r} \sigma^{s}\left[e^{-\frac{1}{\sqrt{3}} \sum_{\bar{a}} \gamma_{\bar{a} r} r_{\bar{a}}}\left(\frac{\rho}{3}+\sqrt{3} \sum_{\bar{b}} \gamma_{\bar{b} r} \pi_{\bar{b}}\right)\right]-\right. \\
& \left.-\delta_{u}^{s} \sigma^{r}\left[e^{-\frac{1}{\sqrt{3}} \sum_{\bar{a}} \gamma_{\bar{a} s} r_{\bar{a}}}\left(\frac{\rho}{3}+\sqrt{3} \sum_{\bar{b}} \gamma_{\bar{b} s} \pi_{\bar{b}}\right)\right]\right)(\tau, \vec{\sigma})- \\
& -\frac{1}{4} \sum_{u} \int_{S_{\tau, \infty}^{2}} d^{2} \Sigma_{u} \phi^{-2}(\tau, \vec{\sigma}) \sum_{v} \int d^{3} \sigma_{1}
\end{aligned}
$$




$$
\begin{aligned}
& {\left[\sigma^{s}\left(e^{-\frac{1}{\sqrt{3}} \sum_{\bar{a}} \gamma_{\bar{a}} r \overline{\bar{a}}(\tau, \vec{\sigma})} \delta_{r(a)} \tilde{\mathcal{T}}_{(a) v}^{u}+e^{-\frac{1}{\sqrt{3}} \sum_{\bar{a}} \gamma_{\bar{a} u} r_{\bar{a}}(\tau, \vec{\sigma})} \delta_{u(a)} \tilde{\mathcal{T}}_{(a) v}^{r}\right)-\right.} \\
& \left.-\sigma^{r}\left(e^{-\frac{1}{\sqrt{3}} \sum_{\bar{a}} \gamma_{\bar{a} s} r_{\bar{a}}(\tau, \vec{\sigma})} \delta_{s(a)} \tilde{\mathcal{T}}_{(a) v}^{u}+e^{-\frac{1}{\sqrt{3}} \sum_{\bar{a}} \gamma_{\bar{a} u} r_{\bar{a}}(\tau, \vec{\sigma})} \delta_{u(a)} \tilde{\mathcal{T}}_{(a) v}^{s}\right)\right] \\
& \left(\vec{\sigma}, \vec{\sigma}_{1}, \tau \mid \phi, r_{\bar{a}}\right]\left(\phi^{-2} e^{-\frac{1}{\sqrt{3}} \sum_{\bar{a}} \gamma_{\bar{a} v} r_{\bar{a}}}\left[\frac{\rho}{3}+\sqrt{3} \sum_{\bar{c}} \gamma_{\bar{c} v} \pi_{\bar{c}}\right]\left(\tau, \vec{\sigma}_{1}\right)\right\}, \\
& J_{A D M, R}^{\tau r}=\hat{J}_{A D M, R}^{\tau r}+\frac{1}{2} \int d^{3} \sigma \sigma^{r} \hat{\mathcal{H}}_{R}(\tau, \vec{\sigma})= \\
& =\frac{\epsilon c^{3}}{8 \pi G} \sum_{u} \int_{S_{\tau, \infty}^{2}} d^{2} \Sigma_{u} \sigma^{r}\left(\phi^{2} e^{-\frac{2}{\sqrt{3}} \sum_{\bar{a}} \gamma_{\bar{a} u} r_{\bar{a}}}\left[-4 \partial_{u} \ln \phi+\frac{1}{\sqrt{3}} \sum_{\bar{b}} \gamma_{\bar{b} u} \partial_{u} r_{\bar{b}}\right]\right)(\tau, \vec{\sigma})+ \\
& +\frac{\epsilon C^{3}}{16 \pi G} \int_{S_{\tau, \infty}^{2}} d^{2} \Sigma_{r}\left(\phi^{-2} \sum_{s} e^{-\frac{2}{\sqrt{3}} \sum_{\bar{a}}\left(\gamma_{\bar{a}}+\gamma_{\bar{a} s}\right) r_{\bar{a}}}\left(\phi^{4} e^{\frac{2}{\sqrt{3}} \sum_{\bar{a}} \gamma_{\bar{a} s} r_{\bar{a}}}-1\right)\left(1-\delta_{s}^{r}\right)\right)(\tau, \vec{\sigma}) .
\end{aligned}
$$

From Eqs.(B1) evaluated with the gauge fixing $\rho(\tau, \vec{\sigma}) \approx 0$, we get the weak ADM Poincaré charges in this special 3-orthogonal gauge

$$
\begin{aligned}
\hat{P}_{A D M, R}^{\tau}= & \epsilon d^{3} \sigma\left(\frac { c ^ { 3 } } { 1 6 \pi G } \left[\phi^{2} \sum_{r} e^{-\frac{2}{\sqrt{3}} \sum_{\bar{a}} \gamma_{\bar{a}} r_{\bar{a}}} \times\right.\right. \\
& \left(8\left(\partial_{r} l n \phi\right)^{2}-\frac{1}{3} \sum_{\bar{b}}\left(\partial_{r} r_{\bar{b}}\right)^{2}-\right. \\
- & \left.\left.\frac{4}{\sqrt{3}} \partial_{r} l n \phi \sum_{\bar{b}} \gamma_{\bar{b} r} \partial_{r} r_{\bar{b}}+\frac{2}{3}\left(\sum_{\bar{b}} \gamma_{\bar{b} r} \partial_{r} r_{\bar{b}}\right)^{2}\right)\right](\tau, \vec{\sigma})- \\
- & \frac{6 \pi G}{c^{3}} \phi^{-2}(\tau, \vec{\sigma})\left[2\left(\phi^{-4} \sum_{\bar{a}} \pi_{\bar{a}}^{2}\right)(\tau, \vec{\sigma})+\right. \\
+ & 4\left(\phi^{-2} \sum_{u} e^{\frac{1}{\sqrt{3}} \sum_{\bar{a}} \gamma_{\bar{a} u} r_{\bar{a}}} \sum_{\bar{b}} \gamma_{\bar{b} u} \pi_{\bar{b}}\right)(\tau, \vec{\sigma}) \times \\
& \int d^{3} \sigma_{1} \sum_{r} \delta_{(a)}^{u} \tilde{\mathcal{T}}_{(a) r}^{u}\left(\vec{\sigma}, \vec{\sigma}_{1}, \tau \mid \phi, r_{\bar{a}}\right]\left(\phi^{-2} e^{-\frac{1}{\sqrt{3}} \sum_{\bar{a}} \gamma_{\bar{a}} r r_{\bar{a}}} \sum_{\bar{b}} \gamma_{\bar{b} r} \pi_{\bar{b}}\right)\left(\tau, \vec{\sigma}_{1}\right)+ \\
+ & \int d^{3} \sigma_{1} d^{3} \sigma_{2}\left(\sum_{u} e^{\frac{2}{\sqrt{3}} \sum_{\bar{a}} \gamma_{\bar{a} u} r_{\bar{a}}(\tau, \vec{\sigma})} \times\right. \\
& \sum_{r} \tilde{\mathcal{T}}_{(a) r}^{u}\left(\vec{\sigma}, \vec{\sigma}_{1}, \tau \mid \phi, r_{\bar{a}}\right]\left(\phi^{-2} e^{-\frac{1}{\sqrt{3}} \sum_{\bar{a}} \gamma_{\bar{a} r} r_{\bar{a}}} \sum_{\bar{b}} \gamma_{\bar{b} r} \pi_{\bar{b}}\right)\left(\tau, \vec{\sigma}_{1}\right) \times \\
& \sum_{s} \tilde{\mathcal{T}}_{(a) s}^{u}\left(\vec{\sigma}, \vec{\sigma}_{2}, \tau \mid \phi, r_{\bar{a}}\right]\left(\phi^{-2} e^{-\frac{1}{\sqrt{3}} \sum_{\bar{a}} \gamma_{\bar{a} s} r_{\bar{a}}} \sum_{\bar{c}} \gamma_{\bar{c} s} \pi_{\bar{c}}\right)\left(\tau, \vec{\sigma}_{2}\right)+ \\
+ & \sum_{u v} e^{\frac{1}{\sqrt{3}} \sum_{\bar{a}}\left(\gamma_{\bar{a} u}+\gamma_{\bar{a} v}\right) r_{\bar{a}}(\tau, \vec{\sigma})}\left(\delta_{(b)}^{u} \delta_{(a)}^{v}-\delta_{(a)}^{u} \delta_{(b)}^{v}\right) \times \\
& \sum_{r} \tilde{\mathcal{T}}_{(a) r}^{u}\left(\vec{\sigma}, \vec{\sigma}_{1}, \tau \mid \phi, r_{\bar{a}}\right]\left(\phi^{-2} e^{-\frac{1}{\sqrt{3}} \sum_{\bar{a}} \gamma_{\bar{a}} r_{\bar{a}}} \sum_{\bar{b}} \gamma_{\bar{b} r} \pi_{\bar{b}}\right)\left(\tau, \vec{\sigma}_{1}\right) \\
& \left.\left.\left.\sum_{s} \tilde{\mathcal{T}}_{(b) s}^{v}\left(\vec{\sigma}, \vec{\sigma}_{2}, \tau \mid \phi, r_{\bar{a}}\right]\left(\phi^{-2} e^{-\frac{1}{\sqrt{3}} \sum_{\bar{a}} \gamma_{\bar{a} s} r_{\bar{a}}} \sum_{\bar{c}} \gamma_{\bar{c} s} \pi_{\bar{c}}\right)\left(\tau, \vec{\sigma}_{2}\right)\right)\right]\right), \\
\hat{P}_{A D M, R}^{r}= & -\int d^{3} \sigma \phi^{-2}(\tau, \vec{\sigma})\left(\phi^{-2}(\tau, \vec{\sigma})\right. \\
&
\end{aligned}
$$




$$
\begin{aligned}
& {\left[e^{-\frac{2}{\sqrt{3}} \sum_{\bar{a}} \gamma_{\bar{a} r} r_{\bar{a}}} \sum_{\bar{b}} \partial_{r} r_{\bar{b}} \pi_{\bar{b}}+\right.} \\
& +2 \sqrt{3} e^{-\frac{2}{\sqrt{3}} \sum_{\bar{a}} \gamma_{\bar{a} r} r_{\bar{a}}}\left(2 \partial_{r} \ln \phi+\frac{1}{\sqrt{3}} \sum_{\bar{b}} \gamma_{\bar{b} r} \partial_{r} r_{\bar{b}}\right) \\
& \left.\sum_{\bar{c}} \gamma_{\bar{c} r} \pi_{\bar{c}}\right](\tau, \vec{\sigma})+ \\
& +\sqrt{3} \int d^{3} \sigma_{1} \sum_{s}\left[-\sum_{u}\left(e^{\frac{1}{\sqrt{3}} \sum_{\bar{a}}\left(\gamma_{\bar{a} u}-2 \gamma_{\bar{a} r}\right) r_{\bar{a}}}\left(2 \partial_{r} \ln \phi+\frac{1}{\sqrt{3}} \sum_{\bar{b}} \gamma_{\bar{b} u} \partial_{r} r_{\bar{b}}\right)\right)(\tau, \vec{\sigma})\right. \\
& \delta_{(a)}^{u} \tilde{\mathcal{T}}_{(a) s}^{u}\left(\vec{\sigma}, \vec{\sigma}_{1}, \tau \mid \phi, r_{\bar{a}}\right] \\
& +\sum_{u v}\left(e ^ { - \frac { 1 } { \sqrt { 3 } } \sum _ { \overline { a } } \gamma _ { \overline { a } u } r _ { \overline { a } } } \left(\delta_{u}^{r}\left(2 \partial_{v} \ln \phi+\frac{1}{\sqrt{3}} \sum_{\bar{b}} \gamma_{\bar{b} u} \partial_{v} r_{\bar{b}}\right)+\right.\right. \\
& \left.\left.+\delta_{v}^{r}\left(2 \partial_{u} \ln \phi+\frac{1}{\sqrt{3}} \sum_{\bar{b}} \gamma_{\bar{b} v} \partial_{u} r_{\bar{b}}\right)\right)\right)(\tau, \vec{\sigma}) \\
& \left.\delta_{(a)}^{u} \tilde{\mathcal{T}}_{(a) s}^{v}\left(\vec{\sigma}, \vec{\sigma}_{1}, \tau \mid \phi, r_{\bar{a}}\right]\right] \\
& \left.\left(\phi^{-2} e^{-\frac{1}{\sqrt{3}} \sum_{\bar{a}} \gamma_{\bar{a} s} r_{\bar{a}}} \sum_{\bar{c}} \gamma_{\bar{c} s} \pi_{\bar{c}}\right)\left(\tau, \vec{\sigma}_{1}\right)\right), \\
& \hat{J}_{A D M, R}^{r s}=\int d^{3} \sigma \phi^{-2}(\tau, \vec{\sigma})\left(\phi^{-2}(\tau, \vec{\sigma})\right. \\
& {\left[e^{-\frac{2}{\sqrt{3}} \sum_{\bar{a}} \gamma_{\bar{a} r} r_{\bar{a}}} \sum_{\bar{b}}\left(\sigma^{r} \partial_{s}-\sigma^{s} \partial_{r}\right) r_{\bar{b}} \pi_{\bar{b}}\right)+} \\
& \left.+2 \sqrt{3} \sum_{u} e^{-\frac{2}{\sqrt{3}} \sum_{\bar{a}} \gamma_{\bar{a} u} r_{\bar{a}}}\left(\sigma^{r} \delta_{u}^{s}-\sigma^{s} \delta_{u}^{r}\right)\left(2 \partial_{u} \ln \phi+\frac{1}{\sqrt{3}} \sum_{\bar{b}} \gamma_{\bar{b} u} \partial_{u} r_{\bar{b}}\right) \sum_{\bar{c}} \gamma_{\bar{c} u} \pi_{\bar{c}}\right](\tau, \vec{\sigma})+ \\
& +\sqrt{3} \int d^{3} \sigma_{1} \sum_{w}\left[\left(-\sigma^{r} \sum_{u}\left(e^{\frac{1}{\sqrt{3}} \sum_{\bar{a}}\left(\gamma_{\bar{a} u}-2 \gamma_{\bar{a} s}\right) r_{\bar{a}}}\left(2 \partial_{s} \ln \phi+\frac{1}{\sqrt{3}} \sum_{\bar{b}} \gamma_{\bar{b} u} \partial_{s} r_{\bar{b}}\right)\right)(\tau, \vec{\sigma})-\right.\right. \\
& \left.-\sigma^{s} \sum_{u}\left(e^{\frac{1}{\sqrt{3}} \sum_{\bar{a}}\left(\gamma_{\bar{a} u}-2 \gamma_{\bar{a} r}\right) r_{\bar{a}}}\left(2 \partial_{r} \ln \phi+\frac{1}{\sqrt{3}} \sum_{\bar{b}} \gamma_{\bar{b} u} \partial_{r} r_{\bar{b}}\right)\right)(\tau, \vec{\sigma})\right) \\
& \delta_{(a)}^{u} \tilde{\mathcal{T}}_{(a) w}^{u}\left(\vec{\sigma}, \vec{\sigma}_{1}, \tau \mid \phi, r_{\bar{a}}\right]- \\
& -\sum_{u v}\left(e ^ { - \frac { 1 } { \sqrt { 3 } } \sum _ { \overline { a } } \gamma _ { \overline { a } u } r _ { \overline { a } } } \left(\left(\sigma^{r} \delta_{u}^{s}-\sigma^{s} \delta_{u}^{r}\right)\left(2 \partial_{v} \ln \phi+\frac{1}{\sqrt{3}} \sum_{\bar{b}} \gamma_{\bar{b} u} \partial_{v} r_{\bar{b}}\right)+\right.\right. \\
& \left.\left.+\left(\sigma^{r} \delta_{v}^{s}-\sigma^{s} \delta_{v}^{r}\right)\left(2 \partial_{u} \ln \phi+\frac{1}{\sqrt{3}} \sum_{\bar{b}} \gamma_{\bar{b} v} \partial_{u} r_{\bar{b}}\right)\right)\right)(\tau, \vec{\sigma}) \\
& \left.\delta_{(a)}^{u} \tilde{\mathcal{T}}_{(a) w}^{v}\left(\vec{\sigma}, \vec{\sigma}_{1}, \tau \mid \phi, r_{\bar{a}}\right]\right] \\
& \left.\left(\phi^{-2} e^{-\frac{1}{\sqrt{3}} \sum_{\bar{a}} \gamma_{\bar{a} s} r_{\bar{a}}} \sum_{\bar{c}} \gamma_{\bar{c} s} \pi_{\bar{c}}\right)\left(\tau, \vec{\sigma}_{1}\right)\right), \\
& \hat{J}_{A D M, R}^{\tau r}=\epsilon \int d^{3} \sigma \sigma^{r}\left(\frac { c ^ { 3 } } { 1 6 \pi G } \left[\phi^{2} \sum_{r} e^{-\frac{2}{\sqrt{3}} \sum_{\bar{a}} \gamma_{\bar{a}} r_{\bar{a}}} \times\right.\right. \\
& \left(8\left(\partial_{r} \ln \phi\right)^{2}-\frac{1}{3} \sum_{\bar{b}}\left(\partial_{r} r_{\bar{b}}\right)^{2}-\right.
\end{aligned}
$$




$$
\begin{aligned}
& \left.\left.-\frac{4}{\sqrt{3}} \partial_{r} \phi \sum_{\bar{b}} \gamma_{\bar{b} r} \partial_{r} r_{\bar{b}}+\frac{2}{3}\left(\sum_{\bar{b}} \gamma_{\bar{b} r} \partial_{r} r_{\bar{b}}\right)^{2}\right)\right](\tau, \vec{\sigma})- \\
& -\frac{6 \pi G}{c^{3}} \phi^{-2}(\tau, \vec{\sigma})\left[2\left(\phi^{-4} \sum_{\bar{a}} \pi_{\bar{a}}^{2}\right)(\tau, \vec{\sigma})+\right. \\
& +4\left(\phi^{-2} \sum_{u} e^{\frac{1}{\sqrt{3}} \sum_{\bar{a}} \gamma_{\bar{a} u} r_{\bar{a}}} \sum_{\bar{b}} \gamma_{\bar{b} u} \pi_{\bar{b}}\right)(\tau, \vec{\sigma}) \times \\
& \int d^{3} \sigma_{1} \sum_{r} \delta_{(a)}^{u} \tilde{\mathcal{T}}_{(a) r}^{u}\left(\vec{\sigma}, \vec{\sigma}_{1}, \tau \mid \phi, r_{\bar{a}}\right]\left(\phi^{-2} e^{-\frac{1}{\sqrt{3}} \sum_{\bar{a}} \gamma_{\bar{a} r} r_{\bar{a}}} \sum_{\bar{b}} \gamma_{\bar{b} r} \pi_{\bar{b}}\right)\left(\tau, \vec{\sigma}_{1}\right)+ \\
& +\int d^{3} \sigma_{1} d^{3} \sigma_{2}\left(\sum_{u} e^{\frac{2}{\sqrt{3}} \sum_{\bar{a}} \gamma_{\bar{a} u} r_{\bar{a}}(\tau, \vec{\sigma})} \times\right. \\
& \sum_{r} \tilde{\mathcal{T}}_{(a) r}^{u}\left(\vec{\sigma}, \vec{\sigma}_{1}, \tau \mid \phi, r_{\bar{a}}\right]\left(\phi^{-2} e^{-\frac{1}{\sqrt{3}} \sum_{\bar{a}} \gamma_{\bar{a} r} r_{\bar{a}}} \sum_{\bar{b}} \gamma_{\bar{b} r} \pi_{\bar{b}}\right)\left(\tau, \vec{\sigma}_{1}\right) \times \\
& \sum_{s} \tilde{\mathcal{T}}_{(a) s}^{u}\left(\vec{\sigma}, \vec{\sigma}_{2}, \tau \mid \phi, r_{\bar{a}}\right]\left(\phi^{-2} e^{-\frac{1}{\sqrt{3}} \sum_{\bar{a}} \gamma_{\bar{a} s} r_{\bar{a}}} \sum_{\bar{c}} \gamma_{\bar{c} s} \pi_{\bar{c}}\right)\left(\tau, \vec{\sigma}_{2}\right)+ \\
& +\sum_{u v} e^{\frac{1}{\sqrt{3}} \sum_{\bar{a}}\left(\gamma_{\bar{a} u}+\gamma_{\bar{a} v}\right) r_{\bar{a}}(\tau, \vec{\sigma})}\left(\delta_{(b)}^{u} \delta_{(a)}^{v}-\delta_{(a)}^{u} \delta_{(b)}^{v}\right) \times \\
& \sum_{r} \tilde{\mathcal{T}}_{(a) r}^{u}\left(\vec{\sigma}, \vec{\sigma}_{1}, \tau \mid \phi, r_{\bar{a}}\right]\left(\phi^{-2} e^{-\frac{1}{\sqrt{3}} \sum_{\bar{a}} \gamma_{\bar{a} r} r_{\bar{a}}} \sum_{\bar{b}} \gamma_{\bar{b} r} \pi_{\bar{b}}\right)\left(\tau, \vec{\sigma}_{1}\right) \\
& \left.\left.\left.\sum_{s} \tilde{\mathcal{T}}_{(b) s}^{v}\left(\vec{\sigma}, \vec{\sigma}_{2}, \tau \mid \phi, r_{\bar{a}}\right]\left(\phi^{-2} e^{-\frac{1}{\sqrt{3}} \sum_{\bar{a}} \gamma_{\bar{a} s} r_{\bar{a}}} \sum_{\bar{c}} \gamma_{\bar{c} s} \pi_{\bar{c}}\right)\left(\tau, \vec{\sigma}_{2}\right)\right)\right]\right)- \\
& -\frac{\epsilon c^{3}}{8 \pi G} \int d^{3} \sigma\left[\phi^{-2} \sum_{u v} \delta_{u}^{r}\left(\delta_{u v}-1\right)\left(\phi^{4} e^{\frac{2}{\sqrt{3}} \sum_{\bar{a}} \gamma_{\bar{a} u} r_{\bar{a}}}-1\right)\right. \\
& \left.e^{-\frac{2}{\sqrt{3}} \sum_{\bar{a}}\left(\gamma_{\bar{a} v}-\gamma_{\bar{a} u}\right) r_{\bar{a}}}\left(\partial_{u} \ln \phi+\frac{1}{\sqrt{3}} \sum_{\bar{b}}\left(\gamma_{\bar{b} v}-\gamma_{\bar{b} u}\right) \partial_{u} r_{\bar{b}}\right)\right](\tau, \vec{\sigma}) .
\end{aligned}
$$

The weak Poincar'e charges of void spacetimes in the 3-orthogonal gauges can be obtained from Eqs.(B1) by putting $r_{\bar{a}}=\pi_{\bar{a}}=0$ (the explicit form of the kernal $\tilde{\mathcal{T}}_{(a) u}^{r}$ is not needed)

$$
\begin{aligned}
\hat{P}_{A D M, R}^{\tau}= & \epsilon \int d^{3} \sigma\left(\frac { 2 \pi G } { 3 c ^ { 3 } } \phi ^ { - 2 } ( \tau , \vec { \sigma } ) \left[\left(\phi^{-4} \rho^{2}\right)(\tau, \vec{\sigma})-\right.\right. \\
- & \frac{2}{3}\left(\phi^{-2} \rho\right)(\tau, \vec{\sigma}) \int d^{3} \sigma_{1} \sum_{n r} \delta_{(a)}^{r} \tilde{\mathcal{T}}_{(a) n}^{r}\left(\vec{\sigma}, \vec{\sigma}_{1}, \tau \mid \phi, 0\right]\left(\phi^{-2} \rho\right)\left(\tau, \vec{\sigma}_{1}\right)- \\
- & \frac{1}{3} \sum_{r s}\left(\delta_{r s} \delta_{(a)(b)}+\delta_{r(b)} \delta_{s(a)}-\delta_{r(a)} \delta_{s(b)}\right) \\
& \int d^{3} \sigma_{1} \sum_{m} \tilde{\mathcal{T}}_{(a) m}^{r}\left(\vec{\sigma}, \vec{\sigma}_{1}, \tau \mid \phi, 0\right]\left(\phi^{-2} \rho\right)\left(\tau, \vec{\sigma}_{1}\right) \\
& \left.\int d^{3} \sigma_{2} \sum_{n} \mathcal{T}_{(b) n}^{s}\left(\vec{\sigma}, \vec{\sigma}_{2}, \tau \mid \phi, 0\right]\left(\phi^{-2} \rho\right)\left(\tau, \vec{\sigma}_{2}\right)\right]+ \\
+ & \left.\frac{c^{3}}{2 \pi G}\left[\phi^{2} \sum_{r}\left(\partial_{r} \ln \phi\right)^{2}\right](\tau, \vec{\sigma})\right) \\
& \rightarrow c_{\rho \rightarrow 0} \frac{\epsilon c^{3}}{2 \pi G} \int d^{3} \sigma\left[\phi^{2} \sum_{r}\left(\partial_{r} \ln \phi\right)^{2}\right](\tau, \vec{\sigma})
\end{aligned}
$$




$$
\begin{aligned}
& \rightarrow_{\phi \rightarrow 1} 0, \\
& \hat{P}_{A D M, R}^{r}=-\frac{2}{3} \int d^{3} \sigma \phi^{-2}(\tau, \vec{\sigma})\left(5\left(\rho \phi^{-2} \partial_{r} \ln \phi\right)(\tau, \vec{\sigma})+\right. \\
& +\sum_{s} \int d^{3} \sigma_{1}\left[-\partial_{r} \ln \phi(\tau, \vec{\sigma}) \sum_{u} \delta_{(a)}^{u} \tilde{\mathcal{T}}_{(a) s}^{u}\left(\vec{\sigma}, \vec{\sigma}_{1}, \tau \mid \phi, 0\right]+\right. \\
& \left.\left.+\left(\delta_{u}^{r} \partial_{v} \ln \phi+\delta_{v}^{r} \partial_{u} \ln \phi\right)(\tau, \vec{\sigma}) \delta_{(a)}^{u} \tilde{\mathcal{T}}_{(a) s}^{v}\left(\vec{\sigma}, \vec{\sigma}_{1}, \tau \mid \phi, 0\right]\right]\left(\phi^{-2} \rho\right)\left(\tau, \vec{\sigma}_{1}\right)\right) \\
& \rightarrow_{\rho \rightarrow 0, \phi \rightarrow 1} 0 \text {, } \\
& \hat{J}_{A D M, R}^{r s}=\frac{10}{3} \int d^{3} \sigma\left[\phi^{-4} \rho\right](\tau, \vec{\sigma})\left[\sigma^{r} \partial_{s} \ln \phi-\sigma^{s} \partial_{r} \ln \phi\right](\tau, \vec{\sigma})+ \\
& +\frac{2}{3} \sum_{w} \int d^{3} \sigma d^{3} \sigma_{1} \phi^{-2}(\tau, \vec{\sigma}) \\
& {\left[\left(\sigma^{r} \partial_{s} \ln \phi-\sigma^{s} \partial_{r} \ln \phi\right)(\tau, \vec{\sigma}) \sum_{u} \delta_{(a)}^{u} \tilde{\mathcal{T}}_{(a) w}^{u}\left(\vec{\sigma}, \vec{\sigma}_{1}, \tau \mid \phi, 0\right]+\right.} \\
& +\sum_{u v}\left(\left(\sigma^{r} \delta_{u}^{s}-\sigma^{s} \delta_{u}^{r}\right) \partial_{v} \ln \phi+\left(\sigma^{r} \delta_{v}^{s}-\sigma^{s} \delta_{v}^{r}\right) \partial_{u} \ln \phi(\tau, \vec{\sigma})\right. \\
& \left.\delta_{(a)}^{u} \tilde{\mathcal{T}}_{(a) w}^{v}\left(\vec{\sigma}, \vec{\sigma}_{1}, \tau \mid \phi, 0\right]\right]\left(\phi^{-2} \rho\right)\left(\tau, \vec{\sigma}_{1}\right) \\
& \rightarrow_{\rho \rightarrow 0, \phi \rightarrow 1} 0 \text {, } \\
& \hat{J}_{A D M, R}^{\tau r}=\epsilon \int d^{3} \sigma \sigma^{r}\left(\frac { 2 \pi G } { 3 c ^ { 3 } } \phi ^ { - 2 } ( \tau , \vec { \sigma } ) \left[\left(\phi^{-4} \rho^{2}\right)(\tau, \vec{\sigma})-\right.\right. \\
& -\frac{2}{3}\left(\phi^{-2} \rho\right)(\tau, \vec{\sigma}) \int d^{3} \sigma_{1} \sum_{n r} \delta_{(a)}^{r} \tilde{\mathcal{T}}_{(a) n}^{r}\left(\vec{\sigma}, \vec{\sigma}_{1}, \tau \mid \phi, 0\right]\left(\phi^{-2} \rho\right)\left(\tau, \vec{\sigma}_{1}\right)- \\
& -\frac{1}{3} \sum_{r s}\left(\delta_{r s} \delta_{(a)(b)}+\delta_{r(b)} \delta_{s(a)}-\delta_{r(a)} \delta_{s(b)}\right) \\
& \int d^{3} \sigma_{1} \sum_{m} \tilde{\mathcal{T}}_{(a) m}^{r}\left(\vec{\sigma}, \vec{\sigma}_{1}, \tau \mid \phi, 0\right]\left(\phi^{-2} \rho\right)\left(\tau, \vec{\sigma}_{1}\right) \\
& \left.\int d^{3} \sigma_{2} \sum_{n} \mathcal{T}_{(b) n}^{s}\left(\vec{\sigma}, \vec{\sigma}_{2}, \tau \mid \phi, 0\right]\left(\phi^{-2} \rho\right)\left(\tau, \vec{\sigma}_{2}\right)\right]+ \\
& \left.+\frac{c^{3}}{2 \pi G}\left[\phi^{2} \sum_{r}\left(\partial_{r} \ln \phi\right)^{2}\right](\tau, \vec{\sigma})\right)+ \\
& +\frac{\epsilon c^{3}}{4 \pi G} \int d^{3} \sigma\left[\phi^{-2}\left(\phi^{4}-1\right) \partial_{r} \ln \phi\right](\tau, \vec{\sigma}) \\
& \rightarrow_{\rho \rightarrow 0} \frac{\epsilon c^{3}}{4 \pi G} \int d^{3} \sigma\left[\phi^{-2}\left(\phi^{4}-1\right) \partial_{r} \ln \phi\right](\tau, \vec{\sigma}) \\
& \rightarrow_{\phi \rightarrow 1} 0 \text {. }
\end{aligned}
$$

Therefore the Poincaré charges vanish in the special 3-orthogonal gauge $\rho(\tau, \vec{\sigma})=0$ by using the solution $\phi(\tau, \vec{\sigma})=1$ of the reduced Lichnerowicz equation in this gauge. Since they 
are gauge invariant, as shown in Section II before Eq.(2.26), they vanish in every gauge. 


\section{REFERENCES}

[1] L.Lusanna and S.Russo, "A New Parametrization for Tetrad Gravity" (gr-qc/0102074).

[2] L.Lusanna and S.Russo, "Tetrad Gravity: I) A New Formulation", Firenze Univ. preprint (gr-qc/9807072).

[3] L.Lusanna and S.Russo, "Tetrad Gravity: II) Dirac's Observables", Firenze Univ. preprint (gr-qc/9807073).

[4] R.De Pietri and L.Lusanna, "Tetrad Gravity III: Asymptotic Poincaré Charges, the Physical Hamiltonian and Void Spacetimes", Firenze Univ. preprint (gr-qc/9909025).

[5] R.Arnowitt, S.Deser and C.W.Misner, Phys.Rev. 117, 1595 (1960); in "Gravitation: an Introduction to Current Research", ed.L.Witten (Wiley, New York, 1962).

[6] L.Lusanna, "The Rest-Frame Instant Form of Metric Gravity", to appear in Gen.Rel. and Grav. (gr-qc/0101048).

[7] P.A.M.Dirac, Can.J.Math. 2, 129 (1950); "Lectures on Quantum Mechanics", Belfer Graduate School of Science, Monographs Series (Yeshiva University, New York, N.Y., 1964).

[8] J.L.Anderson and P.G.Bergmann, Phys.Rev. 83, 1018 (1951). P.G.Bergmann and J.Goldberg, Phys.Rev. 98, 531 (1955).

[9] L.Lusanna, Phys.Rep. 185, 1 (1990); Riv. Nuovo Cimento 14, n.3 (1991), 1; J.Math.Phys. 31, 2126 (1990); J.Math.Phys. 31, 428 (1990). Comtemp.Math. 132, 531 (1992).

[10] L.Lusanna, "Towards a Unified Description of the Four Interactions in Terms of DiracBergmann Observables", invited contribution to the book "Quantum Field Theory: a 20th Century Profile", of the Indian National Science Academy, ed.A.N.Mitra, foreward F.J.Dyson (Hindustan Book Agency, New Delhi, 2000) (hep-th/9907081). "Tetrad Gravity and Dirac's Observables", talk given at the Conf. "Constraint Dynamics and Quantum Gravity 99", Villasimius 1999, eds. V.DeAlfaro, J.E.Nelson, M.Cadoni, M.Cavaglia' and A.T.Filippov, Nucl.Phys. (Proc.Suppl.) B88, 301 (2000) (gr-qc/9912091). "The Rest-Frame Instant Form of Dynamics and Dirac's Observables", talk given at the Int.Workshop "Physical Variables in Gauge Theories", Dubna 1999. "Solving Gauss' Laws and Searching Dirac Observables for the Four Interactions", talk at the "Second Conf. on Constrained Dynamics and Quantum Gravity", S.Margherita Ligure 1996, eds. V.De Alfaro, J.E.Nelson, G.Bandelloni, A.Blasi, M.Cavaglià and A.T.Filippov, Nucl.Phys. (Proc.Suppl.) B57, 13 (1997) (hep-th/9702114). "Unified Description and Canonical Reduction to Dirac's Observables of the Four Interactions", talk at the Int.Workshop "New non Perturbative Methods and Quantization on the Light Cone', Les Houches School 1997, eds. P.Grangé, H.C.Pauli, A.Neveu, S.Pinsky and A.Werner (Springer, Berlin, 1998) (hep-th/9705154). "The Pseudoclassical Relativistic Quark Model in the Rest-Frame Wigner-Covariant Gauge", talk at the Euroconference QCD97, ed. S.Narison, Montpellier 1997, Nucl.Phys. (Proc. Suppl.) B64, 306 (1998).

[11] S.Shanmugadhasan, J.Math.Phys. 14, 677 (1973). L.Lusanna, Int.J.Mod.Phys. A8, 4193 (1993).

[12] M.Chaichian, D.Louis Martinez and L.Lusanna, Ann.Phys.(N.Y.)232, 40 (1994).

[13] K.Kuchar, 1976 J.Math.Phys. 17, 777, 792, 801; 1977 18, 1589.

[14] P.A.M.Dirac, Rev.Mod.Phys. 21 (1949) 392. 
[15] L.Lusanna, Int.J.Mod.Phys. A12, 645 (1997).

[16] H.Crater and L.Lusanna, "The Rest-Frame Darwin Potential from the LienardWiechert Solution in the Radiation Gauge", Firenze univ. preprint 2000, to appear in Ann.Phys.(NY) (hep-th/0001046).

[17] L.Lusanna, 1995 Int.J.Mod.Phys. A10, 3531 and 3675.

[18] C.Møller, 1949 Ann.Inst.H.Poincaré 11, 251; 1957 "The Theory of Relativity" (Oxford Univ.Press, Oxford).

[19] D.Christodoulou and S.Klainerman, "The Global Nonlinear Stability of the Minkowski Space" (Princeton, Princeton, 1993).

[20] M.Nakahara, "Geometry, Topology and Physics" (IOP, Bristol, 1990).

[21] B.O'Neil, "Semi-Riemannian Geometry" (Academic Press, New York, 1983).

[22] D.Bleecker, "Gauge Theory and Variational Principles" (Addison-Wesley, London, 1981).

[23] J.Schwinger, Phys.Rev. 130, 1253 (1963).

[24] G.Longhi and L.Lusanna, Phys.Rev. D34, 3707 (1986).

[25] P.A.M.Dirac, Canad.J.Math. 3, 1 (1951).

[26] T.Regge and C.Teitelboim, Ann.Phys.(N.Y.) 88, 286 (1974).

[27] D.Alba, L.Lusanna and M.Pauri, "Center of Mass and Rotational Kinematics for the Relativistic N-Body Problems in the Rest-Frame Instant Form" (hep-th/0102087) and "Multipolar Expansions for the Relativistic N Body Problem in the Rest-Frame Instant Form (hep-th/0103092).

[28] L.Lusanna and M.Materassi, Int.J.Mod.Phys. A15, 2821 (1999) (hep-th/9904202).

[29] A.J.Hanson and T.Regge, Ann.Phys. (N.Y.) 87, 498 (1974). A.J.Hanson, T.Regge and C.Teitelboim, "Constrained Hamiltonian Systems", in Contributi del Centro Linceo Interdisciplinare di Scienze Matematiche, Fisiche e loro Applicazioni, n.22 (Accademia Nazionale dei Lincei, Roma, 1975).

[30] G.Longhi and M.Materassi, J.Math.Phys. 40, 480 (1999) (hep-th/9803128); Int.J.Mod.Phys. A14, 3397 (1999) (hep-th/9890024).

[31] R.Beig and Ó Murchadha, Ann.Phys.(N.Y.) 174, 463 (1987).

[32] L.Andersson, J.Geom.Phys. 4, 289 (1987).

[33] L.Landau and E.Lifschitz, "The Classical Theory of Fields" (Addison-Wesley, Cambridge, 1951).

[34] S.W.Hawking and G.T.Horowitz, Class.Quantum Grav. 13, 1487 (1996).

[35] B.S.De Witt, Phys.Rev. 160, 1113 (1967).

[36] B.S.De Witt, Phys.Rev. 162, 1195 (1967); "The Dynamical Theory of Groups and Fields" (Gordon and Breach, New York, 1967) and in "Relativity, Groups and Topology", Les Houches 1963, eds. C.De Witt and B.S.De Witt (Gordon and Breach, London, 1964); "The Spacetime Approach to Quantum Field Theory", in "Relativity, Groups and Topology II", Les Houches 1983, eds. B.S.DeWitt and R.Stora (NorthHolland, Amsterdam, 1984). B.S.De Witt and R.W.Brehme, Ann.Phys.(N.Y.) 9, 220 (1960).

[37] D.M.Brill and P.S.Jang, "The Positive Mass Conjecture", in "General Relativity and Gravitation", Vol. 1, ed.A.Held (Plenum, New York, 1980).

[38] H.Stephani, "General Relativity" (Cambridge Univ.Press, Cambridge, 1996).

[39] A.Trautman, in "Gravitation, an Introduction to Current Research", ed.L.Witten (Wi- 
ley, New York, 1962).

[40] V.O.Solov'ev, Theor.Math.Phys, 65, 1240 (1985); Sov.J.Part.Nucl. 19, 482 (1988).

[41] P.G.Bergmann, Rev.Mod.Phys. 33, 510 (1961).

[42] D.Marolf, Class, Quantum Grav, 13, 1871 (1996).

[43] J.Barbour, "General Relativity as a Perfectly Machian Theory", in "Mach's Principle: From Newton's Bucket to Quantum Gravity", eds. J.B.Barbour and H.Pfister, Einstein's Studies n.6 (BirkHäuser, Boston, 1995).

[44] M.Pauri and M.Prosperi, J.Math.Phys. 16, 1503 (1975).

[45] Y.Choquet-Bruhat, A.Fischer and J.E.Marsden, "Maximal Hypersurfaces and Positivity of Mass", LXVII E.Fermi Summer School of Physics "Isolated Gravitating Systems in General Relativity", ed. J.Ehlers (North-Holland, Amsterdam, 1979).

[46] M.H.Soffel, "Relativity in Astrometry, Celestial Mechanics and Geodesy" (Springer, Berlin, 1989).

[47] M.C.Abbati, R.Cirelli, A.Maniá and P.Michor, J.Geom.Phys. 6, 215 (1989).

[48] R.Schmidt, "Infinite Dimensional Hamiltonian Systems" (Bibliopolis, Napoli, 1987). J.Milnor, in "Relativity, Groups and Topology II", Les Houches 1983, (B.S.De Witt and R.Stora, Eds.), Elsevier, Amsterdam, 1984.

[49] D.Bao, J.Isenberg and P.B.Yasskin, Ann.Phys.(N.Y.) 164, 103 (1985).

[50] S.Helgason, "Differential Geometry and Symmetric Spaces" (Academic Press, New York, 1962).

[51] S.Kobayashi and K.Nomizu, "Foundations of Differential Geometry", Vol.I (Interscience, New York, 1963).

[52] A.E.Fischer, "The Theory of Superspace", in "Relativity", eds. M.Carmeli, L.Fickler and L.Witten (Plenum, New York, 1970); Gen.Rel.Grav. 15, 1191 (1983); J.Math.Phys. 27, 718 (1986). M.Rainer, "The Moduli Space of Local Homogeneous 3-Geometries", talk at the Pacific Conf. on Gravitation and Cosmology, Seoul 1996.

[53] S.Timothy Swift, J.Math.Phys. 33, 3723 (1992); 34, 3825 and 3841 (1993).

[54] J.M.Arms, J.E.Marsden and V.Moncrief, Commun.Math.Phys. 78, 455 (1981).

[55] V.Moncrief, J.Math.Phys. 20, 579 (1979). M.Cantor, Bull.Am.Math.Soc. 5, 235 (1981).

[56] H.Cendra, A.Ibort and J.Marsden, J.Geom.Phys. 4, 183 (1987). A.P.Balachandran, G.Marmo, B.S.Skagerstam and A.Stern, "Classical Topology and Quantum States" (World Scientific, Singapore, 1991).

[57] D.Giulini, Helv.Phys.Acta 68, 86 (1995).

[58] J.Lee and R.M.Wald, J.Math.Phys. 31, 725 (1990).

[59] F.Antonsen and F.Markopoulou, "4D Diffeomorphisms in Canonical Gravity and Abelian Deformations", Imperial/TP/96-97/26 (gr-qc/9702046).

[60] C.Teitelboim, "The Hamiltonian Structure of Space-Time", in "General Relativity and Gravitation", ed.A.Held, Vol.I (Plenum, New York, 1980).

[61] K.Kuchar, "Canonical Quantum Gravity" in "General Relativity and Gravitation" Int.Conf. GR13, Cordoba (Argentina) 1992, eds. R.J.Gleiser, C.N.Kozameh and O.M.Moreschi (IOP, Bristol, 1993).

[62] R.Beig, "The Classical Theory of Canonical General Relativity", in "Canonical Gravity: From Classical to Quantum", Bad Honnef 1993, eds. J.Ehlers and H.Friedrich, Lecture Notes Phys. 434 (Springer, Berlin, 1994).

[63] K.Kuchar, Phys.Rev. D4, 955 (1971); J.Math.Phys. 11, 3322 (1970); 13, 768 (1972). 
[64] C.W.Misner, Phys.Rev.Lett. 22, 1071 (1969); Phys.Rev. 186, 1319 and 1328 (1969).

[65] J.W.York jr., "Kinematics and Dynamics of General Relativity", in "Sources of Gravitational Radiation", Battelle-Seattle Workshop 1978, ed.L.L.Smarr (Cambridge Univ.Press, Cambridge, 1979). A.Qadir and J.A.Wheeler, "York's Cosmic Time Versus Proper Time", in "From SU(3) to Gravity", Y.Ne'eman's festschrift, eds. E.Gotsma and G.Tauber (Cambridge Univ.Press, Cambridge, 1985).

[66] C.J.Isham, "Canonical Quantum Gravity and the Problem of Time", in "Integrable Systems, Quantum Groups and Quantum Field Theories", eds.L.A.Ibort and M.A.Rodriguez, Salamanca 1993 (Kluwer, London, 1993); "Conceptual and Geometrical Problems in Quantum Gravity", in "Recent Aspects of Quantum Fields", Schladming 1991, eds. H.Mitter and H.Gausterer (Springer, Berlin, 1991); "Prima Facie Questions in Quantum Gravity" and "Canonical Quantum Gravity and the Question of Time", in "Canonical Gravity: From Classical to Quantum", eds. J.Ehlers and H.Friedrich (Springer, Berlin, 1994).

[67] K.Kuchar, "Time and Interpretations of Quantum Gravity", in Proc.4th Canadian Conf. on "General Relativity and Relativistic Astrophysics", eds. G.Kunstatter, D.Vincent and J.Williams (World Scientific, Singapore, 1992).

[68] K.Kuchar, "Canonical Methods of Quantization", in "Quantum Gravity 2", eds.C.J.Isham, R.Penrose and D.W.Sciama (Clarendon Press, Oxford, 1981).

[69] R.F.Baierlein, D.H.Sharp and J.A.Wheeler, Phys.Rev.126, 1864 (1962).

[70] C.W.Misner, K.S.Thorne and J.A.Wheeler, Gravitation (Freeman, New York, 1973).

[71] R.Parentani, "The Notions of Time and Evolution in Quantum Cosmology", 1997, gr-qc/9710130.

[72] C.Kiefer, "The Semiclassical Approximation to Quantum Gravity" in "Canonical Gravity - from Classical to Quantum", ed.J.Ehlers (Springer, Berlin, 1994). "Semiclassical Gravity and the Problem of Time", in Proc. Cornelius Lanczos Int.Centenary Conf., eds. M.Chu, R.Flemmons, D.Brown and D.Ellison (SIAM, 1994). Nucl.Phys. B475, 339 (1996).

[73] R.Bartnik and G.Fodor, Phys.Rev. D48, 3596 (1993).

[74] D.Giulini, J.Math.Phys. 40, 2470 (1999).

[75] L.Lusanna, Nuovo Cimento, 65B, 135 (1981).

[76] A.Lichnerowicz, J.Math.Pure

Appl.

23 ,

37 (1944). Y.Faures-Bruhat, C.R.Acad.Sci.Paris 226, 1071 (1948); J.Rat.Mech.Anal. 5, 951 (1956); "The Cauchy Problem" in "Gravitation: An Introduction to Current Research", ed.L.Witten (Wiley, New York, 1962).

[77] J.W.York jr, Phys.Rev.Lett. 26, 1656 (1971); 28, 1082 (1972). J.Math.Phys. 13, 125 (1972); 14, 456 (1972). Ann.Ins.H.Poincaré XXI, 318 (1974). N.O'Murchadha and J.W.York jr, J.Math.Phys. 14, 1551 (1972). Phys.Rev. D10, 428 (1974).

[78] Y.Choquet-Bruhat and J.W.York jr., "The Cauchy Problem", in "General Relativity and Gravitation", vol.1, ed. A.Held (Plenum, New York, 1980).

[79] I.Ciufolini and J.A.Wheeler, "Gravitation and Inertia" (Princeton Univ.Press, Princeton, 1995).

[80] R.Schoen and S.T.Yau, Phys.Rev.Lett. 43, 1457 (1979); Commun.Math. Phys. 65, 45 (1979) and 79, 231 (1980). E.Witten, Commun.Math.Phys. 80, 381 (1981). D.M.Brill and P.S.Jang, "The Positive Mass Conjecture", in "General Relativity and Gravi- 
tation", Vol. 1, ed.A.Held (Plenum, New York, 1980). Y.Choquet-Bruhat, "Positive Energy Theorems", in "Relativity, Groups and Topology II", Les Houches XL 1983, eds. B.S.DeWitt and R.Stora (North-Holland, Amsterdam, 1984). G.T.Horowitz, "The Positive Energy Theorem and its Extensions", in "Asymptotic Behaviour of Mass and Spacetime Geometry", ed.F.J.Flaherty, Lecture Notes Phys.202 (Springer, Berlin, 1984). M.J.Perry, "The Positive Mass Theories and Black Holes", in "Asymptotic Behaviour of Mass and Spacetime Geometry", ed.F.J.Flaherty, Lecture Notes Phys.202 (Springer, Berlin, 1984).

[81] J.Isenberg and J.E.Marsden, J.Geom.Phys. 1, 85 (1984).

[82] V.Moncrief, J.Math.Phys. 16, 1556 (1965). J.Arms, A.Fischer and J.E.Marsden, C.R.Acad.Sci.Paris A281, 517 (1975). J.Arms, Acta Phys.Pol. B17, 499 (1986) and Contep.Math. 81, 99 (1988). J.M.Arms, M.J.Gotay anf G.Jennings, Adv.Math. 79, 43 (1990).

[83] J.Isenberg,Phys.Rev.Lett. 59,2389 (1987).

[84] J.Isenberg, Class.Quantum Grav. 12, 2249 (1995).

[85] J.Isenberg and V.Moncrief, Class.Quantum Grav. 13, 1819 (1996).

[86] R.Bartnik, Commun.Math.Phys. 117, 615 (1988). D.Brill, in Proc.Third Marcel Grossman Meeting, ed.H.Ning (North-Holland, Amsterdam,1982).

[87] P.A.M.Dirac, Rev.Mod.Phys. 21, 392 (1949).

[88] R.P.Gaida, Yu.B.Kluchkovsky and V.I.Tretyak, Theor.Math.Phys. 55, 372 (1983); in "Constraint's Theory and Relativistic Dynamics", eds. G.Longhi and L.Lusanna (World Scientific, Singapore, 1987).

[89] L.Lusanna, a) Phys.Rep. 185, 1 (1990). b) Riv. Nuovo Cimento 14, n.3, 1 (1991). c) J.Math.Phys. 31, 428 and 2126 (1990). d) Int.J.Mod.Phys. A8, 4193 (1993). e) Comtemp.Math. 132, 531 (1992).

[90] I.A.Batalin and G.A.Vilkoviski, Nucl.Phys. B 234, 106 (1984).

[91] J.L.Synge, "Relativity: the General Theory" (North-Holland, Amsterdam, 1960).

[92] S.Hwang, Nucl.Phys. B351, 425 (1991).

[93] P.A.M.Dirac, in "Recent Developments in General Relativity", (Pergamon Press, Oxford, and PWN-Polish Scientific Publishers, Warsaw, 1962).

[94] C.J.Isham and K.Kuchar, Ann.Phys.(N.Y.) 164, 288 and 316 (1984). K.Kuchar, Found.Phys. 16, 193 (1986).

[95] E.Cartan, "Lecons sur la Geometrie des Espaces de Riemann", 2nd edn. (GauthierVillars, Paris, 1951).

[96] M.Spivak, "Differential Geometry", vol.2 (Publish or Perish, Boston, 1970).

[97] C.R.Chester, "Techniques in Partial Differential Equations" (McGraw-Hill Kogakusha, Tokyo, 1971).

[98] R.Sugano, Y.Kagraoka and T.Kimura, Int.J.Mod.Phys. A7, 61 (1992).

[99] R.A.d'Inverno and J.Stachel, J.Math.Phys. 19, 2447 (1978). R.d'Inverno, "2+2 Formalism and Applications", in "Relativistic Gravitation and Gravitational Radiation", Les Houches 1995, eds. J.A.Marck and J.P.Lasota (Cambridge Univ.Press, Cambridge, 1997). R.d'Inverno and J.Smallwood, Phys.Rev. D22, 1233 (1980). J.Smallwood, J.Math.Phys. 24, 599 (1983). C.G.Torre, Class.Quantum Grav. 3, 773 (1986). S.A.Hayward, Class.Quantum Grav. 10, 779 (1993).

[100] E.M.Lifshitz and I.M.Khalatnikov, Advan.Phys. 12, 185 (1963). I.M.Khalatnikov and 
E.M.Lifshitz, Phys.Rev.Lett. 24, 76 (1970).

[101] C.Bona, J.Massó, E.Seidel and P.Walker, "Three Dimensional Numerical Relativity with a Hyperbolic Formulation", gr-qc/9804052.

[102] Y.Choquet-Bruhat, J.Isenberg and J.W.York Jr., "Einstein Constraints on Asymptotically Euclidean Manifolds", gr-qc/9906095. A.Anderson, Y.Choquet-Bruhat and J.York Jr., "Einstein's Equations and Equivalent Dynamical Systems", gr-qc/9907099 and "Curvature-Based Hyperbolic Systems for General Relativity", talk at the 8th M.Grossmann Meeting (Jerusalem, Israel, 1997), gr-qc/9802027. A.Anderson and J.W.York Jr., Phys.Rev.Letters 81, 1154 and 4384 (1999). J.W.York Jr., Phys.Rev.Letters 82, 1350 (1999).

[103] K.Kuchar, J.Math.Phys. 13, 768 (1972).

[104] L.Smolin, "The present moment in quantum cosmology: challenges to the arguments for the elimination of time", (gr-qc/0104097).

[105] J.Stewart, "Advanced General Relativity" (Cambridge Univ. Press, Cambridge, 1993).

[106] P.G.Bergmann and A.B.Komar, Phys.Rev.Lett. 4, 432 (1960).

[107] E.Witten, Commun.Math.Phys. 80, 381 (1981).

[108] A.Sen, J.Math.Phys. 22, 1781 (1981); Phys.Lett. 119B, 89 (1982).

[109] A.Sen, Int.J.Theor.Phys. 21, 1 (1982). P.Sommers, J.Math.Phys. 21, 2567 (1980).

[110] A.Ashtekar, "New Perspectives in Canonical Gravity" (Bibliopolis, Napoli, 1988).

[111] R.Penrose and W.Rindler, "Spinors and Space-Time" vol.1 and 2 (Cambridge Univ.Press, Cambridge, 1986).

[112] Y.Choquet-Bruhat and D.Christodoulou, Acta Math. 146, 129 (1981). O.Reula, J.Math.Phys. 23, 810 (1982). O.Reula and K.Todd, J.Math.Phys. 25, 1004 (1984). T.Parker and C.H.Taub, Commun.Math.Phys. 84, 223 (1982). T.Parker, Commun.Math.Phys. 100, 471 (1985). P.Bizón and E.Malec, Class.Quantum Grav. 3, L123 (1986).

[113] J.Frauendiener,Class.Quantum Grav. 8, 1881 (1991).

[114] J.Frauendiener, Class.Quantum Grav. 6, L237 (1989).

[115] A.Einstein, Sitzungsber.Preuss.Akad.Wiss.Phys.Math.Kl. 42, 1111 (1916).

[116] C.Møller, Ann.Phys.(N.Y.) 12, 118 (1961); in Proc.Int. School of Physics E.Fermi, Course XX (Academic Press, New York, 1962).

[117] F.A.E.Pirani, "Gauss' Theorem and Gravitational Energy", in "Les Theories Relativistes de la Gravitation", Proc.Int.Conf. at Royaumont 1959, eds. A.Lichnerowicz and M.A.Tonnelat (CNRS, Paris, 1962).

[118] J.N.Goldberg, Phys.Rev. D37,2116 (1988).

[119] N.Rosen, Phys.Rev. 57, 147 (1940); Ann.Phys.(N.Y.) 22, 1 (1963); Found.Phys. 15, 998 (1986); in "From SU(3) to Gravity", Y.Ne'eman's festschrift, eds.E.Gotsman and G.Tauber (Cambridge Univ.Press, Cambridge, 1985); in "Topological Properties and Global Structure of Space-Time", eds.P.G.Bergmann and V.de Sabbata (Plenum, New York, 1986).

[120] A.Z.Petrov, "Einstein Spaces" (Pergamon, Oxford, 1969).

[121] D.C.Robinson, Class.Quantum Grav. 6, L121 (1989).

[122] I.Bailey and W.Israel, Ann.Phys. (N.Y.) 130, 188 (1980).

[123] W.G.Dixon, J.Math.Phys. 8, 1591 (1967). "Extended Objects in General Relativity: their Description and Motion", in "Isolated Gravitating Systems in General Relativ- 
ity", ed. J.Ehlers (North-Holland, Amsterdam, 1979).

[124] eds. J.Kovalevvsky, I.I.Mueller and B.Kolaczek, "Reference Frames in Astronomy and Geophysics" (Kluwer, Dordrecht, 1989).

[125] W.G.Dixon, Gen.Rel.and Grav. 4, 199 (1973).

[126] B.S.DeWitt and R.W.Brehme, Ann.Phys.(N.Y.) 9, 220 (1960).

[127] J.D.Norton, "What was Einstein's Principle of Equivalence?", in "Einstein and the History of General Relativity: Einstein Studies", Vol. 1, eds. D.Howard and J.Stachel (Birkhäuser, Boston, 1989). Rep.Prog.Phys. 56, 791 (1993).

[128] M.A.Abramowicz, "Inertial Forces in General Relativity", in "The Renaissance of General Relativity and Cosmology", eds. G.Ellis, A.Lanza and J.Miller (Cambridge Univ.Press, Cambridge, 1993). S.Sonego and M.Massar, Class.Quantum Grav. 13, 139 (1996). F.De Felice, Mon.Not.R.Astron.Soc. 252, 197 (1991).

[129] M.Pauri and M.Vallisneri, "Classical Roots of the Unruh and Hawking Effects", Found.Phys. 29, 1499 (1999) (gr-qc/9903052).

[130] L.Lusanna and D.Nowak-Szczepaniak, Int.J.Mod.Phys. A15, 4943 (2000) (hepth/0003095). 